ANL-78-97

ANL-78-97

क) 199

2* DR $2 / 5$

\title{
FX2-TH: A TWO-DIMENSIONAL NUCLEAR REACTOR KINETICS CODE WITH THERMAL-HYDRAULIC FEEDBACK
}

by

R. A. Shober, T. A. Daly, and D. R. Ferguson

BASE TECHNOLOGY

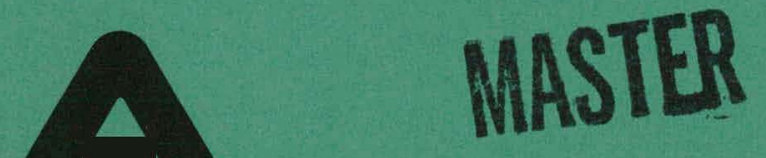

ARGONNE NATIONAL LABORATORY, ARGONNE, ILLINOIS

Prepared for the U. S. DEPARTMENT OF ENERGY

under Contract W-31-109-Eng-38 


\section{DISCLAIMER}

This report was prepared as an account of work sponsored by an agency of the United States Government. Neither the United States Government nor any agency Thereof, nor any of their employees, makes any warranty, express or implied, or assumes any legal liability or responsibility for the accuracy, completeness, or usefulness of any information, apparatus, product, or process disclosed, or represents that its use would not infringe privately owned rights. Reference herein to any specific commercial product, process, or service by trade name, trademark, manufacturer, or otherwise does not necessarily constitute or imply its endorsement, recommendation, or favoring by the United States Government or any agency thereof. The views and opinions of authors expressed herein do not necessarily state or reflect those of the United States Government or any agency thereof. 


\section{DISCLAIMER}

Portions of this document may be illegible in electronic image products. Images are produced from the best available original document. 
The facilities of Argonne National Laboratory are owned by the United States Government. Under the terms of a contract (W-31-109-Eng-38) between the U. S. Department of Energy, Argonne Universities Association and The University of Chicago, the University employs the staff and operates the Laboratory in accordance with policies and programs formulated, approved and reviewed by the Association.

\section{MEMBERS OF ARGONNE UNIVERSITIES ASSOCIATION}

The University of Arizona

Carnegie-Mellon University

Case Western Reserve University

The University of Chicago

University of Cincinnati

Illinois Institute of Technology

University of Illinois

Indiana University

lowa State University

The University of Iowa
Kansas State University

The University of Kansas

Loyola University

Marquette University

Michigan State University

The University of Michigan

University of Minnesota

Universily of Missouri

Northwestern University

University of Notre Dame
The Ohio State University

Ohio University

The Pennsylvania State University

Purdue University

Saint Louis University

Southern Illinois University

The University of Texas at Austin

Washington University

Wayne State University

The University of Wisconsin

NOTICE

This report was prepared as an account of work sponsored by the United States Government. Neither the United States nor the United States Department of Energy, nor any of their employees, nor any of their contractors, subcontractors, or their employees, makes any warranty, express or implied, or assumes any legal liability or responsibility for the accuracy, completeness or usefulness of any information, apparatus, product or process disclosed, or represent.s that. its use would not infringe privately-owned rights. Mention of commercial products, their manufacturers, or their suppliers in this priblication does not imply or connote approval or disapproval of the product by Argonne National Laboratory or the U. S. Department of Energy.

Printed in the United States of America Available from

National Technical Information Service

U. S. Department of Commerce 5285 Port Royal Road

Springfield, Virginia 22161

Price: Printed Copy $\$ 13.00$; Microfiche $\$ 3.00$ 


\title{
ARGONNE NATIONAL LABORATORY \\ 9700 South Cass Avenue \\ Argonne, Illinois 60439
}

\begin{abstract}
FX2-TH: A TWO-DIMENSIONAL NUCLEAR REACTOR KINETICS CODE WITH THERMAL-HYDRAULIC FEEDBACK
\end{abstract}

by

R. A. Shober, T. A. Daly, and D. R. Ferguson*

Applied Physics Division

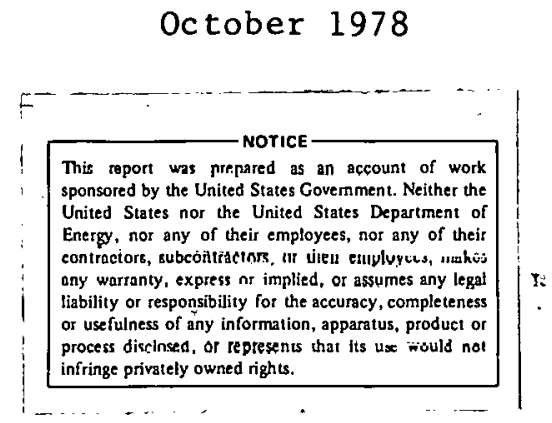

* Reactor Analysis and Safety Division 
THIS PAGE

\section{WAS INTENTIONALLY LEFT BLANK}


TABLE OF CONTENTS

No.

Title

$\underline{\text { Page }}$

ABSTRACT . . . . . . . . . . . . . . . . . . . . . 9

CODE ABSTRACT. . . . . . . . . . . . . . . . . . . . . 9

I. INTRODUCTION. . . . . . . . . . . . . . . . . 12

II. NEUTRONICS EQUATIONS AND THEIR SOLUTION . . . . . . . . . . . . 14

A. The Multigroup Diffusion Equations . . . . . . . . . . . . 14

B. Steady State Solution Strategies . . . . . . . . . . . 16

1. Development of the Steady State Equations . . . . . 16

2. Spatial Discretization. . . . . . . . . . . . 17

3. Outer Iteration Strategies. . . . . . . . . . . 19

4. Inner Iteration Strategies. . . . . . . . . . . . . 20

5. Solution of the Adjoint Flux Equations. . . . . . . 20

C. Time Dependent Solution Strategies . . . . . . . . . . . 21

1. Characteristics of the Time Dependent Problem . . . . . 21

2. Development of the Point Kinetics Equations . . . . . . 22

3. Development of the Quasistatic Method . . . . . . . 26

4. Reactivity Step Calculation Sequence. . . . . . . . 26

a. Reactivity Step Initialization . . . . . . . 26

b. Shape Function Estimation. . . . . . . . . . 27

c. Volume Fraction Calculation. . . . . . . . . 28

d. Point Kinetics Equation Solution . . . . . . . 28

e. Pointwise Precursor Density. . . . . . . . . 29

5. Shape Step Calculation Sequence . . . . . . . . . 30

6. Selection of Time Steps . . . . . . . . . . . . 34

a. Shape Step Selection Criteria. . . . . . . . . . 34

b. Reactivity Step Selection Criteria . . . . . . 35

III. THERMAL-HYDRAULICS EQUATIONS AND THEIR SOLUTION . . . . . . . . . 38

A. The Adiabatic Fuel Temperature Models. . . . . . . . . . 38

1. Zero Heat Transfer Method . . . . . . . . . . . 38

2. Constant Heat Transfer Method . . . . . . . . . . . . 40

3. Calculation of Volumetric Power Generation Rate . . . 40

4. Summary of the Adiabatic Mode1s . . . . . . . . . 41

B. The Detailed Fuel and Coolant Temperature Model. . . . . . 41

1. Derivation of the Fuel Temperature Equations. . . . . 42

2. Solution of the Fuel Temperature Equations. . . . . . 46

3. Derivation of Coolant Temperature Equations . . . . . . 47

4. Steady State and Transient Calculation of Fuel and Coolant Temperatures. . . . . . . . . . . . . 53

5. Calculation of Volumetric Power Generation Rate . . . 56

6. Accuracy Considerations of the Detailed Model . . . . . 56 
TABLE OF CONTENTS

No.

Title

Page

IV. REPRESENTATION OF THERMAL-HYDRAULIC FEEDBACK EFFECTS. • • • . . 58

A. Homogenization of Cross Sections . . . . . . . . . . . 58

B. Temperature Effects Upon Cross Sections. . . . . . . . . 58

C. Overall Solution Strategies for FX2-TH . . . . . . . . 60

1. Steady State Calculation. . . . . . . . . . . 60

2. Time-Dependent Calculation. ............ 63

V. A GUIDE TO USER APPLICATIONS. . . . . . . . . . . . . . . . . 64

A. Description of the Cataloged Procedure ARCSP024. . . . . . . 64

B. Input Data Specifications. . . . . . . . . . . . . . 68

1. Overall Structure of Input Data . . . . . . . . . 68

2. Cross Section Representation. . . . . . . . . . . 70

3. Geometry and Boundary Condition Input . . . . . . . . 72

4. Fixternal Nelitrnn Snurre. . . . . . . . . . . . 72

5. Flux Input. . . . . . . . . . . . . . . . . 72

6. Thermal-Hydraulics Input. . . . . . . . . . 73

C. Computer Core Requirements . . . . . . . . . . . . . . . 74

D. Sample Problems. . . . . . . . . . . . . . 75

E. Érror Messages ...................... 78

F. Restart Capabilities . . . . . . . . . . . . . . 98

1. Bypass Steady-State Calculation . . . . . . . . . 98

2. Transient Restart . . . . . . . . . . . . 98

G. Edits and Output Options . . . . . . . . . . . . . 98

VI. PROGRAMMING INFORMATION . . . . . . . . . . . . . . . . 100

A. Program Structure. . . . . . . . . . . . . . 100

B. Program Flow Charts. . . . . . . . . . . . . . 106

C. Description of Dynamically Allocated Arrays. . . . . . . . 106

1. BPOINTER, $\Lambda$ Dynamic Storage Allocation Program. . . . 106

a. IBM allocation . . . . . . . . . . . . . . . 117

b. CDC allocation................ 119

2. Arrays Permanently In-Core. . . . . . . . . . 119

3. Arrays Which May Be In-Core . ... . . . . . . . . 121

D. Use of Standard Subprograms. . . . . . . . . . . . . 122

1. SEEK. . . . . . . . . . . . . . . . . . 123

2. REED/RITE . . . . . ... . . . . . . . . . 123

3. TIMER ...................... . . 124 
TABLE OF CONTENTS

No.

Title

$\underline{\text { Page }}$

E. Program Implementation . . . . . . . . . . . . . . . 124

1. IBM Code Implementation. . . . . . . . . . . . . . 124

2. CDC Code Implementation. . . . . . . . . . . . . 126

ACKNOWLEDGMENTS . . . . . . . . . . . . . . . . . . . . 128

REFERENCES. . . . . . . . . . . . . . . . . . . . . . . 129 APPENDICES

A. Description of FX2-TH Subroutines. . . . . . . . . . . . . 131

B. Listing of BCD Input files . . . . . . . . . . . . . . . 225

C. Listing of Binary Interface Files. . . . . . . . . . . . . 267

D. Listing of Scratch Files . . . . . . . . . . . . . 303

E. Listing of Common Blocks . . . . . . . . . . . . . . 315

F. Sample Problem 1 output. . . . . . . . . . . . . . 329 
LIST OF FIGURES

No.

Title

$\underline{\text { Page }}$

1. Sequence of Calculations During a Reactivity Step . . . . . . . 27

2. Time Step Lengths . . . . . . . . . . . . . . . . . . 28

3. Fluwchart of Transient Calculations . . . . . . . . . . . 33

4. Mesh Point Placement Within a Fuel Pin. . . . . . . . . . . . 42

5. Overall Flowchart for Thermal-Hydraulic Calculation . . . . . 53

6. Flowchart of Steady-State Calculation of Fuel and Coolant

Temperatures for One Thermal-Hydraulic Channel of the Detailed

Model .. . . . . . . . . . . . . . . . . .

7. Flowchart of Transient Calculation of Fupl and coolant

Tempcratures fus Oue Incrmal-llydraulic Channel of the Detailed

Model . . . . . . . . . . . . . . . . 55

8. Flowchart for the Steary=State Calculation in FX2=TH. . . . . . 62

9. Listing of $\operatorname{ARCSP} 024$. . . . . . . . . . . . . . . 64

10. Input Data Files to FX2-TH. . . . . . . . . . . . . 69

11. Sample Problem 1 Input Deck ................. . 75

12. Sample Problem 2 Input Deck... . . . . . . . . . . 76

13. Sample Problem 3 Input Deck . . . . . . . . . . . . . 79

14. Geiseral overlay structure of FX2-TH . . . . . . . . . . . . 100

15. Macroscopic Flow Diagram. . . . . . . . . . . . . 107

16. BPOINTER Example. . . . . . . . . . . . . . . 118

17. Arrays Permanently In-Core During Steady-State and Transient. . : 120

18. Arrays Whose Dispositions are Determined by the Data

Management Strategy . . . . . . . . . . . . . . . 121

19. Glossary of Terms Used in Figures 17 and 18 . . . . . . . . . 123

20. Creation of $\mathrm{HX} 2-\mathrm{TH}$ Object Code. . . . . . . . . . . . 125

21. Kemoval of ARCSP024 from IBM Program Tape . . . . . . . . . . 126

22. IBM JCL to Execute Sample Problem 3. . . . . . . . . . . 126

23. CDC Control Cards . . . . . . . . . . . . . . . 127 


\section{LIST OF TABLES}

No.

Title

Page

I. Symbolic Parameters for ARCSP024. . . . . . . . . . . . 67

II. Summary of Error Messages for FX2-TH. . . . . . . . . . . . 83

III. Status of Binary files for a Set of Restart Cases . . . . . . . 99

IV. Detailed Overlay Structure of FX2-TH. . . . . . . . . . 101

V. IBM Code Center Tape Description. . . . . . . . . . . . . 127

VI. CDC Code Center Tape Description. . . . . . . . . . . . . 128 
THIS PAGE

\section{WAS INTENTIONALLY LEFT BLANK}


FX2-TH: A TWO-DIMENSIONAL NUCLEAR REACTOR KINETICS CODE WITH THERMAL AND HYDRAULICS FEEDBACK

by

R. A. Shober, D. R. Ferguson, T. A. Daly

\begin{abstract}
FX2-TH is a Lwo-dimensional, time-dependent nurlear reactor kinetics program with thermal and hydraulic feedback. The neutronics model used is multigroup neutron diffusion theory. The following geometry options are available: $x, r, x-y, r-z, \theta-r$, and triangular. FX2-TH contains two basic thermal and hydraulic models: a simple adiabatic fuel temperature calculation, and a more detailed model consisting of an explicit representation of a fuel pin, gap, clad, and coolant. FX2-TH allows feedback effects from both fuel temperature (Doppler) and coolant temperature (density) changes. FX2-TH'will calculate a consistent set of steady state conditions by iterating between the neutronics and thermal-hydraulics until convergence is reached. The time-dependent calculation is performed by the use of the improved quasistatic method. A disk editing capability is available. FX2-TH is operational on IBM system . 360 or 370 computers and on the CDC 7600 .
\end{abstract}

CODE ABSTRACT

1. Program Identification: FX2-TH

2. Computer for which program is designed and others on which it is operable: IBM 370/195, IBM 370/165, CDC 7600, any IBM OS System.

3. Description of Function: FX2-TH solves the steady-state and time-dependent two-dimensional multigroup neutron diffusion equations. These equations are coupled to a solution of the heat conduction equations in a fuel pin, cladding, and non-boiling coolant. The code is designed for nuclear reactor transient analysis.

4. Method of Solution: The multigroup diffusion equations are discretized in space using mesh-centered finite differences. The steady-state solution of these equations is found by accelerating a fission source iteration through the use of Chebyshev polynomialo. The neutron fluxes for each energy group at each power iteration are found using the successive line over-relaxation method. The timedependent diffusion equations are solved using the improved quasistatic method. The shape calculations required by the quasistatic method utilize the same calculational methods as described above for the steady-state calculation. 
5. Restrictions: Variable dimensioning is used throughout the program so that computer core requirements depend on a variety of problem parameters. The amount of core memory required can range from $300 \mathrm{~K}$ bytes for a small problem up to the maximum limit of computer core for very large problems.

6. Running Time: Running times are very dependent on the number of groups, number of mesh points, length of the transient, and dnount of change in the problem variables as the transient progresses.

7. Unusual Features of the Program: A consistent set of steady-state conditions are established by iterating between the steady-state neutronics and thermal-hydraulics equations. The time-dependent solution is then found using the improved quasistatic method. A wide variety of geometry options are available, such as $x, r, x-y, r-z$, $\theta-r$, and triangular. Microscopic cross sections and delayed neutron data are input in the CCCC formats ISOTXS and nT.AYXS, respontivcly. A disk editing capability is included.

8. Related and Auxiliary Programs: Input microscopic cross sections and delayed neutron data may be generated using the $\mathrm{MC}^{2}-2$ program. ${ }^{17}$

9. Status: FX2-TH is operational on the IBM $370 / 195$ at ANL, on an IBM 370/165, and at the CDC 7600 at Lawrence Berkeley Laboratory.

10. References: R. A. Shober, D. R. Ferguson, and T. A. Daly, FX2-TH: A Two-Dimensional Nuclear Reactor Kinetics Code with Thermal and Hydraulic Feedback, ANL-78-97, October 1978.

11. Machine Requirements: FX2-TH will dynamically allocate problem variables either on disk or in core memory depending on the total amount of core available. For any problem, a certain minimum amount of core memory is required; this amount being dependent on the size of the problem. In general, FX2-TH operates more effirient1,y if sufflclent core storage is available to contain moet of the problem variables permanently in core. On the $\operatorname{CDC} 7600$, for large problems as much SCM and LCM (up to a maximum of 131,000 words) as available should be requested.

12. Programming Language Used: FORTRAN-IV

13. Operating System or Monitor Under which Program is Executed: The IBM version of FX2-TH may be compiled under OS or VS operating systems using either FORTRAN $H$ or Extended compilers with the highest level of optimization. The CDC 7600 version of $\mathrm{FX} 2-\mathrm{TH}$ has been implemented at the Lawrence Berkeley Laboratory using the COKE/SCOPE operating system. Directly addressable LCM is used in FX2-TH. The code was compiled using FORTRAN extended compiler under OPT=2 optimization.

14. Any Other Programming or Operating Information or Restrictions: None. 
15. Name and Establishment of Authors: R. A. Shober

Applied Physics Division

D. R. Ferguson

Reactor Analysis and Safety Division

T. A. Daly

Applied Physics Division

Argonne Nationa1 Laboratory

Argonne, Illinois 60439

16. Material Available: Separate tapes are available for the IBM and CDC versions of FX2-TH. The package includes:

1) Source decks

ii) Test problem input decks

iii) Test problem input data sets

iv) Test problem output

v) Reference report

17. Category:

Keywords: kinetics, quasistatic method, multigroup diffusion theory, thermal-hydraulics 


\section{INTRODUCTION}

The FX2-TH code is designed for two-dimensional reactor transient analysis. The neutronics model used is time-dependent multigroup neutron diffusion theory. The thermal-hydraulics model used can be either an "adiabatic" calculation of the fuel temperature, or an explicit solution of the heat conduction equations through a fuel pin, gap, clad and coolant. Although FX2-TH was designed for LMFBR transient analysts, the equations solved and numerical methods used are appropriate for many reactor types.

FX2-TH solves the steady-state and time-dependent multigroup neutron diffusion equations. The steady-state calculation consists of finding both the real and adjoint multigroup neutron flux. These calculations are performed by using an accelerated iteration strategy. The time-dependent diffusion equations are solved by using the improved quasistatic method. 1 This method can be thought of as being a solution of the point kinetics equations with periodic re-calculation of the time-dependent multigrnup neutron fluxes such that the resultant point kinetics solution is an acceptably accurate approximation to a full space-time solution of the diffusion pquatinns. The neutronics equations and solution techniques are described in Chapter II.

The known multigroup neutron fluxes are then used to calculate the fission power generaled in each reactor region. From this fission power, the thermalhydraulics model calculates average fuel and coolant temperatures in this reactor region. Two basic thermal-hydraulics models are available in FX2-TH. The first is an "adiabatic" model, the second a more detailed model. The "adiabatic" model has two variations; the first assumes that no heat transfer occurs between fuel and coolant, the second assumes the rate of heat transfer to be constanl uver the transient. For these models, the coolant temperatures are not calculated. The second thermal-hydraulics model available in FX2-TH is an explicit representation of a fuel pin, gap, clad, and coolant for each reactor region. The heat conduction equations are solved in order to calculate the temperature distribution through the fuel pin, rlad, and conlant. The coolant in each reactor region is assumed nnt to interact with the cool... ant in any other region, and it is assumed not to boll. The effect of wire wraps and the fuel assembly structure on the coolant temperature can also be calculated by defining a lumped "structure" material, and calculating its temperature during the transient. The thermal-hydraulics models present in FX2-'IH are described in detail in Chapter III.

The average temperatures calculated by the thermal-hydraulics models are used in the calculation of the macroscopic cross sections for each reactor region. FX2-TH takes account of feedback effccts from changes in both the average fuel and average coolant temperatures. An increafe in the average fuel temperature causes Doppler broadening of resonances in fuel material, thus changing the microscopic capture and fission cross sections. In FX2-TH, this effect is represented hy a general expansion of the microscopic capture and fission cross sections in terms of the average fuel temperature. An increase in the average coolant temperaturc causes a decrease in the acom density of the coolant. In FX2-TH, this effect is represented by a linear expansion of the atom density in terms of the average coolant temperature. Thus, the macroscopic capture, removal, scattering, and transport cross sections of the coolant are dependent on the average coolant temperature. The details of the cross section representation in FX2-TH are presented in Chapter IV. 
Steady-state conditions are found by iteratively solving for the steady state neutron. fluxes and steady-state fuel and coolant temperatures until a converged set of conditions is found. The transient conditions are calculated by solving the neutronics and thermal-hydraulics equations in tandem within the framework of the improved quasistatic method. The details of the overall solution strategy are presented in Chapter IV.

In Chapter V, information is presented to enable the user to successfully execute the FX2-TH program. A listing of the required input parameters is given, as well as all additional information required to run FX2-TH. Chapter VI presents detailed programming information for those who may be required to modify the code in the future. 


\section{NEUTRONICS EQUATIONS AND THEIR SOLUTION}

\section{A. The Multigroup Diffusion Equations}

The basic neutronics model used in FX2-TH is time-dependent multigroup diffusion theory. Assuming that cross sections and material densities are known, an accurate prediction of the distribution of neutrons in a nuclear reactor requires a solution of the Boltzmann transport equation; an equation which can be derived from first principles. ${ }^{2}$ However, currently available methods for solving this equation for problemis in multidimensional geometry and/or which are time-dependent are extremely long-running even on today's high-speed computers. Therefore, the so-called multigroup diffusion equations are solved in the FX2-'l'H program. To derive these equations from the transport equation, several approximations are required.

One method of solving the transport equation involves expanding the angular flux density in Legendre polynomials. ${ }^{2}$ When only the first two terms of the Legendre expansion are retained, the resulting equations are called the "P-1" equations. The solution of the diffusion equations can be thought of as an approximation to the solution of the P-1 equations. In most fast reactor problems, the error involved in reducing the Boltzmann transport equation to the $\mathrm{P}-1$ equations exceeds that incurred by reducing the $\mathrm{P}-1$ equations to the diffusion equations.

Diffusion theory has been historically the most widely used tool in reactor analysis. This is due to the generally simple structure of the equations, and the fact that sophisticated mathematical techniques exist for solving them. A detailed discussion of the approximations involved. In reducing the transport equation to the diffusion equations is found in $\operatorname{Re} .2$.

The time dependent multigroup diffusion equations, together with the delayed neutron precursor equations, can be written: ${ }^{2}$

$$
\begin{aligned}
& \frac{1}{V_{g}} \frac{\partial}{\partial t} \phi_{g}(\underline{r}, t)=\underline{\nabla} \cdot D_{g}(\underline{r}, t) \underline{\nabla} \phi_{g}(\underline{r}, t)-\sum_{g^{\prime}=1}^{G} \Sigma_{g g^{\prime}}(\underline{r}, t) \phi_{g^{\prime}}(\underline{r}, t) \\
& +\chi_{p g} \underset{g^{\prime}=1}{G}(1-\beta) v \Sigma_{f}(\underline{r}, t) \phi_{g^{\prime}}(\underline{r}, t) \\
& +\sum_{\ell=1}^{L} X_{\left.d g \cdot l^{\lambda} C_{\ell} \underline{\underline{r}}, t\right)}+Q_{g}(\underline{r}, t), \dot{g}=1, \ldots, G \\
& \frac{\partial}{\partial t} C_{\ell}(\underline{r}, t)=-\lambda_{\ell} C_{\ell}(\underline{r}, t)+\sum_{g^{\prime}=1}^{G} B_{\ell^{\nu}} \Sigma_{f_{g^{\prime}}}(\underline{r}, t) \phi_{g^{\prime}}(\underline{r}, t), \ell=1, \ldots L
\end{aligned}
$$


where

$$
\begin{aligned}
& G \text { is the total number of neutron energy groups } \\
& \mathrm{L} \text { is the total number of delayed precursor groups } \\
& \phi_{g}(\underline{r}, t) \quad \begin{array}{l}
\text { is the neutron flux in group } g \text { at point } \underline{r} \text { and time } t \\
\left(\mathrm{n} / \mathrm{cm}^{2} \mathrm{sec}\right)
\end{array}
\end{aligned}
$$

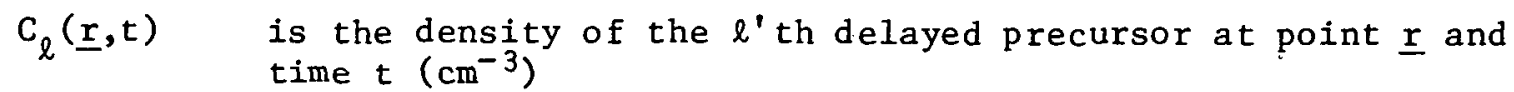

$$
\begin{aligned}
& Q_{g}(\underline{r}, t) \text { is the extcrnal neutron source in group } g \text { at point } \underline{r} \text { and } \\
& \text { time } t\left(\mathrm{n} / \mathrm{cm}^{3} \mathrm{sec}\right) \\
& \mathrm{V}_{\mathrm{g}} \quad \text { is the neutron speed in group } \mathrm{g}(\mathrm{cm} / \mathrm{sec})
\end{aligned}
$$

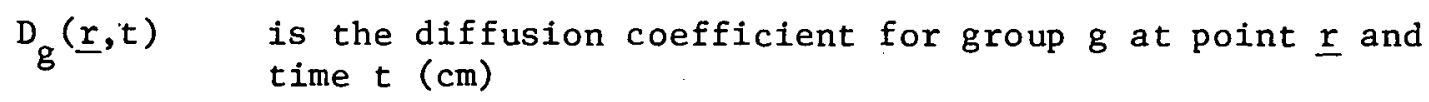

$$
\begin{aligned}
& \begin{array}{ll}
\Sigma_{\mathrm{gg}}{ }^{\prime}(\underline{r}, t) & \text { is the macroscopic transfer cross section from } g^{\prime} \text { to } \\
& g \text { at point } \underline{r} \text { and time } t\left(\mathrm{~cm}^{-1}\right) \text {, where }
\end{array} \\
& \Sigma_{g g}(\underline{r}, t)=\Sigma_{a_{g}}(\underline{r}, t)+\sum_{g^{\prime} \neq g} \sum_{s_{g^{\prime} g}}(\underline{r}, t) \\
& \begin{array}{ll}
\sum_{\mathrm{a}}(\underline{r}, t) \quad \text { is the macroscopic absorption cross section in group } g \text { at } \\
\text { point } \underline{r} \text { and time } t\left(\mathrm{~cm}^{-1}\right)
\end{array} \\
& \begin{array}{ll}
\Sigma_{s_{g}},(\underline{r}, t) & \text { is the macroscopic scattering cross section from group } g^{\prime} \\
\text { to } g \text { at point } \underline{r} \text { and time } t\left(\mathrm{~cm}^{-1}\right)
\end{array} \\
& \Sigma_{g g^{\prime}}(\underline{r}, t)=-\Sigma_{s g^{\prime}}(\underline{r}, t), g^{\prime} \neq g \\
& x_{\mathrm{pg}} \quad \text { is the prompt fission spectrum in group } g \\
& \begin{array}{ll}
\nu \Sigma_{f}(\underline{r}, t) \quad \text { is nu, the number of neutrons per fission, times the macroscopic } \\
\text { fission cross section in group } g \text { at point } \underline{r} \text { and time } t\left(\mathrm{~cm}^{-1}\right)
\end{array} \\
& \beta \quad \text { is the total fractional yield of delayed neutrons per fission } \\
& \lambda_{\ell} \quad \text { is the decay constant of the } \ell \text { 'th group of delayed precursors } \\
& \begin{array}{l}
x_{\mathrm{dg} l} \quad \text { is the fraction of group } \& \text { delayed neutrons which appear in } \\
\text { neutron group } g
\end{array} \\
& \begin{array}{l}
\beta_{\ell} . \quad \text { is the fraction of fissions which produce a delayed precursor } \\
\text { of group } \ell .
\end{array}
\end{aligned}
$$

If the material constants are known as a function of $\underline{r}$ and $t$, and if an initial flux distribution in energy and space is known, then a unique solution to Eqs. (II-1) may be obtained. 
In Eqs. (II.1) above, the term $\nu \Sigma_{f_{g}}(\underline{r}, t)$ should be considered as a sum over all fissionable isotopes. This summation will not be explicitly indicated for the remainder of this report.

\section{B. Steady State Solution Strategies}

\section{B.1 Development of the Steady State Equations}

The time-dependent multigroup diffusion equation (Eq.(II.1a)) constitutes a parabolic partial differential equation in space and time. To obtain a solution to this equation, the initial conditions $\phi_{g}(r, 0)$ and $C_{\ell}(\underline{r}, 0)$ must be known. Generally, a nuclear reactor is assumed to be in a steady state condition at the time a reactor transient analysis is begun. This steady state situation can be achieved in two ways: the reactor can be exactly critical with no external source present, or it can' be subcritical with an external source present. An exactly critical reactor is defined as one in which the total number of neutrons created per second by the fission process throughout the reactor exactly equals to the total number of neutrons lost per second by absorption and leakage out of the reactor. A subcritical reactor is one in which the number of the neutrons lost per second exceeds those created. Both situations (exactly critical, or subcritical with external source) lead to a steady state situation; that is one in which

$$
\begin{aligned}
& \frac{\partial}{\partial t} \phi_{g}(\underline{r}, 0)=0, g=1, \ldots, G \\
& \frac{\partial}{\partial t} C_{\ell}(\underline{r}, 0)=0, \ell=1, \ldots, L
\end{aligned}
$$

Using Eqs. (II.2), we find that Eqs. (II.1) will reduce to

$$
\begin{aligned}
& -\underline{\nabla} \cdot I_{g}(\underline{r}, 0) \underline{\nabla} \phi_{g}(\underline{r}, 0) \neq \stackrel{Z}{G}_{g^{\prime}=1}^{G} I_{g g^{\prime}}(\underline{r}, \tilde{u}) \phi_{g^{\prime}}(\underline{r}, \dot{u}) . \\
& =Q_{g}(\underline{r}, 0)+x_{g} \sum_{g^{\prime}=1}^{G} v \Sigma_{f} g^{\prime}(\underline{r}, 0) \psi_{g^{\prime}}(\underline{r}, 0), g=1, \ldots, G
\end{aligned}
$$

where

$$
x_{g}=x_{p g}(1-\beta)+\sum_{\ell=1}^{L} x_{\mathrm{dg} \ell}{ }^{\beta} \ell
$$

If the external source $Q_{g}(\underline{r}, 0)$ is non zero, Eq. (II.3) is then an inhomogeneous equation, and possesses a unique solution provided the reactor is subcritical. If the external source $Q_{g}(\underline{r}, 0)$ is zero, Eq. (II.3) becomes a homogenous equation, and possesses a solution other than the trivial solution only if the reactor is exactly critical; or, more precisely, if the multigroup diffusion theory model used to calculate the reactor conditions predicts that the reactor is exactly critical. 
In order to insure that a solution to Eq. (II. 3) may always be found, if no external source is present the form of Eq. (II.3) is modified slightly by dividing $\nu$, the number of neutrons per fission, by a number $\lambda$. The number $\lambda$ can then be adjusted in such a way that the reactor model is made exactly critical; and a nontrivial solution found. Once this solution is found, a transient reactor analysis may be begun; with the adjusted value $\frac{\nu}{\lambda}$ used in place of $v$ in Eq. (II.1).

The value $\lambda$ is included into Eq. (II. 3) for the purpose of making the reactor model exactly critical. It is assumed that this operation will not grossly change the neutron flux distribution. This operation compensates for various errors in the reactor model; such as cross section errors, homogenization procedures, the use of the diffusion approximation, etc.

The equation which must be solved to determine the steady state reactor conditions is (omitting the time dependence)

$$
\begin{aligned}
& -\underline{\nabla} \cdot D_{g}(\underline{r}) \underline{\nabla} \phi_{g}(\underline{r})+\sum_{g^{\prime}=1}^{G} \sum_{g g^{\prime}}(\underline{r}) \phi_{g^{\prime}}(\underline{r}) \\
& =\frac{1}{\lambda} \chi_{g} \sum_{g^{\prime}=1}^{G} v \Sigma_{f_{g^{\prime}}}(\underline{r}) \phi_{g^{\prime}}(\underline{r})+Q_{g}(\underline{r}), g=1, \ldots, G
\end{aligned}
$$

\section{B.2. Spatial Discretization}

Since the material constants in Eq. (II.4) are spatially dependent, it is generally not possible to solve Eq. (II. 4) analytically over the domain of the entire reactor. To obtain a solution, Eq. (II.4) is discretized in space by the use of finite difference techniques. This procedure leads. to a matrix eigenvalue problem which may be solved using conventional numerical methods.

To accomplish this discretization, a mesh is superimposed upon the two-dimensional reactor model. The mesh is constructed such that all material buundaries lie on the mesh lines. Thus, inside each mesh cell, the material is homogeneous. Eq. (II.4) is then integrated over the volume of each mesh ce11. For mesh cell k, Eq. (II. 4) becomes:

$$
\begin{aligned}
\int_{k} \underline{J}_{g}(\underline{r}) \cdot d \underline{S} & +\sum_{g^{\prime}=1}^{G} \sum_{g g^{\prime}, k} \int_{k} \phi_{g^{\prime}}(\underline{r}) d V \\
& =\frac{1}{\lambda} x_{g} \sum_{g^{\prime}=1}^{G} v \Sigma_{f_{g^{\prime} k}} \int_{k} \phi_{g^{\prime}}(\underline{r}) d V \\
& +\int_{k} Q_{g}(\underline{r}) d V \quad \begin{array}{l}
k=1, \ldots, K ; \\
g=1, \ldots, G
\end{array}
\end{aligned}
$$


where

$$
\begin{aligned}
& \underline{J}_{g}(\underline{r})=-D_{g}(\underline{r}) \underline{\nabla} \phi_{g}(\underline{r}) \\
& \mathrm{D}_{\mathrm{g}, \mathrm{k}} \quad \text { is the value of } \mathrm{D}_{\mathrm{g}}(\underline{\mathrm{r}}) \text {, in mesh cell } \mathrm{k} \\
& \Sigma_{g g^{\prime}, \mathrm{k}} \quad \text { is the value of } \Sigma_{g g^{\prime}}(\underline{r}) \text { in mesh cell } \mathrm{k} \\
& \nu \Sigma_{\mathrm{f}}, \mathrm{k} \text { is the value of } \nu \Sigma_{\mathrm{f}}(\underline{r}) \text { in mesh cell } \mathrm{k} \text { (representing a sum } \\
& \text { over all fissionable isotopes) }
\end{aligned}
$$

The additional definitions are now made:

$$
\begin{aligned}
& \bar{\phi}_{g, k}=\frac{1}{V_{k}} \int_{k} \phi_{g}(\underline{r}) d V \\
& \bar{Q}_{g, k}=\frac{1}{V_{k}} \int_{k} Q_{g}(\underline{r}) d V \\
& v_{k}=\int_{k} d V .
\end{aligned}
$$

From these definitions, the following equation is obtained:

$$
\begin{aligned}
& \int_{k} \mathrm{~J}(\underline{r}) \cdot d S+\sum_{g^{\prime}=1}^{G} v_{k^{\prime}} g_{g^{\prime}}, k^{\prime} \bar{\phi}_{g^{\prime} k} \\
& =\frac{1}{\lambda} x_{g} \sum_{g^{\prime}=1}^{G} \quad V_{k} \nu \Sigma_{f_{g^{\prime}, k}} \bar{\phi}_{g^{\prime}, k}+\bar{Q}_{g, k} \begin{array}{ll}
k=1, \ldots, k ; \\
g=1, \ldots, G
\end{array}
\end{aligned}
$$

To solve Eq. (II.5), relationships must be specified between the current $\mathrm{J}_{\mathrm{g}}(\underline{r})$ and the average fluxes $\bar{\phi}_{\mathrm{g}, \mathrm{k}}$. The technique used in FX2-TH leads to the "mesh-centered difference cquations," which are the difference equations used in the computer programs VENTURE, ${ }^{3}$ DIF2D, 4 DIF3D, 5 and MEKIN. 6 The difference equations for all geometries are derived in Ref. 4. The resulting equations can be written in the fnllowing matrin form:

$$
\begin{aligned}
& {\left[T_{g}\right]\left[\bar{\phi}_{g}\right]+\left[\Sigma_{r_{g}}\right]\left[\bar{\phi}_{g}\right]-\sum_{\substack{g^{\prime}=1 \\
g^{\prime} \neq g}}^{G}\left[\Sigma_{s_{g g^{\prime}}}\right]\left[\bar{\phi}_{g^{\prime}}\right]} \\
& \left.=\frac{1}{\lambda} x_{g} \sum_{g^{\prime}=1}^{G}\left[\nu \Sigma_{f_{g^{\prime}}}\right]^{T}\left[\bar{\phi}_{g^{\prime}}\right]+\bar{Q}_{g}\right], g=1, \ldots, G
\end{aligned}
$$


Where

$\left[\bar{\phi}_{\mathrm{g}}\right]$ is a column vector of length $\mathrm{K}$ consisting of the average neutron fluxes at every mesh cell for group $g$

[ $\Sigma_{\mathrm{r}}$ ] is a diagonal $\mathrm{K} \times \mathrm{K}$ matrix consisting of the macroscopic removal cross sections at all mesh cells for group $g$ times the volume of the respective mesh cell.

[ $\left.\mathrm{s}_{\mathrm{s}}\right]$ is a diagonal $\mathrm{K} \times \mathrm{K}$ matrix consisting of the macroscopic 'gg' scattering cross sections at all mesh cells for scattering from group $g^{\prime}$ into group $g$ times the volume of the respective mesh cell

$\left[\cup \Sigma_{f}\right]$ is a column vector of length $K$ consisting of nu, the number of neutrons per fission, times the macroscopic fission cross section at all mesh cells for group $g$ times the volume of the respective mesh cell (this term represents a sum over all fissionable isotopes)

$\left[\bar{Q}_{\mathrm{g}}\right]$ is a column vector of length $\mathrm{K}$ consisting of the integrated external source at all mesh cells for group $g$.

$\mathrm{L}_{\mathrm{g}} \quad$ is a banded $\mathrm{K} \times \mathrm{K}$ matrix consisting of the finite difference coefficients at all mesh cells for group $g$.

$\mathrm{K}$ is the total number of mesh cells.

\section{B.3. Outer Iteration Strategies}

If $\left[\bar{Q}_{\mathrm{g}}\right]$ is zero, Eq. (II. 6) is a homogeneous matrix eigenvalue problem. This problem may be solved by using the power method, in which we define:

$$
\left[\mathrm{S}_{\mathrm{g}, \ell}\right]=\frac{1}{\lambda_{\ell}} x_{\mathrm{g}} \underset{\mathrm{g}^{\prime}=1}{\mathrm{G}}\left[\nu_{\mathrm{f}_{\mathrm{g}^{\prime}}}\right]^{\mathrm{T}}\left[\bar{\phi}_{\mathrm{g}^{\prime}}\right]
$$

Where $\ell$ is the outer iteration index, and $\lambda_{\ell}$ and $\left[\bar{\phi}_{g^{\prime}}^{l}\right]$ represent the current estimate of the eigenvalue and average fluxes, respectively, at the $\ell$ th outer iteration. Eq. (II.6) then becomes:

$$
\begin{aligned}
& {\left[L_{g}\right]\left[\bar{\phi}_{g}\right]+\left[\Sigma_{\mathrm{r}_{g}}\right] \cdot\left[\bar{\phi}_{g}\right]} \\
& -\sum_{\substack{g^{\prime}=1 \\
g^{\prime} \neq g}}^{G}\left[\Sigma_{s_{g} g^{\prime}}\right]\left[\bar{\phi}_{g^{\prime}}\right]=\left[s_{g, l}\right], g=1, \ldots, G
\end{aligned}
$$


For outer iteration $\ell$, the sequence of calculations is then:
a) Calculate $\left[\mathrm{S}_{\mathrm{g}, \ell}\right]$ for all groups $\mathrm{g}$
b) Solve the resulting inhomogeneous matrix equation (II.8) for all groups $g$
c) Calculate a new estimate of the eigenvalue $\lambda_{\ell}$.

In FX2-TH, this convergent iterative process is accelerated by the use of Chebyshev polynomials applied to the fission source. This strategy has. proven very successful for fast reactor problems, and is described in detail in Ref. 5.

For problems in which $\left[\bar{Q}_{g}\right]$ is not zero, the parameter $\lambda$ is set to a user-specified value (whose default value is one). Eq. (II.6) is'then solved as an inhomogeneous problem. The solution strategy, however, is very similar to the one used for the homogeneous problem. A fission source is calculated at each "outer" iteration, the fixed source is added, and the resulting matrix equations are solved. The iteration is accelerated by applying Chebyshev polynomials to the fission sources, as was done for the homogeneous case. The solution strategies are described in detail in Ref. 5.

\section{B.4. Inner Iteration Strategies}

In FX2-TH, no "up-scattering" is allowed (that is, $\left[\Sigma_{s_{g g}}\right]$ is zero for all groups $g^{\prime}>g$ ). Thus the solution of Eq. (II.8) involves a single sweep through the energy groups $g=1, \ldots, G$. At each group $g$, an inhomogeneous matrix equation must be solved. The particular structure of the $\left[\mathrm{L}_{\mathrm{g}}\right]$ matrix depends on the geometry of the problem, however $\left[\mathrm{L}_{\mathrm{g}}\right]$ is always a very sparse, symmetric matrix with a regular structure. For two dimensional orthogonal geometries, for example, $\left[\mathrm{L}_{\mathrm{g}}\right]$ is a five striped block tri-diagonal matrix.

Due to the sparseness of the matrix and the large number of unknowns, iterative methods are generally used to solve matrix problems such as (II.8). The particular technique used in FX2-TH is called the "successive line over-relaxation" method, and is a variant of the accelerated Gauss-seidel iterative method. The same technique is used in the DIF3D ${ }^{5}$ program; the details of this scheme are fully described in Ref, 5 .

\section{B.5. Solution of the Adjoint Flux Equations}

FX2-TH uses the improved quasistatic method ${ }^{1}$ to calculate the time dependent neutron fluxes. This method requires that the adjoint neutron fluxes be calculated. The adjoint flux required, $\left[\bar{\phi}_{\mathrm{g}}{ }^{*}\right]$, is the solution of the
following equation: 


$$
\begin{gathered}
{\left[L_{g}\right]\left[\bar{\phi}_{g}^{*}\right]+\left[\Sigma_{r_{g}}\right]\left[\bar{\phi}_{g}^{*}\right] .-\sum_{\substack{g^{\prime}=1 \\
g^{\prime} \neq g}}^{G}\left[\Sigma_{s_{g^{\prime}}}\right]\left[\bar{\phi}_{g^{\prime}} *\right]} \\
=\frac{1}{\lambda^{*}}\left[\nu \Sigma_{f_{g}}\right] \sum_{g^{\prime}=1}^{G} x_{g^{\prime}}\left[\bar{\phi}_{g} *\right], g=1, \ldots, G
\end{gathered}
$$

The same adjoint flux $\left[\bar{\phi}_{g} *\right]$ is calculated independent of whether or not a fixed source is present. It can be seen that Eq. (II.9) is obtained by taking the transpose of the matrix operator which Eq. (II.6) represents. This is called the "physicist's adjoint" (the complex conjugate of the transpose) rather than the "mathematician's adjoint" (the transpose of the matrix of co-factors). This adjoint operator was first introduced by Wigner. The motivation behind the use of this particular form of the adjoint will be discussed in Section $C$ of this Chapter.

\section{Time Dependent Solution Strategies}

\section{C.1. Characteristics of the Time Dependent Problem}

The solution of the time dependent multigroup diffusion equations is a very formidable problem. Once a steady-state flux distribution has been obtained, Eqs. (II.1) must be integrated in time to determine the transient solution. One immediate difficulty with this procedure is the non-linearity introduced by the presence of thermal and hydraulic feedback. This nonlinearity will be discussed in detail in Chapter IV. Another major difficulty lies in the range of time constants present in Eqs. (II.1). The very short time constants present in the neutron flux equations requires that small time steps be taken to integrate them accurately. However, very often the interesting period of a reactor transient can stretch over a period of seconds, thus requiring a very large number of time steps.

The time dependent multigroup diffusion equations are thus difficult to solve first because of the large number of unknowns to be calculated, and secondly because of the large number of time steps for which these unknowns must be calculated.

A number of methods have been proposed for solving the time-dependent multigroup diffusion equations. These methods can be divided into two major categories. The first class of solution methods, here called synthesis methods, at tempt to dramatically reduce the number of unknowns in the problem by using expansions of the solution in known functions. The second class of solution methods, here called space-time nethods, retain the full structure of the equations and attempt to directiy integrate them by one scheme or another.

Several space-time methods have been developed which have proven successful for solving the diffusion equations. These methods often are longrunning even on today's high-speed computers; therefore, where applicable the less costly synthesis methods are preferable. For use in FX2-TH, a method 
called the Improved Quasistatic Method is used. This method will be shown to be a variation of the Points Kinetics Method.

The Point Kinetics Method is one of the simplest of the synthesis methods. This method, which will be developed in the next section, assumes that the shape of the neutron flux distribution (in space and energy) does not change significantly over the course of a reactor transient. If this assumption is valid, the full space-time equations can be reduced to $(1+L)$ coupled ordinary differential equations in time.

Unfortunately, very few reactor transients involve no significant spatial or spectral changes. However, it is possible to significantly improve the Point Kinetics Method by using different approximations to the neutron flux shape at different times during the reactor transient. If these shape functions are the result of a series of static calculations representing various reactor conditions during the transient, the resulting method is called the Adiabatic Method. ${ }^{7}$ If the shape functions are calculated in tandem with the actual point kinetics solution, the method is called the Quasistatic Method. Th1s method, used in the FX2-TH code, w111 be described in the next two sections.

\section{C.2. Development of the Point Kinetics Equations}

To begin the derivation of the point kinetics equations, Eqs. (II.1) will be rewritten in a more compact matrix notation:

$$
\begin{aligned}
& \underline{\nabla} \cdot[D(\underline{r}, t)] \underline{\nabla}[\phi(r, t)]-[A(\underline{r}, t)][\phi(\underline{r}, t)] \\
& \quad+\frac{1}{\lambda_{k}}(1-\beta)\left[\chi_{p}\right]\left[\nu \Sigma_{f}(\underline{r}, t)\right]^{T}[\phi(\underline{r}, t)]+\sum_{\ell=1}^{L} \lambda_{\ell} C_{\ell}(\underline{r}, t)\left[x_{\ell}\right]+[Q(\underline{r}, t)] \\
& \quad=[V]^{-1} \frac{\partial}{\partial t}[\phi(\underline{r}, t)] \\
& \frac{1}{\lambda_{k}} \beta_{\ell}\left[\nu \Sigma_{f}(\underline{r}, t)\right]^{T}[\phi(\underline{r}, t)]-\lambda_{\ell} C_{\ell}(\underline{r}, t)=\frac{\partial}{\partial t} C_{\ell}(\underline{r}, t), \ell=1, \ldots, L
\end{aligned}
$$

where

$$
\begin{aligned}
& {[D(\underline{r}, t)]=\operatorname{diag}\left\{D_{1}(\underline{r}, t), D_{2}(\underline{r}, t), \ldots, D_{G}(\underline{r}, t)\right\}} \\
& {[\phi(\underline{r}, t)]=\operatorname{col}\left\{\phi_{1}(\underline{r}, t), \phi_{2}(\underline{r}, t), \ldots, \phi_{G}(\underline{r}, t)\right\}}
\end{aligned}
$$




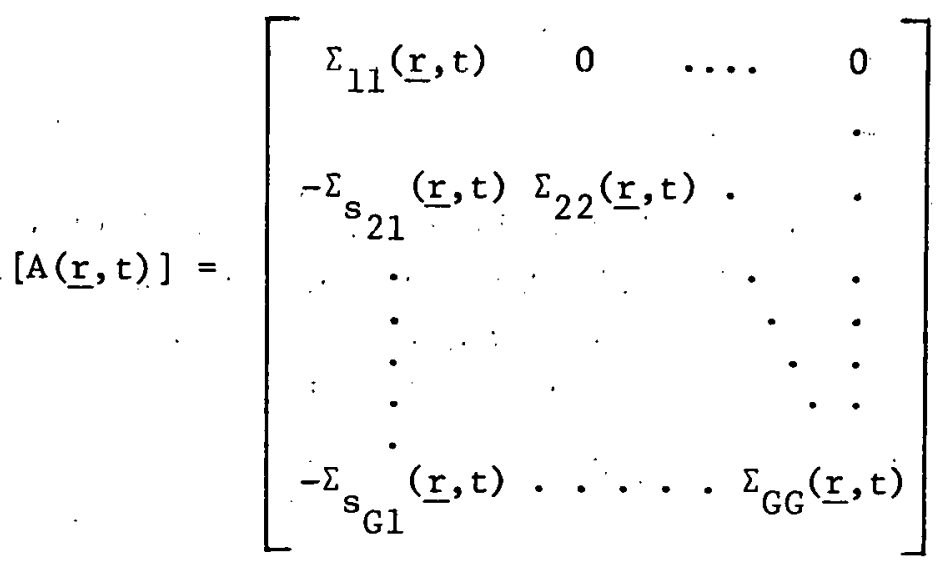

$$
\begin{aligned}
& {\left[x_{p}\right]=\operatorname{col} \quad\left\{x_{p 1}, x_{p 2}, \ldots, x_{p G}\right\}} \\
& {\left[\nu \Sigma_{f}(\underline{r}, t)\right]=\operatorname{col}\left\{\nu \Sigma_{f_{1}}(\underline{r}, t), \nu \Sigma_{f}(\underline{r}, t), \ldots, \nu \Sigma_{f_{G}}(\underline{r}, t)\right\}} \\
& {\left[x_{\ell}\right]=\operatorname{col}\left\{x_{\mathrm{d} 1 \ell}, x_{\mathrm{d} 2 \ell}, \ldots, x_{\mathrm{dGl}}\right\}} \\
& {[Q(\underline{r}, t)]=\operatorname{col}\left\{Q_{1}(\underline{r}, t), Q_{2}(\underline{r}, t), \ldots, Q_{G}(\underline{r}, t)\right\}} \\
& {[V]=\operatorname{diag}\left\{V_{1}, v_{2}, \ldots, v_{G}\right\}}
\end{aligned}
$$

$\lambda_{k}\left\{\begin{array}{l}\text { is the critical eigenvalue } \lambda \text { of } \mathrm{Eq} . \text { (II.4) if no external } \\ \text { source is present } \\ \text { is unity if an external source is present }\end{array}\right.$

The term $v \Sigma_{f}(\underline{r}, t)$ represents a sum over all the fissionable isotopes.

An arbitrary column vector weight function $[W(\underline{r})]$ is now defined:

$$
[W(\underline{r})] \equiv \operatorname{col}\left\{W_{1}(\underline{r}), W_{2}(\underline{r}), \ldots, W_{G}(\underline{r})\right\}
$$

Next define $T(t)$ to be the following weighted integral:

$$
T(t) \equiv \int[W(\underline{r})]^{T}[V]^{-1}[\phi(\underline{r}, t)] d V
$$

The function $T(t)$ is often referred to as the "amplitude function", since it is anticipated that it will determine the primary time dependence of the solution $[\psi(\underline{r}, t)]$.

A column vector shape function is now defined:

$$
[S(\underline{r}, t)] \equiv T(t)-1[\phi(\underline{r}, t)]
$$


such that

$$
\int[W(\underline{r})]^{T}[V]^{-1}[S(\underline{r}, t)] \cdot d V=1
$$

To obtain an equation for $T(t)$, replace $[\phi(\underline{r}, t)]$ by $T(t)[S(\underline{r}, t)]$, multiply the row vector $[\mathrm{W}(\underline{r})]^{\mathrm{T}}$ into. Eq. (II.10a), and integrate over the volume of the vector. To reduce the precursor equations (II.10b), multiply by $[\mathrm{W}(\underline{r})]^{\mathrm{T}}\left[\mathrm{x}_{\ell}\right]$ and integrate over the volume of the reactor. These operations lead to the following equations:

$$
\begin{aligned}
\frac{d}{d t} T(t) & =\left\{\frac{\rho(t)-\beta(t)}{\Lambda(t)}-\frac{\lambda_{s}-1}{\lambda_{s} \Lambda(t)}\right\} T(t) \\
& +\sum_{\ell=1}^{L} \lambda_{\ell} C_{\ell}(t)+Q(t) \\
\frac{d}{d t} C_{\ell}(t) & =\frac{\beta_{\ell}(t)}{\Lambda(t)} T(t)-\lambda_{\ell} C_{\ell}(t), \ell=1, \ldots, L
\end{aligned}
$$

where

$$
\begin{aligned}
& \lambda_{s}\left\{\begin{array}{l}
\text { is unity if there is no external source } \\
\text { is the critical eigenvalue } \lambda * \text { of Eq. (II.9) if } \\
\text { an external source is present }
\end{array}\right. \\
& C_{\ell}(t)=\frac{\int[W(\underline{r})]^{T}\left[x_{\ell}\right] C_{\ell}(\underline{r}, t) d V}{\int[W(\underline{r})]^{T}[V]^{-1}[S(\underline{r}, t)] d V} \\
& Q(t)=\frac{\int[W(r)]^{T}[Q(r, t)] d V}{\int[W(\underline{r})]^{\mathrm{T}}[V]^{-1}[S(\underline{r}, t)] d V} \\
& F(t)=\int[W(\underline{r})]^{T}\left\{(1-\beta)\left[x_{p}\right]+\sum_{\ell=1}^{L} \beta_{\ell}\left[x_{\ell}\right]\right\} \text {. } \\
& \frac{1}{\lambda_{k}}\left[v \Sigma_{f}(\underline{r}, t)\right]^{T}[S(\underline{r}, t)] d v
\end{aligned}
$$




$$
\begin{aligned}
\rho(t) & =\frac{1}{F(t)} \cdot \int[W(\underline{r})]^{T}\{\underline{\nabla} \cdot[D(\underline{r}, t)] \underline{\nabla}[S(\underline{r}, t)] \\
& -[A(\underline{r}, t)][S(\underline{r}, t)]+\left((1-\beta)\left[x_{p}\right]\right. \\
& \left.\left.+\sum_{\ell=1}^{L} \beta_{\ell}\left[x_{\ell}\right]\right) \frac{1}{\lambda_{k}{ }^{\prime}}\left[\nu \Sigma_{f}(\underline{r}, t)\right]^{T}[S(\underline{r}, t)]\right\} d V \\
\Lambda(t) & =\frac{1}{F(t)} \int[W(\underline{r})]^{T}[V]^{-1}[S(\underline{r}, t)] d V \\
B(t) & =\frac{1}{F(t)} \int[W(\underline{r})]^{T} \frac{1}{\lambda_{k}} \beta_{\ell}\left[x_{\ell}\right]\left[\nu \Sigma_{f}(\underline{r}, t)\right]^{T}[S(\underline{r}, t)] d V \\
\beta(t) & =\sum_{\ell=1}^{L} \beta_{\ell}(t)
\end{aligned}
$$

The term $\lambda_{s}$ was introduced such that the reactivity $\rho(0)$ would equal zero for the case with an external source.

In most reactor transients, spatial and spectral changes in [S(r,t)] have a fairly small effect on the value of terms such as $\beta_{\ell}(t)$ and $\Lambda(t)$. The reactivity $(\rho(t))$, however, is sensitive to such changes since its numerator is a difference of two quantities which are almost equal. Therefore, it is of prime importance to minimize errors in $\rho(t)$ resulting from the fact that an approximation to the shape function will be used in practice. The weight function $[W(\underline{r})]$ is chosen in such a way that first order errors in $[S(\underline{r}, t)]$ are eliminated. This is accomplished by using the time-independent adjoint flux $\left[\bar{\phi}_{g}^{*}\right]$ (the solution of Eq. (II.9)) as thc weight function. 2

The Point Kinetics Equations derived in this section are the most widely used equations in the field of reactor kinetics. They can be derived from the multigroup diffusion equations (or from the transport equation) with no approximations. In practice, their accuracy depends on the method used to calculate $\beta_{\ell}(t), \Lambda(t)$, and especially $\rho(t)$. The accuracy of $\rho(t)$ is primarily dependent on the accuracy of the shape function [S $(\underline{r}, t)]$. In the next section, an accurate method for the calculation of the shape function will be described. 


\section{C.3. Development of the Quasistatic Method}

The Quasistatic Method 18 can be thought of as a solution to the point kinetics equations in which the shape functions used to calculate the point kinetics parameters are calculated in tandem with the point kinetics solution. This method will be shown to be derived from the factorization defined in Eq. (II.12), and to be capable of reproducing the results of an exact temporal integration of Eqs. (I.I.10) in the limit of small time steps. Therefore, the quasistatic method achieves the dramatic reduction in the number of unknowns characteristic of a synthesis method, and also possesses the reliability of a full space-time method.

The following factorization is defined:

$$
[\phi(\underline{r}, t)] \equiv T(t)[S(\underline{r}, t)]
$$

Th1s equation is substituted into Eq. (II. 10a) to yield:

$$
\begin{aligned}
\underline{V} \cdot[D(\underline{r}, t)] \underline{\nabla}[S(r, t)]-[A(\underline{r}, t)][S(\underline{r}, t)] \\
-[V]^{-1} T(t)^{-1} \frac{\partial}{\partial t} T(t)[S(\underline{r}, t)]+\frac{1}{\lambda_{k}}(1-B)\left[x_{p}\right]\left[\nu \Sigma_{[}(\underline{r}, t)\right]^{T}[S(\underline{r}, t)] \\
\quad+\sum_{\ell=1}^{L} \lambda_{\ell} T(t)^{-1} C_{\ell}(\underline{r}, t)\left[x_{\ell}\right]+T(t)^{-1}[Q(\underline{r}, t)] \\
=[V]^{-1} \frac{\partial}{\partial t}[S(\underline{r}, t)]
\end{aligned}
$$

In the quasistatic method, Eqs. (II.14) are solved to determine the primary temporal behavior of the solution. At various times during the transient, Ey. (II.15) 1s solved to ralrulate a now chapc funetion [G(L,L)]. Thereforé, two basic time step units are required. $\Lambda$ reactivity step is a unit of lime over which Eqs. (II.14) are solved given a certain known set of point kinetics parameters. A shape step is a unit of time, typically several. reactivity steps long, which elapses between solutions of Eq. (II.15); that is, between shape re-calculations. The next sections will describe these calculations in more detail.

\section{C.4. Reactivity Step Calculation Sequence.}

\section{C.4.a. Reactivity Step Initialization}

Across a reactivity step, the point kinetics equations (Eqs. (II.14a, b)) and the thermal-hydraulics equations (see Chapter ITI) are solved. Although these equations are nonlinear, they are solved in tandem across each reactivity step. This approach has proved successfui for several multidimensional space-time kinetics programs. ${ }^{6}, 9,10$ In addition to these computations, several other initialization procedures are performed at the beginning of each reactivity step. These computations are described below. Figure 1 shows a flow chart of the calculations performed at each reactivity step. 


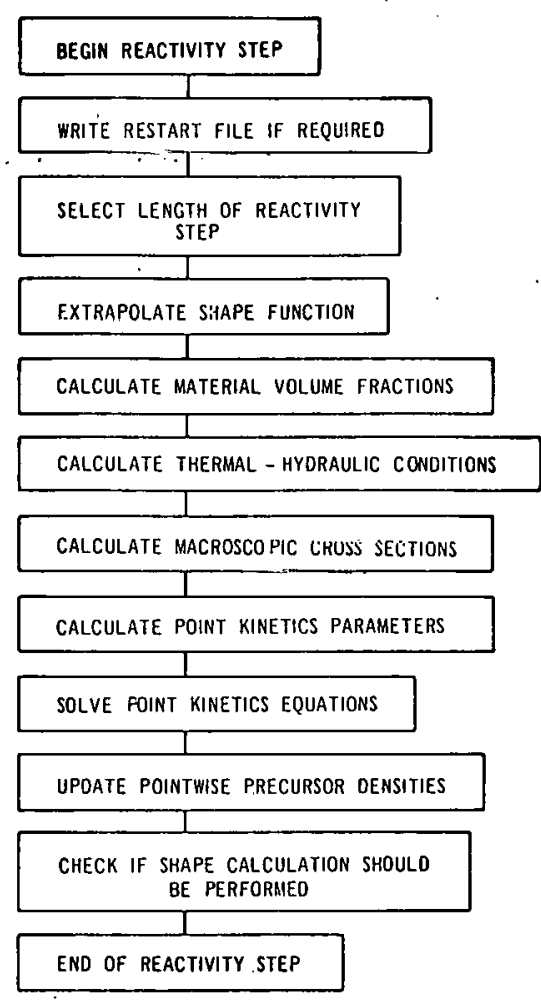

Fig. 1

Sequence of Calculatione During a Reactivity Step

The length of the reactivity step is first calculated. The selection of the step sizes is based on how rapidly system parameters are changing. The selection criteria for both shape steps and reactivity steps are discussed in Section II.C.6. The first reactivity step in a transient is computed as a user-specified factor times the prompt neutron lifetime. The first shape step is chosen to be this same length, thus forcing a shape calculation at the end of the first reactivity step of the transient.

\section{C.4.b. Shape Function Estimation}

In order to evaluate the point kinetics parameters (Eqs. (II.14 $\mathrm{f}, \mathrm{g}, \mathrm{h}, \mathrm{i})$ ), an estimate of the shape function is required. In FX2-TH, the shape is assumed to vary linearly between shape calculations. Therefore, the shape functions obtained by the solution of Eq. (II.15) at two previous shape calculations are linearly extrapolated to obtain the required shape function. Let $t_{j}$ be the time at the beginning of shape step $j$. The shapes $[S(\underline{r}, t)]$ calculated at times $t_{j-1}$ and $t_{j}$ are used to extrapolate the shape to form the shape estimate at $t_{j, i+1}$ used for reactivity scep 1. Figure 2 ohow the relationship of these time step levels.

To calculate the thermal-hydraulic conditions at the end of the reactivity step, an estimate of the amplitude function during the reactivity step is required. The amplitude values calculated at times $t_{j, i-2}$, $t_{j, i-1}$, and $t_{j, i}$ are fit to a quadratic function; and this quadratic is integrated from $t_{j, i}$ to $t_{j, i+1}$ to calculate the average value of the amplitude over this reactivity step. 


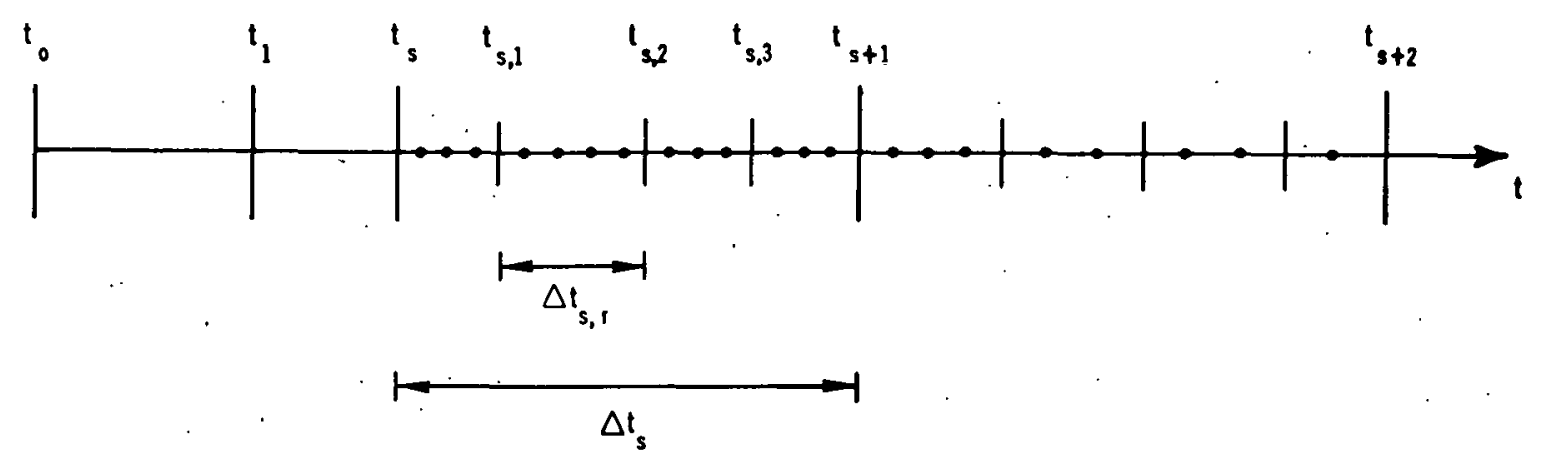

$\Delta t_{S}=$ SHAPE STEP LENGTH

$\Delta \mathrm{t}_{s, \mathrm{i}}=$ REIACTIVITY STEP LEMGTH

Fig. 2. Time Stap Lcvcls

\section{C.4.c. Volume Fraction Calculation}

The volume fractions appropriate for this reactivity step are next calculated. Volume fractions can be changed during the transient by use of driving functions. A driving function forces the volume fraction of some material $\mathrm{m}$ in a region $e, V F(e, m, t)$ to change over a time interval from $t_{S T}$ to $t_{S P}$ according to the formula

$$
V F(e, m, t)=V F\left(e, m, t_{S T}\right)+C L \cdot\left(t-t_{S T}\right)+C Q \cdot\left(t-t_{S T}\right)^{2},\left(t_{S T} \leq t_{\leq} t_{S P}\right) .
$$

The user may set $t_{S T}=t_{S P}$, in which case CL is added to VF in a stepwise fashion at time $t_{S T}$, as $V F(e, m, t)=V F\left(e, m, t_{S T}\right)+C I_{,}\left(t>t_{S T}\right)$.

The driving functions are used to start and modify a transient.

The therma1-hydraulic conditions for Llis reactivity step are next calculated. The details of the thermal-hydraulic models are given in Chapter III. Next, with the volume fractions and thermal-hydraulic conditions known, the macroscopic cross sections for this reactivity step can be calculated. Details of the dependence of the macroscopic cross sections on temperatures are given in Chapter IV.

\section{C.4.d. Point Kinetics Equation Solution}

Using the estimated shape function, the cross sections, and volume fractions, the point kinetics parameters (Eqs. (Ir.14 f,g,h,i)) are then calculated. The point kinetics equations (Eqs. (II.14a,b)) may then be integrated over this reactivity step. The integration technique used is an extension ${ }^{11}$ of a method originally devised by Kaganove. ${ }^{12}$ The method involves a scheme for integrating the point kinetics equations over subdivisions of the reactivity step. An automatic time step halving and doubling scheme is used to achieve a specified precision. 


\section{C.4.e. Pointwise Precursor Density}

After the amplitude function has been calculated, the pointwise precursor densities may be updated. Rewriting Eq. (II.10b):

$$
\frac{\beta_{\ell}}{\lambda_{k}}\left[\nu \Sigma_{f}(\underline{r}, t)\right]^{T}[\phi(\underline{r}, t)]-\lambda_{\ell} C_{\ell}(\underline{r}, t)=\frac{\partial}{\partial t} C_{\ell}(\underline{r}, t), \ell=1, \ldots, L
$$

where

$L$ is the total number of delayed precursor gruups

This equation is integrated from $t_{j, i}$ to $t_{j, i+1}$ to obtain:

$$
\begin{aligned}
& c_{\ell}\left(\underline{r}, t_{j, i+1}\right)=C_{\ell}\left(\underline{r}, t_{j, i}\right) \exp \left(-\lambda_{\ell} \Delta t_{j, i}\right) \\
& +\int_{t}^{t}{ }_{j, i}+1 \frac{\beta_{\ell}}{\lambda_{k}}\left[\nu \Sigma_{f}(\underline{r}, t)\right]^{T}[S(\underline{r}, t)] T(t) ! \\
& \quad \exp \left[\lambda_{\ell}\left(t_{j, i+1}-t\right)\right] d t, \ell=1, \ldots, L
\end{aligned}
$$

where

$$
\begin{aligned}
& \Delta t_{j, i}=t_{j, i+1}-t_{j, i} \\
& {[\phi(\underline{r}, t)]=[S(\underline{r}, t)] T(t)}
\end{aligned}
$$

In order to evaluate the integral in Eq. (II.16), it is assumed that the product $\left[\nu \Sigma_{f}(\underline{r}, t)\right]^{T}[S(\underline{r}, t)]$ is a linear function of time over the reactivity step. Therefore, the integral can be replaced by a sum of two terms each involving a known estimate of $\left[\nu \Sigma_{f}(\underline{r}, t)\right]^{T}[S(\underline{r}, t)]$ at an endpoint of $\Delta t{ }_{j, i}$; therefore:

$$
\begin{gathered}
C_{\ell}\left(\underline{r}, t_{j, i+1}\right)=C_{\ell}\left(\underline{r}, t_{j, i}\right) \exp \left(-\lambda_{\ell} \Delta t_{j, i}\right) \\
\quad+I_{1}^{\ell}+I_{\eta}^{\ell}, \ell=1, \ldots, L
\end{gathered}
$$

where

$$
\begin{aligned}
& I_{1}^{\ell}=\frac{\beta_{\ell}}{\Delta t_{j, i} \lambda_{k}}\left[v \Sigma_{f}\left(\underline{r}, t_{j, i}\right)\right]^{T}\left[S\left(\underline{r}, t_{j, i}\right)\right] \cdot \\
& \int_{t_{j, i}}^{t_{j, i+1}} T(t) \exp \left[-\lambda_{\ell}\left(t_{j, i+1}-t\right)\right] \cdot\left(t_{j, i+1}-t\right) d t
\end{aligned}
$$




$$
\begin{aligned}
& I_{2}^{\ell}=\frac{\beta_{\ell}}{\Delta t_{j, i} \lambda_{k}}\left[\nu \Sigma_{f}\left(\underline{r}, t_{j, i+1}\right)\right]^{T}\left[s\left(\underline{r}, t_{j, i+1}\right)\right] . \\
& \int_{t_{j, i}}^{t_{j, i+1}} T(t) \exp \left[-\lambda_{\ell}\left(t_{j, i+1}-t\right)\right]\left(t-t_{j, i}\right) d t
\end{aligned}
$$

The integrals in Eqs. (II.17b,c) are evaluated by numerical integration in which $T(t)$ is expressed as a piecewise quadratic function over the subsets. 11 This completes the calculations required for this reactivity step.

\section{C.5. Shape Step Calculation Sequence}

After each reactivity s tep is compicted, FX2-TH determines if a shipe calculation should be performed at this point. These tests are described in Section II.C.6. To calculate a new shape function, Eq. (II.15) must be solved. This is done by using the fully implicit time integration method. 13 obtained:

When this method is applied to Eq. (II.15), the following equation is

$$
[B][S]-[F][S]=[W]
$$

where

$$
\begin{aligned}
& {[S]=\left[S\left(\underline{r}, t_{j+1}\right)\right]}
\end{aligned}
$$

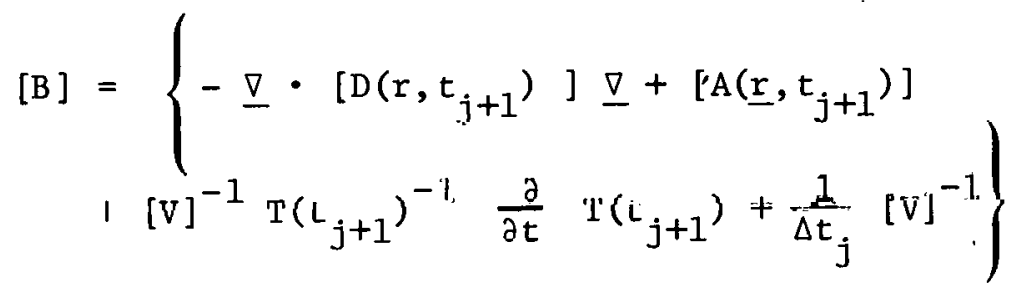

$$
\begin{aligned}
& {[F]=\frac{1}{\lambda_{k}}(I-\beta)\left[\chi_{\mu}\right]\left[\nu \Sigma_{f}\left(\underline{r}_{,} t_{j+1}\right)\right]^{T}} \\
& {[W]=\sum_{\ell=1}^{L} \lambda_{\ell} T\left(t_{j+1}\right)^{-1} C_{\ell}\left(\underline{r}, t_{j+1}\right)\left[x_{\ell}\right]} \\
& +T\left(t_{j+1}\right)^{-1}\left[Q\left(\underline{r}, t_{j+1}\right)\right]+\frac{1}{\Delta t_{j}}[V]^{-1}\left[S\left(\underline{\underline{r}}, t_{j}\right)\right]
\end{aligned}
$$

and $L$ is the total number of delayed neutron families. 
The amplitude function $T\left(t_{j+1}\right)$ is known from the solution of the point kinetics equations, its time derivative at $t_{j+1}$ is known from evaluating the right hand side of Eq. (II.14a). The pointwise precursor densities are known from the solution of Eqs. (II.17). Therefore, Eq. (II.18a) can be solved for the shape function [S].

For the remainder of this Section, the variables of interest wili be written as continuous functions of $\underline{r}$. In the FX2-TH program; these variables are spatially discretized as described in Section II.B.2. This leads to matrix problems which are solved numerically.

During the sequence of reactivity steps, the estimate of the shape function used to calculate the point kinetics parameters was made by linearly extrapolating the results of two preceeding shape function calculations. The new shape function $\left[S\left(\underline{r}, t_{j+1}\right)\right]$ will not, in general, be equal to the linearly extrapolated shape function used to calculate the point kinetics parameters.

Therefore, an iterative procedure called the "shape step iteration" will be performed. This iteration will consist of first re-calculating the point kinetics solution at each reactivity step within this shape step. This re-calculation is performed by approximating the time dependence of the point kinetics parameters $\frac{\rho}{\Lambda}, \frac{\beta}{\Lambda}, \frac{1}{\Lambda}$, and $\frac{\beta}{\Lambda}$ as being linear over the shape step. Let $\mathrm{n}$ denote the shape step iteration index; and $\mathrm{h}^{\mathrm{n}}$ denote the $\mathrm{n}$ iteration value of any of the above ratios. Then

$$
h^{n}\left(t_{j, i}\right)=h^{1}\left(t_{j, i}\right)+\alpha\left(t_{j, i}\right)\left[h^{n}\left(t_{j+1}\right)-h^{1}\left(t_{j+1}\right)\right]
$$

where

$$
\alpha\left(t_{j, i}\right)=\frac{t_{j, i}-t_{j}}{t_{j+1}-t_{j}}
$$

The ratios $h^{1}\left(t_{j, i}\right)$ and $h^{1}\left(\dot{t}_{j+1}\right)$ are already known and $h_{n}\left(t_{j+1}\right)$ is found using the $n^{\text {th }}$ shape estimate $\left[s^{n}\left(\underline{x}, t_{j+1}\right)\right]$. For the purposes of the shape step iteration, it is assumed that the thermal-hydraulic effects and pointwise precursor densities calculated during the first sequence of reactivity steps are sufficiently accurate.

The second portion of the shape step iteration is a recalculation of the shape function using the newly calculated values of ' $I$ ' $\left(t_{j+1}\right)$ and $i$ ts time derivative. Eq. (II.18a) is solved to calculate the $(n+1)^{\text {th }}$ estimate of the shape function. 
In the development of the quasistatic method, it was observed that for some problems the solution of Eq. (II.18a) poses numerical difficulties. When the system approaches prompt critica1, $T\left(t_{j+1}\right)$ becomes very large. In addition, for typical shape step sizes the operator $\frac{1}{\Delta t_{j}}[\mathrm{~V}]^{-1}$ is small compared to the magnitude of [B] and [F]. Therefore, the term [W] becomes very small, and Eq. (II.18a) becomes almost homogeneous. In that case, the coefficient matrix $([B]-[F])$ becomes almost singular, and the iterative convergence very slow.

To mitigate these effects, Eq. (II.18a) is modified to resemble an eigenvalue problem

$$
\text { [B] }[\mathrm{S}]=\frac{1}{\gamma}[\mathrm{F}][\mathrm{S}]+[\mathrm{W}]
$$

where $\gamma$ is a scalar.

Letting $m$ be the outer iteration index, the equation sn pred to calculato the $(n+1)^{\text {Ll }}$ shape estimate is:

$$
\left.\left[B^{n}\right]\left[s^{n+1}, m+\right]\right]=\frac{1}{\gamma^{n+1, m}}\left[F^{n}\right]\left[s^{n+1, m}\right]+\left[W^{n}\right]
$$

where

$$
\gamma^{n+1, m}=\int_{V}[\phi *(\underline{r})]^{T}[V]^{-1}\left[s^{n+1, m}\right] d V
$$

The converged solution of Eq. (II.19a), divided by $\gamma^{n+1}$ (to satisfy the constraint condition), is used as the new shape estimate.

The solution of Fq. (TI, 19a) uses the same numerical strategies as the steady-state solution with a nonzero external source. These solution strategies are described in detail in Ref. 5 .

After the new shape function is calculated by solving Eq. (II.19a), the point kinetics equations are re-solved over each reactivity step in this shape step as described above. When this calculation is complete, the value $\gamma^{n}$ calculated for the $n^{\text {th }}$ shape step iteration is examined. If the amplitude $T\left(t_{j+1}\right)$ and $i$ ts time derivative used in forming [B] and [W] are "correct" (in the sense that they form a correct solution to the coupled set of equations (II.14) and (II.15)), then the value $\gamma^{\text {n }}$ calculated above will be un1ty. If $\gamma^{n}$ is within a user-specified value of being unity, then this shape step is completed; and a new shape step is begun. If $\gamma^{n}$ is not sufficiently close to unity, Eq. (II.19a) is re-solved using the newly calculated values of $\mathrm{T}\left(\mathrm{t}_{j+1}\right)$ and $i$ ts time derivative. The shape step iteration procedure then continues until either a converged value $\gamma^{n}$ is found, or until a user-specified number of shape step iterations are 
completed. The convergence of $\gamma^{n}$ to unity with shape step iteration is generally very rapid; the quantity $\left|\gamma^{n}-1\right|$ is usually reduced by an order of magnitude with each iteration. Figure 3 shows a detailed flowchart of the transient solution strategy for FX2-TH.

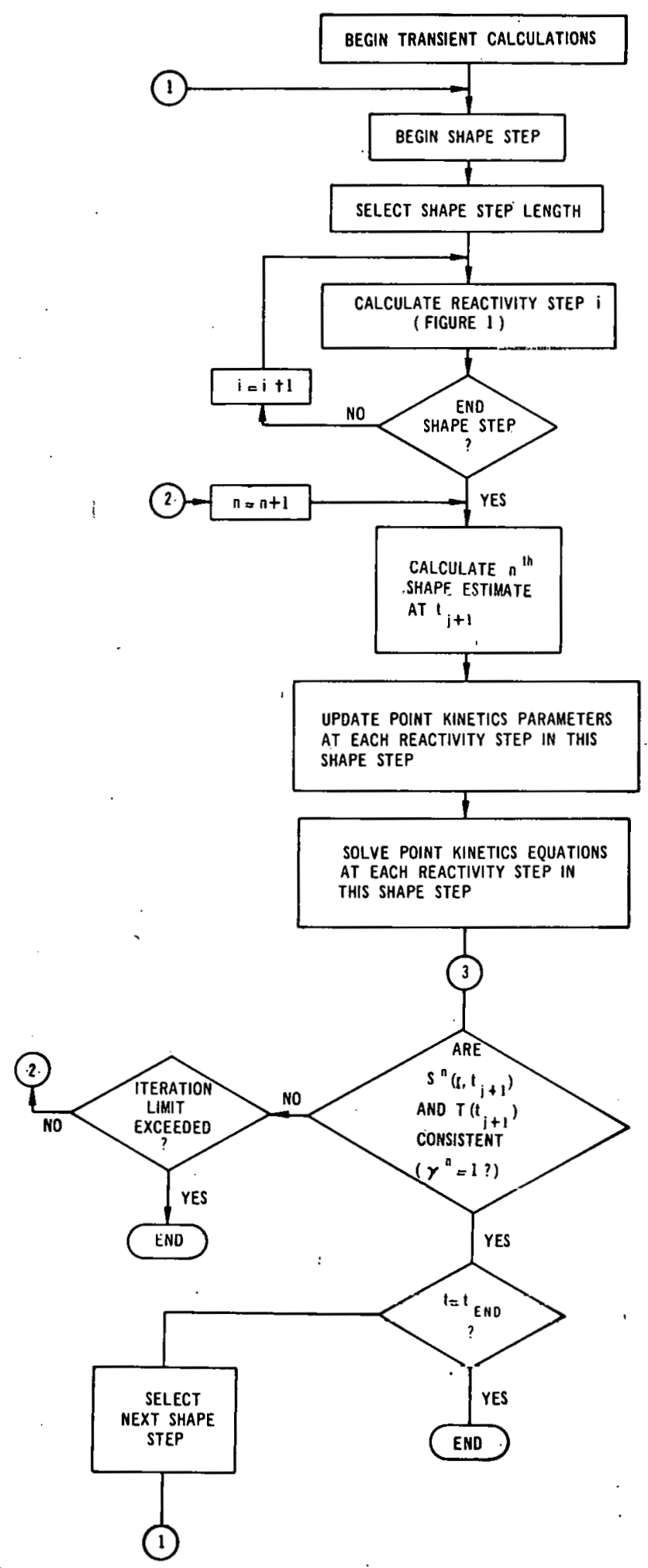

Fig. 3. Flowchart of Transient Calculations 
The shape step iteration procedure can be seen to be a convergent method of simultaneously solving Eqs. (II.14) and (II.15). The only approximations made are those previously noted; that the thermal-hydraulic effects and pointwise precursor densities calculated during the first reactivity steps pass are sufficiently accurate. In the limit of small reactivity and shape step lengths, FX2-TH $c$ an be expected to reproduce the results of an exact temporal numerical integration.

In summary, the calculational sequence at a shape step is divided into two sections. First, for each reactivity step within this shape step the point kinetics equations and thermal-hydraulics equations are solved in tandem (see Figure 1). The second part involves only solutions of the point kinetics equations (Eq. (II.14a,b)) and the shape equation (Eq. (II.19a)). These equations are solved until a converged solution is reached; that is, until $\gamma^{\mathrm{n}}$ is acceptably close to unity. (see Figure 3).

\section{C.6. Selection of Time Steps}

The purpose of the time step selertion algorithm is to provida acceptably accurate results for the lowest computation cost. Ultimately, the user must determine the acceptability of a certain solution. Therefore, FX2-TH allows the user to input a number of parameters which determine maxluum allowablè changes in certain system parameters. Through these input variables, the user is able to control the time step selection.

In the vicinity of prompt critical, it has been found desirable to reduce the user-specified limits by a calculated factor. This factor is

$$
f=1.0-\left(1.0-f_{\min }\right) \exp \left[\begin{array}{ll}
-\frac{1}{2} & \left.\left(\frac{\rho-\beta}{\beta \sigma}\right)^{2}\right]
\end{array}\right.
$$

where $f_{\text {min }}$, the minimum value of $f$, is an input parameter. The constant $\sigma$ is such that the exponential equals 0.5 when

$$
\frac{|\rho-\beta|}{\beta}=0.277 \text {. }
$$

C.6.a. Shape Step Selection Criteria

The shape step criteria are tested at two points in the program; at the beginning of each shape step, and at the end of each reactivity step. The shape step length is then taken as the largest time interval which meets all of the required constraints.

At the beginning of each shape step (other than the firat) the program computes three step lengths implied by maximum allowable parameter magnitudes or changes. The smallest of these values is found, and tested for compliance with two more constraints.

The shape step size, $\Delta t_{j}{ }^{(1)}$, implied by the maximum. allowed change in the flux shape is estimated by assuming that the shape function is a linear function from $t_{j-1}$ to $t_{j+1}$. The expression for $\Delta t_{j}{ }^{(1)}$ is 


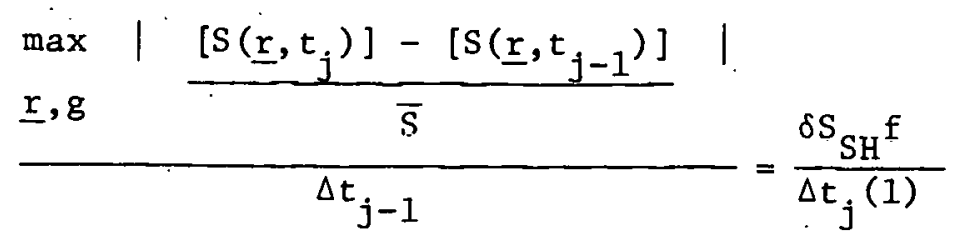

where $\left[S\left(\underline{r}, t_{j}\right)\right]$ is the shape function at $\underline{r}$ at time $t_{j}$ for all groups $\mathrm{g}=1, \ldots, \mathrm{G}, \mathrm{S}$ is the average shape element over a11 groups and points, and $\delta \mathrm{S}_{\mathrm{SH}}$ is the maximum allowed change over a shape step in the point and group-dependent relative shape function.

The second shape step length is $\Delta t_{j}{ }^{(2)}=\delta t_{S H}$, where $\delta t_{S H}$ an input maximum shape step length. Finally, the shape step must not be more than ten times the previous shape step length; more precisely

$$
\Delta t_{j}{ }^{\prime(3)}=10 \Delta t_{j-1} \frac{f_{j}}{f_{j-1}}
$$

where $\mathrm{f}_{\mathrm{j}-1}$ is the reduction factor at the last shape step selection.

If the problem end time, $t=t_{E N D}$, falls within the smallest of the three step lengths, $\Delta t_{j}$, then this step length is reduced such that $t_{j+1}=t_{E N D}$. Finally, if $t_{S T}$ and $t_{S P}$ of a driving function are equal and if this time falls within $\Delta t_{j}$, then the shape $s$ tep is made to end at $t_{S T}$. Thus, stepwise driving functions can be used to force shape steps to end at predetermined times.

The shape step will be terminated at the end of a reactivity step if any of five constraints are violated at that point. First, the shape step is ended if the current time coincides with the end time predicted at the beginning of the shape step. Second, a fuel temperature change over the step exceeding an input maximum will terminate the step. Third, the step is ended if the amplitude change thus far over the shape step does not satisfy the inequality:

$$
-\frac{10}{\delta T_{S H}} \leq \frac{T\left(t_{j+1}\right)}{T\left(t_{j}\right)} \leq \frac{10}{\delta T_{S H}},
$$

where $\delta \mathrm{T}_{\mathrm{SH}}$ is an input parameter. Fourth, the step is ended if the fixed source was altered at the beginning of this reactivity step. Finally, the number of reactivity steps in the shape step must not exceed an input maximum number.

\section{C.6.b. Reactivity Step Selection Criteria}

To determine the reactivity step length, the program computes four step lengths implied by maximum allowed magnitudes or changes 
in parameters. The smallest of these lengths is selected and is checked against four additional constraints.

The first step length is two times the previous step length,

$$
\Delta t_{j, i}(1)=2 \Delta t_{j, i-1} \frac{f}{f_{i, j-1}} .
$$

The second limit is an input maximum reactivity step length.

The fuel temperature rise over the reactivity step is restricted to be not more than the input value $\delta \mathrm{T}_{\mathrm{RH}}$. For the purposes of calculating the reactivity step length, the temperature rise is assumed to be proportional to the fission energy released over the time interva1. The energy released is approximated by a constant times the amplitude at the beginning of the interval times the time interval. The expression for the fuel-temperature limited. step length is

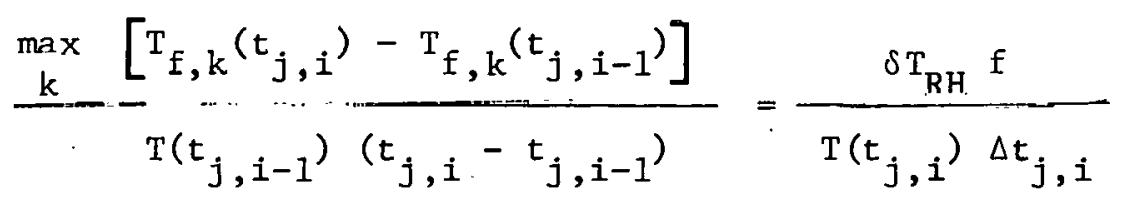

where $T_{f, k}\left(t_{j, i}\right)$ is the $f u e l$ temperature in mesh cell $k$ at time $t_{j, i}$, An amplitude-limited step length is determined by the requirement that the amplitude change over the reactivity step must satisfy the inequality

$$
-\frac{10}{\delta N_{R H}} \leq \frac{T\left(t_{j, i \mid 1}\right)}{T\left(t_{j, i}\right)} \leq-\frac{10}{\delta N_{R H}}
$$

where $\delta \mathrm{N}_{\mathrm{RH}}$ is an input parameter. The time step required is obtained by approximating the amplitude function by a quadratic and linearizing the resulting expression.

The smallest of the four limiting step lengths is used as the reactivity step if it does not contain any of the following times:

1)

$$
t_{S T} \text { or } t_{S P} \text { of a driving function, }
$$

the endpoint of a shape step predicted at the beginning of the shape step,

the time that the external source is to be modified, and

the problem end time. 
If the time step contains any of these times, then the step length is adjusted so that the smallest of the times which fell within the step becomes the endpoint of the step. 


\section{THERMAL-HYDRAULICS EQUATIONS AND THEIR SOLUTION}

The objective of the thermal-hydraulics models present in FX2-TH is to provide realistic reactor feedback calculations for off-normal conditions. For LMFBR applications, accurate pre-disassembly feedback is desired. The models described in this chapter are intended to provide the required feedback without a large expense in computation time.

Two basic models will be described in this chapter. The first model, called the "adiabatic" model, has two variations; and is used to calculate transient fuel temperatures only. The second model, called the "detailed" model, calculates steady-state and transient fuel and coolant temperatures.

In order to solve the multigroup diffusion equations, the reactor volume was divided into "mesh cells" (Section III.B.2). For the solution of the thermal-hydraulics equations, an additional spatial grid io placed over the mesh cell grid to define thermal-hydraulics regions. A thermal-hydraulic region may consist of one spatial mesh cell, or more than one mesh cell; and a region need not be contiguous. For each thermalhydraulic region, it is assumed that accurate feedback effects can be obtained by using a single average value of fuel and coolant temperature.

Generally, a thermal-hydraulic region is chosen to correspond to a volume in the reactor taken up by a fuel assembly (with appropriate axial subdivision). The user may elect to place more than one thermal-hydraulic region within each fuel assembly; however for most problems this corresponds to a negligible increase in accuracy. The user may also choose to define a thermal-hydraulic region over more than one fuel assembly; however this practice may be dangerous, as significant spatial effects may be overlooked.

In FX2-TH, the thermal-hydraulic regions are considered to be completely separate; that is, there is no heat or mass transfer hetwenn them. The particular thermal-hydraulic model chosen is used to calculate the average thermal-hydraulic conditions within each thermat-hydroulis region.

A. The Adiabatic Fuel Temperature Models

l'he thermal-hydraulic models present in FX2-TH are designed to calculate an accurate value of the average fuel and coolant temperature in this thermal-hydraulic region. For the adiabatic models, the coolant temperature is assumed to be independent of time; therefore, no coolant temperatures are calculated. During the entire calculation, the coolant temperatures remain at their user-specified initial values: Within the adiabatic model, there are two alternate methods for calculating the transient fuel temperatures. These methods will now be described.

\section{A.1 Zero Heat Transfer Method}

In the first method, it is assumed that no heat transfer exists between the fuel and coolant. The heat conduction equation 14 then reduces to

$$
\rho_{f} C_{f} \frac{\partial T_{f}(\underline{r}, t)}{\partial t}=q(\underline{r}, t)
$$


where

$$
\begin{array}{ll}
\mathrm{T}_{\mathrm{f}}(\underline{r}, t) & \begin{array}{l}
\text { is the average fuel temperature at point } \underline{r} \\
\text { and time } t\left({ }^{\circ} \mathrm{C}\right)
\end{array} \\
\rho_{\mathrm{f}} & \text { is the density of the } \mathrm{fuel}\left(\mathrm{g} / \mathrm{cm}^{3}\right) \\
\mathrm{C}_{\mathrm{f}} & \text { is the specific heat of the fuel (watt-sec/g } \left./ \mathrm{g}^{\circ}\right) \\
\mathrm{q}(\underline{\mathrm{r}}, \mathrm{t}) & \begin{array}{l}
\text { is the volumetric power generation rate in the } \\
\text { fuel (watts } \left./ \mathrm{cm}^{3}\right)
\end{array}
\end{array}
$$

Since no unique steady-state condition exists for Eq. (III.1), the user must enter the initial fuel temperatures $T_{f}(\underline{r}, 0)$. The quantities $\rho_{f}$ and $C_{f}$ are both dependent on the fuel temperature, therefore in FX2-TH the following correlation is made

$$
\begin{aligned}
& \rho_{f} C_{f}\left(T_{f}\right)=\rho_{f} C_{f}{ }^{0}+\rho_{f} C_{f}^{1}\left(T_{f}\right) \\
& +\rho_{f} C_{f}^{2}\left(T_{f}\right)^{2}+\rho_{f} C_{f}^{3}\left(T_{f}\right)^{3}+\rho_{f} C_{f}{ }^{4}\left(T_{f}\right)^{4}
\end{aligned}
$$

where

$$
\begin{array}{ll}
\rho_{\mathrm{f}} \mathrm{C}_{\mathrm{f}}\left(\mathrm{T}_{\mathrm{f}}\right) & \text { is the specific heat density of the fuel } \\
& \text { (watt-sec/cm } \left.{ }^{\circ} \mathrm{C}\right) \\
\mathrm{T}_{\mathrm{f}} & \text { is the fuel temperature in this thermal-hydraulic } \\
& \text { region }\left({ }^{\circ} \mathrm{C}\right)
\end{array}
$$

At each reactivity step, Eq. (III.1) must be solved to calculate the fuel temperature at the end of the reactivity step. First, the temperature $\mathrm{T}_{f}$ at the beginning of the reactivity step is used in Eq. (III.2) to evaluate $\rho_{f} C_{f}$. This value is assumed to hold for this reactivity step. Then, Eq. (III.1) is integrated from $t_{j, i}$ to $t_{j, i+1}$ to obtain

$$
T_{f}\left(\underline{r}, t_{j, i+1}\right)=T_{f}\left(\underline{r}, t_{j, i}\right)+\frac{\int_{t_{j, i}}^{t_{j, i+1}} q(\underline{r}, t) d t}{\rho_{f} C_{f}}
$$

To evaluate the integral in Eq. (III. 3), the amplitude function $T(t)$ is fit to a quadratic function from its values at $t_{j, i}, t_{j, i-1}$, and $t_{j, i-2}$. This quadratic function is then integrated numerically from $t_{j, i}$ to $t_{j, i+1}$ to obtain the required integral. The calculation of $q(\underline{r}, t)$ is described in Sec. III.A.3. 


\section{A.2 Constant Heat Transfer Method}

In the second method, it is assumed that the rate of heat transfer from fuel to coolant remains constant during the transient. In this case, the heat conduction equation ${ }^{14}$ becomes

$$
\rho_{f} C_{f} \frac{\partial T_{f}(\underline{r}, t)}{\partial t}=q(\underline{r}, t)-q(\underline{r}, o)
$$

where

$$
\begin{aligned}
& q(\underline{r}, 0) \quad \text { is the volumetric power generation rate in the } \\
& \text { fuel at point } \underline{r} \text { and time zero. }
\end{aligned}
$$

All other terms are as defined for Eq. (III.1).

To solve this equation over a reactivity step, the same methods are used as for Eq. (III.1). The quantity $\rho_{f} C_{f}$ is calculated from Eq. (III.2), then the equation integrated from $t_{j, i}$ to $t_{j, i+1}$ to obtain.

$$
\begin{aligned}
& T_{\underline{f}}\left(\underline{r}, t_{j, i+1}\right)=T_{f}\left(\underline{r}, t_{j, i}\right) \\
& +\frac{\int_{t_{j, i}}^{t_{j, i+1}} q(\underline{r}, t) d t-q(\underline{r}, t) \Delta t_{j, i}}{\rho_{f} C_{f}}
\end{aligned}
$$

The first integral in Eq. (III.5) is evaluated exactly as was done in Eq. (III.3).

\section{A.3 Calculation of Volumetric Power Generation Rate}

In the above two methods, the volumetric power generation rate $q(\underline{r}, t)$ is required. In practice, $F^{\prime} X 2-I^{\prime} H$ calculates an average value of $q(\underline{r}, t)$ over each thermal-hydraulic region. Let $q_{k}(t)$ be the average volumetric power generation rate in thermal-hydraulic region $k$. 'l'hen

$$
\begin{aligned}
& \left(3.20 \times 10^{-11}\right) \sum_{g=1}^{G} \int_{V_{k}} \sum_{f}(\underline{r}, t) \phi_{g}(\underline{r}, t) d V \\
& q_{k}(t)=\frac{V F_{f} \int_{v_{k}} d V}{}
\end{aligned}
$$

where

$$
\begin{array}{ll}
V_{k} & \text { is the volume of thermal-hydraulic region } k \\
V F & \text { is the volume fraction of } f \text { uel within thermal-hydraulic } \\
& \text { region } k
\end{array}
$$


and $3.20 \times 10^{-11}$ is a conversion factor converting fissions/second to watts. The fuel volume fraction $\mathrm{VF}_{\mathrm{f}}$ is a user-input number.

\section{A. 4 Summary of the Adiabatic Model}

The adiabatic fuel temperature models described above are capable of calculating transient fuel temperatures very inexpensively. The assumptions made to derive these models are most likely to be satisfied by a very rapid transient in which the primary feedback mechanism is Doppler feedback. The drawback of these methods is that they require the user to supply the steady-state solution. It is sugges ted that a steady-state calculation using the detailed model (to be described in the next Section) could be used to obtain estimates of the initial fuel and coolant temperatures.

\section{B. The Detailed Fuel and Coolant Temperature Model}

This model is designed to calculate realistic fuel and coolant temperatures for both steady-state and transient conditions. The heat conduction equations in both the fuel and coolant are solved to calculate the required temperatures. It is assumed that within each thermal-hydraulic region, an average fuel pin is defined. This fuel pin is assumed to be cylindrical; with a gas gap, cladding, and surrounding coolant flowing in the $+z$ direction. The following additional assumptions are made:

1) The coolant is assumed not to boil.

2) The coolant specific heat, density, and thermal conductivity are taken as polynomial functions of the average coolant temperature.

3) No coolant flow reversal is allowed.

4) The fuel thermal conductivity and specific heat density are taken as polynomial functions of the fuel temperature.

5) All heat is deposited in the fuel pin.

6) No fuel motion is allowed, nor is fuel (or clad) expansion.

7) Heat generation in the fuel is radially uniform.

8) Axial and azimuthal heat transfer in the fuel pin are neglected.

The reactor is assumed to be made up of fuel pins which traverse the entire length of the axial ( $\mathrm{Z}$ ) direction. Each fuel pin is referred to as a thermal-hydraulic channel. : Each channel contains the fuel pin and its associated coolant, flowing upwards in the $+Z$ direction. The axial direction is divided into axial mesh regions. An axial mesh region may be composed nf. one or more fine-mesh intervals (neutronics mesh intervals), and must be contiguous. The reactor core is therefore divided into thermal-hydraulic channels, and each channel into axial mesh regions. A thermal-hydraulic region (within which a single value of average fuel and coolant temperatures are used for feedback purposes) is therefore a particular axial mesh region within a particular thermal-hydraulic channel. 
When FX2-TH is run using $r-Z$ geometry, the region of solution is inherently three-dimensional, and the above scheme for dividing the reactor core is appropriate. When any other geometry is used, the one- or twodimensional region of solution is assumed to be perpendicular to the axial direction. In this case, the one- or two-dimensional region of solution is divided into thermal-hydraulic channels as described above, however only. one axial mesh region is used. The height of this axial mesh region is. taken as the height of the active core.

\section{B.1 Derivation of the Fuel Temperature Equations}

For the purpose of calculating the temperature distribution within the fuel pin and cladding, first assume that the cladding outer surface temperature, $\mathrm{T}_{c s}$, is known. The details of this calculation will be presented in Section III.B.3. Then, I equally spaced nodes are placed within the fuel pin, one node at the inside of the clad, and one node at the middle of the clad. I is a user-specified parameter. There are, therefore, (I+2) unknowns to be found. Figure 4 shows the mesh point placement with a fuel pin.

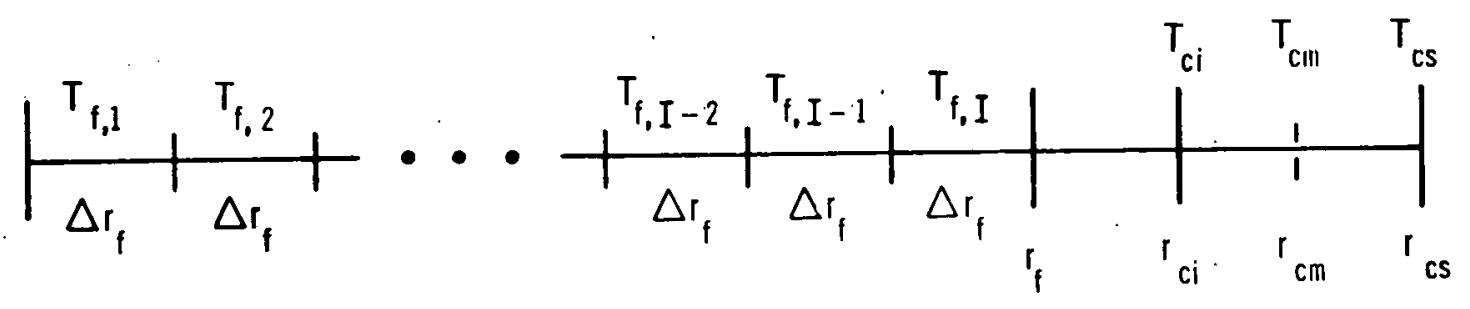

Fig. 4. Mesh Point Placement Within a Fuel Pin

The heat conduction equation in the fuel can he written 14

$$
\rho_{f} C_{f} \frac{\partial}{\partial t} T_{f}(r, t)=\frac{1}{r} \frac{\partial}{\partial r} r k_{f} \frac{\partial}{\partial r} T_{f}(r, t)+q(r ; t)
$$

where

$$
\begin{array}{ll}
\mathrm{T}_{\mathrm{f}}(\mathrm{r}, \mathrm{t}) & \begin{array}{l}
\text { is the fuel temperature at point } \mathrm{r} \text { along } \\
\text { the fuel pin radius and time to }\left({ }^{\circ} \mathrm{C}\right)
\end{array} \\
\mathrm{k}_{\mathrm{f}} & \begin{array}{l}
\text { is the fuel thermal conductivity (watts } / \mathrm{cm}^{\circ} \mathrm{C} \text { ) } \\
\mathrm{q}(\mathrm{r}, \mathrm{t}) \quad
\end{array} \\
\begin{array}{l}
\text { is the volumetric power generation rate at } \\
\text { point } \mathrm{r} \text { along the fuel pin radius and time } \mathrm{t}
\end{array} \\
\rho_{\mathrm{f}} \mathrm{C}_{\mathrm{f}} & \begin{array}{l}
\text { is the } \mathrm{fuel} \mathrm{sm}^{3} \text { ) } \\
\text { (watt-sec } / \mathrm{cm}^{3}{ }^{\circ} \mathrm{C} \text { ) }
\end{array}
\end{array}
$$

In the FX2-TH program, the fuel thermal conductivity and specific heat density are correlated as polynomial functions of the fuel temperature. The specific heat density correlation is given in Eq. (III.2), evaluated at 
each point $r$ along the fuel pin radius. The thermal conductivity is correlated as

$$
k_{f}(r)=k_{f} 0+k_{f}^{1} \quad\left(T_{f}(r, t)\right)+k_{f}^{2}\left(T_{f}(r, t)\right)^{2}
$$

where $T_{f}(r, t)$ is in ${ }^{\circ} \mathrm{C}$. The calculation of $q(r, t)$ will be described in Section III.B. 5 .

It is desired to form difference equations over each region $\left(r_{i}<r<r_{i+1}\right)$, where $T_{f, i}(t)$ denotes the average temperature within this interval. This is accomplished by multiplying Eq. (III.7) by r, and integrating it from $r_{i}$ to $r_{i+1}$ :

$$
\begin{aligned}
& \frac{\partial}{\partial t} \int_{r_{i}}^{r_{i+1}} r \rho_{f} C_{f}(r) T_{f}(r, t) d r \\
& =\int_{r_{i}}^{r_{i+1}} \frac{\partial}{\partial r} r k_{f}(r) \frac{\partial}{\partial r} T_{f}(r, t) \cdot d r+\int_{r_{i}}^{r} r q(r, t) d r
\end{aligned}
$$

To obtain difference equations from Eq. (III.9), the same technique is used as was done in Section II.B. 2. The difference equations derived inside the fuel pin are the "mesh-centered difference equations." $3,4,5,6$ These equations are derived in the following manner.

Let $T_{f, i}(t)$ denote the average $f u e l$ temperature in region $i, \rho_{f} C_{f, i}$ denote $\rho_{f} C_{f}(r)$ in region $i$, and $k_{f, i}$ denote $k_{f}(r)$ in region $i$. Then Eq.' (III.9) becomes

$$
\begin{gathered}
\rho_{f} C_{f, i} \frac{\left(r_{i+1}{ }^{2}-r_{i}^{2}\right)}{2} \frac{\partial}{\partial t} T_{f, i}(t)=\left.r_{i+1} k_{f, i} \frac{\partial}{\partial r} T_{f}(r, t)\right|_{r_{i+1}} \\
-\left.r_{i} k_{f, i} \frac{\partial}{\partial r} T_{f}(r, t)\right|_{r_{i}}+q_{i} \frac{\left(\underline{r}_{1+1}{ }^{2}-r_{1}{ }^{2}\right)}{2}
\end{gathered}
$$

where the calculation of $q_{i}$ is described in Section III.B.5. To obtain difference equations, a relationship must be found between the derivative of the fuel temperature at a point and the adfacent average temperatures. The following coupling relationship is obtained: 


$$
\begin{aligned}
& \left.k_{f, i-1} \frac{\partial}{\partial r} T_{f}(r, t)\right|_{r_{i-1}}= \\
& \frac{2 k_{f, i-1} k_{f, i}}{\Delta r_{f}\left(k_{f, i-1}+k_{f, i}\right)}\left(T_{f, i}(t)-T_{f, i-1}(t)\right)
\end{aligned}
$$

The difference equation for the innermost fuel node is therefore

$$
\begin{aligned}
& \rho_{\mathrm{f}} C_{\mathrm{f}, 1} \frac{\Delta \mathrm{r}_{\mathrm{f}}^{2}}{2} \frac{\partial}{\partial t} \mathrm{~T}_{\mathrm{f}, 1}(\mathrm{t})=\frac{2 \mathrm{k}_{\mathrm{f}, 1_{\mathrm{f}, 2}}}{\left(\mathrm{k}_{\mathrm{f}, 1}+\mathrm{k}_{\mathrm{f}, 2}\right)} \cdot \\
& \cdot\left\{\mathrm{T}_{\mathrm{f}, 2}(\mathrm{t})-\mathrm{T}_{\mathrm{f}, 1}(\mathrm{t})\right\}+\mathrm{q}_{1} \frac{\Delta \mathrm{r}_{\mathrm{f}}^{2}}{2}
\end{aligned}
$$

The difference equation for an internal fuel node is

$$
\begin{aligned}
& \rho_{f} C_{f, i} \frac{\left(r_{i+1}^{2}-r_{i}^{2}\right)}{2} \frac{\partial}{\partial t} \cdot T_{f, i}(t)=
\end{aligned}
$$

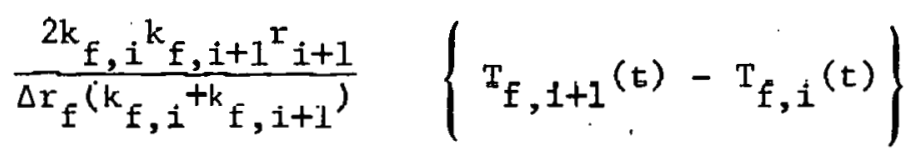

$$
\begin{aligned}
& -\frac{2 k_{f, i-1} k_{f, i} r_{i}}{\Delta L_{f}\left(k_{f, i-1}+k_{f, i}\right)} \quad\left\{T_{f, 1}(t)-T_{f, i-1}(t)\right\}+q_{i} \frac{\left(r_{i+1}^{2}-r_{i}^{2}\right)}{2}
\end{aligned}
$$

To obtain the difference equation for $T_{f, I}(t)$, the derivative at $r=r_{f}$ must be evaluated. Note that $\mathrm{T}_{\mathrm{f}, \mathrm{I}}(\mathrm{t})$ is located $i$ in the middle of the last. fuel node. Beginning with Fourier's equation

$$
q^{\prime \prime}\left(r_{f}\right)=-\left.k_{f} \frac{\partial T}{\partial r}\right|_{r_{f}}
$$

To evaluate $q$ " at $r_{f}$, the following equation is used

$$
q^{\prime \prime}\left(r_{f}\right)=\frac{T_{f, I}(t)-T_{c i}(t)}{R_{g}}
$$


where $\mathrm{R}_{\mathrm{g}}$ is the effective gap resistance

$$
R_{g}=\frac{1}{h_{g a p}}+\frac{\Delta r_{f}}{2 k_{f, I}}
$$

Therefore

$$
\left.\frac{\partial T}{\partial r}\right|_{r_{f}}=-\frac{T_{f, I}(t)-T_{c i}(t)}{k_{f, I} R_{g}}
$$

Therefore the difference equation for $T_{f, I}(t)$ is

$$
\begin{aligned}
& \rho_{f} C_{f, I} \frac{\left(r_{f}^{2}-r_{I-I}^{2}\right)}{2} \frac{\partial}{\partial t} T_{f, I}(t)=-\frac{r_{f}}{R_{g}}\left(T_{f, I}(t)-T_{c i}(t)\right) \\
& -\frac{2 r_{I-1} k_{f, I-1} k_{f, I}}{\Delta r_{f}\left(k_{f, I-1}+k_{f, I}\right)}\left(T_{f, I}(t)-T_{f, I-1}(t)\right)+q_{i} \frac{\left(r_{f}{ }^{2}-r_{I-I}{ }^{2}\right)}{2}
\end{aligned}
$$

where $r_{f}=r_{I}$ and $r_{I-1}=r_{f}-\Delta r_{f}$.

Inside the cladding, the analog to Eq. (III.7) is

$$
\rho_{c} c_{c} \frac{\partial}{\partial t} T_{c l}(r, t)=\frac{1}{r} \frac{\partial}{\partial r} k_{c} \frac{\partial}{\partial r} T_{c l}(r, t)
$$

where

$$
\begin{aligned}
& \mathrm{T}_{\mathrm{cl}}(\mathrm{r}, \mathrm{t}) \text { is the cladding temperature at radius } \mathrm{r} \text { and } \\
& \text { time } \mathrm{t}\left({ }^{\circ} \mathrm{C}\right)
\end{aligned}
$$

and the subscript $c$ l denotes cladding.

The cladding is divided into three unknown temperatures; $T_{c i}(t)$ at the inside of the cladding, $\mathrm{T}_{\mathrm{cm}}(\mathrm{t})$ at the middle of the cladding, and $\mathrm{T}_{\mathrm{cs}}(\mathrm{t})$ at the outside of the clad (assumed known). Using the same techniques as described above, the difference equations for the unknown cladding tempcraturce will now be written:

$$
\begin{aligned}
& \rho_{c} c_{c} \frac{\left(r_{c m l}^{2}-r_{c i}^{2}\right)}{2} \frac{\partial}{\partial t} T_{c i}(t)=\frac{2 \cdot r_{c m l} k_{c}}{\Delta r}\left(T_{c m}(t)-T_{c i}(t)\right) \\
& +\frac{r_{c i}}{R_{g}}\left(T_{f, I}(t)-T_{c i}(t)\right)
\end{aligned}
$$




$$
\begin{aligned}
& \rho_{c} c_{c} \frac{\left(r^{2}{ }_{c m}-r^{2}{ }_{c m l}\right)}{2} \frac{\partial}{\partial t} T_{c m}(t)=\frac{2 r_{c m 2} k_{c}}{\Delta r}\left(T_{c s}(t)-T_{c m}(t)\right) \\
& -\frac{2 r_{c m l} k}{\Delta r}\left(T_{c m}(t)-T_{c i}(t)\right)
\end{aligned}
$$

where

$$
\begin{aligned}
& r_{c m 1}=r_{c i}+.25\left(r_{c s}-r_{c i}\right) \\
& r_{c m 2}=r_{c i}+.75\left(r_{c s}-r_{c i}\right)
\end{aligned}
$$

\section{B.2 Solution of the Fue1 Temperature Equationo}

The fuel thermal conductivity and specific heat density are polynomial functions of the average fuel temperature in this fuel region. In steady state, the fuel temperatures are solved iteratively until a converged solution is found. For the transient solution, the equations derived above are differenced according to the fully implicit method. The value $q$ used in the difference equatione io calculated from the integral over this reactivity step of a quadratic function of the amplitude $T(t)$ (as described in Section III.A.1). Initially, the values $\rho_{f} C_{f}$ and $k_{f}$ are estimated by solving Eqs. (III.2) and (III.8) using the fuel temperatures present at the beginning of the reactivity step. These values are then used for the remainder of this reactivity step. The equations are then solved using the above-described modification of the fully implicit method.

The fuel and cladding equations comprioe a set of ( $I+2)$ equations in $(I+2)$ unknowns, whose matrix structure is tridiagonal. This matrix problem is easily factorized and solved. ${ }^{6}$

For feedback purposes, the average fuel temperature is required. This temperature is calculated from

$$
T_{f, a v}(t)=\frac{\sum_{i=1}^{I}\left(r_{i-1}+r_{i}\right) T_{f, i}(t)}{\sum_{i=1}^{I}\left(r_{i-1}+r_{i}\right)}
$$




\section{B.3 Derivation of Coolant Temperature Equations}

The development of the coolant temperature equations follows closely the development given in Ref. 15. The average coolant properties for each axial mesh region will be calculated; and if the user requests, the average structure temperature also.

As described above, the axial dimension is split into a series of axial mesh regions, with the assumption being made that the material properties are uniform over each segment. If some segment $k$ has zero fission power, the fluid simply transports upward with no change in temperature.

The fuel temperatures calculated above represent average fuel temperatures over each axial mesh region. It will be convenient, however, to calculate coolant temperatures only at the interfaces $k$ and $k+1$ below and above segment $k$. To obtain the average coolant temperature, the below and above temperatures are averaged. equations: ${ }^{15}$

The development begins with the continuity of mass and energy

$$
\begin{aligned}
& \frac{\partial \rho_{\ell}(z, t)}{\partial t}+\frac{\partial G(z, t)}{\partial z}=0 \\
& \\
& \frac{\partial}{\partial t}\left[\rho_{\ell}(z, t) H(z, t)\right]+\frac{\partial}{\partial z}[G(z, t) H(z, t)]=\gamma \psi(z, t)
\end{aligned}
$$

where

$$
\begin{aligned}
& \rho_{\ell}(z, t) \quad \text { is the coolant density }\left(\mathrm{g} / \mathrm{cm}^{3}\right) \\
& G(z, t) \quad \begin{array}{l}
\text { is the coolant mass flow rate for this channel } \\
\left(\mathrm{g} / \mathrm{cm}^{2} \mathrm{sec}\right)
\end{array} \\
& \mathrm{H}(\mathrm{z}, \mathrm{t}) \quad \text { is the coolant enthalpy (watt-sec/g) } \\
& \gamma \quad \begin{array}{l}
\text { is the ratio of the surface area of cladding } \\
\text { to the volume of the coolant (cm } 1)
\end{array} \\
& \left.\psi(z, t) \quad \text { is the heat. flux (watts } / \mathrm{cm}^{2}\right)
\end{aligned}
$$

The assumptions are made that $G(z, t)$ and $\rho_{\ell}(z, t)$ are constant within this axial mesh region, therefore combining the above equations gives

$$
\rho_{\ell} \frac{\partial}{\partial t} H(z, t)+G \frac{\partial}{\partial z} H(z, t)=\gamma \psi(z, t)
$$


For a non-boiling coolant,

$$
\mathrm{dH}(z, t)=\mathrm{C}_{\ell} \mathrm{dT}_{\mathrm{c}}(z, t)
$$

where

$$
\begin{aligned}
& \mathrm{C}_{\ell} \quad \text { is the coolant specific heat } \\
& \text { (watt-sec/g } /{ }^{\circ} \mathrm{C} \text { ) } \\
& T_{c}(z, t) \text { is the coolant temperature }\left({ }^{\circ} \mathrm{C}\right)
\end{aligned}
$$

The quantity $C_{\ell}$ is also assumed to be a constant over this axial mesh region. Combining Eqs. (III.18) and (III.19) yields

$$
\rho_{\ell} C_{\ell} \frac{\partial}{\partial t} T_{C}(z, t)+G C_{\ell} \frac{\partial}{\partial z} T_{C}(z, t)=\gamma \psi(z, t)
$$

The time dependent equation for the structure temperature is ${ }^{15}$

$$
\tau_{s} \rho_{s} C_{s} \frac{\partial}{\partial t} T_{s}(z, t)=h_{s}\left[T_{c}(z, t)-T_{s}(z, t)\right]
$$

where

$$
\begin{aligned}
& \mathrm{T}_{\mathrm{S}}(\mathrm{z}, \mathrm{t}) \text { is the structure temperature }\left({ }^{\circ} \mathrm{C}\right) \\
& \tau_{s} \text { is the ratio of structure volume to surface } \\
& \text { area in contact with coolant (cm) } \\
& \begin{array}{l}
\rho_{s} C_{s} \quad \text { is the specific heat density for the structure } \\
\text { (watt-sec/ } / \mathrm{cm}^{3}{ }^{\circ} \mathrm{C} \text { ) }
\end{array} \\
& h_{s}=\left(\frac{1}{h_{c}}+R_{s t}\right)^{-1} \\
& R_{\text {st }} \quad \text { is the thermal resistance of the structure } \\
& h_{c} \quad \text { is the convection-film heat transfer coefficient }
\end{aligned}
$$

FX2-TH allows the user to specify a time dependence for the coolant mass flow rate $G(z, t)$.

Let

$$
G(z, t)=G_{m} \cdot f
$$

where

$$
\begin{aligned}
& \mathrm{G}_{\mathrm{m}} \quad \text { is the initial mass flow rate for thermal- } \\
& \text { hydraulic channel } \mathrm{m}
\end{aligned}
$$


and

$$
f=1.0+\alpha_{1}\left(t-t_{s}\right)+\alpha_{2}\left(t-t_{s}\right)^{2}
$$

where

$$
\begin{array}{ll}
\alpha_{1}, \alpha_{2} & \text { are input variables } \\
t_{s} & \text { is the time the coolant flow rate variation } \\
& \text { is to begin. }
\end{array}
$$

For all times $t<t_{S}$, $f$ is set to unity.

In the steady-state, Eq. (III. 20) reduces to

$$
G C_{\ell} \frac{\partial}{\partial z} T_{C}(z, 0)=\gamma \psi(z, 0)
$$

In steady-state, all power goes toward heating the coolant. Therefore the following expression is correct:

$$
\gamma \psi(z, 0)=\frac{P(j)}{\Delta z_{j} A_{c}}
$$

where

$$
\begin{aligned}
& P(j) \quad \text { is the total power produced in this fuel pin in } \\
& \text { axial mesh region } j \text { (watts) } \\
& \Delta z_{j} \quad \text { is the length of axial mesh region } j \\
& A_{C} \quad \text { is the coolant flow area }\left(\mathrm{cm}^{2}\right)
\end{aligned}
$$

All the data required to solve Eq. (III.22) are now known, therefore integrate it from $\mathrm{Z}_{\mathrm{k}}$ to $\mathrm{Z}_{\mathrm{k}+1}$ to obtain:

$$
T_{c}\left(z_{k+1}, 0\right)=T_{c}\left(z_{k}, 0\right)+\frac{\Delta z_{k} \gamma \psi(z, 0)}{G C_{\ell}}
$$

where $\gamma \psi(Z, 0)$ is given by Eq. (III.23). The steady-state average coolant temperature in axial mesh region $k$ is then

$$
T_{c, k}(0)=\frac{T_{c}\left(z_{k+1}, 0\right)+T_{c}\left(z_{k}, 0\right)}{2}
$$

and the steady-state averageistructure temperature is

$$
\mathrm{T}_{s, k}(0)=\mathrm{T}_{\mathrm{c}, \mathrm{k}}(0)
$$

During a translent, the heat flux has components from both the fuel pin and the structure: 


$$
\psi(7, t)=\psi_{c}(z, t)+\gamma_{2} \psi_{s}(z, t)
$$

where

$$
\begin{aligned}
& \psi_{c}(Z, t)=\frac{T_{c m}(Z, t)-T_{c}(Z, t)}{R_{e c}(Z, t)} \\
& \psi_{s}(Z, t)=\frac{T_{s}(Z, t)-T_{c}(Z, t)}{R_{s c}(Z, t)}
\end{aligned}
$$

and

$$
\begin{aligned}
& \mathrm{T}_{\mathrm{cm}}(\mathrm{Z}, \mathrm{t}) \text { is the clad midpoint temperature at } \mathrm{Z} \text { and } \mathrm{t}\left({ }^{\circ} \mathrm{C}\right) \\
& \left.T_{c}(z, t) \text { is the coolant bulk temperature at } z \text { and } t r^{\circ} \mathrm{r}\right) \\
& R_{e c}(z, t)=\frac{1}{h_{c}}+\frac{\Delta r_{c}}{2 k_{c}} \\
& \begin{array}{l}
\text { he is the convection-film heat transfer coneficiont } \\
\text { (watts } / \mathrm{cm}^{2}{ }^{\circ} \mathrm{C} \text { ) }
\end{array} \\
& \Delta \mathrm{r}_{\mathrm{c}} \text { is the clad thickness }(\mathrm{cm}) \\
& k_{c} \text { is the clad thermal conductivity (watt } / \mathrm{cm}{ }^{\circ} \mathrm{C} \text { ) } \\
& \mathrm{T}_{s}(\mathrm{Z}, \mathrm{t}) \quad \text { is the structure temperature at } \mathrm{Z} \text { and } \mathrm{t}\left({ }^{\circ} \mathrm{C}\right) \\
& R_{s c}(z, t)=\frac{1}{h_{c}}+R_{s t} \\
& R_{s t} \quad \text { is the thermal resistance of the structure } \\
& \gamma_{2} \quad \text { is the ratio of the surface area of structure } \\
& \text { to the surface area of cladding. }
\end{aligned}
$$

Eqs. (III. 20) and (III. 21) are now solved for this reactivity step. The values $\rho_{\ell}$ and $C_{\ell}$ are each expanded as polynomial functions of the average coolant temperature, as

$$
\begin{aligned}
& \rho_{\ell, k}(t)=\rho_{\ell}{ }^{0}+\rho_{\ell}{ }^{1}\left(T_{c, k}(t)\right)+\rho_{\ell}{ }^{2}\left(T_{c, k}(t)\right)^{2} \\
& C_{\ell, k}(t)=C_{\ell}{ }^{0}+C_{\ell}{ }^{1}\left(T_{c, k}(t)\right)+C_{\ell}{ }^{2}\left(T_{c, k}(t)\right)^{2}
\end{aligned}
$$


The average coolant temperature used to evaluate these expressions is extrapolated from past history. Let $T_{c, k}^{i}$ be the coolant temperature at axial level $k$ and reactivity step $i$. Then the extrapolated average coolant temperature at reactivity step $i+1$ is:

$$
T_{c, k+\frac{1}{2}}^{i+1}=T_{c, k}^{i+1}+\frac{1}{2} \quad\left\{T_{c, k+1}^{i}-T_{c, k}^{i}\right\}
$$

Eqs. (III. 20) and (III. 21) are then solved using the fully implicit method, to yield:

$$
\begin{aligned}
& T_{c, k+1}^{i+1}=\left\{\frac{\rho_{\ell} C_{\ell}}{\Delta t_{j, i}}+\frac{G C_{\ell}}{\Delta Z_{k}}\right\}^{-1} \\
& \cdot\left\{\frac{\rho_{\ell} C_{\ell}}{\Delta t_{j, i}} T_{c, k+1}^{i}+\frac{G C_{\ell}}{\Delta Z_{k}} T_{c, k}^{i+1}+\gamma \psi(k, i+1)\right\} \\
& T_{s, k}^{i+1}=\left\{\frac{\tau_{s} \rho_{s} C_{s}}{\Delta t_{j, i}}+h_{s}\right\}-1\left\{\frac{\tau_{s} \rho_{s} C_{s}}{\Delta t_{j, i}} T_{s, k}^{i}+h_{s} T_{c, k}^{i+1}\right\}
\end{aligned}
$$

The fuel temperature and coolant temperature calculations are linked by two properties, the heat flux and the clad surface temperature. The clad surface temperature is found from

$$
T_{c s}(z, t)=T_{c}(z, t)+\psi_{c}(z, t) / h_{c}
$$

The heat flux $\psi_{c}(z, t)$ calculated above can be used with the calculated value of $T_{c}(z, t)$ to calculate the new clad surface temperature $T_{c s}(Z, t)$. The fuel temperature equations can then be solved.

To calculate the heat transfer coefficient $h_{c}$, a general correlation is used which is applicable to several coolants. Let

$$
\mathrm{Nu}=\mathrm{C}_{1}+\mathrm{C}_{2}(\mathrm{Re})^{\mathrm{C}_{3}}(\mathrm{Pr})^{\mathrm{C}_{4}}
$$

where

$$
C_{1}, C_{2}, C_{3}, C_{4} \text { are user-specifled constants }
$$

$\mathrm{Nu} \quad$ is the Nusselt number

$\operatorname{Re}$ is the Reynolds number

$\operatorname{Pr} \quad$ is the Prandt1 number 
These dimensionless quantities are defined as

$$
\begin{aligned}
& \mathrm{Nu}=\frac{h_{c} D_{e}}{k} \\
& \operatorname{Re}=\frac{D_{\dot{e}} G}{\mu} \\
& \operatorname{Pr}=\frac{C_{p} \mu}{k}
\end{aligned}
$$

where

$$
\begin{aligned}
& \mathrm{h}_{\mathrm{c}} \quad \begin{array}{l}
\text { is the heat transfer coefficient } \\
\text { (watt } / \mathrm{cm}^{2}{ }^{\circ} \mathrm{C} \text { ) }
\end{array} \\
& \mathrm{D}_{\mathrm{e}} \quad \text { is the hydraulic diameter }(\mathrm{cm}) \\
& \mathrm{k} \quad \text { is the thermal conductivity of the coolant (watt } / \mathrm{cm}{ }^{\circ} \mathrm{C} \text { ) } \\
& \mathrm{G} \quad \text { is the mass flow rate of the coolant }\left(\mathrm{g} / \mathrm{cm}^{2} \mathrm{sec}\right. \text { ) } \\
& \mu \quad \text { is the viscosity of the coolant (g/cm sec) } \\
& \left.\mathrm{C}_{\mathrm{p}} \quad \text { is the specific heat of the coolant (watt-sec/g }{ }^{\circ} \mathrm{C}\right)
\end{aligned}
$$

The hydraulic diameter $\mathrm{D}_{\mathrm{e}}$ is calculated from

$$
D_{e}=\frac{4 A_{c}}{2 \pi r_{c s}\left(I+r_{2}\right)}
$$

where

$A_{c}$ is the area of the coolant $\left(\mathrm{cm}^{2}\right)$

$$
A_{c}=\frac{V_{f c} \pi r^{2} c s}{1-V_{f c}}
$$

$\mathrm{V}_{\mathrm{fc}}$ is the volume fraction of the coolant.

The coolant viscosity is also represented as a polynnmial function of the average coolant temperature, as

$$
\mu_{k}(t)=\mu(0)+\mu^{(1)}\left(T_{c, k}(t)\right)+\mu^{(2)}\left(T_{c, k}(t)\right)^{2}
$$




\section{B.4 Steady State and Transient Calculation of Fuel and Coolant Temperatures}

In steady state, the heat flux is calculated from Eq. (III.23). is an analytical (and therefore exact) relationship. Once the transient begins, Eqs. (III.25, 26, 27) are used to calculate the heat flux. These expressions are dependent on the cladding midpoint temperature, which is found using a finite difference approximation to the heat conduction equation. Therefore, the two expressions are not in complete agreement. In order for there to be no spurious "drift" when the transient is begun, the steady state solution strategy has been devised such that the fuel temperatures and coolant temperatures are 1 terated to convergence, using Eqs. (III.25, 26, 27) to calculate the heat flux.

For the transient calculation, the heat flux at this reactivity step is first found. This is done by assuming that the heat flux determined by Eqs. (III. 25, 26, 27) using temperatures present at the beginning of the reactivity step is sufficlently accurate for the calculation of the coolant temperatures across this reactivity step. The SAS $2 A$ code ${ }^{15}$ uses an extrapolated value of heat $f l u x$ based on past history. Numerical tests performed on the FX2-TH detailed model using such an extrapolation demonstrated that when large reactivity steps were taken, the coolant temperatures calculated could be significantly in error. therefore, an extrapolation of this kind is not performed in FX2-TH. Once the coolant properties for this reactivity step are found, the heat transfer coefficient $\left(h_{c}\right)$ and cladding surface temperature $\left(T_{c s}\right)$ are calculated, and the resulting fuel temperatures calculated.

Figure 5 shows a flowchart of the overall thermal-hydraulic solution strategy. Figure 6 shows a flowchart of the steady-state solution strategy for the detailed model. Figure 7 shows a flowchart of the transient solution strategy for the detailed model.

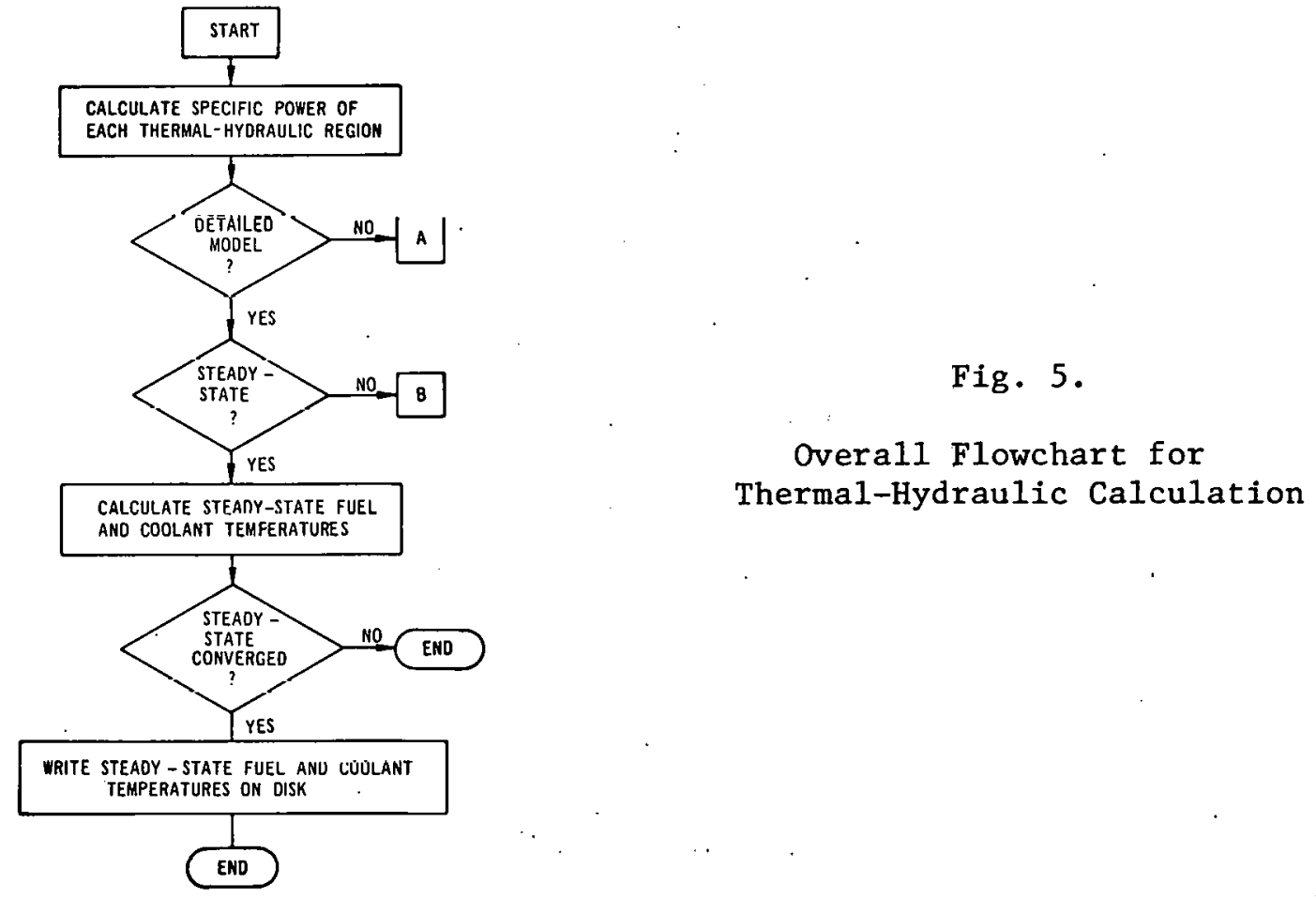



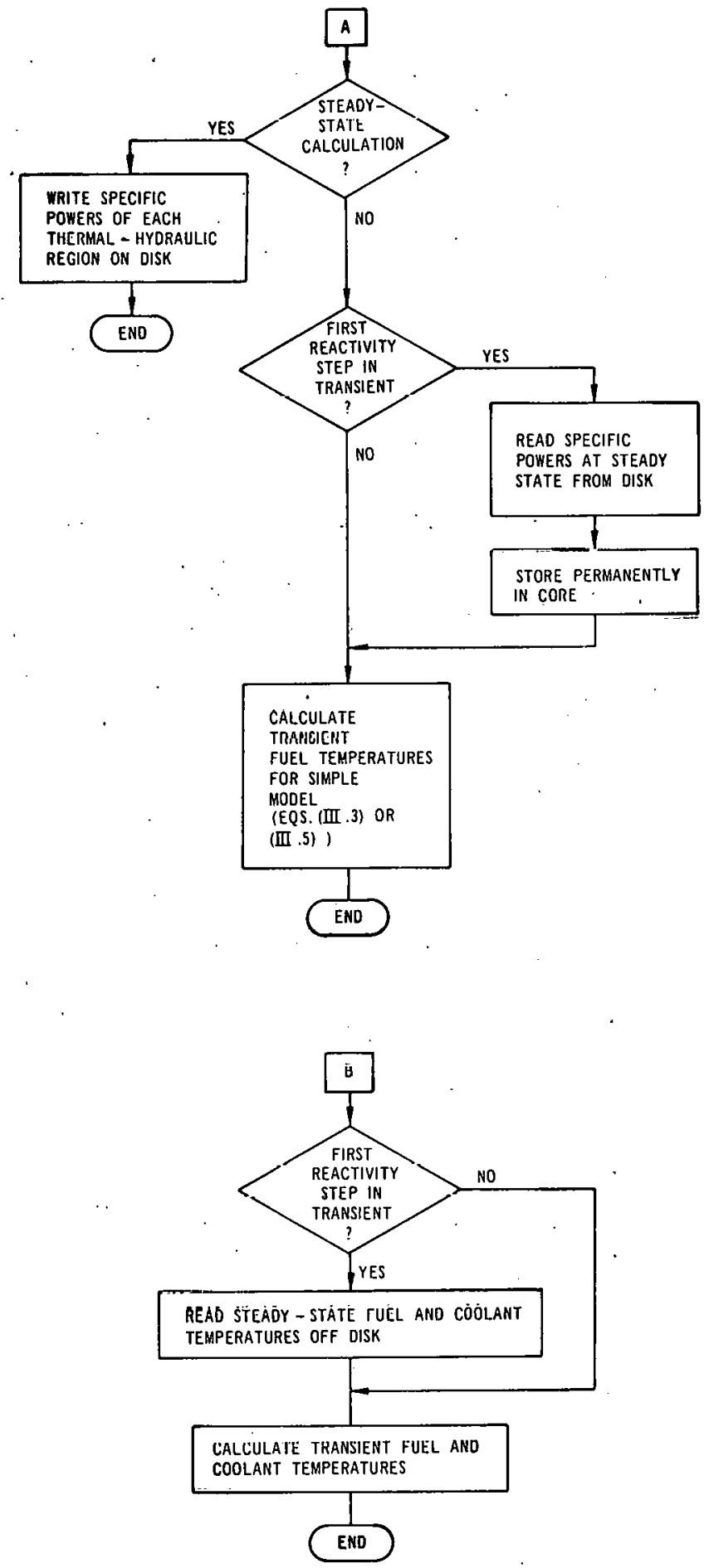

Fig. 5. (Contd) 


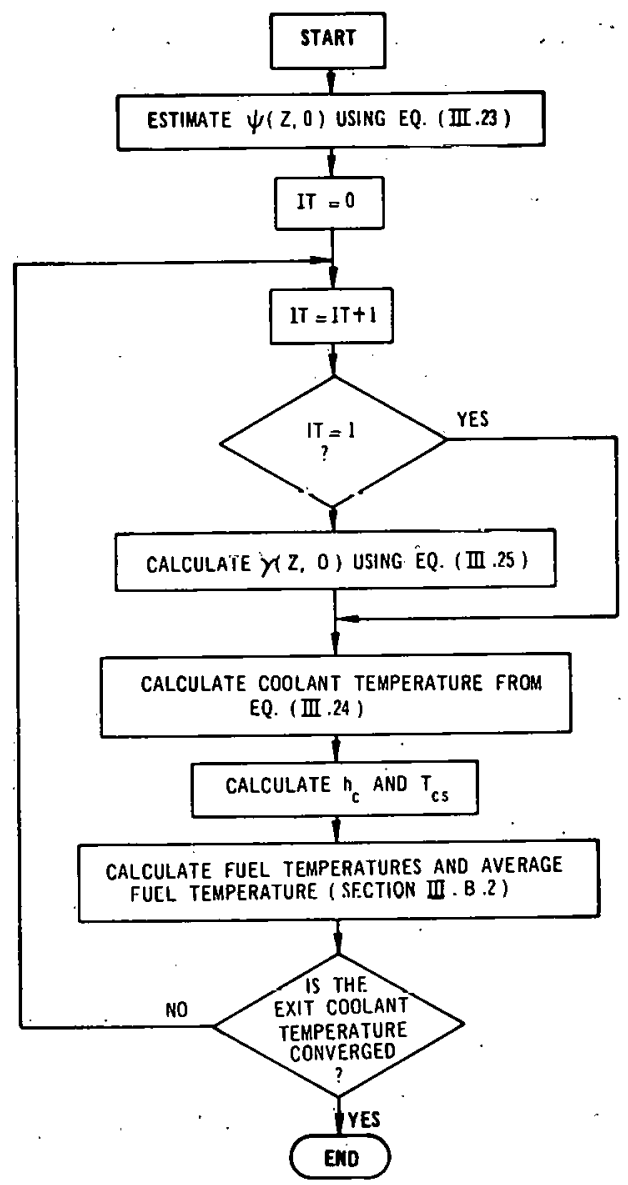

Fig. 6. Flowchät of Steady-State Calculation of Fuel and Coolant Temperatures for One Thermal-Hydraulic Channel of the Detailed Model

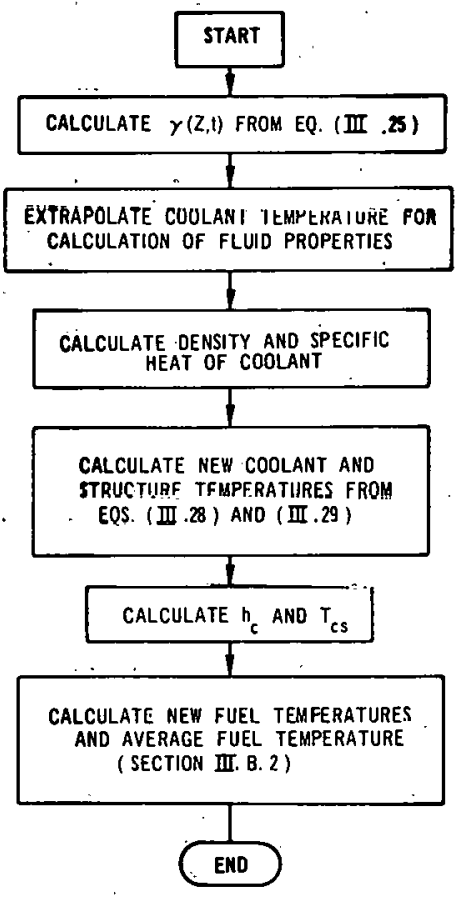

Fig. 7. Flowchart of Transient Calculation of Fuel and Coolant Temperatures for One Thermal-Hydraulic Channel of the Detailed Model 


\section{B.5. Callculation of Volumetric Power Generation Rate}

In the fuel temperature equations for the detailed model, the volumetric power generation rate $q(r, t)$ is $r$ equired. For each thermal-hydraulic region $k$, the following equation is used

$$
q_{k}(t)=\frac{\left(3.20 \times 10^{-11}\right) \sum_{g=1}^{G} \int_{v_{k}} \Sigma_{f g}(\underline{r}, t) \phi_{g}(\underline{r}, t) d V}{N P I N \cdot h_{k} \cdot \pi r_{f}{ }^{2}}
$$

where

$$
\begin{aligned}
& \text { NPIN is the total number of fuel pins in thermal-hydraulic } \\
& \text { region } k \\
& h_{k} \quad \text { is the height (axially) nf thermal-hydraulic regiun } k \\
& r_{f} \quad \text { is the radius of the fuel pin }
\end{aligned}
$$

In equations such as (III, 10) and (III. 12), the value $q_{i}$ is required, where $q_{i}$ is the volumetric power generatinn rate in fucl node $i$. Bluce the power is assumed to be generated uniformly in the fuel pin, $q_{i}=q_{k}$ for all fuel
nodes $i$.

\section{B.6 Accuracy Considerations of the Detailed Mode1}

In the development of the detailed thermal-hydraulic model for FX2-TH, an extensive model verification procedure was undertaken. The results of this procedure will be summarized in this Section. Errors present in the detailed model can come from two basic sourcca; errors inlroduced due to the ascumptions made to derive the model, and errors iulruduced due to the approximate solution of the pquatinns. Tho moot gigntficaul crrors discovered in this investigation will now be discussed.

The most significant error inherent in the model generally comes from the restriction that no fuel or cladding motion dip to thermal crpansion is allowed. Comparison of results betwcen FX2-TII and $S \Lambda S 2 \Lambda^{15}$ reveals that care must be taken in setting up the thermal-hydraulic model in FX2-TH so that the results using FY2-TH will be iil yuud agreement with more detailed calculations. The most significant parameters which must be matched between FX2-TH and a more accurate therma1-hydraulics calculation are the width of the gap ( $\tau_{\text {gap }}$ ) and the conductivity of the gip ( $h_{\text {gap }}$ ). In particular, a fuel pin located in a very high-power region of the reactor core will probably have a smaller $\tau_{g a p}$ (and hence a large $h_{\text {gap }}$ ) than a similar pin located in a low-power region of the core. FX2-TH has the capability of allowing more than one set of thermal-hydraultc input parameters, and this is useful in matching the proper fuel pin dimensions to their approprlate position in the core. 
In addition, the power distribution of a reactor can have significant axial variations; thus a fuel pin's outer radius can vary axially. To account for this variation, FX2-TH allows the user to input a set of "axial correction Factors" to the gap conductivity hap. The value of "$h_{\text {gap }}$ used for axial location $k$ is then

$$
\mathrm{h}_{\text {gap, } \mathrm{k}}=\mathrm{h}_{\mathrm{gap}} / \mathrm{h}_{\operatorname{corr}, \mathrm{k}}
$$

where

$$
\begin{aligned}
& h_{\text {gap }} \text { is the input value of } h_{\text {gap }} \text { for this fuel pin } \\
& h_{\text {corr }, k} \text { is the correction factor for axial level } k
\end{aligned}
$$

One set of correction factors is entered for all fuel pins. Therefore, through the combination of adjusting the $f u e l$ pin dimensions and the axial correction factors, the user can adjust the FX2-TH input parameters to match more detailed calculations. Also, these input values can be set in such a way as to guarantee a conservative safety analysis result.

The most significant error incurred in the numerical solution of the thermal-hydraulic equations is the effect of a finite difference approximation to the heat conduction equation inside the $f$ uel pin. It has been found that four or five radial fuel nodes are sufficient to keep errors in average fuel temperature below one or two percent for the duration of the transient. 


\section{REPRESENTATION OF THERMAL-HYDRAULIC FEEDBACK EFFECTS}

\section{A. Homogenization of Cross Sections}

The FX2-TH program accepts microscopic cross section data from the CCCC standard interface files ISOTXS and DLAYXS. 16 If upscatter cross sections are present in the ISOTXS file, they will be read correctly by the FX2-TH input processor; however the calculation will not use the upscatter cross sections. Neither a set matrix nor an isotope matrix for the fission spectrum is allowed. Also, no co-ordinate dependent transport cross sections are read by FX2-TH.

The FX2-TH homogenization routines use information from the files ISOTXS, DLAYXS, and FXHMOG to generate the unique material macroscopic cross sections and the temperature correction coefficients. The material cross sections are mixed in the usual way

$$
\Sigma_{m, g}=\sum_{i=1}^{\text {NISO }} \sigma_{i, g} N_{i, m}
$$

i.e. the product of the atom density and micrnsonpis cross ocction are summed over all isotopes comprising that unique material. The specific form of the terms $\Sigma_{m, g}$ and $N_{i, m}$ in the presence of temperature feedback will be discussed in the next section. The material prompt fission spectrum is computed as follows

$$
x_{m, g}=\frac{\sum_{i=1}^{N I S O} x_{i, g}{ }^{N} i, m g_{g^{\prime}=1}^{N G}\left(v \sigma_{f}\right)_{g}^{i}}{N_{i, m} \sum_{g^{i}=1}^{N G}\left(v \sigma_{f}\right)_{m, g^{\prime}}}
$$

This weighting assumes that the flux is constant in each region. $m$ is the unique material number, $i$ is the isotope number, and $g, g^{\prime}$ are energy group indices.

\section{B. Temperature Effects Upon Cross Sections}

In FX2-TH, feedback effects due to both fuel and coolant temperature changes are calculated. These feedback cffccts will be discussed separately.

The fuel temperatures calculated in FX2-TH affect the microscopic cross sections through Doppler broadening of the resonances. ${ }^{2}$ In FX2-TH, Doppler broadening is assumed to affect only the microscopic capture and fission cross sections. FX2-TH requires that the ISOTXS file contain cross sections for a particular isotope at four different values of fuel temperature. From these values, a general correlation relating the microscopic cross section to the fuel temperature is evaluated: 


$$
\begin{aligned}
& \sum_{\mathrm{m}, \mathrm{g}}^{\mathrm{P}}\left(\mathrm{T}_{\mathrm{f}}\right)=\sum_{\mathrm{m}, \mathrm{g}_{\text {base }}}^{\mathrm{P}}+\mathrm{C}_{\mathrm{m}, \mathrm{g}}^{\mathrm{P}, 1} \cdot\left(\sqrt{\mathrm{T}_{\mathrm{f}}}-\sqrt{\left.\mathrm{T}_{\mathrm{f}_{\text {base }}}\right)}\right. \\
& \quad+\mathrm{C}_{\mathrm{m}, \mathrm{g}}^{\mathrm{P}, 2} \cdot \ln \left(\mathrm{T}_{\mathrm{f}} / \mathrm{T}_{\mathrm{f}_{\text {base }}}\right)+\mathrm{C}_{\mathrm{m}, \mathrm{g}}^{\mathrm{P}, 3} \cdot\left(\frac{1}{\mathrm{~T}_{\mathrm{f}}}-\frac{1}{\mathrm{~T}_{\mathrm{f}_{\text {base }}}}\right)
\end{aligned}
$$

where

$$
\begin{aligned}
& \begin{array}{ll}
\sum_{m, g}^{P}\left(T_{f}\right) & \text { is the macroscopic cross section for process } P \\
& \text { (capture or fission) for malerial } m \text { and group } g
\end{array} \\
& \text { at temperature } \mathrm{T}_{\mathrm{f}} \\
& \sum_{\mathrm{m}, \mathrm{g}_{\text {base }}}^{\mathrm{P}} \\
& \begin{array}{l}
\text { is the macroscopic cross section } \Sigma_{m, g}^{P}\left(T_{f_{\text {base }}}\right) \\
\text { evaluated at } T_{f}
\end{array} \\
& C_{m, i}^{P, i} \\
& \text { f base } \\
& \text { is the } i^{\text {th }} \text { fuel temperature feedback coefficient } \\
& \text { for process } P \text {, material } \mathrm{m} \text { and group } \mathrm{g} \text {. } \\
& \mathrm{T}_{\text {fiase }} \\
& \text { is the base fuel temperature }
\end{aligned}
$$

The feedback coefficients are calculated from the known values of $\Sigma_{m, g}^{P}\left(T_{f}\right)$ at four values of $\mathrm{T}_{\mathrm{f}}$. Each such value $\Sigma_{\mathrm{m}, \mathrm{g}}^{\mathrm{P}}\left(\mathrm{T}_{\mathrm{f}}\right)$ represents a sum over all isotopes in this material, such as Eq. (IV.1).

To represent changes in the coolant temperature, the atom density $\left(\mathrm{N}_{i, \mathrm{~m}}\right)$ of each coolant isotope is allowed to be a function of the coolant temperature, as

$$
N_{i, m}\left(T_{c}\right)=N_{i, m}^{0}+N_{i, m}^{1}\left(T_{c}-T_{c_{b a s e}}\right)
$$

where

$$
\begin{aligned}
& \mathrm{N}_{\mathrm{i}, \mathrm{m}}\left(\mathrm{T}_{\mathrm{c}}\right) \quad \begin{array}{l}
\text { is the atom density for isotope } i \text { in material } \mathrm{m} \\
\text { at temperature } \mathrm{T}_{c}
\end{array} \\
& \mathrm{~N}_{\mathrm{i}, \mathrm{m}}^{0} \quad \begin{array}{l}
\text { is the a tom density } \mathrm{N}_{1, \mathrm{~m}}\left(\mathrm{~T}_{\mathrm{c}_{\text {base }}}\right) \text { evaluated at } \\
\mathrm{T}_{\mathrm{c}_{\text {base }}}
\end{array} \\
& \dot{\mathrm{N}}_{1, \mathrm{~m}}^{1} \quad \text { is the first derivative of the atom density with } \\
& \text { material m. This value is user input. } \\
& \mathrm{T}_{\mathrm{c}_{\mathrm{b}}} \text { is the base coolant temperature }
\end{aligned}
$$


The macroscoplc cross section and coolant temperature feedback coefficient are then calculated as:

$$
\Sigma_{\mathrm{m}, \mathrm{g}}^{\mathrm{P}}\left(\mathrm{T}_{\mathrm{c}}\right)=\Sigma_{\mathrm{m}, \mathrm{g}_{\text {base }}}^{\mathrm{P}}+\mathrm{D}_{\mathrm{m}, \mathrm{g}}^{\mathrm{P}} \cdot\left(\mathrm{T}_{\mathrm{c}}-\mathrm{T}_{\mathrm{c}_{\text {base }}}\right)
$$

where

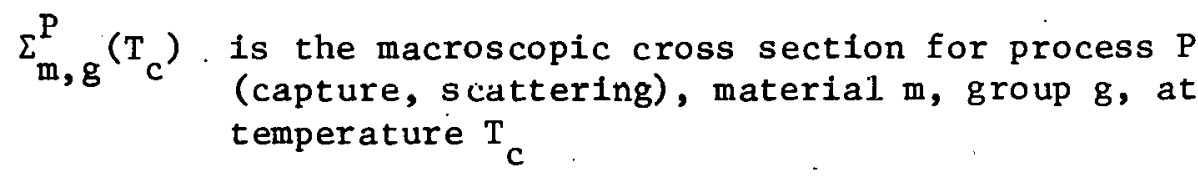

$$
\begin{aligned}
& \Sigma_{m, g_{\text {base }}}^{P}=\sum_{i=1}^{N I S O} \sigma_{i, g}^{P} N_{i, m}^{0} \\
& \mathrm{D}_{\mathrm{in}, \mathrm{g}}^{\mathrm{P}}=\sum_{i=1}^{\mathrm{NISO}} \sigma_{i, g}^{\mathrm{P}} \mathrm{N}_{i, \mathrm{~m}}^{1}
\end{aligned}
$$

Therefore, for materials m representing fuel matcrial, the macruscupic capture and fission cross sections are functions of the fuel temperature through Eq. (IV.3). For materials m representing coolant material, the macroscopic capture and scattering cross sections are functions of the coolant temperature through Eq. (IV.5).

To calculate the temperature dependent transport cross section, the variations from capture and fissinn from both fuel and tcmpcrature are added to the base transport cross section. The diffusion coefficient is then calculated from $\mathrm{D}=1 / 3 \Sigma_{\mathrm{rr}}$.

\section{Overall Solution Strategies for FX2-TH}

\section{C.1 Steady State Calculation}

FX2-TH must calculate the steady-state real and adjoint neutron fluxes, and the steady state thermal-hydraulic conditions. As a result of the thermal-hydraulic feedback equations given in Chapter ITI, the calculation of a consistent set of steady-state conditions is, in general, an iterative process. A flowchart of the steady-state solution strategy used in FX2-TH is given in Figure 8 .

If no thermal-hydraulics calculations are being performed, or if one of the adiabatic fuel temperature models are being used, then the steady-state fuel and coolant temperatures are known from the problem input; and an iteration is not necessary (see Figure 8). If the detailed model is being used, however, then the steady-state real neutron fluxes and thermalhydraulic conditions are solved consecutively until the pointwise reactor powers from two successive iterations agree to within a user-specified amount. When this occurs, the final set of real and adjoint neutron fluxes and thermal-hydraulic conditions are calculated. 
During this steady-state iterative process, the neutron fluxes are being calculated from macroscopic cross sections which were found from the previous (approximate) set of thermal-hydraulic conditions. Therefore, there is no need to converge the neutron flux equations to a high degree of convergence during this iterative process. Before the start of the steady-state iteration, the steady-state convergence criteria are modified. The criterion which determines the maximum change in pointwise fission source vector, the most restrictive of the convergence criteria, is set as

$$
\varepsilon_{f s}=0.5 \varepsilon_{t h}
$$

where

$$
\begin{array}{ll}
\varepsilon_{\mathrm{fs}} & \begin{array}{l}
\text { is the pointwise fission source vector } \\
\text { convergence criterion }
\end{array} \\
\varepsilon_{\text {th }} & \begin{array}{l}
\text { is the user-specified polntwise power } \\
\text { convergence criterion for the steady-state } \\
\text { iteration }
\end{array}
\end{array}
$$

After a converged set of steady-state conditions are found, the steady-state convergence criteria are returned to their user-specified values. The tightly converged real and adjoint neutron fluxes are then calculated, as well as the final set of thermal-hydraulic conditions (see Figure 8 ).

It has been assumed that the 1 terative process described above is a convergent one. If this is not the case, then no unique steady-state condition exists for the given set of FX2-TH input. The iterative process can be guaranteed to converge if the temperature coefficients.

$\frac{\partial \rho}{\partial T_{f}}$ and $\frac{\partial \rho}{\partial T_{c}}$ are both negative (these temperature coefficients are assumed

to represent the overall response of the reactor to changes in temperature). It is possible, however, for a reactor to have one of the above coefficients, for instance $\frac{\partial \rho}{\partial T_{c}}$, be positive; while the other be negative. This situation could occur in a PWR at beginning-of-life with a relatively high initial concentration of soluble boron present in the coolant. Such a reactor could still exhibit an overall negative temperature coefficient if the negative of the fuel temperature coefficient was dominant over that of the coolant temperature. To determine this, a "power coeffictent" is helpful, where $\frac{\partial p}{\partial P_{i}}$ represents the change $i n . \rho$ for a change in reactor power $P_{i}$ at any point 1 in the reactor. This quantity, which includes the effects of fuel and coolant temperature feedback, must be negative for the iterative strategy to converge. (It also must be negative for the reactor to be licensable). 


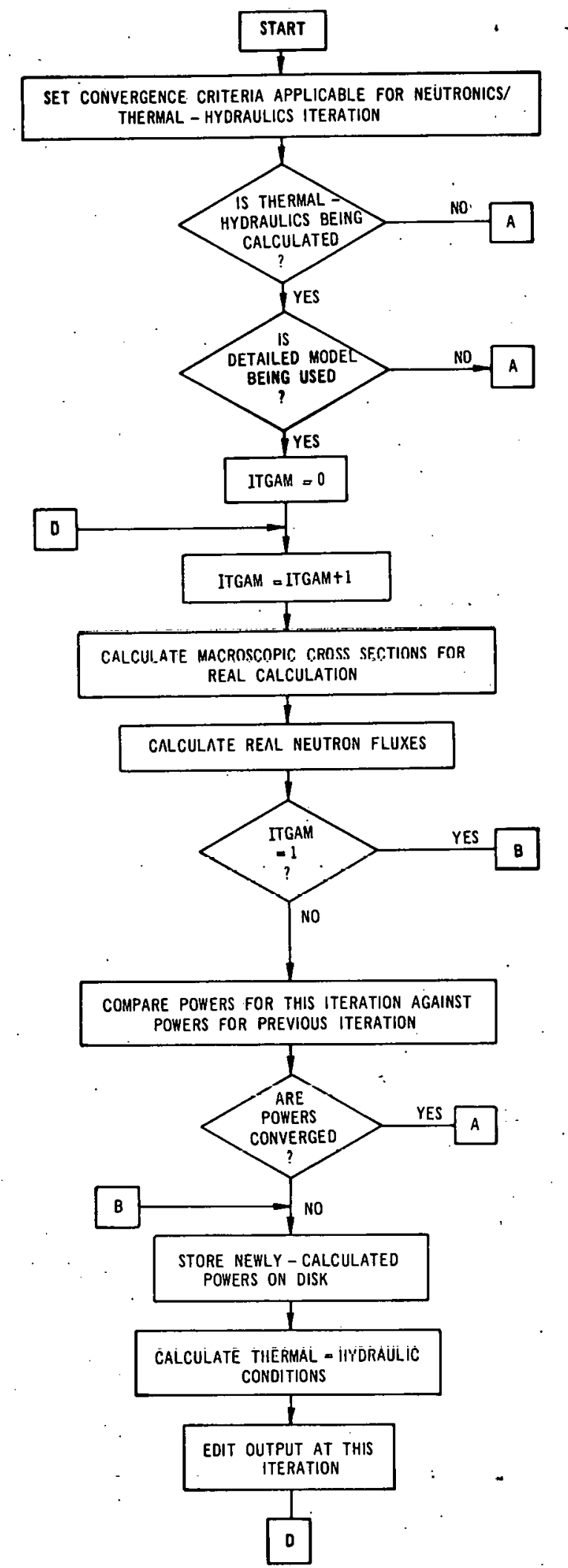

Fig. 8. Flowchart for the Steady-State Calculation in FX2-TH 


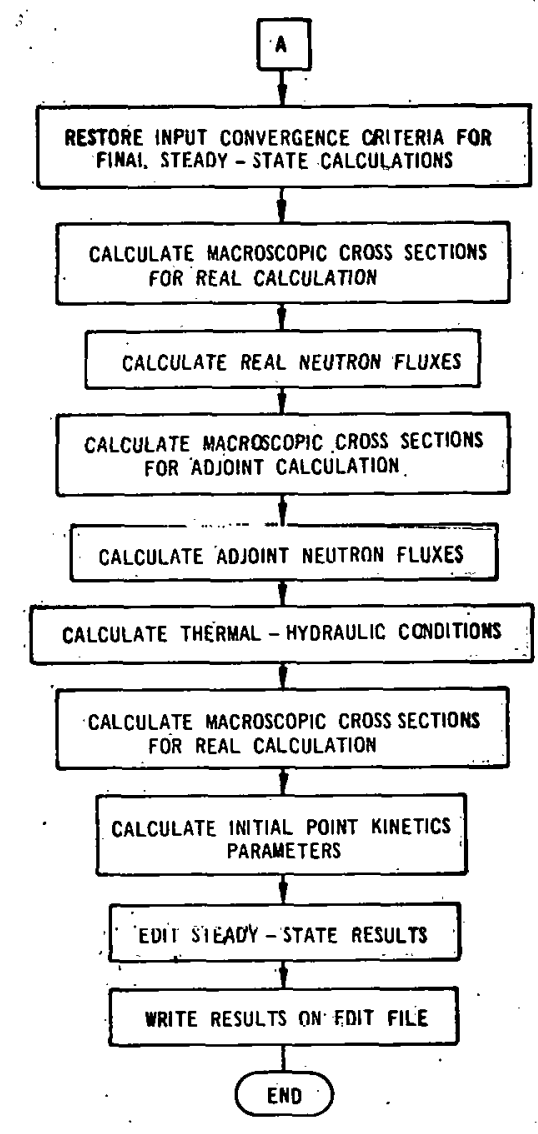

Fig. 8. (Contd)

\section{B.2 Time-Dependent Calculation}

During the transient calculation, the point kinetics equations and thermal-hydraulics equations are solved in tandem at each reactivity step, thus eliminating the need for costly iterations between the neutronics and thermal-hydraulics equations. This approach has proven to work well for several other multidimensional space-time kinetics programs. $6,9,10$ 


\section{A GUIDE TO USER APPLICATIONS}

\section{A. Description of the Cataloged Procedure ARCSP024}

The FX2-TH program is a stand-alone load module. To assist the user in executing this program, the cataloged procedure ARCSP024 has been written to relieve much of the burden of coding job control language when using FX2-TH on an IBM 360 or 370 series computer. A listing of this procedure is given in Figure 9. A description of the symbolic parameters in this procedure is given in Table I.

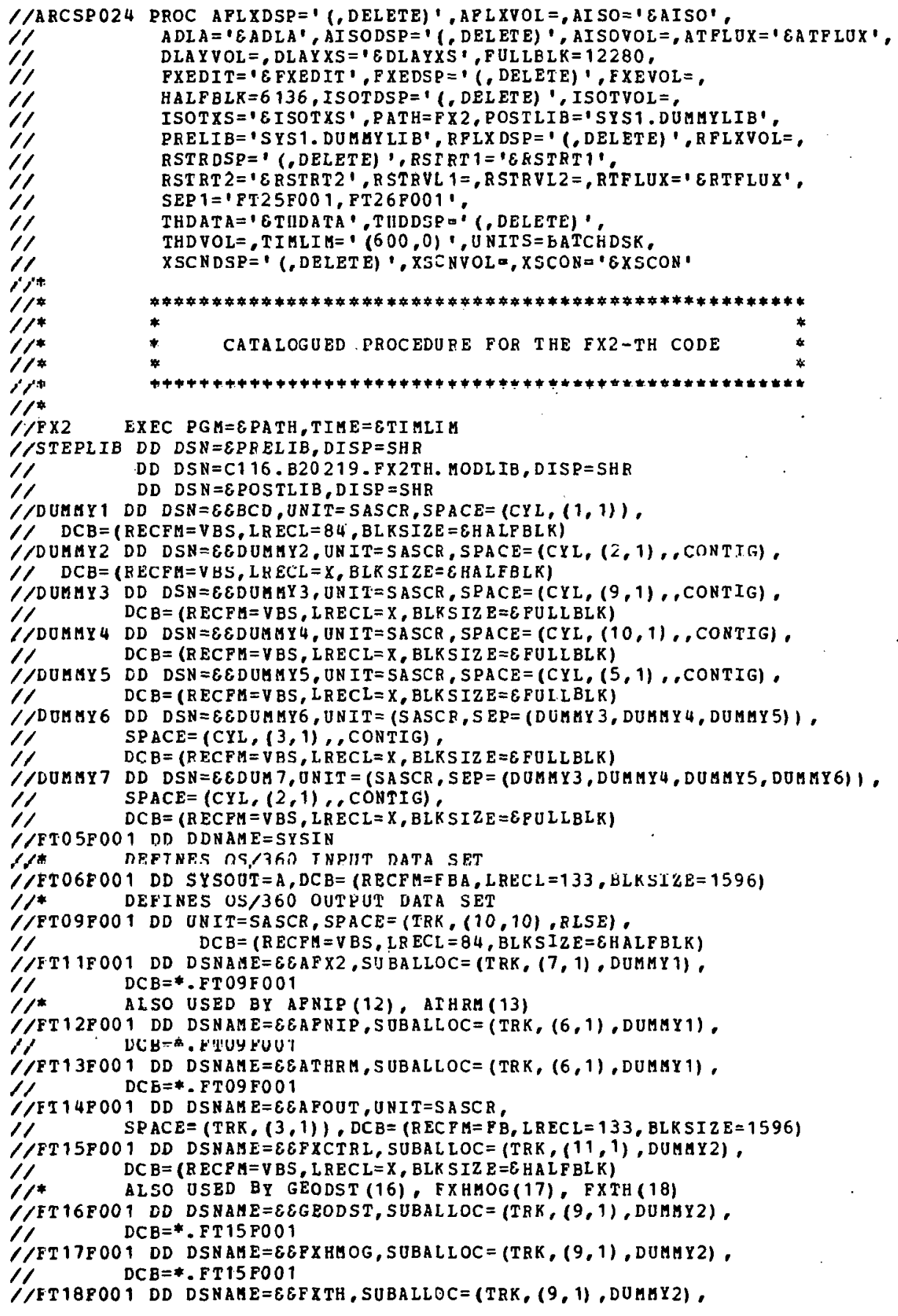

Fig. 9. Listing of Cataloged Procedure ARCSP024 


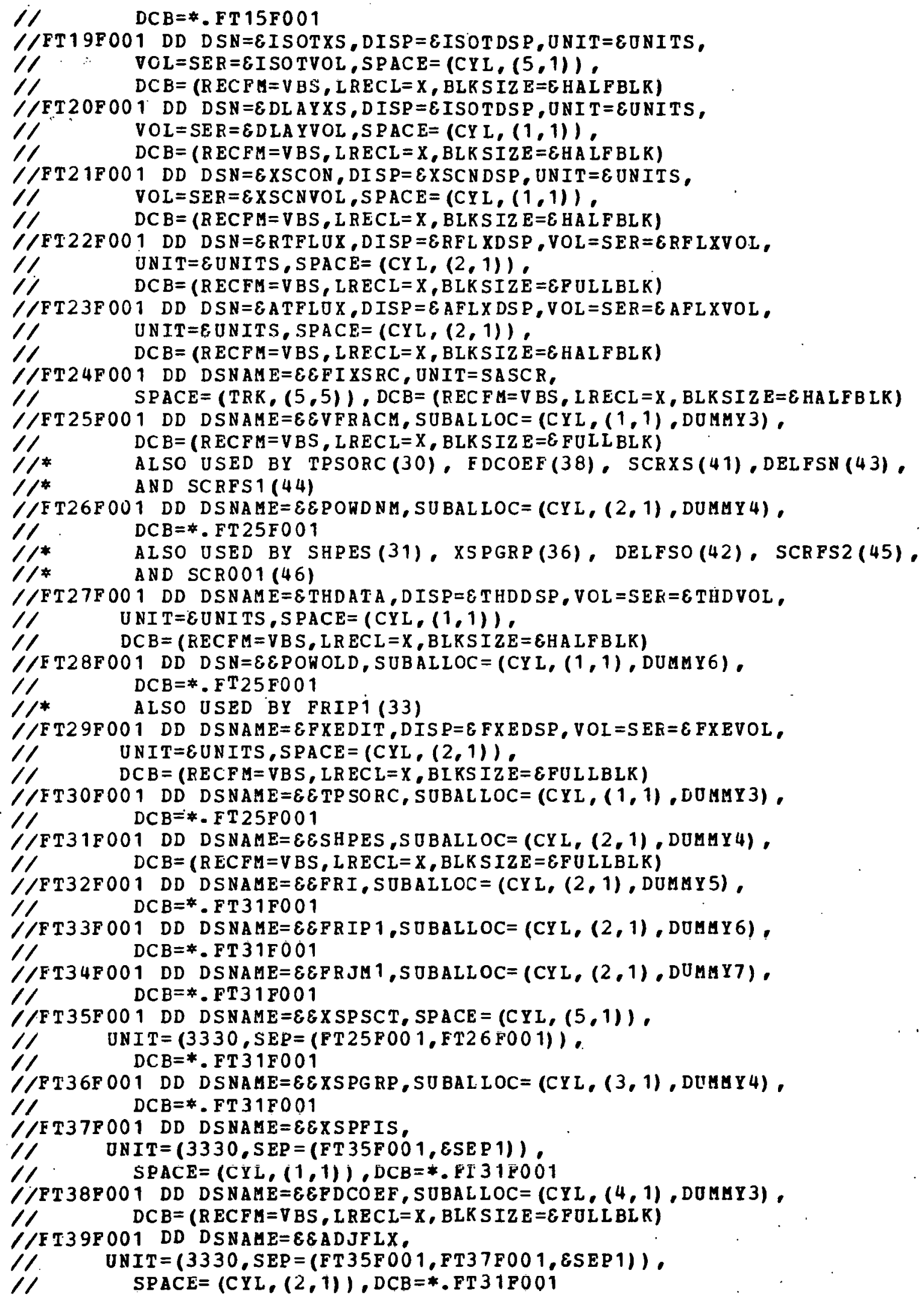

Fig. 9: (Contd) 


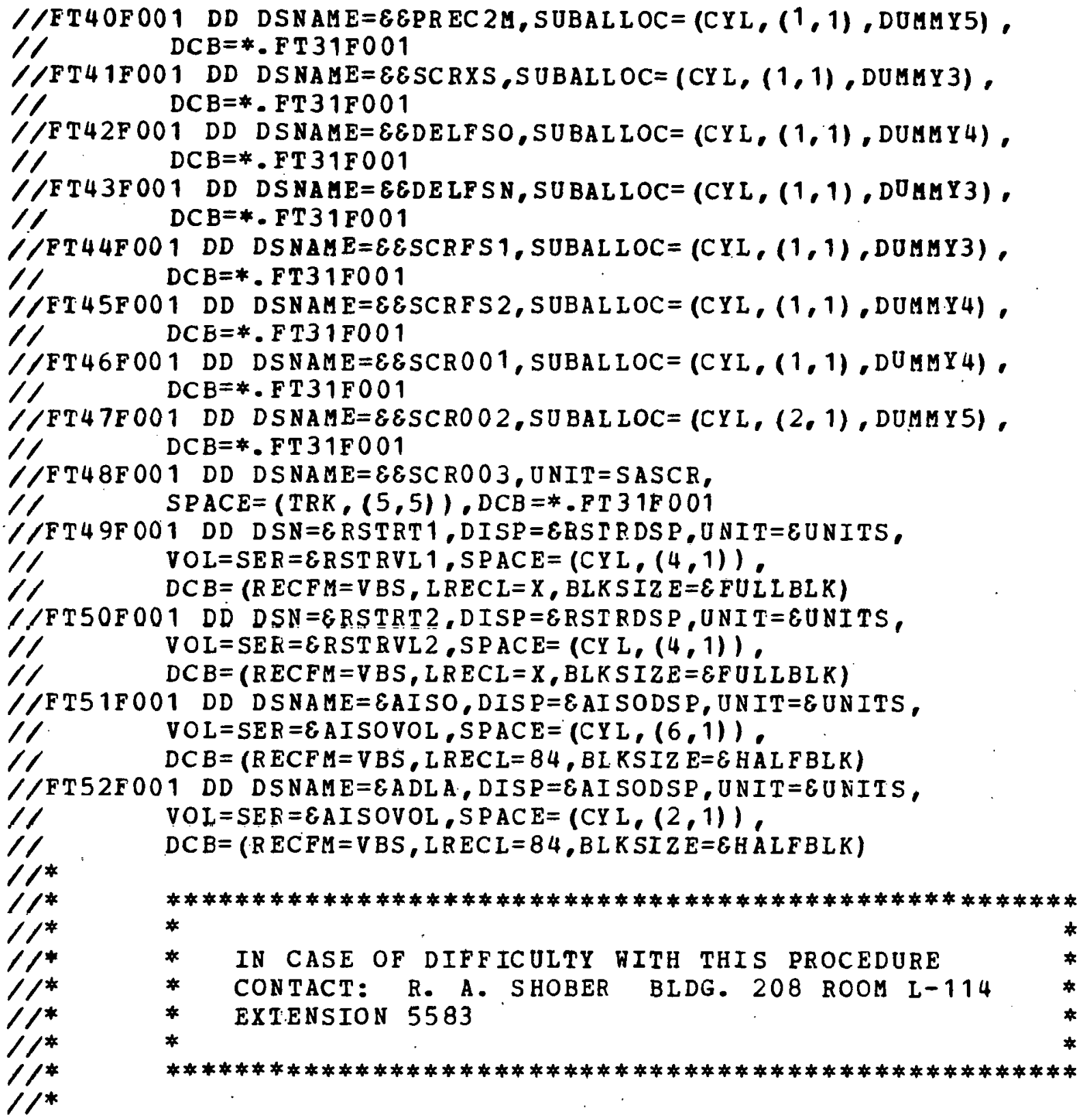

F1g. 9. (Contd) 
TABLE I. Symbolic Parameters for ARCSP024

\begin{tabular}{|c|c|c|c|}
\hline Parameters & Description & Default & FTNNFO01 \\
\hline AFLXDSP & disposition for ATFLUX & (, DELETE) & 23 \\
\hline AFLXVOL & volume for ATFLUX & no volume & 23 \\
\hline AISO & DSN for A.ISO & ¿AISO & 51 \\
\hline ADLA & DSN for A. DLA & $£$ ADLA & 52 \\
\hline AISODSP & disposition for A.ISO & (, DELETE) & 51,52 \\
\hline AISOVOL & volume for A.ISO & no volume & 51,52 \\
\hline ATFLUX & DSN for ATFLUX & \&ATFLUX & 23 \\
\hline DLAYVOL & volume for DLAYXS & no volume & 20 \\
\hline DLAYXS & DSN for DLAYXS & (, DELETE) & 20 \\
\hline FULLBLK & $\begin{array}{l}\text { block length for full-track } \\
\text { blocking }\end{array}$ & 12280 & \\
\hline FXEDIT & DSN for FXEDIT & $\&$ FXEDIT & 29 \\
\hline FXEDSP & disposition for FXEDIT & (, DELETE) & 29 \\
\hline FXEVOL & volume for FXEDIT & no volume & 29 \\
\hline HALFBLK & $\begin{array}{l}\text { block length for half-track } \\
\text { blocking }\end{array}$ & 6136 & \\
\hline ISOTDSP & $\begin{array}{l}\text { disposition for DLAYXS and } \\
\text { ISOTXS }\end{array}$ & SHR & 19,20 \\
\hline ISOTVOL & volume for ISOTXS & no volume & 19 \\
\hline ISOTXS & DSN for ISOTXS & (, DELETE) & 19 \\
\hline PATH & name of member to be executed & FX2 & EXEC \\
\hline POSTLIB & step library & SYS1. DUMMY LIB & STEPLIB \\
\hline PRELIB & step 1ibrary & SYS1. DUMMYLIB & STEPLIB \\
\hline RFLXDSP & disposition for RTFLUX & $($, DELETE) & 22 \\
\hline RFLXVOL & volume for RTFLUX & no volume & 22 \\
\hline RSTRDSP & $\begin{array}{l}\text { disposition for FX2RS1 and } \\
\text { FX2RS2 }\end{array}$ & $($, DELETE $)$ & 49,50 \\
\hline RSTRT1 & $\mathrm{DSN}$ for FX2RS1 & \&RSTRT1 & 49 \\
\hline RSTRT2 & DSN for FX2RS2 & \&RSTRT2 & 50 \\
\hline RTFLUX & DSN for RTFLUX & \&RTFLUX & 22 \\
\hline SEP1 & separation parameter & FT25F001,FT26F001 & \\
\hline THDATA & DSN for THDATA & \&THDATA & 27 \\
\hline THDDSP & disposition for THDATA & (, DELETE) & 27 \\
\hline THDVOL & volume for THDATA & no volume & 27 \\
\hline TIMLIM & time limit & $(600,0)$ & EXEC \\
\hline UNI TS & generic unit name & BATCHDSK & \\
\hline XSCNDSP & disposition for XSCON & (,DELETE) & 21 \\
\hline XSCNVOL & volume for XSCON & no volume & 21 \\
\hline XSCON & DSN for XSCON & $\& X S C O N$ & 21 \\
\hline
\end{tabular}


The parameters HALFBLK, FULLBLK, and UNITS which are used in many of the datasets are defined with the other symbolic parameters. The block size defaults of 6136 and 12280 for HALFBLK and FULLBLK are consistent with the use of 3330 disk packs. The use of SASCR and BATCHDSK for the UNIT parameter in the procedure correspond to ANL conventions for generic names. SASCR is used to designate permanently resident disk packs for scratch purposes. BATCHDSK corresponds to both permanently resident and setup disk packs, and is used for those files which may be saved on user option for later use. The PRELIB and POSTLIB parameters allow the user to access other load modules than the one permanently referred to. They default to empty partitioned data sets.

The use of the cataloged procedure allows $\mathrm{FX} 2-\mathrm{TH}$ problems to be executed with a minimum of job control language requirements. Examples of the necessary JCL are given in Section V.D.

\section{B. Input Data Specifications}

Input data for FX2-TH may be entered as either BCD input files or as binary interface files stored on disk. If desired, all the input data may be on BCD input files, or all the input data may be on binary interface files. In practice, neither of these modes of operation is very convenient. In general, cross section input resides on binary interface files, while the rest of the input resides on $B C D$ input files.

\section{B.1 Overall Structure of Input Data}

The input deck for FX2-TH is divided into two parts, or "blocks". The first block is labeled "BLOCK=OLD", while the second block is labeled "BLOCK=STP014". In the BLOCK=OLD section, the user specifies files, by their FX2-TH file name, which have previously been saved and are now resident on disk as binary files. Such files generally include microscopic or macroscopic cross sections. The files are specified with a card which says, starting in column 1, DATASET=fillename. There is one such data card for each file so specified.

The cross sections may be input in either microscopic or macroscopic form. If microscopic cross sections are to be entered, two data sets must be present; one containing the microscopic cross sections (ISOTXS), the other containing the delayed neutron parameters (DLAYXS). These files must be in the format spcified by the CCCC, 16 although they may be present as binary interface files (ISOTXS and DLAYXS), or as BCD input files (A.ISO and A.DLA). If the files are entered as binary files, FX2-TH wil1. use them directly. If they are entered as BCD input files FX2-TH will first convert them to binary files, and then use them directly. These files can be obtained by running a code such as $M C^{2}-2.17$ If macroscopic cross sections are to be entered, the data set XSCON must be present as a binary file on disk. The XSCON file contains the result of the cross section homogenization procedure. It also contains the fuel and coolant temperature feedback coefficients. This data set may be obtained in one of two ways. The user may construct it himself and write it on disk using a user-written program. Or, the XSCON file written by FX2-TH after successful completion of the cross section homogenization procedure of Section IV.A and calculation of the required temperature coefficients may be saved on disk. 
This file may then be used for future cases instead of FX2-TH performing the cross section homogenization again.

In $B L O C K=S T P 014$, the user inputs the BCD input cards, broken into files. Each BCD input file is preceeded by a card which says, starting in column 1, DATASET=filename. The file A.FX2 contains various control parameters and convergence criteria. The file A.FNIP describes the geometry of the problem and specifies the homogenization of the microscopic cross sections. The file A. THRM provides input for the thermal-hydraulics calculation.

As stated above, some data required by $\mathrm{FX} 2-\mathrm{TH}$ can originate either as $B C D$ input files or as binary interface files. Figure 10 shows a diagram 1llustrating the data flow between these input data files. Any file listed under the column "Files Used Directly by FX2-TH" may be saved on disk for a future run, where it would be placed in BLOCK=OLD. The user must determine the mode of operation which is most convenient for himself.

If BCD input data sets A.ISO and A.DLA are present in the input job stream, they. must be preceeded by a card which says NOSORT=A.ISO, or NOSORT=A. DLA. This takes the place of the DATASET=A:ISO or DATASET=A.DLA.

$\begin{array}{lcc}\text { INPUT } & \text { BINARY } & \text { FILES USED } \\ \text { R } . D & \text { INTERFACE } & \text { DIRECTLY BY } \\ \text { FILES } & \text { FILES } & \text { FX2 }- \text { TH }\end{array}$

A. FX2<smiles>CCC</smiles>

A. FNIP

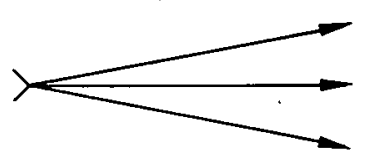

A.THRM

$\gamma$

A. ISO

A. OLA

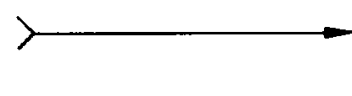

FXCTRL

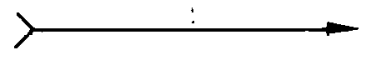

FXHMOG

GEODST

FIXSRC

FXTH
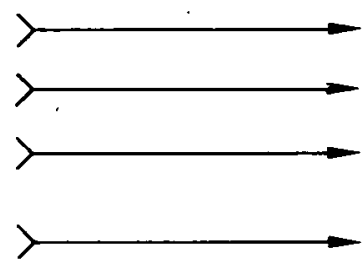

FXTH

ISOTXS

DLAYXS

FXCTRL

FXHMOG

GEODST

FIXSRC

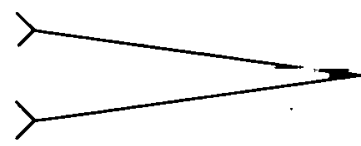

XSCON

Fig. 10. Input Data Files to FX2-TH 


\section{B.2 Cross Section Representation}

A multigroup representation of a nuclear reactor requires a great deal of data manipulation and storage. The strategies used in FX2-TH were designed to provide full user flexibility while at the same time making optimal use of available core storage. The various cross section homogenization schemes in FX2-TH will now be discussed.

The cross sections in FX2-TH undergo two stages of homogenization. In the first stage, microscopic cross sections representing each physical isotope are smeared together to form a unique material. Then, the unique materials are smeared together to form compositions. These two stages will be discussed separately.

In the first stage of homogenization, isotopes are mixed together to form unique materials. This homogenization stage is performed when FX2-TH accepts ISOTXS and DLAYXS files, homogenizes them, and writes an XSCON f1le. The type 13 cards of the BCD input file A.FNIP controls this homogenization. stage.

FX2-TH allows the user to enter any number of physical isotopes which then will be homogenized into a unique material. For example, the fuel in a specific region of the reartinr might he composed of the iootopes ${ }^{23.5} \mathrm{U}$, ${ }^{23{ }^{2}} \mathrm{U}$, and ${ }^{2}{ }^{39} \mathrm{Pu}$. FX2-TH would then allow the user to define a specific type of fuel material, consisting of a specific isotopic content of the above three isotopes. This isotopic content is assumed fixed for the duration of the FX2-TH calculation. The cross sections and chi vectors are calculated as shown in Eqs. (IV.1, 2). Each unique material also has a set of fuel and coolant temperature coefficients. To calculatc the fuel temperature coefficients, FX2-TH allows the user to enter sets of microscopic cross sections representing the same physical isotope at different values of fuel temperature. The general correlation shown in Eq. (IV.3) is then evaluated by considering the cross section values at the four temperaturoe. To cal.. culate the coolant temperature coefficients, FX2-TH allows the user to enter, for each physical coolant isotope, the first derivative of the atom density of this isotope with respect to coolant temperature. The coolant temperature feedback coefficient is then calculated as in Eq. (IV.5). When the cross section homogenization and feedback coefficient calculation has been accomplished for each unique material, the XSCON file is written with all of this information written on it.

At this point, further clarification of the unique material concept is required. In FX2- TH, the reactor is thought of as being composed of physical materials such as uranium oxide, plutonium oxide, stainless steel, etc. Each of these materials represents one of the "unique materials" described above. However, it is recognized that the cross sections for a particular physical material at one point in the reactor core may not be the same as the cross sections of the same physical material at another point in the core (from reactor burnup, for example). In an attempt to minimize computer storage requirements, FX2-TH employs a generic material unique'material concept. That is, a number of generic materials are defined (such as plutonium oxide, stainless steel, etc.). At a specific point in the reactor core, the generic material is composed of a specific unique material (which may be different from the unique material present at a different point in the core). Therefore, there are several unique 
materials, each with a separate cross section set and unique material label, all of which however share the same generic material label. Under this scheme, uranium oxide in the inner and outer core regions might be represented as UO2-IC and UO2-OC, respectively, with both sharing the generic material label U02. Since core storage requirements and computing times are much more dependent on the number of generic materials than on the number of unique materials, it is in the user's interest to attempt to minimize the number of generic materials.

In the second stage of homogenization, the unique materials which make up the actual reactor material at a specific point in the reactor are homogenized. This is performed by FX2-TH when it reads in the XSCON file and multiplies the unicue material cross sections and temperature coefficients by the appropriate volume fractions. The volume fractions are input on the type 14 cards of the BCD input file A.FNIP. The composition so generated, represented by a particular region label, can then be placed at any point in the reactor as the user desires.

For materials in which Doppler feedback is present, the macroscopic cross section for process $P$ in group $g$ is calculated as follows. First, the cross sections $\Sigma_{\mathrm{m}, \mathrm{g}}^{\mathrm{P}}\left(\mathrm{T}_{\mathrm{f}}\right)$ are evaluated from $\mathrm{Eq}$. (IV.3) using the fuel temperature present in this region. Then, these cross sections are summed over each material m making up this composition:

$$
\Sigma_{g}^{P}\left(T_{f}\right)=\sum_{m=1}^{M} V F_{m} \Sigma_{m, g}^{P}\left(T_{f}\right)
$$

where

$$
\begin{array}{ll}
M & \text { is the total number of unique materials in this } \\
\text { composition }
\end{array}
$$

For materials in which coolant temperature feedback is present, the required macroscopic cross section is calculated in a similar manner. First, the cross sections $\Sigma_{m, g}^{P}\left(T_{c}\right)$ are evaluated from Eq. (IV.5) using the coolant temperature present in this region. Then, the cross sections are summed over each material m making up this composition:

$$
\Sigma_{g}^{P}\left(T_{c}\right)=\sum_{m=1}^{M} V F_{m} \Sigma_{m, g}^{P}\left(T_{c}\right)
$$

For materials in which neither fuel nor coolant feedback is present, the following expression is used

$$
\Sigma_{g}^{P^{\prime}}=\sum_{m=1}^{M} V F_{m} \Sigma_{m, g_{\text {base }}}^{P}
$$


In FX2-TH, perturbations are initiated by allowing the user to alter the values of $V F_{m}$ for any $m$ in any composition. This modification can be a step, ramp, or quadratic function. The modifications to $\mathrm{VF}_{\mathrm{m}}$ are controlled by the type 10 cards of the BCD input file A.FX2.

\section{B.3 Geometry and Boundary Condition Input}

The FX2-TH code allows a variety of one- and two-dimensional geometric configurations. These are
a) $\operatorname{slab}(\mathrm{x})$
b) cylinder $(r)$
c) $\mathrm{x}-\mathrm{y}$
d) $r-z$
e) $\theta-r$
f) triangular

For the one-dimensional geometries and all two-dimensional geometries; except the triangular mesh, the region, and mesh data are supplied on type 06 or 09 cards of dataset A.FNIP. For the triangular mesh geometries, the region and mesh data are supplied on type 29, 30, and 31 cards of dataset A. FNIP. The transverse half-height and extrapolation distance are specified on a type 12 card of A. FNIP.

The boundary condition types that can be specified are
a) $\phi=0$
b) $\phi^{\prime}=0$
c) $\phi^{\prime}+\frac{\mathrm{A}}{\mathrm{D}} \quad \phi=0$
d) repeating periodic with next adjacent face

where $D$ is the diffusion coefficient in the mesh interval immediately within the reactor boundary. For orthogonal geometries (except $\theta-r$ ); options a), b), and c) above may be used. For $\theta-r$ geometry and triangular geometry, any of the above options may be used.

The above boundary conditiuns can be dependent on the neutron energy group. The data are supplied on the type 04 and 05 cards of dataset A. FNIP.

\section{B.4 External Neutron Source}

If there is an external isotropic neutron source, the data are supplied on the type 29 card of dataset A. FNIP. The external source is allowed to be time dependent in FX2-TH. A very simple time dependence is specified; the user is allowed to multiply the fixed source (at all mesh points and energy groups) by an input number at a specific time during the transient. This time, which can be equal to zero, is also input by the user.

\section{B.5 Flux Input}

If no flux files are specified as being in BLOCK=OLD, FX2-TH uses a flat flux guess (for each mesh point and energy group) to begin the steady state calculation. At the conclusion of the steady state calculation, the real 
and adjoint fluxes are written on the standard interface files RTFLUX and ATFLUX respectively. If files RTFLUX and ATFLUX are specified in BLOCK=OLD, they will be used as the initial flux estimates for the steady-state calculation.

\section{B.6 Thermal-Hydraulics Input}

The input to the thermal-hydraulics models in $\mathrm{FX} 2-\mathrm{TH}$ is given in dataset A.THRM. In this dataset, the user selects which thermal-hydraulics model is to be used, and the relevant input data for that model.

For the purposes of the thermal-hydraulic calculation, the reactor core is broken into thermal-hydraulic channels, and each channel into axial mesh regions. In the dataset A. THRM, the correspondence between the neutronics mesh and the thermal-hydraulics mesh is specified in a very general manner. The user is given three options:
a) each neutronics region may become one thermal-hydraulics region, or
b) each neutronics mesh cell may become one thermal-hydraulics region, or
c) the thermal-hydraulics mesh structure may be defined in an arbitrary manner.

These options are accomplished by using type 03, 04, and 05 cards of A.THRM.

In FX2-TH, it is recognized that different regions of the core may contain different thermal-hydraulics data values. Each therma1-hydraulics channel is assigned a data type number, and the user inputs different sets of input data values for each data type. The input data are read on card type 06 of A.THRM. Each "set" of data (corresponding to a particular data type number) consists of an entire set of data values given in the list under card type 06 of dataset A.THRM ( 6 values for the simple models, 34 for the detailed model). All data for thermal-hydraulics data set IT must be entered before any data from data set (IT+1) can be entered. Within a particular data set IT, the individual datum values may be input in any order. Before each datum value, the datum number (either 1 to 6 or 1 to 34, depending on whi.sh mndel is being used) is entered. When all of the data for any particular data set IT have been entered, a new type 06 card should be used to begin to specify the data for data set (IT+1). When any particular data set IT has been completely entered, the code will "copy" the data of data set IT into the locations reserved for data set $(\mathrm{IT}+1)$. Then, only those values for data set $(I T+1)$ which are different from those of data set IT need be entered. The correspondence between the thermal-hydraulic channels and the data types is made on the type 09 cards of dataset A. THRM.

On card types 13 and 14 of A.THRM, the user may enter the initial values of the average coolant and fuel temperatures. If one of the simple thermalhydraulic models is being used, these temperatures represent the steadystate temperatures. A coolant and fuel temperature may be entered for every thermal-hydraulic channel and axial mesh region. If the detailed thermalhydraulic model is being used, these temperatures are used to begin the steady-state iteration between neutronics and thermal-hydraulics. If type 13 and 14 cards are not entered, the initial coolant and fuel temperatures are taken to be the base coolant and fuel temperatures, respectively. 


\section{Computer Core Requirements}

Previous versions of the FX2 code used one large block of, core memory to contain the problem variables. When FX2 was executed on a two-level storage computer such as the CDC 7600 , only a small amount of small core memory (SCM) was available for data storage, requiring FX2 to perform a great deal of disk I/O. To relieve this problem and also allow for larger problems to be executed on the CDC 7600, a two-level data storage technique has been implemented into FX2-TH. In this technique, FX2-TH uses two levels of core storage, a "small core" block, and a "large core" block. On the CDC 7600, this corresponds to SCM and LCM, respectively. On IBM 360 or 370 series computers, both blocks are contained in directly addressable core memory. The user must input the amount of small core and the amount of large core required for this calculation. FX2-TH provides edits of the amount of core storage required for both the small core and the large core blocks. A small test run can be used together with these edits to determine the amount of storage required.

The small core storage block is used to contain small blocks of data. A certain minimum amount of large core storage is required for each problem. FX2-TH then examines the amount of additional large core storage remalning and determines which additional data blocks may be kept permanently in core. There are eight blocks of data which FX2-TH will attempt to fit into the large core block; in the following order:
a) Thermal-hydraulic data
b) Fission cross sections
c) Scattering cross sections
d) Removal and transport cross sections
e) Flux file
f) Volume fractions
g) Precureor deneitiec
h) Finite ditterence coctticiente.

If insufficient room is available for a particular file, then that file is read from and written to the disk as needed. Generally, FX2-TH is more cost efficient if as many of the above files are permanently contained in large core as are possible. On the CDC 7600 , storage in the large core block is accessed directly using LEVEL 2 statements. In order to execute FX2-TH, the CDC 7600 system must support this directly addressable LCM concept.

The overal1 core requirements of $\mathrm{FX} 2-\mathrm{TH}$ will now be discussed. On IBM 360 or 370 series computers, the FX2-TH overlayed program requires $150 \mathrm{~K}$ of core. In-core buffers require from $105 \mathrm{~K}$ to $156 \mathrm{~K}$, depending on the problem. If all files are permanently contained in large core, $105 \mathrm{~K}$ of buffer space is required. If none of the above files can be core contained, $156 \mathrm{~K}$ of buffer space is required. The core taken up by the small core and large core blocks are then added to determine to total FX2-TH requirement.

For the CDC 7600 computer, the core requirements of both SCM and LCM must be considered. The FX2-TH overlayed program plus SCM buffers requires 28,700 words of SCM. This leaves roughly 20,000 words of SCM for the small core block (this is dependent on the particular operating system). The large core storage block must not exceed 131,000 words. 
D. Sample Problems

Three sample problems have been defined to demonstrate the use of the FX2-TH code. The first sample problem is an $r-z$ model of an LMFBR in four neutron energy groups. No feedback is included in this sample problem. The transient is induced by removing some $s$ tainless steel from the inner core. A listing of the input data for this sample problem is given in Figure 11. The output for this sample problem is given in Appendix VI.

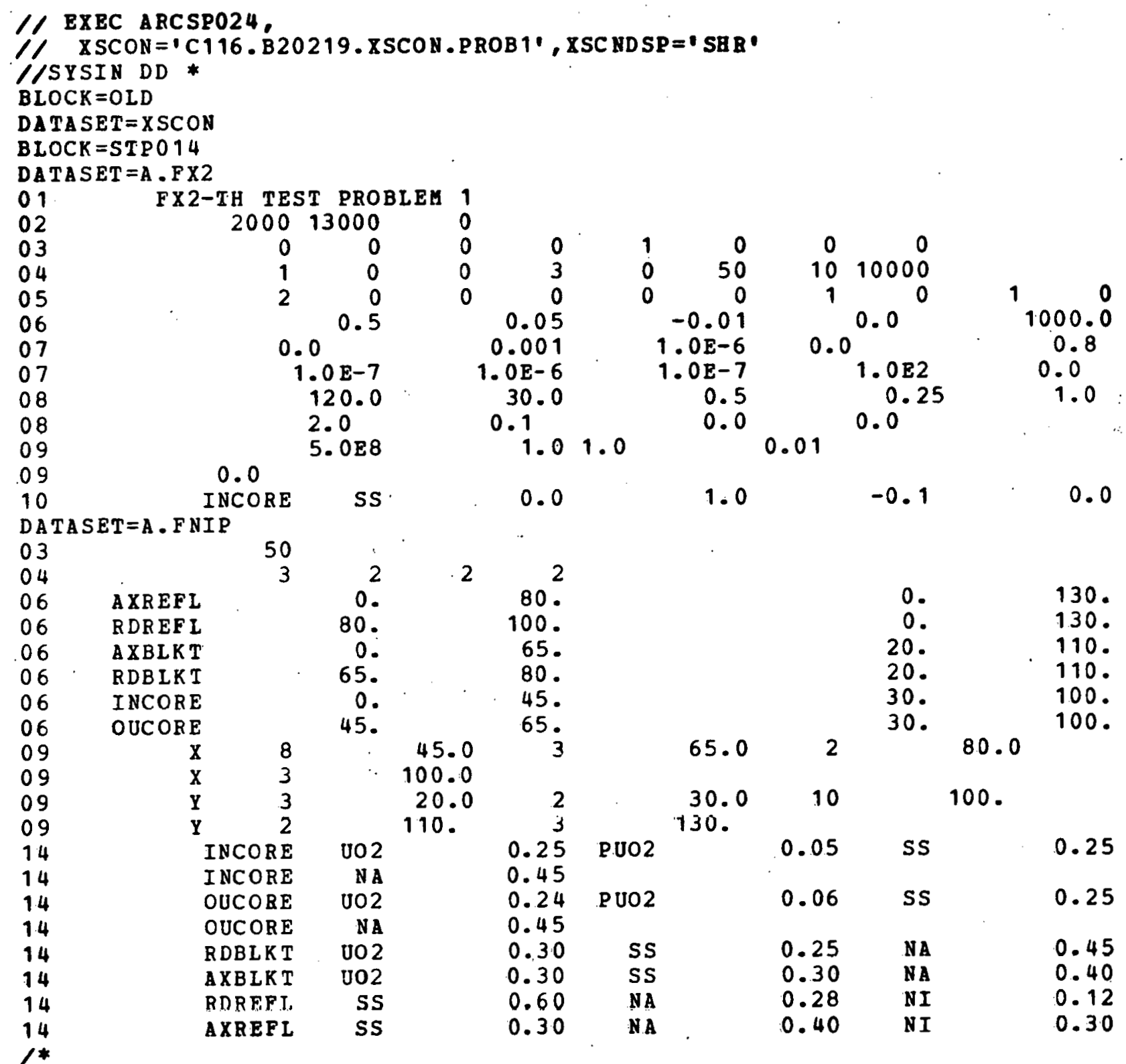

Fig. 11. Sample Problem 1 Input 
Sample problem 2 is ail $x-y$ model of a BWR in two neutron energy groups. The feedback model used is the adiabatic model with the constant heat transfer option. The steady-state coolant and fuel temperatures are input in the dataset A. THRM. These temperatures were obtained by running a steady-state FX2-TH solution using the detailed thermal-hydraulics model. The temperatures input into sample problem 2 are the converged coolant and fuel temperatures found from the detailed model. The results of the steady-state and transient calculations in sample problem 2 are almost identical to the same problem when using the detailed model. A listing of the input data for sample problem 2 is given in Figure 12.

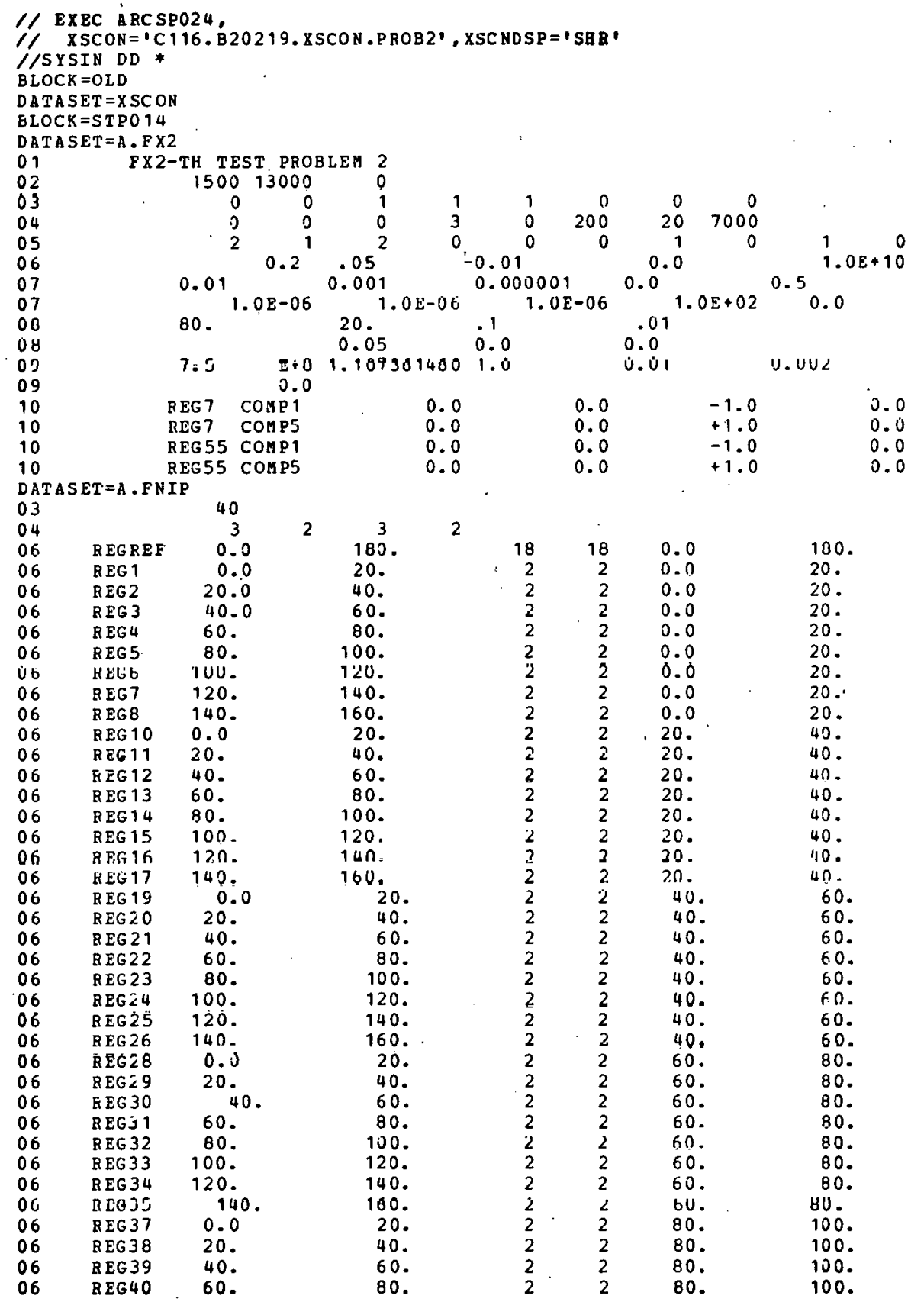

Fig. 12. Sample Problem 2 Input 


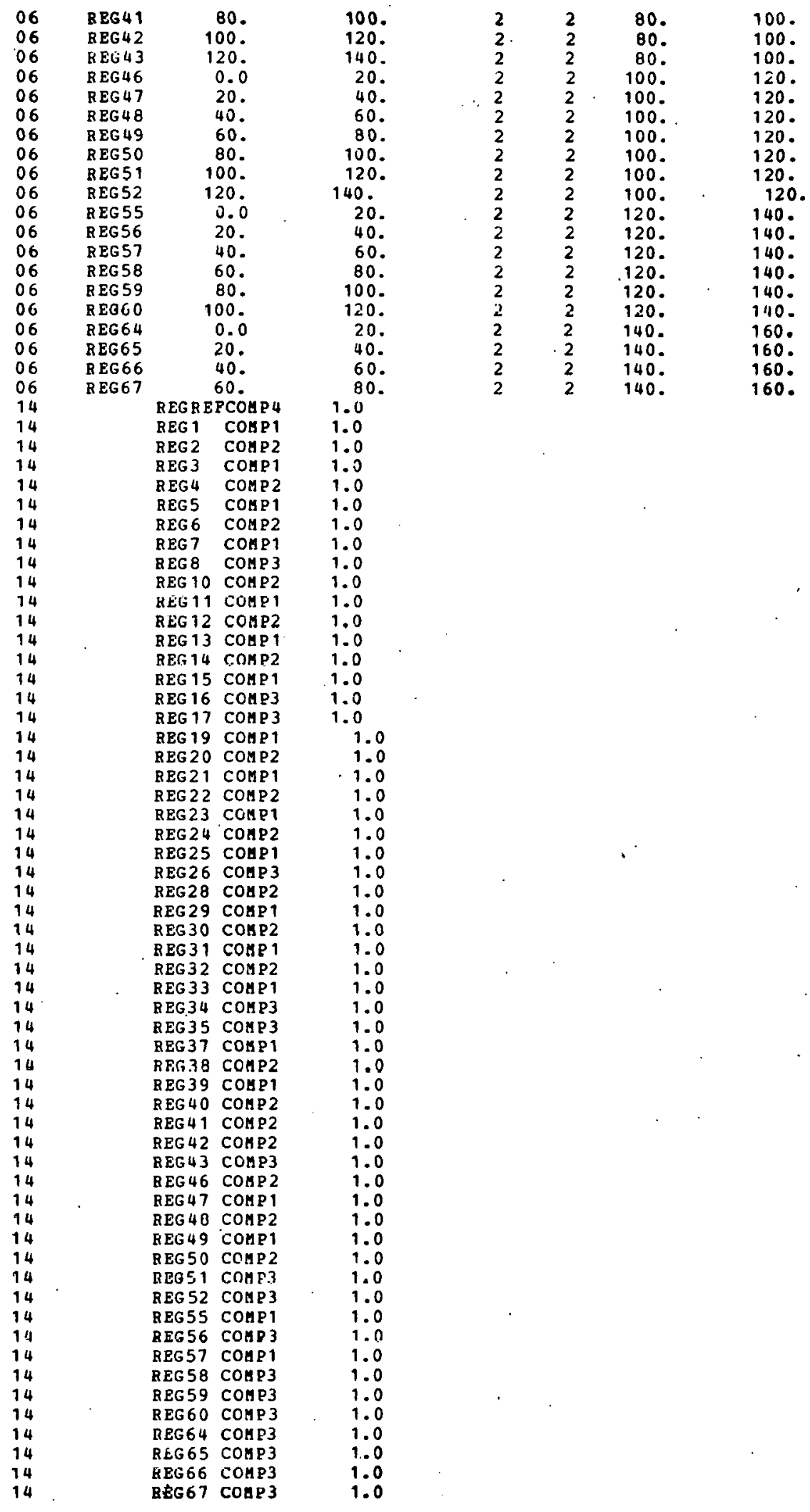

Fig. 12, (Contd) 


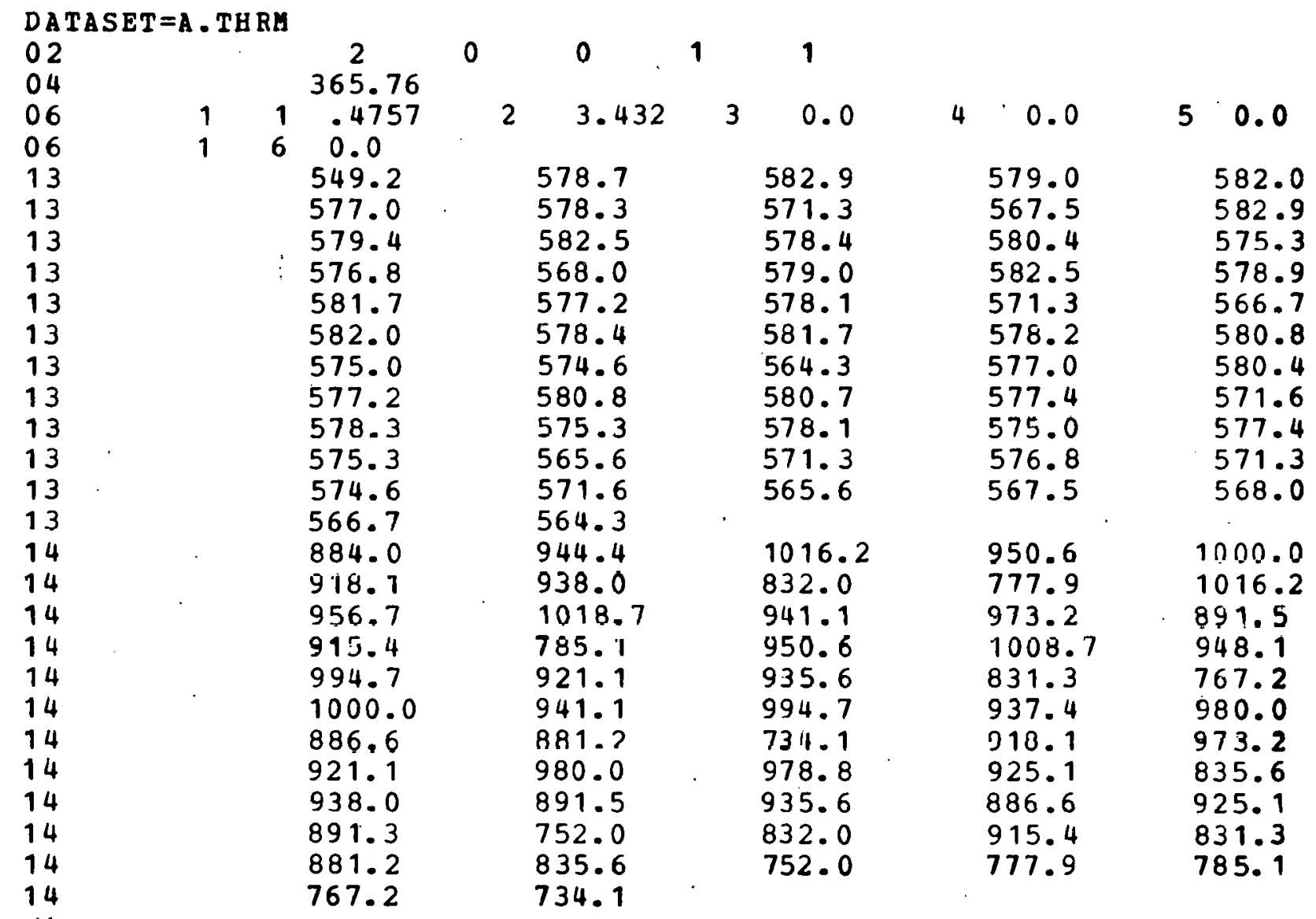

Fig. 12. (Contd)

Sample problem 3 is an $r-z$ motel of an LMFBR in nine neutivu tultey groups. The detailed thermal-hydraulics model is used for this problem. For the steady-state solution, a converged set of coolant and fuel temperatures and power densities are found. A short transient is then run. This problem demonstrated the generally rapid convergence of the overall steadystate iterative process which appears to be typiral of LMFBR problcmo. A listing of the BCD input data for sample problem 3 is given in Figure 13. lhe BCD input data sets A.ISO and A.DLA are not shown in Figure 13.

\section{E. Error Messages}

A 1ist of the error messages provided by FX2-TH is given in Table II. The error messages are arranged by subroutine, which are grouped in alphabetical order.

There are two main types of errors in FX2-TH; non-fatal and fatal errors. In a non-fatal error, the subroutine ERROR is called with a nonnegative integer argument. In a fatal error, the integer input is negative. When a fatal error occurs, processing is generally ended. 


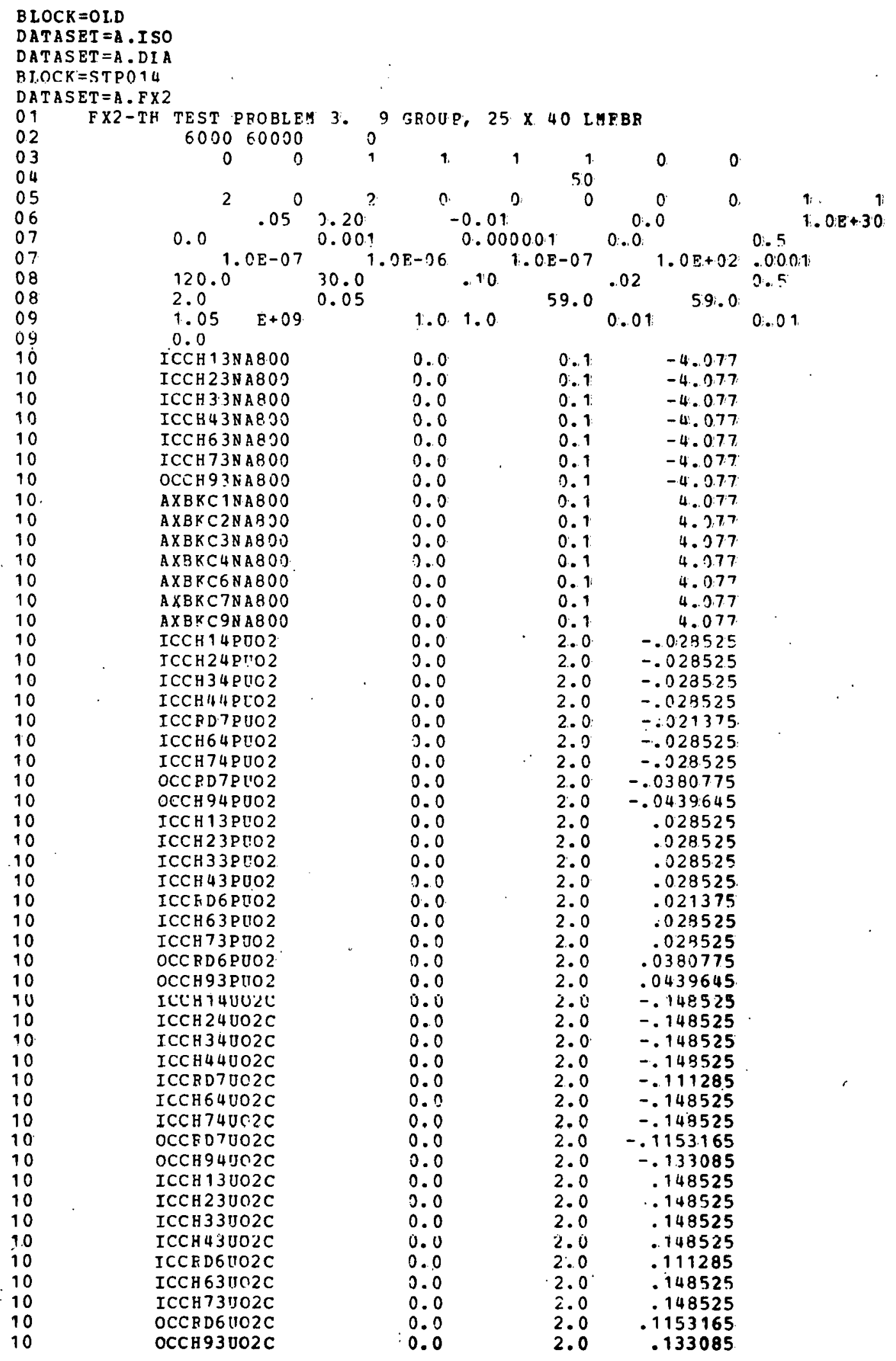

Fig. 13. Sample Problem 3 Input 


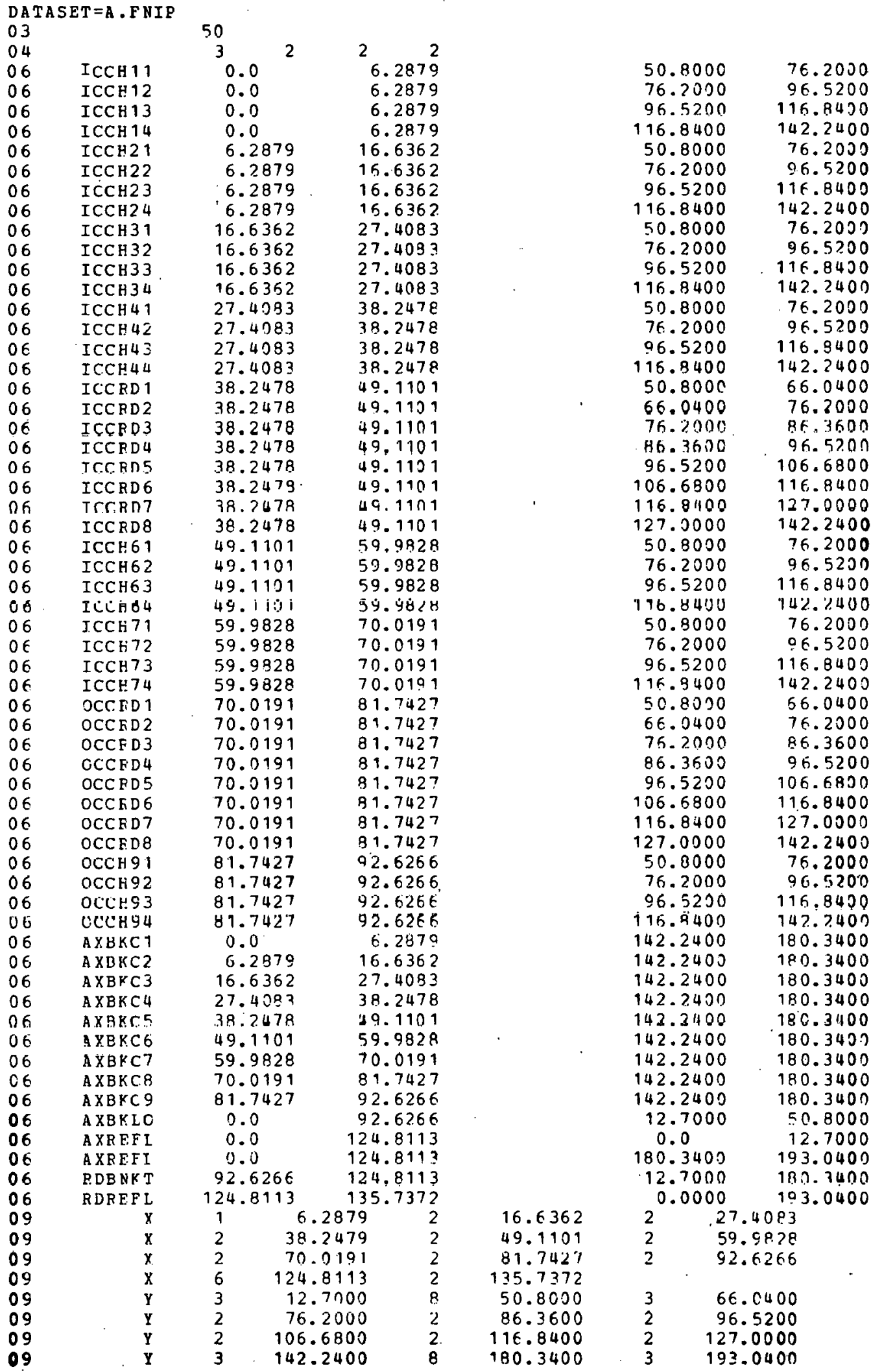

Fig. 13. (Contd) 


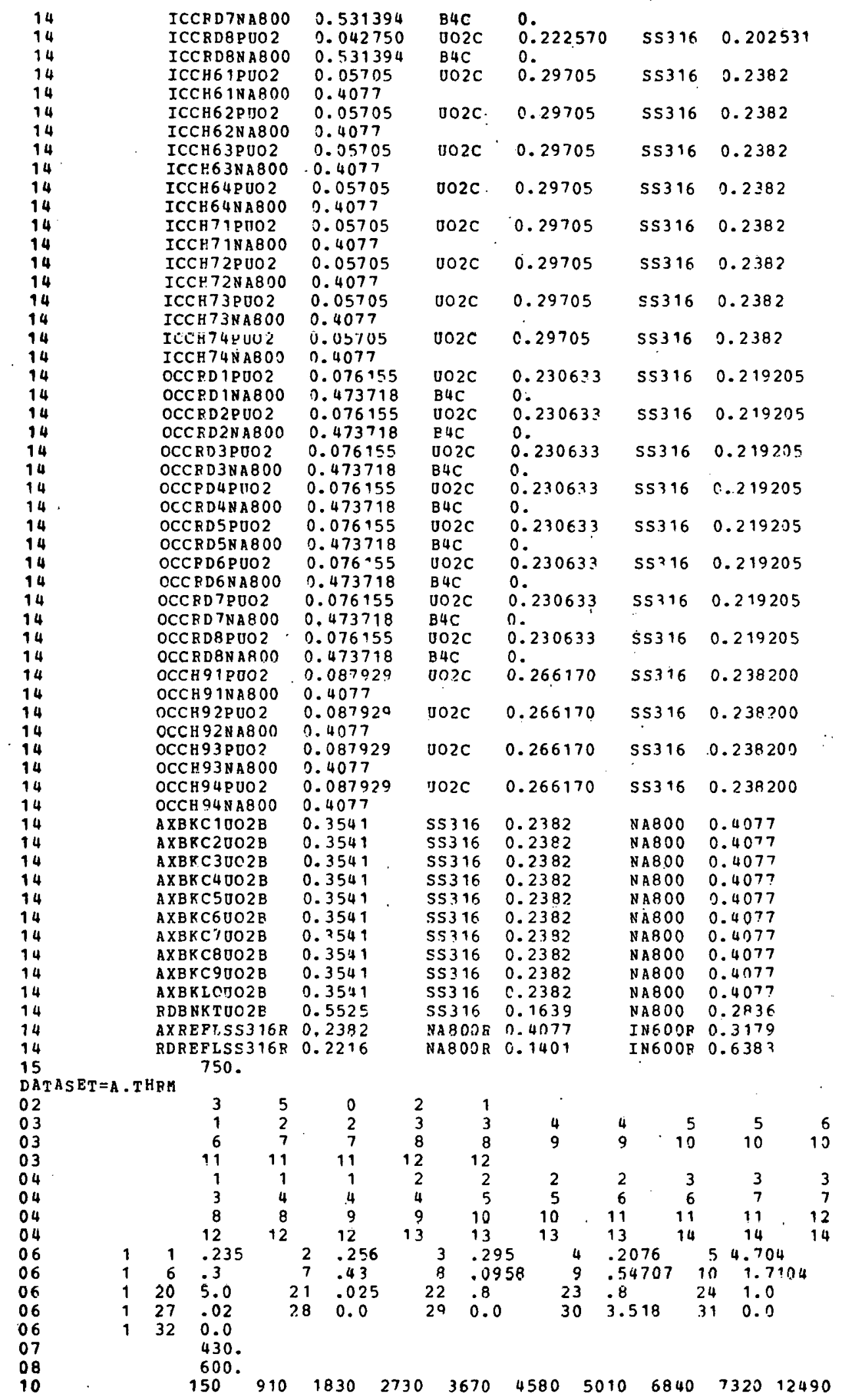

Fig. 13. (Contd) 


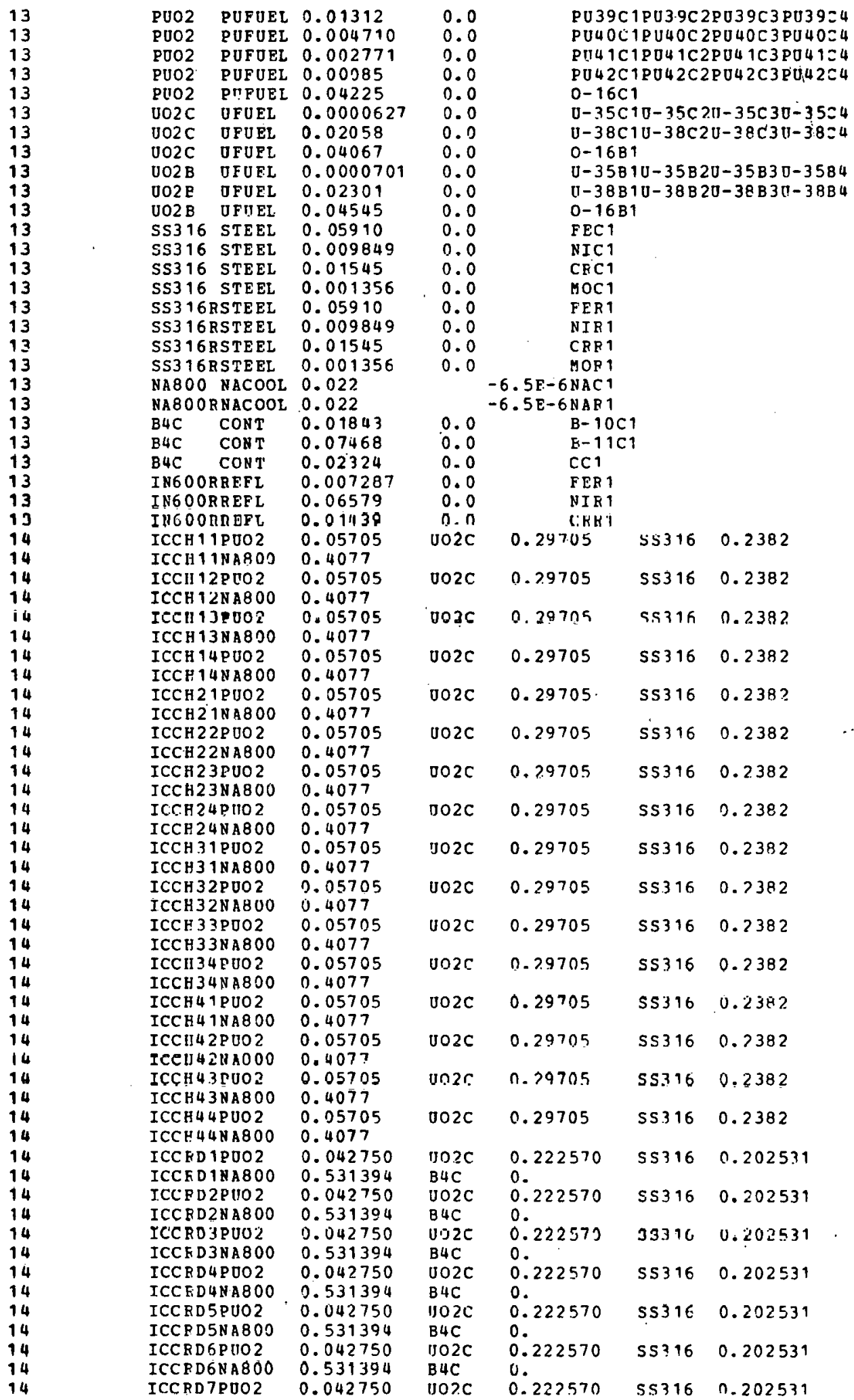

Fig. 13. (Contd) 
TABLE II. Summary of Error Messages for FX2-TH

\section{Subroutine AFX210}

$\begin{array}{lll}\text { Error } & -100 . & \begin{array}{l}\text { A driving function was assigned a blank region } \\ \text { label. }\end{array} \\ \text { Error } & -110 . & \begin{array}{l}\text { A driving function was assigned a blank material } \\ \text { label. }\end{array} \\ \text { Error } & -120 . & \begin{array}{l}\text { A driving function was assigned an ending time } \\ \text { less than zero }\end{array} \\ \text { Error } & -130 . & \begin{array}{l}\text { Adriving function was assigned an ending time } \\ \text { less than its starting time. }\end{array}\end{array}$

\section{Subroutine ANIPHX}

\begin{tabular}{|c|c|c|}
\hline Error & 100 & No type 29 cards were entered. \\
\hline Error & 110. & Distance along hex flat cannot be non-positive \\
\hline Error & 120. & No type 30 cards entered. \\
\hline Error & 130 & A blank region label was entered. \\
\hline Error & 140. & Hex ring number entered as negative \\
\hline Error & 150 . & $\begin{array}{l}\text { Initial and/or final hex position entered as } \\
\text { negative. }\end{array}$ \\
\hline Error & 160 & $\begin{array}{l}\text { Background region label identical to another } \\
\text { region label already entered. }\end{array}$ \\
\hline Error & 170 & Zone label entered as blank \\
\hline Error & 180. & $\begin{array}{l}\text { Region label entered on type } 33 \text { card does not } \\
\text { match any region label on a type } 30 \text { card. }\end{array}$ \\
\hline
\end{tabular}

\section{Subroutine ANIP03}

$\begin{array}{lll}\text { Error } & 100 . & \text { No type } 03 \text { card entered. } \\ \text { Error } & 110 . & \text { Invalid geometry type entered. }\end{array}$

\section{Subroutine ANIP0 4}

Error $100 . \quad$ No type 04 card entered.

Error $\quad 110$.

Invalid boundary condition on type 04 card. 
TABLE II. (Contd.)

\section{Sub routine ANIP05}

$\begin{array}{ll}\text { Error } & 110 . \\ \text { Error } & 120 . \\ \text { Error } & 130 . \\ \text { Error } & 140 .\end{array}$

Invalid boundary designator on type 05 card.

Negative energy group number entered on type 05 card.

Same as error 120.

Lower energy group number is less than the higher energy group number.

\section{Subroutine ANIP06}

\begin{tabular}{|c|c|c|}
\hline Error & 110. & Blank region 1 abel entered on type 06 card. \\
\hline Error & 120 . & $\begin{array}{l}\text { Invalid } x \text {-coordinate region boundary on type } \\
06 \text { enra: }\end{array}$ \\
\hline Error & 130. & $\begin{array}{l}\text { Invalid } y \text {-coordinate region boundary on type } \\
06 \text { card. }\end{array}$ \\
\hline Error & 140 & $\begin{array}{l}\text { Negative number of intervals specified on type } \\
06 \text { card. }\end{array}$ \\
\hline
\end{tabular}

\section{Subroutine ANIP08}

Error $\quad 100$.

Blank zone 1 abel on type 08 card.

Error 110 .

Regiun label given on type 08 card does not match any label given on type 06 card.

\section{Subroutine ANIP09.}

$\begin{array}{ll}\text { Error } & 110 . \\ \text { Error } & 120 . \\ \text { Error } & 130 . \\ \text { Error } & 140 . \\ \text { Error } & 150 . \\ \text { Error } & 160 . \\ \text { Error } & 170 . \\ \text { Error } & 180 . \\ \text { Error } & 190 . \\ \text { Error } & 200 .\end{array}$

Invalid coordinate entcred on type 09 card.

Discrepancy. in number of mesh intervals on type 09 card.

Zero or negative value was given for first lower coordinate on type 09 card.

Zero or negative value was given for number of intervals on type 09 card.

Negative value given as number of intervals on the type 09 card.

Upper coordinates on type 09 cards are not monotonically increasing.

Two successive upper coordinates on type 09 cards are equal.

Same as Error 120.

Same as Error 130.

Same as Error 140 . 
TABLE II. (Contd.)

Subrout ine ANIP09 (Contd.)

\begin{tabular}{|c|c|c|}
\hline Error & 210 . & Same as Error 1.50. \\
\hline Error & 220 . & Same as Error 16 \\
\hline Erro & 230 & Same as Error \\
\hline
\end{tabular}

Subroutine ANIP12

\begin{tabular}{|c|c|c|}
\hline Error & 110. & $\begin{array}{l}\text { Region } 1 \text { abel on type } 12 \text { card does not match any } \\
\text { region } 1 \text { abel on type } 06 \text { or } 30 \text { card. }\end{array}$ \\
\hline Error & 120 & $\begin{array}{l}\text { Region label is blank, so only one type } 12 \text { card is } \\
\text { allowed. More than one was entered. }\end{array}$ \\
\hline Error & 130. & Non-positive value given for transverse half-height. \\
\hline Erro & 140 . & given for transverse extrapolation \\
\hline
\end{tabular}

Subroutine ANIP13

Error $\quad 110$

Blank material label on type 13 card.

Error 120 .

Blank absolute material label on type 13 card.

Error 130 .

First isotope label on type 13 card is blank.

Error 140.

Error 150.

Identical label found on type 13 card.

Input atom density is negative.

\section{Subroutine ANIP14}

$\begin{array}{lll}\text { Error } & 110 . & \begin{array}{l}\text { Material label given on type } 14 \text { card does not match } \\ \text { any label given on type } 13 \text { card. } \\ \text { Material label given on type } 14 \text { card does not match } \\ \text { any label on UMNAME array of XSCON file. }\end{array} \\ \text { Error } & 120 . & \begin{array}{l}\text { Negative volume fraction entered on type } 14 \text { card. } \\ \text { Error }\end{array} \\ \text { Error } & 130 . & \begin{array}{l}\text { Same as Error } 110 \% \\ \text { Region label appears more than once in the set of } \\ \text { type } 14 \text { cards. }\end{array}\end{array}$

\section{Subroutine ANIP19}

Error 120.

Negative value entered for an energy group number on type 19 cards.

Error 130.

Label entered on type 19 cards does not match any region label. 
TABLE II. (Contd.)

Subroutine ANIP19 (Contd.)

\begin{tabular}{|c|c|c|}
\hline Error & 140 & Same as Error 120. \\
\hline Error & 150 & $\begin{array}{l}\text { Higher energy group number is greater than lower } \\
\text { energy group number on type } 19 \text { cards. }\end{array}$ \\
\hline Error & 160. & $\begin{array}{l}\text { Negative value given for lower coordinate on } \\
\text { type } 19 \text { card. }\end{array}$ \\
\hline Error & 170 & Same as Error 160. \\
\hline
\end{tabular}

\section{Subroutine ANIP34}

$\begin{array}{ll}\text { Error } & 100 . \\ \text { Error } & 110 . \\ \text { Error } & 120 . \\ \text { Error } & 130 . \\ \text { Error } & 140 .\end{array}$

Cannot have blank zone 1 abel on type 34 card if more than one type 34 card is entered.

Zone label on type 34 card does not match any zone label on type OB ar 33 rava:

Higher group number on type 34 card is negative.

Lower group number on type 34 card is negative.

Lower group number is less than higher group number on this type 34 card.

\section{Subroutine CHKHOM}

$\begin{array}{ll}\text { Error } & -100 . \\ \text { Error } & -110 . \\ \text { Error } & -120 . \\ \text { Error } & -130 . \\ \text { Error } & -140 . \\ \text { Error } & -150 . \\ \text { Error } & -160 .\end{array}$

Region label is blank, not allowed.

Label for unique material from FXHMOG is blank, not allowed:

Label for gener1c material from FXHMOG 1s blank, not allowed.

Label from material mixing table not found in list

of unique material labels.

A geometry region has not had its composition specified.

Entry in volume fraction array is negative not allowed.

Sum of elements of MRSPEC array does not equal

material mixing table length.

\section{Subroutine CHKXSC}

Error $\quad-100$

Decay constant less than zero.

Error -110 .

Neutron velocity less than zero.

Error $\quad-120$.

Error in LTOLP array.

Error -130 .

Error in LPTOL array.

Error -140 .

Unique materià in FXHMOG not found in XSCON. 
TABLE II., (Contd.)

Subroutine CHKXSC (Contd.)

$\begin{array}{lll}\text { Error } & -150 . & \text { Generic material in FXHMOG not found in XSCON. } \\ \text { Error } & -160 . & \text { Material number is too large. }\end{array}$

\section{Subroutine CRED}

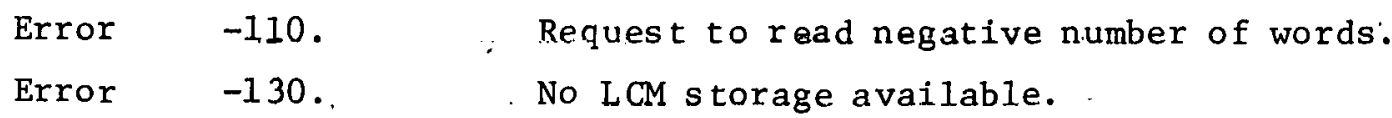

\section{Subroutine CRIT}

Error $\quad-120$.

Request to write negative number of words.

Error $\quad-140$.

No LCM storage available.

Subroutine DIMXCK

Error -100. Inconsistency in isotopes - do not share same common absolute isotope label.

\section{Subroutine DRIVEF}

$\begin{array}{lll}\text { Error } & -100 & \text { Driving function material label not a valid } \\ \text { cross section label. } & \\ \text { Error } & -110 & \text { Driving function region label not a valid region } \\ & & \text { label. }\end{array}$

\section{Subroutine EXTBC}

\begin{tabular}{|c|c|c|}
\hline Error & $\begin{array}{l}100 \\
110 .\end{array}$ & $\begin{array}{l}\text { Must specify boundary conditions for upper and } \\
\text { lower } x \text {-boundaries for one-dimensional geometries. } \\
\text { Boundary condition at } x \text {-boundary is incorrect. }\end{array}$ \\
\hline Error & 120. & 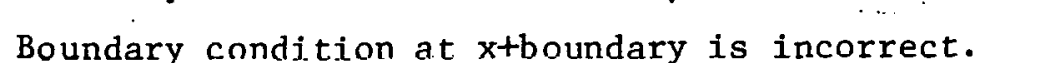 \\
\hline Error & 130 & $\begin{array}{l}\text { Must specify boundary conditions for four } \\
\text { boundaries in two-dimensional geometries. }\end{array}$ \\
\hline Error & 140 & Same as Error 110 \\
\hline Error & 150 & Same as Error 120. \\
\hline Erro & 160 & Boundary condition at $\mathrm{y}$-boundary is incorrect. \\
\hline Error & 170 . & 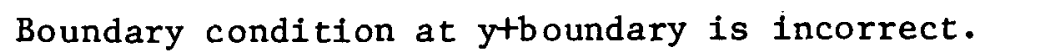 \\
\hline
\end{tabular}


TABLE II. (Contd.)

Subroutine FFIXSR

Error -100. Insufficient storage to allocate arrays.

Subroutine FFXHMO

$\begin{array}{lll}\text { Error } & -100 . & \text { Insufficient storage to allocate arrays. } \\ \text { Error } & -110 . & \text { Same as Error }-100 . \\ \text { Error } & -120 . & \text { Insufficient large core storage available to } \\ \text { Error } & -130 . & \text { allocate arrays. }\end{array}$

Subroutine FGEODS

Error $(-110)$ to $(-180)$. Insufficient storage to allocate arrays.

Subroutine FORMSH

$\begin{array}{lll}\text { Error } & -100 . & \begin{array}{l}\text { Total number of fine-mesh intervals specified } \\ \text { cannot be less than } 2 .\end{array} \\ \text { Error } & -110 . & \text { Same as Error }-100 . \\ \text { Error } & -120 . & \text { Zero or negative interval number not allowed. } \\ \text { Error } & -130 . & \text { Coarse-mesh interval assigned negative width. } \\ \text { Error } & -140 . & \text { Same as Error }-120 . \\ \text { Error } & -150 . & \text { Same as Er ror }-130 .\end{array}$

Subroutine FXINIT

$\begin{array}{lll}\text { Error } & -100 . & \text { Fatal error in processing input binary files. } \\ \text { Error } & -110 . & \text { Fatal error in initializing steady-state calculation. } \\ \text { Error } & -120 . & \text { Fatal error in initializaing transient calculation. }\end{array}$

Subroutine FXINPD

Error $(-100)$ to $(-120)$. Insufficient storage to allocate arrays. 
TABLE II. (Contd.)

\section{Subroutine FXINPR}

\begin{tabular}{ll} 
Error & -100 \\
Error & -110 \\
Error & -120 \\
Error & -130 \\
Error & -140 \\
Error & -150. \\
Error & -160 \\
Error & -170 \\
Error & -180 \\
Error & -190 \\
Error & -200 \\
Error & -210. \\
Error & -220. \\
Error & -230. \\
Error & -240. \\
Error & -250. \\
Error & -260 \\
Error & -300 \\
Error & -310 \\
Error & -320 \\
Error & -330 \\
\hline
\end{tabular}

Neither ISOrXS or XSCON exist.

Dataset A.FNIP does: not exist and it should.

Fatal error in reading A. FNIP.

Same as Error -120 .

Cannot find GEODSI in SEEK tábles while-preparing to write it.

Error in forming GEODST file.

Cannot find FXHMOG in SEEK tables while preparing to write it.

Error in forming FXHMOG file.

Cannot find RTFLUX in SEEK tables while preparing to write it.

Cannot find ATFLUX in SEEK tables while preparing to write it.

Cannot $f$ ind FIXSRC in SEEK tables while preparing to write it.

Error in forming FIXSRC file.

BCD dataset A. THRM is not present and should be.

Cannot find FXTH in SEEK tables while preparing to write it.

Error in A.THRM input.

Error in forming FXTH file.

File FXTH must be in BLOCK=OLD and it is not.

BCD dataset A.FX2 is needed but does not exist.

Cannot find FXCTRL in SEEK tables while preparing to write it.

Error in A.FX2 input make it impossible to write FXCTRL.

Same as Error -320 .

\section{Subroutine FXINXS}

Error $\quad-100$.

File FXHMOG must exist and it does not. ...

Error $\quad-110$.

File DLAYXS must exist and it does not.

Error $\quad-120$.

File ISOTXS must exist and it does not.

Error $\quad-130$.

File XSCON must exist and it does not.

Error -140 .

F1le FXCTRL must exist and 1t does not.

Error $\quad-150$.

Error in processing cross section input. 
TABLE II. . (Contd.)

Subroutine FXOV31

Error -100. Insufficient space to allocate arrays.

Subroutine FXOV32

Error - -100 Insufficient large core space to allocate arrays.

Error $-110 . \quad$ Same as Error -100 .

Error $\quad-120$. Same as Error -110 .

Subroutine FXov33

Error - Ins ufficient large core, spare to a 11nrate arrays,

Subroutine FXoV41

Error $(-110)$ to $(-180)$. File is required but has not been supplied.

Error $(-120)$ to $(-280)$. Error incurred while reading and checking file.

Subroutine FXOV42

Error -100. Insufficient space to allocate arrays.

Subroutine FXPTXS

Error $(-100)$ to $(-130)$. Insufficient large core spare to a11ncate arrays.

Sub routine FXSHAP

Error -100. Insufficient space to perform shape calculation.

Subroutine INITSS

Error $\quad-100 . \quad$ Insufficient space to perform steady-state calculation.

Subroutine INITTI

Error $-100 . \quad$ Insufficient space to perform transient calculation. 
TABLE II. (Contd.)

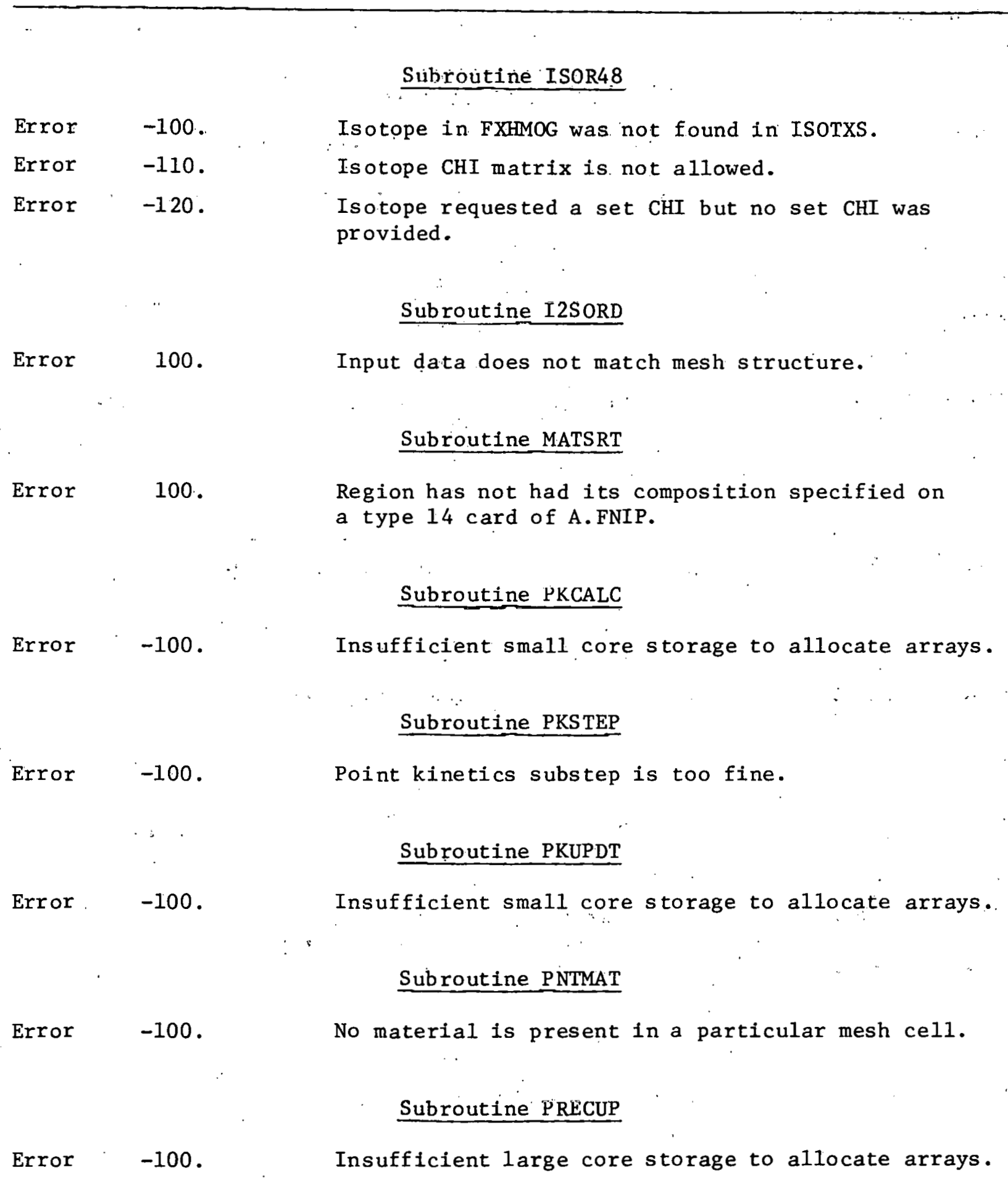

\section{Subroutine RAFNIP}

$\begin{array}{lll}\text { Error } & 110 . & \text { No type } 06 \text { cards supplied in A.FNIP. } \\ \text { Error } & 120 . & \text { No type } 13 \text { cards supplied in A.FNIP. }\end{array}$ 
TABLE II. (Contt.).

Subroutine RAFNIP (Contd.)

$\begin{array}{lll}\text { Error } & 130 . & \text { Insufficient small core storage space to allocate } \\ \text { arrays. } & \end{array}$

\section{Subroutine RAFX2}

$\begin{array}{lll}\text { Error } & -100 . & \text { No type } 01 \text { card in A.FX2. } \\ \text { Error } & -120 . & \text { Unacceptable input variable: } \\ \text { Error } & -140 . & \text { Ins ufficient core storage space to allocate arrays }\end{array}$

Suhroutine RATFLX

Error -100. Number of intervals inconsistent with. GEODST.

Ellul $=110$. ATFLUX supplied is not une- or two-dimensional.

Subroutine RATHRM

Error -100. No type 01 card present in A.THRM.

\section{Subroutine RCMESH}

\begin{tabular}{|c|c|c|}
\hline Error & 100 & $\begin{array}{l}x \text { coordinate spectfied on type } 06 \text { card is incon- } \\
\text { sistent with XMESH array. } \\
\text { Same as Error } 100 \text {. }\end{array}$ \\
\hline DILOL & 110. & \\
\hline Error & 130 & $\begin{array}{l}\text { Y coordinate specified on type } 06 \text { card is } \\
\text { inconsistent with the YMESH array. }\end{array}$ \\
\hline Error & 140 & $\begin{array}{l}\text { A mesh point did not receive a region specification. } \\
\text { Subroutine RDLYR4 }\end{array}$ \\
\hline Error & -100 & $\begin{array}{l}\text { Isotope does not occur in DLAYXS. } \\
\text { Subroutine RESTRT }\end{array}$ \\
\hline Error & -100 & $\begin{array}{l}\text { Insufficient large core storage space to allocate } \\
\text { arrays. }\end{array}$ \\
\hline Error & -120 & $\begin{array}{l}\text { Old and new values of amount of small and large } \\
\text { core available are not compatible. } \\
\text { Same as Error - } 100 \text {. }\end{array}$ \\
\hline
\end{tabular}


TABLE II. . (Contd.)

Subroutine RESTRT (Contd.)

Error $\quad-150$.

Insufficient small core storage space to allocate arrays.

\section{Subroutine RFIXSR}

$\begin{array}{ll}\text { Error } & -100 . \\ \text { Error } & -120 . \\ \text { Error } & -130 . \\ \text { Error } & -140 .\end{array}$

FIXSRC file inconsistent with GEODST file.

FIXSRC supplied is not one- or two-dimensional.

FIXSRC supplied is not for P-O diffusion theory.

Insufficient core storage space for allocating arrays.

\section{Subroutine RFXCTL}

$\begin{array}{lll}\text { Error } & -100 . & \text { Input variable IPK has incorrect value. } \\ \text { Error } & -110 . & \text { Input variable NTHF has incorrect value. } \\ \text { Error } & -120 . & \text { Input variable NFDBK has incorrect value. } \\ \text { Error } & -130 . & \text { Input variable ICMPFL has incorrect value. } \\ \text { Error } & -140 . & \text { Input variable NTMPCR has incorrect value. } \\ \text { Error } & -150 . & \text { Input variable IPTYPE has incorrect value. } \\ \text { Error } & -160 . & \text { Input value NDOSS has incorrect value. } \\ \text { Error } & -180 . & \text { Input value NGAMN or NOUTMX has incorrect value. } \\ \text { Error } & -190 . & \text { Input array IEDF has an incorrect value. } \\ \text { Error } & -200 . & \text { Record } 4 \text { of FXCTRL has an incorrect value. } \\ \text { Error } & -210 . & \text { Insufficient core storage space to allocate arrays. } \\ \text { Error } & -220 . & \text { Starting time for a driving function is less than } \\ \text { Error } & -230 . & \text { zero. } \\ & & \text { Ending time for a driving function is less than }\end{array}$

\section{Subroutine RFXHMO}

$\begin{array}{lll}\text { Error } & -100 . & \begin{array}{l}\text { Number of regions for which materials have been } \\ \text { specified is less than number of geometry regions. } \\ \text { Number of regions from FXHMOG is not equal to } \\ \text { number of geometry regions. }\end{array} \\ \text { Error } & -110 . & \begin{array}{l}\text { Number of zones from FXHMOG is not equal to } \\ \text { number of geometry zones. }\end{array} \\ \text { Error } & -120 . & \text { Insufficient core storage space to allocate arrays. }\end{array}$


TABLI II. (Contd.)

\section{Subroutine RFXH35}

$\begin{array}{ll}\text { Error } & -110 . \\ \text { Error } & -120 . \\ \text { Error } & -130 . \\ \text { Error } & -140 . \\ \text { Error } & -150 . \\ \text { Error } & -160 . \\ \text { Error } & -1,70 .\end{array}$

Length of material mix table is not greater than zero.

Number of isotopes in FXHMOG is not greater than zero.

Number of generic materials in FXHMOG is not greater than zero.

Number of unique materials in FXHMOG is not greater than zero.

Number of unique and generic materials are not compatible.

Insufficient core storage space to allocate arrays.

Same as Error -160 .

Subroutine RFXTH

Error $\quad-100$

Insufficient core storage to allocate arrays.

\section{Subroutine RGEODS}

Error $\quad-100$.

Insufficient core storage space to allocate arrays.

\section{Subroutine RHOBEG}

Error $\quad-100$.

Error $\quad-110$.

Insufficient core storage space to allocate arrays. Same as Error -100 .

\section{Subrriut ine ROMRT}

Error

1.

Order 15 exceeded in Romberg integration.

\section{Subroutine RRTFLX}

Error $\quad-100$

Number of first or second dimension intervals or number of groups of RTFLUX is inconsistent.

Error $\quad-110$. RTFLUX specified is not one- or two-dimensional.

Subroutine RWIS $\emptyset \mathrm{T}$

Error -600. Insufficient sma11 core storage space to perform A.ISO to ISOTXS transformation. 
TABLE II. (Contd.)

\section{Subroutine RXSCON}

$\begin{array}{lr}\text { Error } & -100 . \\ \text { Error } & -110 . \\ \text { Error } & -120 . \\ \text { Error } & -130 . \\ \text { Error } & -140 .\end{array}$

Number of generic materials on XSCON is inconsistent.

Number of unique materials on XSCON is inconsis tent. Variables NFMAT or NTFMAT are inconsistent with NMAT.

NFAM is greater than six.

Insufficient core storage space to allocate arrays.

\section{Subroutine SCAN}

Error -18

Error in $B C D$ input cards.

Error $\quad-22$.

Same as Error -18 .

Error 24. .

File is not in SEEK tables.:

Subroutine SHPEND

Error $\quad 100$

Number of gamma factor iterations exceeded.

Subroutine START

Error -100. Unable to allocate the requested number of words.

\section{Subroutine STUFF}

Error

1.

Error in subroutine STUFF.

\section{Subroutine STUFF1}

Error - 8 .

Error - 14.

Error $\quad-22$.

Error $\quad-48$.
End of file encountered on ARC input file.

Record containing the word end was expected.

Subroutine STUFF1 expects a keyword.

Error in subroutine STUFFl.

\section{Subroutine TIMDEP}

Error $\quad-100$.

Insufficient core storage space to allocate arrays. 
TABLIF II. (Conta:)

\section{Subroutine TRIGOM}

Error 100 .

A row in the MR array has no non-zero entries.

Error 110.

A row in the MR array has a hole In it.

\section{Subroutine XDENCK}

Error $\quad-100$.

Energy bounds of ISOTXS and DLAYXS do not agree.

Error $\quad-110$.

Same as Error -100 .

\section{Subroutine XDRDR2}

\begin{tabular}{|c|c|c|}
\hline Error & -100 & $\begin{array}{l}\text { Number of energy groups in ISOTXS is not greater } \\
\text { than zero. }\end{array}$ \\
\hline Error & -110 & $\begin{array}{l}\text { Number of 1sotopes. In ISOTXS is not greater } \\
\text { than zero. }\end{array}$ \\
\hline Error & -120 & $\begin{array}{l}\text { Maximum scattering order must not be less than } \\
\text { zero. }\end{array}$ \\
\hline Error & -130 & Error in blocking of scattering data. \\
\hline Error & -140 & Number of isotopes in DLAYXS must be positive. \\
\hline Error & -150 & $\begin{array}{l}\text { Number of energy groups of ISOTXS and DLAYXS } \\
\text { must agree. }\end{array}$ \\
\hline Error & -160 & $\begin{array}{l}\text { Number of delayed families in DLAYXS is not } \\
\text { positive and less than } 7 \text {. }\end{array}$ \\
\hline Error & -170 & Set fission spectrum is a matrix - not allowed. \\
\hline
\end{tabular}

\section{Subroutine XDRDR3}

Error $\quad-100$.

Error $\quad-110$.

Insufficient core storage space to allocate arrays.

Same as Error -100 .

\section{Subroutine XSCTSV}

Error $\quad=100$

Scattering matrix type must be $0,1,2$, or 3 .

Subroutine XSFIPR

$\begin{array}{lll}\text { Error } & -100 . & \text { Denominator on term for prompt chi was zero. } \\ \text { Error } & -110 . & \text { Denominator in term for prompt nu was zero. } \\ \text { Error } & -120 . & \text { Denominator in term for delayed nu was zero. }\end{array}$


TABLE II. (Contd.)

Subroutine XSUPDT

Error $\quad-100 . \quad$ Array in SPEC was inconsistent.

Error -110. Temperature of isotope is not within five percent of required temperature. 
After a fatal error occurs, a flag is set in subroutine ERROR. Subsequently. if a call is made to ERROR with FATAL substituted for the subroutine name, the flag is checked to see if a fatal error has occured. If such an error has occured, then processing is terminated.

\section{F. Restart Capabilities}

FX2-TH has two different ways in which a problem may be restarted. These will be discussed separately.

\section{F.1 Bypass Steady-State Calculation}

FX2-TH allows the user to bypass the steady-state calculation if the converged steady state real and adjoint fluxes and thermal-hydraulic conditions are available as binary files. Therefore, the files RTFLUX, ATFLUX, and (if thermal-hydraulics is being used) THDATA must have been previously saved and avilable as binary files in BLOCK=0LD. The FX2-TH code will then begin the calculation with the time-dependent section. The cross sections may be input in the smae manner on both runs.

\section{F.2. Transient Restart}

A transient $\mathrm{FX} 2-\mathrm{TH}$ problem may be restarted at a certain time in the transient. A restart file is written periodically (under user control) during the transient. Periodic dumps are written every $C$ minutes of computing time and $P$ seconds of problem time. A restart dump is also written at normal problem termination time. The restart file is written alternately. on two datasets; a message appears in the printed edit to indicate which dataset the file was last written on.

I'o submit a problem which begins trom a restart file, the dataset XSCON must be placed in BLOCK=OLD, and the dataset FX2RS1 or FX2RS 2 must also be placed in BLOCK=OLD. Use FX2RS1 if the restart file is written on file 49, and FX2RS2 if the restart file is written on file 50. In dataset A. FX2 the restart flag on the type 03 card must be set to unity. Other input data in the dataset A.FX2 may be changed on a restart (see the input data description). If a fixed source file (FIXSRC) is written using the type 19 cards of A. FNIP, that file must be saved and the name FIXSRC placed in $B L O C K=O L D$ for the restart case.

Table III shows the status of the files for a hypothetical set of runs. The first run calculates the steady-state conditions with the microscopic cross sections originating on $\mathrm{BCD}$ input data sets. The second run bypasses the steady-state calculation and performs part of a transient. The final restart file was written on FT49F001. The third run restarts the transient using the restart file written above.

\section{G. Edits and Output Options}

The edits produced by the FX2-TH program are controlled entirely by the type 05 card of BCD input data set A.FX2. There are three kinds of edits produced. The first is the standard printed edit file printed as the 
TABLE III STATUS OF BINARY FILES FOR A SET OF RESTART CASES

\begin{tabular}{l|l|l|l}
\hline & $\begin{array}{l}\text { INITIAL } \\
\text { STEADY } \\
\text { STATE } \\
\text { SOLUTION }\end{array}$ & $\begin{array}{l}\text { FIRST } \\
\text { TRANSIENT } \\
\text { CASE }\end{array}$ & $\begin{array}{l}\text { TRANSIENT } \\
\text { RESTART } \\
\text { CASE }\end{array}$ \\
\hline A. ISO & OLD & TEMPORARY & TEMPORARY \\
A. DLA & OLD & TEMHUKARY & TEMFORARY \\
ISOTXS & NEW, KEEP & TLD, DELETE & TEMPORARY \\
DLAYXS & NEW, KEEP & TEM, DELETE & TEMPORARY \\
XSCON & NEW, DELETE & OLD \\
RTFLUX & OLD, KEEP & TEMPORARY \\
ATFLUX & NEW, KEEP & OLD, DELETE & TEMPORARY \\
THDATA & NEW, KEEP & OLD, DELETE & TEMPORARY \\
FX2RSI & NEW, KEEP & OLD, DELETE & OLD \\
FX2RS2 & TEMPORARY & NEW, KEEP & TEMPORARY \\
FIXSRC & TEMPORARY & NEW, KEEF & OLO \\
\hline
\end{tabular}

calculation proceeds. The second is a log of certain key parameters during the transient which is written on data set A.FOUT in BCD format. When the problem reaches a normal termination, the file A.FOUT is printed out as a summary of the transient. This edit tabulates several key variables as a function of reactivity step, and flags each shape step with an asterisk. The third is a binary edit file, FXEDIT. This file can be written in brief, standard, or extended edit form. This file is written to assist the user in interpreting FX2-TH output, and allow the user to plot key variables easily. With the extended edit, the user has available a summary of various problem parameters, the pointwise powers, regionwise temperatures, and multigroup neutron fluxes. The FXEDIT description in Append1x III sliuws tie format of this binary file. The output from the standard printed output for Sample Problem 1 is shown in Appendix VI.

If an FXEDIT file is being written and a transient restart case is performed, the edit files may be concatenated from the two jobs. This may be done by specifying DISP=(MOD,KEEP) on the restart job for the FXEDIT dataset. Care should be taken to insure that the restart file taken to begin the restart job was the last such file written by the initial job and that the calculation of the initial job terminared lmmedialely after writing the restart file. This will insure that duplicate entries for the same time step do not appear on the FXEDIT file. On the CDC 7600 , the FXEDIT file should be skipped to the end of information at the beginning of the restart job. 


\section{PROGRAMMING I NFORMATION}

\section{A. Program Structure}

The FX2-TH program is structured along the lines of the overlays which compose it. The program consists of a root segment, and ten primary overlays. Four of the primary overlays are further subdivided into secondary overlays. A general chart showing the overlay structure is given in Figure 14 . A detailed Table listing all subroutines contained in each overlay is given in Table IV.
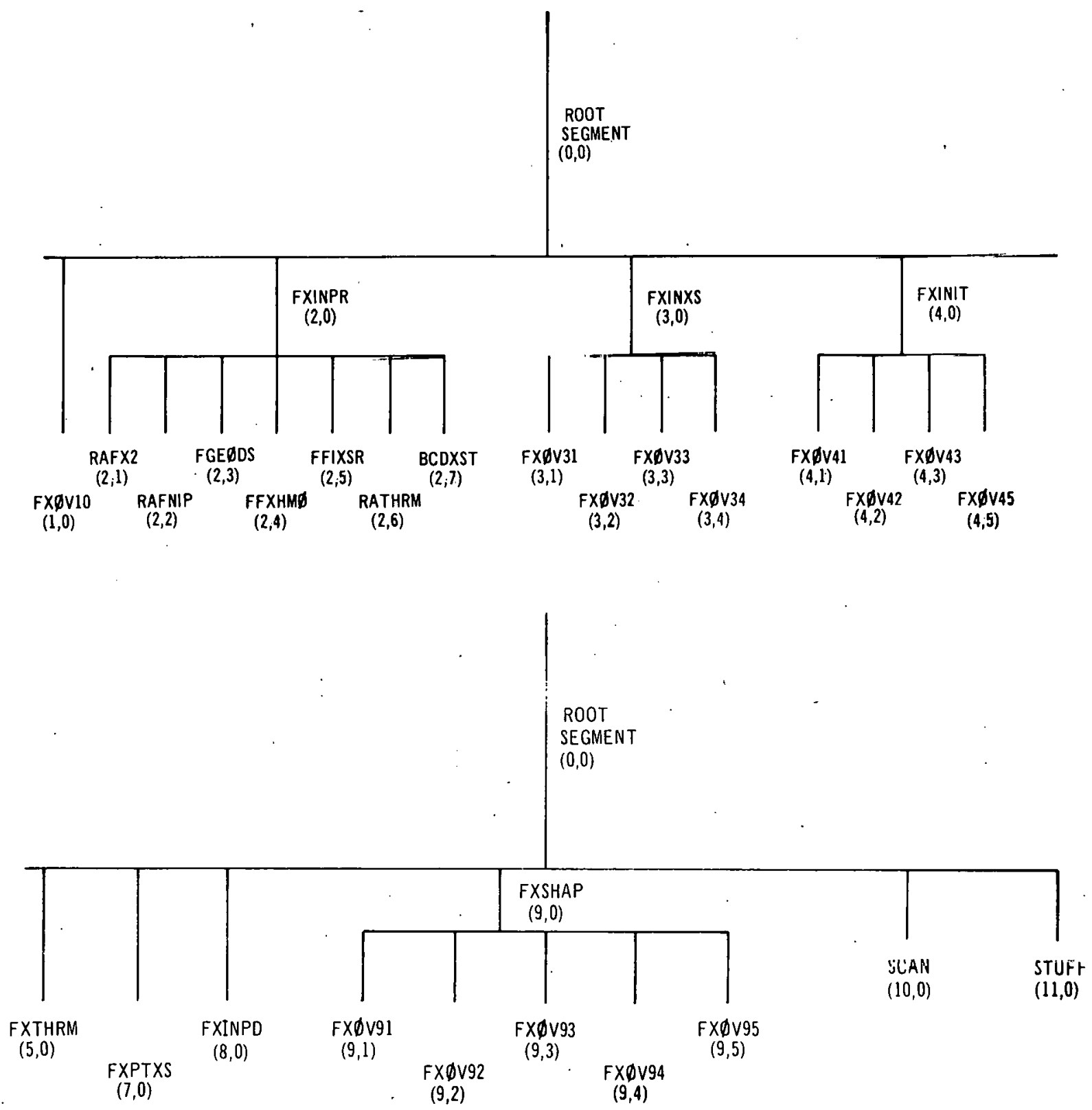

Fig. 14. Genera1 Overlay Structure of FX2-TH 
TABLE IV. Detalled Overlay Structure of FX2-TH

LEVEL $(0,0)$

MAIN

START

SSTATE

TIMDEP

SSEDI T

CALFSP

SUMARY

TIMSUM

BUOPEN BUCLOS

IEQUAT

FEQUAT

FLTSET

INTSET

LINES

DSKEDT

ERROR

TWODPR

TWOPR2

VOLUME

PAR

EDTFTC

IEQUT2

FEQUT2

INTS T2

FLTST 2

SQUEZ2

LCOPEN

LCREED

LCCLOS

FEQUT4

IEQUT4

CODE

SEEK

REED

TIMER

CRED

ECMV

IRED LRIT

ABEND ABS TOP

POINTR

PUTPNT PUTBLK

BULK

FREE

WIPOUT CLEAR

GETPNT GETN

1 GE'l'

IPT2

PUTM PUTB

IPTERR NNAMSF

ILAST ILASTB

REDEF
REDEFM REDEFB

PURGE PURGEB

STATUS

PRTI 1

PRTI 2

PRTRI

PRTR 2

PRTECM

SPACE

SQUEZE

ALLOCl

ALLOC2 ${ }^{\circ}$

FREEl

EREE2 
TABLE IV. (Contd.)

$\underline{\operatorname{LEVEL}(1,0)}$

FXovi0

RHOBEG

RHOEND

SHPEND

TIMSTP

DRI VE $F$

EXTFLX

EDITS

PRECUP

PRCUPD

PRCMAP

PKCALC

PKSTEP

PKSTOR

PKUPDT

LEVEL $(2,0)$

FXINPR

FRTFLX

FATFLX

LEVEL $(2 ; 1)$

RAFX2

AFX? 10

WFXCTL

LEVEL $(2,2)$

RAFNIP

REPORT

ANIPO 3

NNIPHX

ANIPO 4

ANI.POS

ANIP0 6

ANIP08

ANIP09

ANIT12

ANIP1 3

ANIP14

ANIP19

AN1PJ4

GSCAN

LEVEL $(2,3)$

FGEODS

NUMREG

MESHC

MESHV

MSHORD

RCMESH

LOCHEX

TRIGOM

DZONE

BUCK

VOLREC

EXTBC

WGEODS

LEVEL $(2,4)$

FFXHMO

ISOSRT

MATSRT

WFXHMO 
TABLE IV. (Contd.)

LEVEL $(2,5)$

FFIXSR

I2SORD

GRPSOR

LEVEL $(2,6)$

RATHRM

THRMRZ

THRMO 3

THRMO 4

THRM05

THRMO 6

WFXTH

LEVEL $(2,7)$

BCDXST

RWISOT

RWDELY

RWDY 1

RWDY 2

LEVEL $(3,0)$

FXINXS

SDEQT

SDSEQT

SINEQT

SINSET

FARSET

SDRCST

IPT 3

PRNTEL

PRNTIA

PRNTII

PRNT1E

PRNT2E

PRNTID

PRNT2D

SINST2

SDEQT4

SDSEQ4

3INEQ4

LEVEL $(3,1)$

FXOV31

FHEADR

RFXH 35

XDRDR2

XDRDR 3

XDENCK

LEVEL $(3,2)$

FXov 32

XSCTSV

XSUPDT

DIMXCK

ISOR 48 
TABLE IV. (Contd.)

LEVEL $(3,3)$

FXov33

RDLYR4

DLUPDT

DLY 23

EDLYR4

XSURDK

DCRNCH

XSFIPR

DOPCAL

LEVEL $(3,4)$

FXOV 34

XS CTL

XSCTLI

LEVEL $(4,0)$

FXINIT

LEVEL $(4,1)$

FXOV41

RFXCTL

RGEODS

FORMSH

RFXHMO

CHKHOM

RFXTH

RXS CON

CHKXSC

RRTFLX

RATFLX

RFIXSR

LEVEL $(4,2)$

FXOV 42

INEDIT

EUXSLU

REGMAP

INI TSS

LEVEL $(4,3)$

FXov 43

INITT1

MAXMN F

LEVEL $(4,5)$

FXov45

RES TR T

COPYDS

CHNGIN

LEVEL $\left(5, U^{\prime}\right)$

FXTHRM

PWCAL

SIMYL

DETAL

THCALC

THFT

HCAIC.

ROMBI 
TABLE IV. (Contd.)

- LEVEL $(7,0)$

FXPTXS

PNTMAT

FISSON

SCTTER

FISFIX

XSFIX

LEVEL $(8,0)$

EXINPD

PASS1

PASS2

NROTAT

LEKIP

TLEKIP

GETBND

AREAP

LEVEL $(9,0)$

FXSHAP

CHEBE

DACOSH

HYPCOS

ROWSRC

TRISRC

INVRT

NGS UB

SOLVP

LEVEL $(9,1)$

FXOV91

SRCCAL

SRCMOD

LEVEL $(9,2)$

FXOV92

SFDGEN

SFDCEL

TFDCEL

SGTBND

AREAS

LEVEL $(9,3)$

FXoV93

FISINT

OMEGCA

OMEGIN

INNDS

TOMEGN

LEVEL $(9,4)$

FXov94

NITF.RS

GSORCI

GSORC2

INNERS

INNDO

INNEK' 1

GNDOTS

UPDFS

EXTRPO 
TABLE IV. (Contd.)

LEVEL $(9,5)$
FXOV95
SHPOUT
WRTFLX
WATFLX
LEVEL $(10,0)$
SCAN
LEVEL $(11,0)$
STUFF
STUFE1

In the root segment, routines such as the main program; SSTATE, and TIMDEP reside. In addition, the BPOTNTER and REED/RITE routines as well as various other common subroutines reside there. Overlay level $(1,0)$ performs the solution of the neutronics, pquations at each reactivity step. Overlay level $(2,0)$ reads $B C D$ input cards, and writes binary interface files. Overlay level $(3,0)$ converts the files ISOTXS and DLAYXS to macroscopic form, and writes the file XSCON. Overlay level $(4,0)$ performo variuus initializing functions for the steady state and transient, as well as restart capability. Overlay level $(5,0)$ solves the steady state and transient thermal-hydraulics equations. Overlay level $(7,0)$ calculates the temperaturedependent macroscopic cross sections present at this time. Overlay level $(8,0)$ calculates the inner products required to evaluate the point kinetics parameters. Overlay level $(9,0)$ calculates the inultigroup neutron flux distribution. Overlay levels $(10,0)$ and $(11,0)$ read the input data and form the $\mathrm{BCD}$ input data sets.

In Appendix I, a listing is provided of all subroutines in FX2-TH. In addition, a brief objective is present, as well as a 1 isting of all other routines called by and called from this routine.

\section{B. Program Flow Charts}

In Figure 15, macroscopic flow sharts for FX2 TII are provided. These flow thas sh show the overai.1 program logic for FX2-TH.

C. Description of Dynamically Allocated Arrays

\section{C.1 BPOINTER, A Dynamic Storage Allocation Program}

BPOINTER is a FORTRAN subprogram package which was developed to alleviate bookkeeping chores associated with the use of dynamic storage allocation techniques.

Programs which use the BPOINTER capability tend to be structured in subroutine form. A control routine is used to define one or two large blocks of storage (called the container arrays), and make the appropriate calls to BPOINTER to control the allocation of storage within these block(s). Calls to calculational subroutines transmit pointers corresponding to appropriate array locations through the calling sequences. All BPOINTER 
MAIN

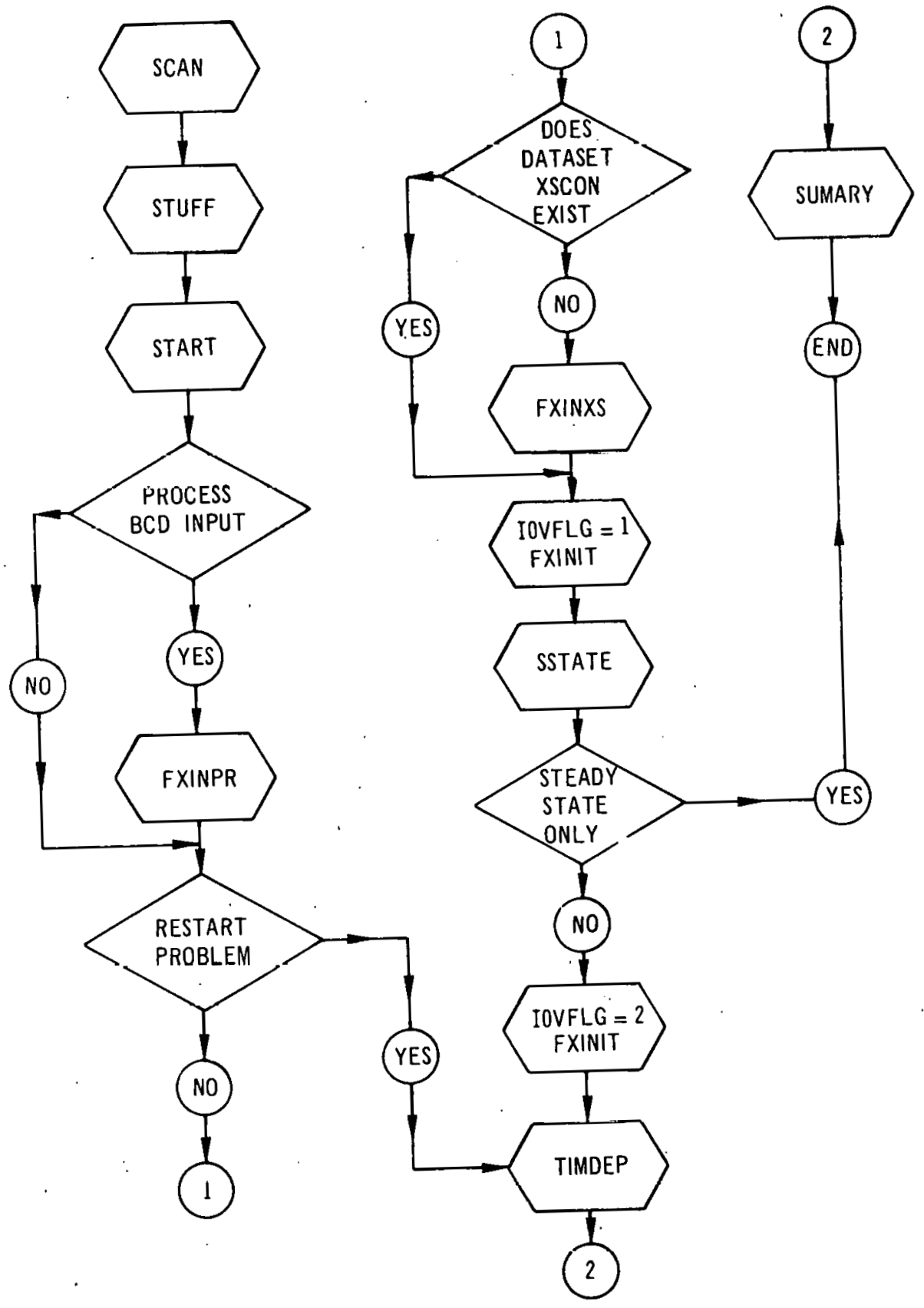

Fig. 15. Macroscopic Flow Diagram 
START

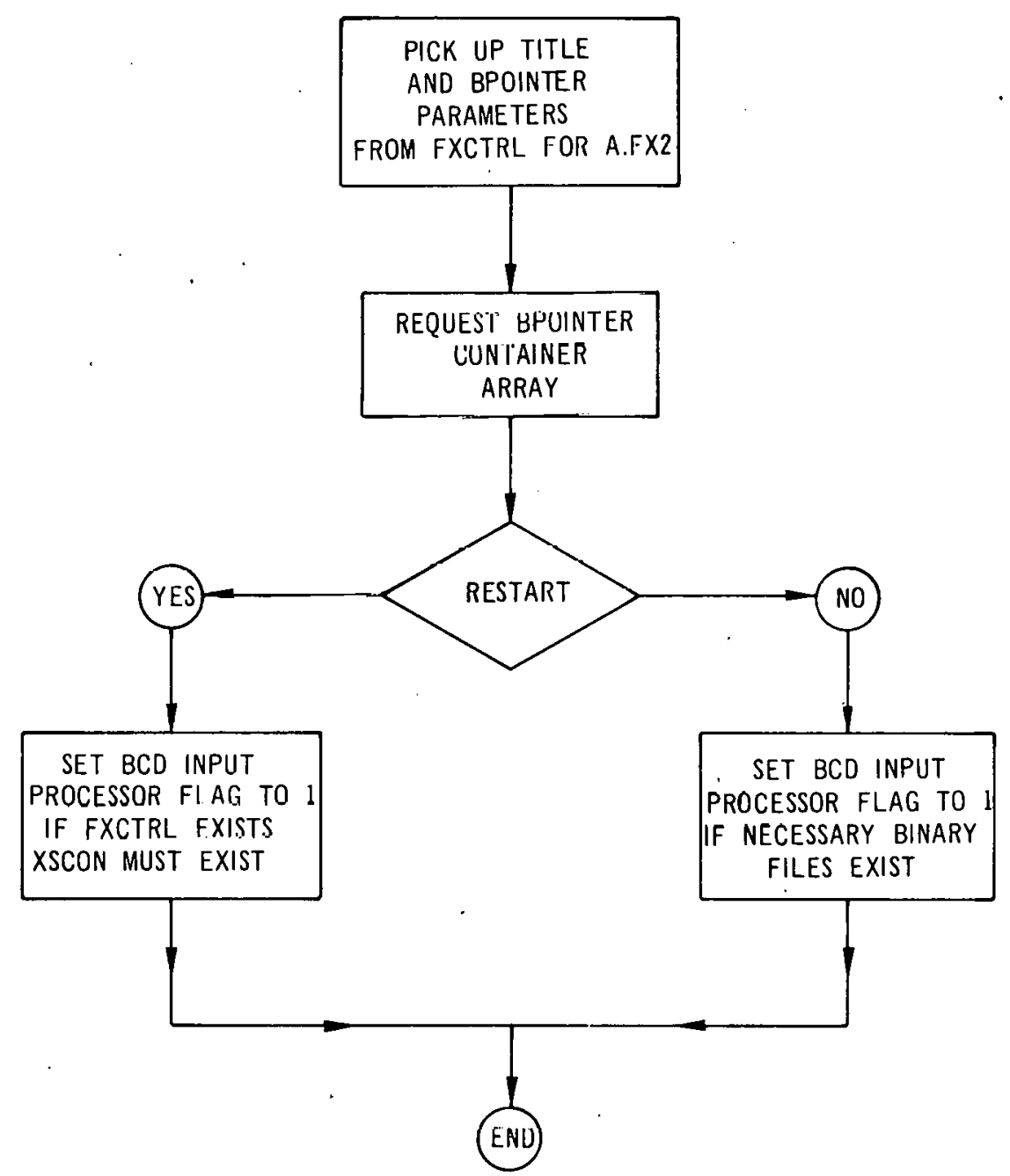

Fig. 15. (Contd) 


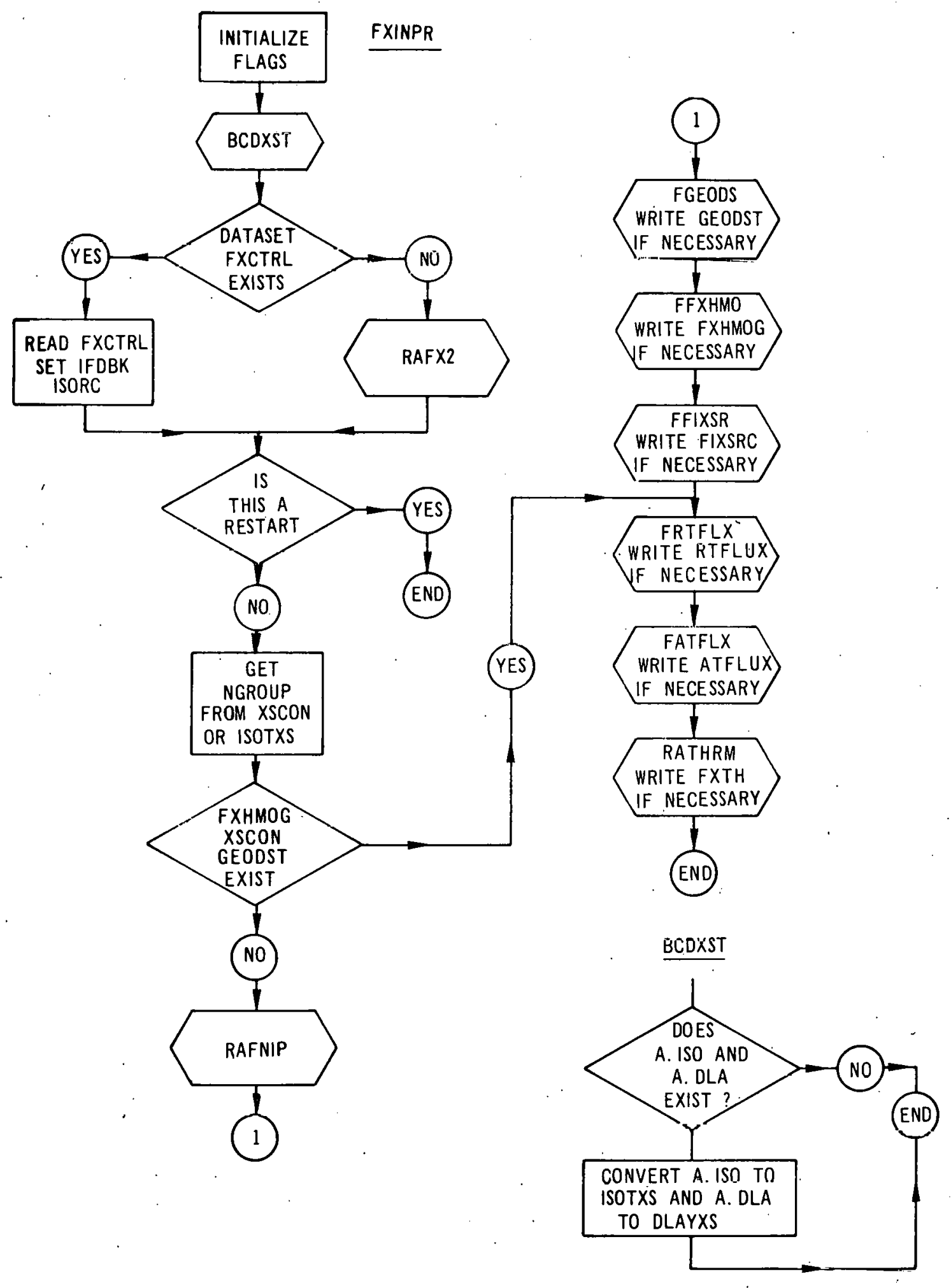

Fig. 15. (Contd) 


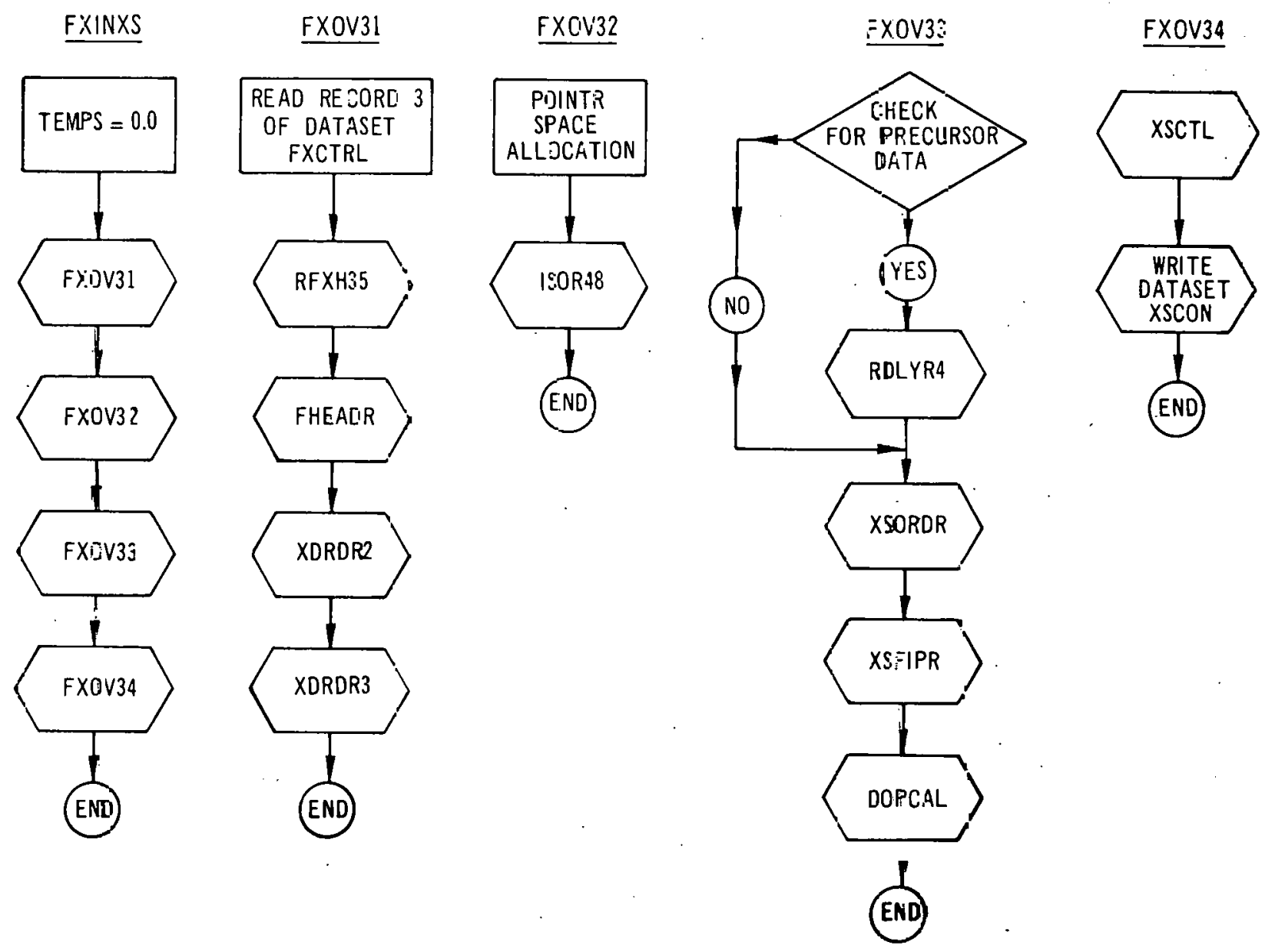

Fig. 15. (Contd! 
FXINIT
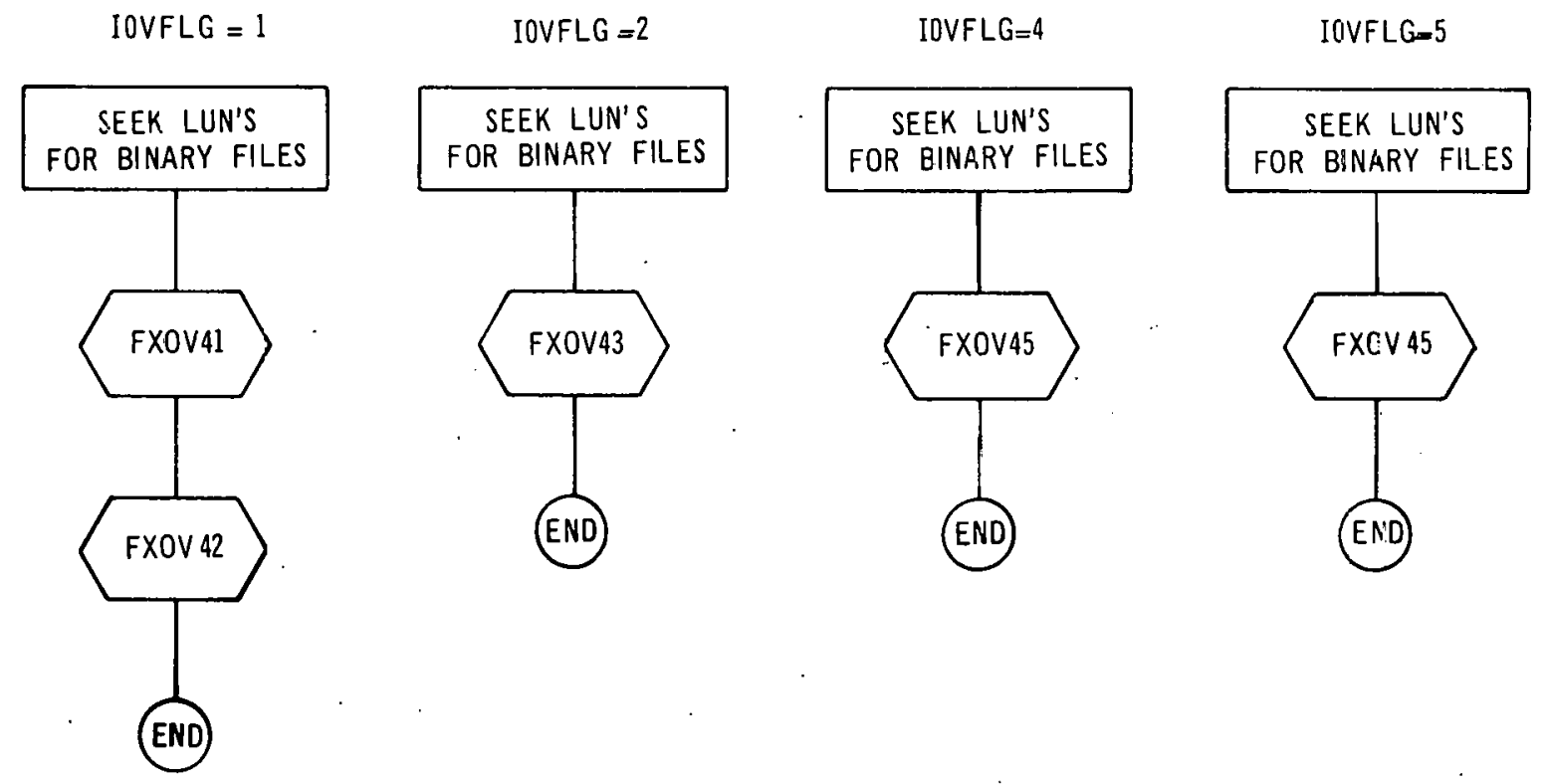

Fig. 15. (Contd) 


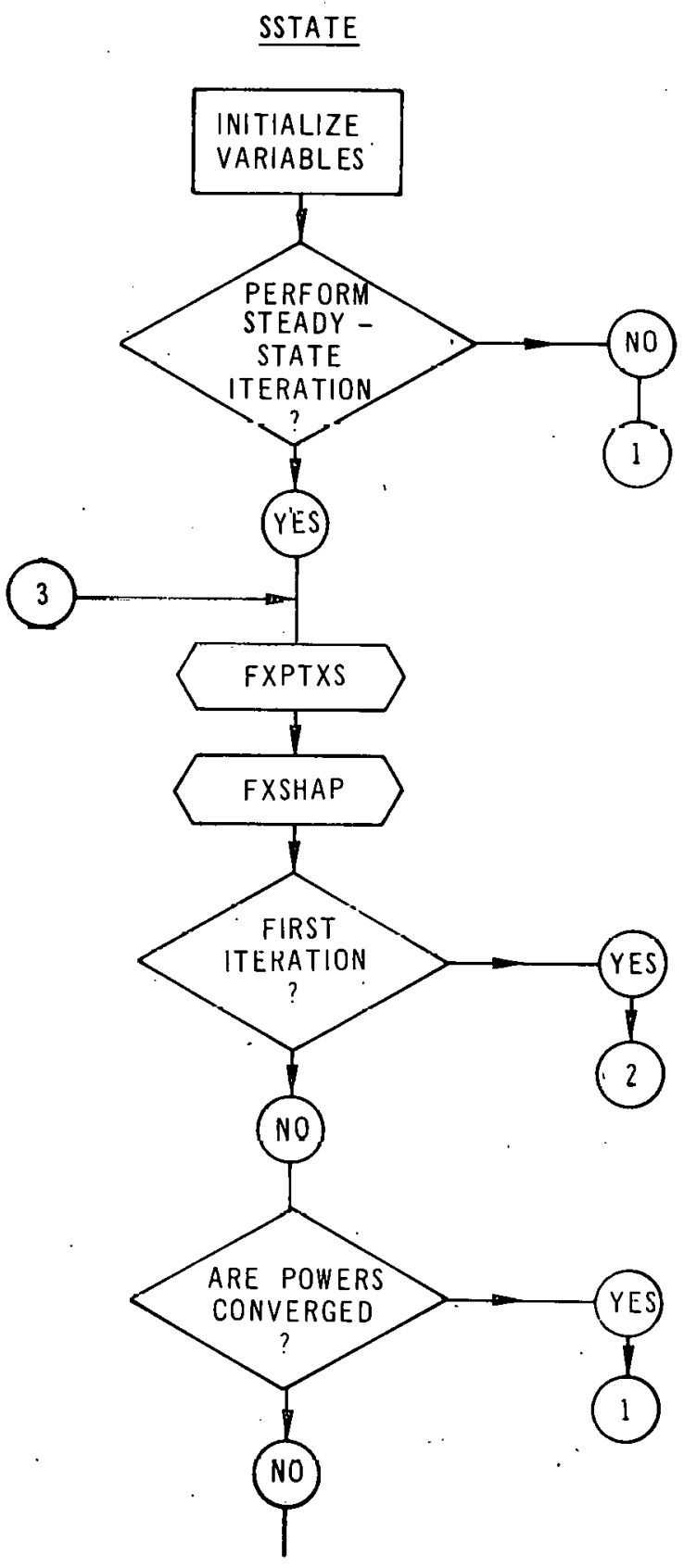

Fig. 15. (Contd) 

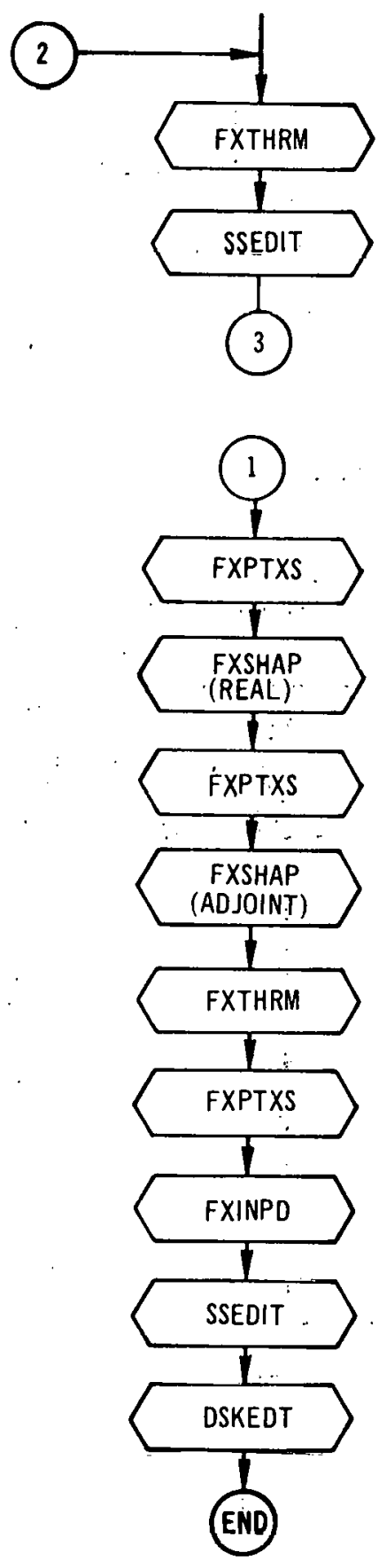

Fig. 15. (Contd) 


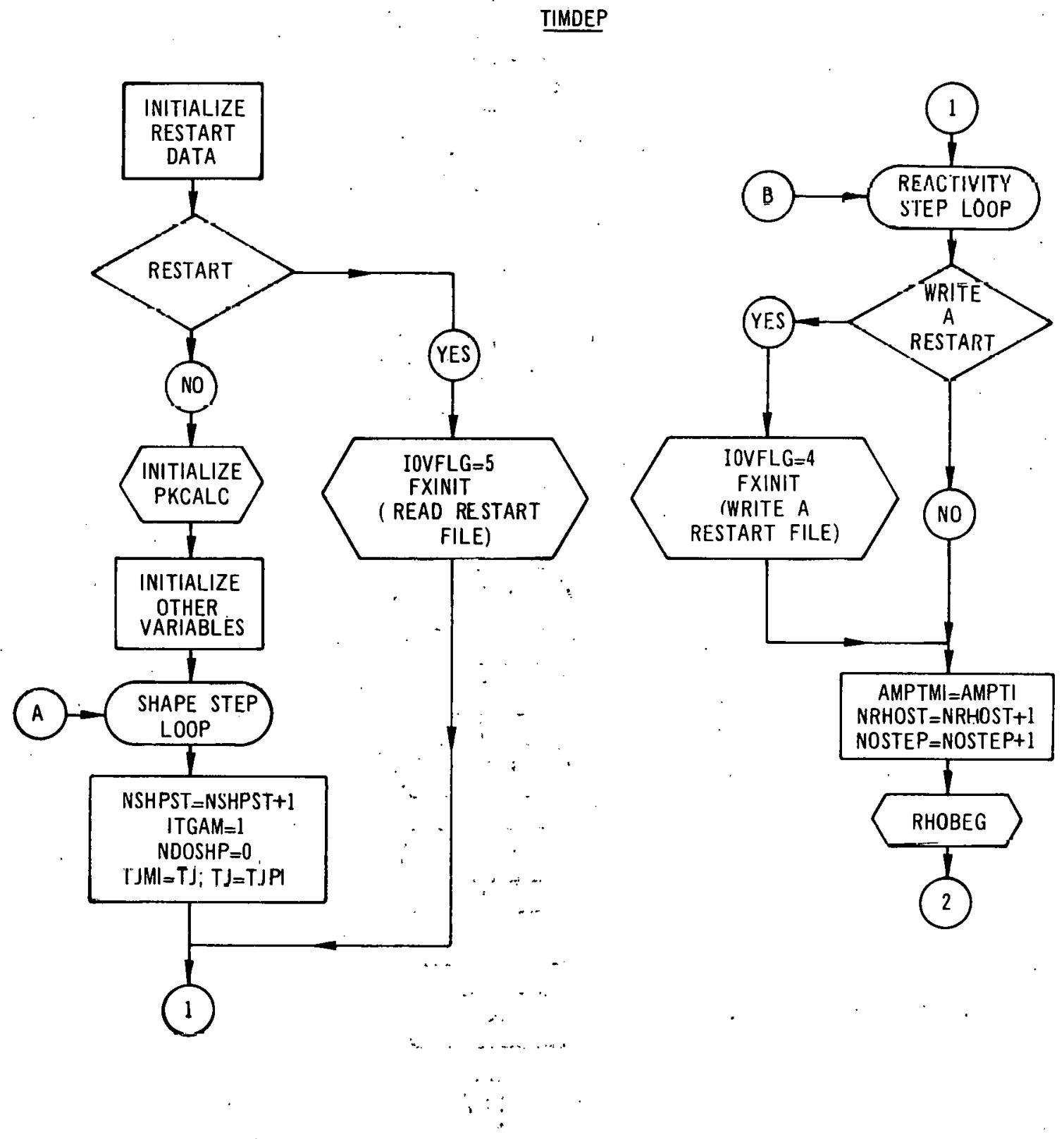

Fig. 15. (Contd) 


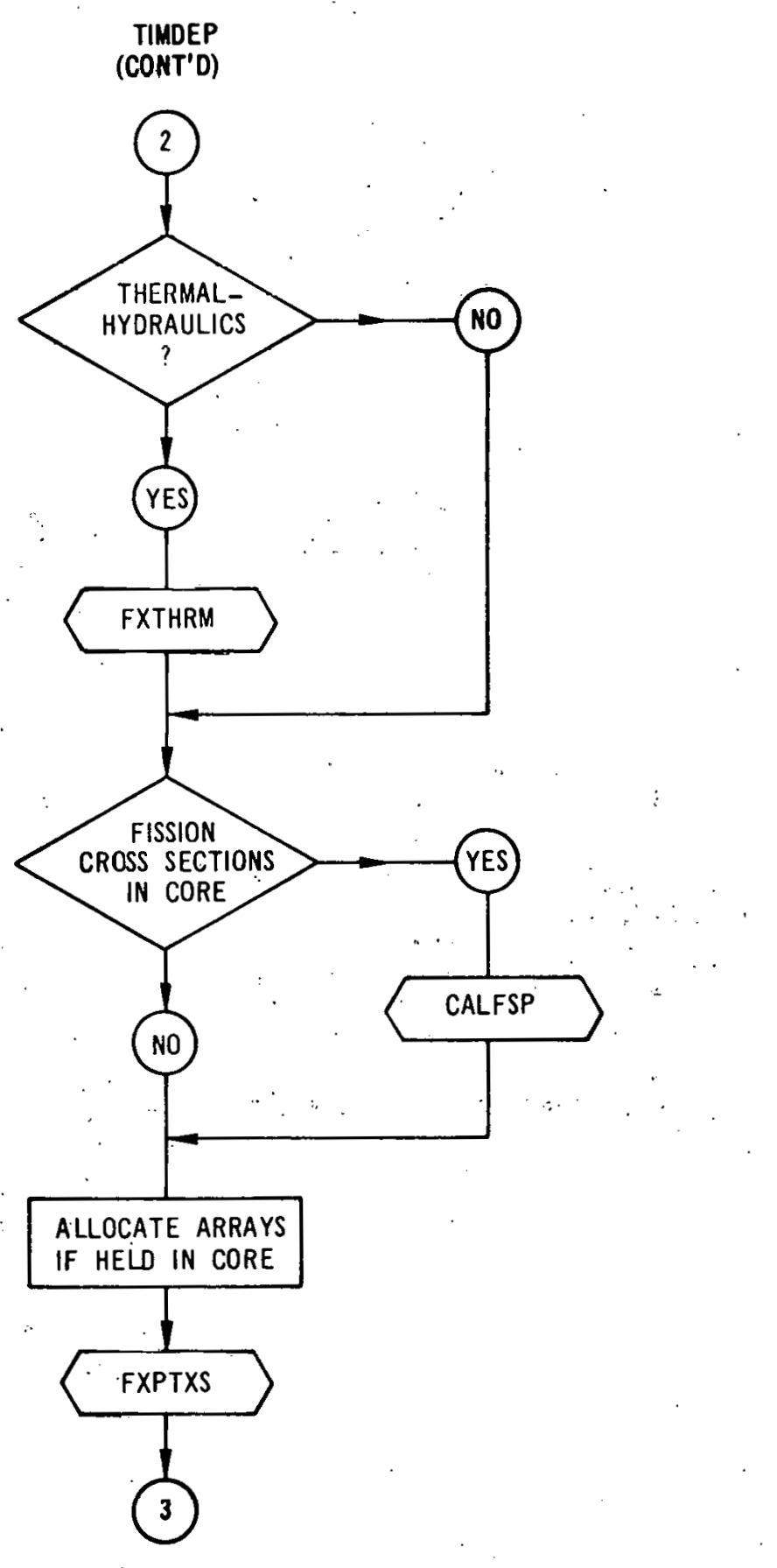

Fig. 15. (Contd) 


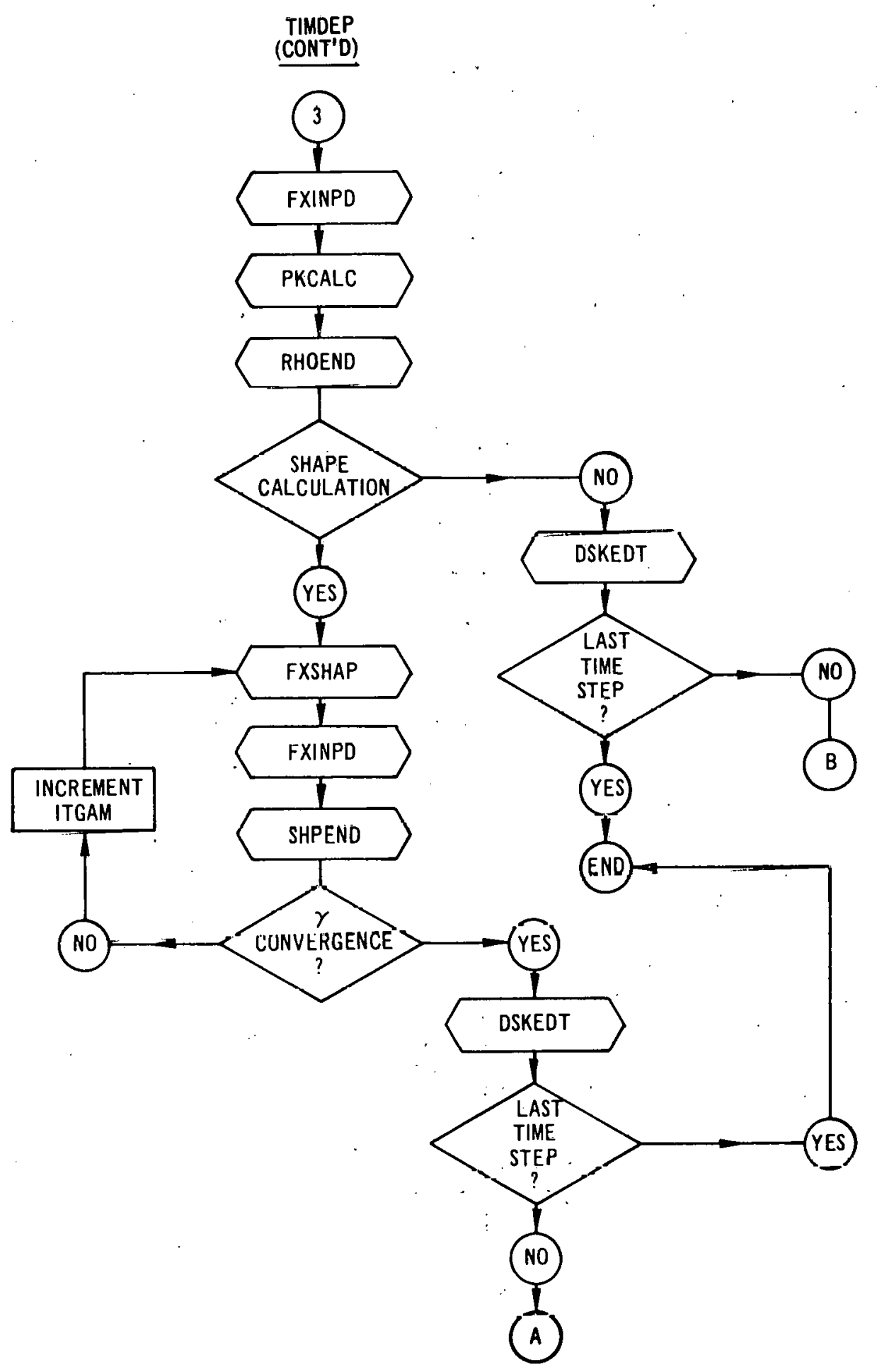

Fig. 15. (Contd) 
capabilities are accessed through an appropriate call to an entry point, subroutine, or function subprogram. The following capabilities are available in the BPOINTER system:

(a) Storage of data in and retrieval of data from the container array, via user defined variable arrays.

(b) Purge of variable arrays stored in the container array.

(c) Automatic "cleanup" of the container array when more storage is required.

(d) Re-definition of array sizes without loss of data already stored in the array.

(e) Array dump of selected integer, floating point or BCD arrays in a prescribed format.

(f) Trace dumps of BPOINTER activities.

(g) Status reports of the BPOINTER tables.

Detailed program documentation including flow charts, common block information and subprogram descriptions is available in Reference.18. This Section is intended to provide a brief description of how the program package operates. The major differences between the IBM and CDC standalone versions of the program package are also noted.

The short example listed in Figure 16 is intended to illustrate the structure of a program using the BPOINTER package. This example demonstrates the manner in which a container is allocated, pointers defined and used, and the container released.

Brief descriptions of the BPOINTER subroutines and functions are given in the subroutines descriptions in Appendix I.

All dynamically allocated arrays are addressed relative to the common block /ARRAY/ which contains a single array element, BLK(1). In the IBM version of the code the element must be declared as DOUBLE PRECISION. A second common block /ARRAY2/ is used in the CDC version of BPOINTER to address arrays allocated to a large core memory container. This common block also contains a single array element BLK2(1) which must be declared a LEVEL 2 variable. The equivalent of the large core memory container on IBM equipment is a second container which may be given a HIARCHY 1 location but is addressed in precisely the same manner as the first (SCM) container. The one word assigned to the container by the source language program provides a reference address. At execution time machine language routines (ALLOCl, ALLOC2 on IBM, MEMGET1, MEMGET2 on CDC) are used to obtain the addresses of core which are avilable to the program for the allocation of data arrays. These blocks of core are allocated in the following manner.

\section{(a) IBM allocation}

The standard IBM macro instructions GETMAIN and FREEMAIN are used to allocate and free consecutive words of core which are available to the program. The designations subpool 1 and 2 are assigned to the bulk (LCM) and fast (SCM) containers respectively. Since allocations are performed in units of 256 (eight byte) words, it is most efficient to request blocks of core in such multiples. 


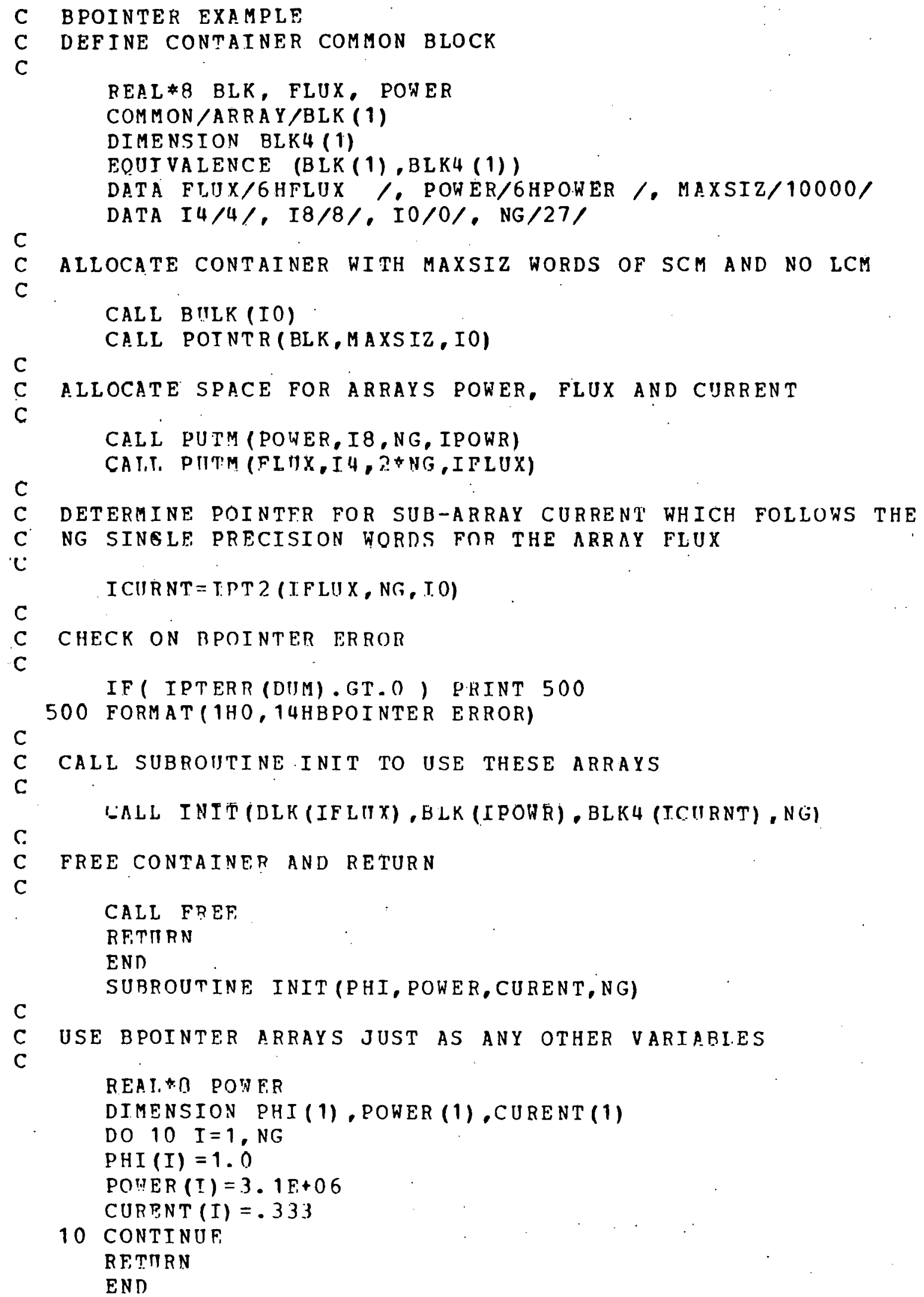

Fig. 16. BPOINTER Example 


\section{(b) CDC allocation}

The COMPASS routine MEMGET uses the standard CDC macro instruction MEMORY to determine the jobs SCM and LCM field length. The core available as a container for BPOINTER SCM arrays is determined by subtracting the address of blank common from the SCM field length. Thus a program using the CDC version of BPOINTER should not use blank common. Blank common is used for this purpose because of the CDC loader convention which places blank common after all other program sections in core. Although blank common is used in this manner to determine the available core for a container, arrays in the container are addressed relative to the common block/ARRAY/ as noted above. The BPOINTER program package accounts for the offsets between the address of /ARRAY/ in core and the address of the container. It should also be noted that the conventions used by BPOINTER are such that the first word of the container is not in general set to the first word of blank common. This is important since the loader convention noted above is not adhered to by all CDC Systems. The user is therefore responsible for providing enough SCM memory to accommodate the program, any SCM buffers, and the BPOINTER container as there is currently no effective check to make sure that data stored in the BPOINTER container do not overlap code. It is assumed by the CDC version of BPOINTER that the LEVEL 2 common block /ARRAY2/ is addressed as the first word of LOM and the entire LOM field length is assumed to be available to BPOINTER for its LCM container.

The letters $M$ and $B$ are used as neumonics within BPOINTER to designate routines which operate on the $S C M$ and LCM containers respectively. Thus PUTM allocates an array in the SCM container while PUTB allocates an array which must be referenced on CDC equipment as a LEVEL 2 array. On IBM equipment without HIARCHY support (e.g. 370/195) the two containers are equivalent. The distinctions noted above between the two dynamic containers are important on CDC equipment where the containers are addressed quite differently and on IBM equipment with HIARCHY support where access to the BULK core container (HIARCHY 1 , subpoo 1 ) is significantly slower than access to the MAIN core container (HIARCHY 0 , subpool 2).

\section{2 Arrays Permanently In-Core}

In FX2-TH, arrays are allocated for three basic purposes:

a) Permanently in-core arrays

b) Arrays which may be in-core depending on the data management scheme

c) Temporary a rrays

Arrays which reside permanently in-core are discussed in this Section, and arrays which may be in-core depending on the data management scheme are discussed in the next Section.

In each FX2-TH $r$ un, a certain number of arrays are allocated permanently in-core. These arrays are described in Figure 17. The name, arguments, function, word length, and core block indicator are given for each array. Most of these arrays are allocated in overlay $(4,1)$ and remain throughout the rest of the calculation. These arrays can be divided into the following categories: 

a) Driving function (DFRLAB, DFMLAB, DRF)
b) Geometry (NZNR, MR, XMESH, YMESH, IS, IE, ZONLAB, REGLAB)
c) Materials (BSQ, BNDC, CHIP, PNU, CHID, DNU, VEL, NDN, . LTOLP, LPTOL, UMNAME, AMNAME, NUMAM, MATREG)
d) Thermal-Hydraulics (MTH, TF, TC, ITYP, DAT, NPIN, TCIN, FLOW, AXTH, HCORR, PWOLD)
e) Neutronics (NINGR, OMER, POWERM, PKBUF)

Categories a), b), c), and e) are present for all cases. If no thermalhydraulic feedback is used, category d) is not present. If one of the adiabatic thermal-hydraulic models is used, the following arrays are present in category d): MTH, TF, TC, ITYP, DAT, AXTH, PWOLD. The entire category d) listed above is present if the detailed model is used.

\begin{tabular}{|c|c|c|c|}
\hline Name & Function & $\begin{array}{l}\text { Word Length } \\
\text { (Bytes) }\end{array}$ & $\underset{(0 / .1)}{\text { Sma11/Large }}$ \\
\hline $\begin{array}{l}\text { DFRLAB (NREGD) } \\
\text { DFMLAB (NREGD) }\end{array}$ & $\begin{array}{l}\text { Region labels for diving functions } \\
\text { Material labels for driving }\end{array}$ & 8 & 0 \\
\hline & functions & 8 & 0 \\
\hline $\mathrm{DRF}(4$, INREGD $)$ & $\begin{array}{l}\text { Driving function data }\left(\mathrm{T}_{\mathrm{SI}}, \mathrm{T}_{\text {END }}\right. \\
\text { (I., m) }\end{array}$ & 8 & 0 \\
\hline BSQ (NZONE, NGROUP) & Values of. $\mathrm{B}^{2}$ & 8 & 1 \\
\hline BNDC (NBCS) & Boundary conditim constants & 8 & 1 \\
\hline NZNR (NREG) & Region to zone assignment & 4 & 1 \\
\hline MR(NINTI, NINTJ) & Neutronics mesh region assignments & 4 & 1 \\
\hline XMESII (NINTI) & $\mathrm{x}$-d1rection mesh locations & 8 & 0 \\
\hline YMESH (NINTJ) & Y-direction mesh locations & 8 & 0 \\
\hline IS(NINTJ) & $\begin{array}{l}\text { Mesh starting locations } \\
\text { (if triangular) }\end{array}$ & 4 & 0 \\
\hline IE (NINTJ) & $\begin{array}{l}\text { Mesh ending locations } \\
\text { (1f triangular) }\end{array}$ & 4 & 0 \\
\hline REǴLAB (NREG) & Region labels & 8 & 1 \\
\hline ZONLAB (NZONE) & Zone labels & 8 & 1 \\
\hline CHIP (NTFMAT, NGROUP) & Prompt CHI values & 8 & 1 \\
\hline PNU(NTFMAT, NGROUP) & Prompt NU values & 8 & 1 \\
\hline CHID(NFAM, NGROUP) & Delayed CHI values & 8 & 1 \\
\hline DNU(NFAM, NTFMAT, & & & \\
\hline NGROUP) & Delayed NU values & 8 & 1 \\
\hline VEL(NGROUP) & Neutron velocities & 8 & 1 \\
\hline NDN(NGROUP) & Number of downscatter groups & 4 & 1 \\
\hline LTOLP (NMAT) & Generic material numbers & 4 & 1 \\
\hline LPTOL (NFMAT) & Generic fissionable material number & 4 & 1 \\
\hline UMAME (NTMAT) & Unique material label & 8 & 1 \\
\hline AMNAME (NMAT) & Genertc material label & 8 & 1 \\
\hline NUMAM(NTMA') & $\begin{array}{l}\text { Position in AMNAME array of } \\
\text { unique material m }\end{array}$ & 4 & 1 \\
\hline MATREG (NMAT, NREG) & $\begin{array}{l}\text { Unique material number assigned } \\
\text { to a generic material in a specific } \\
\text { material }\end{array}$ & 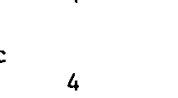 & 1 \\
\hline MTH (NINTI, NINTJ) & $\begin{array}{l}\text { Thermal-Hydraullcs mesh region } \\
\text { assignment }\end{array}$ & 4 & 1 \\
\hline TF $(\mathrm{NAX}, \mathrm{NCH})$ & Average fuel temperature & 8 & 1 \\
\hline $\mathrm{TC}(\mathrm{NAX}, \mathrm{NCH})$ & Average coolant temperature & 8 & 1 \\
\hline $\operatorname{ITYP}(\mathrm{NCH})$ & Data type number & 4 & 1 \\
\hline DAT (LEN, I TYPE) & Thermal-Hydraulics data array & 8 & 1 \\
\hline NPIN (NCH) & Number of pins & 4 & 1 \\
\hline 'I'CLN(NCH) & Inlet coolant temperature & 8 & 1 \\
\hline FLOW (NCH) & Mass flow rate & 8 & 1 \\
\hline $\operatorname{AXTH}(\mathrm{NAX})$ & Axial mesh spacing & 8 & 1 \\
\hline HCORR (NAX) & H-gap correction factor & 8 & 1 \\
\hline NINGR (NGROUP) & Number of inner iterations & 4 & 0 \\
\hline OMER (NGROUP) & Over relaxation-factor & 8 & 0 \\
\hline POWERM(NINTI, NINTJ) & Pointwise power density & 8 & 1 \\
\hline PKB UF (NPKM) * & $\begin{array}{l}\text { Storage for point kinetics update } \\
\text { parameters }\end{array}$ & 8 & 0 \\
\hline PWOLD (NAX, NCH) & old thermal-hydraulics power & 8 & 1 \\
\hline
\end{tabular}

*NPKM $=67+($ NROSMX -1.1$) \star 24$

Fig. 17. Arrays Permanently In-Core During Steady-State and Transient 


\section{3 Arrays Which May Be In-Core}

Depending on the amount of core memory provided to FX2-TH, the data management strategy decides which files may be contained in core while they are being used, and which must be read from and written to the disk as the calculation progresses. These files are described in Figure 18.

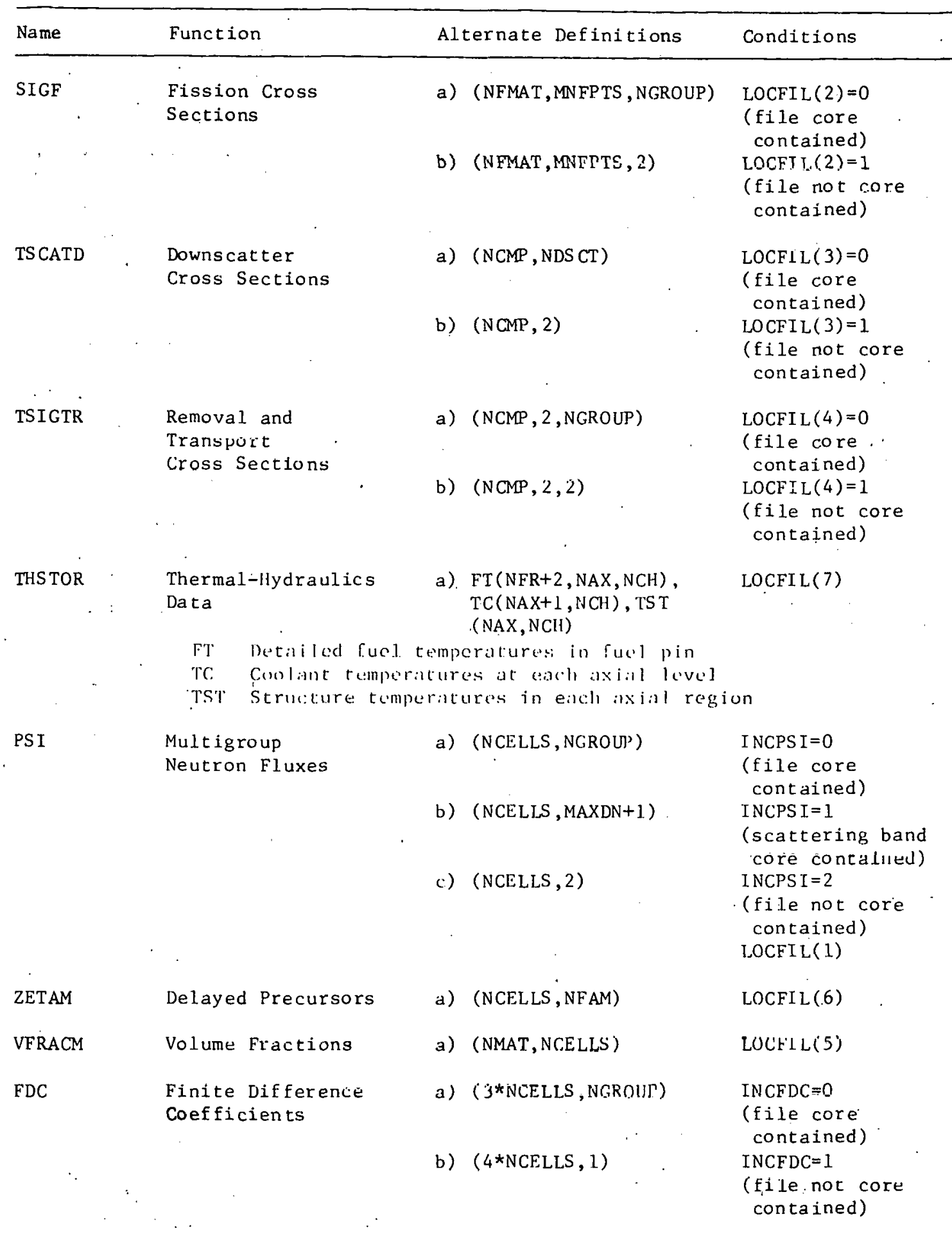

Fig. 18. Arrays Whose Dispositions are Determined by the Data Management Strategy 
The arrays SIGF, TSCATD, and TSIGTK have different structures depending on the data management decisions. If sufficient storage is avallable to contain them, the entire record (a11 NGROUP or NDSCT records) are allocated. This mode is indicated by the appropriate entry in the LOCFIL array being set to zero. If insufficient space is available, two records are allocated, so that the file may be double buffered as it is read from or written to the disk.

If sufficient space exists to permanently contain the THSTOR array, then it is allocated at the beginning of the steady state calculations. Regardless of the data management decision, THSTOR is written to disk and deleted at the end of the steady state calculation. It is then read from the disk and re-allocated at the beginning of the transient. The array THSTOR is made up of the arrays FT, TC, and TST; consisting of the detailed fuel temperatures, coolant temperature at each axial level, and structure temperatures in each axial region. If the detailed thermal-hydraulics model is not used, this file is not present.

The array PSI has three different configurations depending on available corc space. If sufficient core is available, the entire array will be allocated in core. If a scattering band of fluxes can only be contained, then that will be allocated. Finally, if a scattering band cannot be allocated, then two records wlll be allocated to provide double buffering of the file.

The files ZETAM and VFRACM have only one configuration. They are either present in core or read from and written to the disk depending on the appropriate LOCFIL entry. The array FDC also has two configurations, a full array if INCFDC $=0$, and a reduced array if INCFDC $=1$. The extra NCELLS array is to provide duuble buffering of the file.

and 18 .

Figure 19 is a glossary of the various terms used in Figures 17

In addition to the arrays mentioned in Figures 17 and 18, a large number of other files are allocated to temporarily contain data. These files are later deleted, and the space purged. Descriptions of these arrays can be found in the comments at the beginning nf eash oubroutiute

D. Use of Standard Subprograms

The FX2-TH code uses the BPOINTER routines to manage core memory. The BPOINTER routines are described in SecLiun VI.C..1. The BCD input data follows the ARC system conventinns, 18 and is discueocd in jucllon $V . B$. The l'URTRAN routines SCAN and STUFF read and process the input as discussed in Reference 18.

'I'he Committee on Computer Code Coordination has specified a number of standard routines ${ }^{16}$ to be used in RRD funded code development. In principle these routines may be installation dependent. The FX2-TH code uses the standard routines REED, RITE, LRED, LRIT, TIMER and SEEK as specified in Reference 16. While it is possible to substitute installation dependent code, simple versions of these routines are provided with both the IBM and $\mathrm{CDC}$ versions of $\mathrm{FX} 2-\mathrm{TH}$. 


\begin{tabular}{ll}
\hline Name & Description \\
NREGD & Number of regions which have driving functions \\
NZONE & Number of zones \\
NGROUP & Number of neutron energy groups \\
NBCS & Number of entries in BNDC array \\
NREG & Number of regions \\
NINTI & Number of X-dimension mesh intervals \\
NINTJ & Number of Y-dimension mesh intervals \\
NTFMAT & Number of fissionable unique materials \\
NFAM & Number of delayed neutron families \\
NMAT & Number of generic materials \\
NFMAT & Number of fissionable generic materials \\
NTMAT & Number of unique materials \\
NAX & Number of axial thermal-hydraulic regions \\
NCH & Number of thermal-hydraulic channels \\
LEN & Length of one set of thermal-hydraulic data \\
ITYPE & Number of different thermal-hydraulic data types \\
NROSMX & Maximum number of reactivity steps per shape. step \\
MNFPTS & Number of mesh cells with fissionable material in them \\
NCMP & Number of compositions \\
NDSCT & Number of downscatter components \\
NFR & Number of regions in fuel pin \\
NCELLS & Number of mesh cells \\
MAXDN & Number of downscatter groups \\
&
\end{tabular}

Fig. 19. Glossary of Terms Used in Figs. 17 and 18

\section{1 SEEK}

The subroutine SEEK is used by FX2-TH to return the logical unit number associated with a named data file. With only two exceptions, all files referenced by $\mathrm{FX2}-\mathrm{TH}$ axe assigned unit numbers through calls to subroutine SEEK. The two exceptions are the BCD files input (5) and printed output (6). While it should be pussible to uee installation dependent versions of SEEK, it is obvious that any changes in unit allocation must also be reflected in the Job Control Language required for the IBM version of FX2-TH or the program card of the CDC version. A single initialization call to SEEK is made from the main program driver of FX2-TH.

\section{2 REED/RITE}

The standard routines REED and RITE are used to perform the non-formatted (binary) I/O operations for FX2-TH. The ARC systeni version of REED/RITE make use of three access methods, standard FORTRAN, asynchronous FORTRAN, and a special random access I/O package SIO. ${ }^{19}$ The IBM and CDC standalone versions of FX2-TH contain far simpler versions of REED/RITE which use standard FORTRAN I/O. REED and RITE are designed to read data directly into the small core container (SCM on the CDC 7600). To allow data to be read into the large core container (LCM) directly, 
the routines LRED and LRIT were written. These routines are identical to KEED and RITE except that they directly address the large core container. All records of a file must be read (or written) using the same subroutine; i.e., calls to REED (or RITE) and LRED (or LRIT) may not be intermixed in a particular processing of a file. A file may be processed using one set of routines at one point in the code, and later processed using the other set of routines; as long as all records of the file are processed in the same way.

\section{3 TIMER}

The standard subroutine TIMER is used by $\mathrm{FX} 2-\mathrm{TH}$ to provide timing and problem identification information. These data are not essential to the execution of the FX2-TH program. As a consequence, the CDC version of TIMER is in large part a dummy program. It calls only the standard cns. system code SECOND to provide GPU timiul dara. The IBM version of TIMER makes use of a number of assembler language routines to provide time and edit information.

\section{E. Program Implementation}

Both the IBM and CDC versions of the program FX2-TH are avaj.lablo from the Argonne Cote Canter. A descripelon of the tapes along with a step-by-step implementation strategy for each version of the program is described below.

\section{E.1 IBM Code Implementation}

The IBM program tape is written as described in Table $V$. The oource code for FX2-TH is writtcn ou Flles 1 and 2 of the program tape. The FORTRAN routines are contained on File 1 , while the ASSEMBLER routines are contained on File 2. File 3 contains the SYSIN ata carda for tlit linkage editor; this lefines the overlay structure. The cataloged procedure ARCSP024 is contained in File 4. Files $5-7$ contain the input data for Sample Problem 3. File 8 contains the output from Samplc Probleil 3. File $y$ contains documentation information found in this manual.

To implement FX2-TH, first the object code should be created, then the cataloged procedure obtained, finally Sample Problem 3 should be executed. To create the object code, the job shown in Figure 20 should be executed. This job is composed of five steps:

i) Allocation of disk space for the compiled and link-edited source code from F1le 1.

i1) Compilation and link-edit of FORTRAN source code from File 2 .

iii) Assembly and link-edit of ASSEMBLER source code from File 2 .

iv) Allocation of disk space for the object program.

v) Link-edit of complied code from steps ii) and iii). 
//STEP 1 EXEC PGM=IEFBR 14

/ /OBJECT DD DSN=C116.B20219. FX2ACC.SEGLIB,DISP=(NEW,CATLG).

11 ONIT=SHRT 3330, CCB $=B L K S I Z E=6144, \operatorname{SPACE}=(\mathrm{CYI},(6,3,1))$

$/ /$ STEP 2 EXEC FTXCEF, OPTIONS= 'OPT=2, XREF', EDTOPTS='LET, NCAL',

// OBJROOH =' (CYL, $(10,2))^{\prime}$, ISIZE=' $(500 \mathrm{~K}, 100 \mathrm{~K})^{\prime}$

//FTX.S YSPRINT DD ONIT=TAPE 1600, LABEL $=(1, \mathrm{NL})$,

1) DISP $=$ (NEN, PASS), $\mathrm{VOL}=S E R=135706$,

$1 / D C B=(R E C F M=F B, I R E C L=133$, BLKSIZE=1596, DEN=2)

$/ / P T X$.S YSIN DO ONIT $=$ TAPE800, VOL $=S E R=135536$,

// LABEL $=(1, N L), D I S P=(O L D, K E E P)$,

$1 / D C B=(R E C F M=P B, L R E C L=80, B L K S I Z B=3200, D E N=2)$

//BDT.SYSLMOD DD DSN=C116.B20219.FX2ACC.SBGLIB (FORT), DISP = (OLD,KEEP)

//EDT.STERLIB DD DISP=SHR, DSN=B99960.SYS1.LINKLIB

//STEP 3 EXEC ASMCEP, EDTOPTS='LIST, MAP, DCBS'

/ ASH.SYSIN DD UNIT TARE8OO, VOI =SER=135536,

/ I L ABEL $=(2, \mathrm{NL}), \mathrm{DISP}=(\mathrm{OLD}, \mathrm{KEEP})$.

$1 / D C B=(R E C F E=F B, I R E C L=80, B L K S I Z E=3200, D E N=2)$

//EDT.SYSLMOD DD DSN=C116.B20219.FX2ACC.SEGLIB (ASSM),DISP=(OLD,KEEP)

//STEP4 EXEC PGM=IEFBR 14

$/ / D D 1$ DD DSN=C116.B20219. F X2ACC. $M O D L I B, D I S P=(N E,, C A T L G)$,

/ UNIT=LONG3330, DCB=BLKSIZE=6144, SPACE $=(C Y L,(4,1,1)$, RLSE $)$

//STEP5 EXEC FTXEP,EDTOPTS=OVLY, LSIZE=' $(250 \mathrm{~K}, 64 \mathrm{~K})$ '

//EDT.SYSLMOD DD DSN=C116.B20219.F X2ACC. MODLIB,DISP= (OLD,KEEP)

$/ /$ EDT.SEGLIE DD DSN=C116.B20219. FX 2ACC. SEGLIB, DISP=(OLD, KEEP)

$/ / E D T . S I S I N$ DD UNIT $=$ TAPEBD0, VOL $=S E R=135536$,

1) IABEL $=(3, N L), D I S P=(O L D, K E E P)$,

1) $\mathrm{DCB}=(\mathrm{RECF}=\mathrm{FB}, \mathrm{LRECL}=80, \mathrm{BLKSIZE}=3200, \mathrm{DEN}=2)$

Fig. 20. Creation of FX2-TH Object Code

The user may specify certain JCL parameters to correspond to his particular installation. The compilation of File 1 is performed using FORTRAN IV EXTENDED compiler using OPT $=2$ optimization. If this compiler is not available, FORTRAN IV H with OPT=2 optimization is adequate. The assembly of File 2 coding is performed in the usual manner.

Next, the cataloged procedure ARCSP024 should be removed from file 4 of the program tape. The job shown in Figure 21 should be executed to remove this procedure from the tape and punch cards. The cataloged procedure may then be added to the PROCLIB of the user's installation.

Finally, Sample Problem 3 should be executed. The problem can be run without having to transfer the data from the program tape. This is done by overriding certain of the DD cards. The necessary JCL is shown in Figure 22. This problem will execute in 5 minutes (CPU plus WAIT time) on a $370 / 195$ and requires $900 \mathrm{~K}$ of core storage.

The output from Sample Problem 3 is stored on File 8. This output may be printed by using the same JCL as shown in Figure 21, with two exceptions: substitute $\mathrm{LABEL}=(8, \mathrm{NL})$, and let

$$
\text { //SYSUT2 DD SYSOUT=A, DCB= ( RECFM=FB , // LRECL=133, BLKSIZE=1596) }
$$

File 9 contains a description of the $B C D$ input files, the binary interface files, and the COMMON blocks. This file may be printed as in the example above except that $L R E C L=80, B L K S I Z E=3200$, and $L A B E L=(9, N L)$. 
// EXEC PGM=IEBGFNER

//SYSPRINT DD SYSOUT $=\Lambda$

//SYSUT1 DD UNIT=TAPE800, VOL=SER=135536,

// $\mathrm{LABEL}=(4, \mathrm{NL}), \mathrm{DISP}=(\mathrm{OLD}, \mathrm{PASS})$,

// $\mathrm{DCB}=(\mathrm{RECFM}=\mathrm{FB}, \mathrm{LRECL}=80, \mathrm{BLKSIZE}=3200$,

// $\mathrm{DEN}=2$ )

//SYSUT2 DD SYSOUT $=\mathrm{B}$

Fig. 21. Removal of ARCSP024 from IBM Program Tape

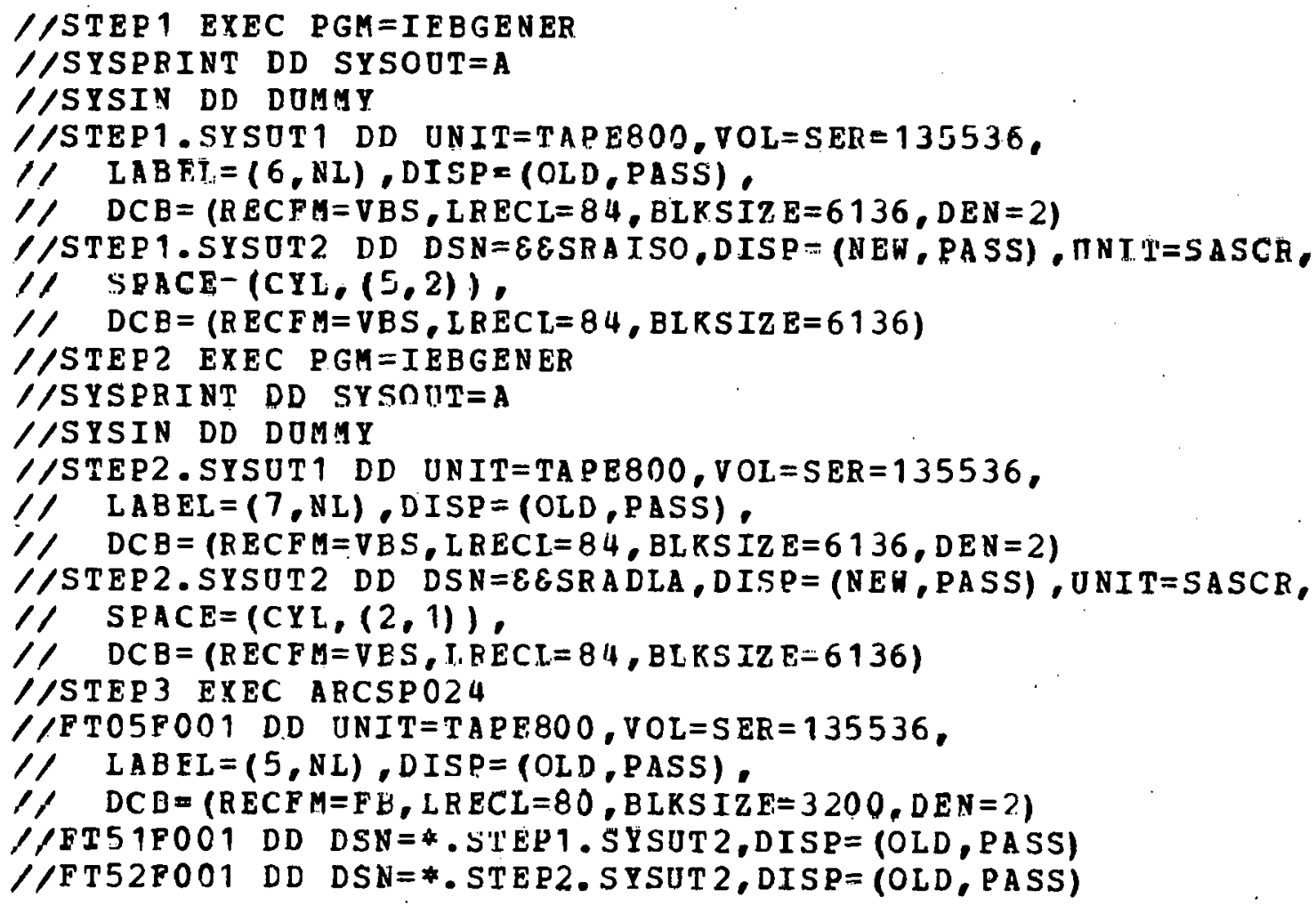

Fig. 22. IBM JCLL to Exerute Sample Probliem 3

\section{E.2 CDC Code Implementation}

The FX2-TH code has been implemented on a CDC 7600 computer at the Lawrence Berkeley Laboratory. The 7600 at LBL has 65,636 (60-bit) words of small core memory and $572, n 00$ ( 60 -hit) worde of large cure memory. The examples given in this Section use the control cards suitable at LBL. The essential nature of each step should be system independent.

The CDC program tape is written as described in Table V. The source has intermixed COMPASS routinco with the FORTRAN. The FORTRAN EXTENDED Version 4 compiler (FTN4) available at LBL is capable of processing coding mixed in this way. If the FORTRAN compiler available at a particular installation is not capable of this, the COMPASS routines must be separated out, assembled, and then replaced in their original position in the LGO file. The FORTRAN EXTENDED compiler is required since FX2-TH makes use of LEVEL 2 statements. Full optimization (OPT-2) is recommended. 
TABLE V. IBM Code Center Tape Description

Program Tape (Non-labelled, 9 track, 800 bpi)

\begin{tabular}{|c|c|c|c|c|}
\hline File & Description & RECFM & LRECL & BLKSIZE \\
\hline 1 & $\begin{array}{l}\text { Fortran Source } \\
\text { (EBCDIC) }\end{array}$ & $\mathrm{FB}$ & 80 & 3200 \\
\hline 2 & $\begin{array}{l}\text { Assembler Source } \\
\text { (EBCDIC) }\end{array}$ & FB & 80 & 3200 \\
\hline 3 & $\begin{array}{l}\text { Linkage Editor Directive } \\
\text { Cards (EBCDIC) }\end{array}$ & FB & 80 & 3200 \\
\hline 4 & $\begin{array}{l}\text { Cataloged Procedure } \\
\text { ARCSP024 (EBCDIC) }\end{array}$ & FB & 80 & 3200 \\
\hline 5 & $\begin{array}{l}\text { Sample Problem } 3 \\
\text { Input Data (EBCDIC) }\end{array}$ & $\mathrm{FB}$ & 80 & 3200 . \\
\hline 6 & $\begin{array}{l}\text { Sample Problem } 3 \\
\text { A.ISO (EBCDIC) }\end{array}$ & VBS & 84 & 6136 \\
\hline 7 & $\begin{array}{l}\text { Sample Problem } 3 \\
\text { A.DLA (EBCDIC) }\end{array}$ & VBS & 84 & $6136^{\circ}$ \\
\hline 8 & $\begin{array}{l}\text { Sample Problem } 3 \\
\text { Output (EBCDIC) }\end{array}$ & FB & 133 & 1596 \\
\hline 9 & Manual Documentation & FB & 80 & 3200 \\
\hline
\end{tabular}

The required PROGRAM and OVERLAY cards are positioned as required for the CDC overlay structure, therefore no special overlay control cards are required. The source file may be compiled, loaded, and executed without special instructions. In Figure 23, the CDC control cards required to process the program tape, compile and load FX2-TH, and execute Sample Problem 3 are given. The control cards are specific to the LBL installation, however, their general function may be duplicated at any installation.

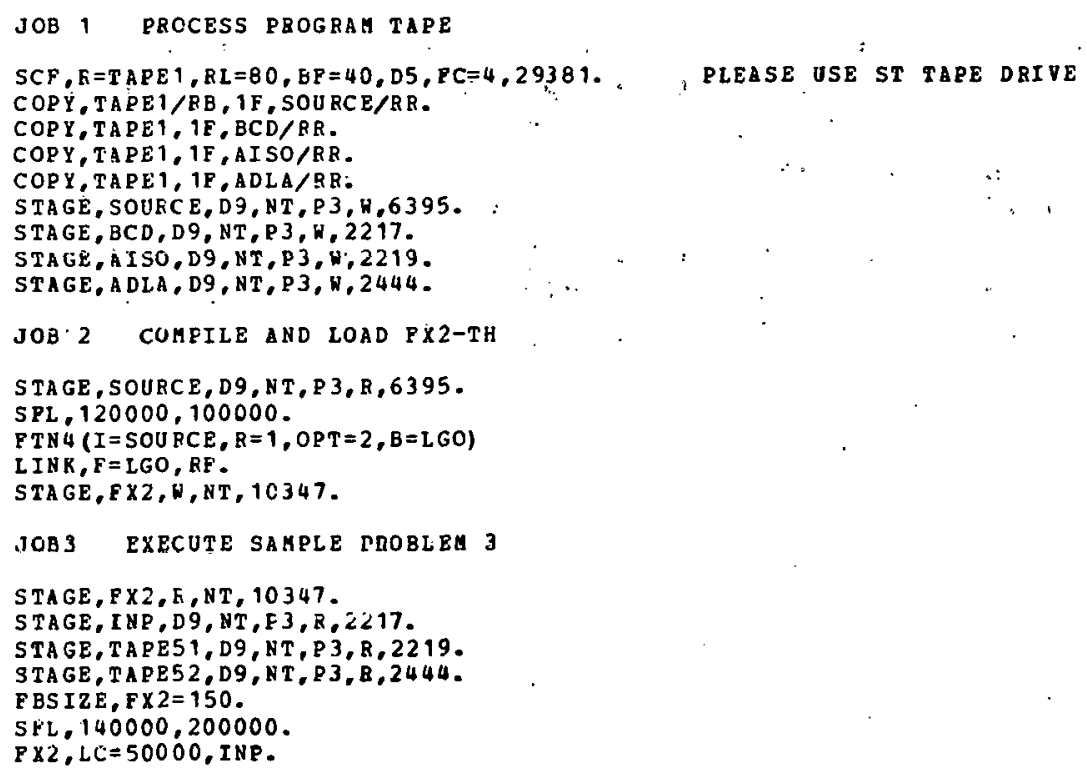

Fig. 23. CDC Control Cards 
TABLE VI. CDC Code Center Tape Description

Program Tape (7-track, 556 bpi)

\begin{tabular}{ccccc}
\hline File & Description & RECFM & LRECL & BLKSIZE \\
\hline 1 & FX2-TH Source & FB & 80 & 3200 \\
2 & $\begin{array}{c}\text { Sample Problem 3 } \\
\text { Input Data (BCD) }\end{array}$ & FB & 80 & 3200 \\
3 & $\begin{array}{c}\text { Sample Problem 3 } \\
\text { A.ISO (BCD) }\end{array}$ & FB & 80 & 3200 \\
4 & $\begin{array}{l}\text { Sample Problem 3 } \\
\text { A.DLA (BCD) }\end{array}$ & FB & 80 & 3200 \\
5 & Manual Documentation & FB & 80 & 3200 \\
\hline
\end{tabular}

\section{ACKNOWLEDGMENTS}

A number of people have contributed significantly to the development of the FX2-TH code: The original development of the quasistatic method used in this code is due to Dan Meneley and his associates at Argonne. An internal report (FRA-TM-87) was prepared by D. Ferglisnn, T. Na1,y, and R. W. Schaefer in 1976 documenting a version of FX2 coupled with hydrodynamics calculations. This work was guided in large measure by W. M. Stacey, Jr. The capability to solve problems on a triangular mesh was added by H. Henryson, II. The work of Lyn Young in typing this report is greatly appreciated a.lso. 


\section{REFERENCES}

1. D. A. Meneley, et al., A Kinetics Model for Fast Reactor Analysis in Two Dimensions, Dynamics of Nuclear Systems, The Iniversity of Arizona Press, Tucson, Arizona 483-500 (1972).

2. A. F. Henry, Nuclear-Reactor Analysis, Cambridge, Mass.: MIT Press, (1975).

3. D. R. Vondy, T. B. Fowler, G. W. Cunningham, VENTURE: A Code Block for Solving Multigroup Neutronics Problems Applying the FiniteDifference Diffusion-Theory Approximation to Neutron Transport; ORNL-5062, Oak Ridgc National Laboratory (1975).

4. T. A. Daly et al., The ARC System Two-Dimensional Diffusion Theory Capability, DARC2D, ANL-7716, Argonne National Laboratory (1972).

5. D. R. Ferguson, K. L. Derstine, Optimized Iteration Strategies and Data Management Considerations for Fast Reactor Finite Difference Diffusion Theory Codes, Nuc1. Sci. Eng., 64, 593 (1977).

6. MEKIN: MIT-EPRI Nuclear Reactor Kinetics Code, EPRI-227,. E1ectric Power Research Institute (1975).

7. A. F. Henry, The Application of Reactor Kinetics to the Analysis of Experiments, Nuc1. Sci. Eng. 3, 58 (1958).

8. D. A. Meneley, K. O. Ott, E. S. Weiner, Further Developments of the Quasistatic Neutron Kinetics Model, ANL-7410, Argonne National Laboratory, 398-400, (1969).

9. P. E. Rohan, C. M. Kang, R. A. Shober, S. G. Wagner, A Multi-Dimensional Space-Time Kinetics Code for PWR Transients, CoNF-750413, Charleston, South Carolina, VI-69, Apri1 15-17, 1975.

10. J. B. Yasinsky, M. Natelson, L. A. Hageman, TWIGL - A Program to Solve the Two-Dimensional, Two-Group, Space-Time Neutron Diffusion Equations with Temperature Feedback, WAPD-TM-743, Bettis Atomic Power Laboratory (r'ebruary, 1968).

11. E. L. Fuller, The Point Kinetics Algorith for FX2, ANL-7910, Argonne National Laboratory, 503-508 (1972).

12. J. J. Kaganove, Nromerical Solution of the One-Group Space-Independent Reactor Kinetics Equations for Neutron Density Given by Excess Reactivity, ANL-6132, Argonne National Laboratory (1960).

13. W. M. Stacey Jr., Space-lime Nuclear Reactor Analysis, New York: Academic Press, (1969).

14. M: M. El-Wakil, Nuclear Heat Transport, Scranton, Penn.: International Textbook Company (1971). 
15. F. E. Dunn et al., The SAS2A LMFBR Accident-Analysis Computer Code, ANL-8138, Argonne National Laboratory (1974).

16. R. D. 0'De1I, Standard Interface Files and Procedures for Reactor Physics Codes, Version IV, T-1-77-3090, Los Alamos Scientific Laboratory (1977). 
APPENDIX A

Description of FX2-TH Subroutines 


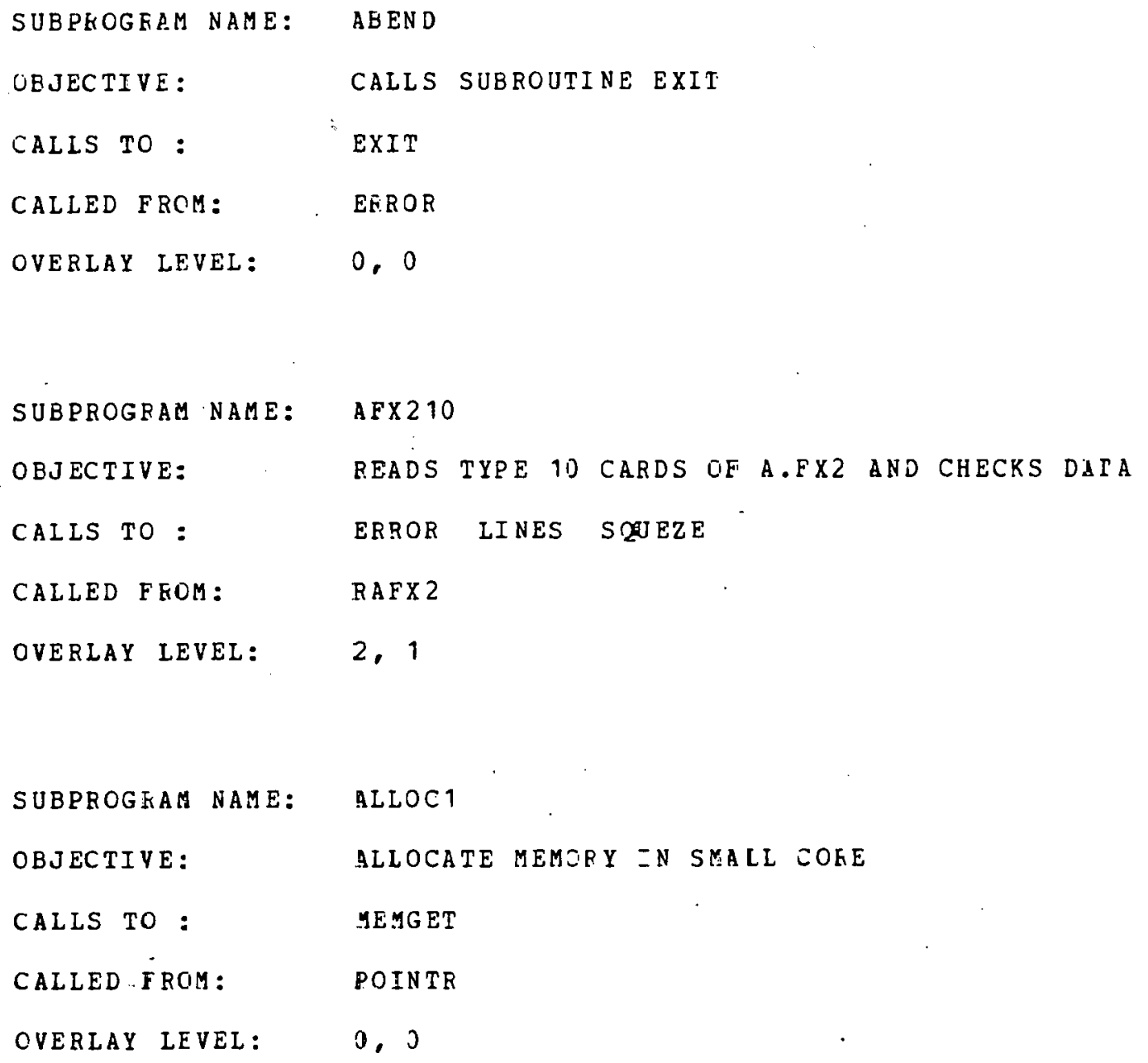




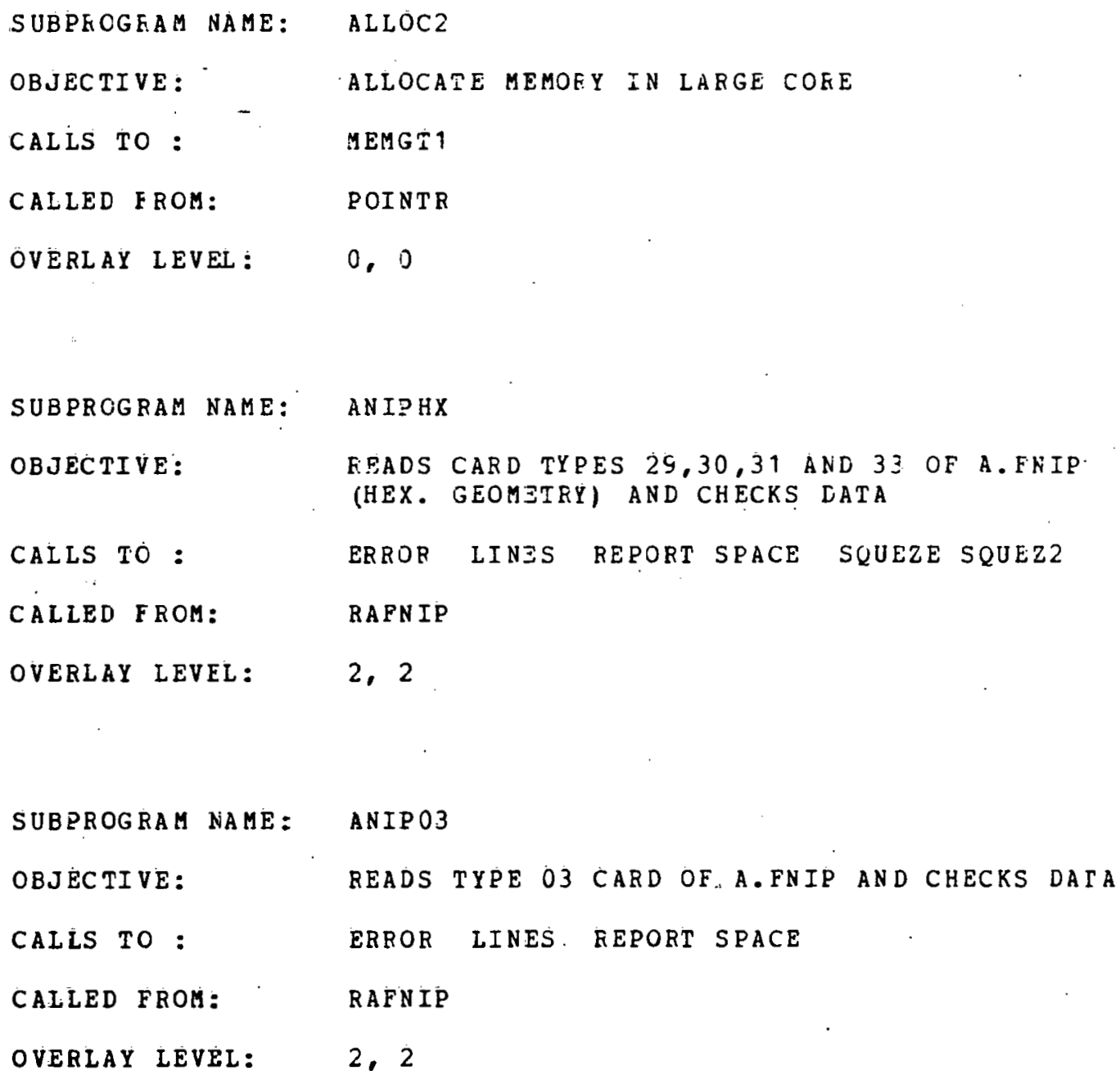




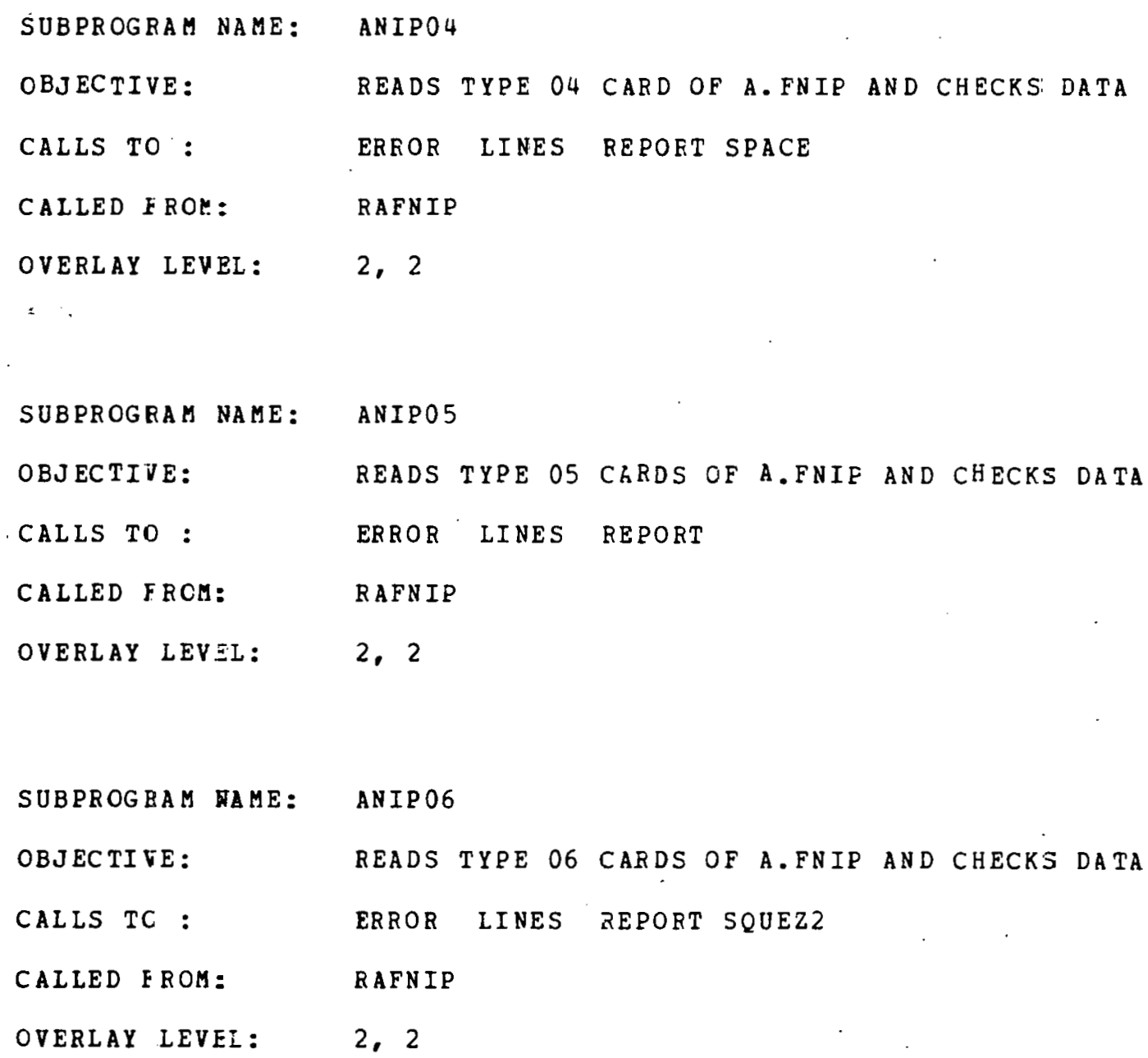

SUBPROGBAM HAME: ANIPO6

OBJECTIVE: READS TYPE 06 CARDS OF A.FNIP AND CHECKS DATA

CALLS TC : ERROR IINES REPORT SQUEZ2

CALLED FROM: RAFNIP

OVERLAY LEVEI: $\quad 2,2$ 


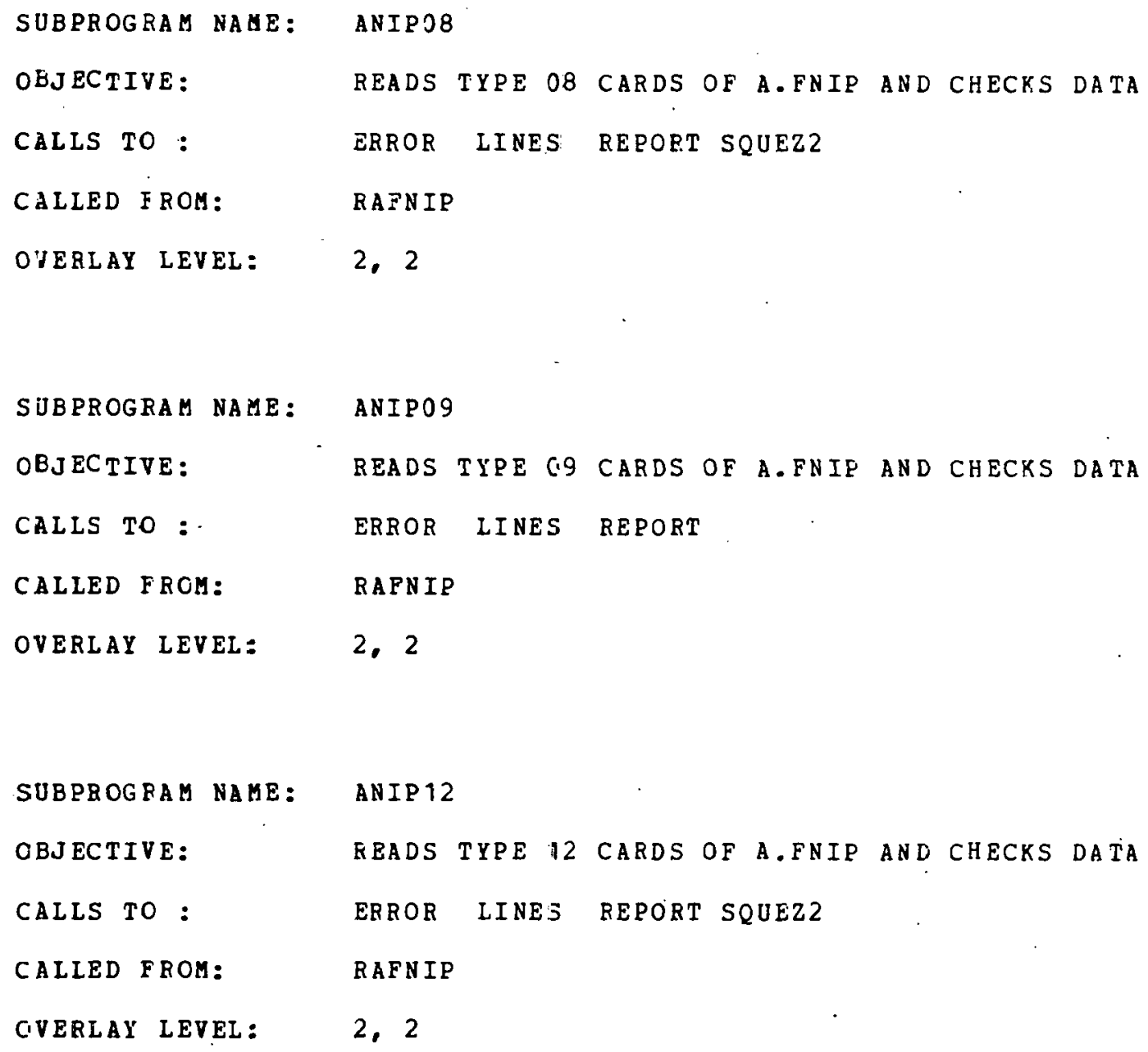




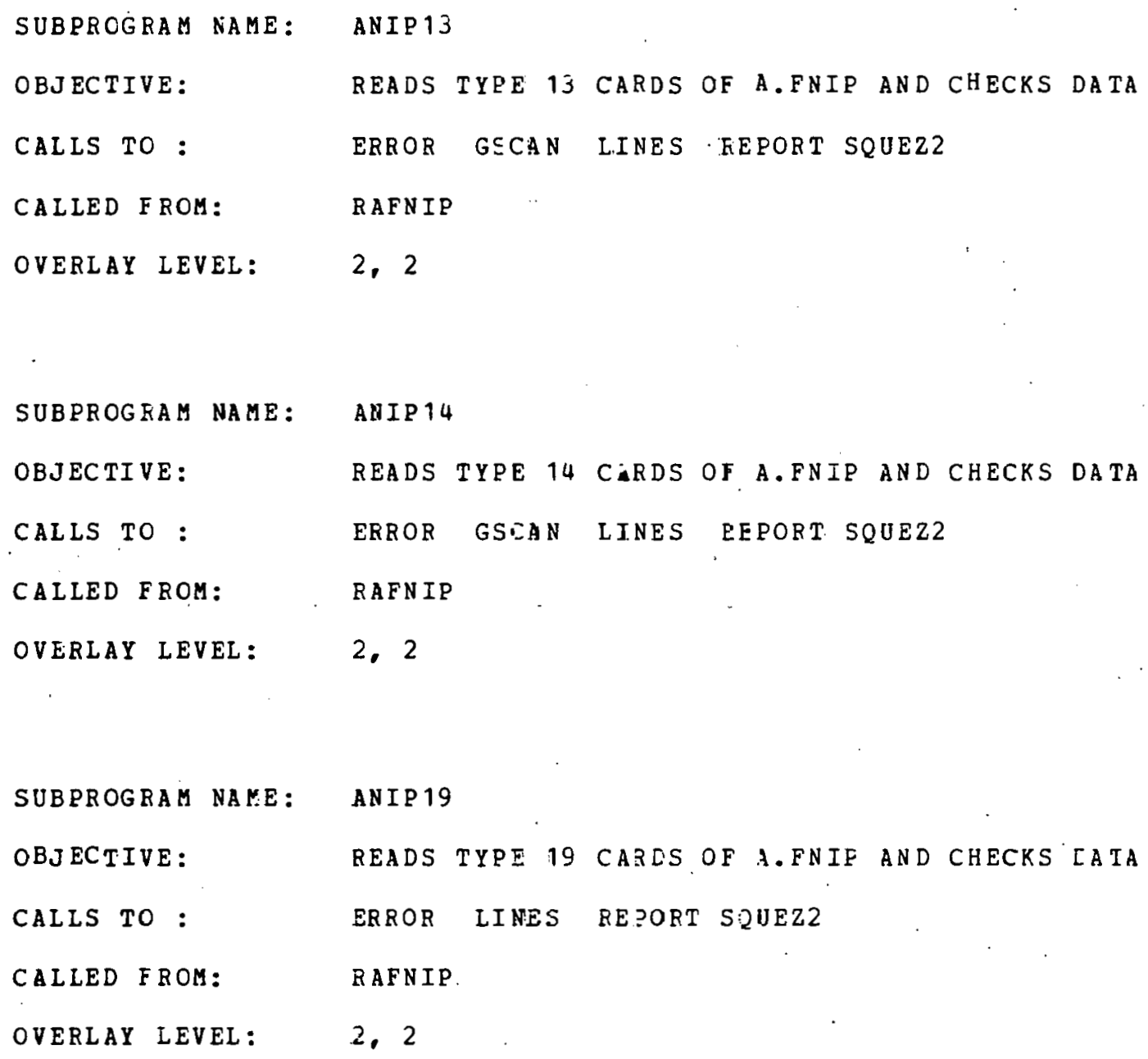

SUBPROGRAM NAME: ANIP19

OBJ ECTIVE :

READS TYPE 19 CARES OF A.FNIF AND CHECKS LATA

CALLS TO :

ERROR LINES RE?ORT SPUEZ2

CALLED FROM: RAFNIP.

OVERLAY LEVEL: $\quad 2,2$ 
SUBPROGRAM NAME: ANIP34

OBJECTIVE: READS TYPE 34 CARDS OF A.FNIP AND CHECKS DATA

CALLS TO: $\quad$ ERROR LINES REPORT SQUEZ2

CALLED FROM: RAFNIP

OVERLAY LEVEL: $\quad 2,2$

SUBPROGRAM NAME: REAP

OBJECTIVE: CalCULATES SURFACE AREAS OF EACH MESH CELl

CALLS TO: PEQUAT FLTSET FITST2

CALLED FROM: PASS 2

OVERLAY LEVEL: $\quad 8,0$

SUBPROGRAM NAME: AREAS

OBJECTIVE:

CalCulates SURface aRea OF EaCH MESH CELL

CALLS TO: : FEUAR FLEET FLTST2

CALLED FROM: SEDGE

OVERLAY LEVEL: 9,2 


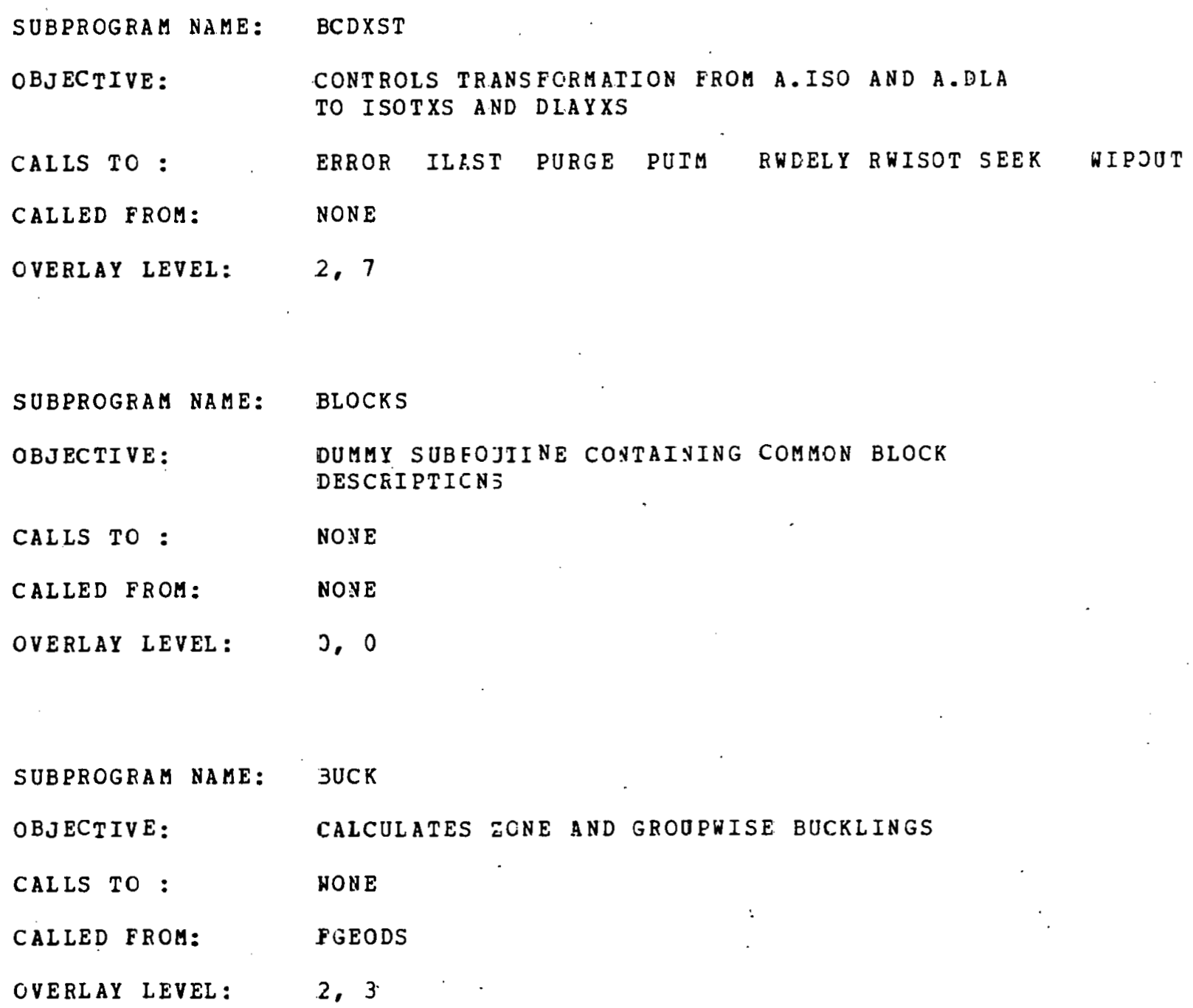




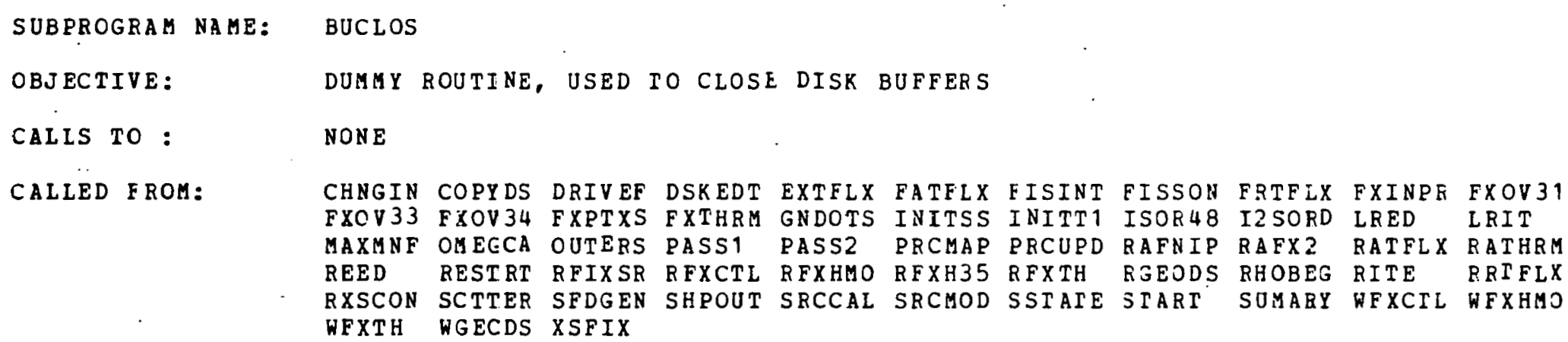

OVERLAY IEVEL: $\quad 0,0$

SUBPROGFAM NHME: BUOPEN

OBJECTIVE:

DUMMY ROUTINE, USED TO OPEN DISK EUFPERS

CALLS TO :

NONE

CALLED FROM: CHNGIN COPYDS DRIVEF DSKEDT EXTFLX FATFLX FISINT EISSON FRTELX FXINPR FXOV31 FXOV 34 FXOV 41 FXPTXS FXTHRM GNDOTS GSORC2 INITSS INITT1 I2SORD LRED LRIT MAXMNF OMEGCA OUTERS PASS1 PASS2 PRCMAP PRCUPD RATHRM REED RESTRT RFXH35 RHOBEG RITE SCTTER SFDGEN SHPOUT SRCCAL SECMOD SSTATE START SUMARY WATFLX WPXCTL WFXHMO WFXTH WGEODS WRTFIX XDRDR 2 XSFIX

OVERLAY LEVEL: $\quad 0,0$

SOBPROGRAM NAME: CALFSP

OBJECTIVE:

COMPOTES NUMBER OF FISSIONABIE COMPOSITIONS PRESENT

CALIS TO:

NONE

CALLED FROH:

IN ITSS

OVERLAY LEVEL: 0,0 
SUBPROGRAM NAME:

OBJ EC IV E:

CALLS TO:

CALLED FROM:

OVERLAY LEVEL:

SUBPROGRAM NAME:

OBJECTIVE:

CALLS TO:

CALLED FROM:

OVERLAY - LEVEL :

SUBPROGRAM NAME :

OBJECTIVE:

CALLS TO:

CALLED FROM:

OVERLAY LEVEL:
CHE BE

COMPUTES CHEBYSHEV ACCELERATION COEFFICIENTS

DACOSH HYPOS

GNDOTS

9,0

CHKHOM

CHECKS FILE FXHMOG FOR CONSISTENCY

ERROR LIN IS

RFXHMO

4. 1

CaKXSC

CHECKS FILE XSCON FOR CONSISTENCY

ERROR FLTST2 INTST2 LINES

RXSCON

4. 1 


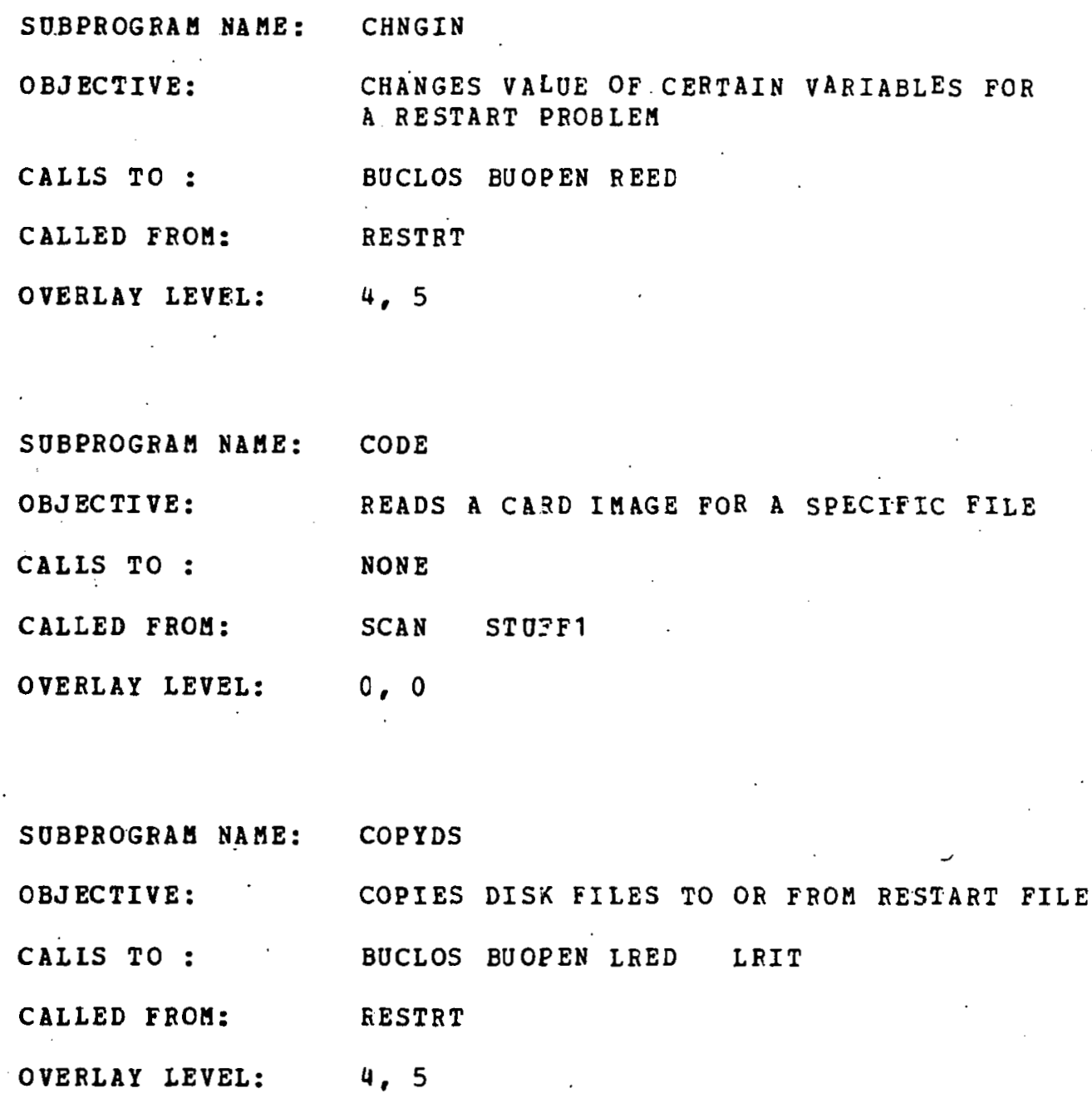

SUBPROGRAG NAME: COPYDS

OBJECTIVE: - COPIES DISK FILES TO OR FROM RESTART FILE

CALIS TO: BUCLOS BUOREN IRED LRIT

CALLED FROH: $\quad$ GESTRT

OVERLAY LEVEL: $\quad 4,5$ 
SUBPROGRAM NAME:

OBJECTIVE :

CALLS TO:

CALLED FROM:

OVERLAY LEVEL: . 0,0

SUBPROGRAM NAME:

OBJECTIVE:

CALLS TO:

CALLED FROM:

OVERLAY LEVEL:

SUBPROGRAM NAME:

OBJECTIVE:

CALLS TO:

CALLED FROM:

OVERLAY LEVEL:

CRED

CRIT

0,0

9,0
TRANSFERS DATA FROM LARGE COKE TO SMALL COZE

ERROR LINES READER WRITER

ECMV PRTECM REDEFB REDEEM

TRANSFERS DATA FROM SMALL CORE TO LARGE DOE

ERROR LINES READER WRITES

ECU ECZERO FATFLX FRTFLX REDEFB REDEEM

DACOSH

COMPUTES INVERSE HYPERBOLIC COSINE

BLOG DSQRT

THEBE GNDOTS 


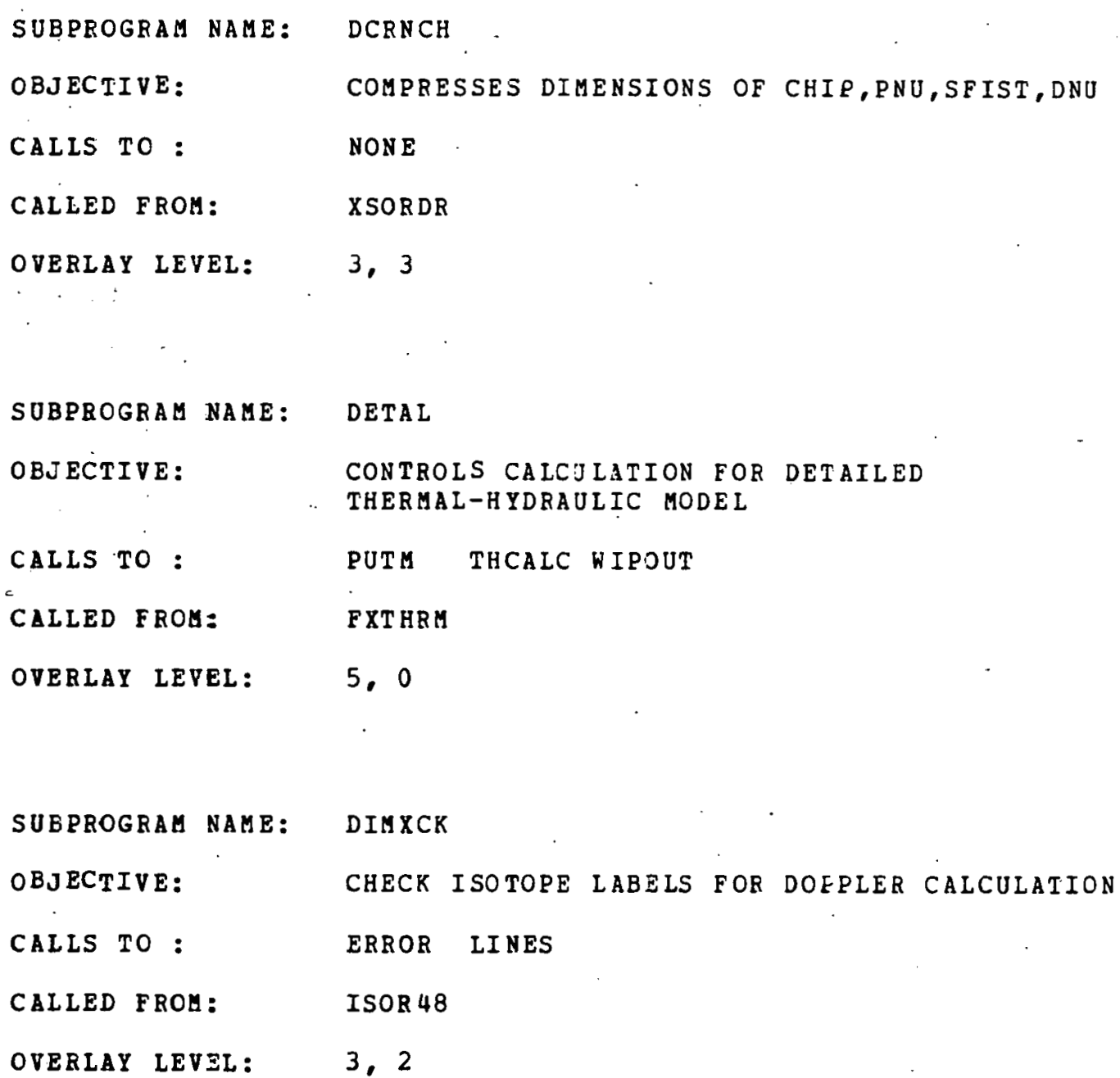




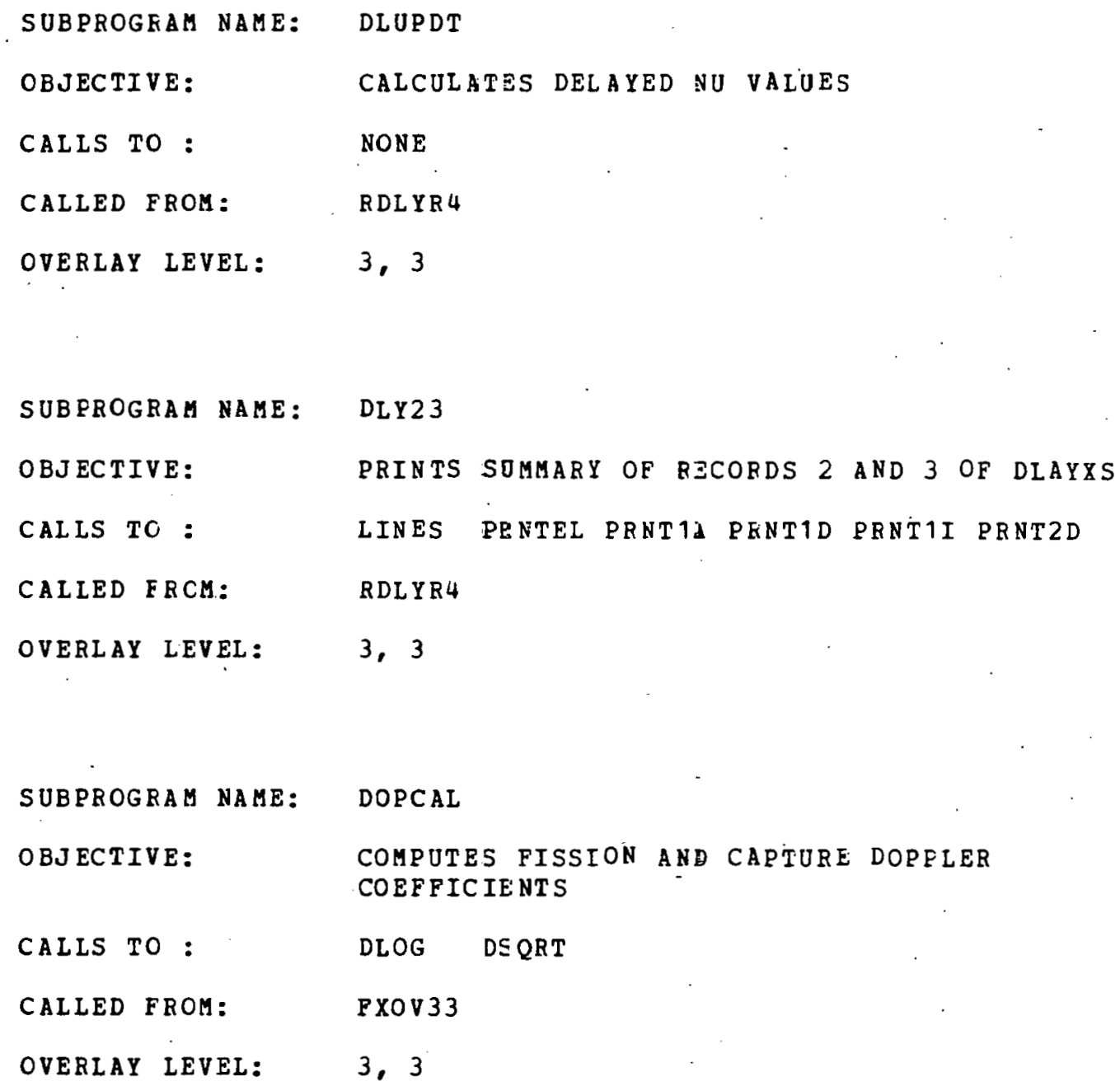


SUBPROGRAM NAME: DRIVER

OBJECTIVE: CALCULATES NEW VOLUME FRACTIONS AT EACH REACTIVITY TIME STEP

CALLS TO: BUCLOS BUGPEN ERROR LINES IED LIT SEEK

CALLED FROM:

OVERLAY LEVEL: $\quad 1,0$

SUBPROGRAM NAME: DSKEDT

OBJECTIVE: WRITES DISK EDIT FILE FY EDIT AT EACH

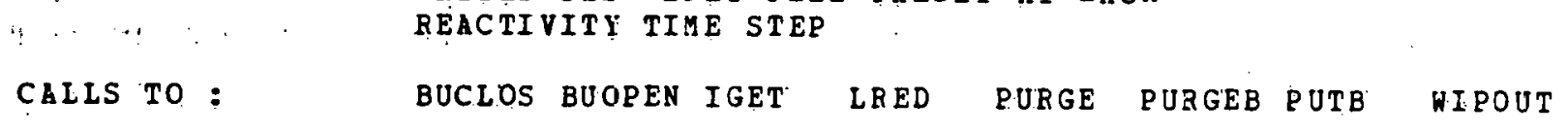




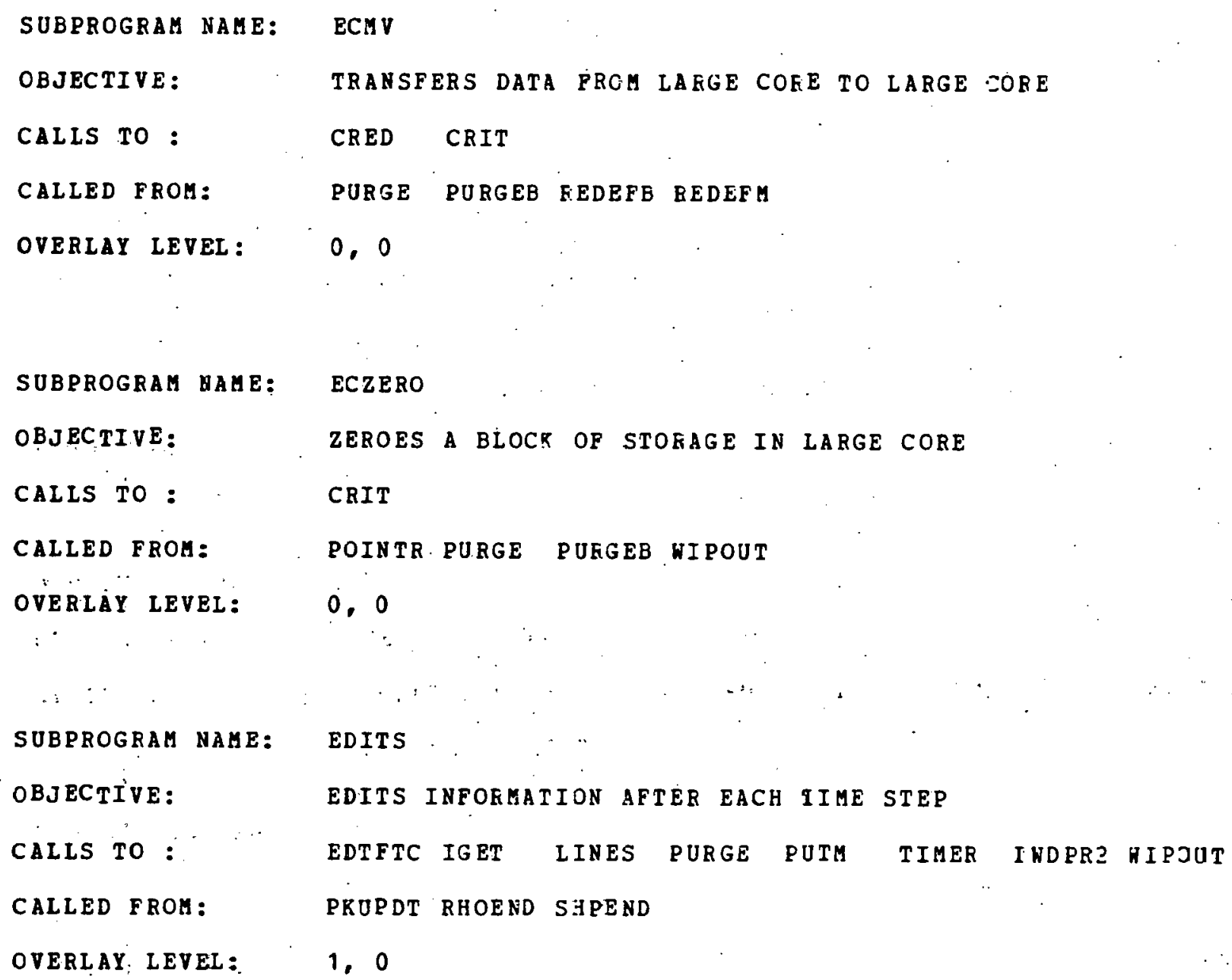




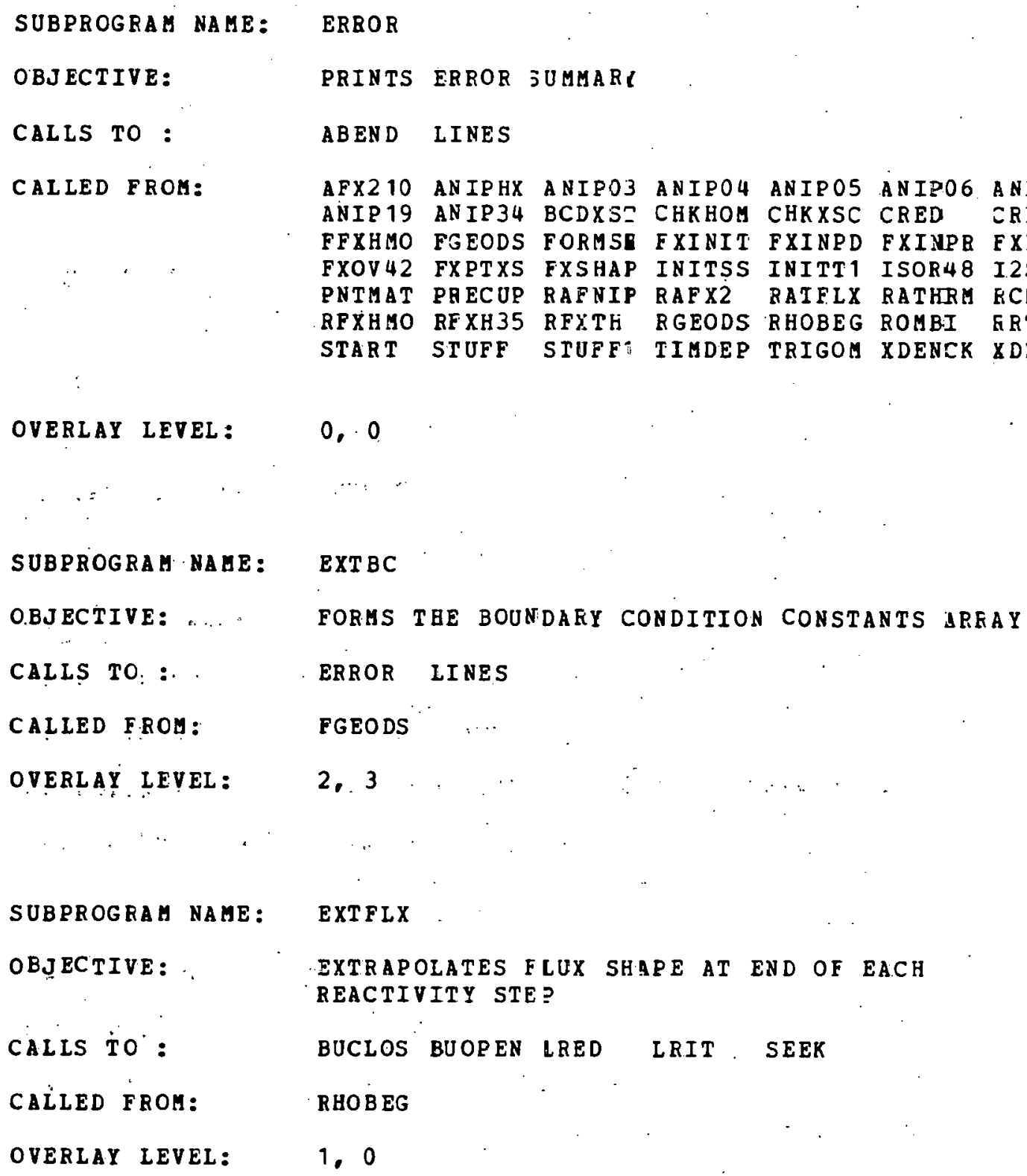


SUBPROGRAM NAME: EXTRPO

OEJECTIVE: EXTRAPOLATES FISSION SOURCE IN CHEBYSHEV

EALLSTO: NONE.

CALLED FROB: GNDOTS

OVERLAT L̇EVEEL : 9," 4

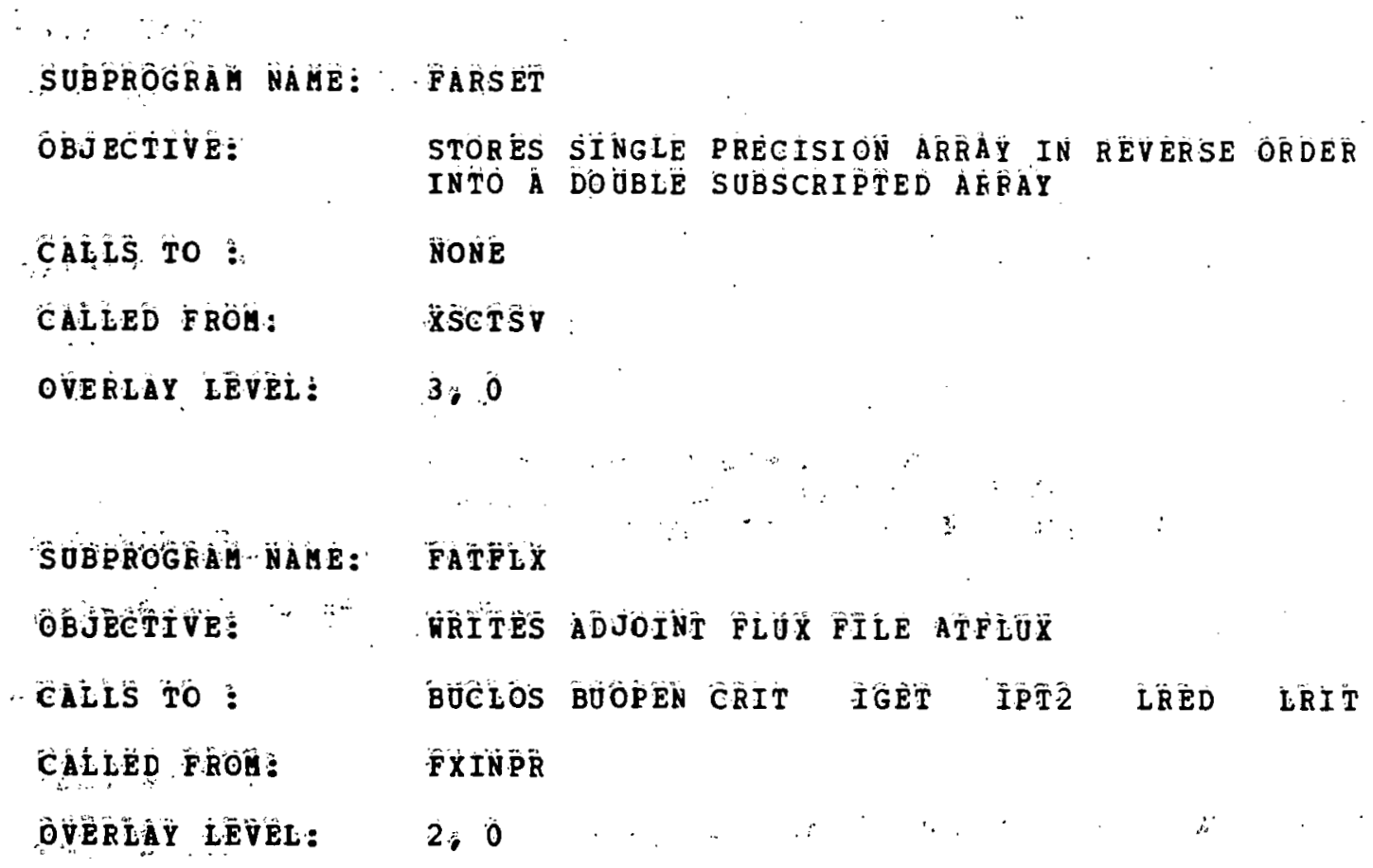




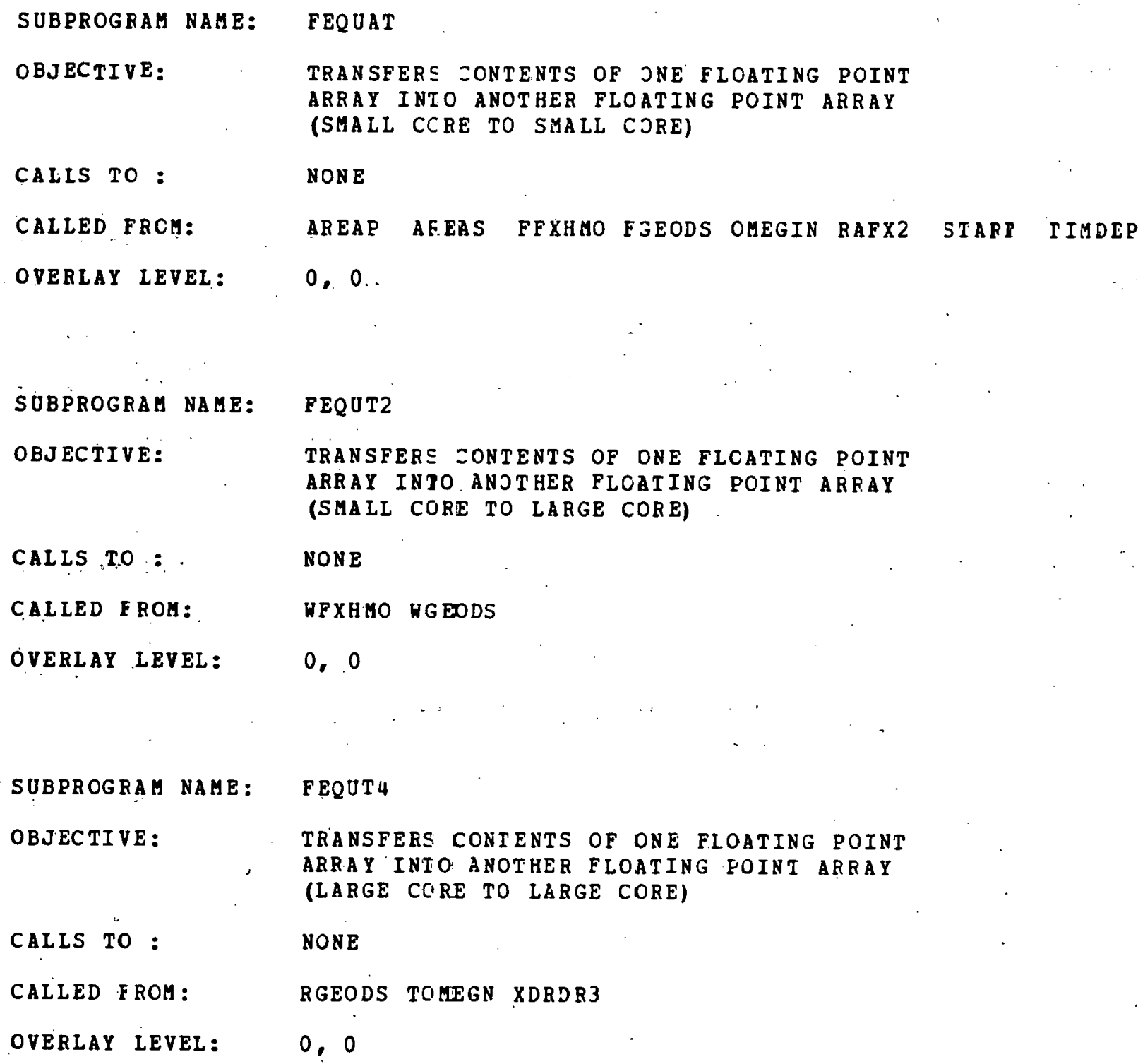




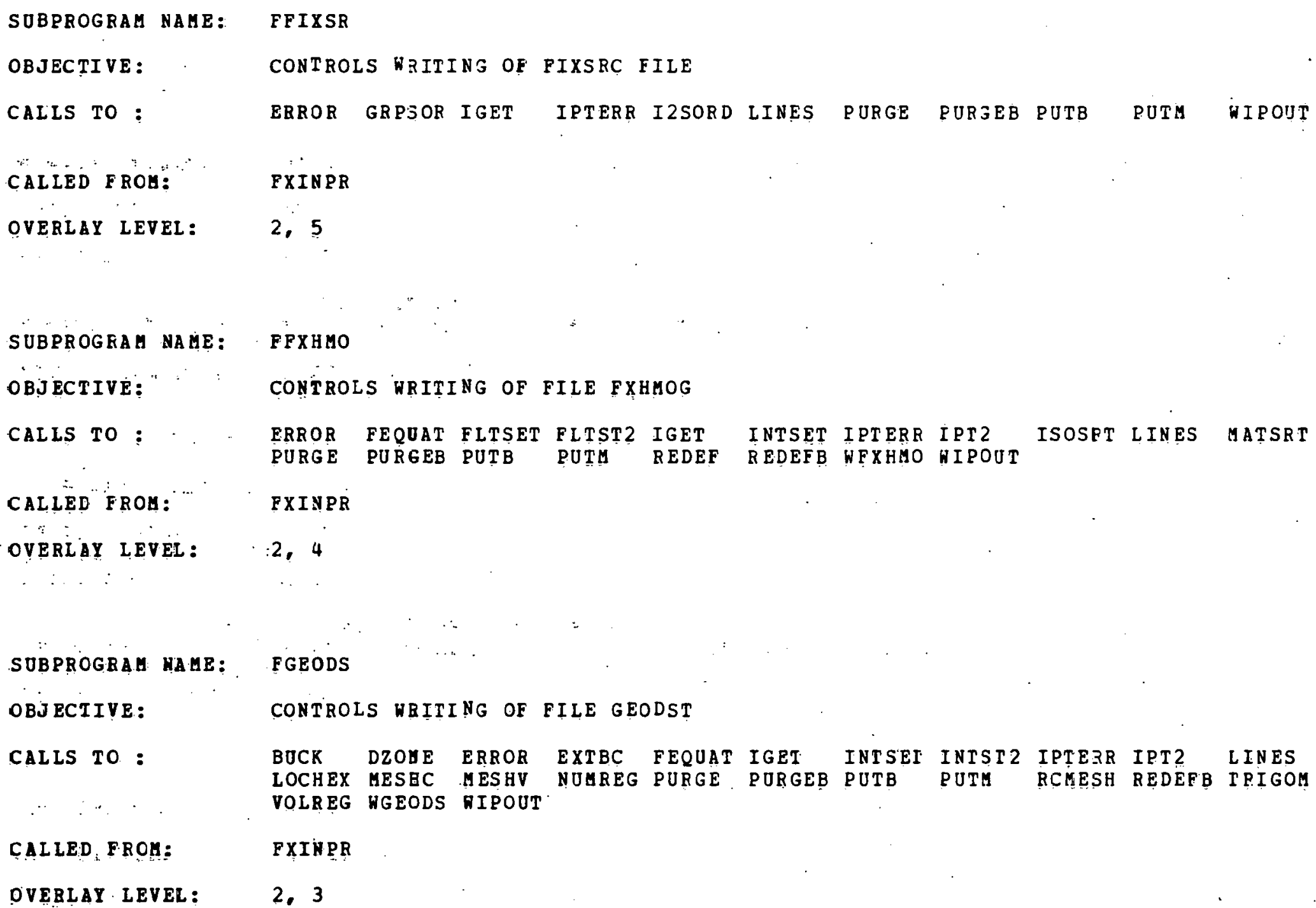


SUBPROGRAM NAME: PHEADR

OBJECTIVE: PRINTS SUMMARY OF ISOTOPE-TO-MATERIAL MIXING TABLE

CALLS TC: $\quad$ LINES

CALLED FROM: $\quad$ PXOV3̣1

OVERLAY LEVEL: $\quad 3,1$

SUBPROGGAM NAME: FILES 1

OBJECTIVE: DUMMY SUBROUTINE CONTAINING DESCRIPTION OF BCD AND DINAR: INTERFACE FILES

CALLS TC: NONE

CALLED FROM: NONE

OVERLAY LEVEL: : 0,0

SUBPROGFAM NAME: FILES 2

OBJECTIVE: DUMMY SUBROUTINE CONTAINING DESCRIPTION OF SCRATCH FILES

CALLS TC : NONE

CALLED FROM: NONE

OVERLAY LEVEL: $\quad 0,0$ 


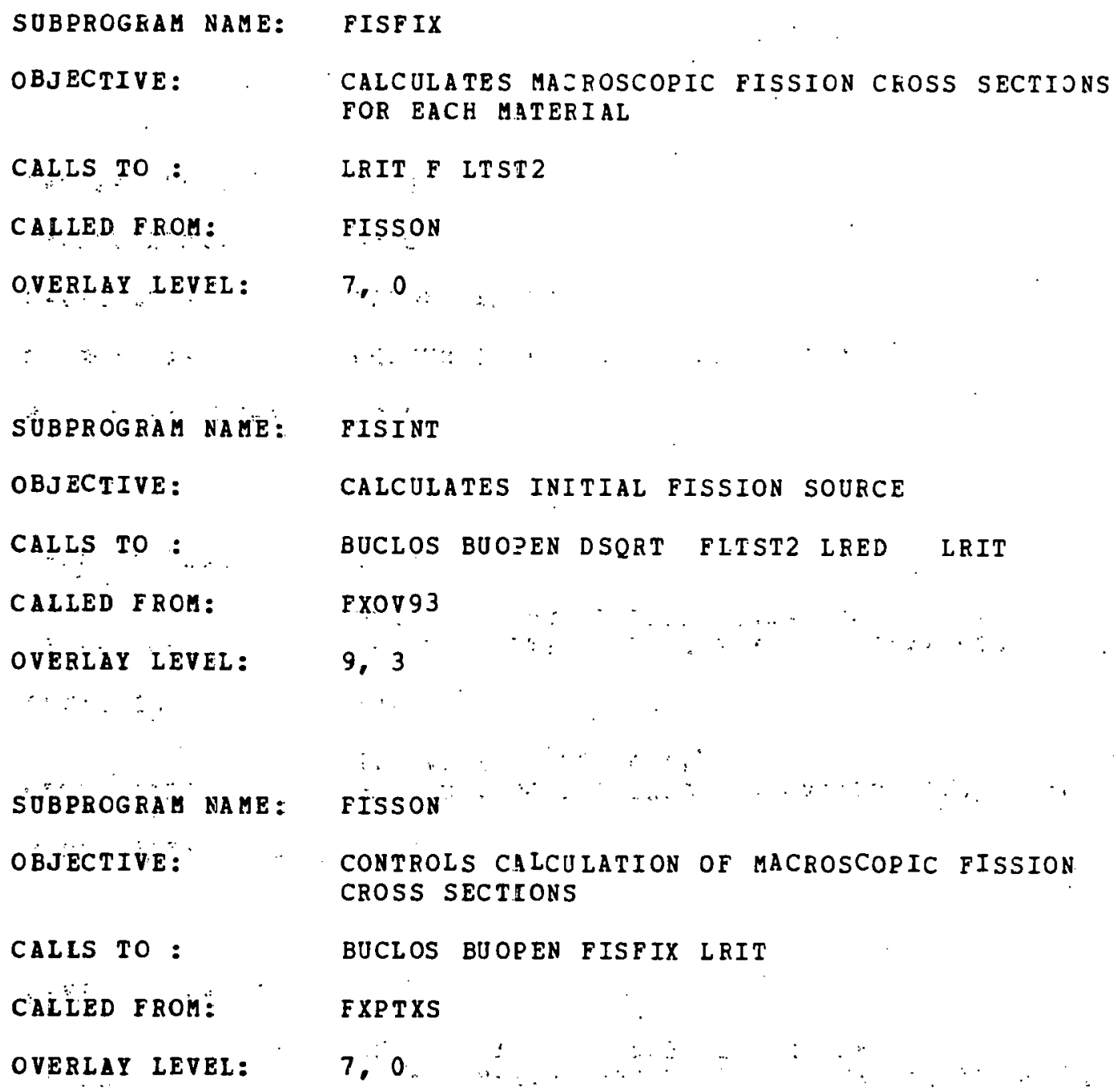




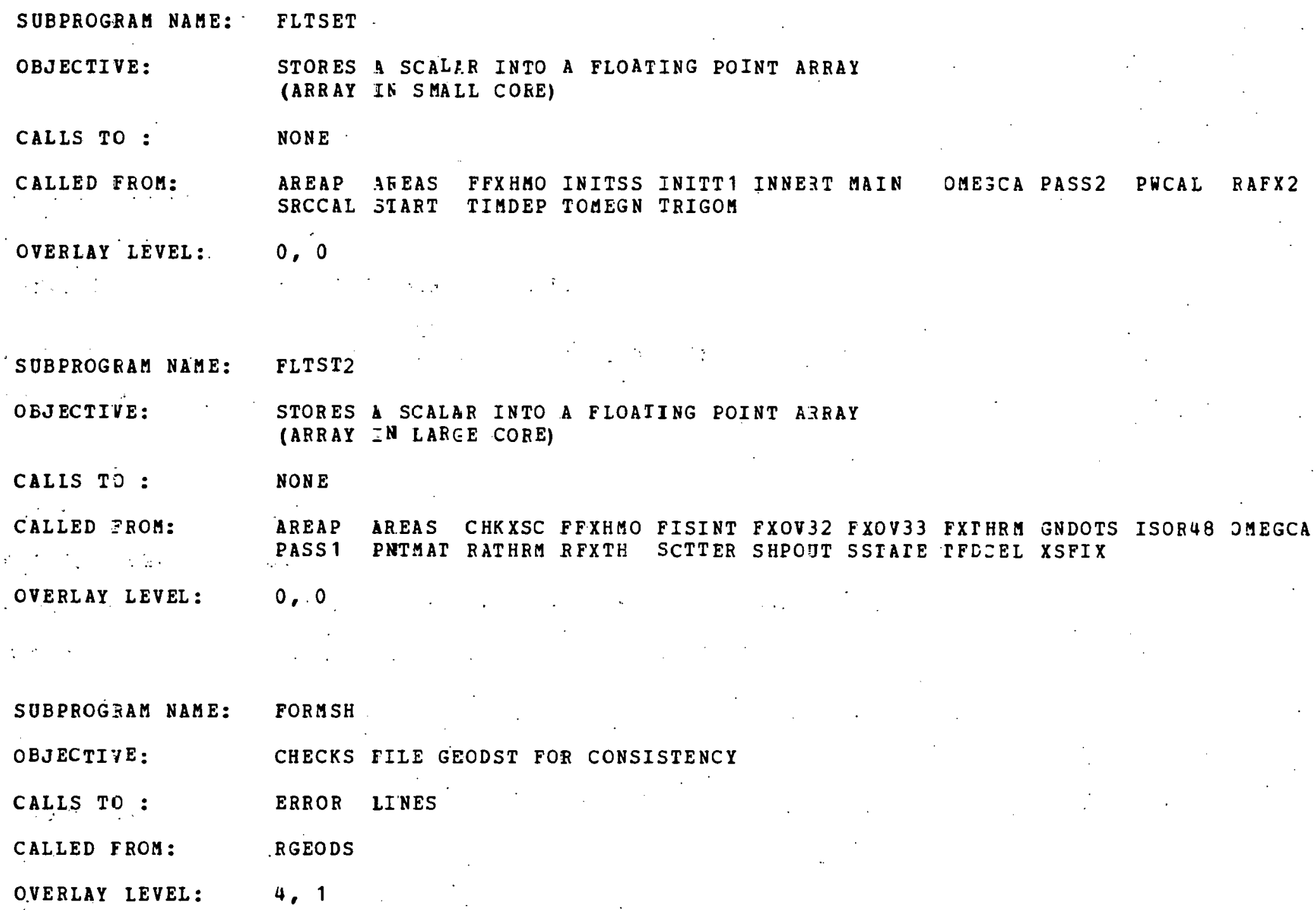




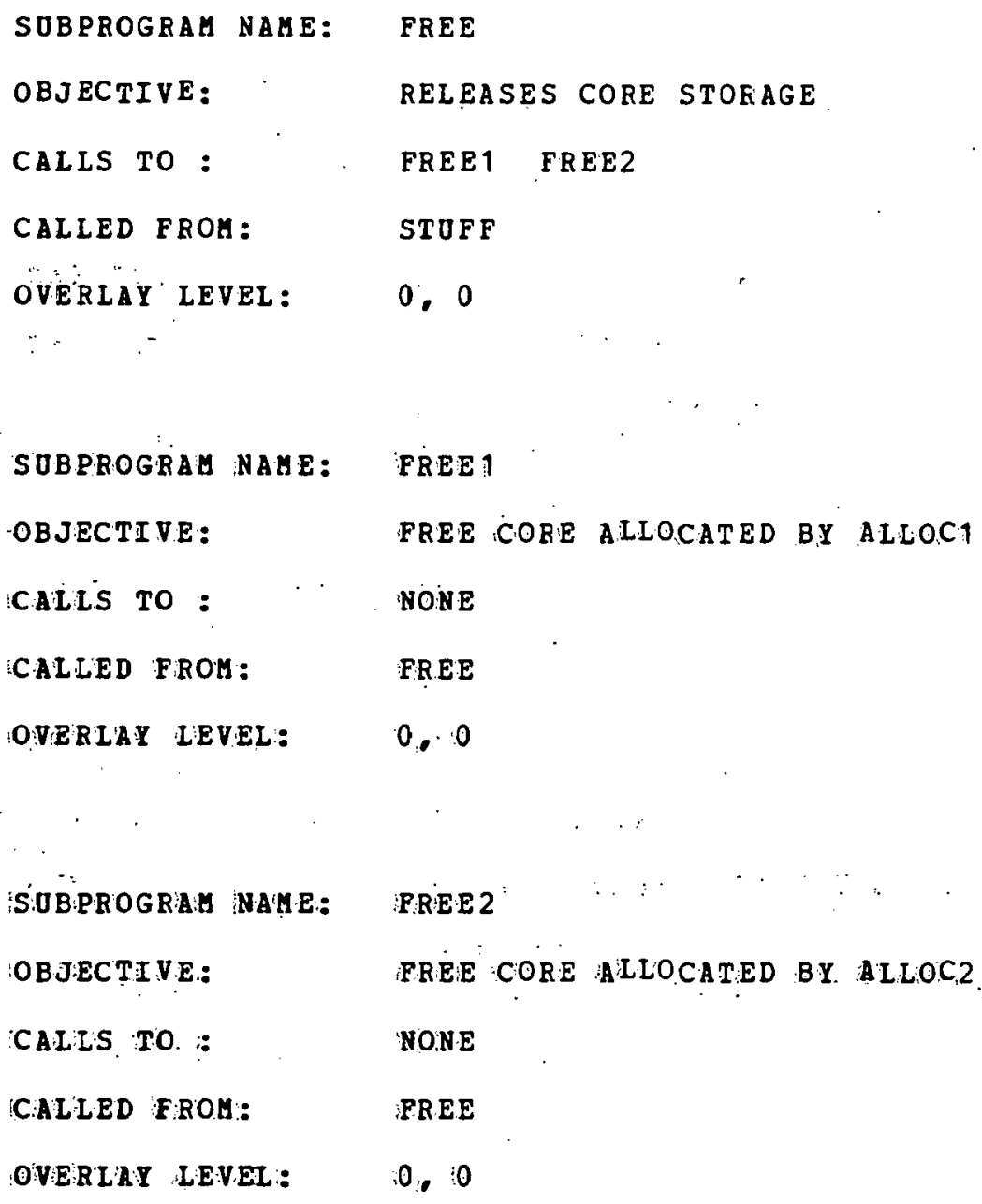




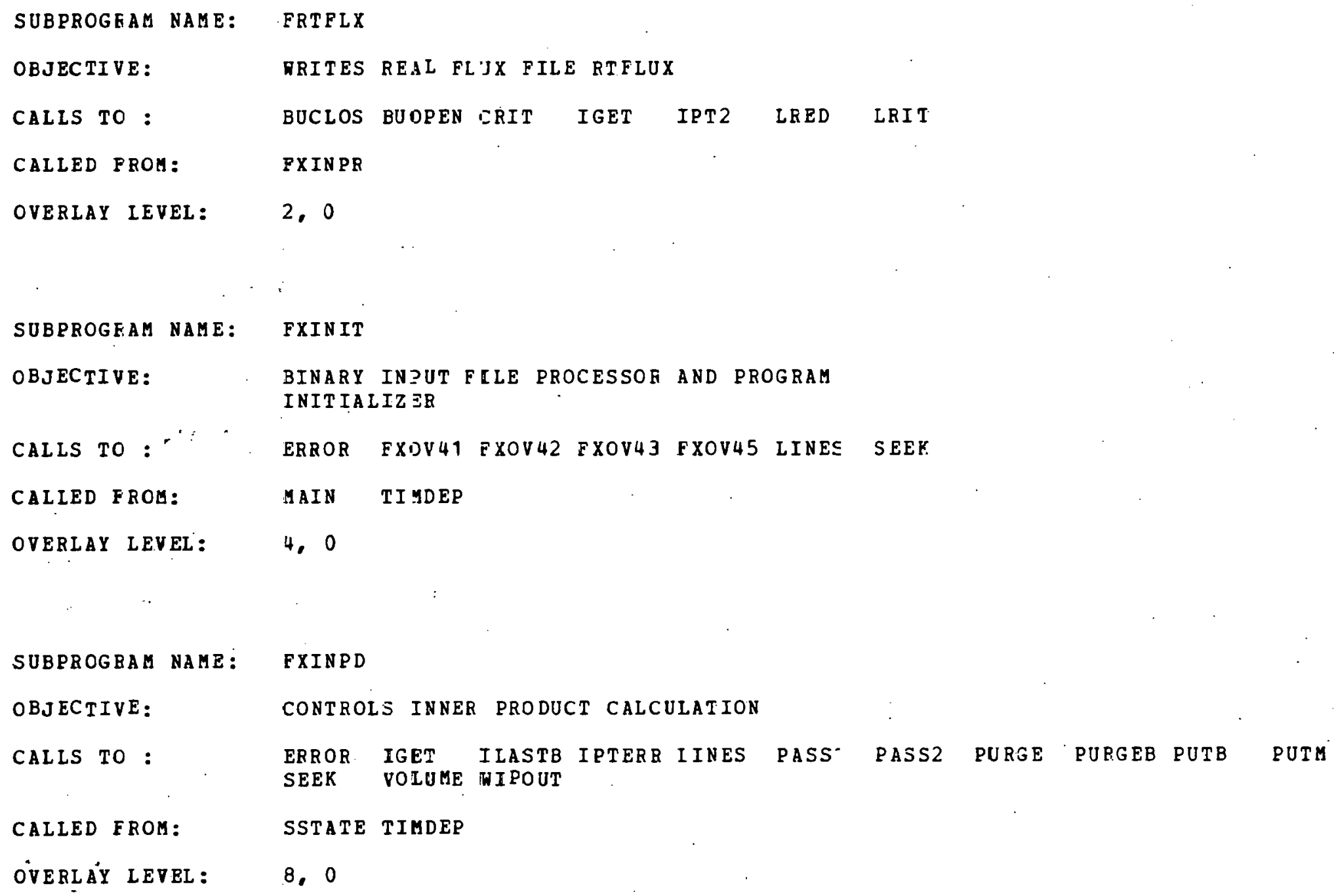




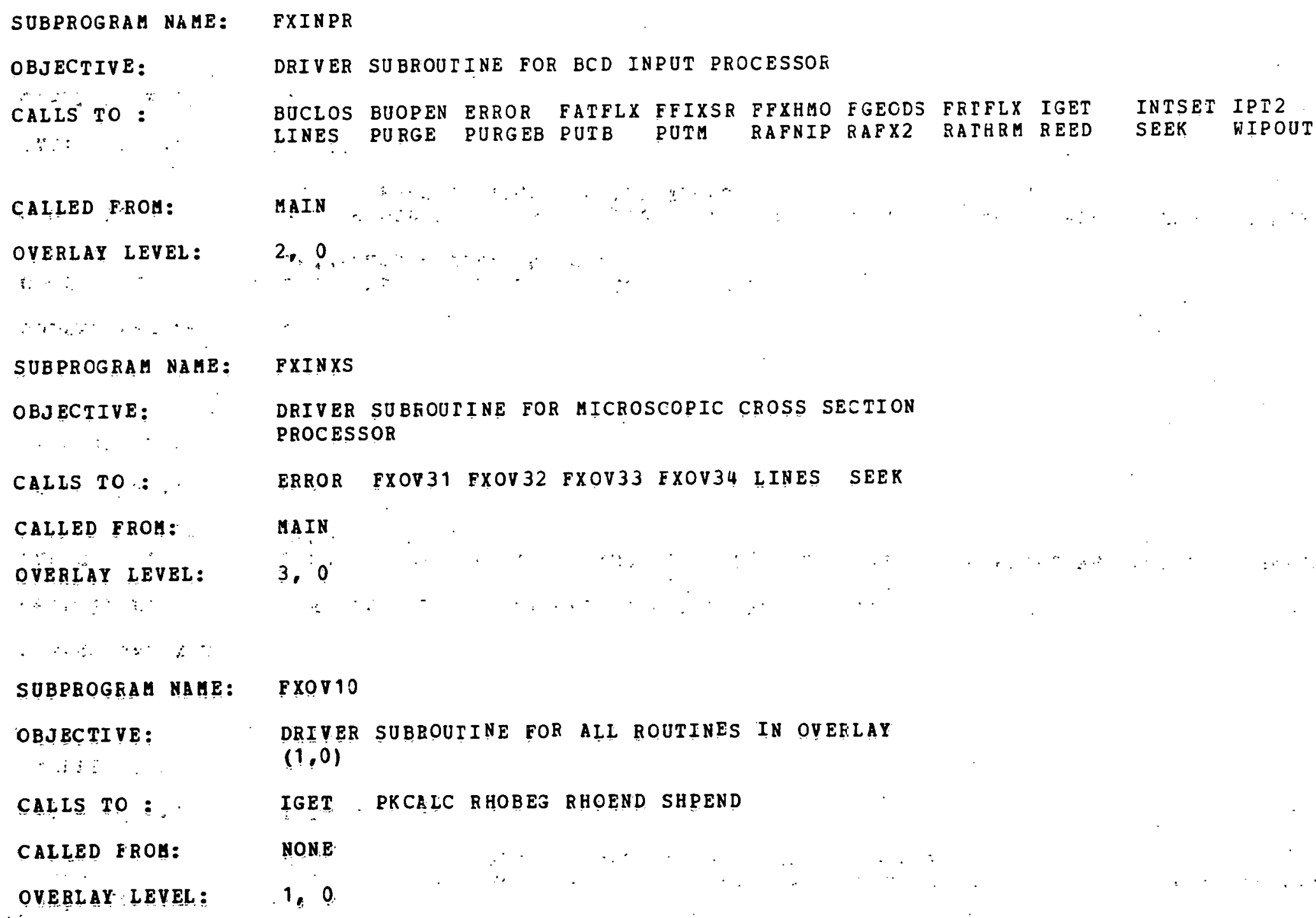




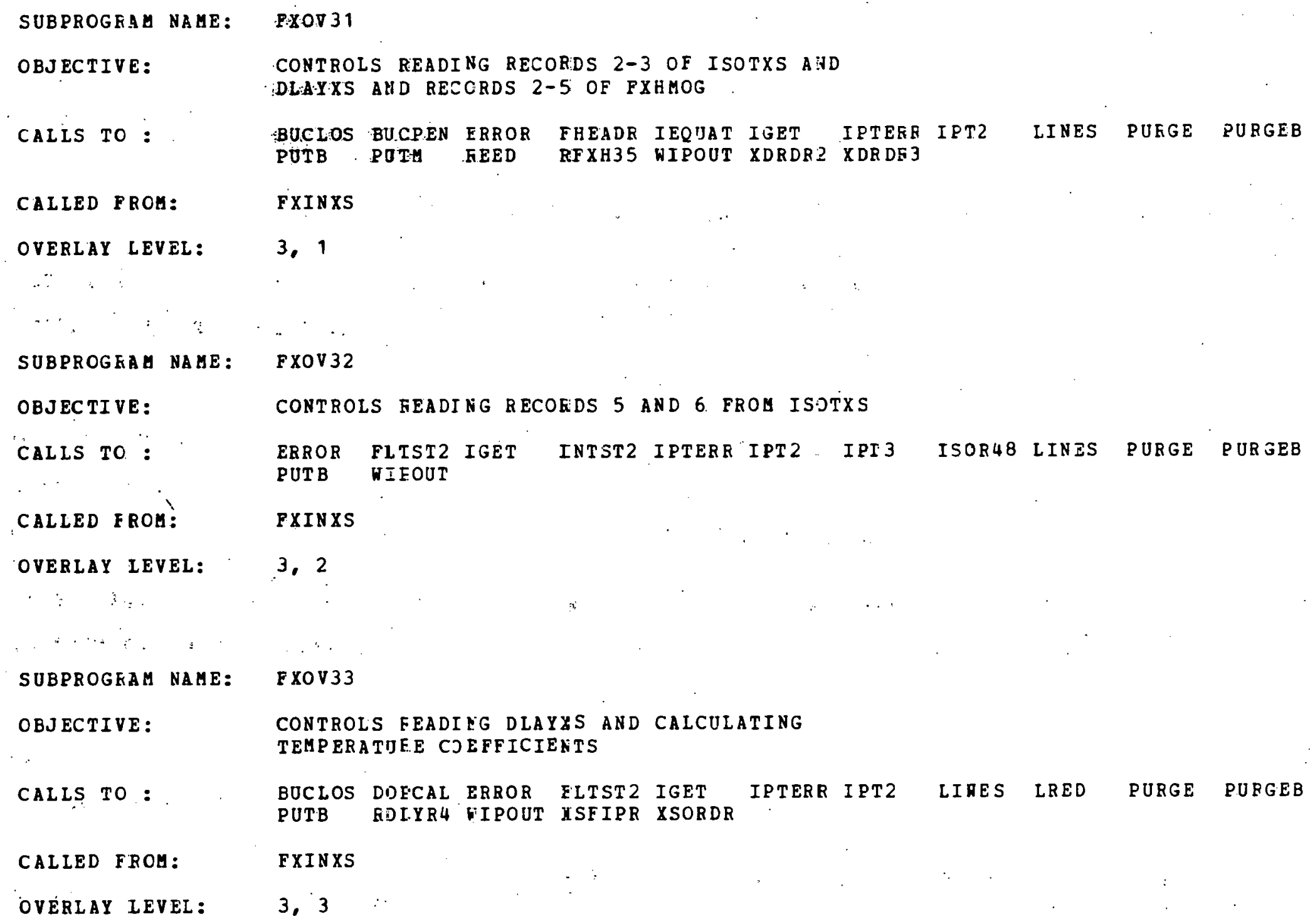




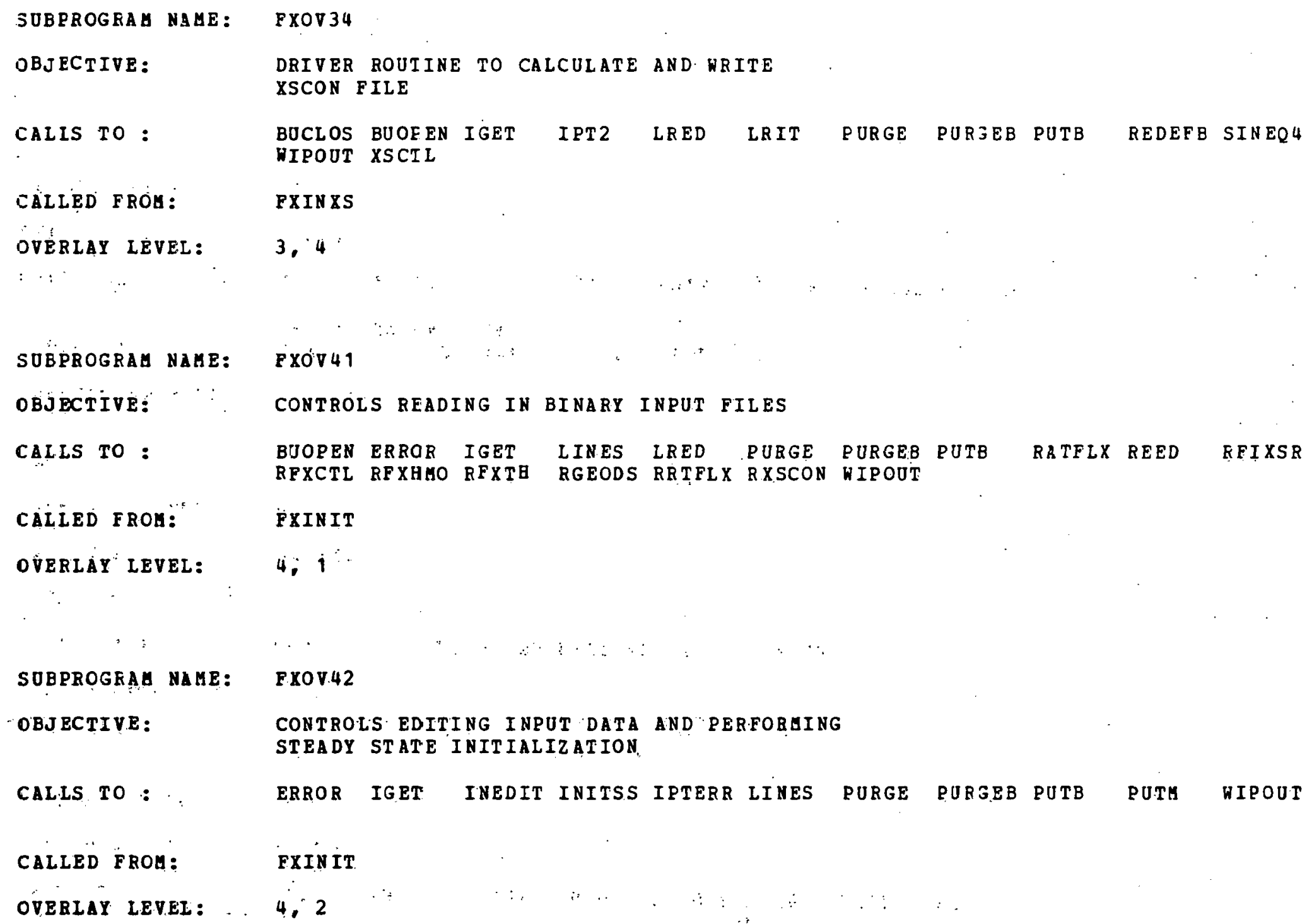




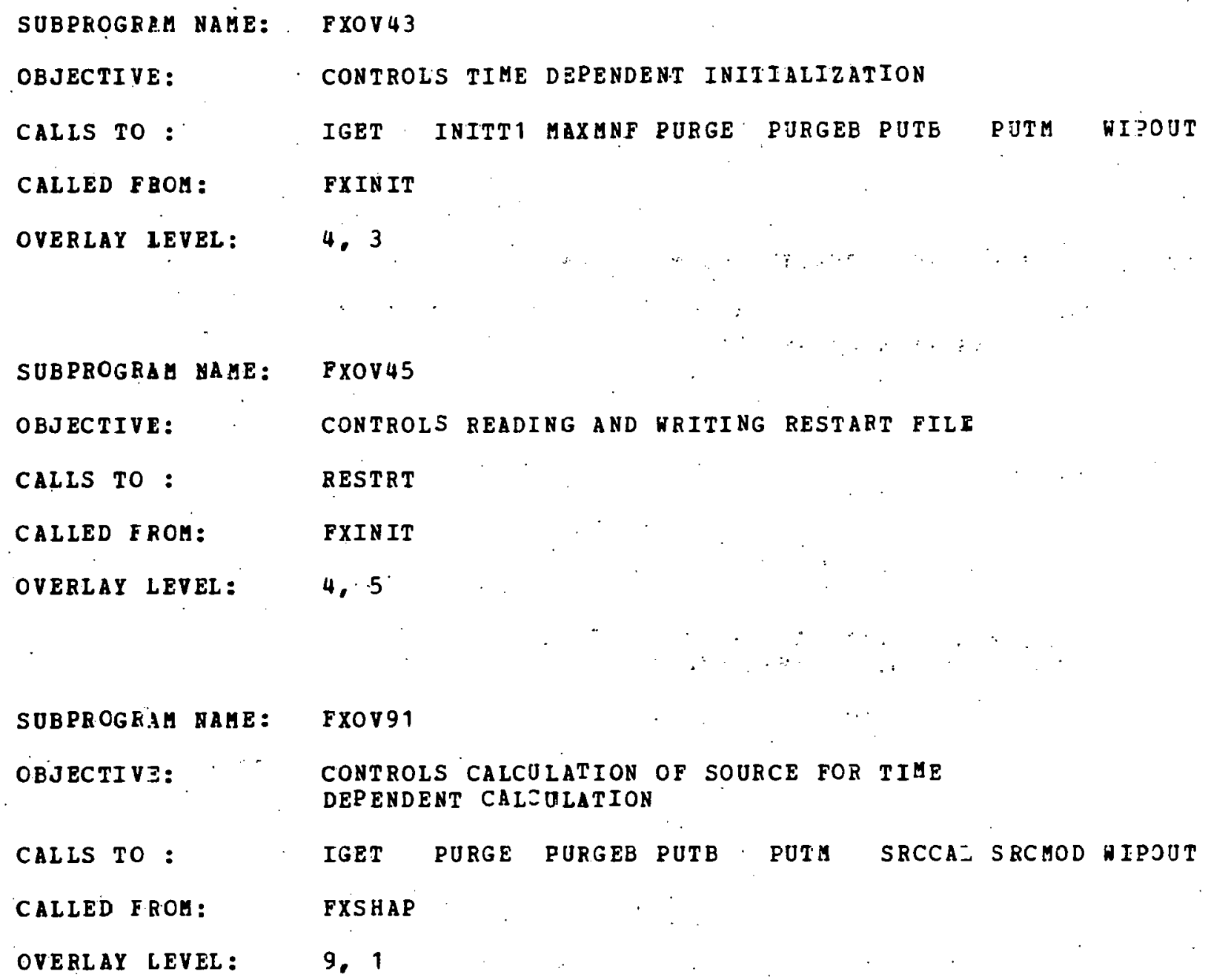


SUBPROGRAM NAME: FXOV.92

CBJECTIVE: CONTROLS CALZULATION OF FINITE DIFFERENC COEPFICIENTS

CALLS TO: IGET SFDGEN VOLUME

CALLED FROM: $\quad$ FXSHAP

OVERLAT IEVEL: $\quad 9,2$

SUBPROGRAM NGHE: FXOV93

OBJECTIVE: CONTROLS CALCULATION OF OVER-EELAXATION FACTORS

CALLS TO: FISINT IGET LCOPEN OMEGCA

CALLED FROU:

DVERLAY LEVEL: $\quad 9,3$

SUBFROGRAM NAGE: FXOV94

DBJECTIVE: CONTROLS CELCULATION OF FIUX SHAPE

CALLS TO : IGET OUTERS

CALLED FROM: $\quad$ FXSHAP

OVERLAT LEVEL: $\quad 9,4$ 


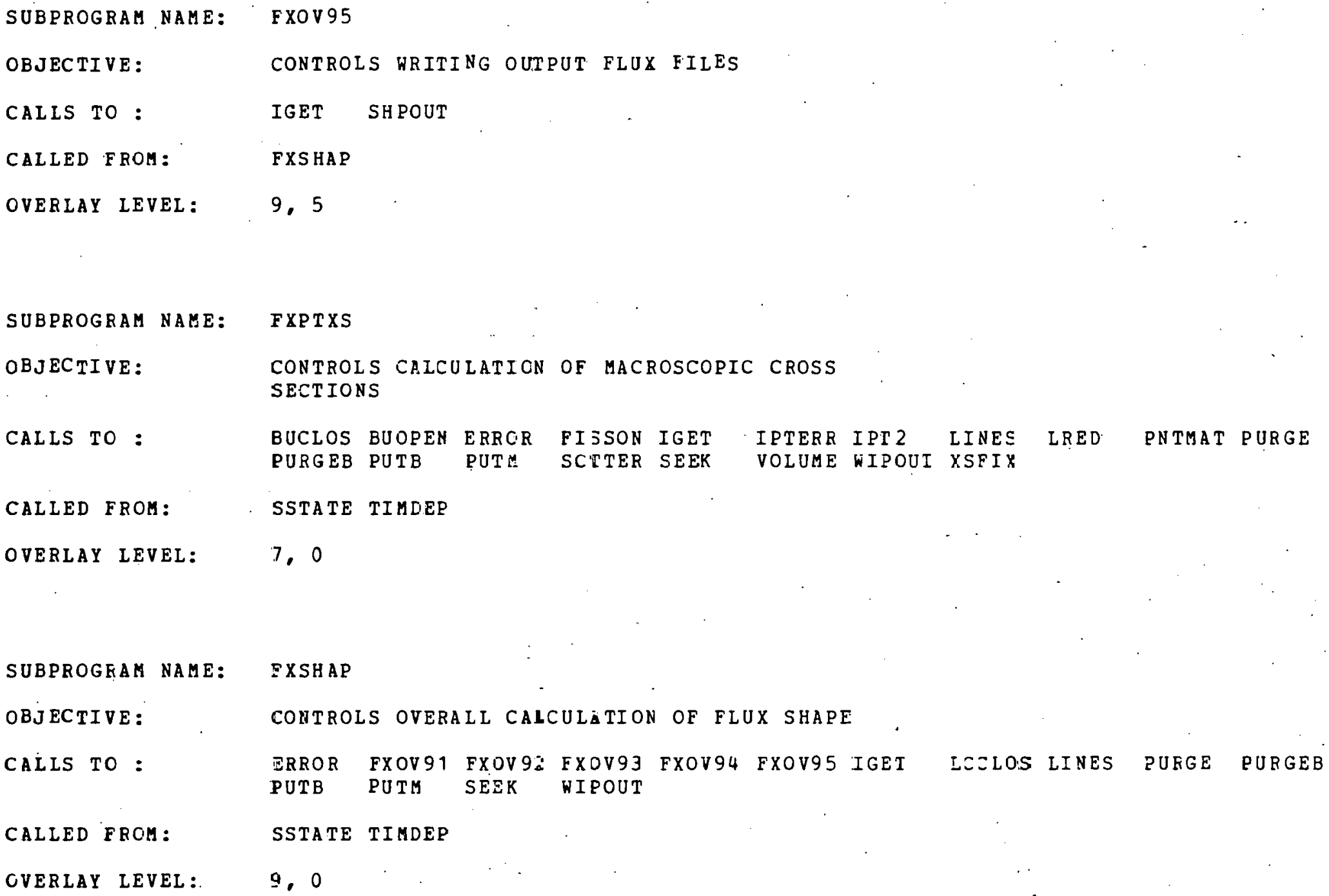




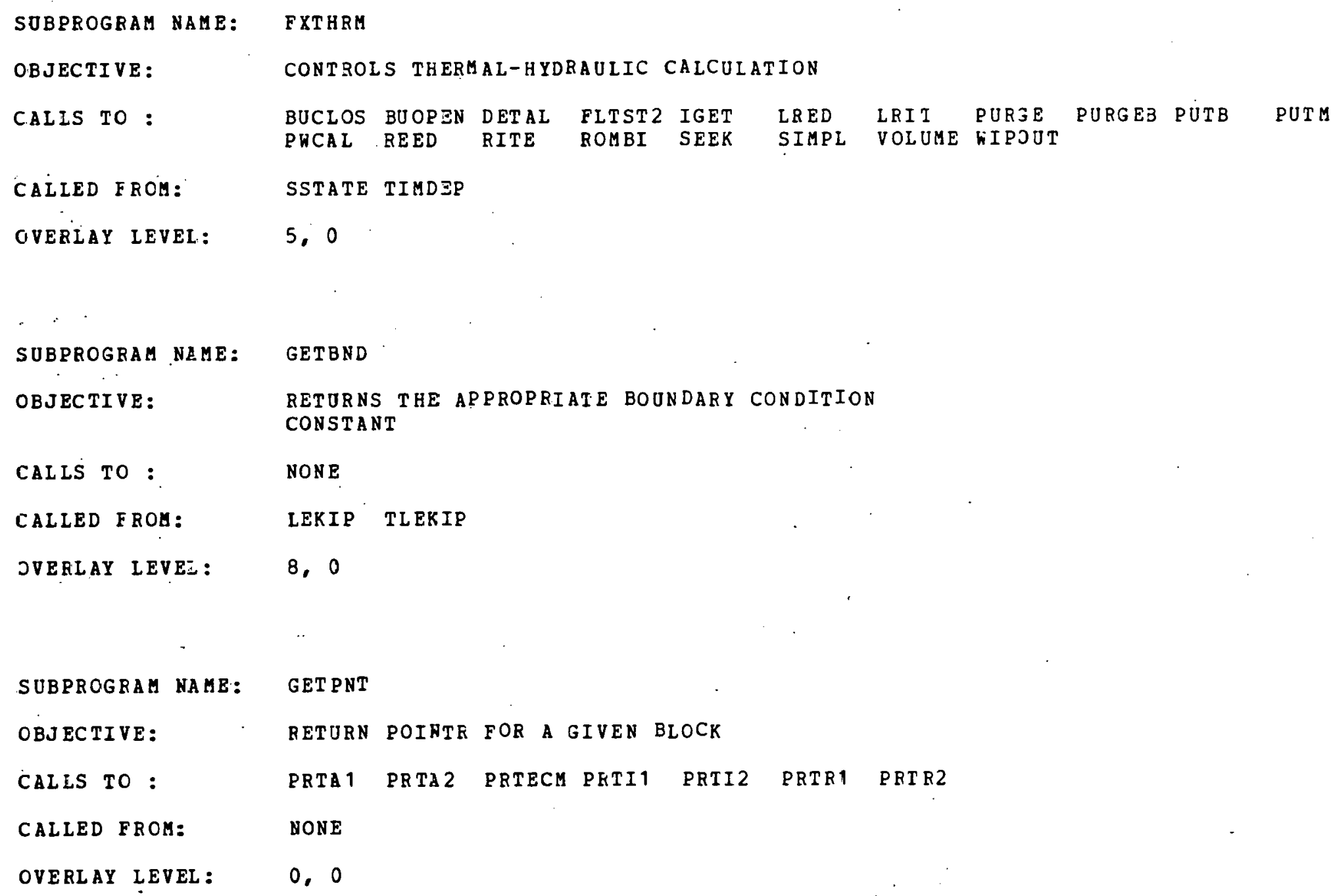




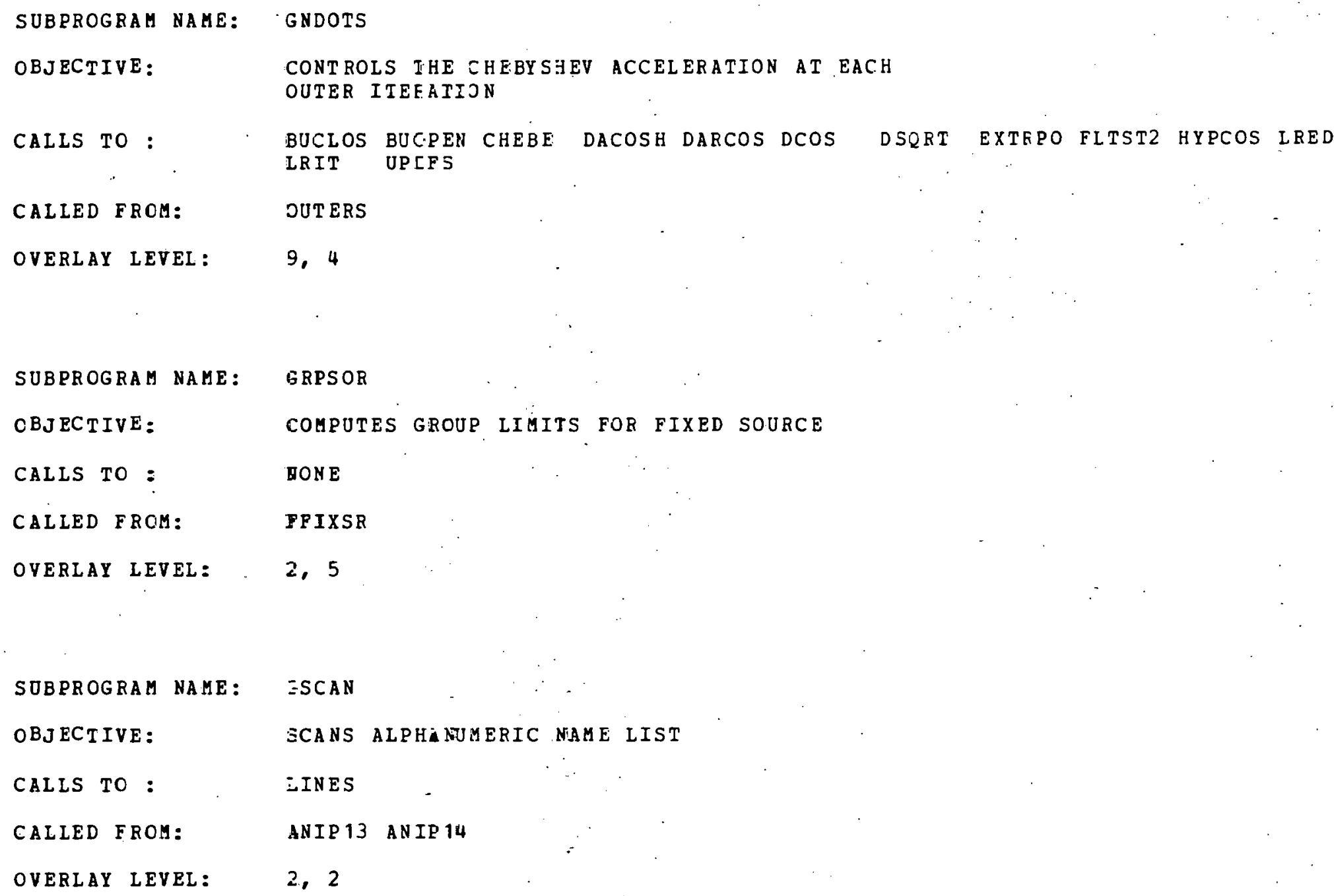




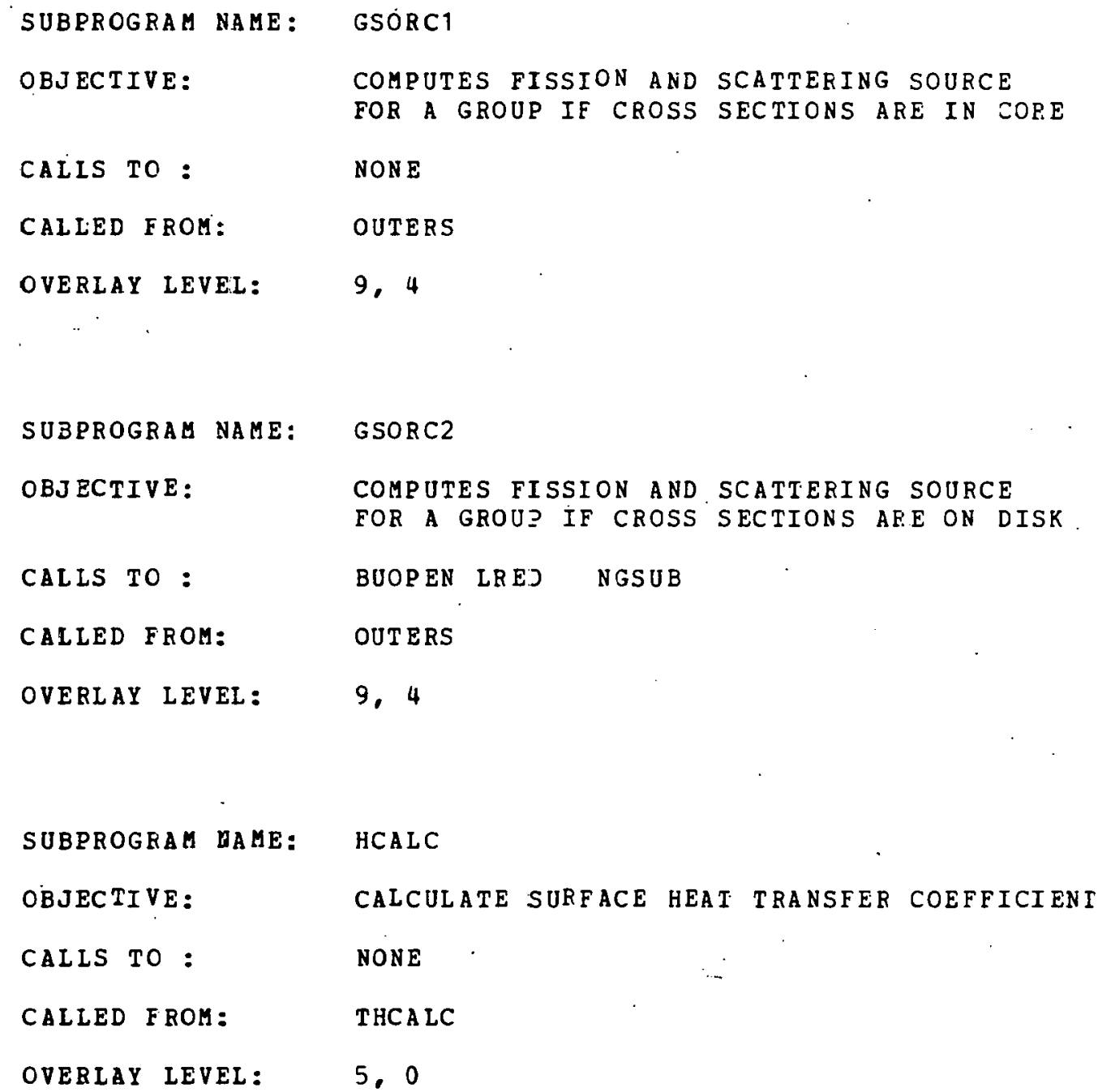




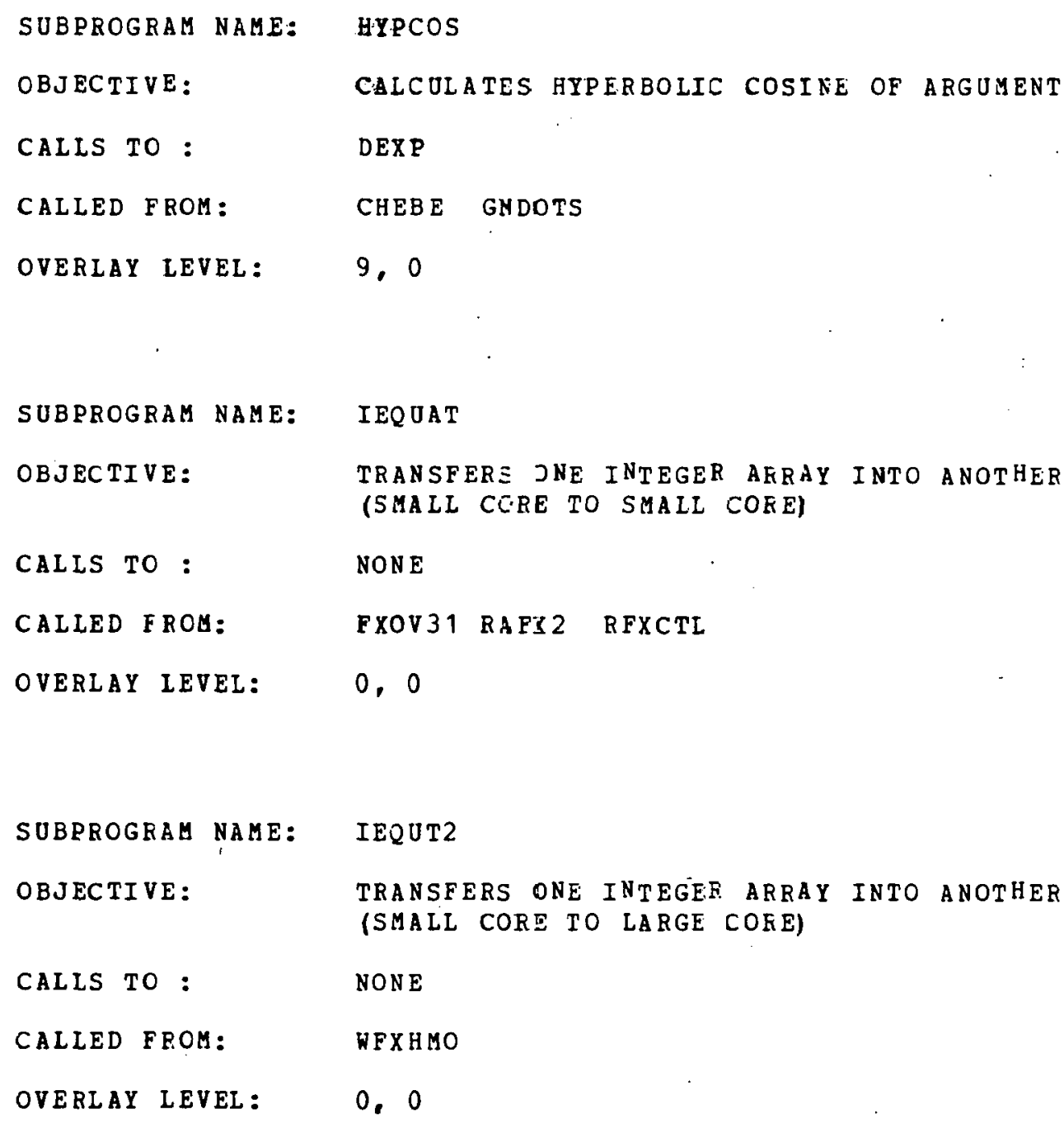




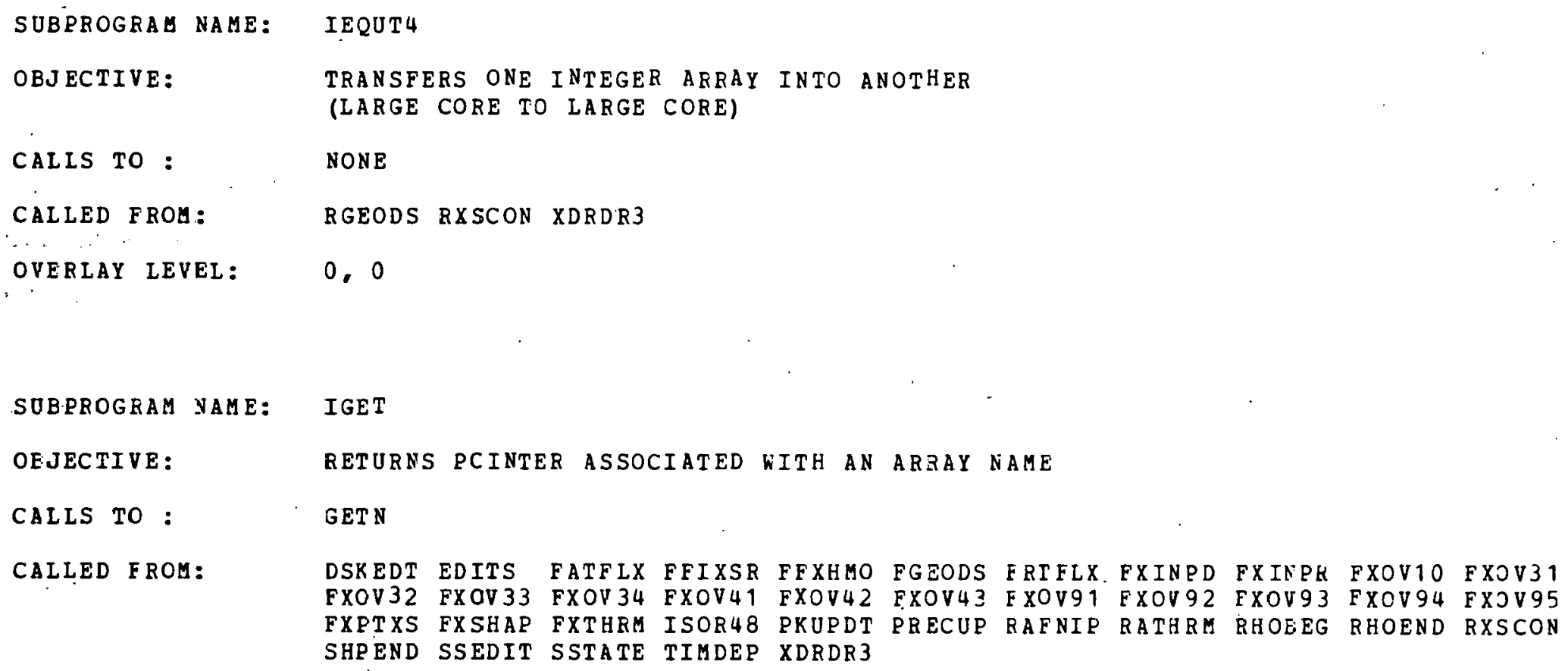




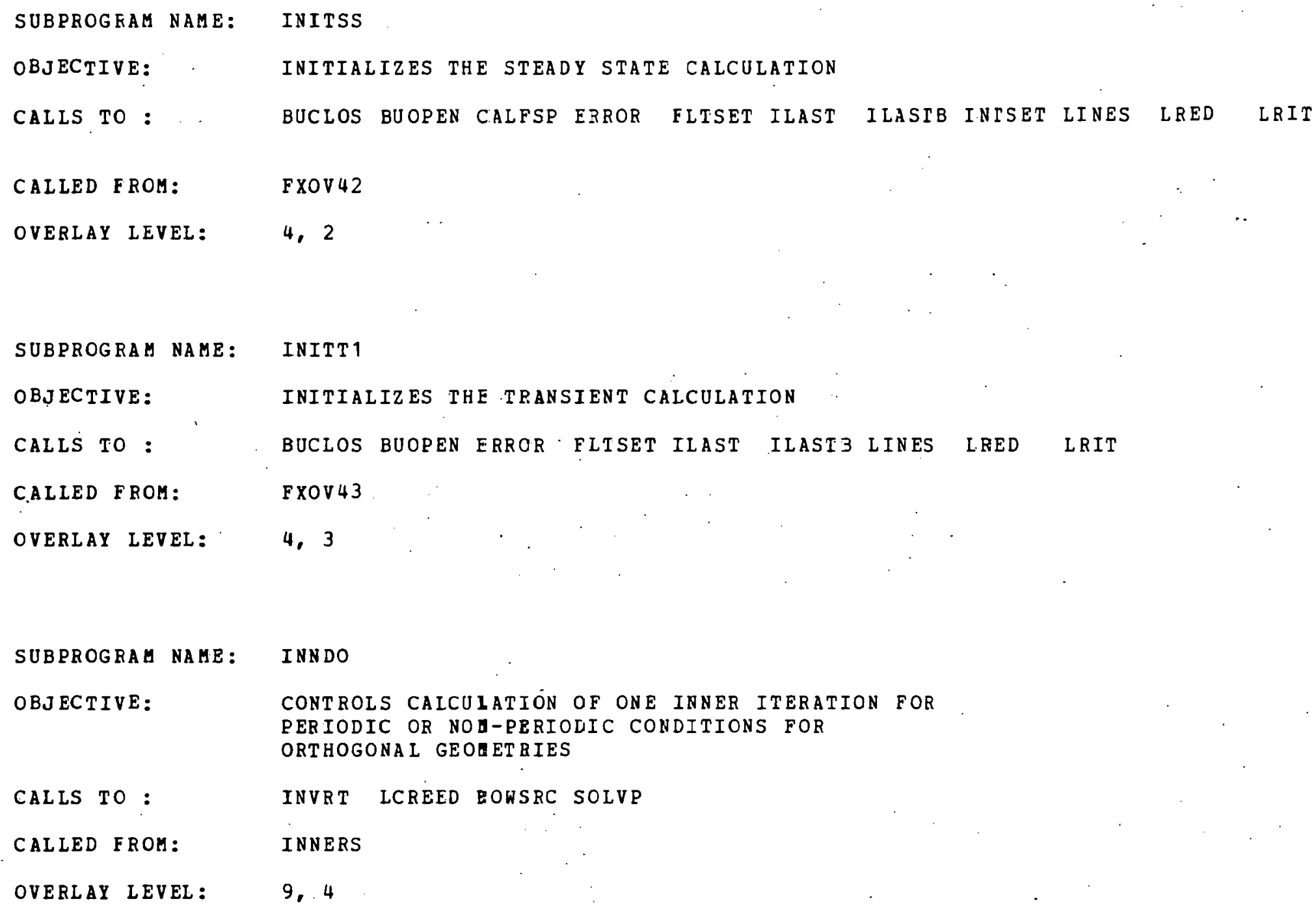




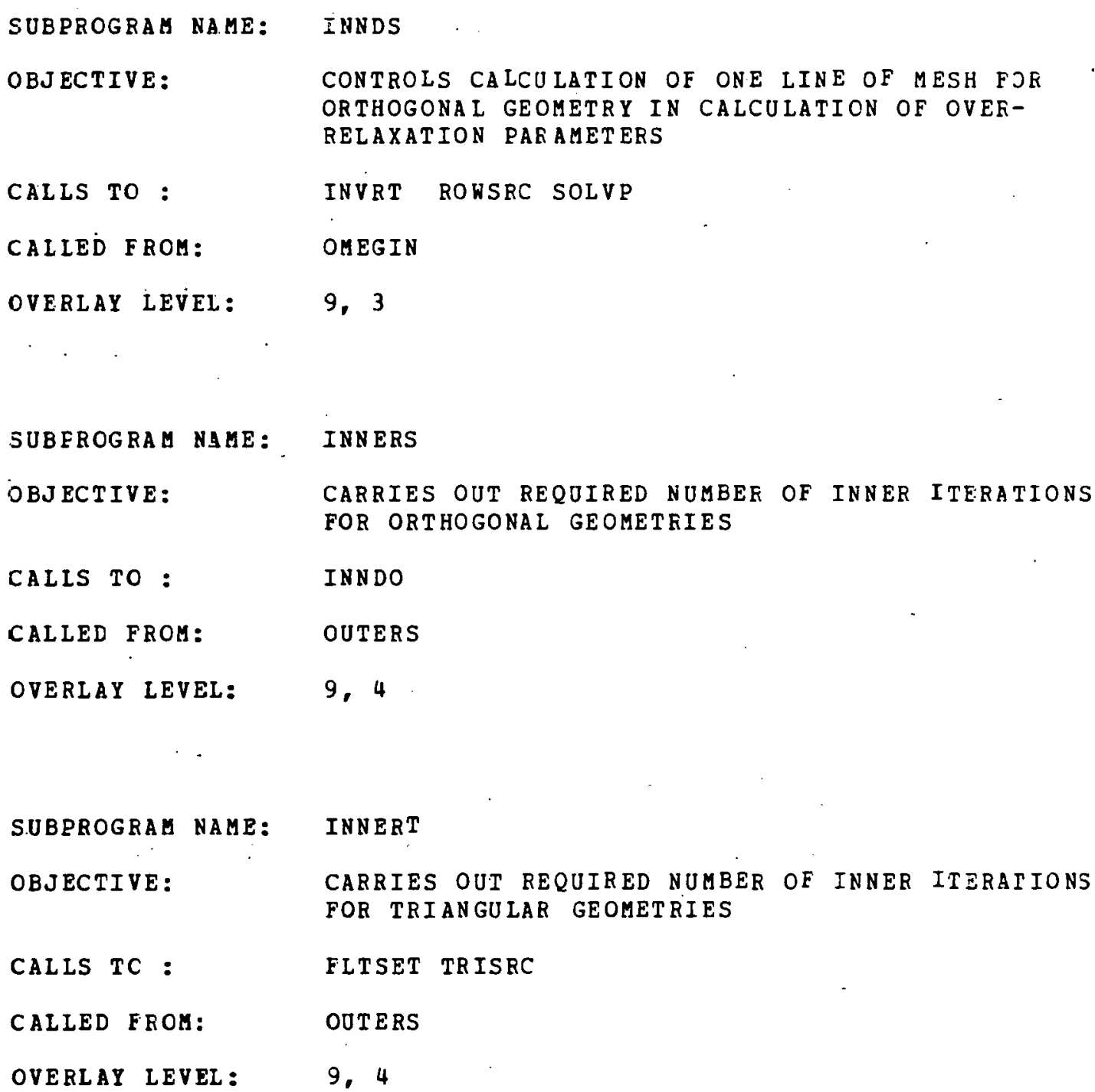




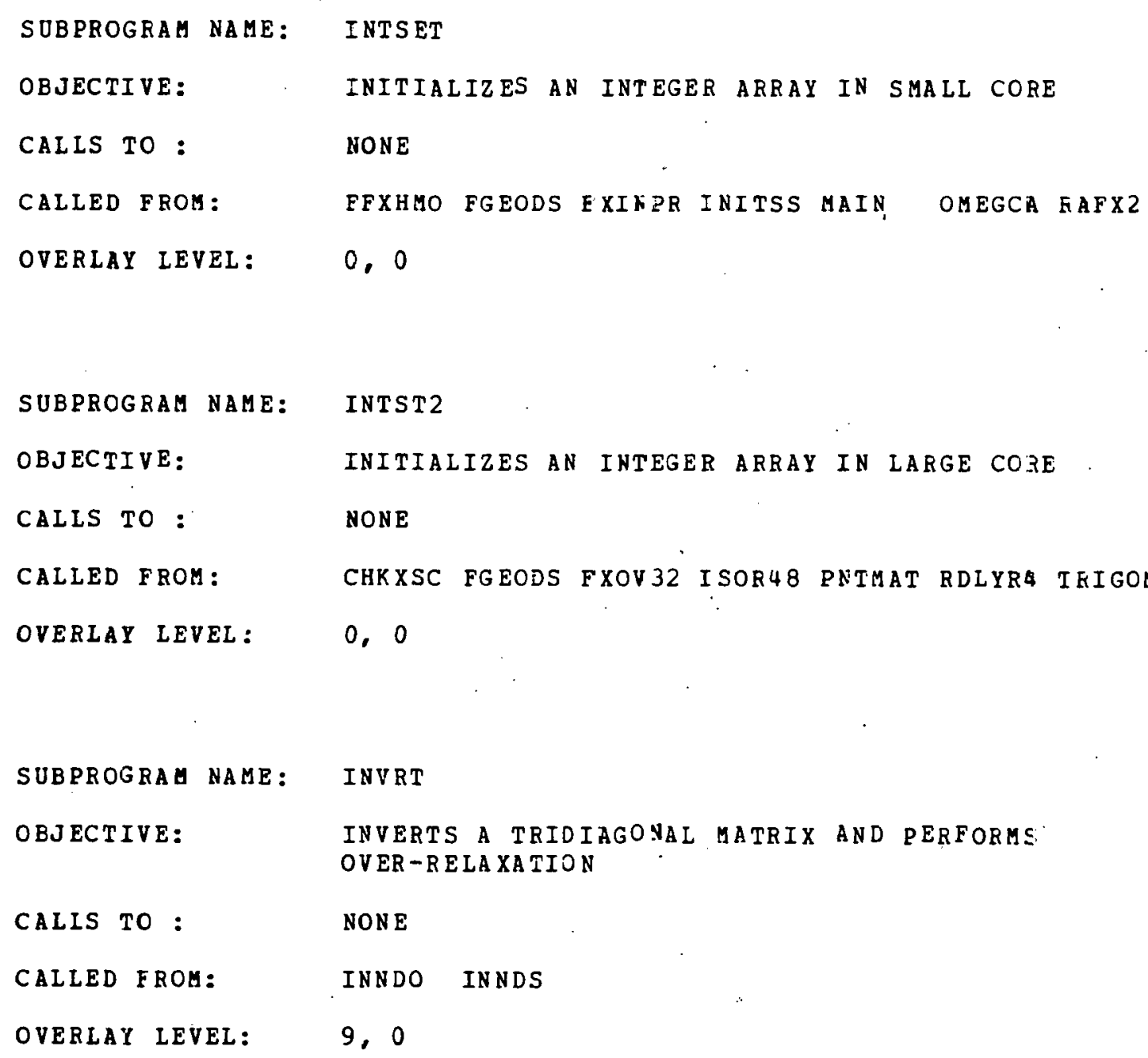




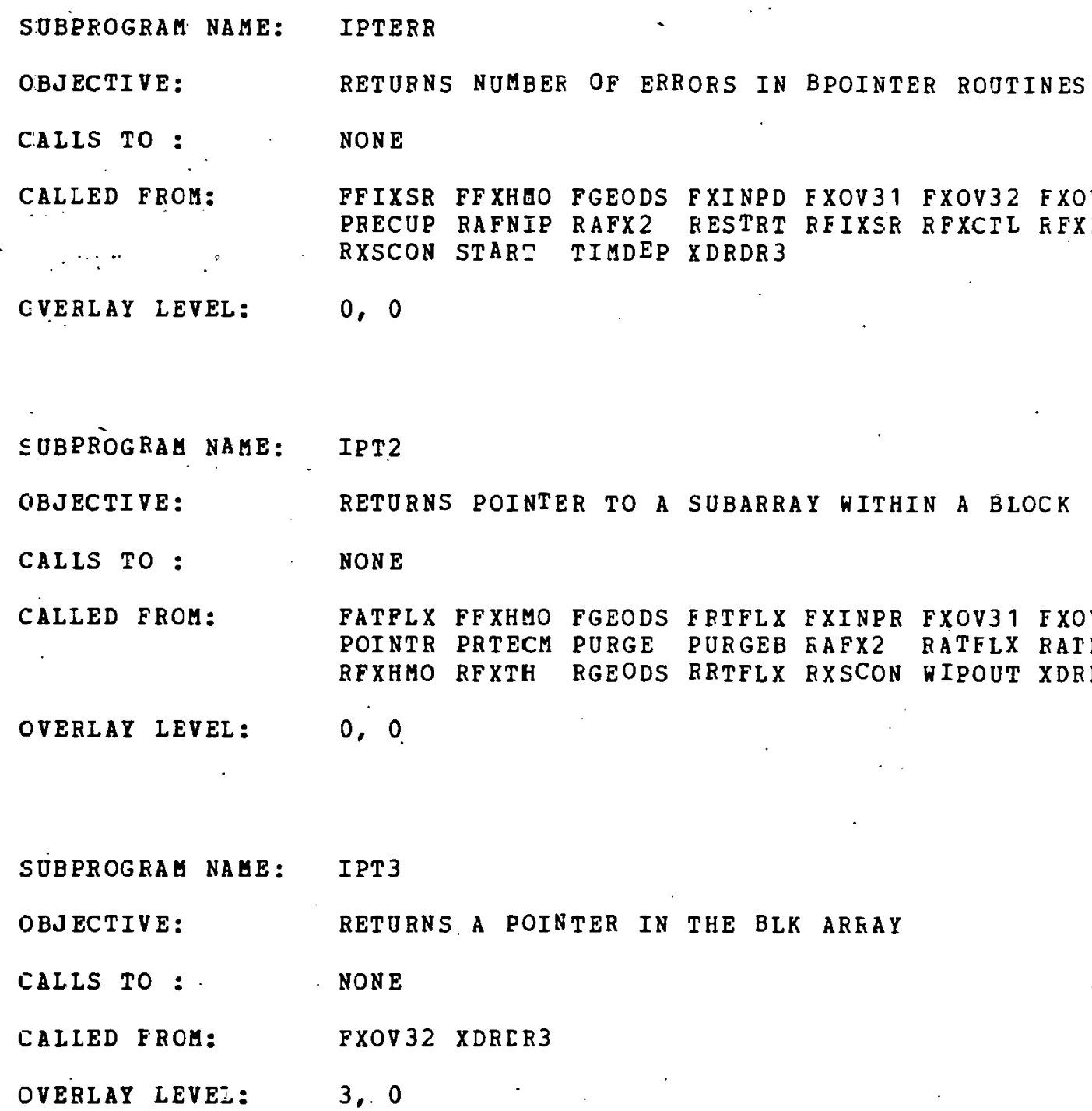




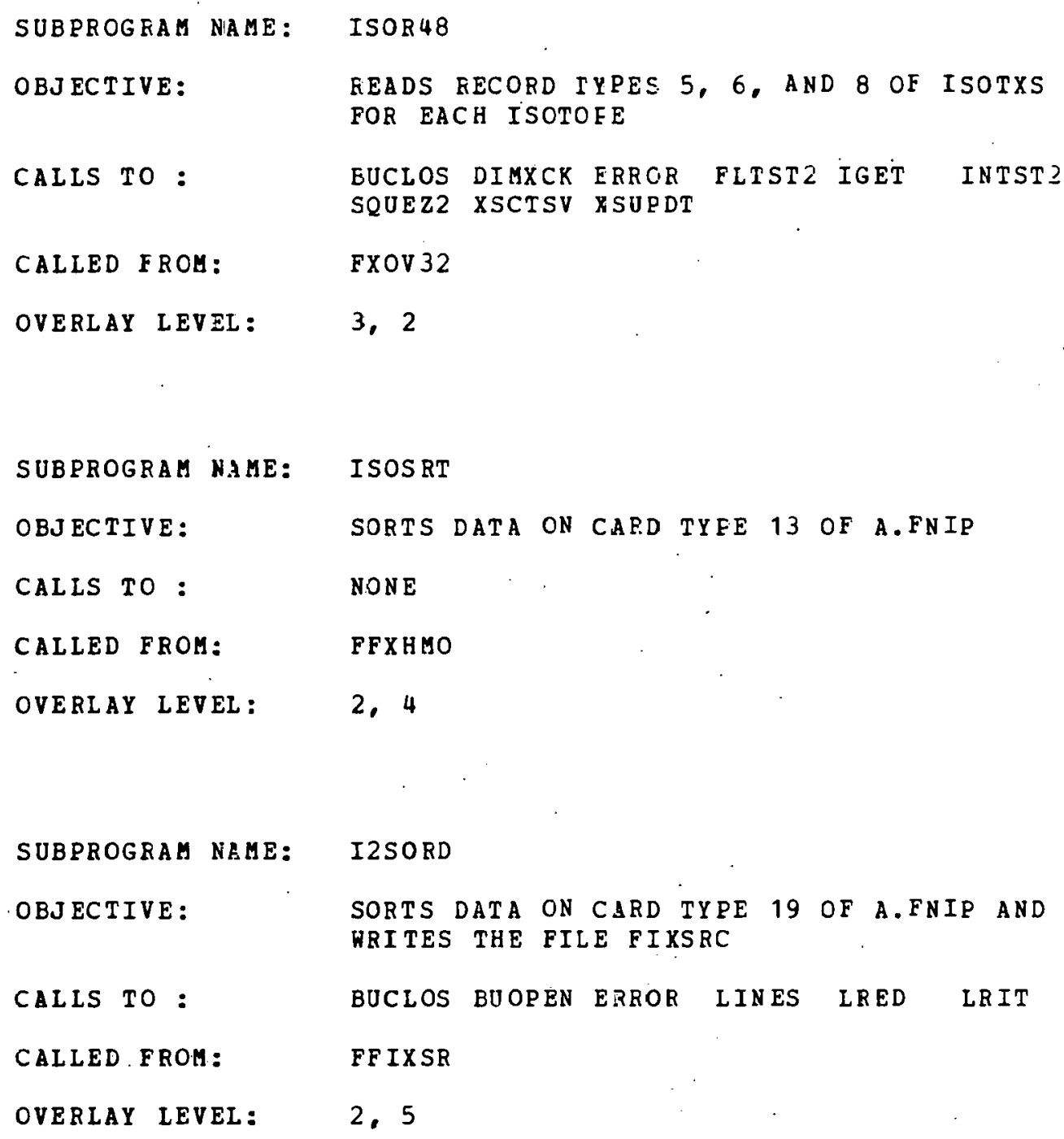


SUBPROGRAM NAME: LACLOS

OBJECTIVE: PERFORMS A WIPOUT ON FILE FILNAM

CALLS TO: WI POUT

CALLED FROM: FXSHAP

OVERLAY LEVEL: $\quad 0,0$

SUBPROGRAM NAME: LCOPEN

OBJECTIVE: CALLS PUT TO OPEN A FILE IN SMALL CORE

CALLS TO: - PUT

CALLED FROM: $\quad$ FXOV 93

OVERLAY LEVEL: $\quad 0,0$

SUBPROGRAM NAME: LCEEED

OBJECTIVE: READS DATA FROM LARGE CORE TO SMALL CORE

CALLS TO : . NONE

CALLED FROM: $\quad$ INNDO

OVERLAY LEVEL: $\quad 0,0$ 
SUBPROGFAM NAME: LCRITE

OBJECTIVE: WRITES DATA FROM SMALI CCRE TO LARGE CORE

CALLS TO : NONE

CALIED FROM: $\quad$ NONE

OVERLAY LEVEL: $\quad 0,0$

SUBPROGRAM NAME: LEKIP

OBJECTIVE: COMPUTES IEAKAGE PLUS REMOVAL INNER PRODJCT

FOR ORTHOEONAI GEOMETFIES

CALLS TO: DCOS, DSIN GETBND

CALLED FROM: PASS2

OVERLAY LEVEL: $\quad 0,0$

SUBPROGFAM NAME: LINES

OBJECTIVE:

KEEPS TRACK OF LINES PRINTED ON THE PAGE

CALIS TO :

NONE

CALLED FROM:

\begin{tabular}{|c|c|c|c|c|c|c|c|c|c|c|}
\hline & & & & & & & & & & \\
\hline & & $M$ & C & CRE & CF I & & & $\nabla E F$ & & \\
\hline $\mathrm{TT}$ & $5 \mathrm{CO}$ & $\therefore P$ & EXTBC & FFIX & & & & & & \\
\hline & & & & & & & & & & \\
\hline & & & & & & & & & & \\
\hline & PF & & & & & & & & & \\
\hline & & & & & & & & & & \\
\hline Dון & REIS & & $S H P$ & & & & MARY & I MDEP & TIMSTP & 1 \\
\hline & & & & & & & & & & \\
\hline
\end{tabular}

OVERLAY LEVEL: $\quad 0,0$ 
SUBPROGRAM NAME:

OBJECTIVE:

CALLS TO:

CALLED FROM:

OVERIAY LEVEL :

SUBPROGEAM NAME:

OBJECTIVE:

CALLS TO:

CALLED FROY:

SUBPROGKAM NAME:

OBJECTIVE:

CALIS TO :

CALLED FROK:

OVERLAY LEVEL:

\section{LOCHEX}

LOCATES A PARTICULAR HEX CELL IN A

PARTICULAR RING

NONE

FGEODS TREGOM

2,3

LRED

GENERAL PORPOSE FILE READ SUBPCUTINE (TO

LARGE CORE). MEETS CCCC SPECIFICATIONS

BUCLOS BUOPEN ZEROIO

COPYDS DR ZVEF DSKEDT EXTFLX FATFLX FISINT FRTFLX FXOV 33 FXOJ34 FXOV41 FXPTXS FXTHRM GNDOTS GSORC2 INITSS INITT 1 ISOE48 I2SORD MAXMNF OMEGCA OUTERS PASS1. PASS2 PRCUPD RATFLX RATHRM RDLYF 4 RESTRT FFIXSR RFXHMO RFXTH RGEODS RRTFLX RXSCON SFDGEN SHPOUT SRCCAL SRCMOD SSTATE WFXHMO WFXTY WGEDDS XDRDR2 XDEDR3

0,0

LRIT

GENERAL PURPOSE FILE READ SUBROUTINE (FROM LARGE COR I) - MEETS CCCC SPECIFICATIONS

BUCLOS BUOPEN ZEROIO

COPYDS DRIVEF EXTFLX FATFLX FISINT FISSON FRTFLX FXOV34 FXTARM GNDOTS INITSS INITT1 I2SORD OUTERS PASS2 PRCMAP RESTRT SCTTER SFDGEN SHPDIT SSTATE WFXHMO WFXTH WG $Z O D S$ XSFIX

0,0 


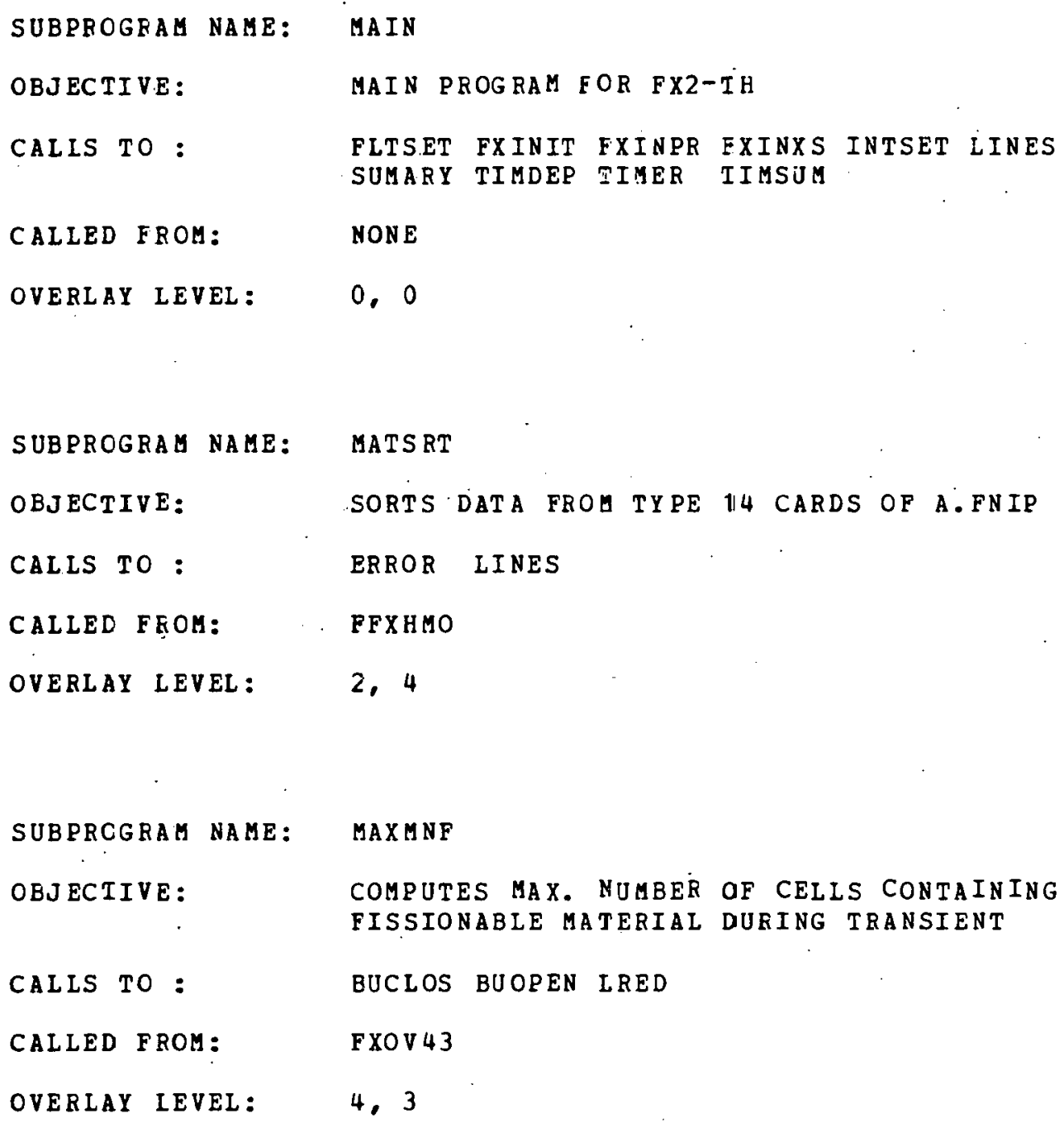




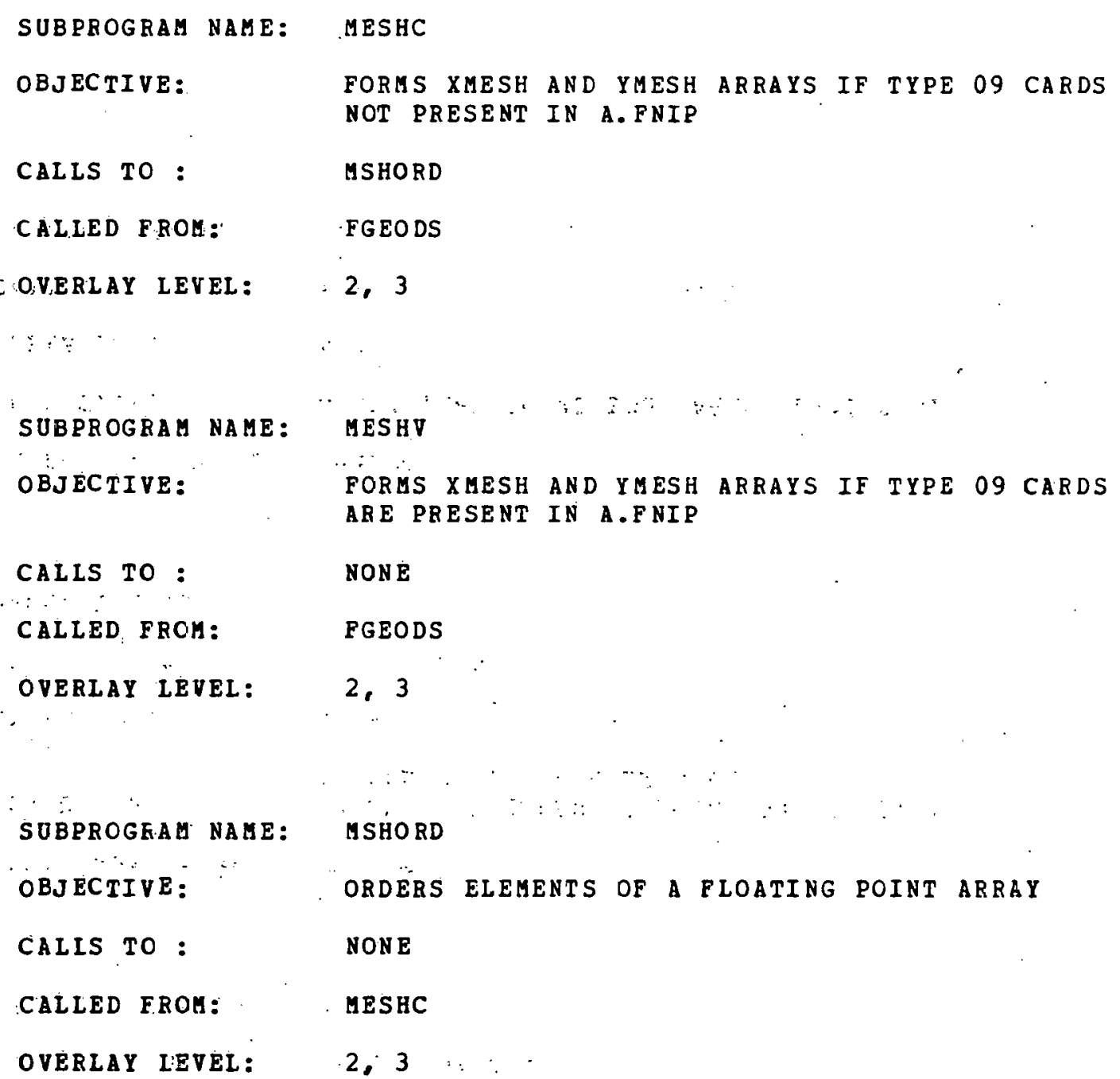




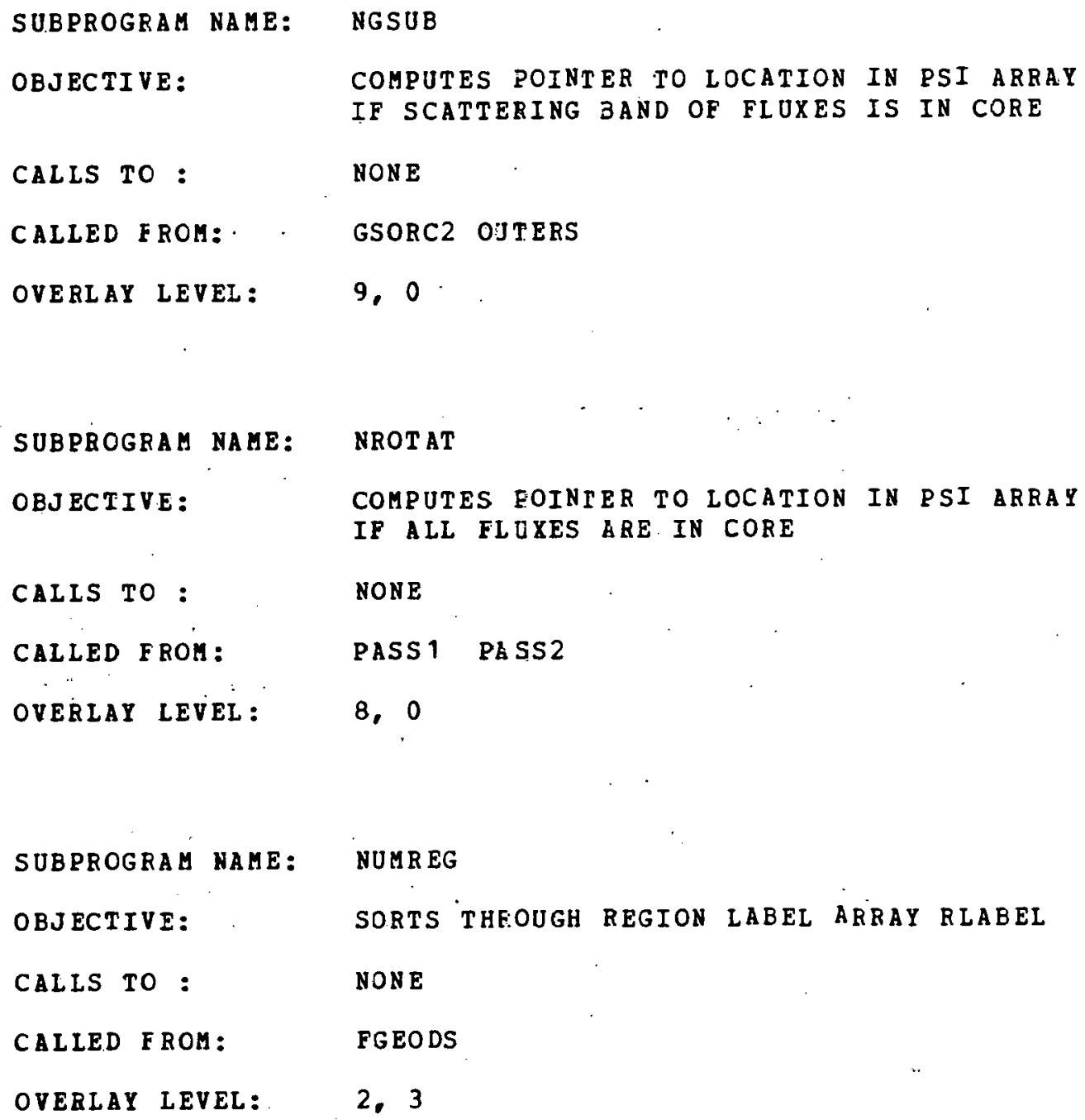




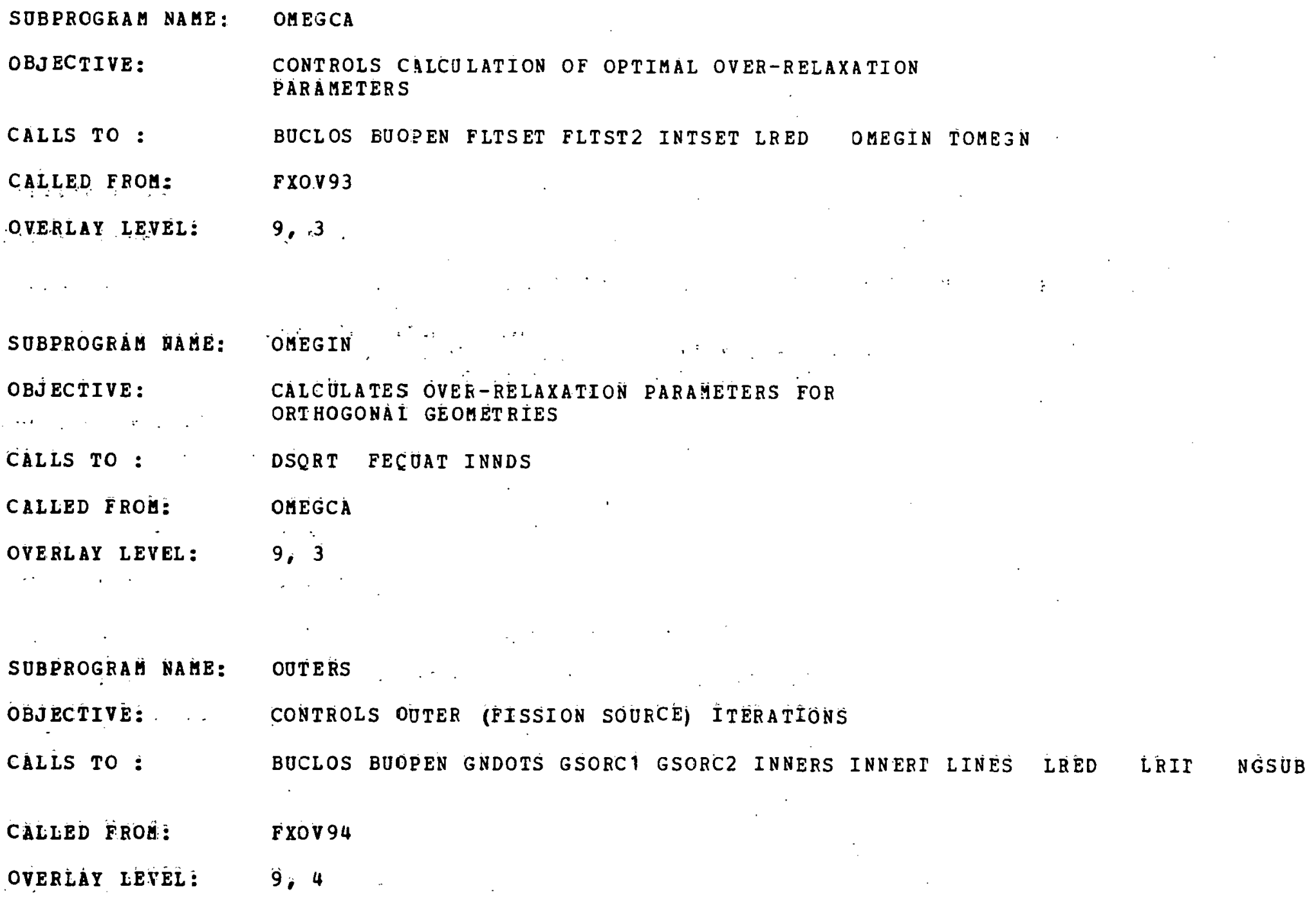




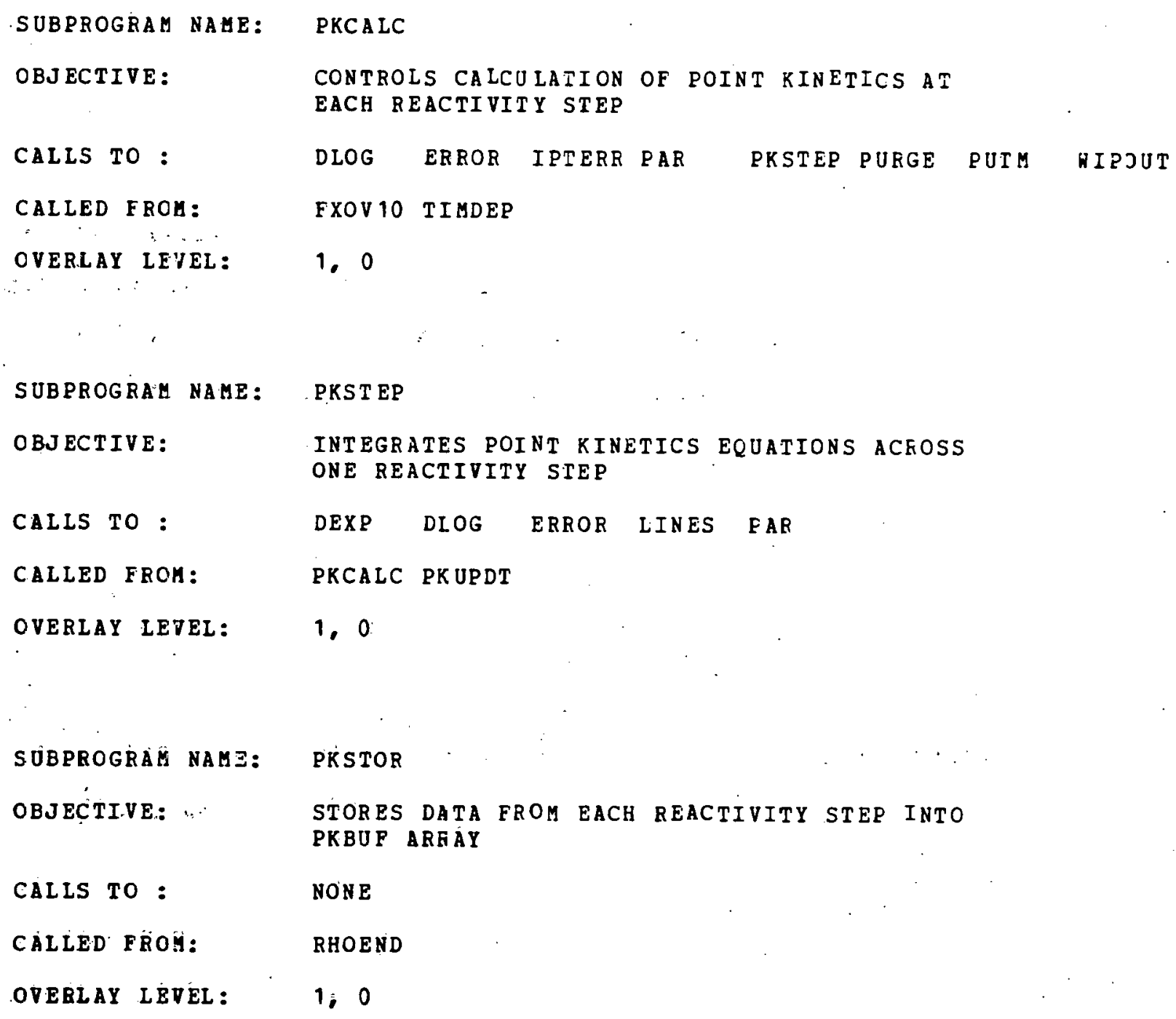




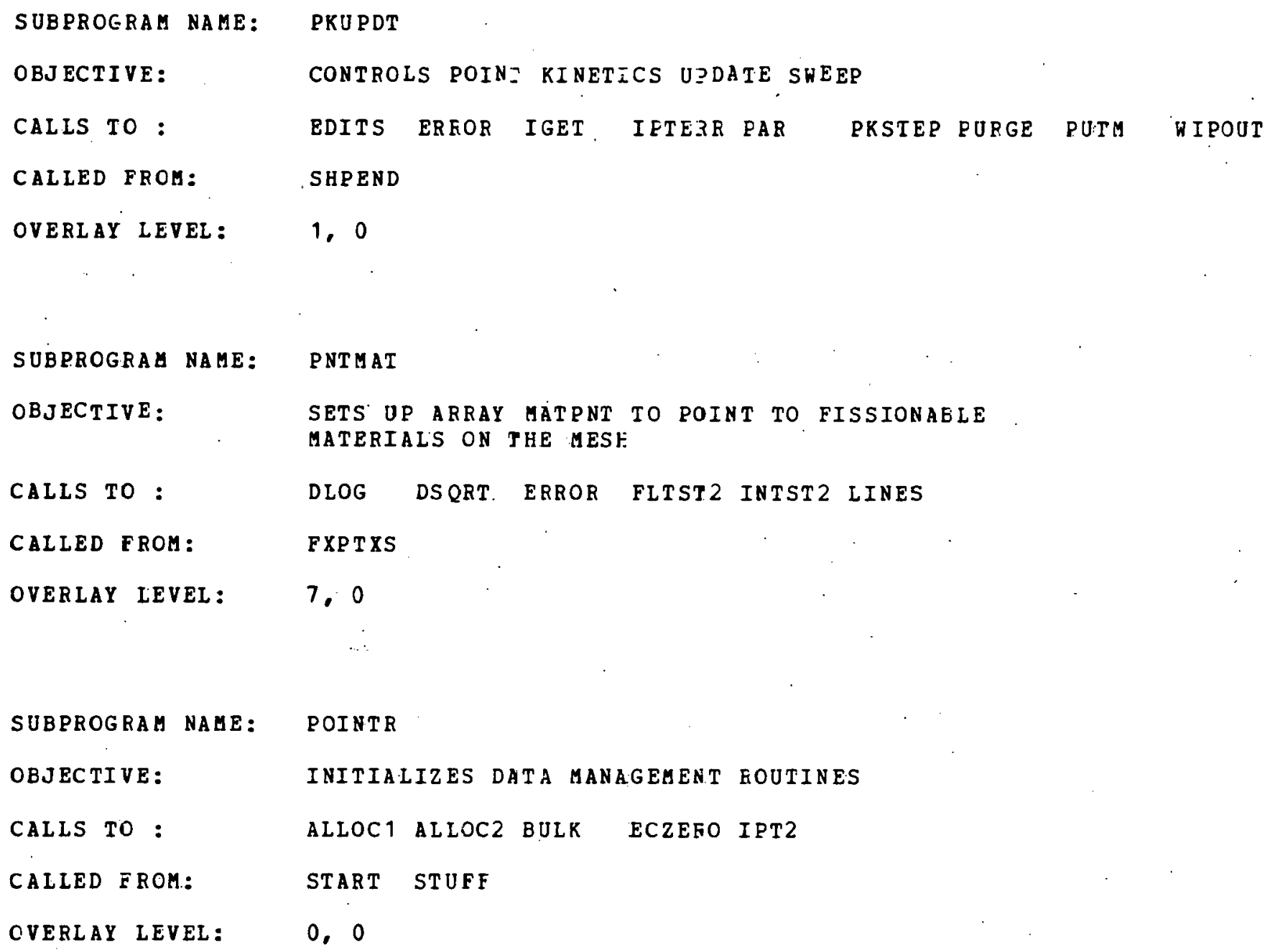




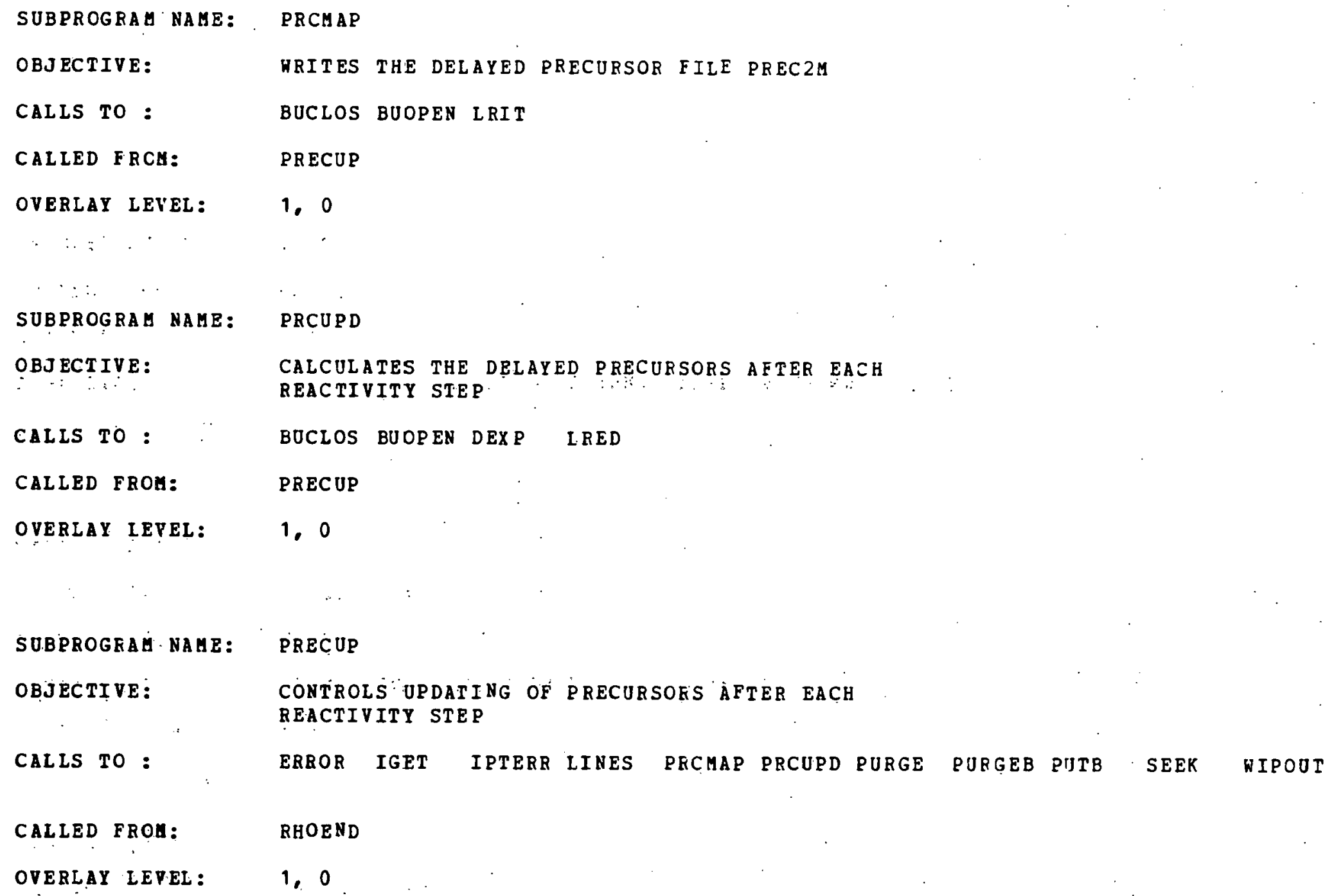

SUBPROGEAE NAHE: PRECCUP

OBJECTIVE: CONTROLS UPDATING OF PRECURSOES ÁFTER EACH REACTIVITY STEP

CALIS TO:

ERROR

IGET

IPTERR LINES

PECMAP PRCUPD PURGE PURGEB PIJTB

SEEK WIPOOT

CALIED FROH:

RHOEND

OVERLAY IEPEL:

1. 0 
SUBPROGRAM NAME: RATEL

OBJECTIVE: PRINTS A ONE-DIMENSIONAL ARRAY IN AG FORMAT

CALLS TO: LINES

CALLED FROM: DLY23 XDRDR3

OVERLAY LEVEL: $\quad 3,0$

SUBPROGRAM NAME: PRNT1A

OBJECTIVE: PRINTS ONE-DIEENSIONAL ARRAY WITH INDEXING

CALLS TO: LINES

CALLED FROM: PLY 23 XDEDR3

OVERLAY LEVEL: $\quad 3,0$

SUBPROGRAM NAME: PRNT1D

OBJECTIVE: - PRINTS ONE-DIUENSIONAL ARRAY WITH INDEXING

Calls TO: LINES.

CALLED FROM: DLY23

OVERLAY LEVEL: $\quad 3,0$ 
SUBPROGRAM NAE: PRATE

OBJECTIVE: $\quad$ PRINTS ONE-DIMENSIONAL ARRAY WITH INDEXING

CALLS TO:

CALLED FROM: . XDRDR3

OVERLAY LEVEL: $\quad 3,0$

SUBPROGRAM NAME: . PRAT II

OBJECTIVE: $\quad$ PRINTS ONE-DIMENSIONAL ARRAY WITH INDEXING

CALLS TO: . I INES

CALLED FROE: DLY23

OVERLAY LEVEL: $\quad 3 ; 0$

SUBPROGRAM NAME: PRNT2D

OBJECTIVE: PRINTS TWO-DIMENSIONAL ARRAY WITH INDEXING

CALLS TO : LINES

CALLED FEOH: · DY 23

OVERLAY LEVEL: 3,0 


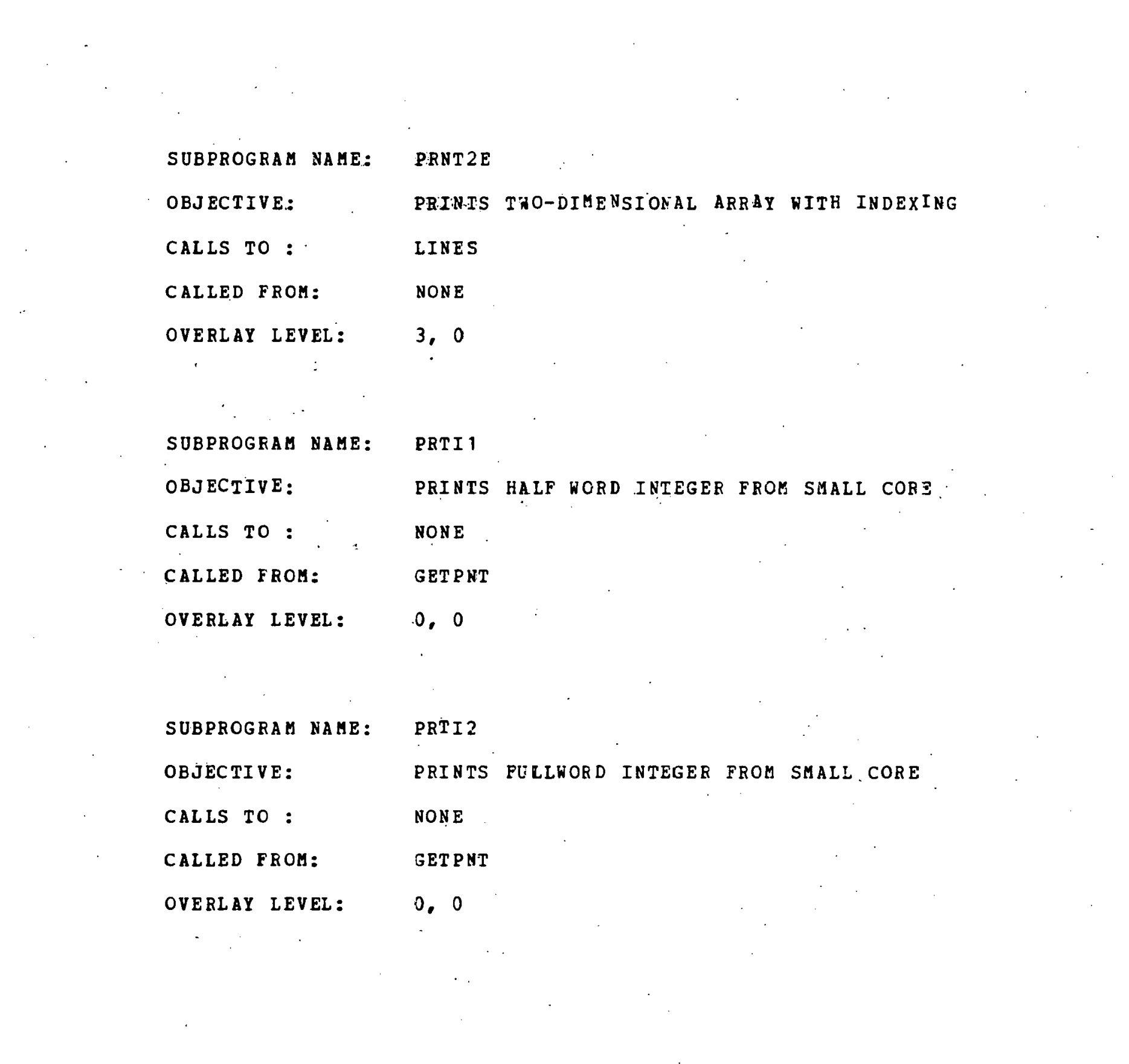




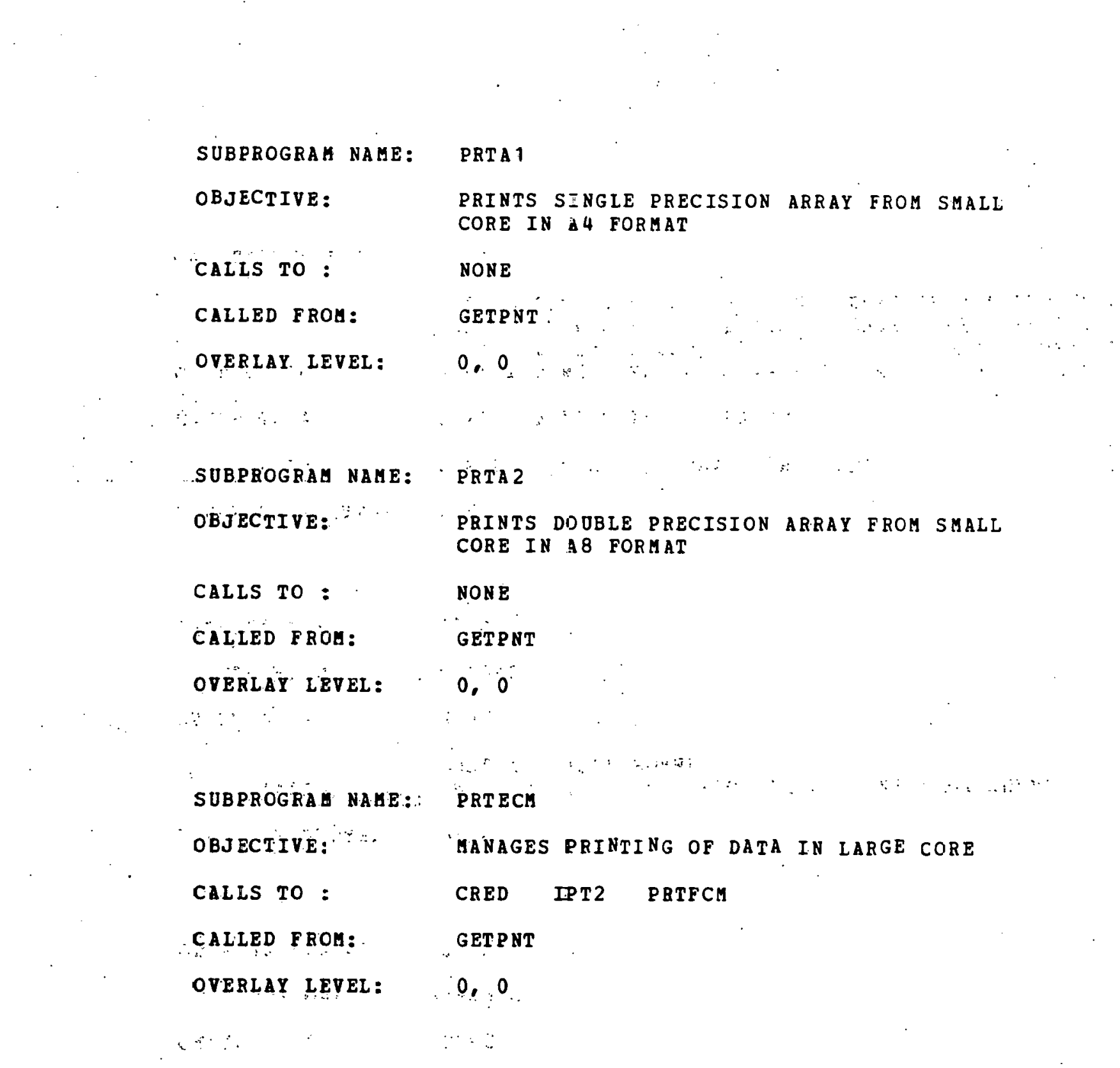




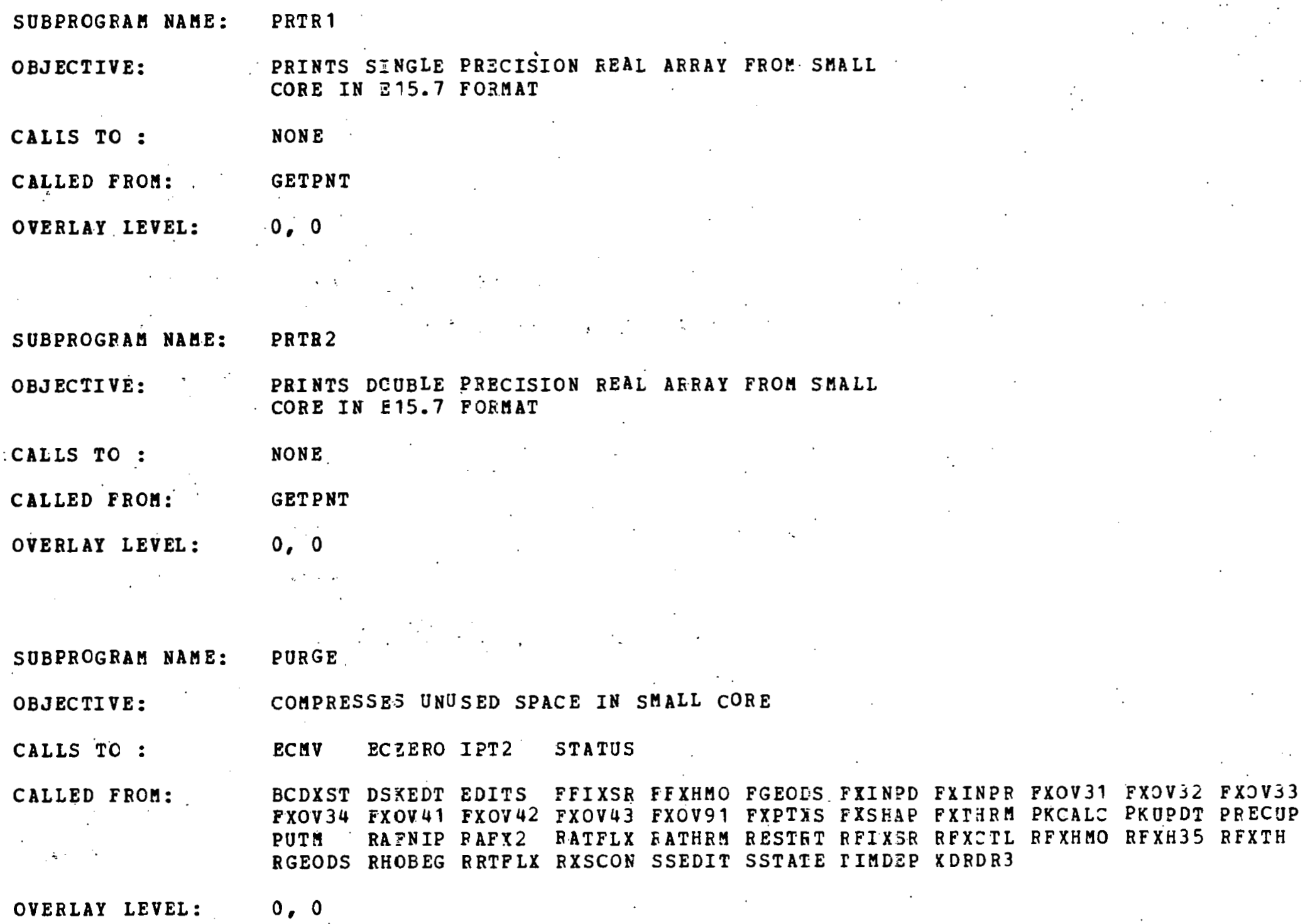

OVERLAY LEVEL: 0,0 


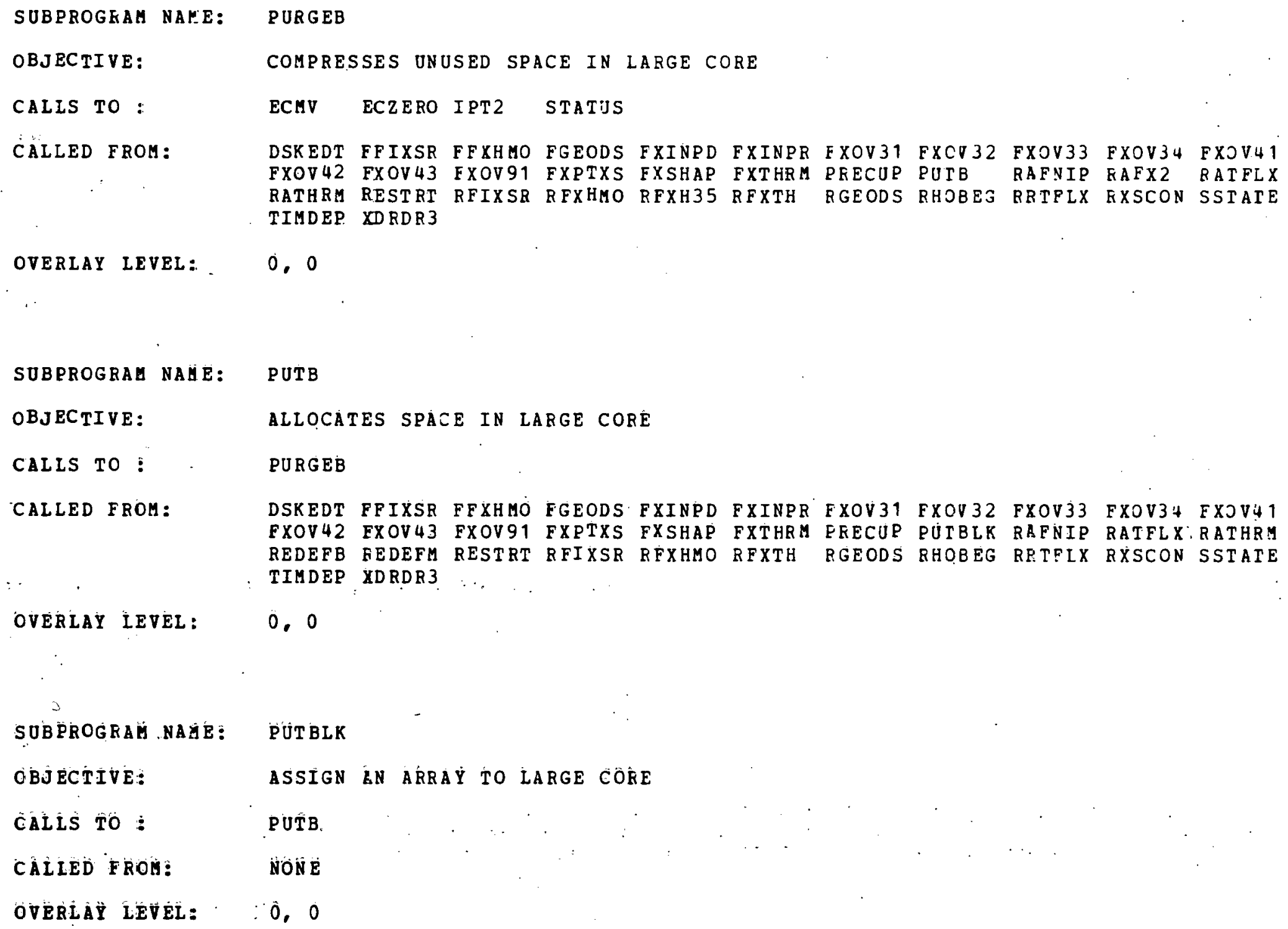




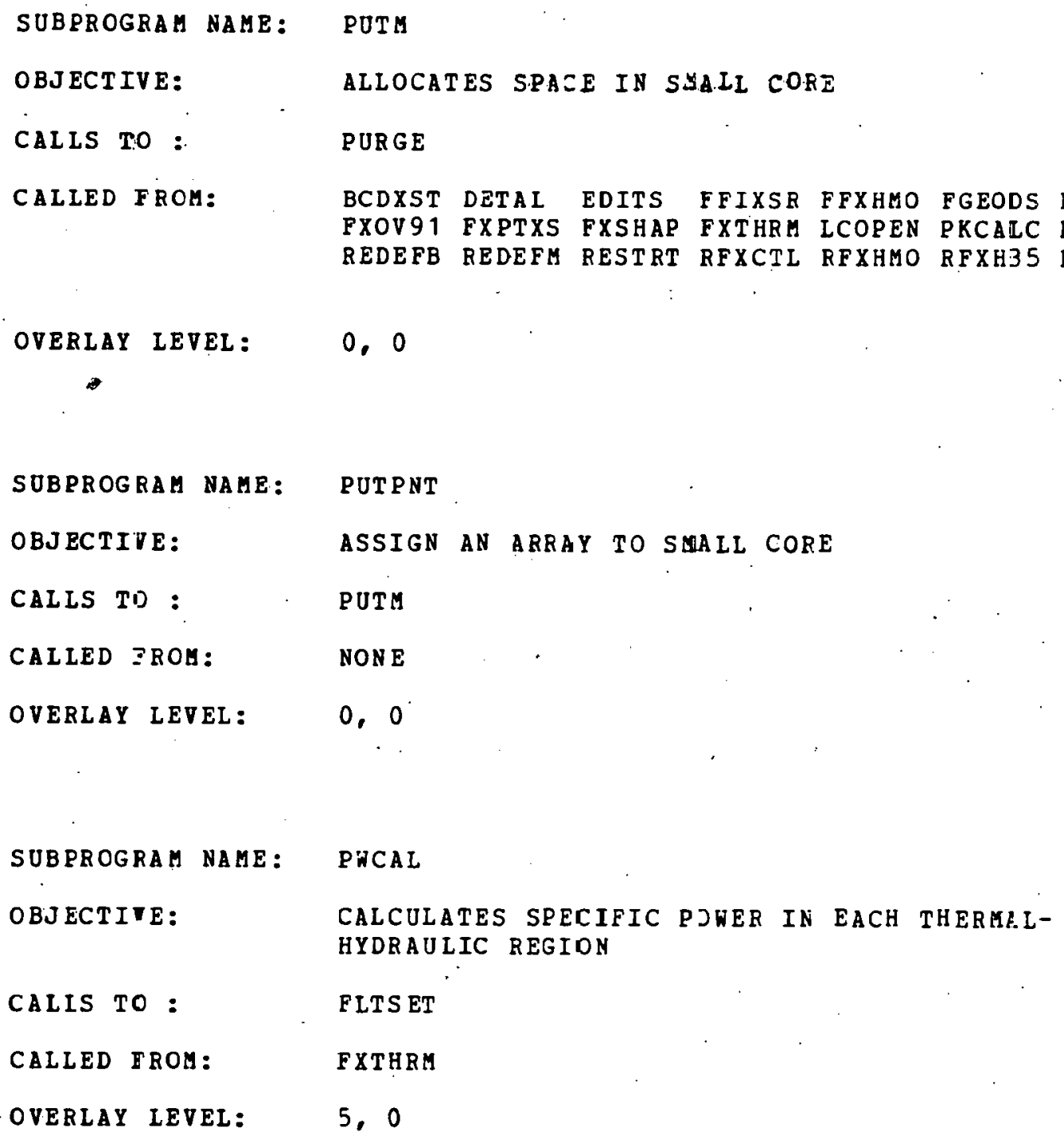




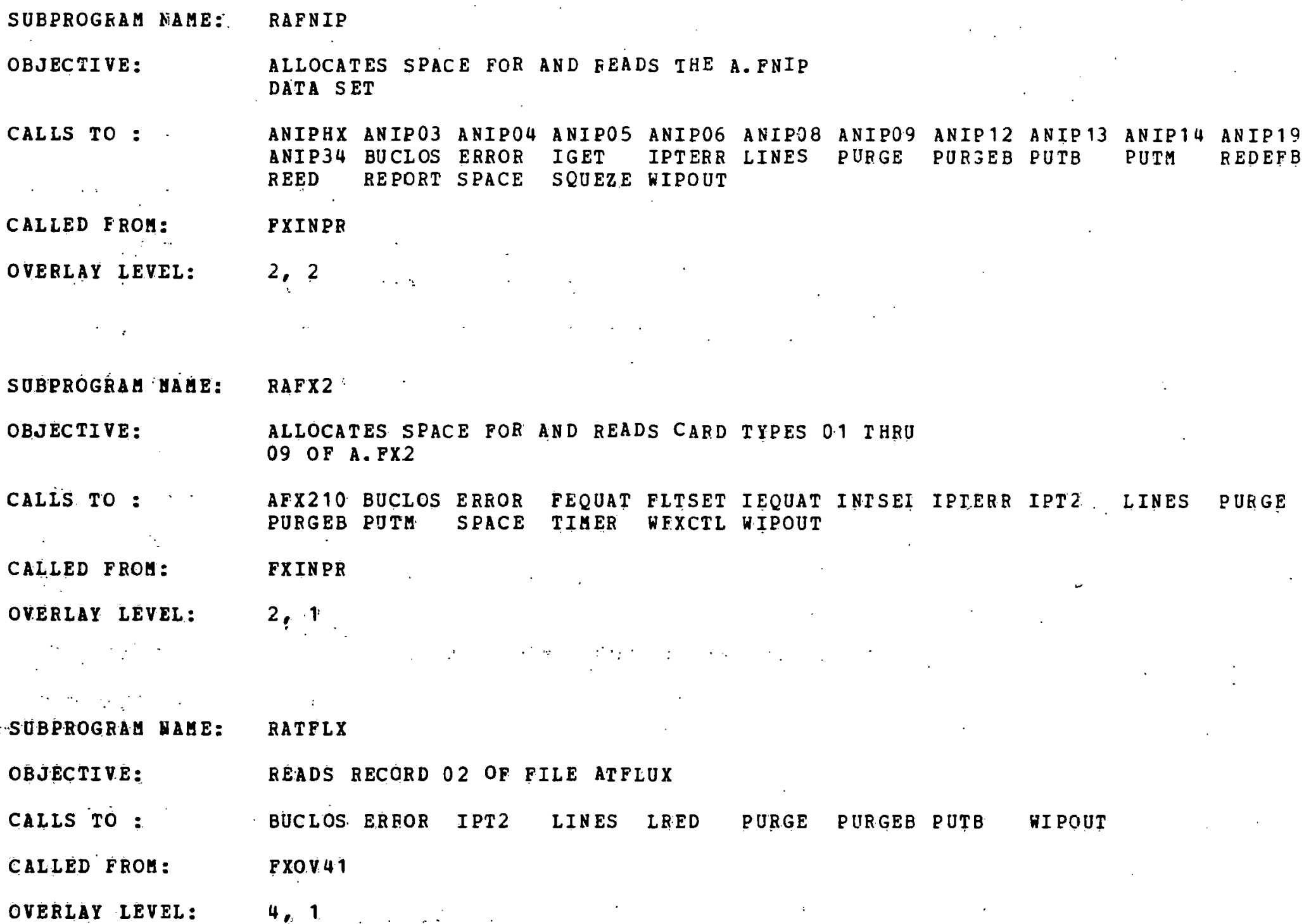




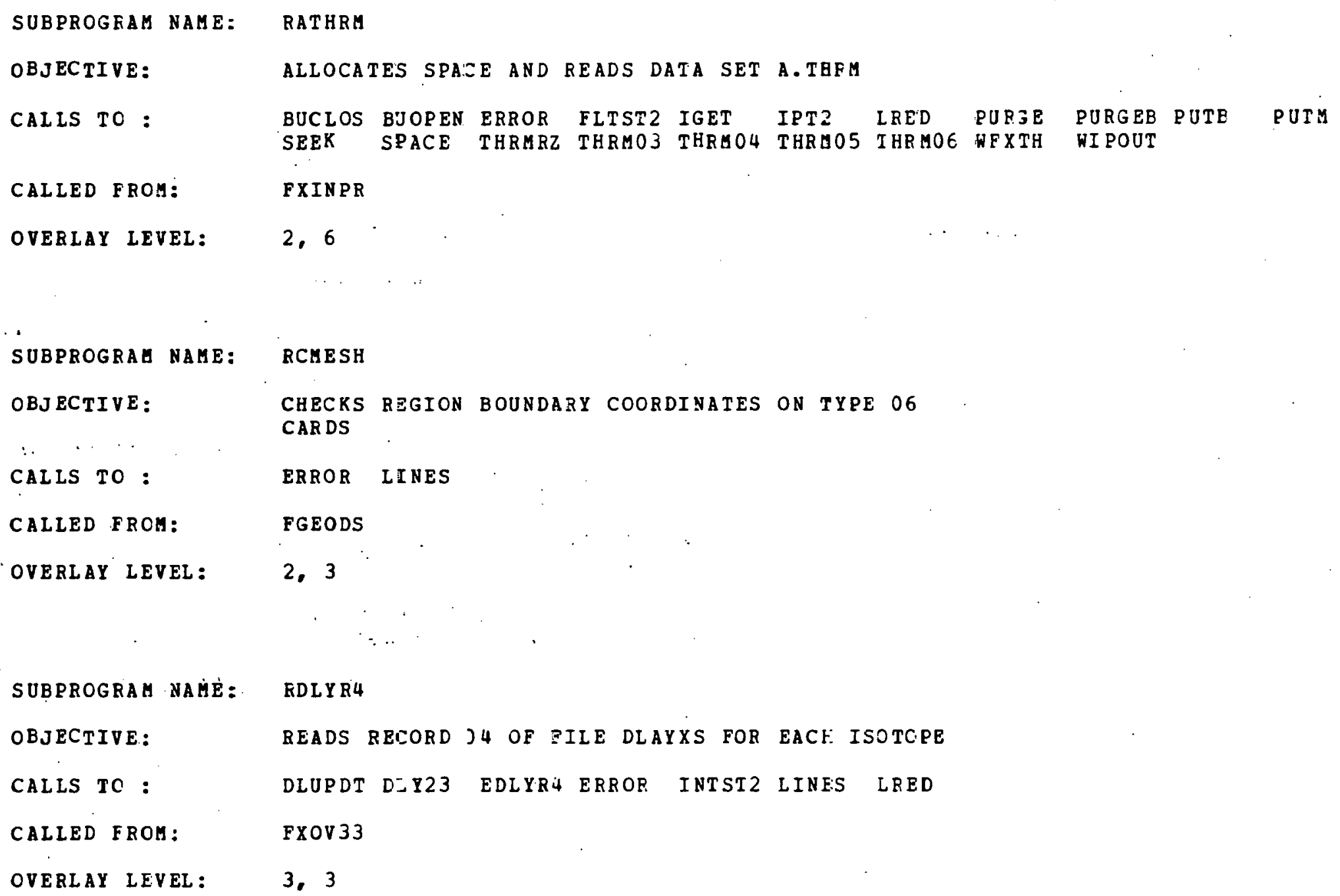


SUBPROGRAM NAME: READER

OBJECTIVE: TRANSFER FROM LARGE CORE TO SMALL CORE

CALLS TO: NONE

CALLED FROE: CRED CRIT

OVERLAY LEVEL: $\quad 0,0$

SUBPROGRAM NAME: REDE

OBJECTIVE: $\quad$ REDEFINES LENGTH OF ARRAY IN SMALL CORE

CALLS TO: REDEEM

CALLED FROE:

OVERLAY LEVEL: $\quad 0,0$

SUBPROGRAM NAME: REDEFB

OBJECTIVE: REDEFINES LENGTH OF ARRAY IN LARGE CORE

CALLS TO: CRED CRIT ECMV GET IPT2 PUT FUT

CALLED FROM: $\quad$ FFXHMO FGEODS FXOV 34 RAFNIP

OVERLAY LEVEL: $\quad 0,0$ 


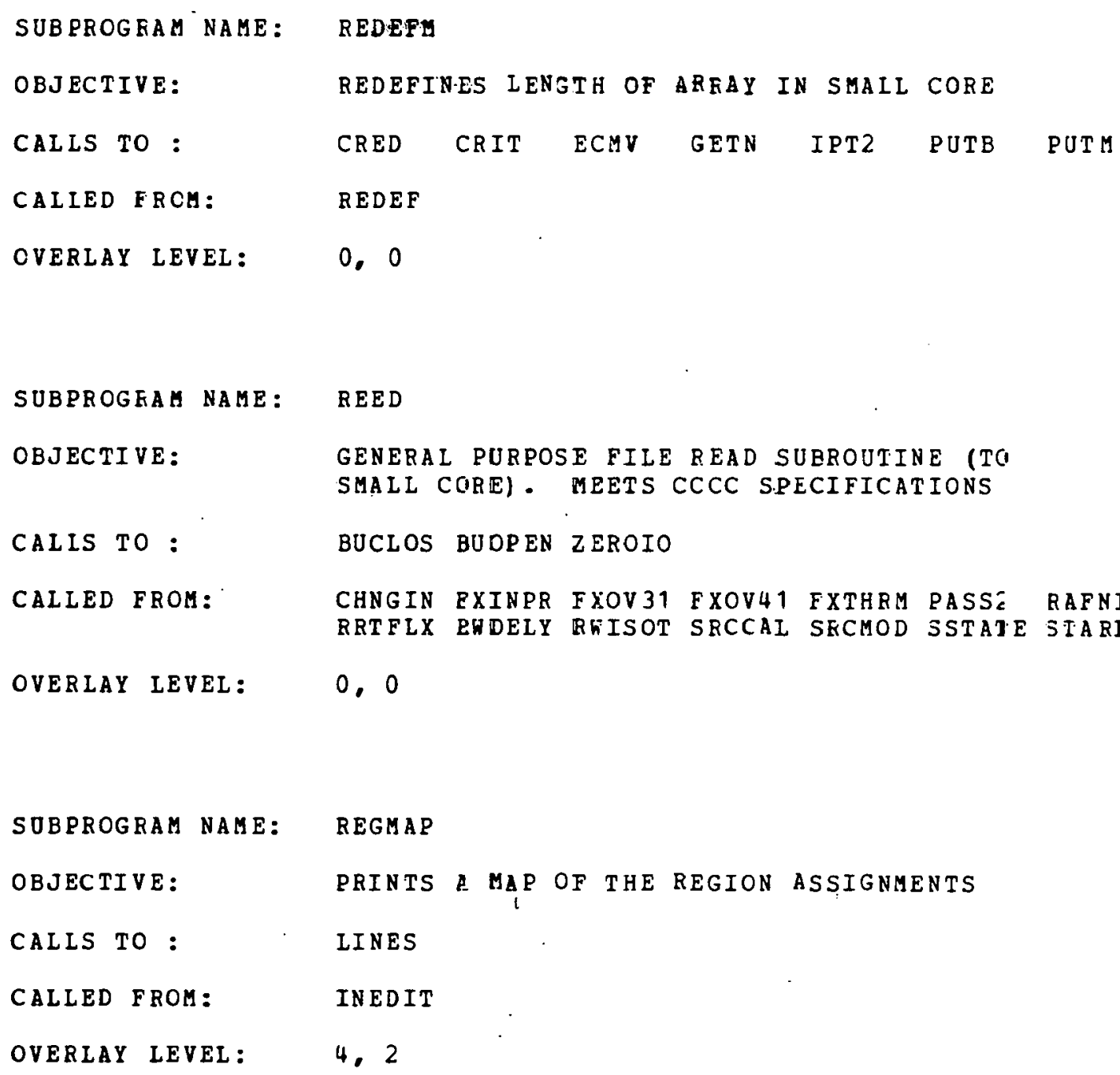

SOBPROGRAM NAME: REGMAP

OBJECTIVE:

PRINTS MaP OF THE REgION ASSIGNMENTS

CALLS TO :

LINES

CALLED FROM:

INEDIT

OVERLAY LEVEL: $\quad 4,2$ 


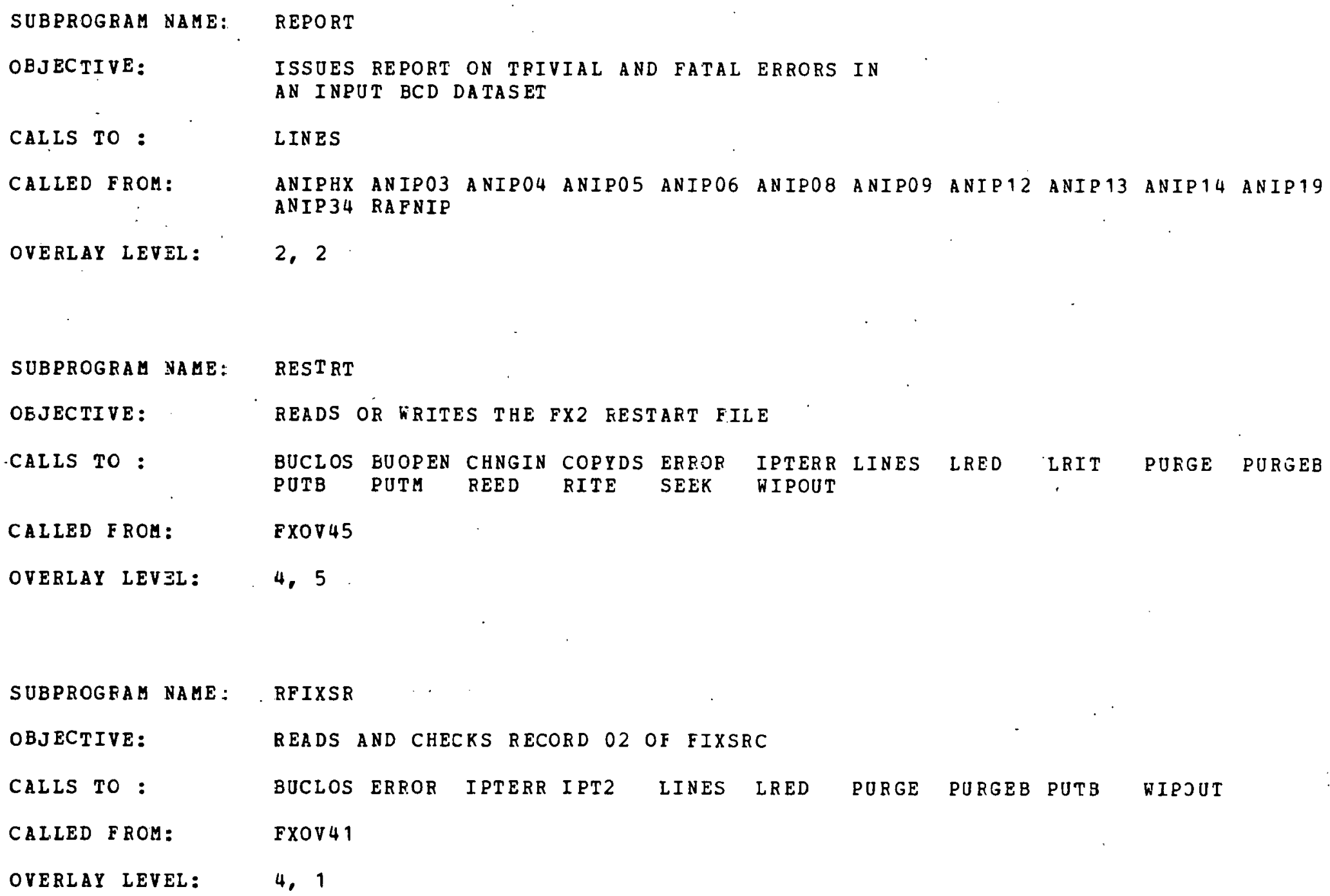




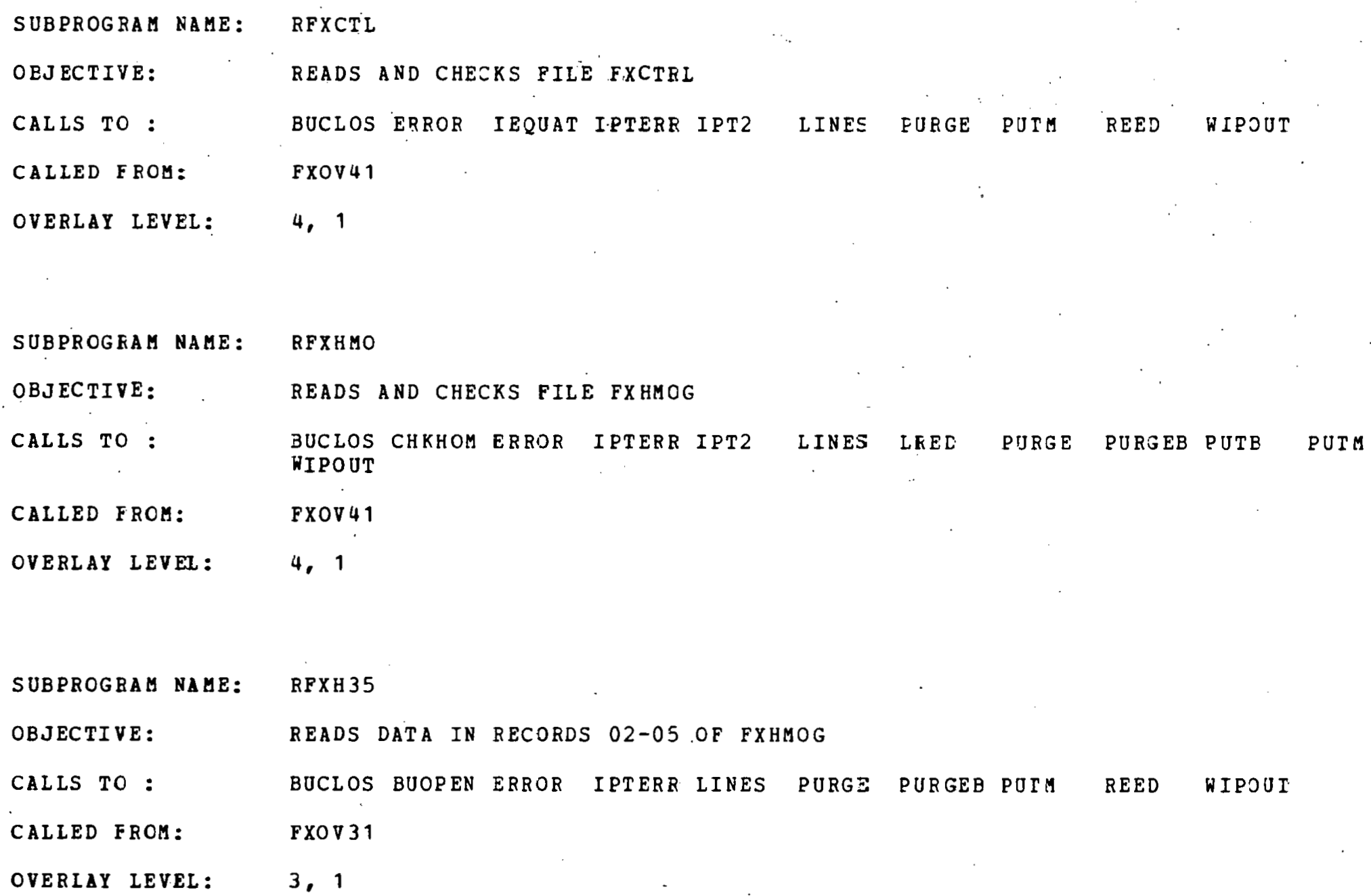




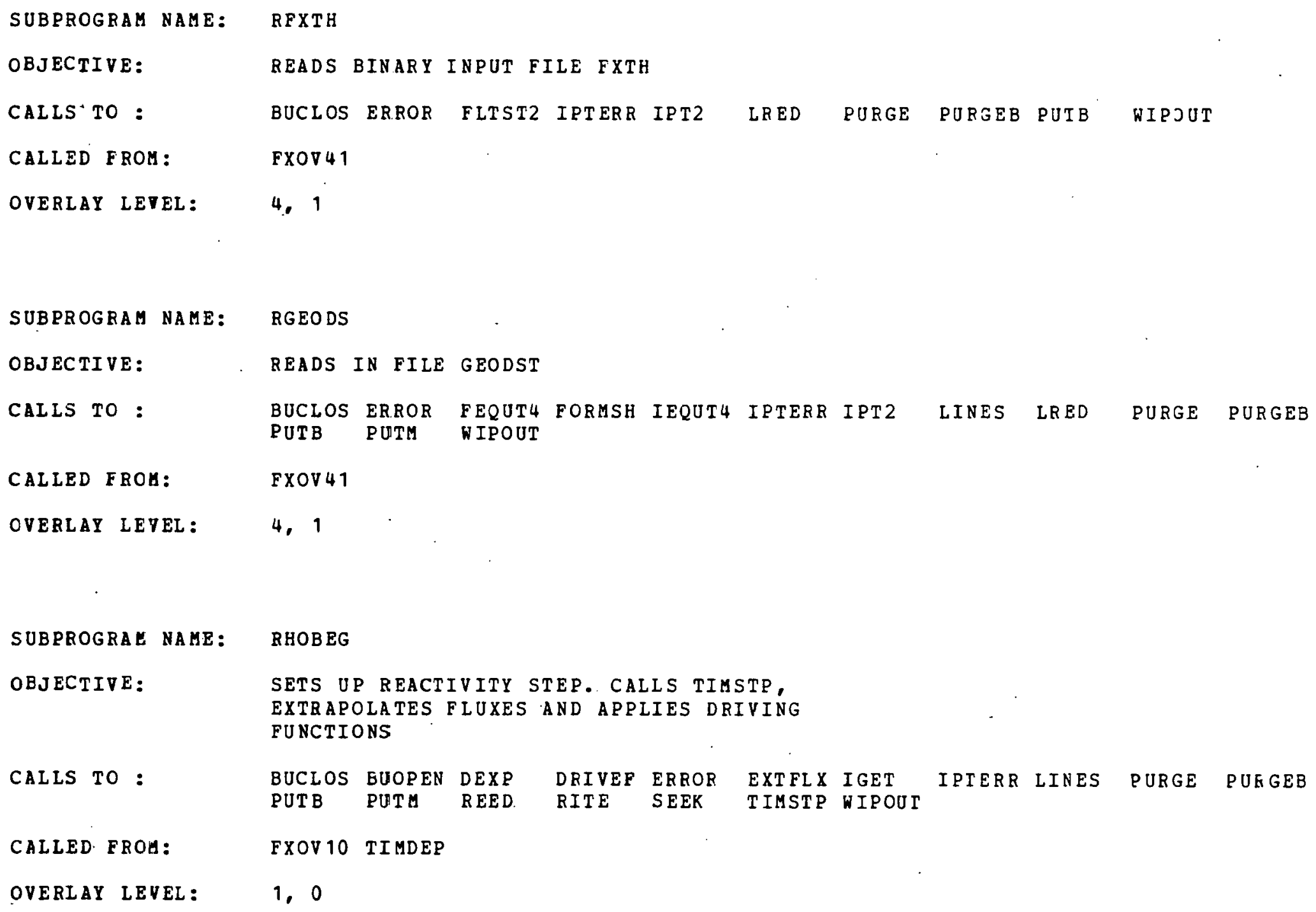

SUBPROGRAE NAME: RHOBEG

OBJECTIVE:

SETS UP REACTIVITY STEP. CALLS TIMSTP, EXTRAPOLATES FLUXES AND APPLIES DRIVING FUNCTIONS

CALLS TO :

BUCLOS BUOPEN DEXP DRIVEF ERROR EXTFLX IGET PUTB PUTA REED. RITE SEEK TIMSTP WIPOUT

IPIERR LINES PURGE PUEGEB

CALLED FROH:

FXOV 10 TIMDEP

OVERLAY LEVEL: 1,0 


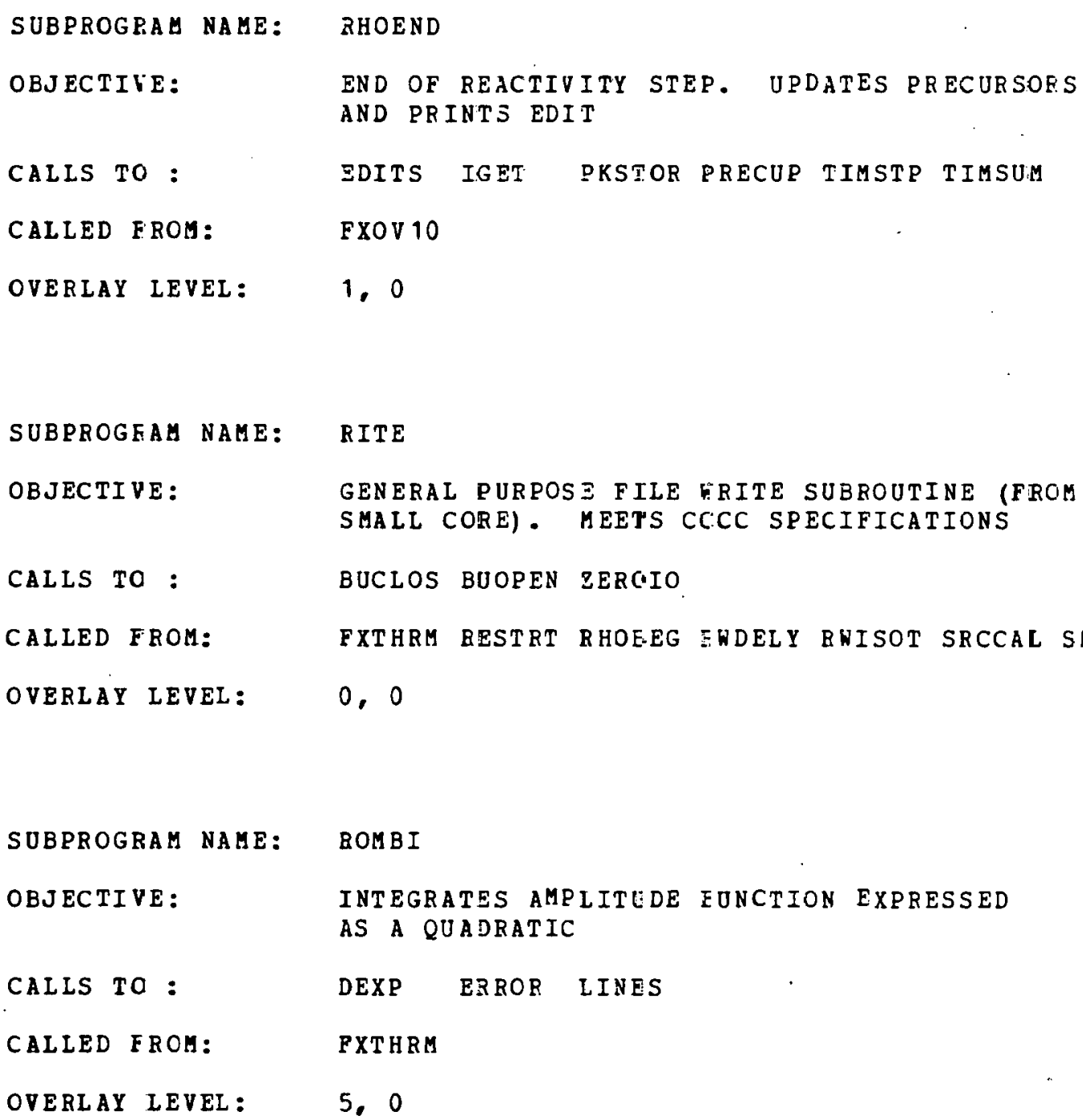

OVERLAY LEVEL: 0,0 


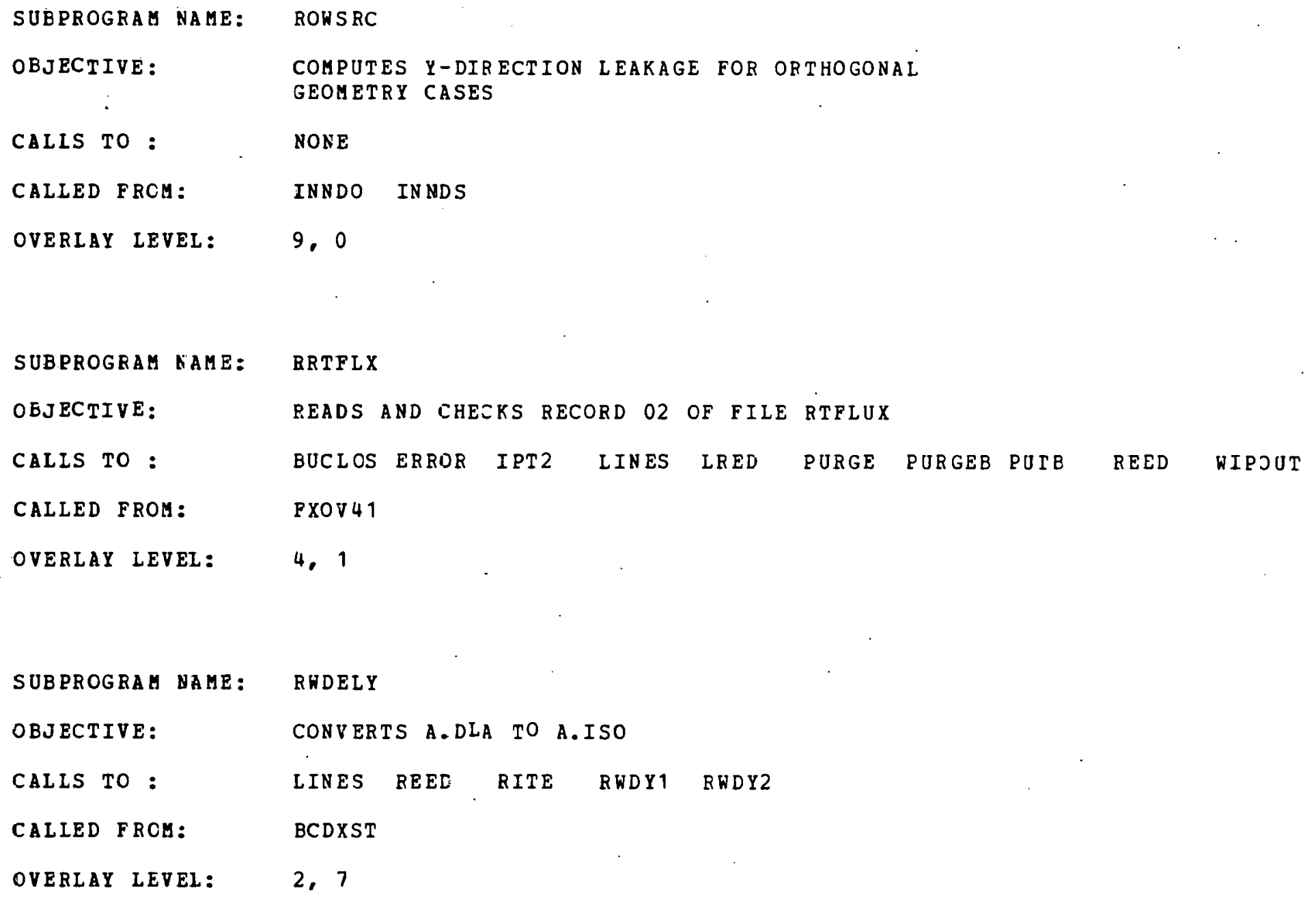

\section{SUBPROGRAM NAME: RHDELY}

OBJECTIVE: CONVERTS A.DIA TO A.ISO

CALLS TO: LINES REEE RITE RWDY1 RWDY2

CALIED FRCM: $\quad$ BCDXST

OVERLAY LEVEL: $\quad 2,7$ 


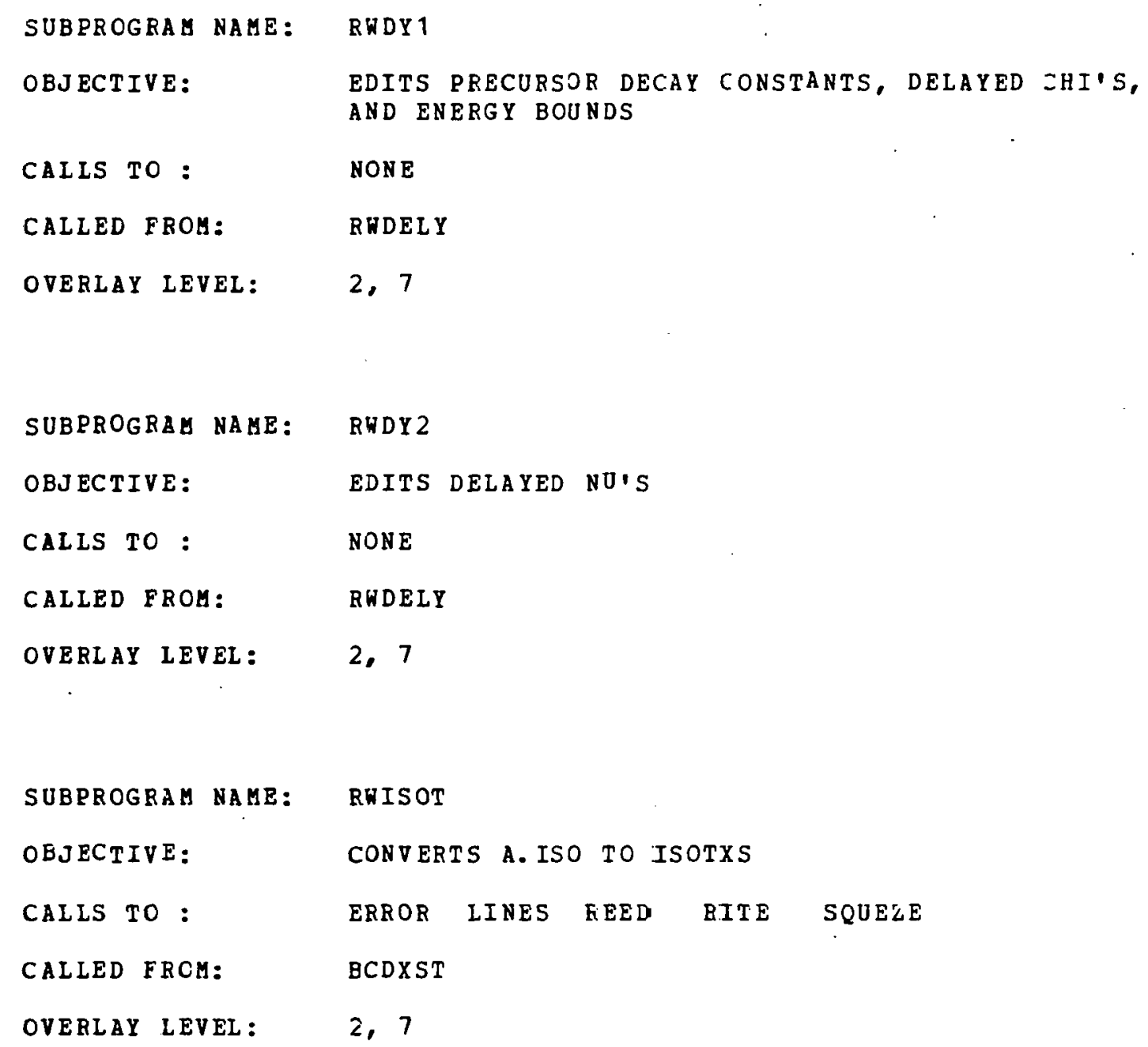

SUBPROGRAM NAME: RWISOT

OBJECTIVE: CONVERTS A. ISO TO ISOTXS

CALLS TO: ERROR LINES FEED BITE SQUELE

CALLED FRCM: $\quad$ BCDXST

OVERLAY LEVEL : $\quad 2,7$ 


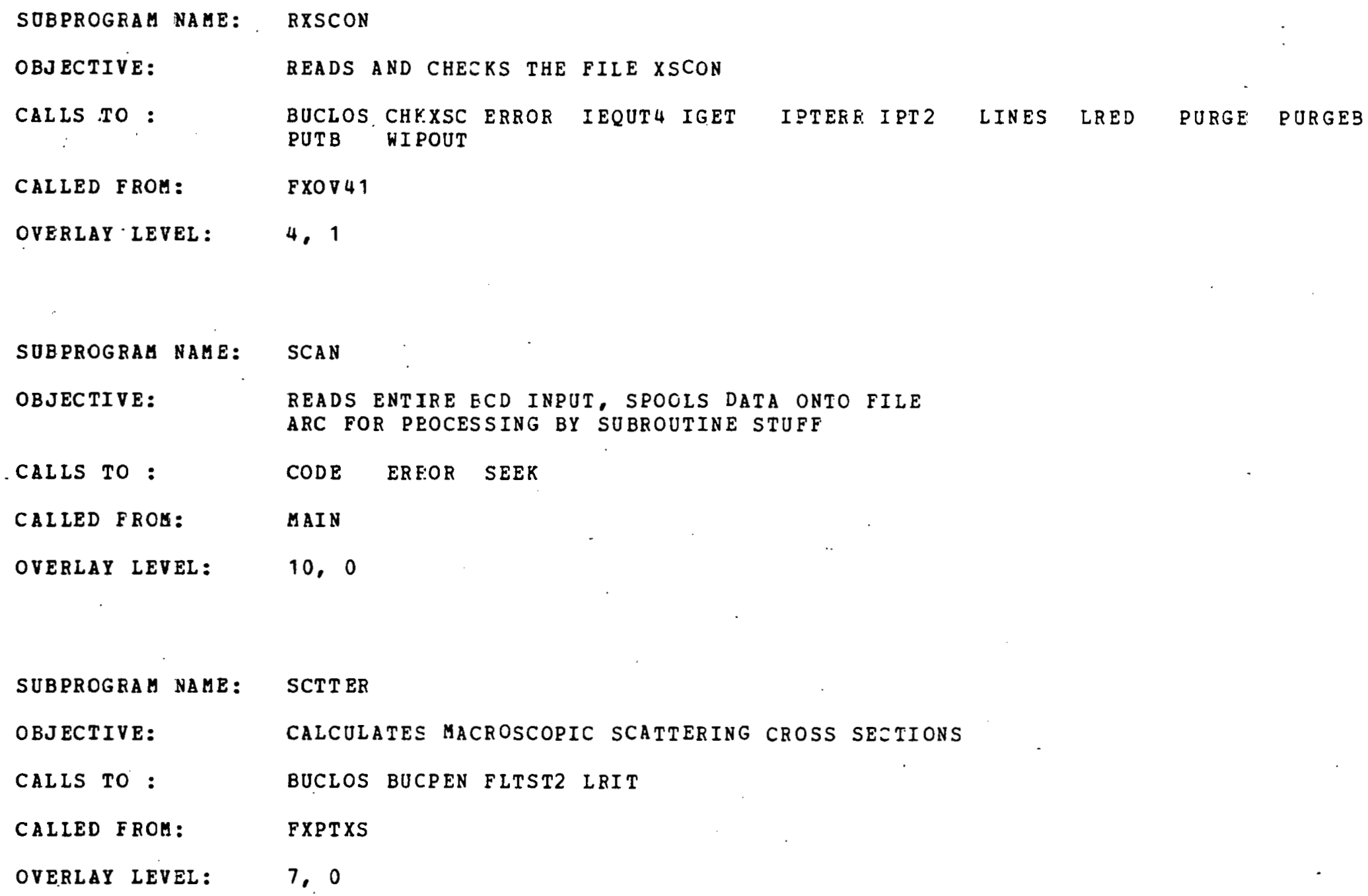




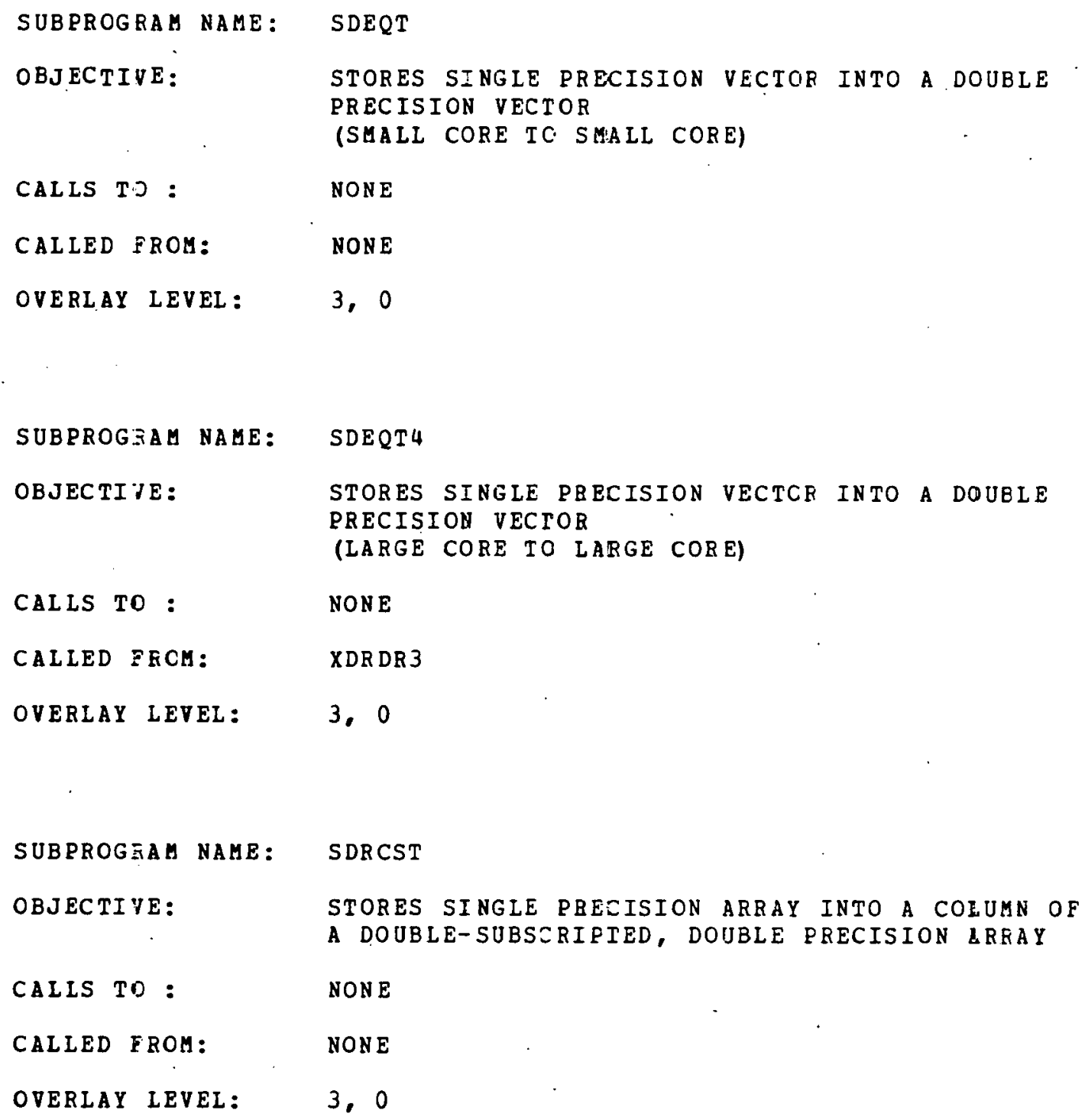




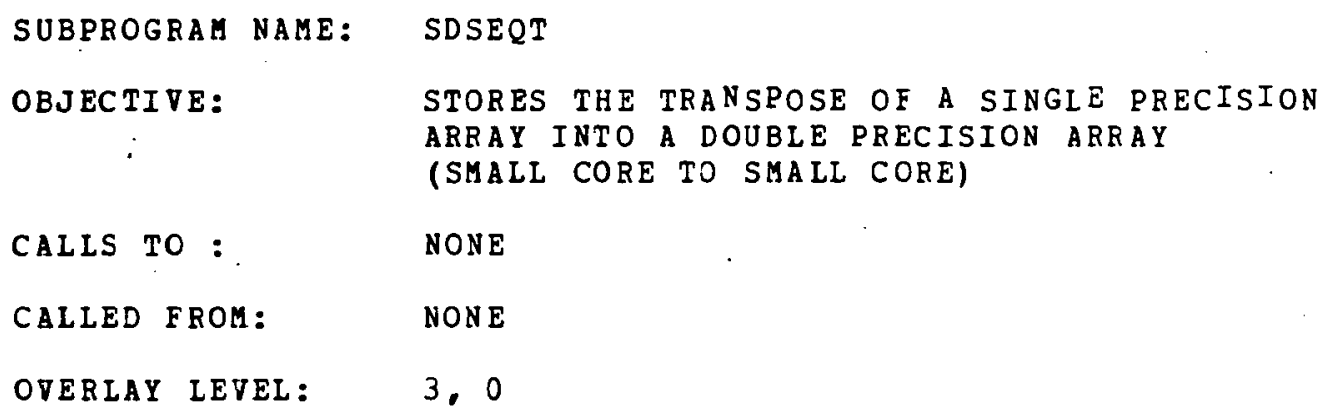

CALIS TO :

NONE

CALLED FRCM:

XDRDR 3

OVERLAY LEVEL: $\quad 3,0$

SUBPROGRAM NAUE: SEEK

OBJECTIVE: RETURNS LUN VALUE OF FILE. FOUTINE FUNCTION DESCRIBED IN LA-5486-MS

CALIS TO:

NONE

CALLED FROM:

BCDXST DRIVEF EXTFLX FXINIT FXINPD FXINPR FXINXS FXPIXS FXSHAP FXTHRM YAIN PRECUP RATHRM RESTRT RHOBEG SCAN SSTATE START STUFF STUFF 1

OVERLAY LETEL: $\quad 0,0$ 
SUBPROGRAM NAME: SFDCEL

OBJECTIVE: COMPUTES THE FINITE DIFFERENCE COEFFICIENTS

IN ORTHOGONAL GEOMETEY

CALLS TO: DOS DIN SGTBND

CALLED FROM: $\quad$ SFDGEN

OVERLAY LEVEL: . 9,2

SUBPROGRAM NAME: SFDGEN

OBJECTIVE: CONTROLS COMPUTATION OF FINITE DIFFERENcE COEFFICIENTS

CALLS TO: AREAS 3UCLOS. BJJOPEN LED LIT SFDCEL TFDCEL

CALLED FROM: $\quad$ FXOV 92

OVERLAY LEVEL: $\quad 9,2$

SUBPROGRAM NAME: SGTBND

OBJECTIVE: RETURNS BOUNDARY CONDITION CONSTANT FOR EXTERNAL MESH CELL

CALLS TO: NONE

CALLED FROM: $\quad$ SFDCEL TFDCEL

OVERLAY LEVEL: $\quad 9,2$ 


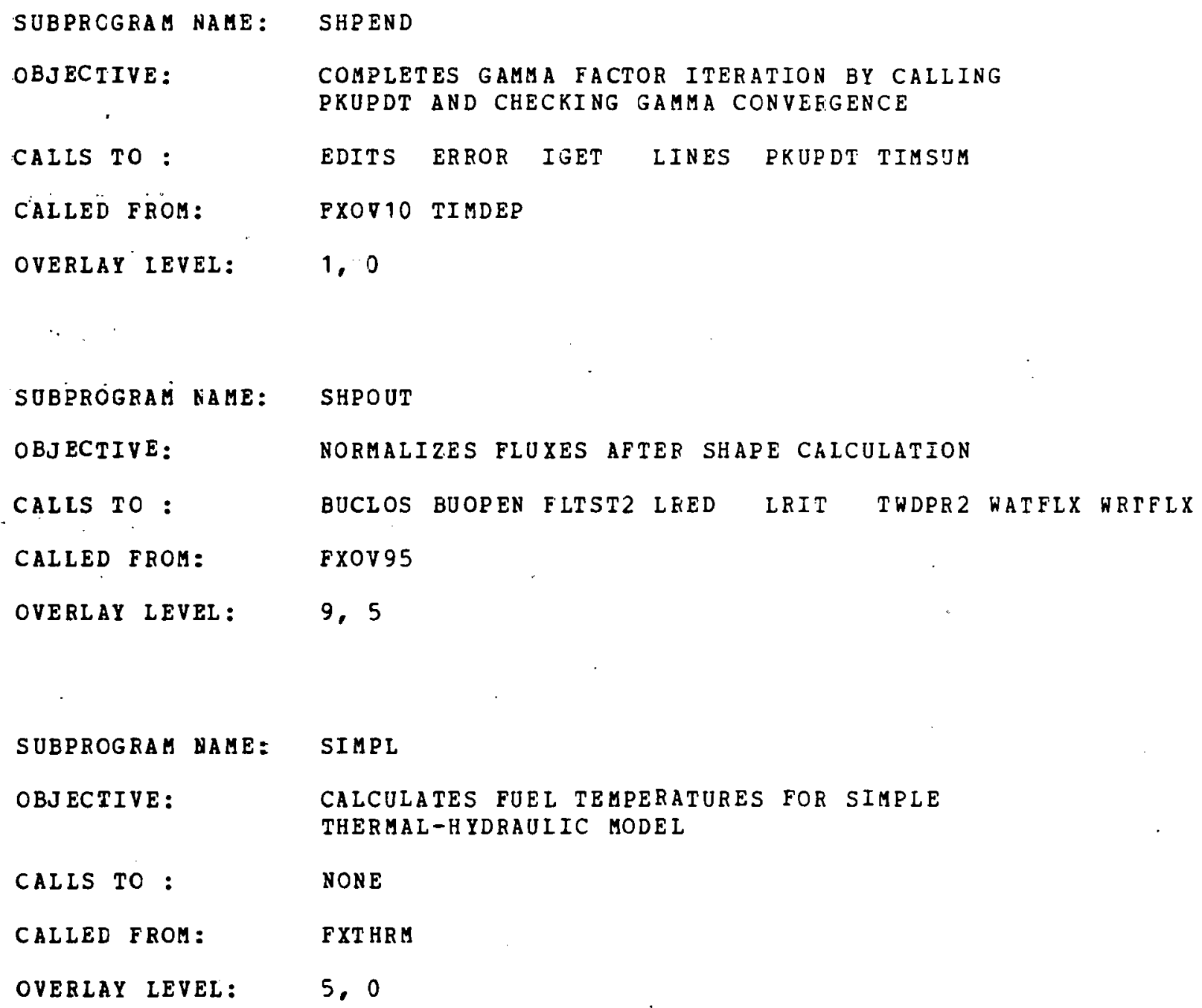


SUBPROGEAM NAME:

OBJECTIVE:

CALIS TO:

CALLED FROM:

OVERLAY LEVEL:

SUBPROGRAM NAME:

OBJECTIVE :

CALLS TO :

CALLED FROM:

OVERLAY LEVEL:

SUBPROGFAM NAME:

OBJECTIVE:

CALLS TO:

CALLED FRCH:

OVERLAY LEVEL:

\section{SINEQT}

STORES ONE SINGLE PEECISION VECTOR INTO ANOTHER SINGLE PRECISION VECTOR

(SMALL CORE TO SMALL CORE)

NONE

NONE

3. 0

SINEQ4

STORES ONE SINGLE PRECISIDN VECTOR INTC ANCTHER

SINGLE PRECISION VECTOR

(LARGE COFE TO LARGE COFE;

NONE

FXOV 34 ISOR 48 XDRDR3

3,0

\section{SINSET}

STORES SCALAR INTO A SINGLE PRECISION VECTOR (IN SMALL CORE)

NONE

NON E

3. 0 


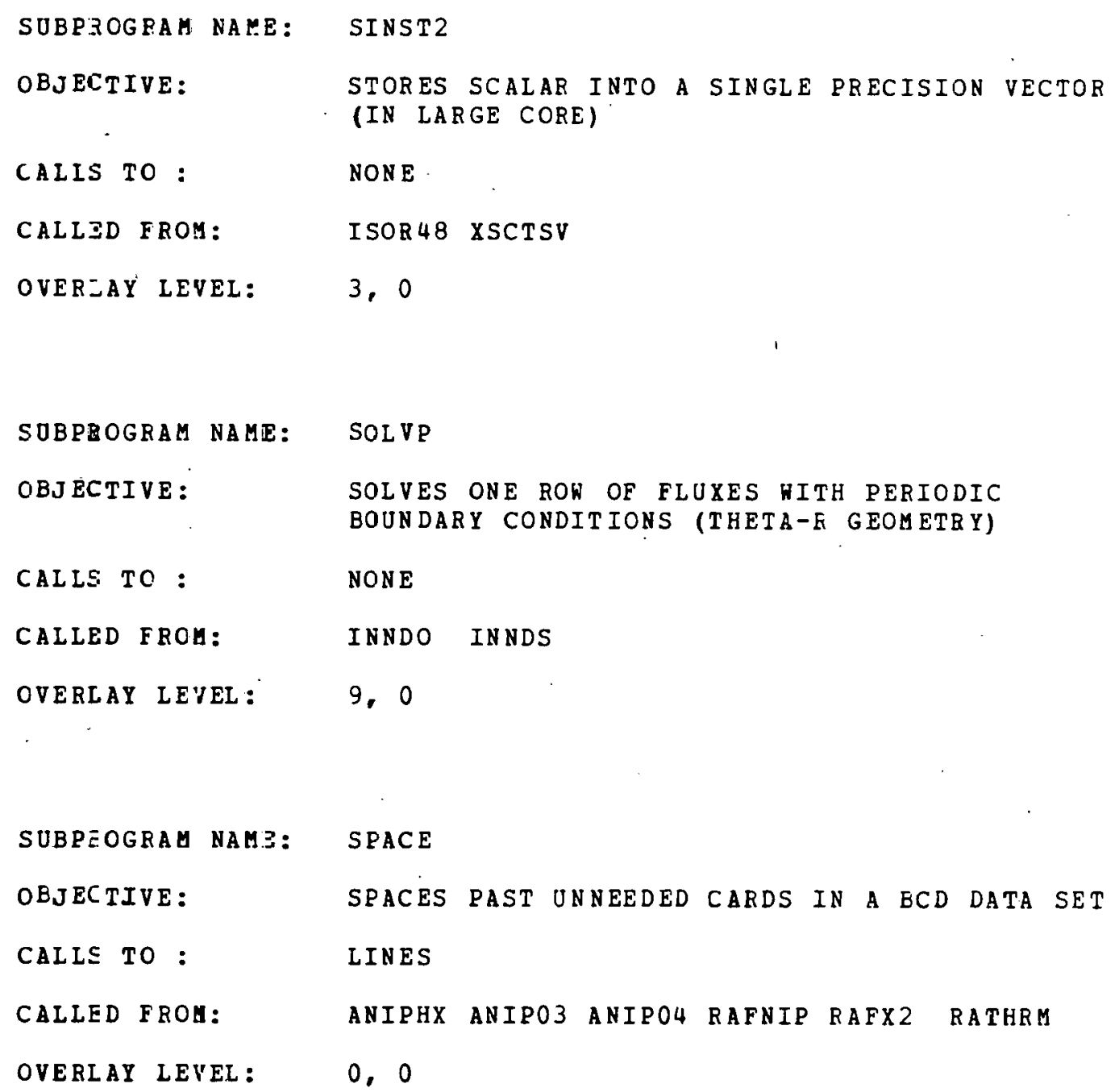




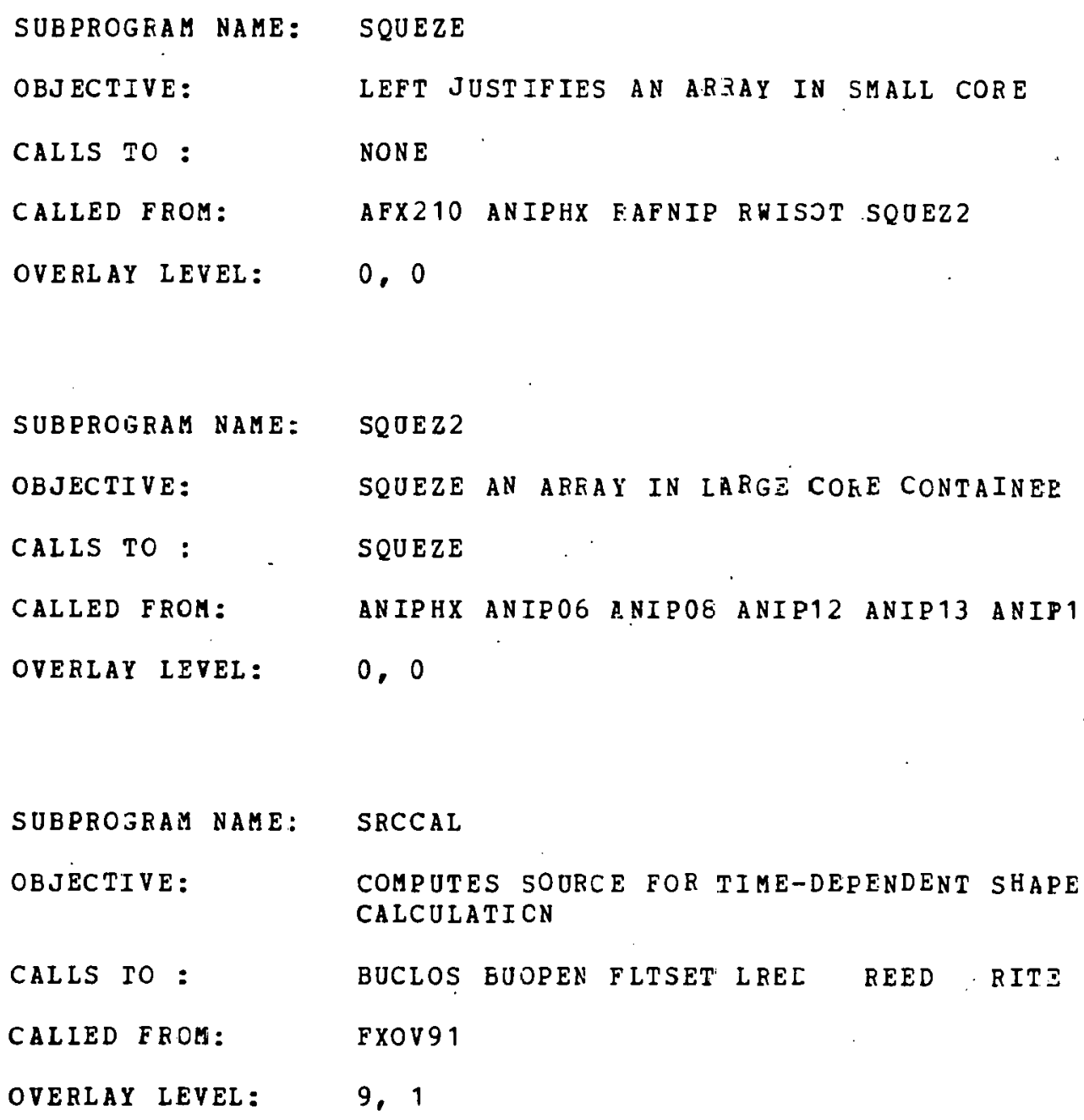




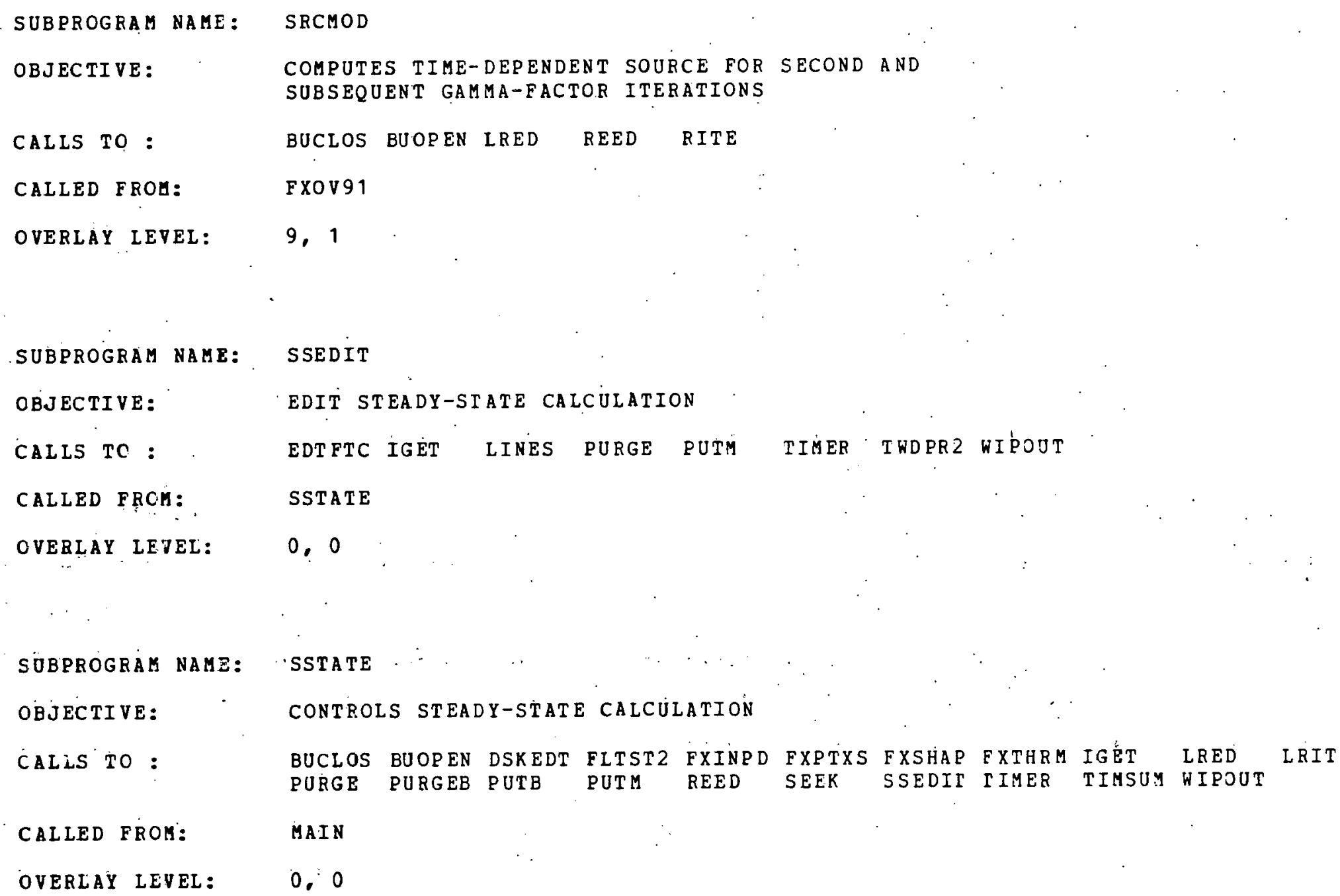




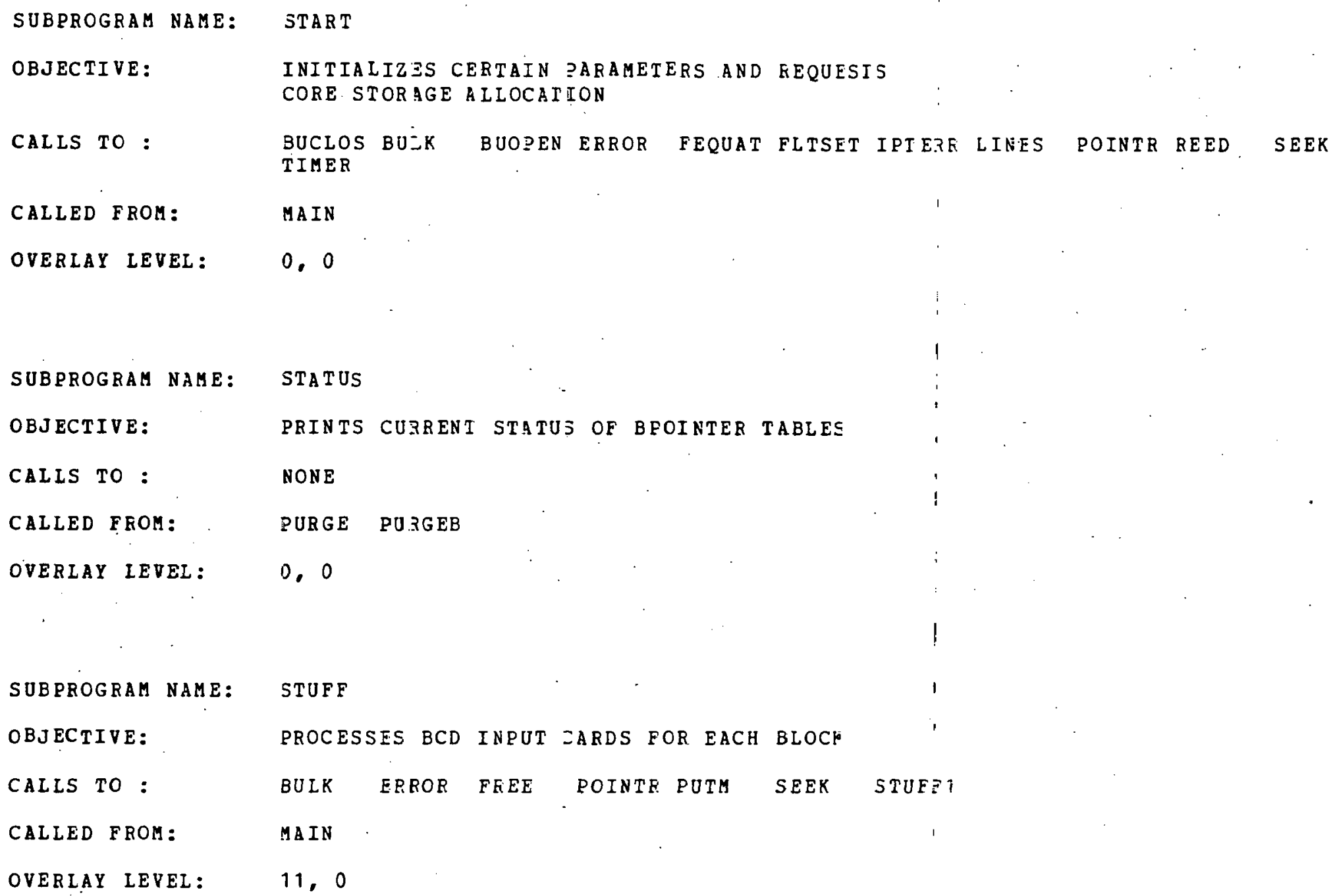




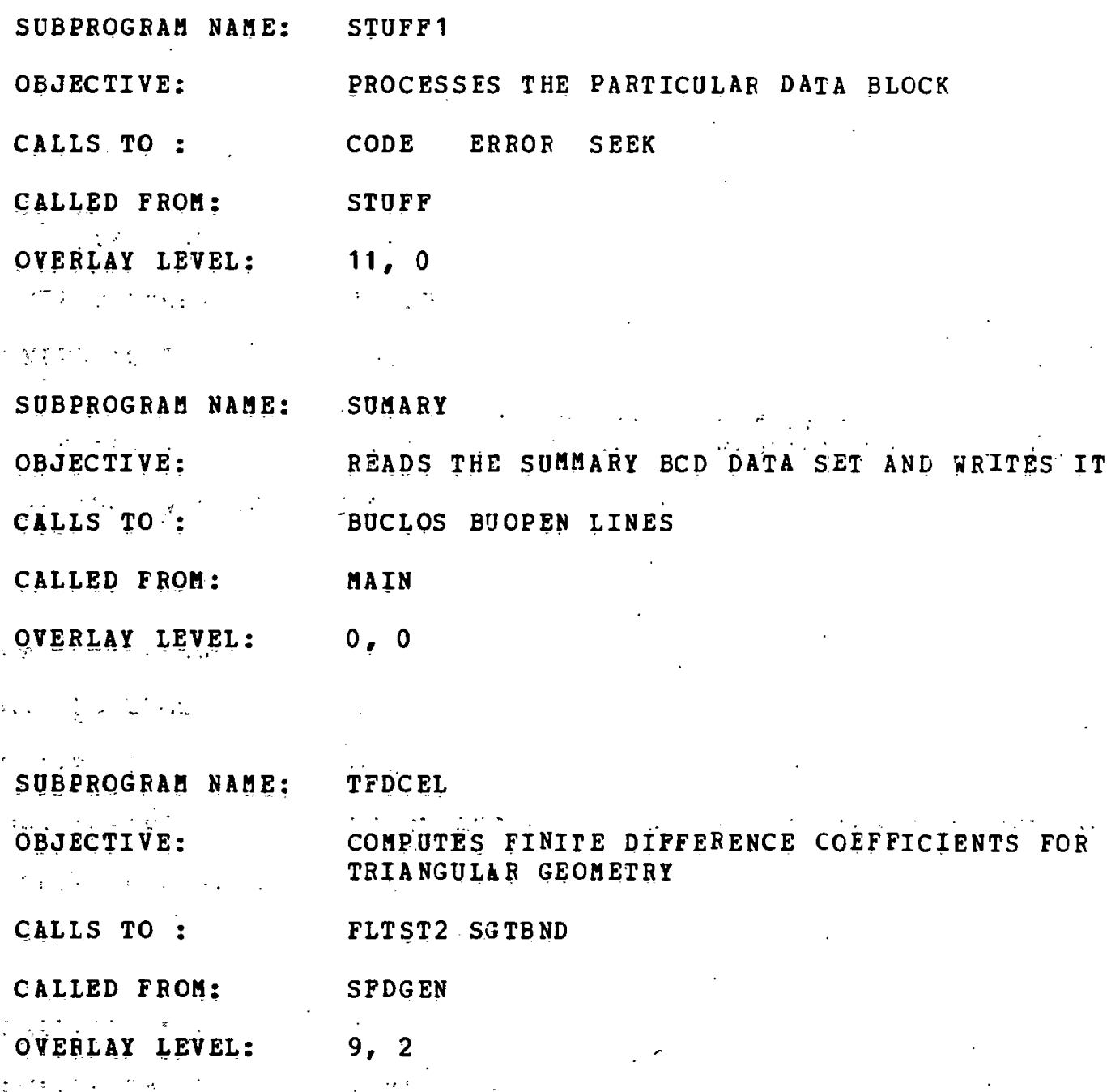




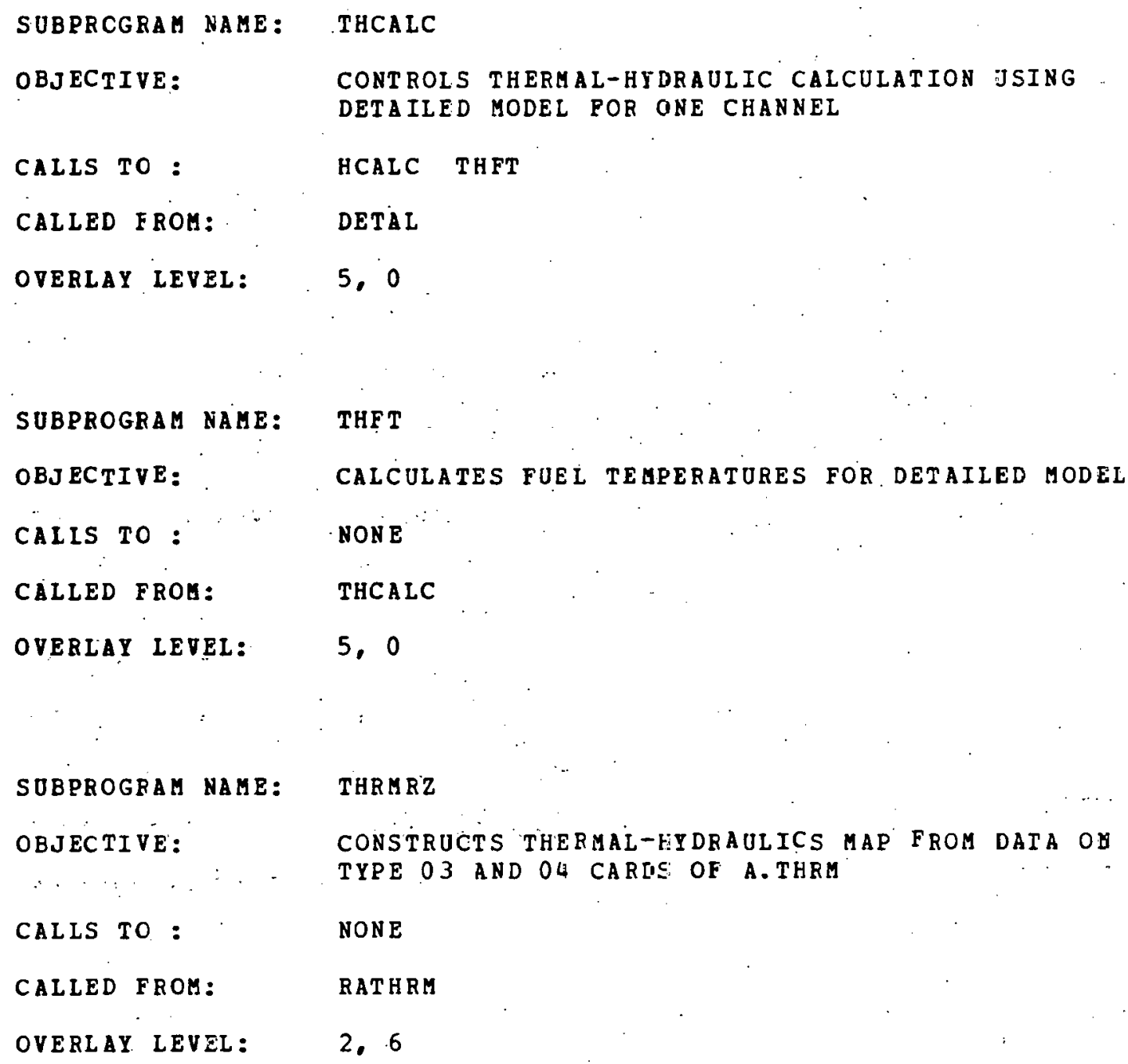


SUBPROGRAM NAME: THRMO3

OBJECTIVE: $\quad$ READ TYPE 03 ARD OF A.THRM

CALLS TO : $\quad$ NONE

CALLED FROM: $\quad$ RATHRM

OVERLAY LEVEL: 2,6 :

SUBPROGRAM NAME: THRMO4

OBJECTIVE: - READ TYPE $04 \approx A R D$ OF A.THRM

CALLS TO: NONE

CALLED FROM: $\quad$ RATHE

OVERLAY LEVEL: 2,6

SUBPROGRAM NAME: THRMO5

OBJECTIVE: READ TYPE OS CARD OF A.THRA. • .

CALLS TO : $\cdots$ NONE

CALLED FROM:

OVERLAY LEVEL: $\quad 2,6$ 


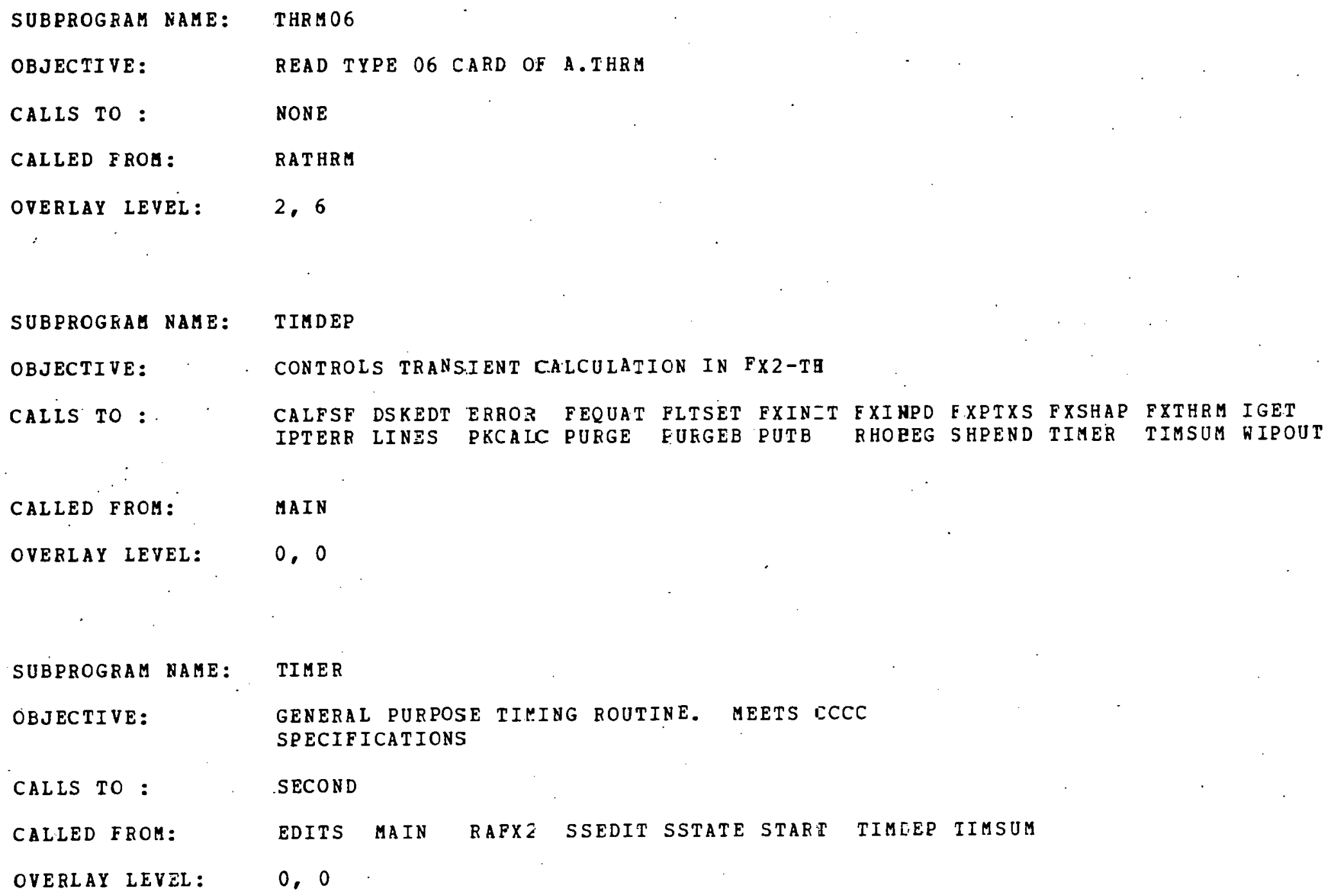


SUBPROGRAM NAME: TIMSTP

OBJECTIVE: SELECT TI AE STEP SIZES

CALLS TO: DEXP BLOG LINES

CALLED FROM: RHOBEG RHOEND

OVERLAY LEVEL : $\quad 1,0$

SUBPROGRAM NAME: TILSUM, :

OBJECTIVE: COMPUTES ELAPSED TIME BETWEEN CALLS TO IT

CALLS TO: TIMER

CALLED FROM: MAIN FHOEND SHEND STATE TIMDEP

OVERLAY LEVEL: $\quad 0,0$

$\stackrel{N}{\mathfrak{E}}$

SUBPROGRAM NAME: TLEKIP

OBJECTIVE: COMPOTES LEAKAGE PLUS REMOVAL INNER "PRODUCT

FOR TRIANGULAR GEOMETRY

CALLS TO: $\quad$ GETBND

CALLED FROM: $\quad$ PASS 2

OVERLAY LEVEL: 8,0

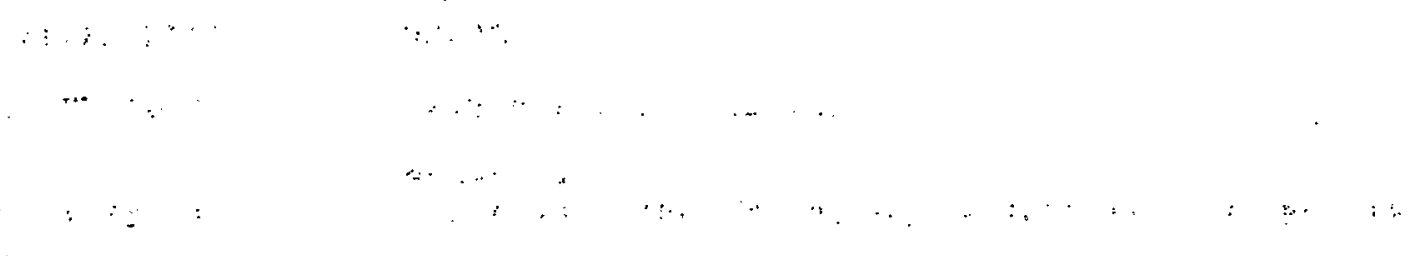


SUBPROGRAM NAME: TOMEGN

OBJECTIVE: COMPUTES OVER-RELAXATION FACTORS FOR TRIANGULAR GEOMET RY

CALLS TO : FEQUT4 FIST TRISRC

CALLED FROM:

OMEGA

OVERLAY LEVEL: $\quad 9,3$

SUBPROGRAM NAME: TRIGON

OBJECTIVE: CONSTRUCTS GEOMETRY FOR TRIANGULAR GEOMETRY

CALLS TO: ERROR FLTSET INST LINES LOCHEX

CALLED FROM: : GEOD

OVERLAY LEVEL: 2,3

SUBPROGRAM NAME: TRISRC

OBJECTIVE: COMPOTES THE Y -LEAKAGE FOR B ROW IN TRIANGULAR GEOMETRY

CALLS TO : NONE

CALLED FROM: INERT TOMEGN

OVERLAY LEVEL: 9,0 
SUBPROGRAM NAME: THDPR2.

OBJECTIVE: PRINTS A TWO-DIMENSIONAL ARRAY FROM LARGE ZOE

CALLS TO: $\quad$ LINES

CALLED FROE: $\quad$ EDITS SPOUT SSEDIT

OVERLAY LEVEL: $\quad 0,0$

SUBPROGRAM NAME: TWODPR

OBJECTIVE: $\quad$ PRINTS A TWO-DIMENSIONAL ARRAY FROM SMALL LORE

CALLS TO: : $: \cdots$ LINES

CALLED FROM: $\quad$ EDTFTC

OVERLAY LEVEL: $\quad 0,0$.

SUBPROGRAM NAME: ODES

OBJECTIVE: COMPUTES PROMPT AND DELAYED FISSION SOURCE

FROM ONE GROUP

CALLS TO:

CALLED FROE: GNDOTS

OVERLAY LEVEL: 9,4 


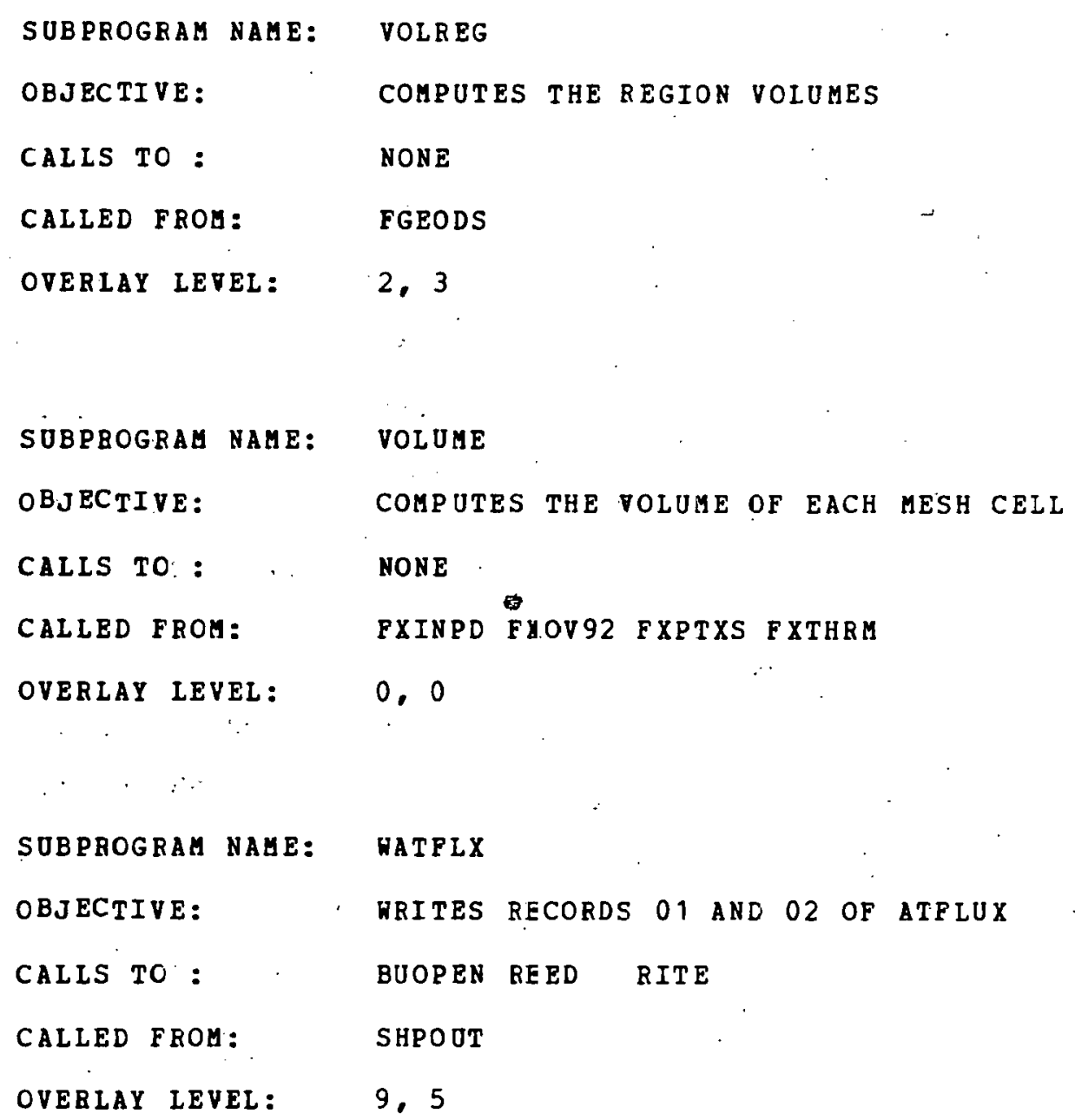


SUBPROGRAM NAME: WFXCTL

OBJECTIVE: WRITES THE FILE FXCTRL

CALLS TO: BUCLOS BUOPEN REED RITE

CALLED FROM: RAF XI

OVERLAY. LEVEL: $\quad 2 ; 1$

SUBPROGRAM NAME: WFXHMO

OBJECTIVE: WRITES TEE FILE FXHMOG

CALLS TO: BUCLOS BUOPEN FEQUT2 IEQUT2 IED LIT

CALLED FROM:

OVERLAY LEVEL: 2,4

SUBPROGRAM NAME: WFXTH

OBJECTIVE: WRITES THE FILE FXTH

CALLS TO: BOLOS BLIOREN IRE RIT

CALLED FROM: RATH RM

OVERLAY LEVEL: 2,6 


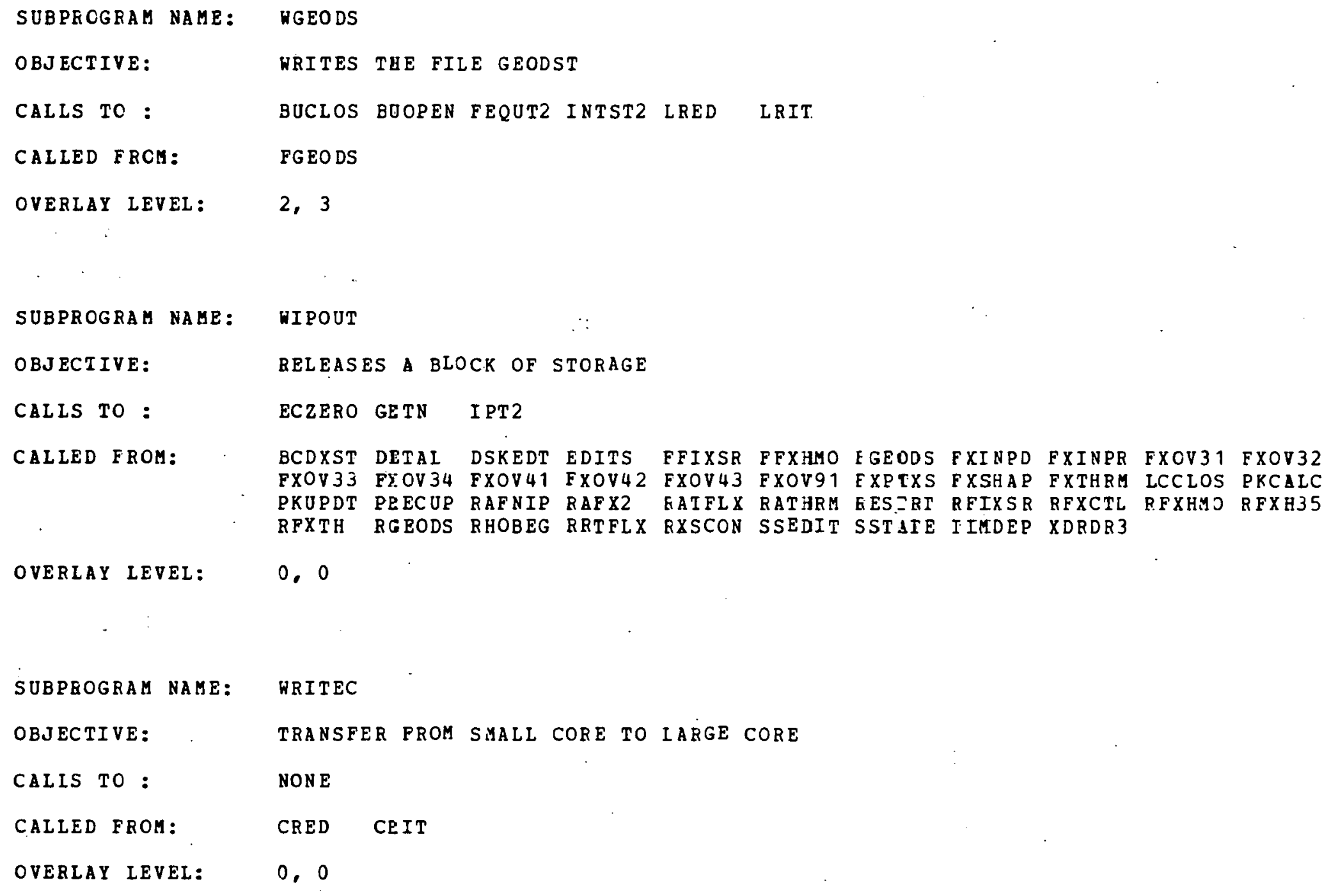

OVERLAY LEVEL: $\quad 0,0$

$\begin{array}{ll}\text { SUBPEOGRAM NAME: } & \text { WRITEC } \\ \text { OBJECTIVE: } & \text { TRANSFER FROM SMALL CORE TO IARGE CORE } \\ \text { CALIS TO : } & \text { NONE } \\ \text { CALLED FROM: } & \text { CRED CEIT } \\ \text { OVERLAY IEVEL: } & 0,0\end{array}$




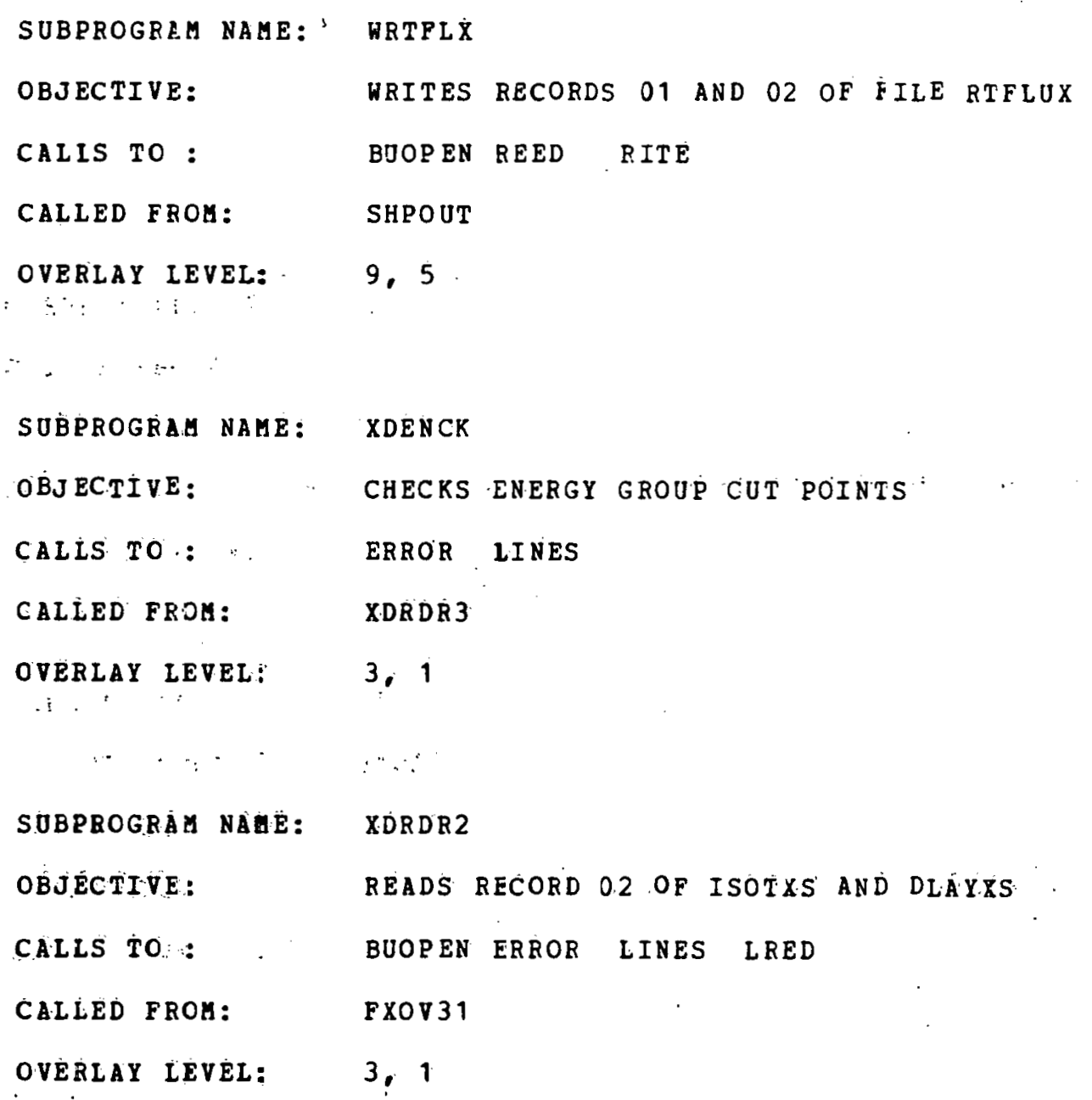




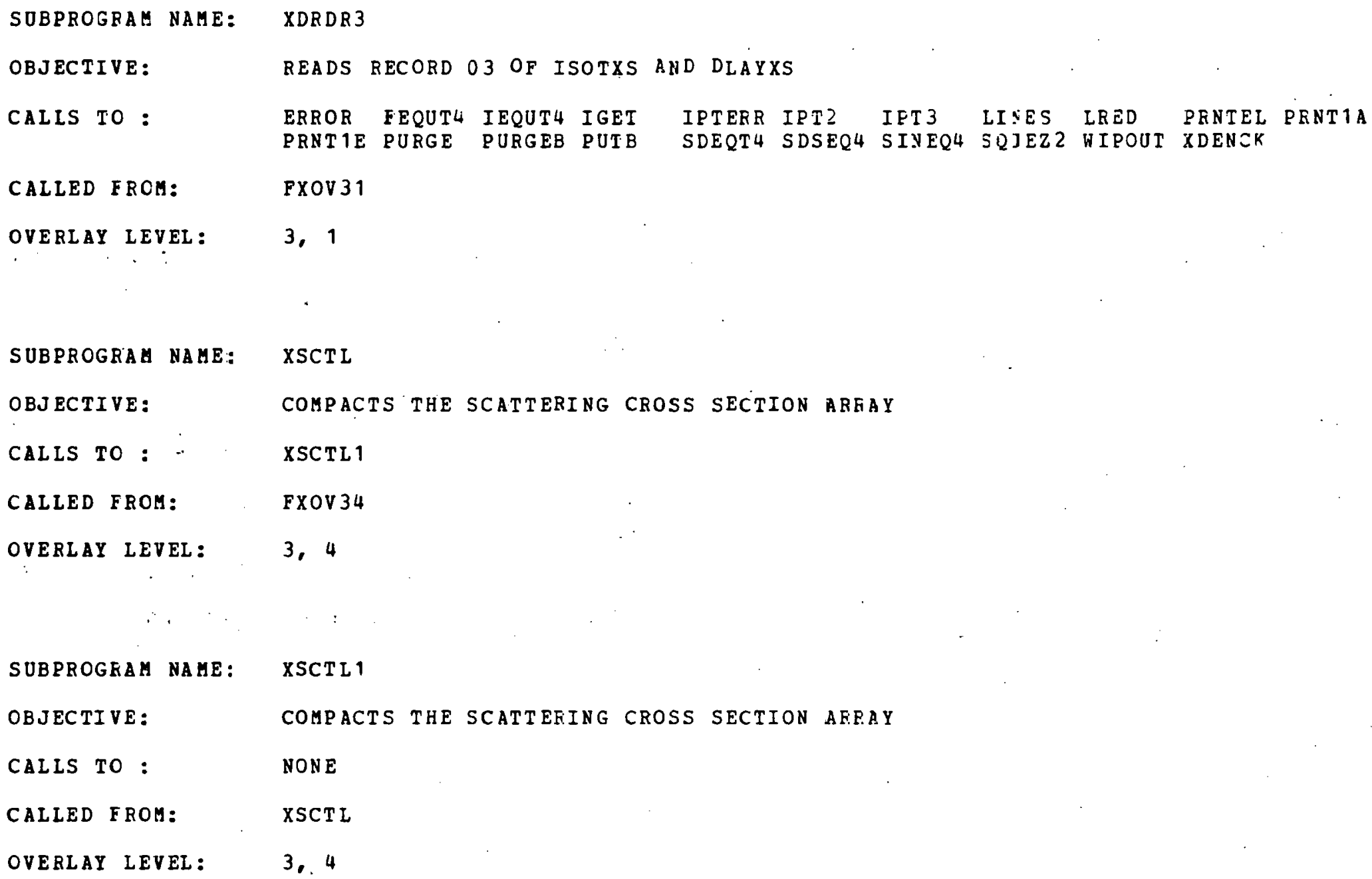

SUBPROGEAM NAHE: XSCTL1

OBJECTIVE:

COMPACTS THE SCATTERING CROSS SECTION AFRAY

CALIS TO: NONE

CALLED FROM: XSCTL

OVERLAY LEVEL: 3,4 


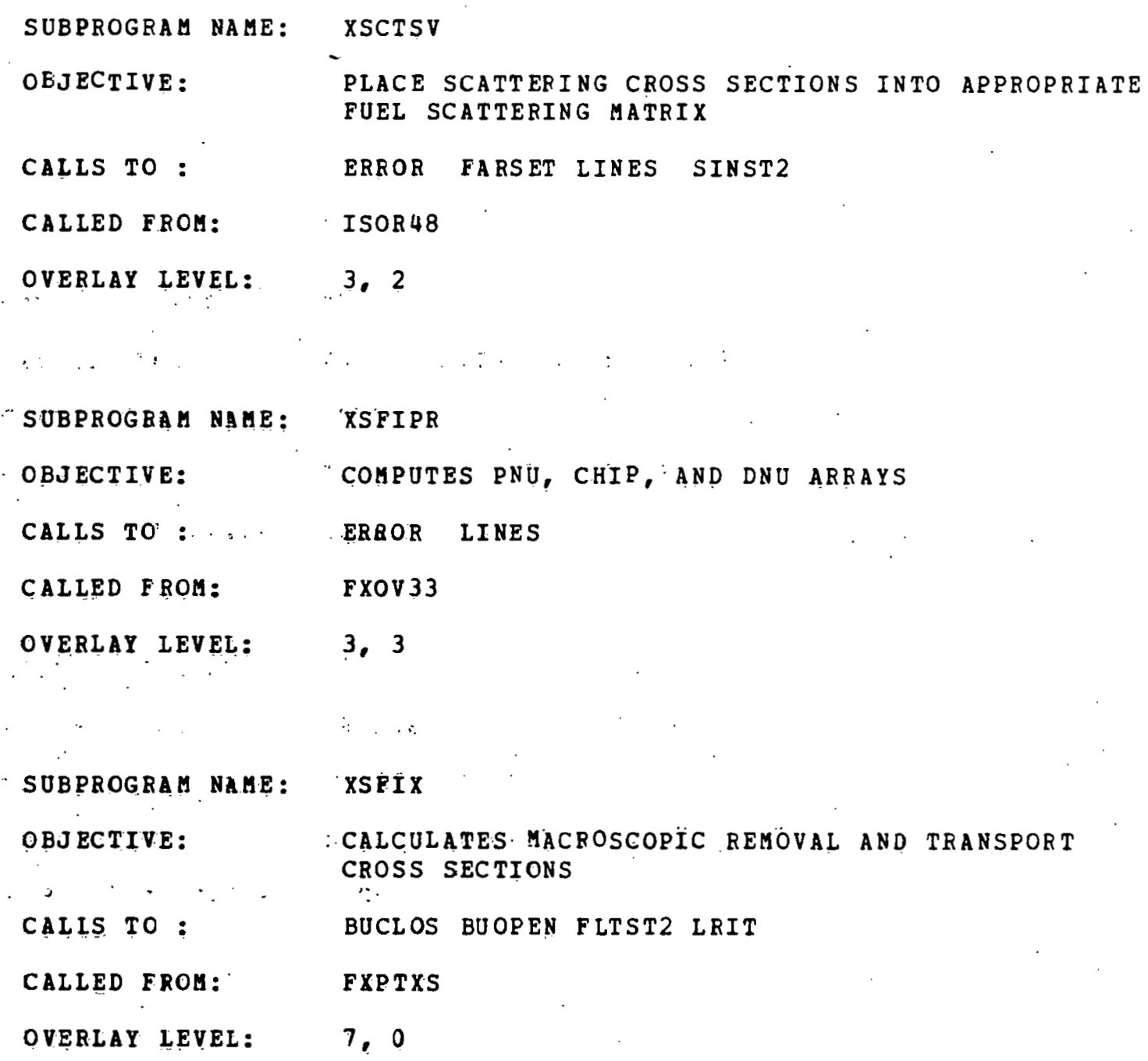




$\begin{array}{ll}\text { SUBPRCGRAM NAME: } & \text { XSORDR } \\ \text { OBJECTIVE: } & \text { REORDERS MACROSCOPIC CROSS SECTION ARRAY } \\ \text { CALLS TC: } & \text { DCRNCH } \\ \text { CALLEL FROM: } & \text { FXOV33 } \\ \text { OVERLAY LEVEL: } & 3,3\end{array}$

SUBPRCGEAM NAME: XSUPDT

OBJECIIVE: UPDATES MACROSCOPIC CRCSS SECTIONS

CALIS TC : $\cdot \because$ ERRÓR IINES

CALLEC FROM: ISOR 48

CVERLAY LEVEL: $\quad 3,2$

SUBPRCGRAM NAME: ZEROIO

OBJECIIVE: . INITIALIZES I/O FLASS

CALIS TO : $\because \quad$ NONE

CALLEE FROM: LRED LRIT REED RITE

OVERLAY IEVEL: $\quad 0,0$ 


\section{APPENDIX B}

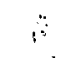

Listing of $\mathrm{BCD}$ Input Files 


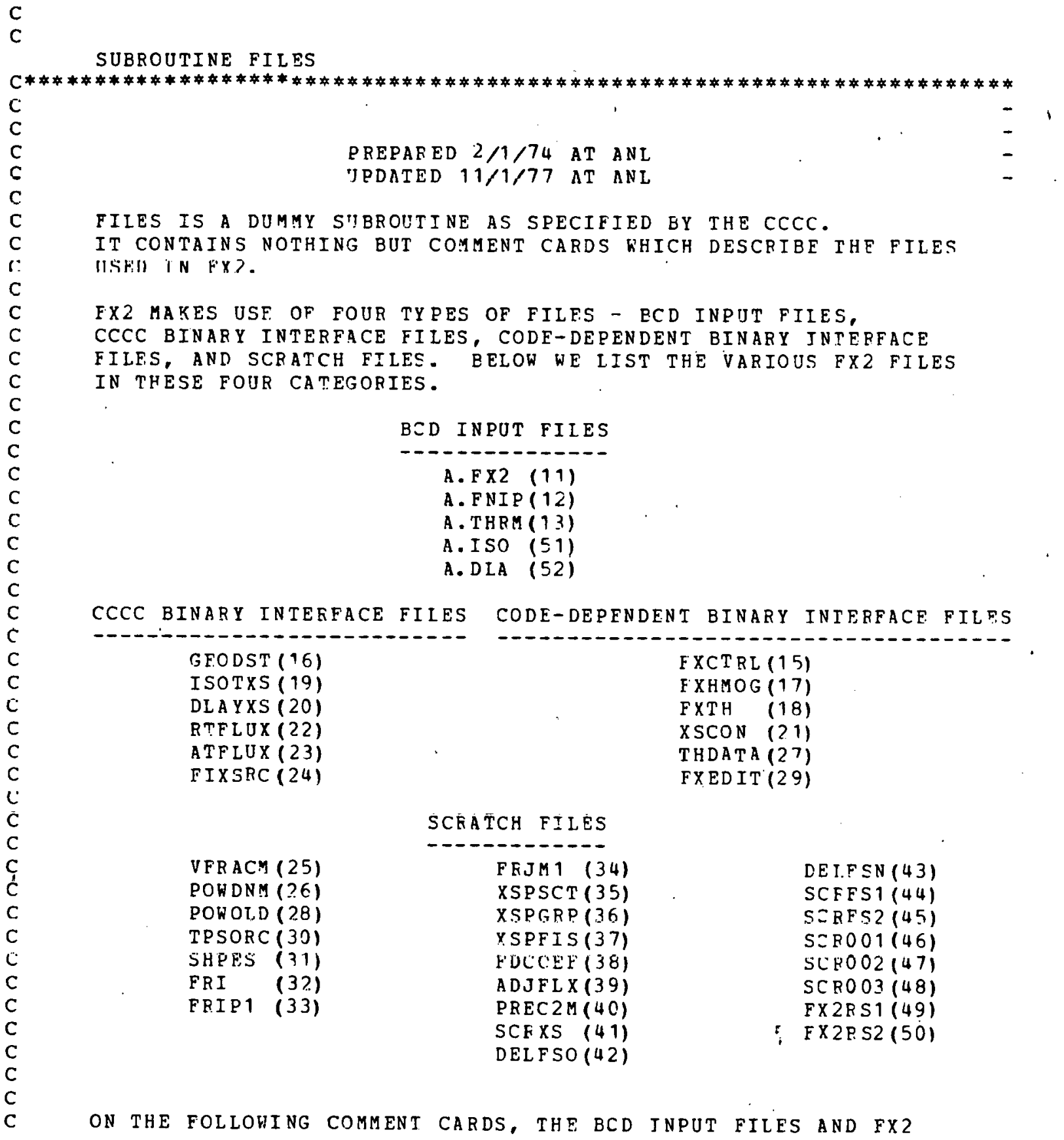




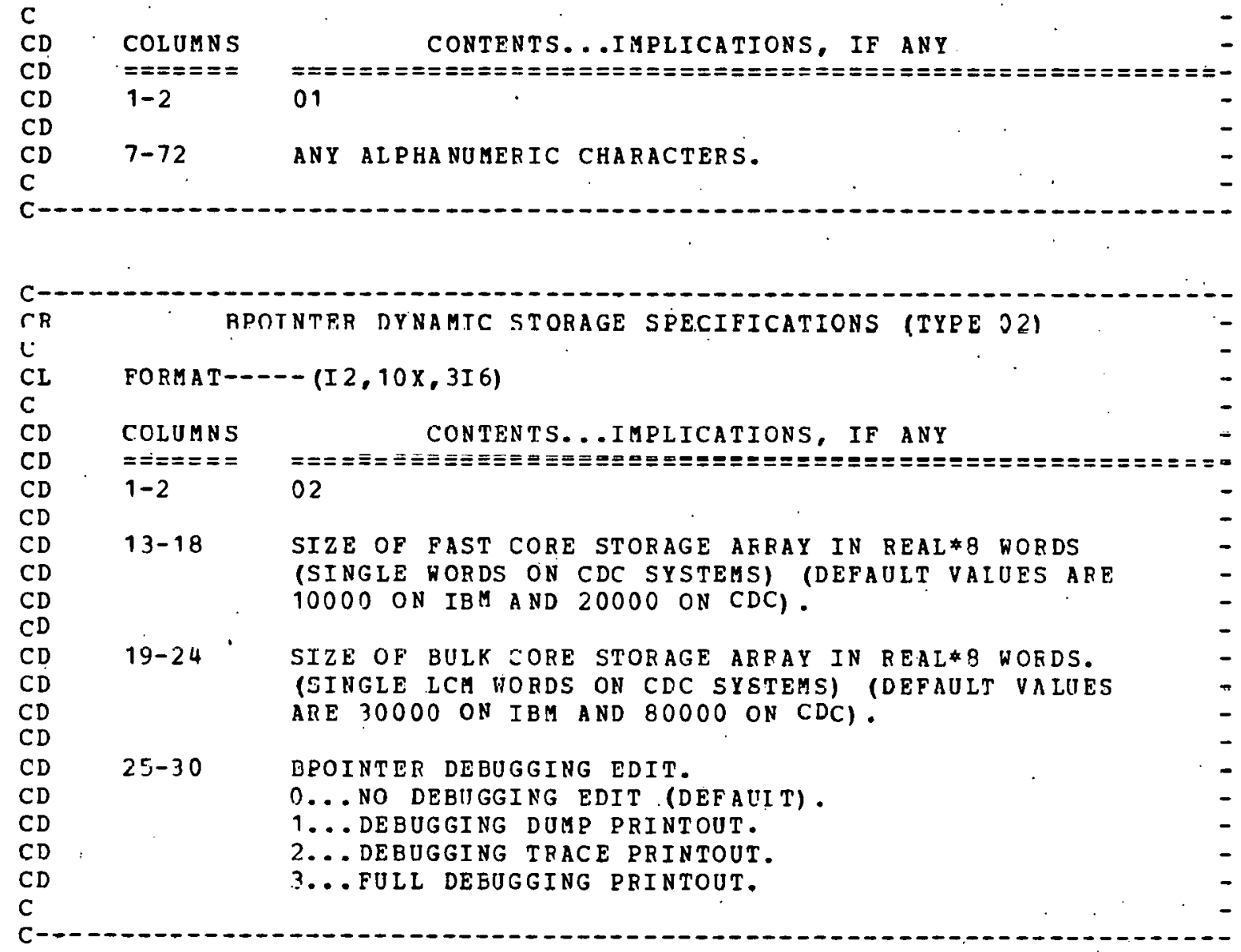

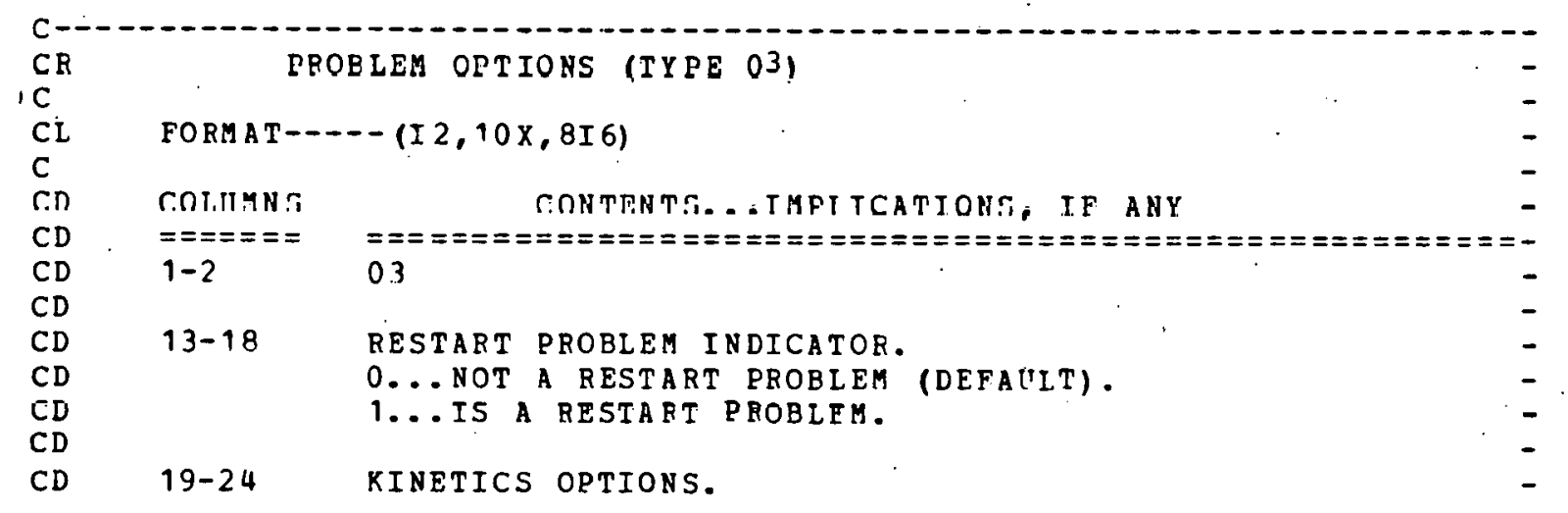




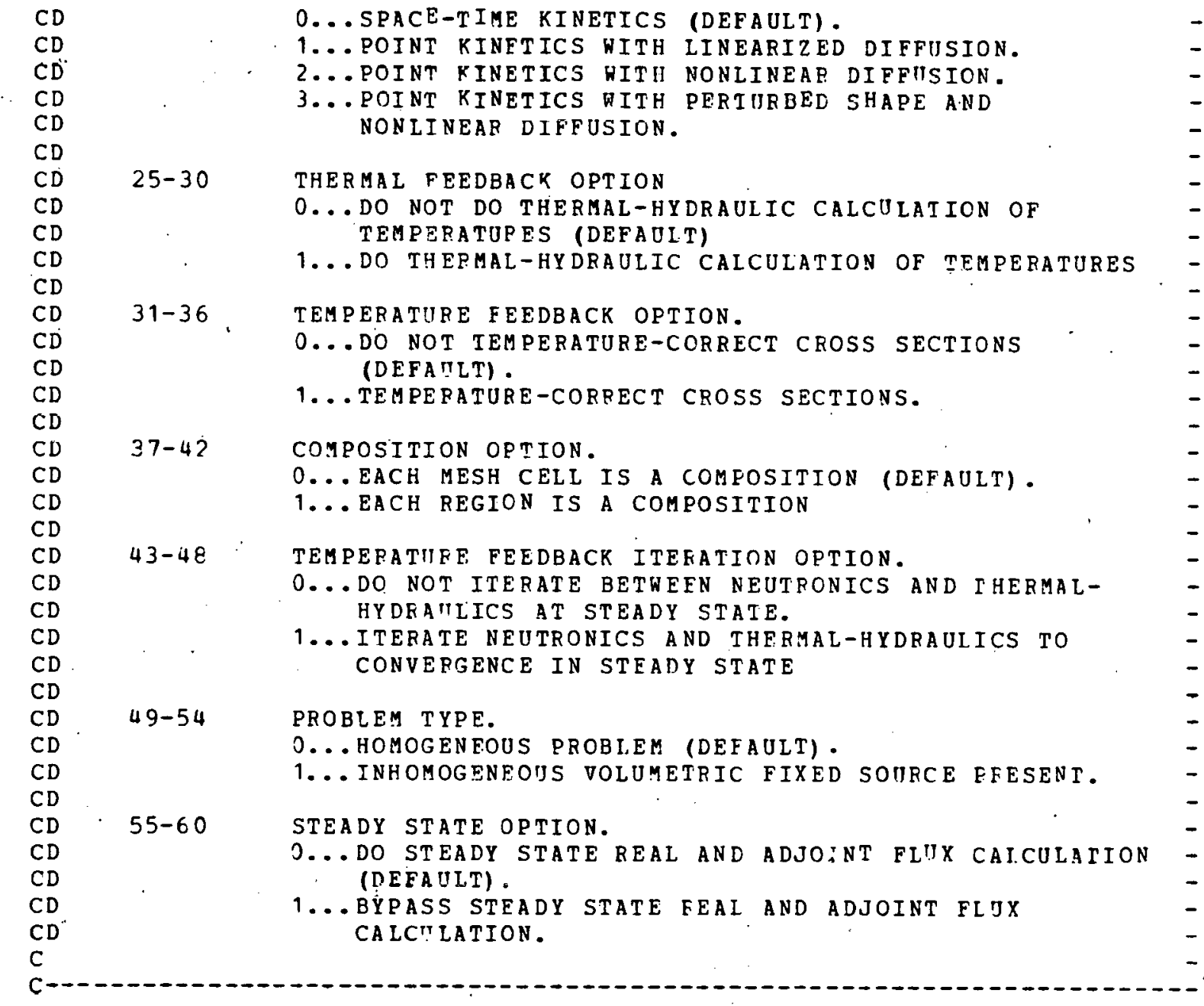

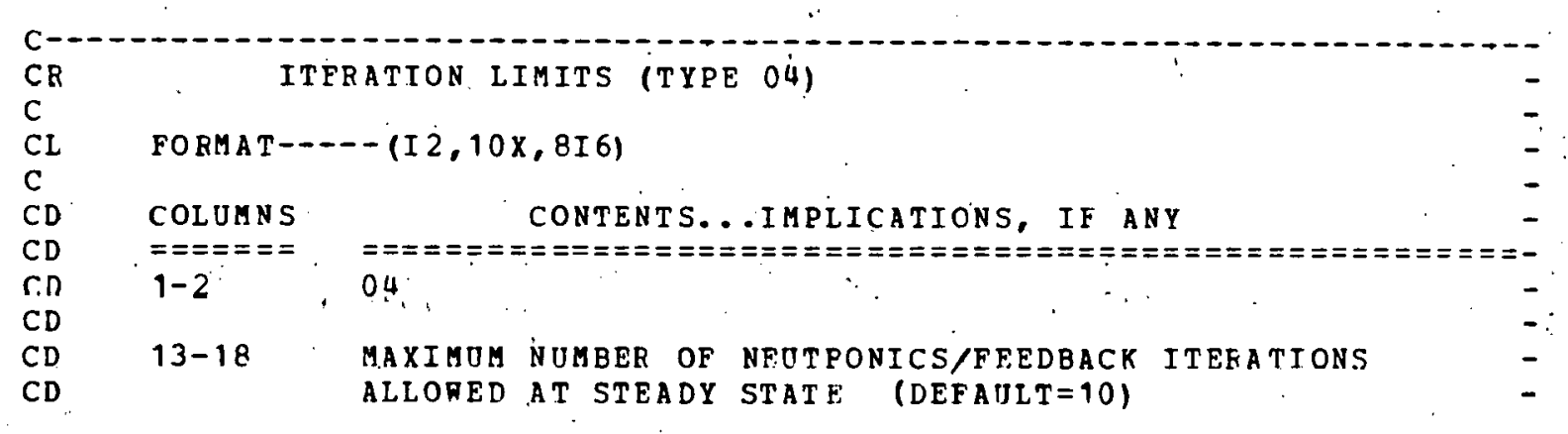




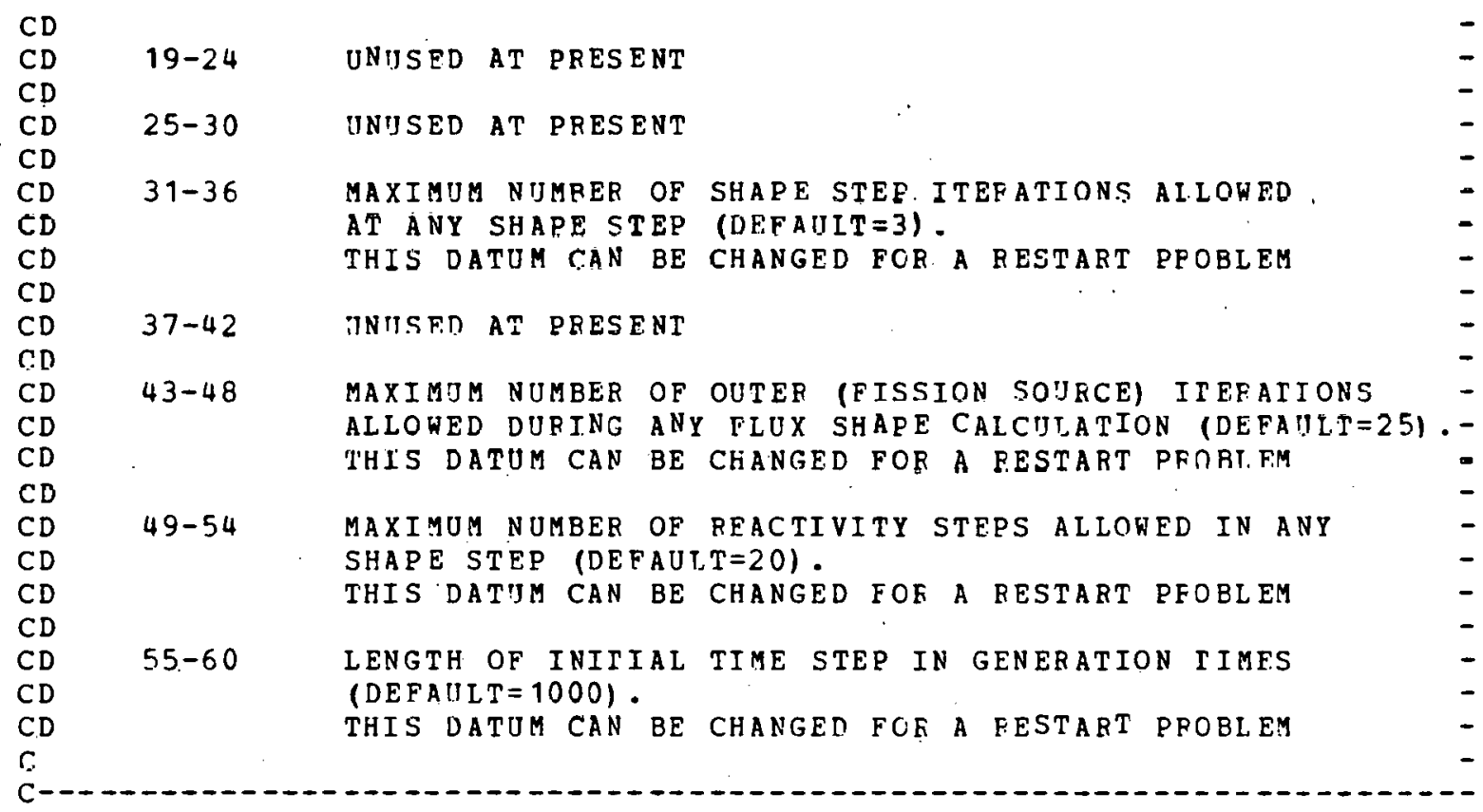

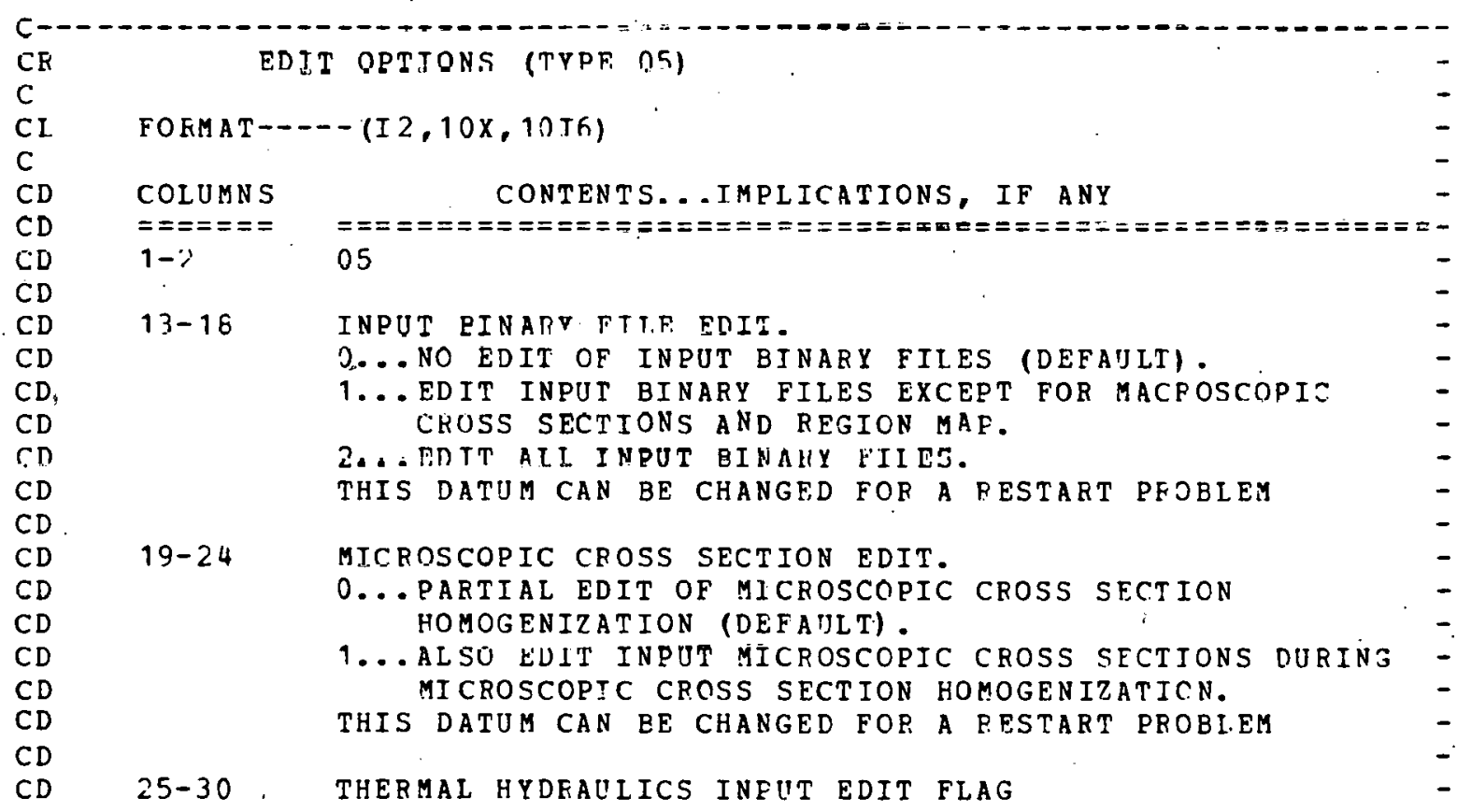


$C D$

CD

$C D$

$C D$

$C D$

$C D$

$C D$

$C D$

$C D$

$C D$

$C D$

$C D$

$C D$

$C D$

$C D$

$C D$

$C D$

$C D$

$C D$

$C D$

$C D$

$C D$

$C D$

$C D$

$C D$

$C D$

CD

CD

$C D$

$C D$

$C D$

$C D$

$C D$

$C D$

$C D$

$C D$

$C D$

$C D$

$C D$

$C D$

$C D$

$C D$

$C D$

CD

$C D$

CD

C

0...DO NOT EDIT THERMAI. HYDRAULICS (DEFAOLI). 1 ... PARTIAL EDIT OF THERMAL HYDRAULICS INPUT 2...FULI EDIT OF THERMAL HYDRAULICS INPUT THIS DATUM CAN BE CHANGED FOR. A FESTART PROBLEM

31-36 UNOSED AT PRESENT

37-42 DISK EDIT FIAG

O... NO DISK EDIT

1 ... BRIFF, DISK EDIT

2... DETAILED DISK EDIT

(POINTHISE POWERS, COOLANT ANE FUEL TEMPERAIURES)

3... EXTENDED DETAILED DISK FDIT

(ALI EDITS ABOVE PIUS POINTWISE FLDXES)

43-48 FUEL AND CONLANT TEMPERATURE EDIT FLAG

O... DO NOT EDIT FUEL AND COOLANT TEMPERATUFES

1... EDJT FUEL AND COOIANT TEMPERATURES AFTER

EACH SHAPE. STEP

49-54 REACTIVITY STEP SUMMARY EDIT.

, 0...BRIEF EDIT (DEFACIT).

1... NORMAL EDIT.

2... DETAILED EDIT.

THIS DATJM CAN BE CHANGED FOR A FESTART PPOBLEM

55-60 POWER DENSITY EDIT (REACTIVITY STEP)

O...DO NOT EDIT POWER DENSITY AFTER

REACTIVITY STEPS (DEFAULT).

N... EDIT POWER DENSITY AFTER EVERY

N REACTIVITY" STEPS.

THIS DAT'M CAN BE CHANGED FOF A RESTART PROBLEM

61-66 FLJX SHAPE CALCULATION EDIT.

0... MINIMUM EDIT. OF EACH FLUX SHAPE CALCULATICN (DEFAULT) .

1... EDIT ITERATION HISTORY AT EACH FLOX SHAPE

CALCIJ LATION.

2... EDIT ITERATION HISTOPY AND MULTIGROUP ELUX SHAPE AT EACH FLUX SHAPE CALCUIATION.

67-72 POWER DENSITY EDIT (SHAPE STEPS)

O... DO NOT EDIT POWER DENSITIES AFTER

SHAPE STEPS (DEFAULT).

N. . EDIT POWER DENSITIES AFTER EVERY N SHAPE STEPS.

THIS DATUM CAN BE CHANGED FOF A FESTART PROBLEM 


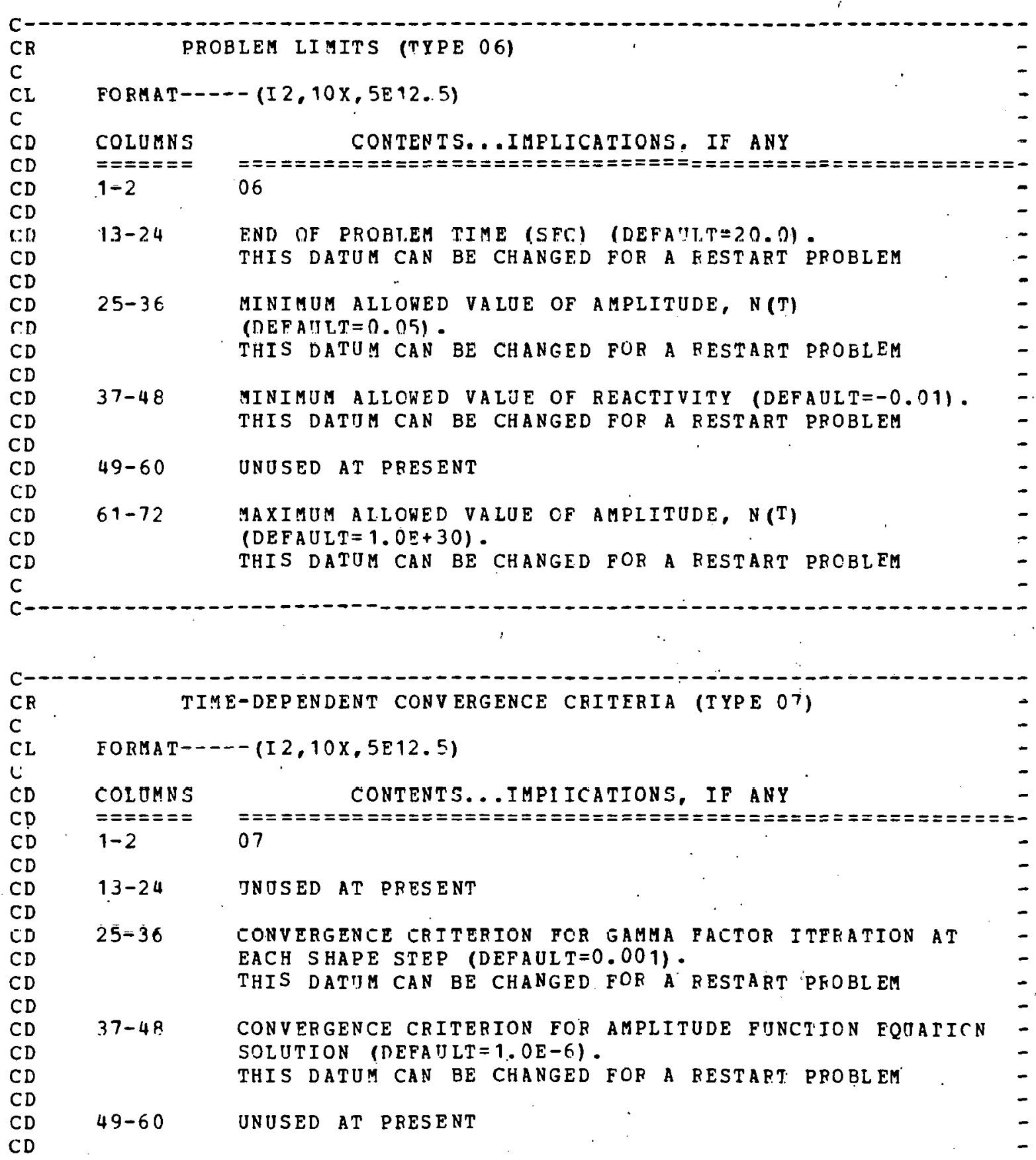




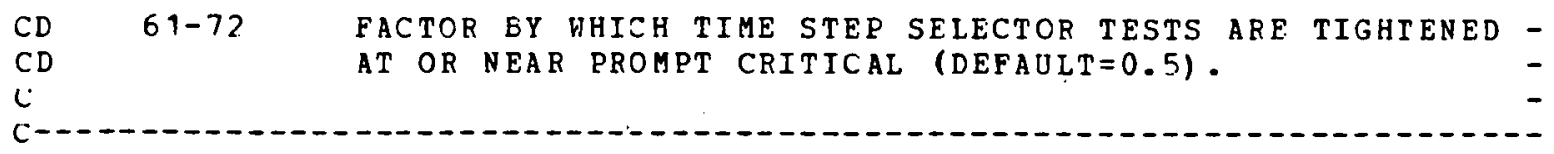

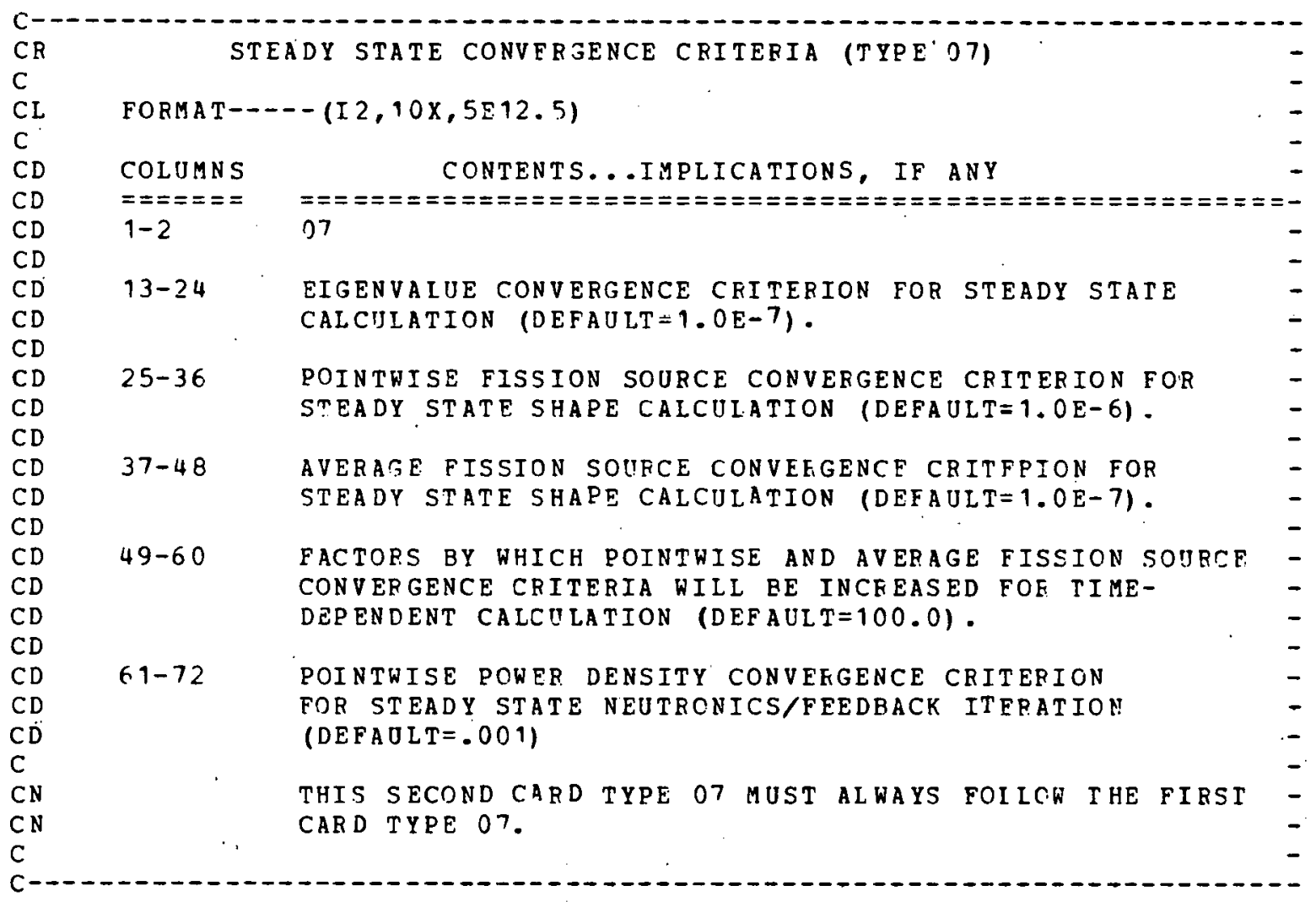

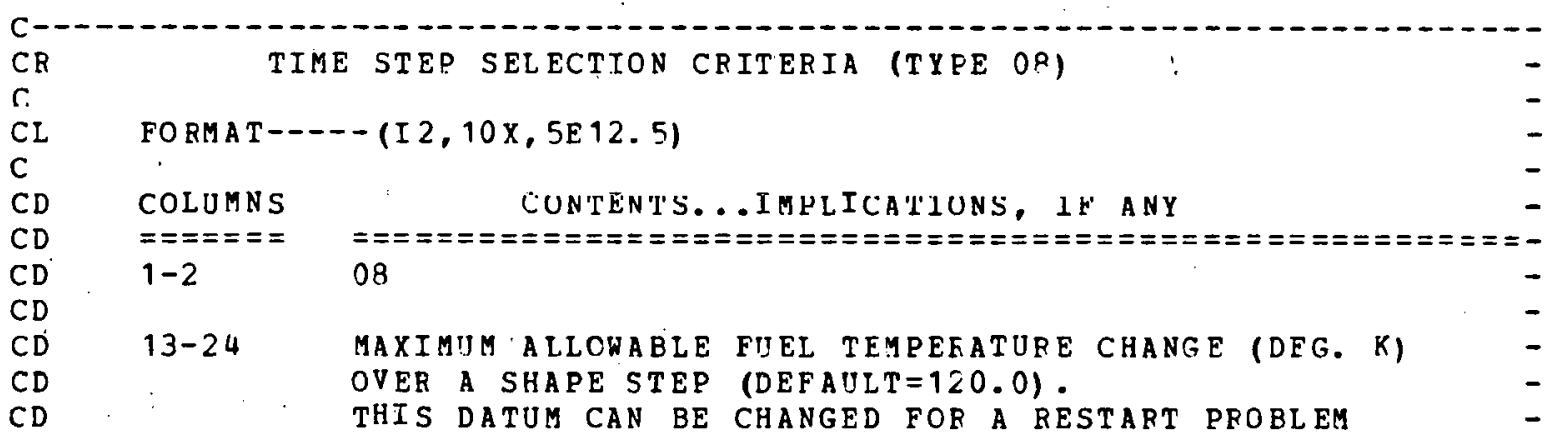




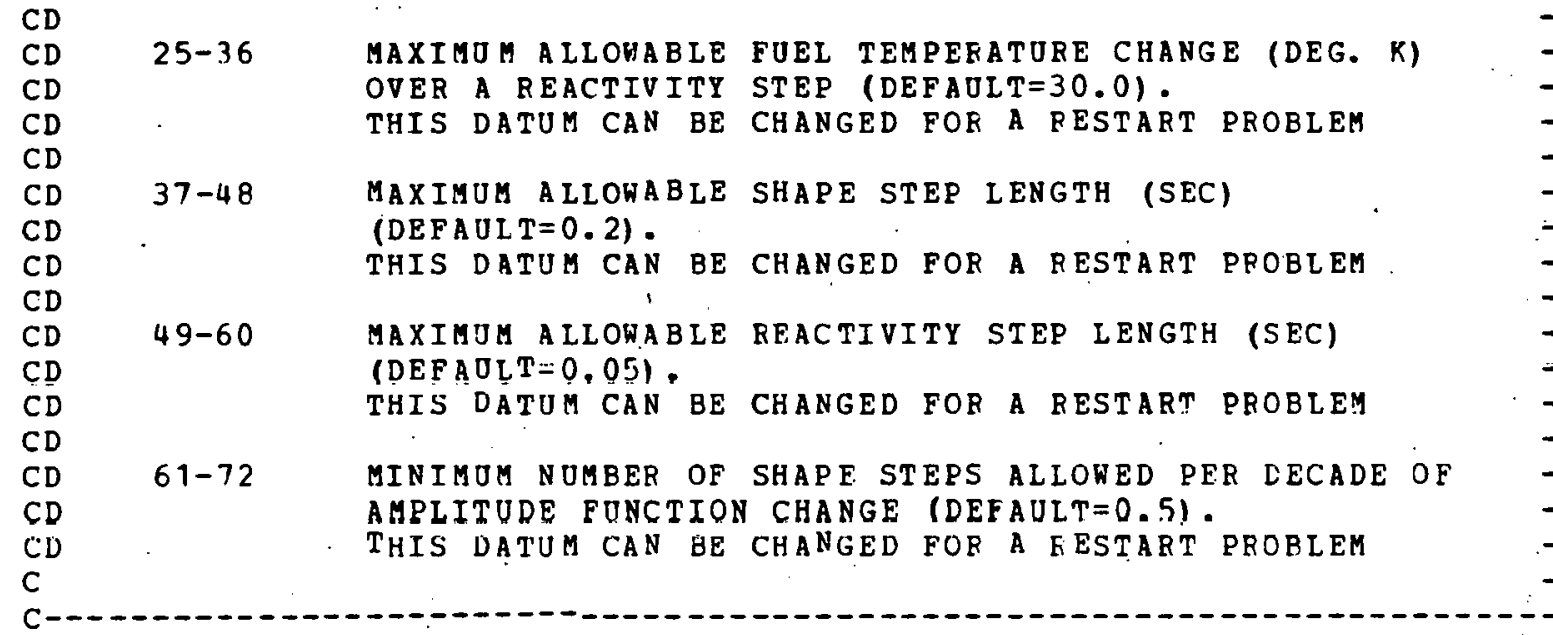

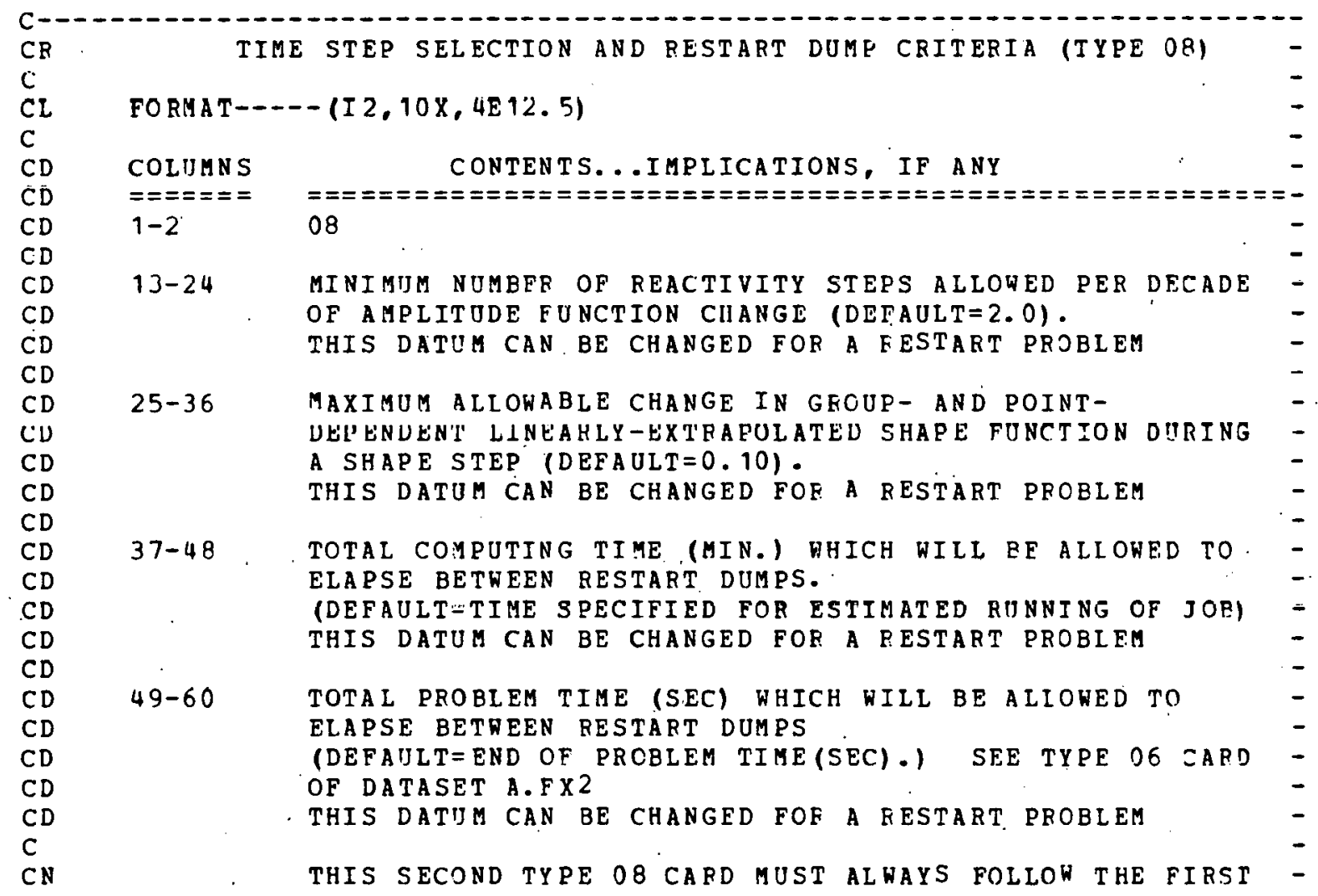




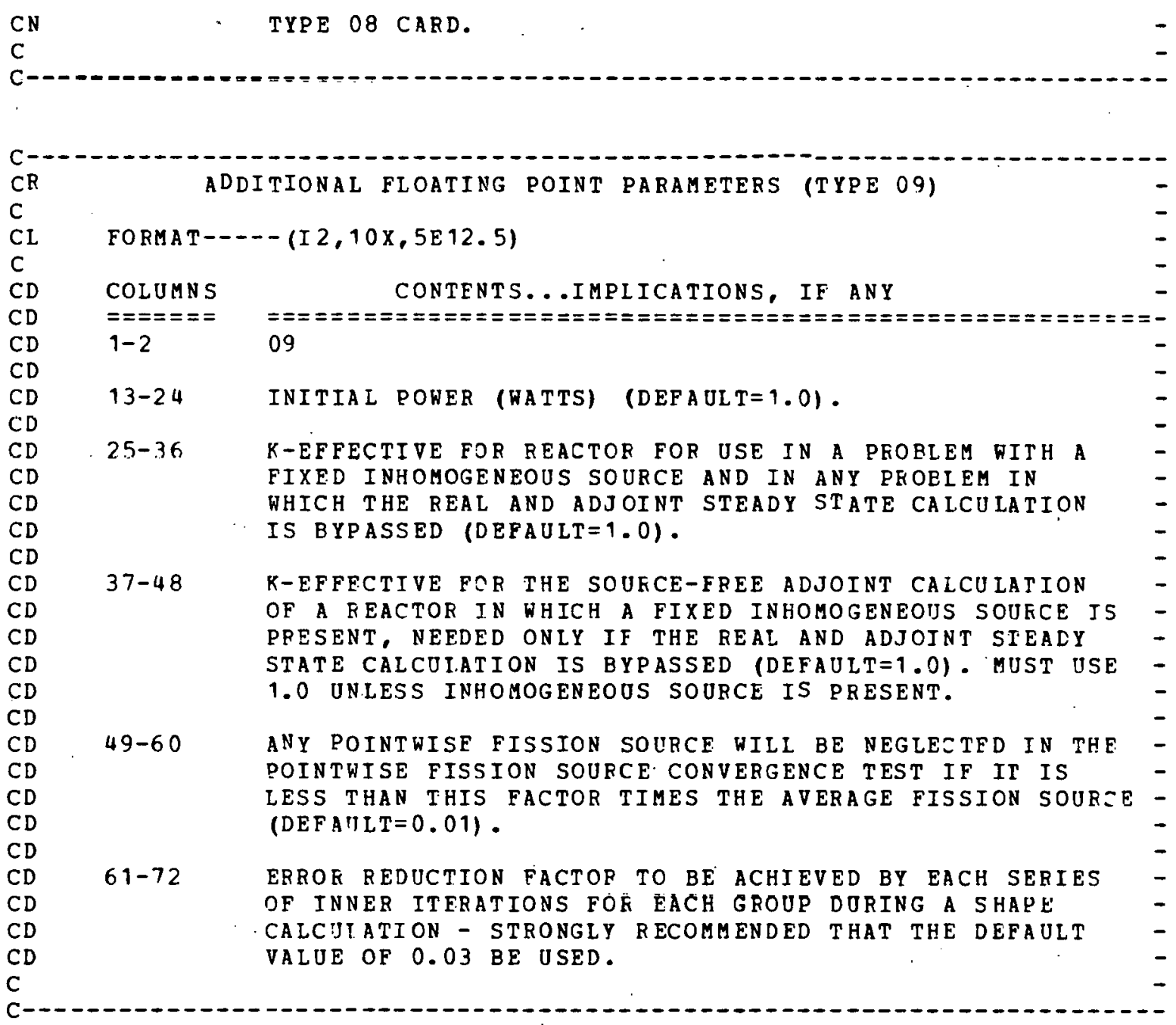

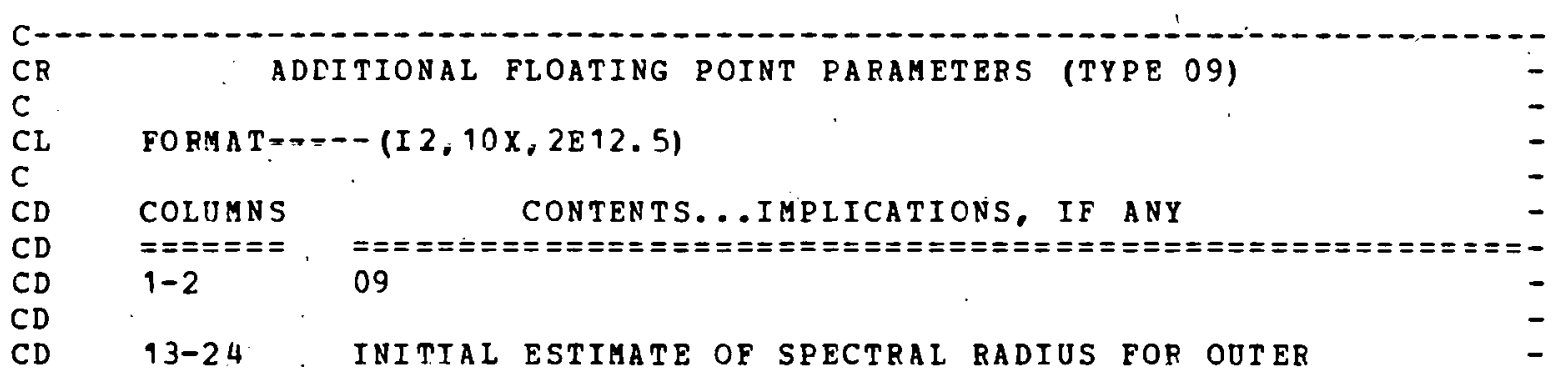




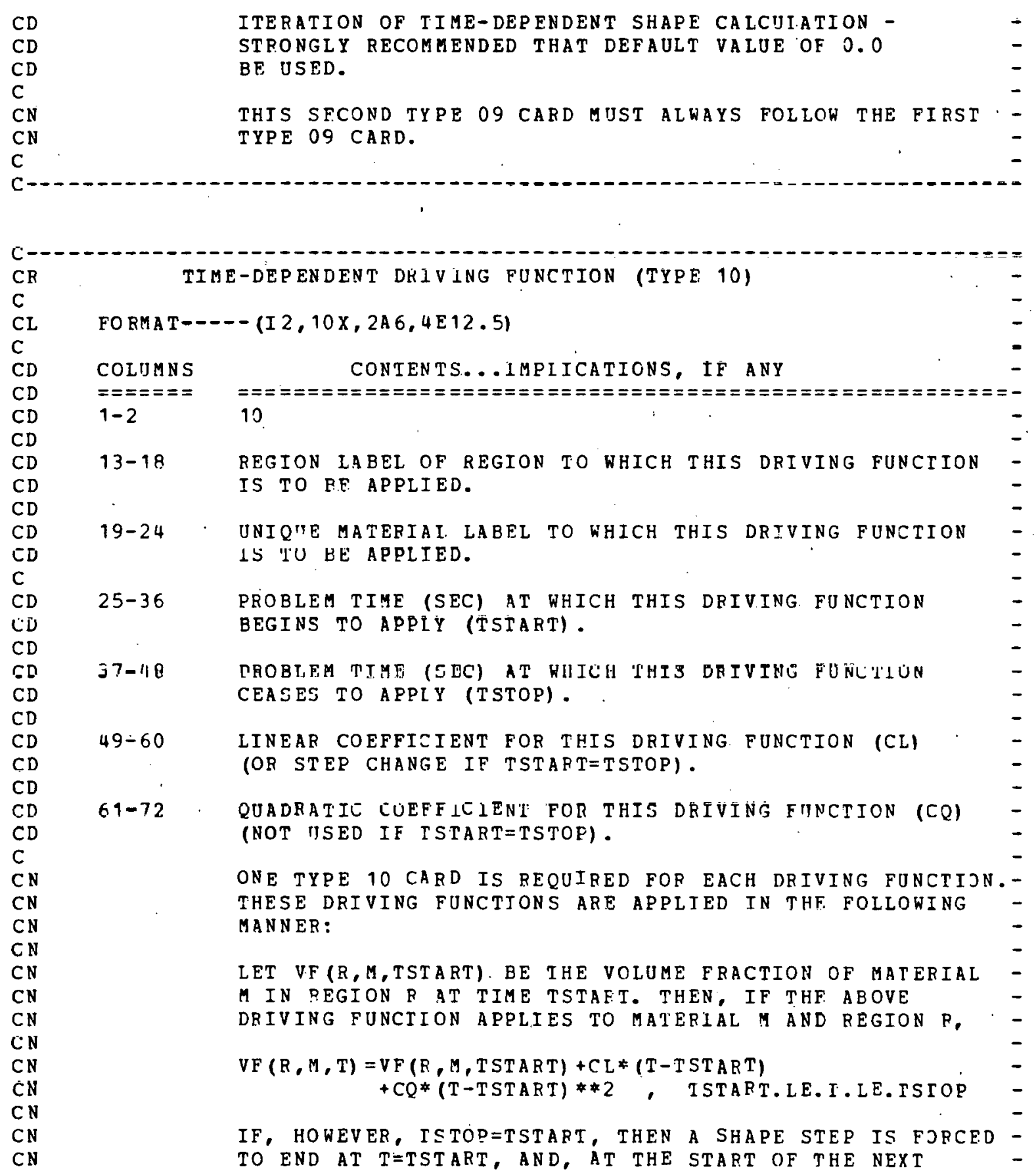




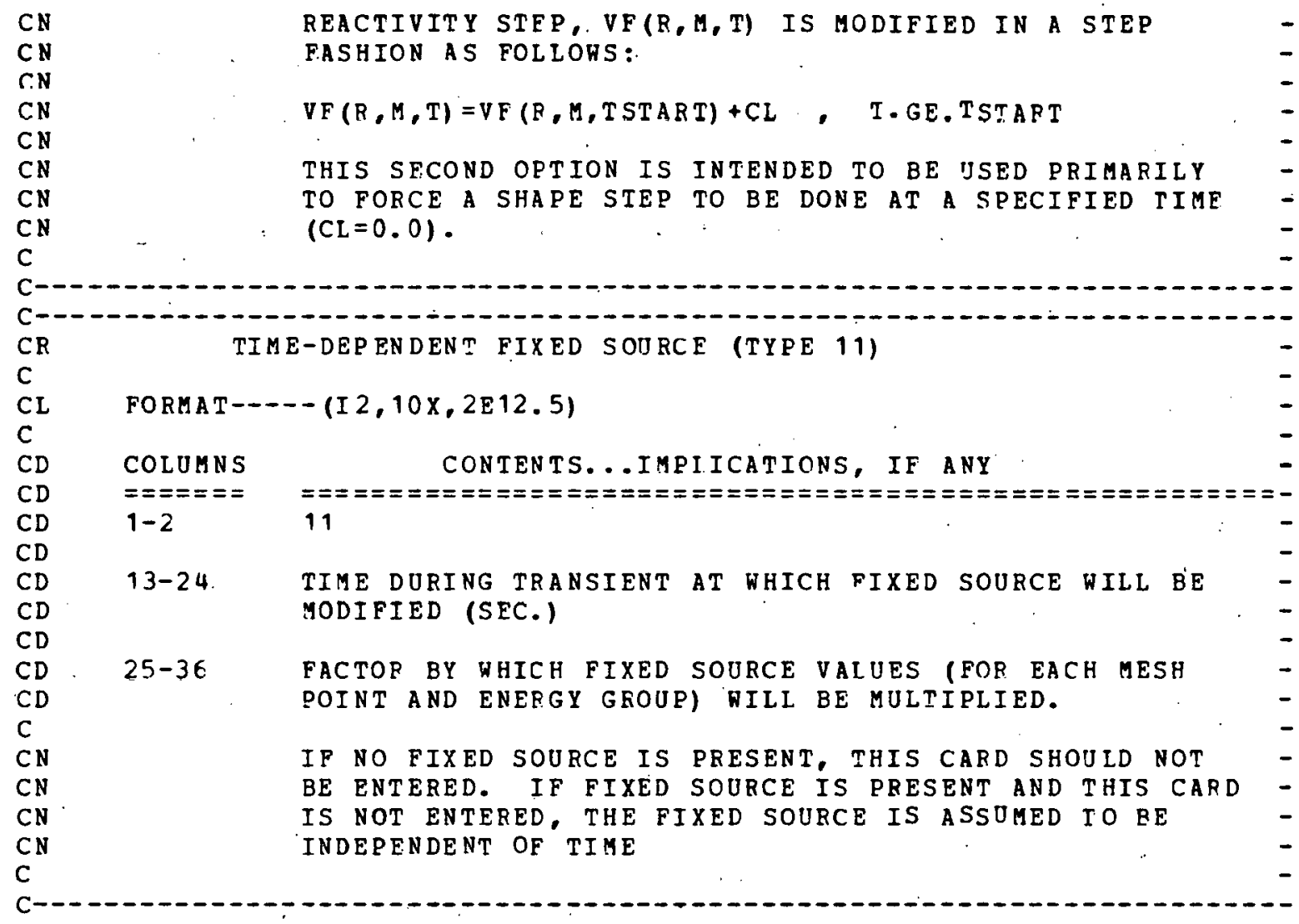

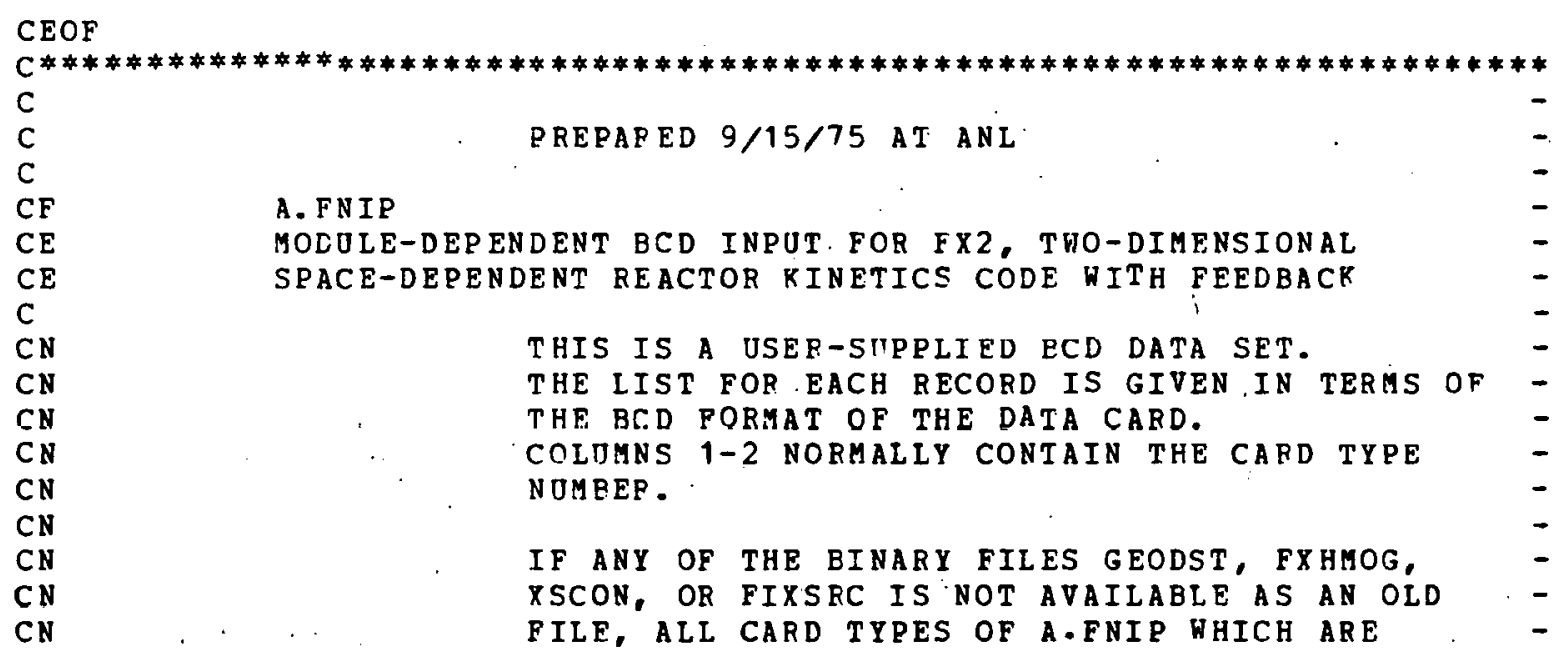


$C N$
$C N$
$C N$
$C N$
$C N$
$C N$
$C$

REQTIRED TO DESCRIBE THE PROBLEM ARE REQUIRED TO BE PRESENT. THE ONE EXCEPTION IS THAT IF THE MACROSCOPIC CROSS SECTION FILE, XSCON, IS PRESENT AS OLD FILE, CARD TYPE 13 MAY BE OMITTED FROM A.FNIP IF IT IS NEEDED TO CON - STROCT GEODST, FXHMOG, AND/OR FIXSRC.

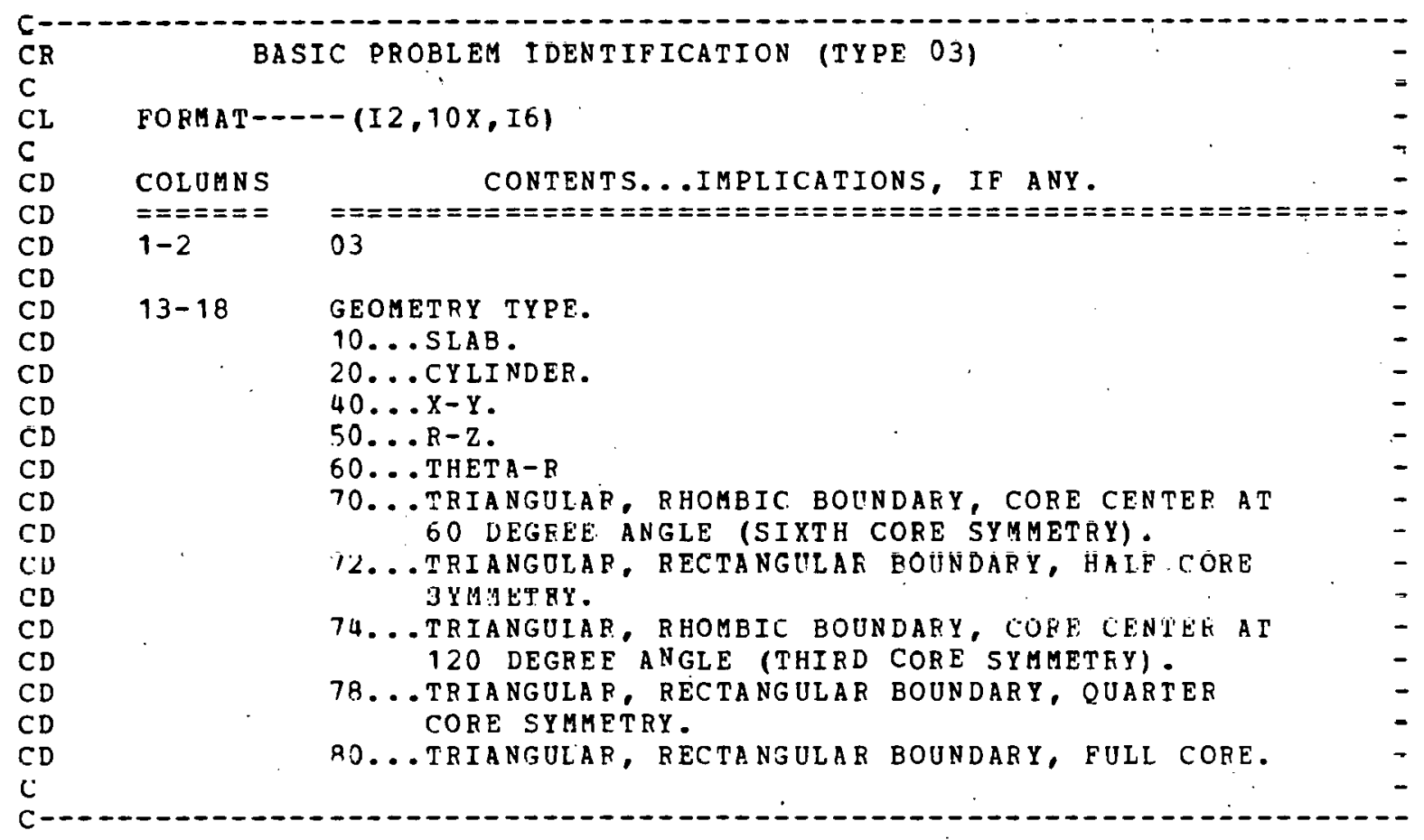

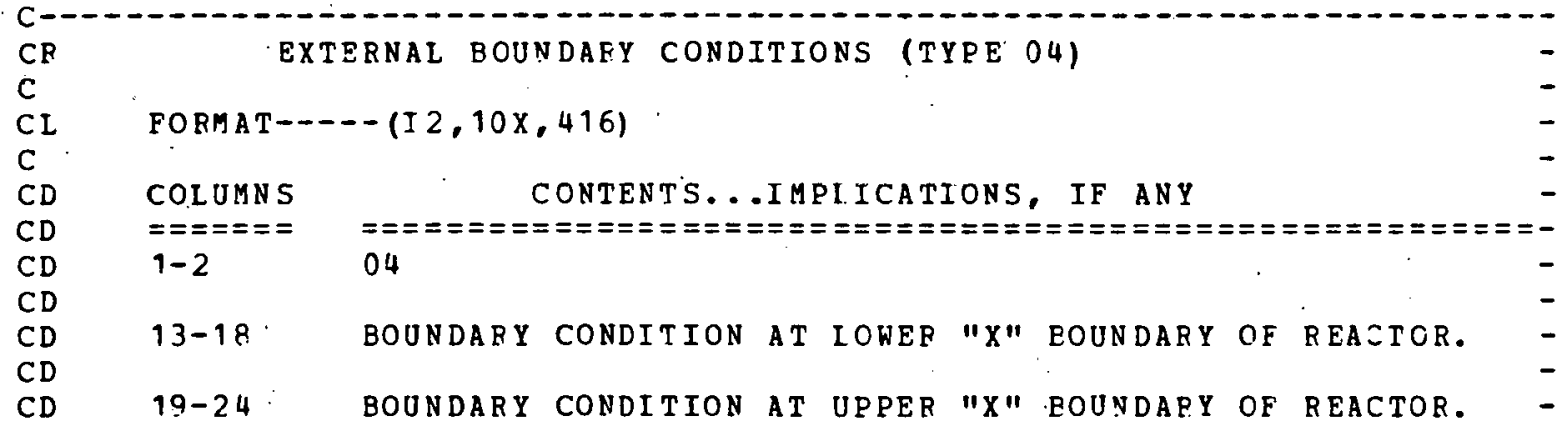


<smiles></smiles> 


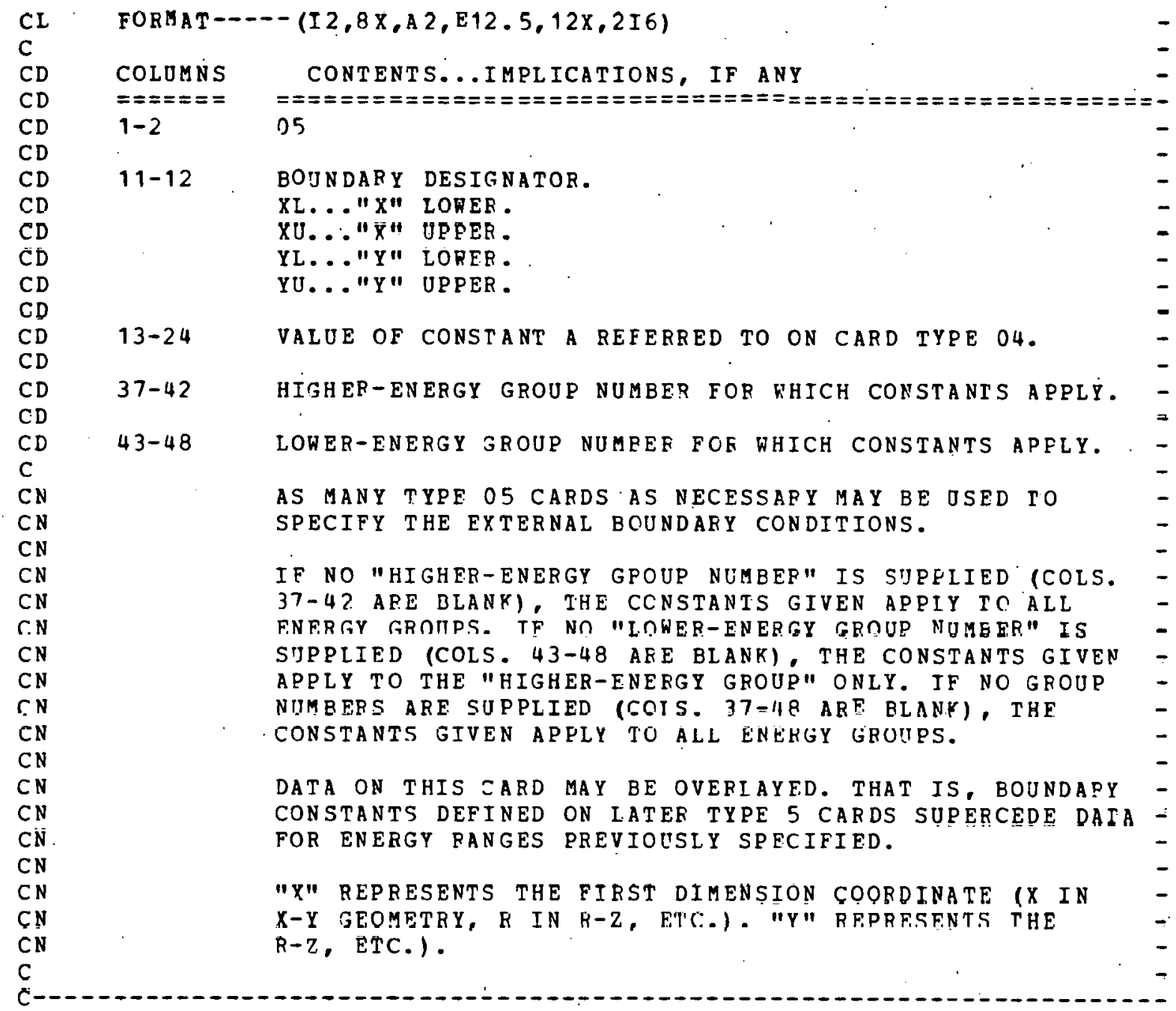

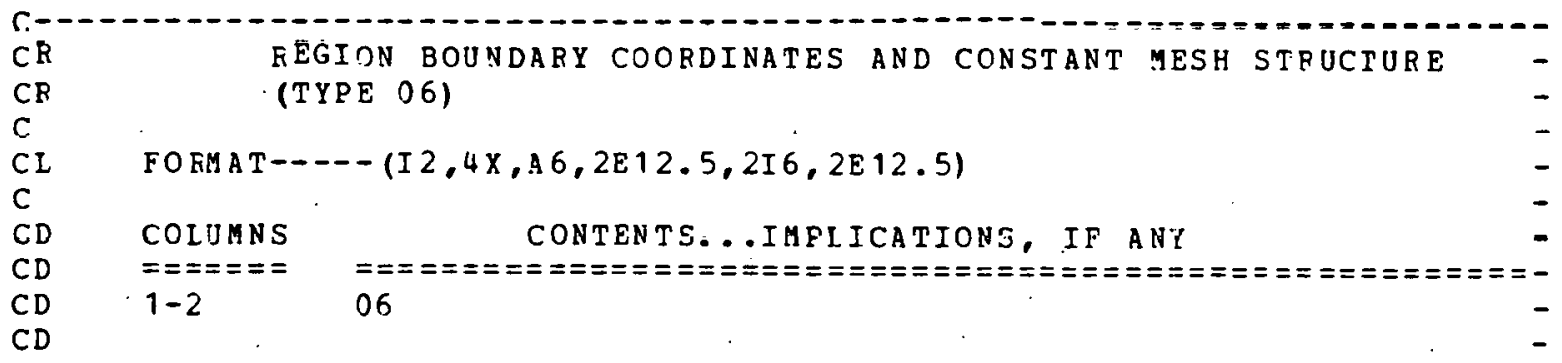




\begin{tabular}{|c|c|c|}
\hline $\begin{array}{l}C D \\
C D\end{array}$ & $7-12$ & REGION LABEL (REPEATED ON ADDITIONAL TYPE 06 CARDS). \\
\hline $\begin{array}{l}C D \\
C D\end{array}$ & $13-24$ & "X"-DIRECTION LOWER-BOUNDARY COOFDINATE. \\
\hline $\begin{array}{l}C D \\
C D\end{array}$ & $25-36$ & "X"-DIRECTION UPPER-BOUNDARY COOPDINATE. \\
\hline $\begin{array}{l}C D \\
C D\end{array}$ & $37-42$ & NUMBER OF INTERVALS IN "X" DIRECTION. \\
\hline $\begin{array}{l}C D \\
C D\end{array}$ & $43-48$ & NUMBER OF INTERVALS IN "Y" DIRECTION. \\
\hline $\begin{array}{l}C D \\
C D\end{array}$ & $49-60$ & "Y" - DIRECTION LOWER-BOUNDARY COORDINATF. \\
\hline $\begin{array}{l}C D \\
C\end{array}$ & $61-72$ & "Y" - DIRECTION UPPER-BOUNDARY COORDINATE. \\
\hline $\begin{array}{l}\mathrm{CN} \\
\mathrm{CN}\end{array}$ & & "X" REPPESENTS $X$ OF R. "Y" REPRESENTS Y OR Z. \\
\hline $\begin{array}{l}C N \\
C N \\
C N\end{array}$ & & $\begin{array}{l}\text { CARD TYPE } 06 \text { IS NOT PERTINENT FOE TRIANGJIAR } \\
\text { GEOMETRIES. }\end{array}$ \\
\hline $\begin{array}{l}C N \\
C N\end{array}$ & & $\begin{array}{l}\text { REGIONS MAY BE DEFINED USING THE OVERLAY FROCEDURE, } \\
\text { WITH THE LATEST REGION ASSIGNMENT OVERTAYING THE }\end{array}$ \\
\hline $\begin{array}{l}\mathrm{CN} \\
\mathrm{CN}\end{array}$ & & $\begin{array}{l}\text { PREVIOUS CONFIGURATION, OR USING THE USIJAL PROCEDURE, } \\
\text { WITH EACH REGION'S BOUNDARIES GIVEN EXPIICITLY. }\end{array}$ \\
\hline $\mathrm{CN}$ & & REGION LABELS MUST BE NON-BLANK. \\
\hline $\begin{array}{l}\mathrm{CN} \\
\mathrm{CN}\end{array}$ & & $\begin{array}{l}\text { THE MESH FOR A DIRECTION MUST BE COMPLETEIY } \\
\text { SPECIFIED EITHER ON THE TYPE O6 OR O9 CARDS. IF MESH }\end{array}$ \\
\hline $\begin{array}{l}\mathrm{CN} \\
\mathrm{CN}\end{array}$ & & $\begin{array}{l}\text { DATA ARE SUPPI.IED ON BOTH TYPE } 26 \text { AND } 09 \text { CARDS, THE } \\
\text { TYPE } 09 \text { DATA VILL BE USED. FOR ONE-DIMENSIONAL }\end{array}$ \\
\hline $\begin{array}{l}\mathrm{CN} \\
\mathrm{CN}\end{array}$ & & $\begin{array}{l}\text { PROBLEMS, ONLY THE "X"-DIRECTION UPPER BOUNDARIES NEED } \\
\text { BE GIVEN FOR REGIONS AFTER THE FIRST. }\end{array}$ \\
\hline C & & \\
\hline
\end{tabular}

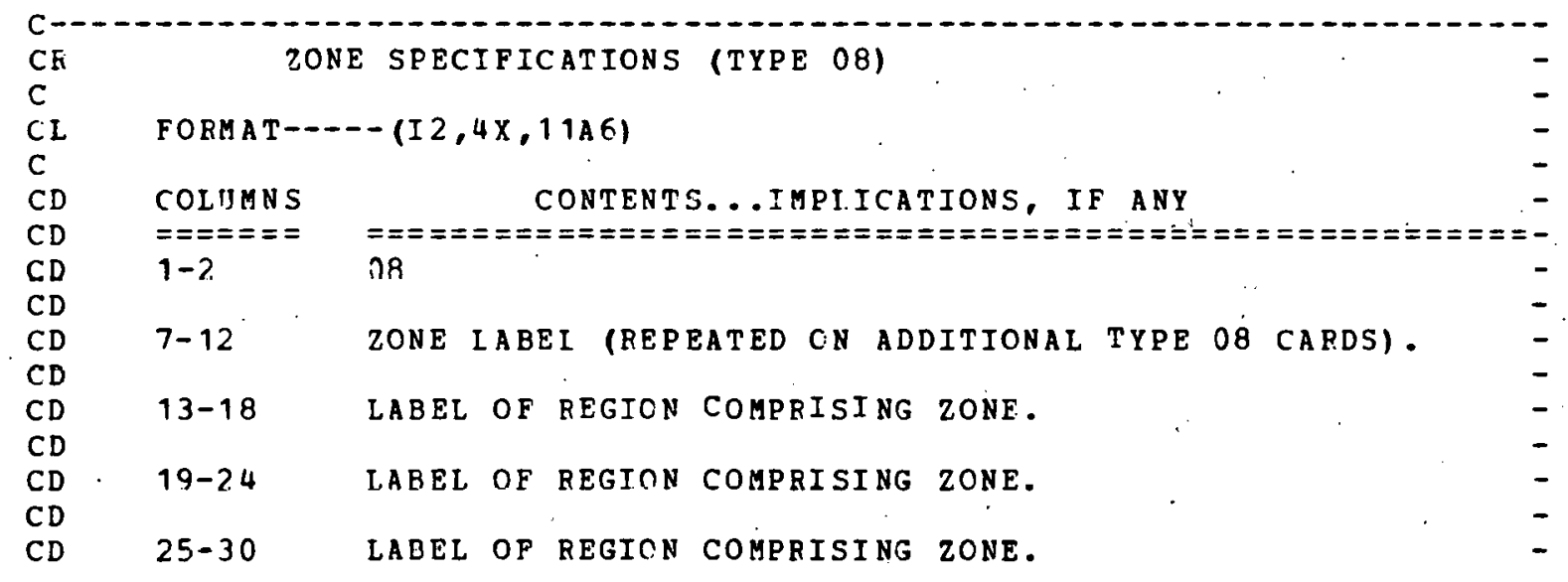




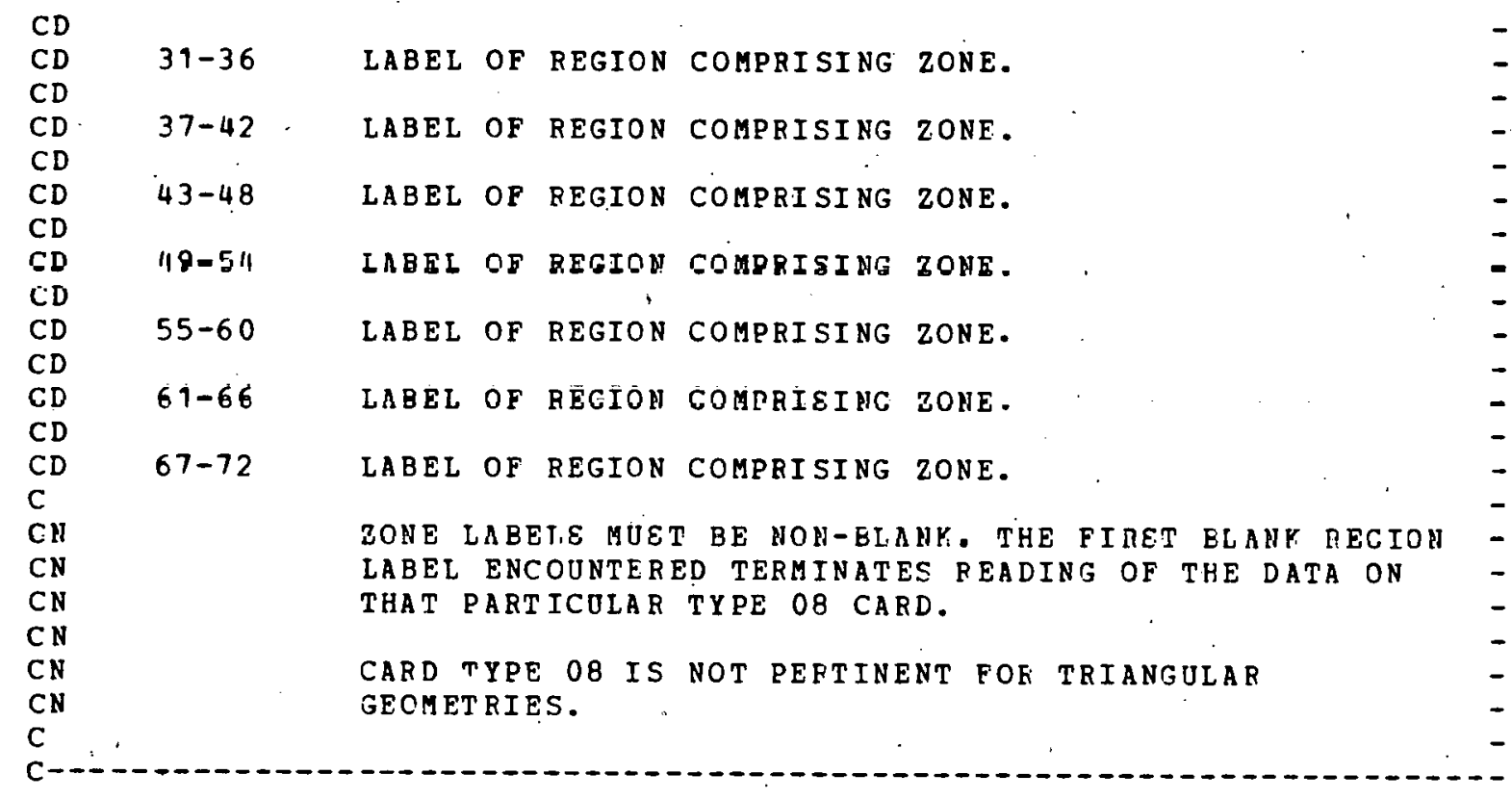

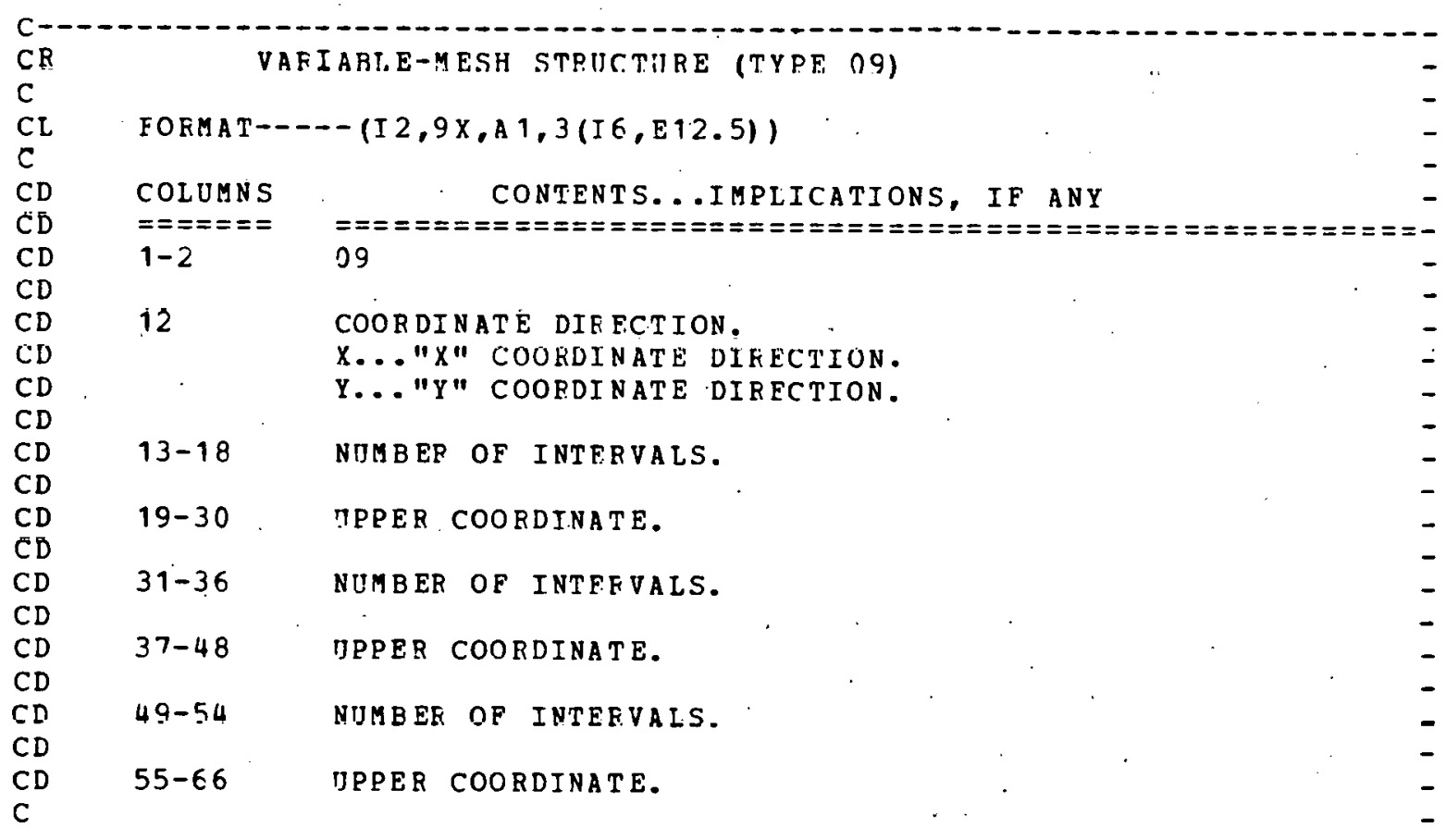




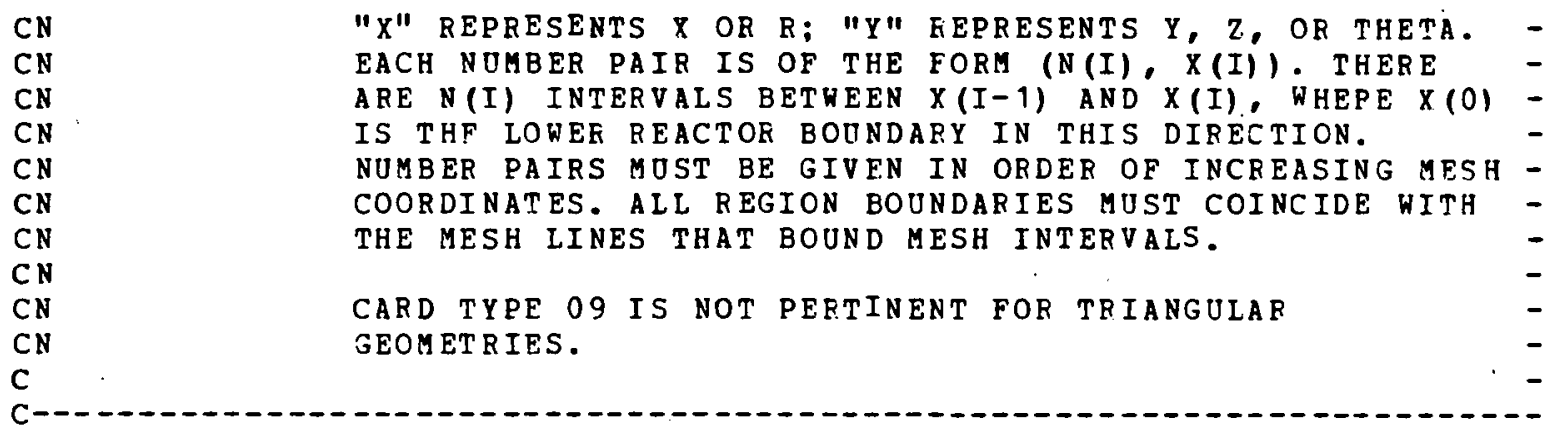

\begin{tabular}{|c|c|c|c|}
\hline $\begin{array}{l}C R \\
C\end{array}$ & & ITE-GEOMETRY TRANVERSE DISTANCE (TYPE 12) & - \\
\hline CL & FORMAT-- & $--(I 2,4 X, A 6,2 E 12.5)$ & - \\
\hline c & & & - \\
\hline CD & COLUMNS & CONTENTS... IMPLICATIONS, IF ANY & - \\
\hline$C D$ & $==ニ=ニ=$ & 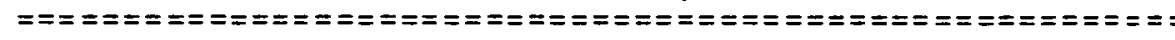 & $=-$ \\
\hline CD & $1-2$ & 12 & - \\
\hline CD & & & - \\
\hline CD & $7-12$ & REGION LABEL. & - \\
\hline$C D$ & & & - \\
\hline CD & $13-24$ & ACTUAL TRANSVERSE HALF-HEIGHT OR RADIUS. & - \\
\hline CD & & & - \\
\hline CD & $25-36$ & TRANSVERSE EXTRAPOLATION DISTANCE. & - \\
\hline $\mathrm{C}$ & & & . \\
\hline CN & & IF THERE IS NO REGION LABEL (COLS. $7-12$ ARE RLANK), & - \\
\hline $\mathrm{CN}$ & & THE DATA ON THE CARD APPLY TO ALI REGIONS OF THE & - \\
\hline CN & & REACTOR. IF THERE IS NO REGION LABEL AND IF THERE ARE & - \\
\hline CN & & NO TYPE 34 CARDS (ZONE AND GROUP DEPENDENT BUCKLING & $\boldsymbol{-}$ \\
\hline $\mathrm{CN}$ & & SPECIFICATIONS), THE CATA ON THIS CARD WIIL EE USED & - \\
\hline $\mathrm{CN}$ & & TO CALCULATE A SPACE- AND ENFRGY-INDEPENDENT BUZKLIN3 & - \\
\hline $\mathrm{CN}$ & . & AND TO CALCULATE REGION VOLUMES. IN THIS MODE OF INPUT. & - \\
\hline CN & & ONIY ONE TYPE 12 CARD SHOULD BE SUPPLIED. & - \\
\hline $\mathrm{CN}$ & & & - \\
\hline $\begin{array}{l}\mathrm{CN} \\
\mathrm{CN}\end{array}$ & & $\begin{array}{l}\text { IF MORE THAN ONE TYPE } 12 \text { CARD IS PRESENT (EACH CARD } \\
\text { WITH A VIID REGION LABEL IN COLS. 7-12), THE }\end{array}$ & $\overline{-}$ \\
\hline $\mathrm{CN}$ & & DATA ON THE CARDS HILI BE USED TO CALCULATE REGION & - \\
\hline $\mathrm{CN}$ & & VOLUMES. IF BUCKLINGS ARE REQUIRED, THEY MUST BE & - \\
\hline $\mathrm{CN}$ & & SPECIFIED ON TYPE 34 CARDS. & - \\
\hline $\mathrm{CN}$ & & | & - \\
\hline CN & & REGION VOLUMES ARE CALCULATED USING THE HALF-HEIGHT & - \\
\hline $\mathrm{CN}$ & · & (EXCLUDING THE EXTRAPOIATION DISTANCE). & - \\
\hline CN & & & - \\
\hline $\mathrm{CN}$ & & $\begin{array}{l}\text { DATA ON THIS CARD MAY BE OVERLAYED. THAT IS, } \\
\text { TRANSVERSE DISTANCES DEFINED ON IATER TYPE } 12\end{array}$ & - \\
\hline $\mathrm{CN}$ & & CARDS SUPERCEDE DATA FCR REGIONS PREVIOUSLY & - \\
\hline
\end{tabular}




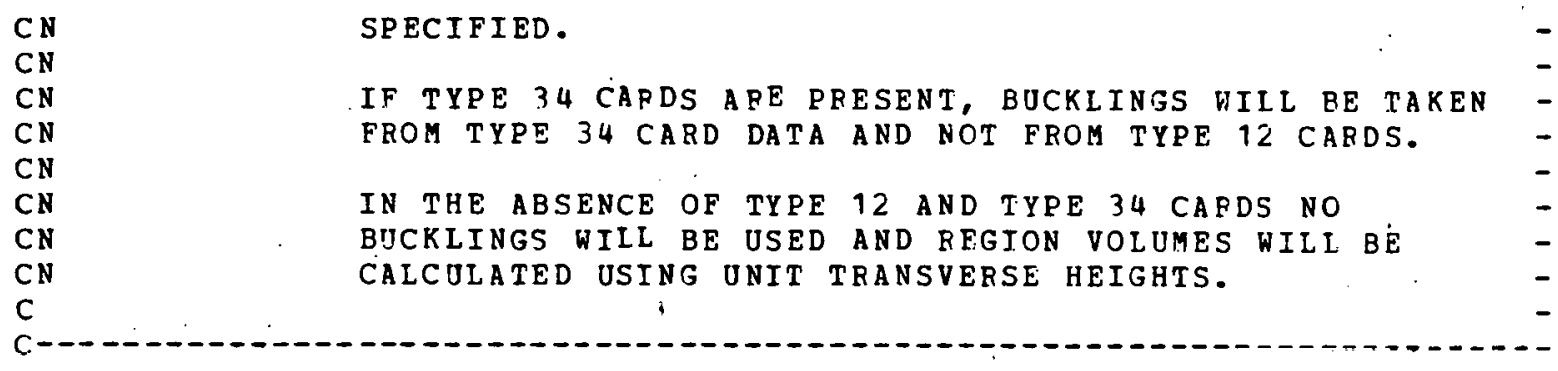

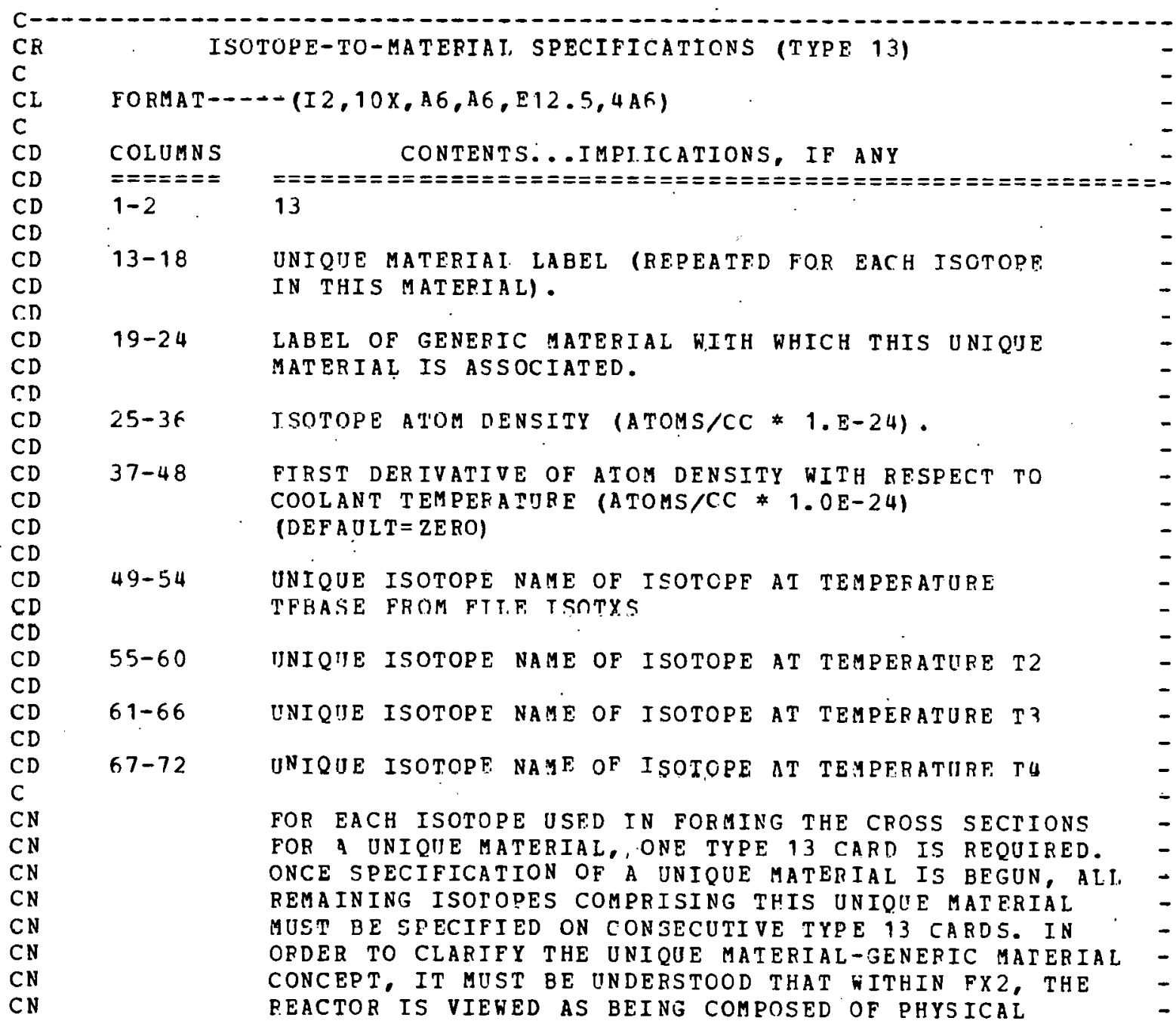


MATERIALS SUCH AS URANIUM OXIDE, PLTTONIUM OXIDE, STAINLESS STEEL, BOBON CARBIDE, ETC. EACH PHYSICAL MATERTAL IS ASSUMED TO HAVE A FIXED ISOTOPIC CONTENT DURING A TRANSIENT.

SINCE FX2 EMPLOYS THE MULTIGROUP APPROXIMATION, IT WILI OFTEN BE DESIRABLF TO IISE DIFFERENT CROSS SECTIONS FOR A PARTICULAR PHYSICAL MATERIAL IN DIFPERENT REGIONS OF THE REACTOR. THIS IS ACCOMPIISHFD IN PX2 BY CEEATING SEVEKAL UNIQUE MATERIALS, EACH. WIIH ITS OWN CROSS SECTIONS AND UNIQUE MATERIAI. LABEL, BUT ALL SHARING THE SAME GENERIC MATERIAL LABEL. THIS, URANIUM OXIDE IN THE INNER AND OUTER COFE FEGIONS MIGHT BE DESIGNATED BY THE UNIQUE MATERIAI LABELS UO2-IC AND UO2-OC, RESPECTIVELY, WITH BOTH SHARING THE GENERIC MATFRIAL LABEL UO2. BECAUSE CORE STORAGE REQUIREMENTS AND COMPUTING TIMES ARE DEPENDENT ON THE NUMBER OF GENERIC MATERIALS MUCH MORE STRONGLY THAN ON THE NUMBER OF UNIQUE MATERIALS, IT IS IN THE ISSER'S INTEREST TO ATTEMPT TO MINIMIZE THE NUMBER OF GENERIC MATERTALS.

IN CONSTRUCTING EACH UNIQUE MATERIAL, ISOTOPES ARE IDENTIFIED BY THEIR UNIQUE ISOTOPE NAMES (FFOM THE HISONM ARRAY) ON THE ISOTXS FILE. EITHEP ONE UNIQUE ISOTOPE NAME OR FOUP UNIQUE ISOTOPE NAMES MUST APPEAR ON EACH TYPE 13 CARD. IF FOUP APPEAR ON A CARD, THEY MUST SHARE A COMMON ABSOLUTE ISOTOPE NAME (HABSIDI ON ISOTXS.

IF THE CAPTURE AND FISSION CFOSS SECTIONS OF A PARTICULAR UNIQUE MATERIAL ABE TO BE TEMPERATUREDEPENDENT, AT LEAST ONF OF THE ISOTOPES COMPRISING IT MUST HAVE FOUR INIQUE ISOTOPE NAMES SPFCIFIED ON ITS TYPE 13 CARD. PX2 CCNSTFUCTS MACROSCOPIC CAPTURE AND FISSION CPOSS SECTICNS AT TEMPERATUEES TBASE, T2, T3. AND T4 FOR THIS TNIQUE MATERIAL AND EXTRACTS THEIR TEMPERATURE DEPENDENCE IN THE FORM OF FITTING COEFFICIENTS. FOR EACH GROUP, G, COEFFICIENTS A1, A2, A3. A 4, 5, AND A6 ARE COMPUTED SO THAT THE CAPTURE AND FISSION CROSS SECTIONS FOR THIS GROUP AT SOME TEMPERATURE T, $\operatorname{SIGCAP}(G, T)$ AND SIGFIS $(G, T), C A N B E$ COMPUTED AS FOLLOWS:

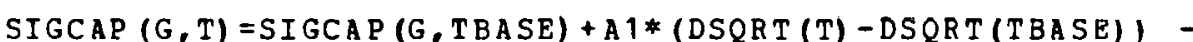
$+A 2 *(D L O G(T / T B A S E))+A 3 *(T B A S E-T) /(T B A S E * T)$

SIGFIS (G, T) =SIGFIS (G,TBASE) + A4* (DSQRT (T) - DSQRT (TBASE)) $+A 5 *(D L O G(T / T B A S E))+A 6 *(T B A S E-T) /(T B A S E * T)$ - 


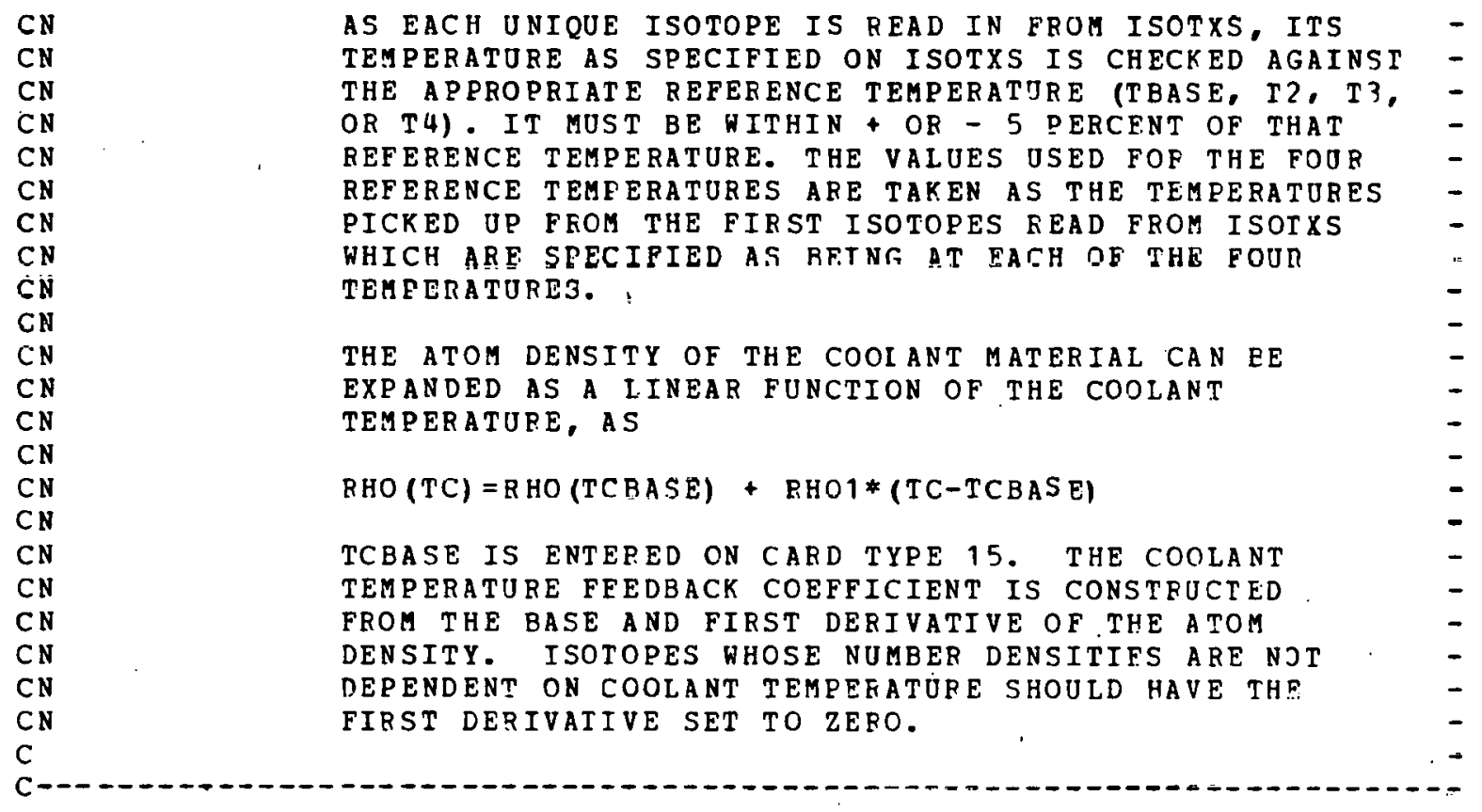

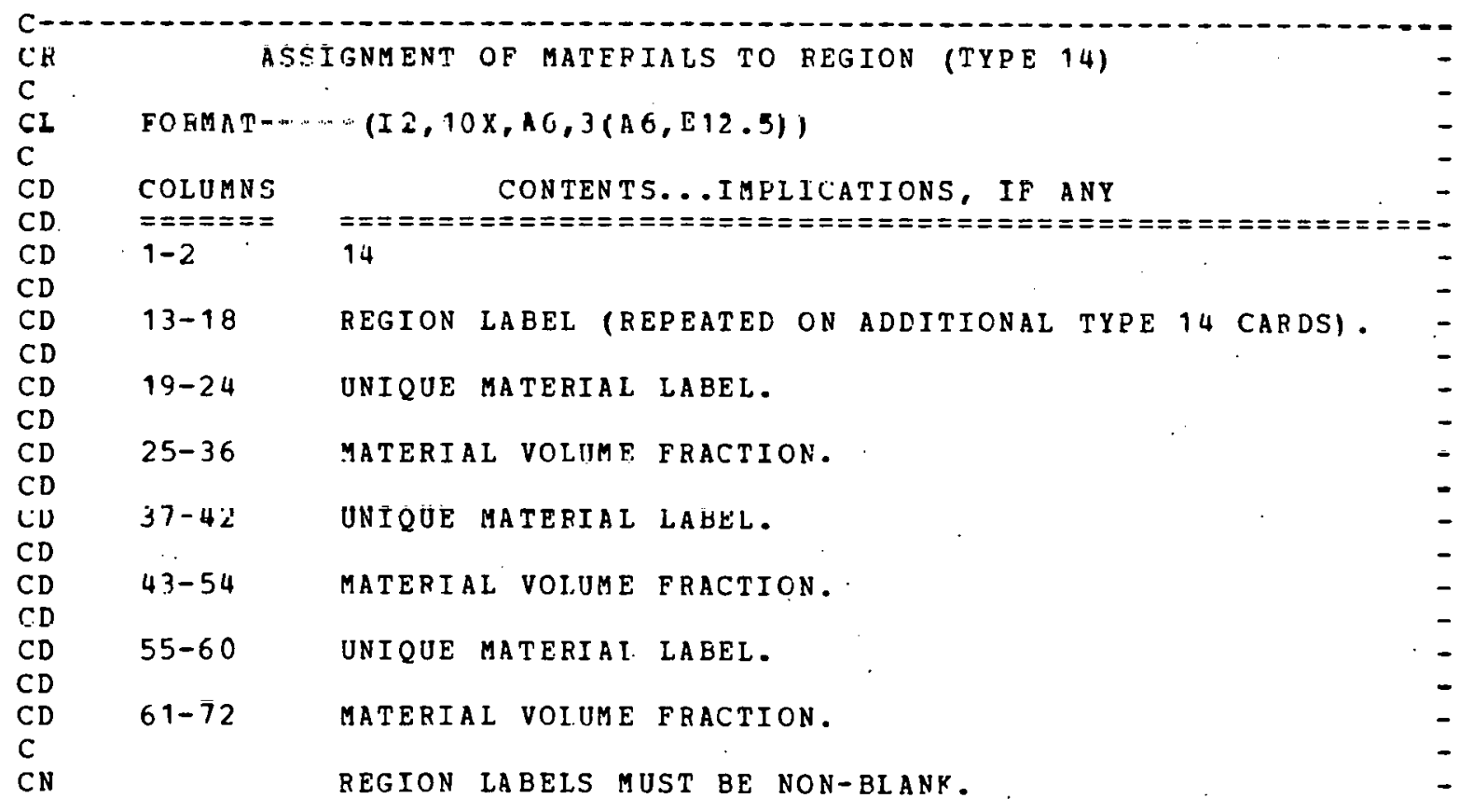




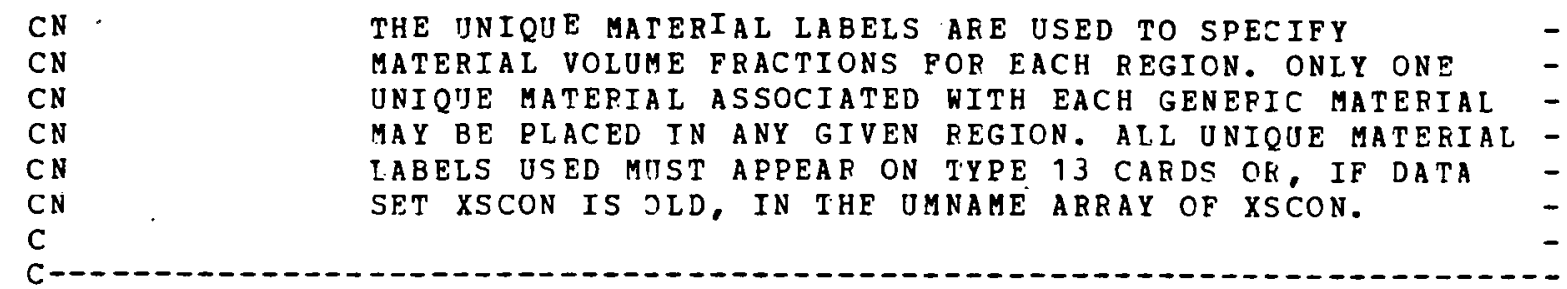

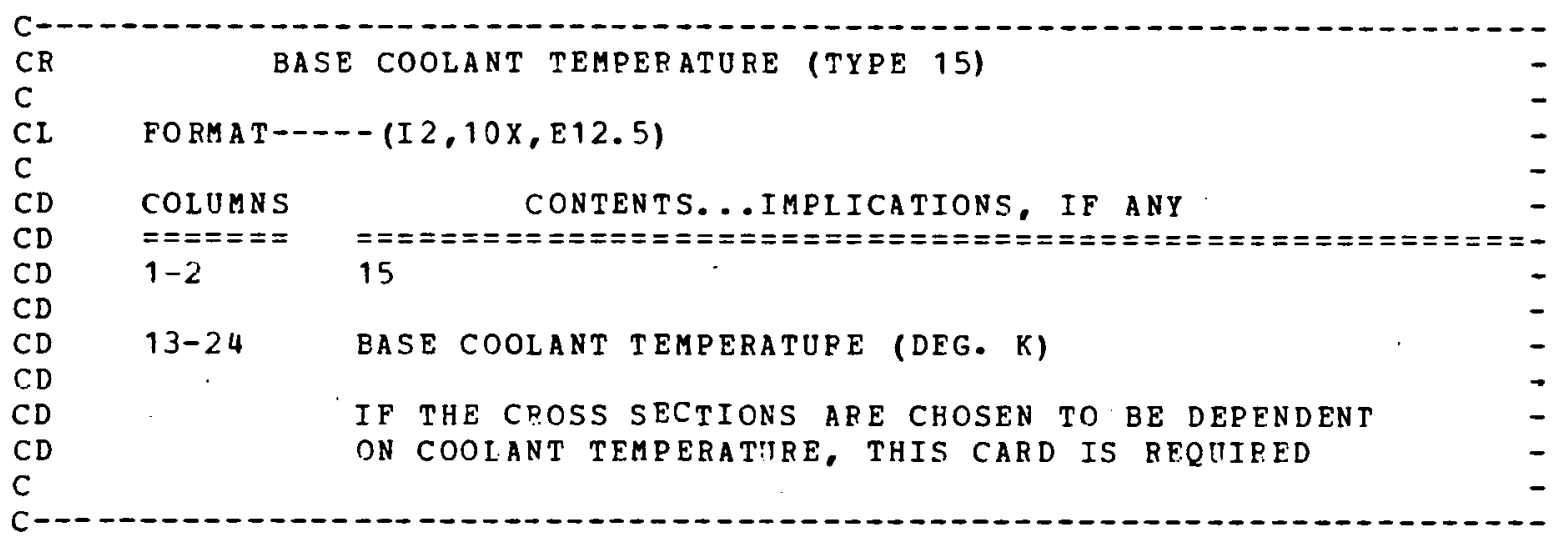

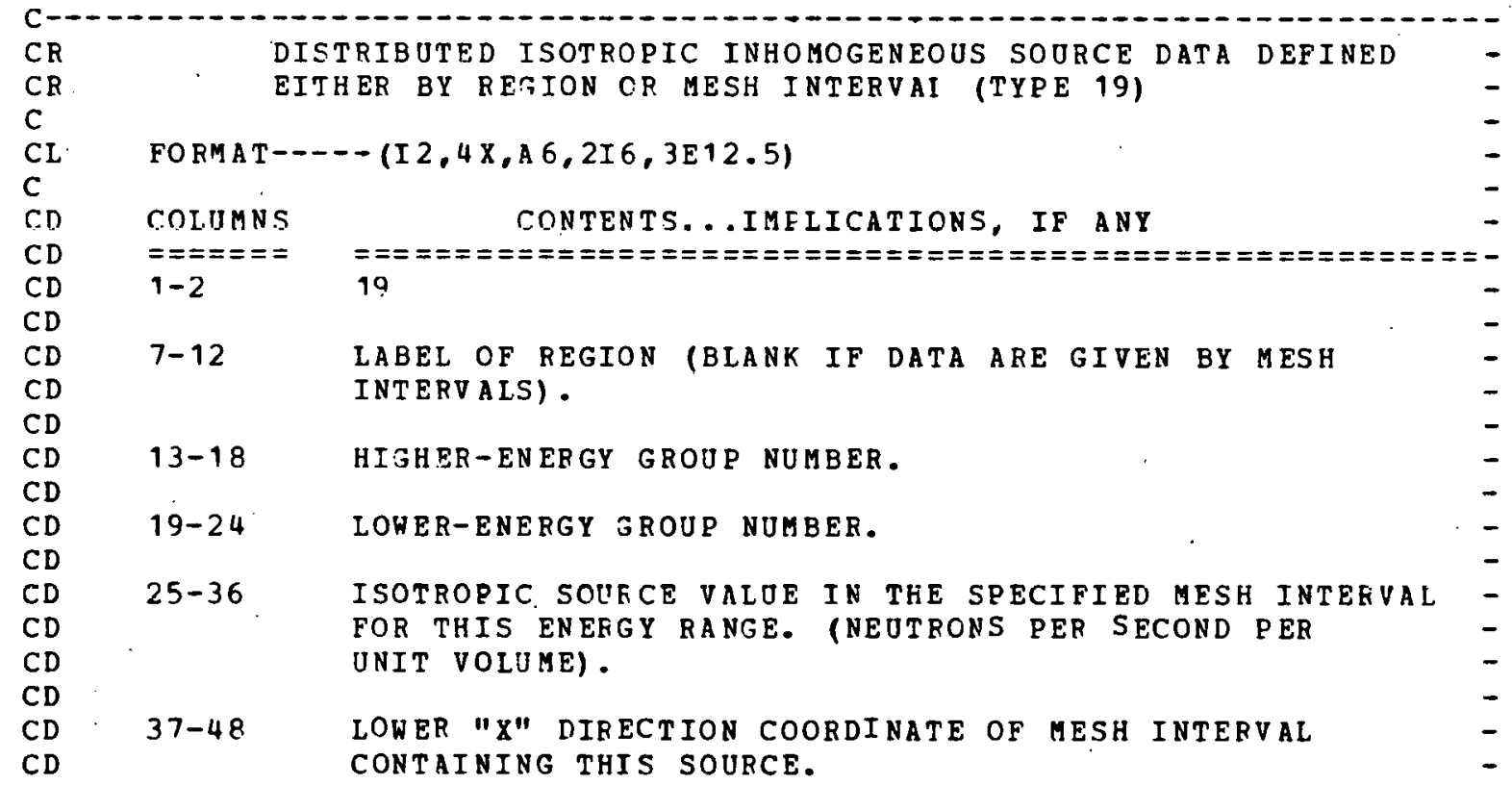




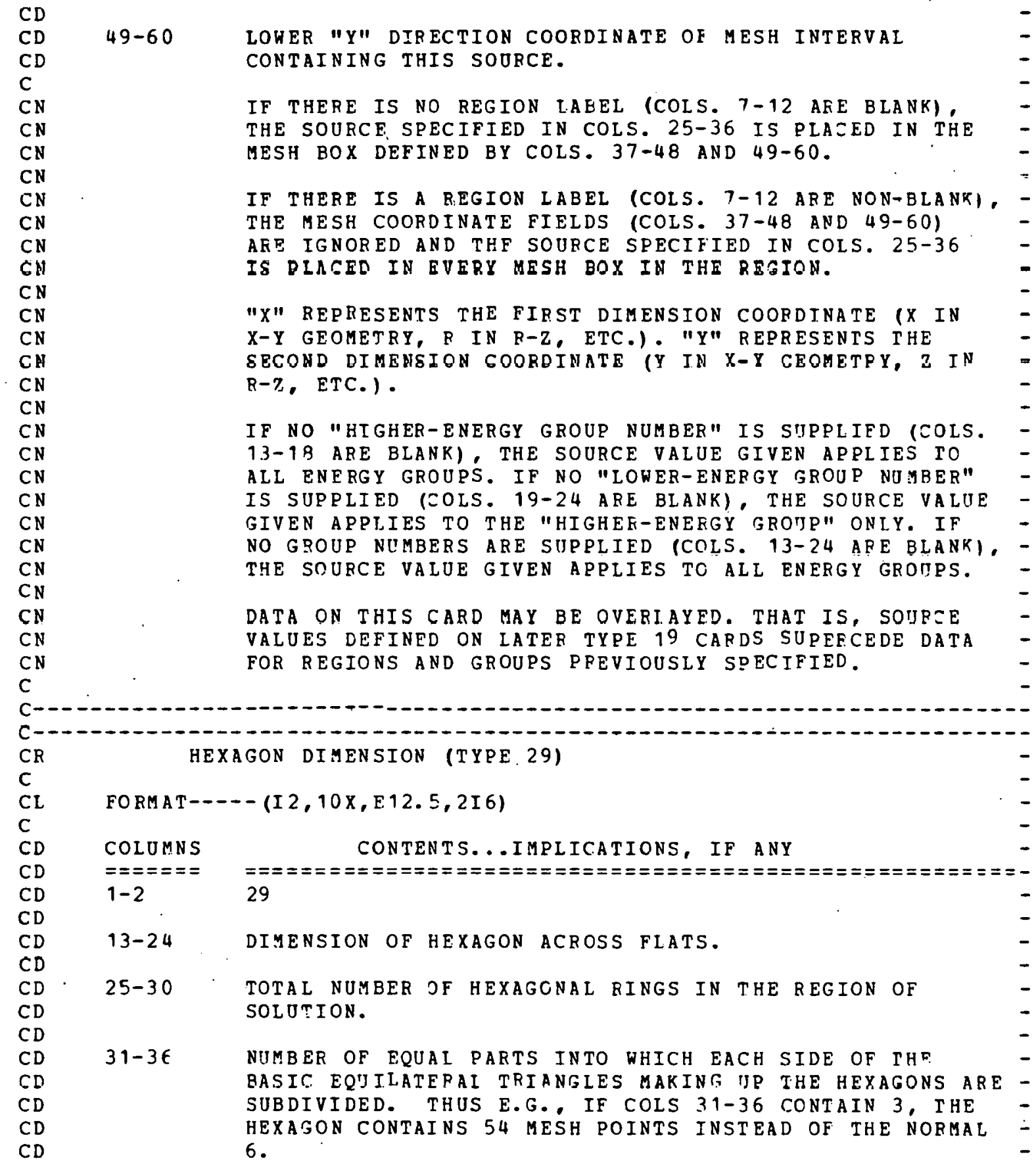


C

$\mathrm{CN}$

CN

CN

CN

$\mathrm{CN}$

$\mathrm{CN}$

$\mathrm{CN}$

CN

$\mathrm{C}$

$$
\text { c }
$$

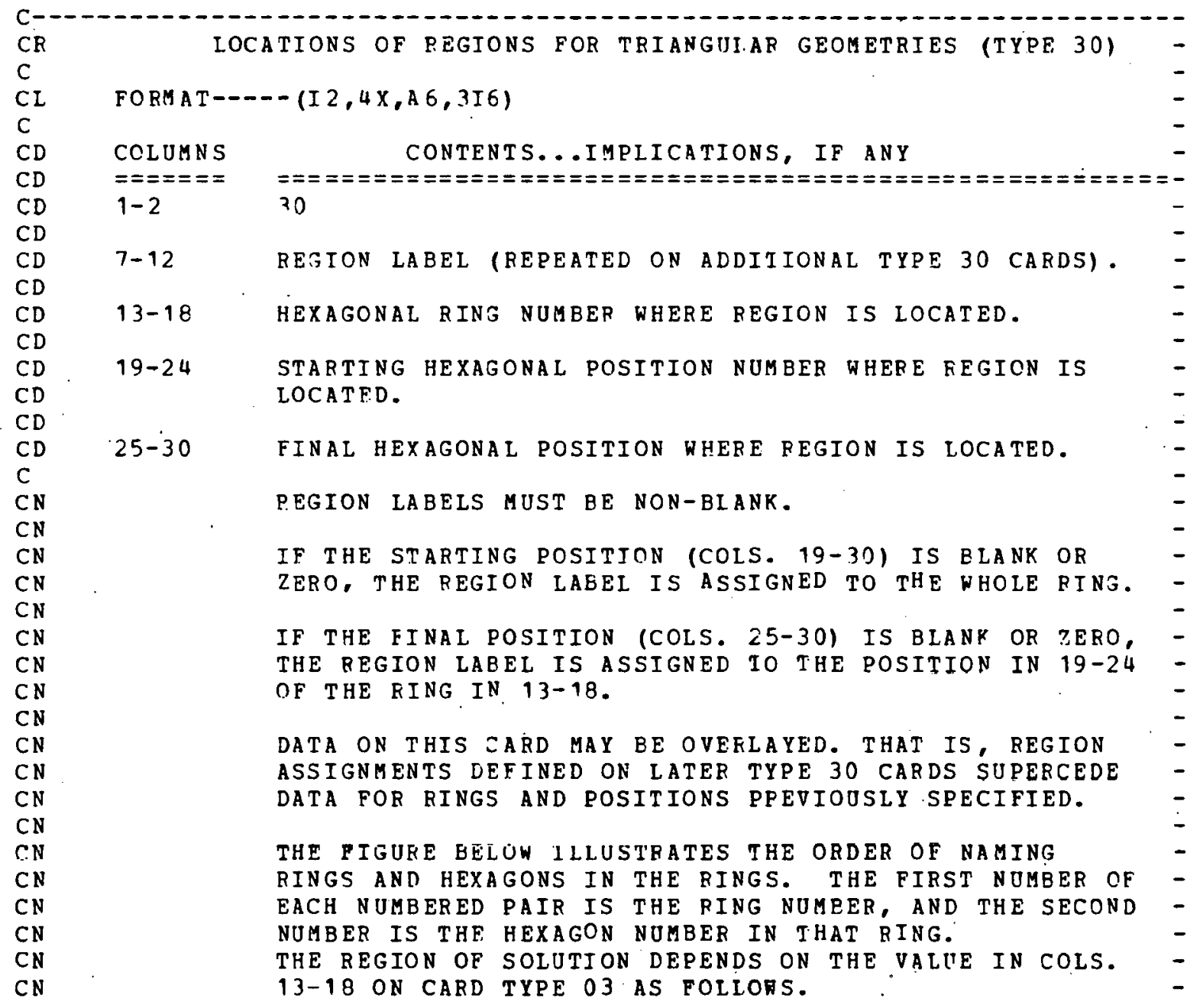

IF THE NIMBER OF RINGS IS NOT PROVIDED IN COLS. 25-30, IT IS DERTVED FROM THE TYPE 30 CARDS.

IF COLS. 31-36 ARE BLANK, THE TRIANGLES APE NOT SUBDIVIDED.

THE TYPE 29 CARD IS PERTINENT ONLY IF COLS. $13-18$ ON CARD TYPE 03 ARE GREATER THAN EI (TRIANGULAR GEONETPY).- 


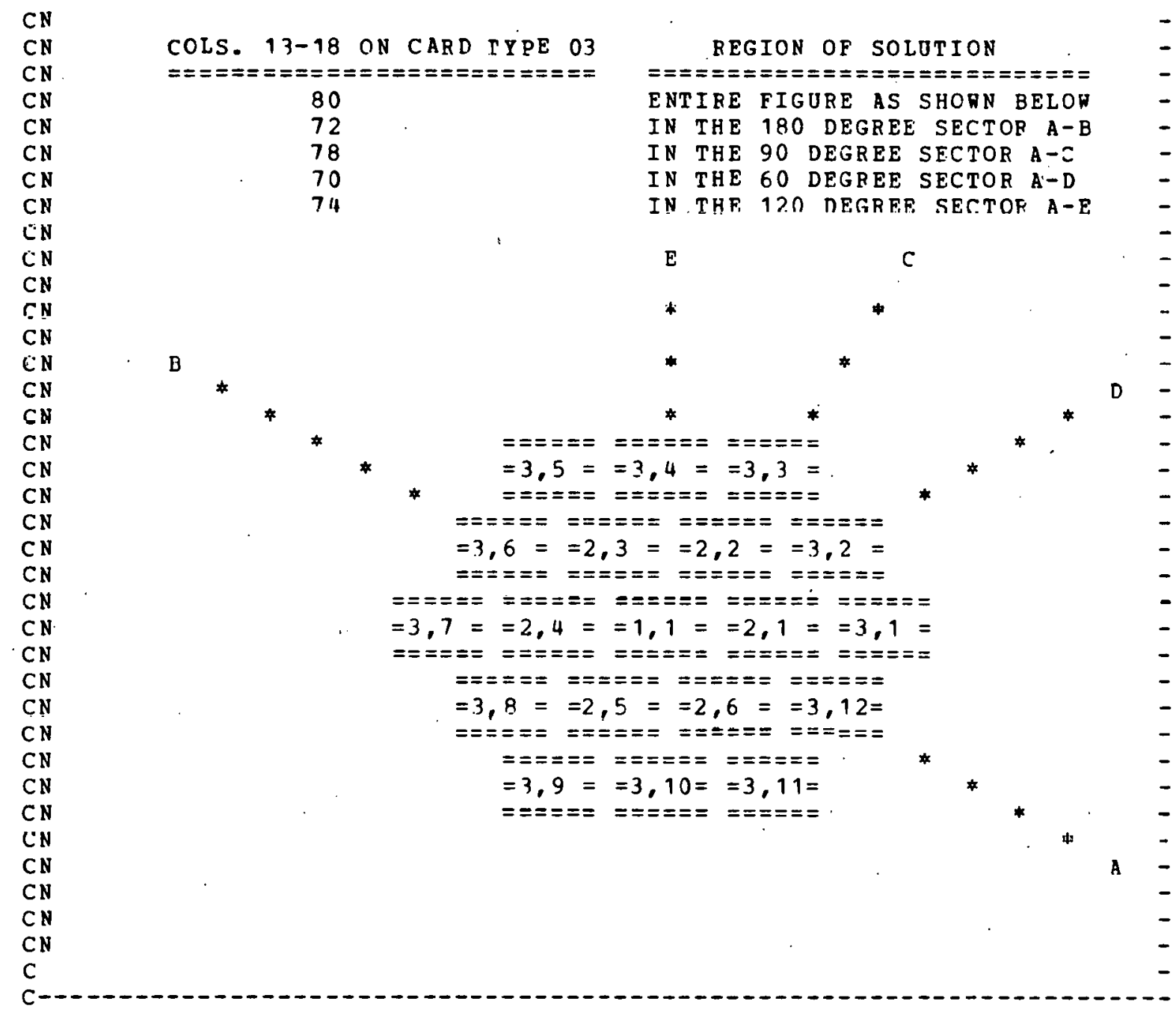

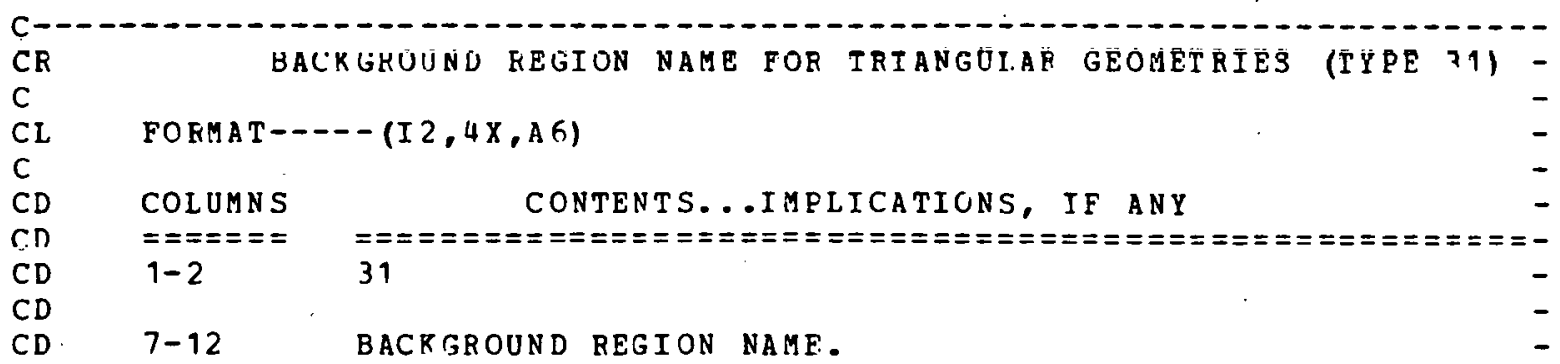




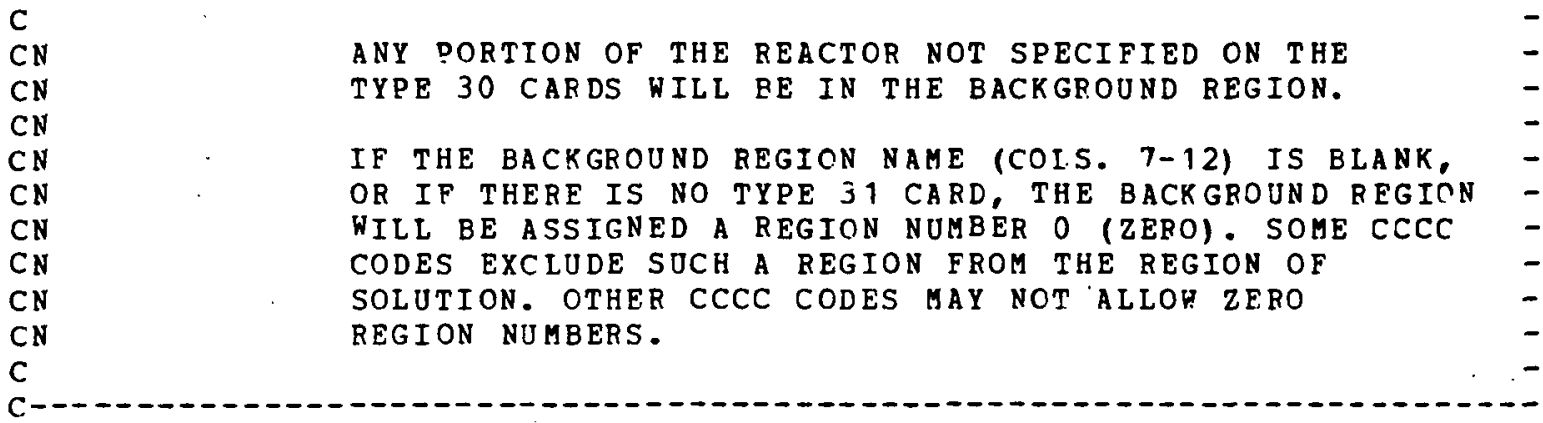

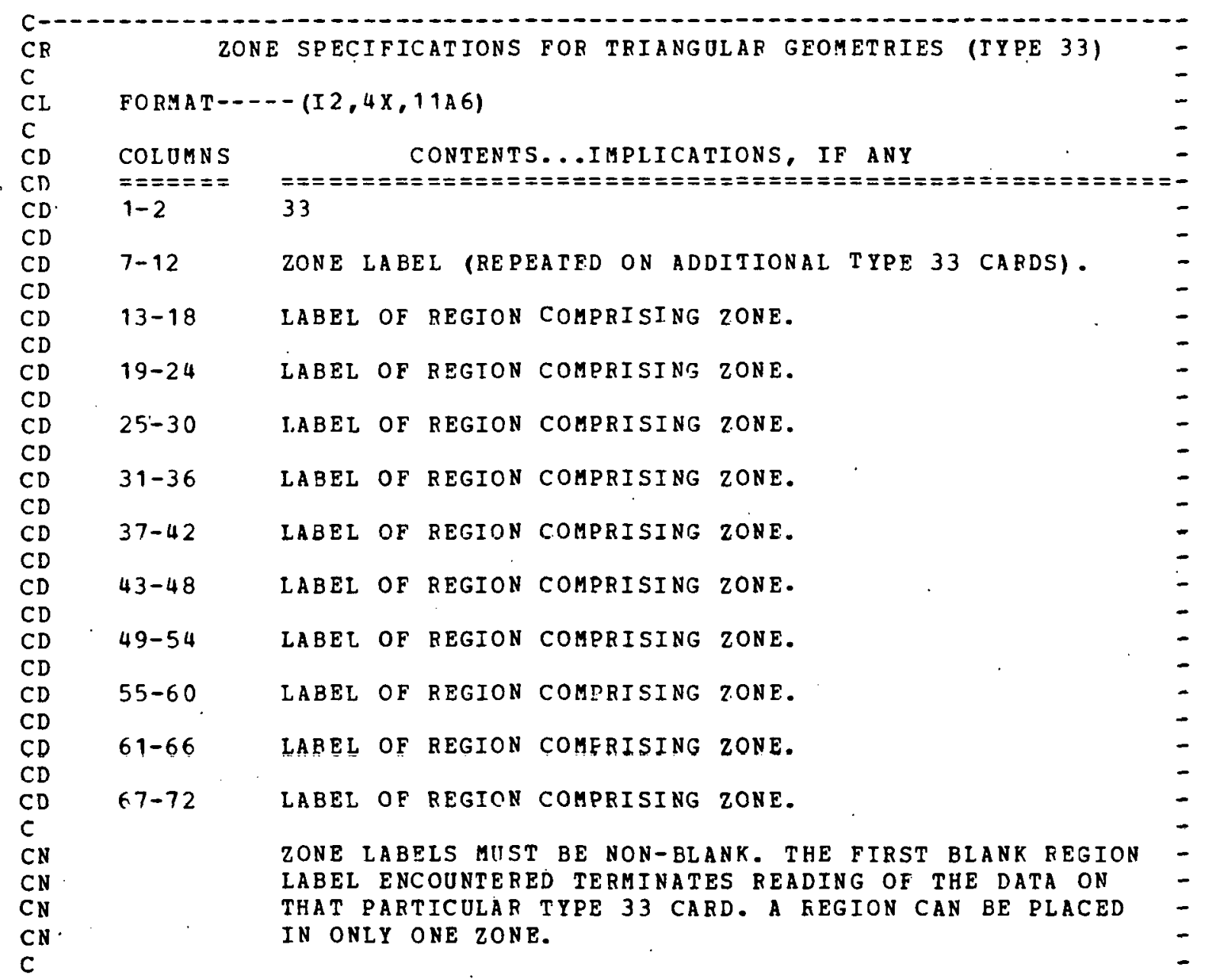




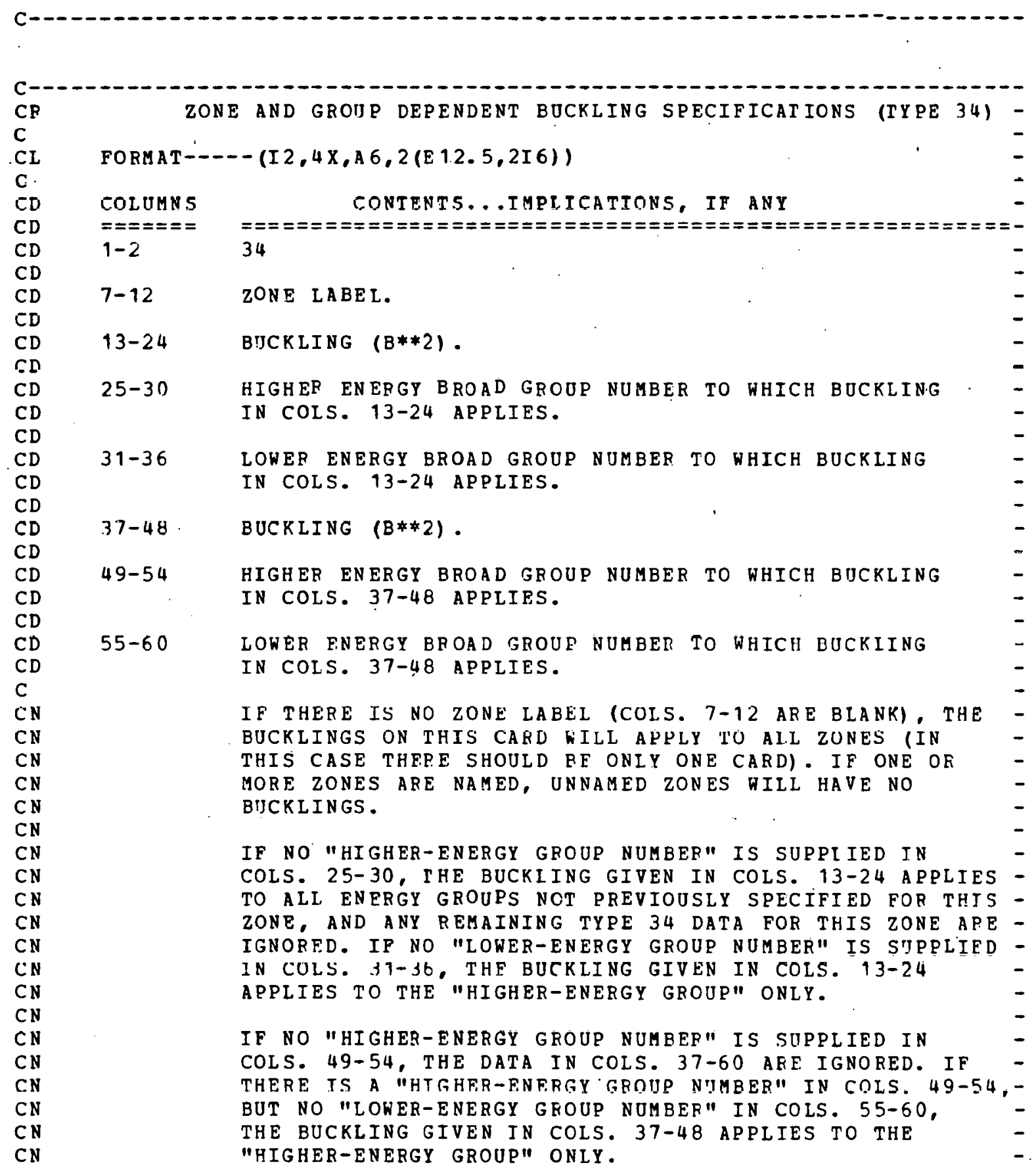




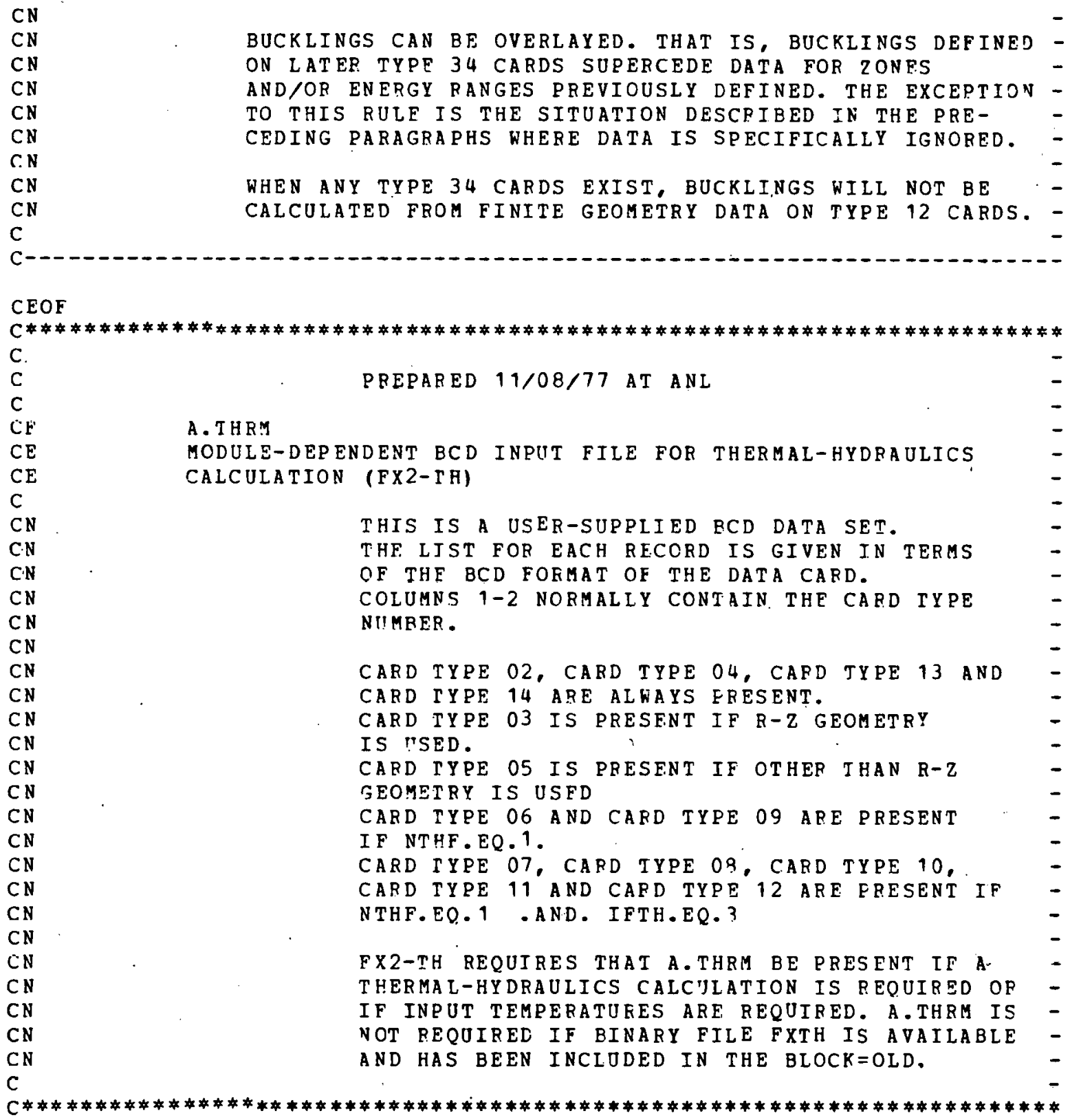

BUCKLINGS CAN BE OVERLAYED. THAT IS, BUCKIINGS DEFINED AND/OR ENERGY RANGES PREVIOUSLY DEFINED. THE EXCEPTION TO THIS RULE IS THE SITUATION DESCPIBED IN THE PRECEDING PARAGRAPHS WHERE DATA IS SPECIFICAILY IGNORED.

WHEN ANY TYPE 34 CARDS EXIST, BUCKLINGS WILL NOT BE CALCULATED FROM FINITE GEOMETRY DATA ON TYPE 12 CARDS. -

CEOF

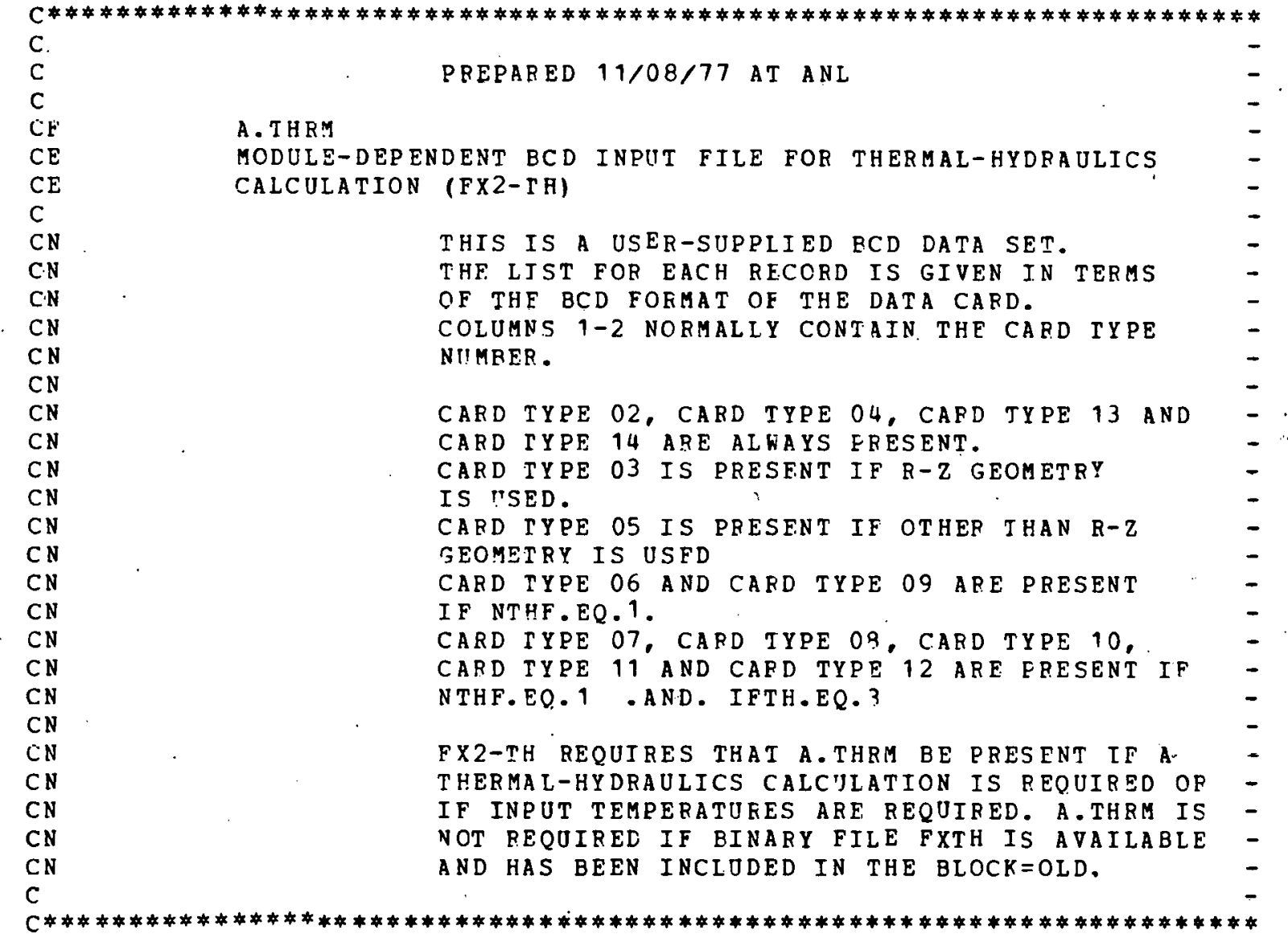

CR INTEGER CONTROL (TYPE 02) 


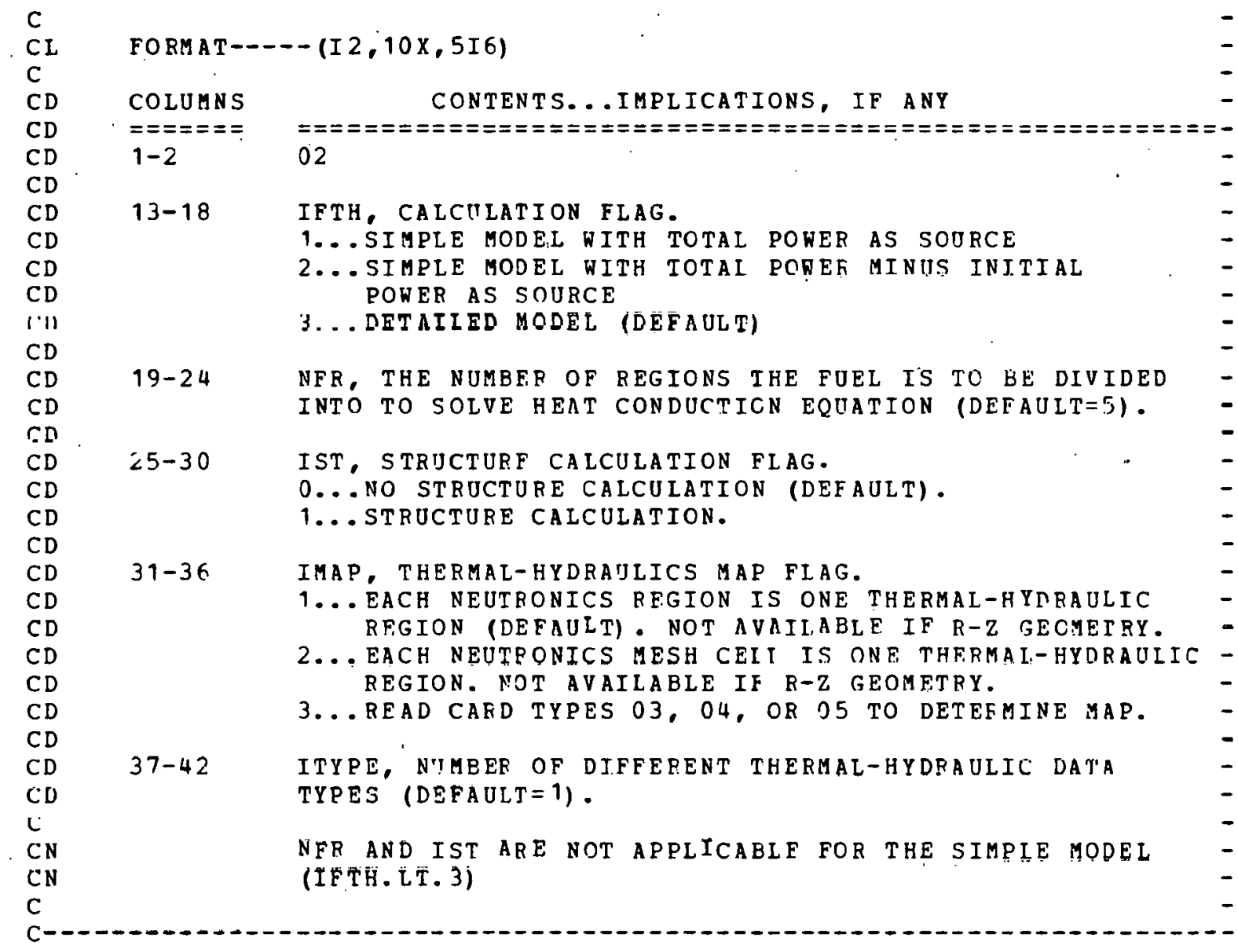

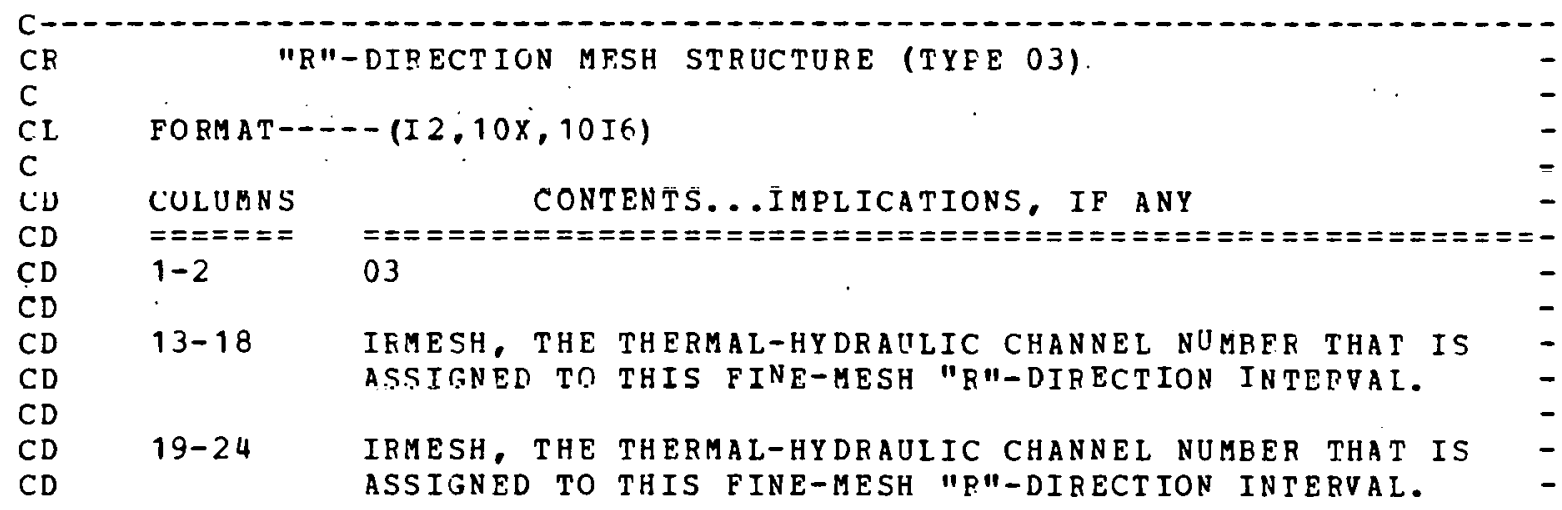


$C D$

$C D$

CD

$C D$

CD

CD

$C D$

$C D$

$C D$

$C D$

$C D$

$C D$

$C D$

$C D$

$C D$

$C D$

$C D$

CD

$C D$

$C D$

$C D$

$C D$

$C D$

C

$\mathrm{CN}$

$\mathrm{CN}$

$\mathrm{CN}$

$\mathrm{CN}$

$\mathrm{CN}$

$\mathrm{CN}$

$\mathrm{CN}$

$\mathrm{CN}$

$\mathrm{CN}$

CN

C$$
\text { C }
$$

$25-30$

IRMESH, THE THERMAL-HYDRAULIC CHANNEL NUMBER THAT IS ASSIGNED TO THIS FINE-MESH "R"-DIRECTION INTERVAL.

$31-36$

TPMESH, THF THERMAI-HYDRAJIIC CHANNEL NUMEER THAT IS ASSIGNED TO THIS FINE-MESH "R"-DIRECTION INTERVAL.

$37-42$

IRMESH, THE THERMAL-HYDRAULIC CHANNEL NUMBER THAT IS ASSIGNED TO THIS FINE-MESH "R"-DIRECTION INTERVAI.

$43-48$

IFMESH, THE THERMAL-HYDRAUI IC CHANNEL NUMBER THAT IS ASSIGNED TO THIS FINE-MESH "R"-DIRECTION INTERVAL.

$49-54$

IRMESH, THE THERMAL-HYDRAJIIC CHANNEL NUMBER THAT IS ASSIGNED TO THIS FINE-MESH "F"-DIRECTION INTERVAL.

$55-60$

IRMESH, THE THERMAL-HYDRAULIC CHANNEL NUMEER THAT IS ASSIGNED TO THIS FINE-MESH "P"-DIBECTION INTERVAL.

$61-66$

IRMESH, THE THERMAL-HYDRAUIIC CHANNEL NUMBER THAT IS ASSIGNED TO THIS FINE-MESH "P"-DIRECTION INTEPVAL.

$67-72$

IRMESH, THE THERMAL-HYDRAULIC CHANNEL NUMBER THAT IS ASSIGNED TO THIS FINE-MESH "E"-DIRECTION INTERVAL.

TYPE $O 3$ CARDS ARE ONLY KEAD IF R-Z GEOMETRY IS IJSED AND IMAP IS FNTERED AS 3. IF NO TYPE 03 CARDS ARE PRFSENT (AND GEOMETRY IS R-Z), THEN JSE ONE THERMALHYDRAULIC CHANNEL FOR EACH FINE MESH INTERVAI. IF IRYESH IS INPUT AS 0, THEN THIS "R"-DIRECTION INTERVAL DOES NOT BELONG TO ANY THERMAI-HYDRADLIC CHANNEL.

AS MANY TYPE 03 CARDS ARE USED AS ARE NECESSARY TO SPECIFY THE "R"-DIRECTICN MESH STROCTURE FOR ALL MESH INTERVALS IN THE "R"-DIEHC'ILUN. 


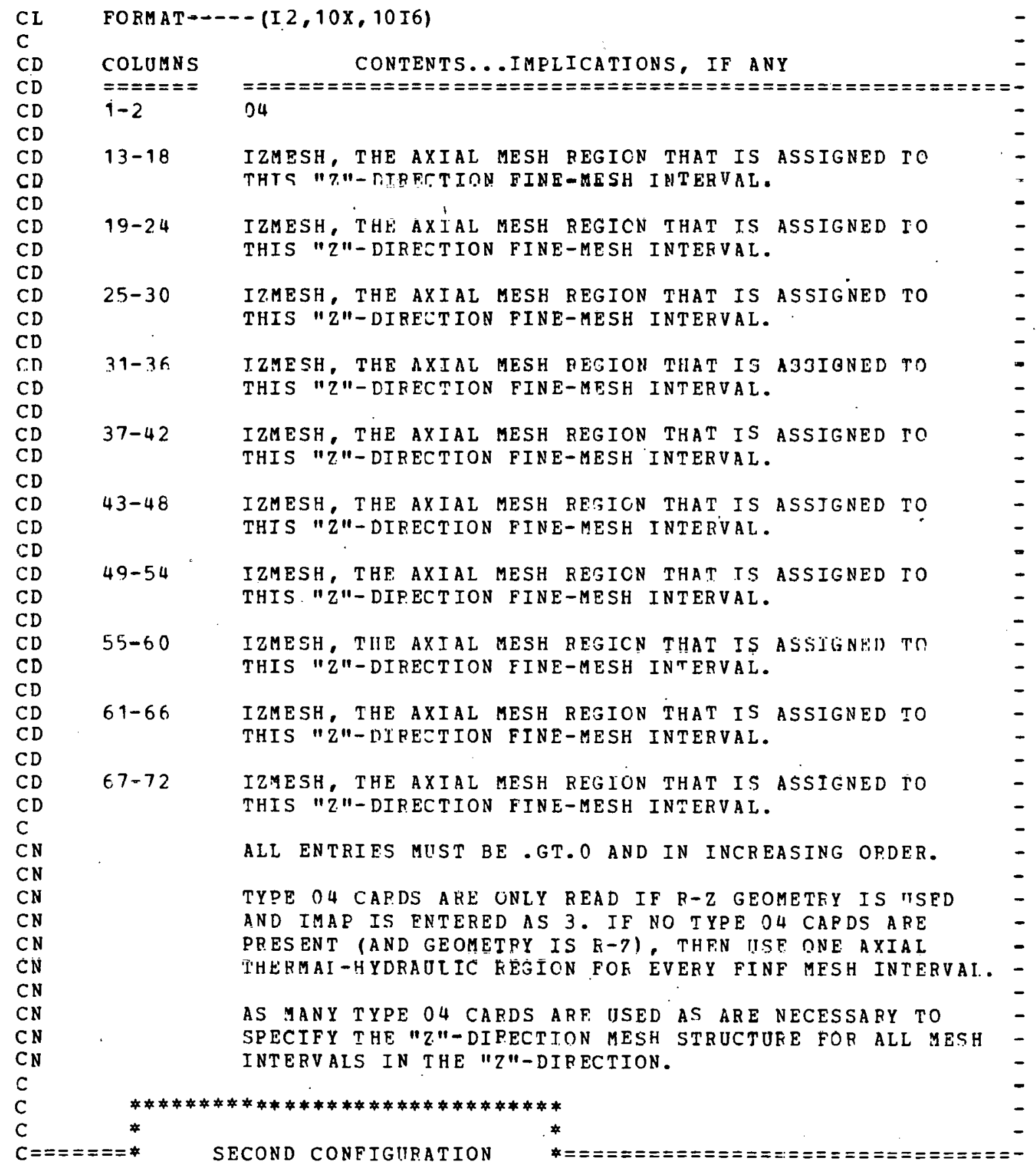




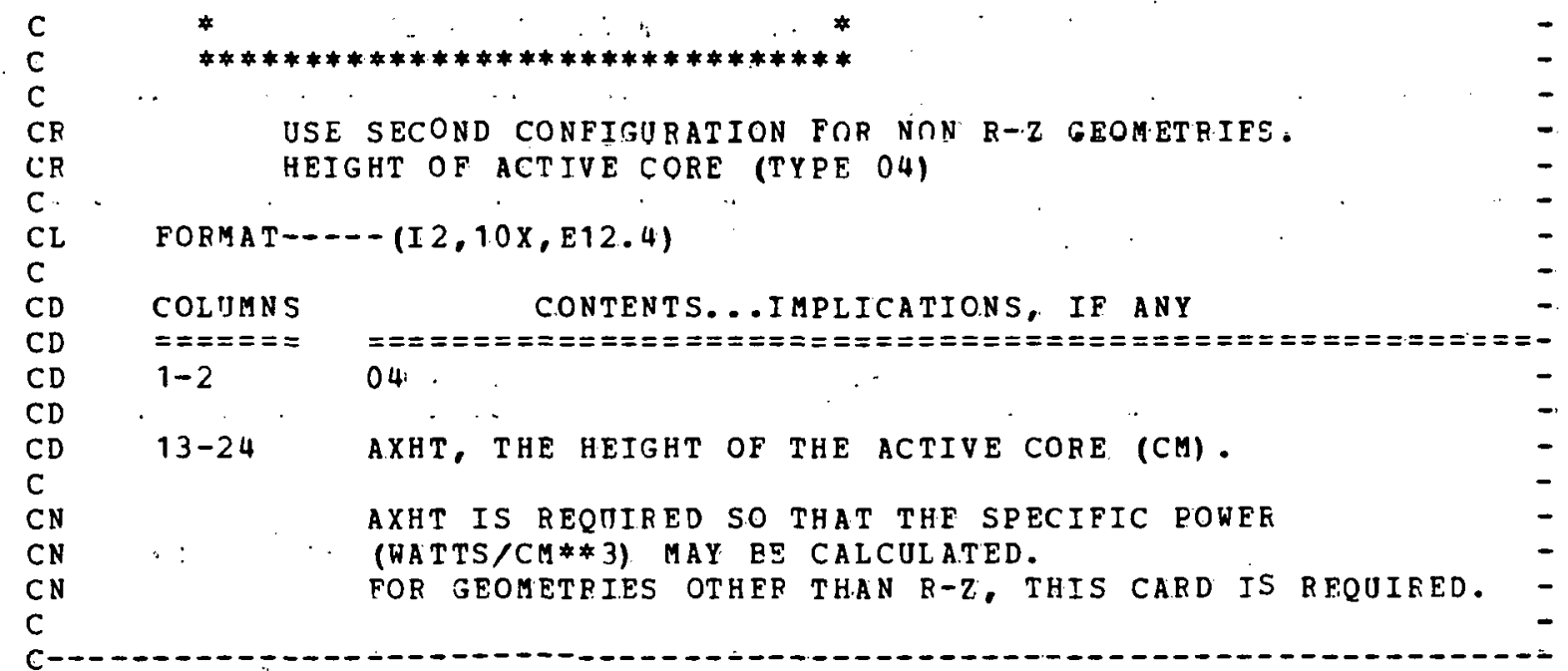

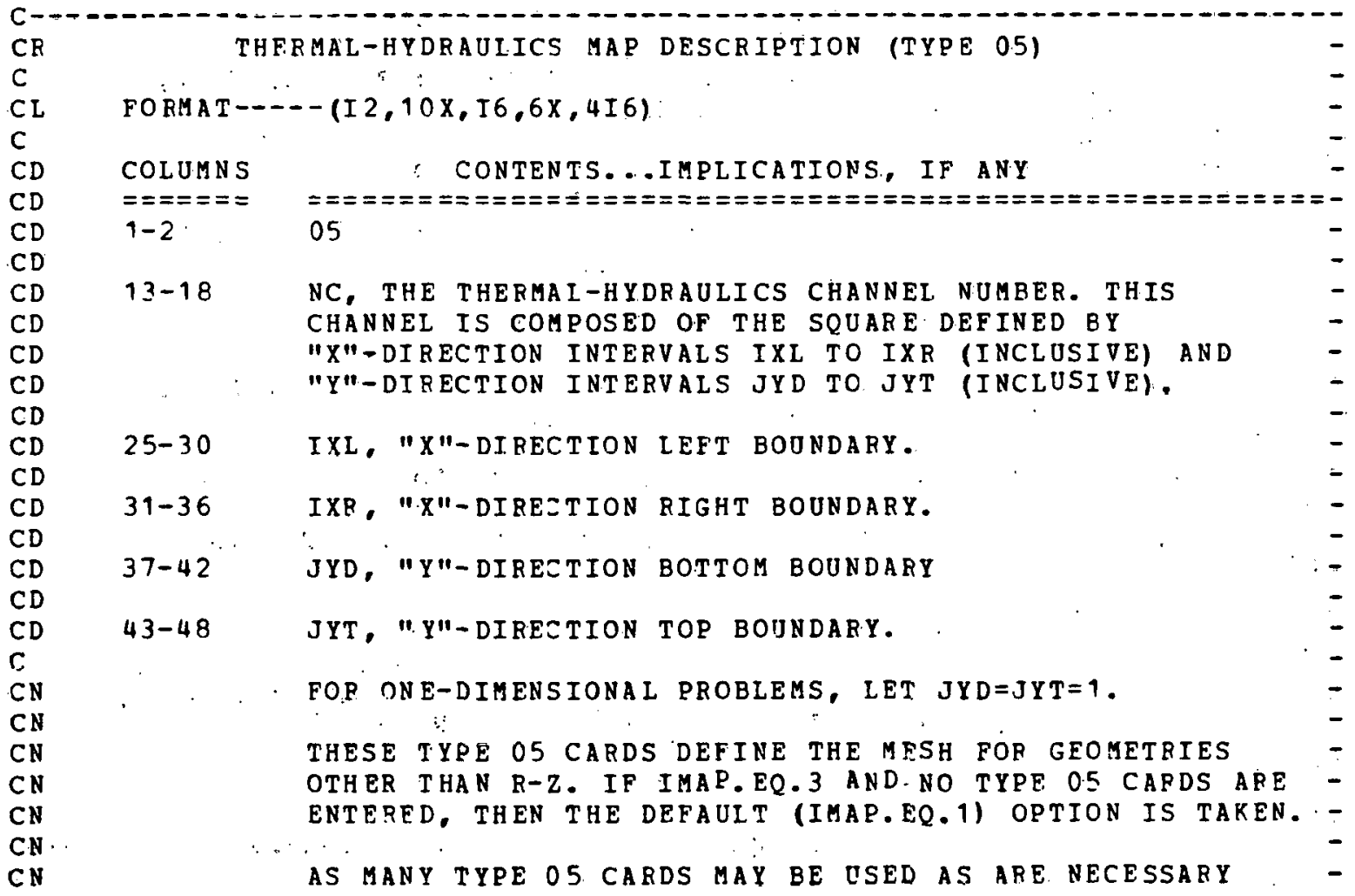




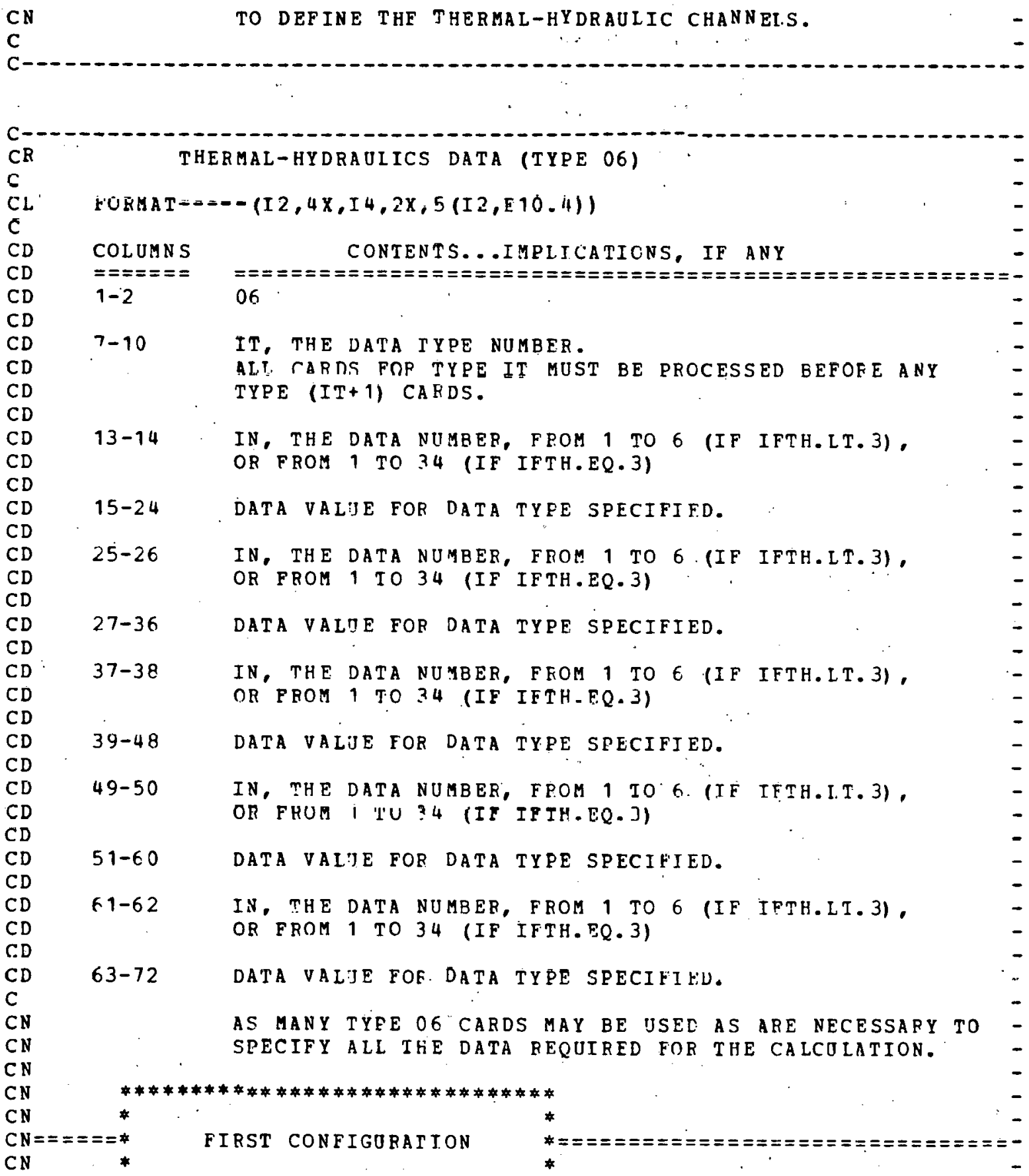




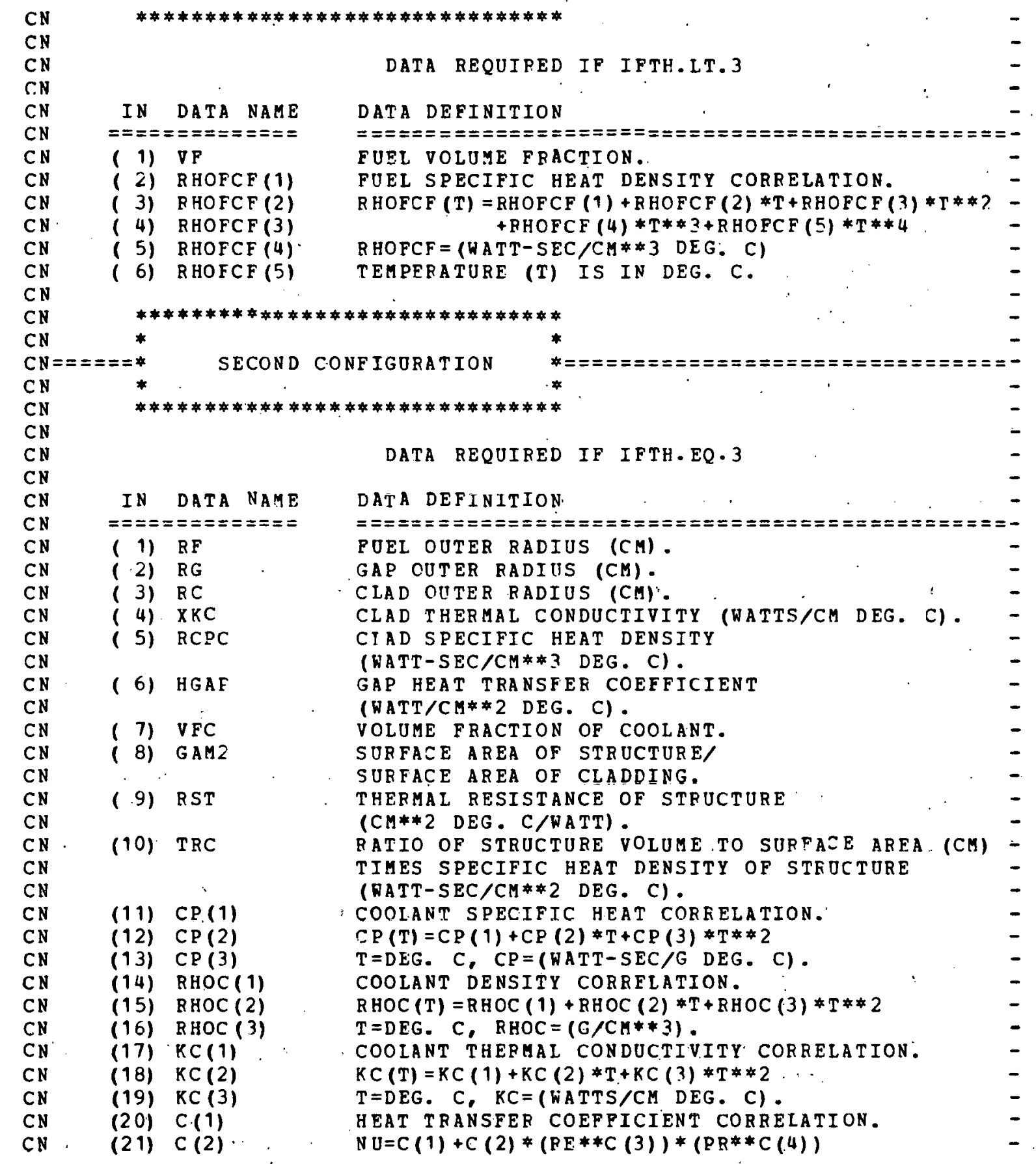




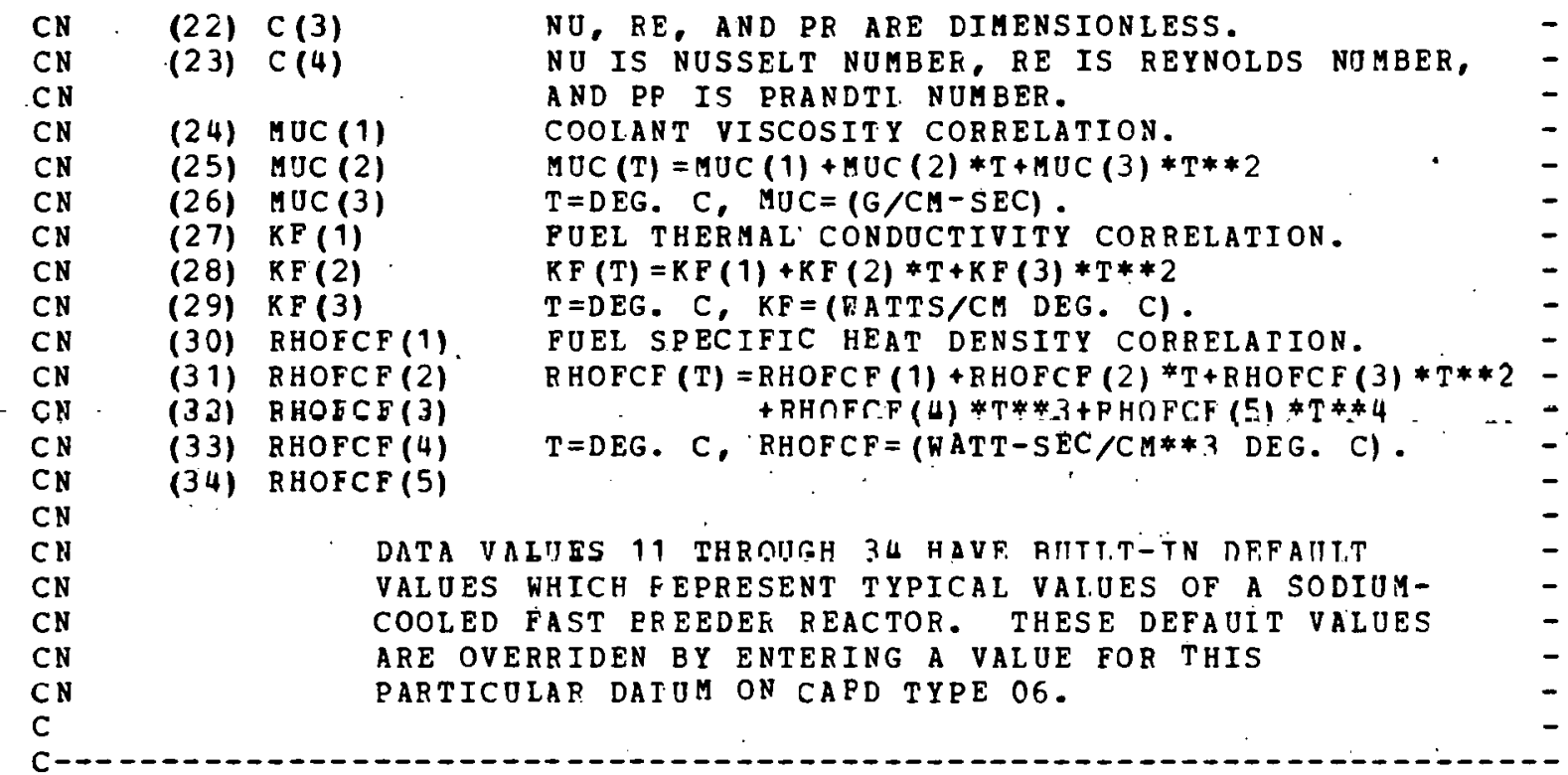

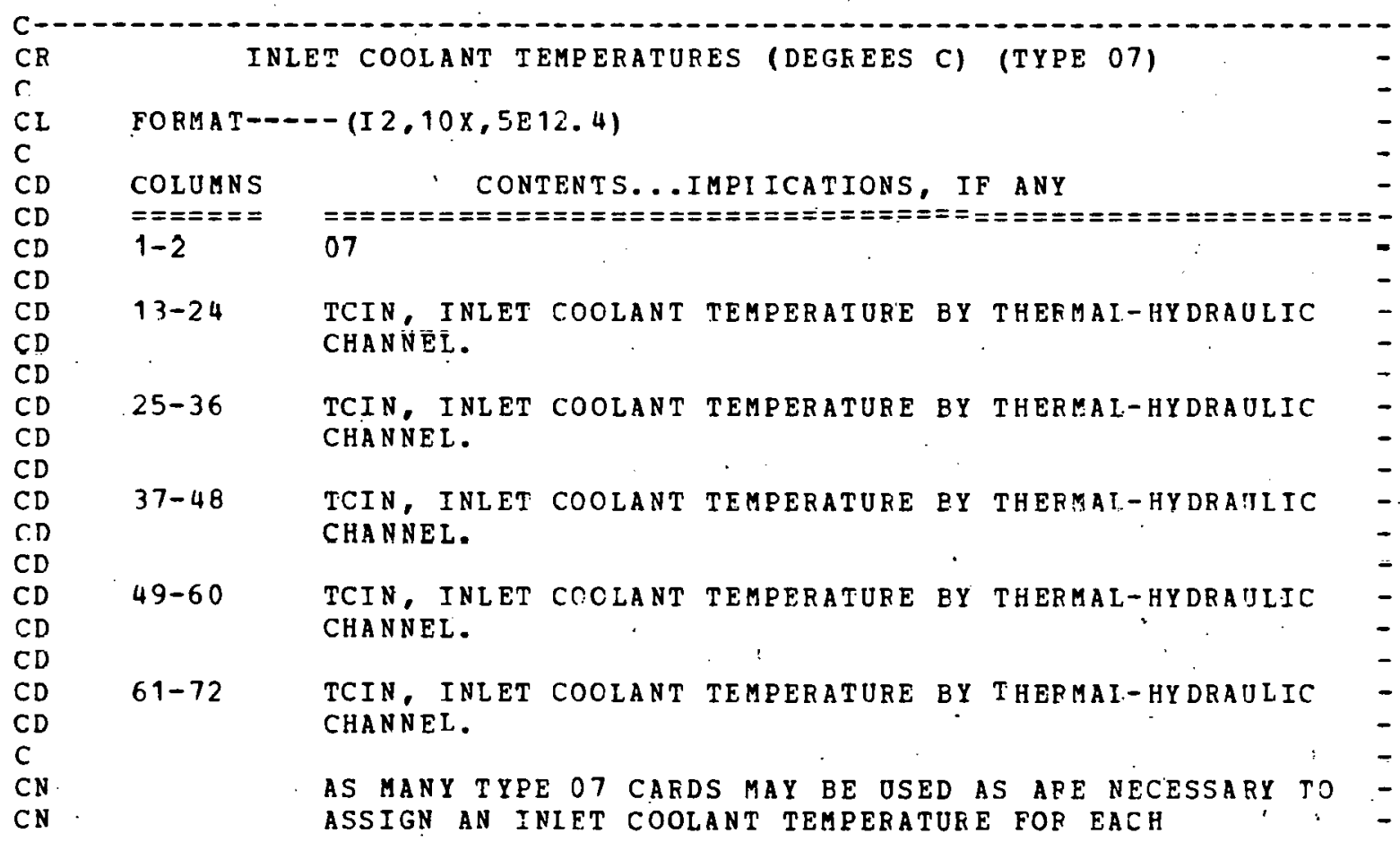


THERMAL - HYDRAULIC CHANNEL.

IF ONLY ONE INLET COOLANT TEMPERATURE IS READ IN, THAT VALUE IS USED FOR ALL INLET TEMPERATURES.

TYPE 07 CARDS ARE NOT REQUIRED IF IFTH. LT. 3

$\mathrm{C}$

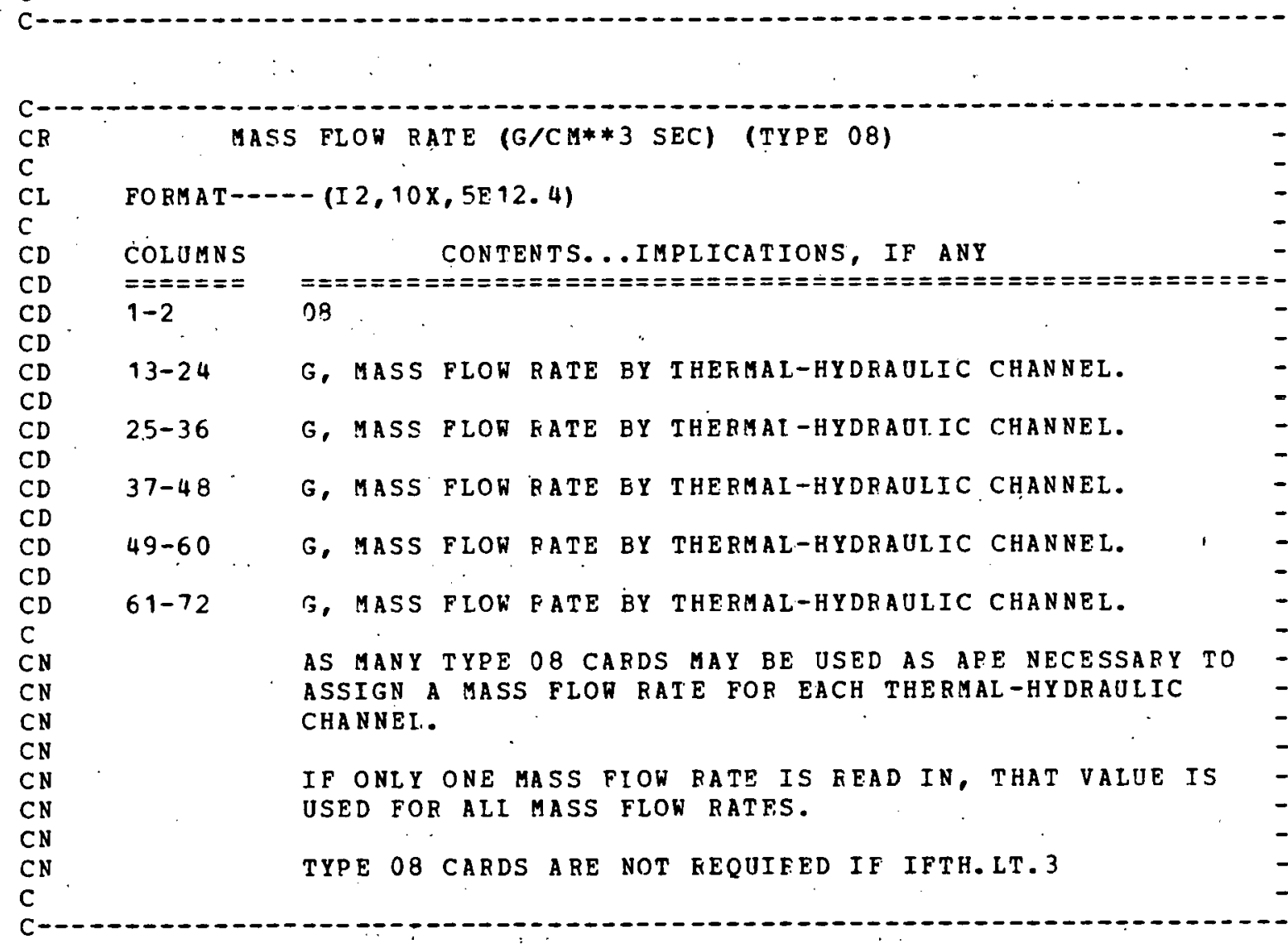

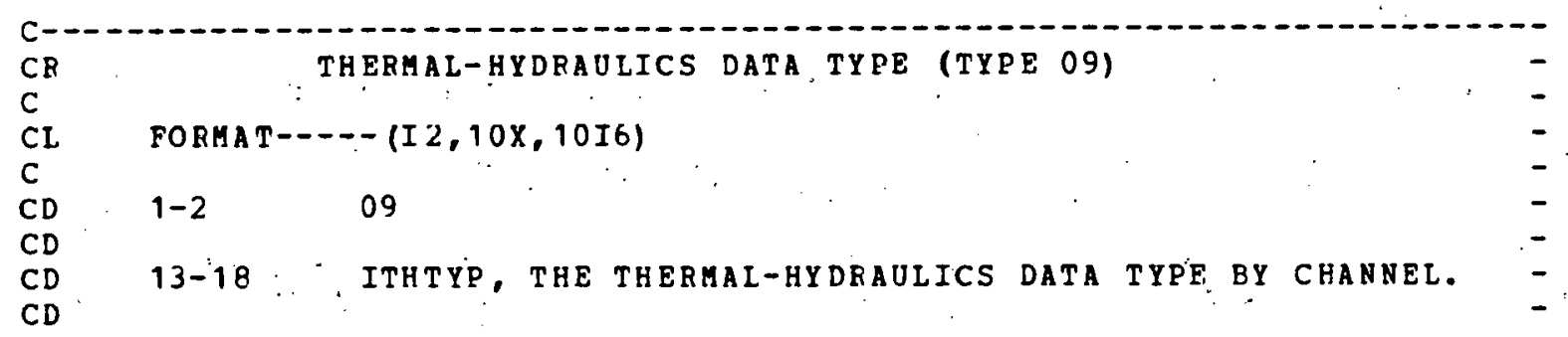




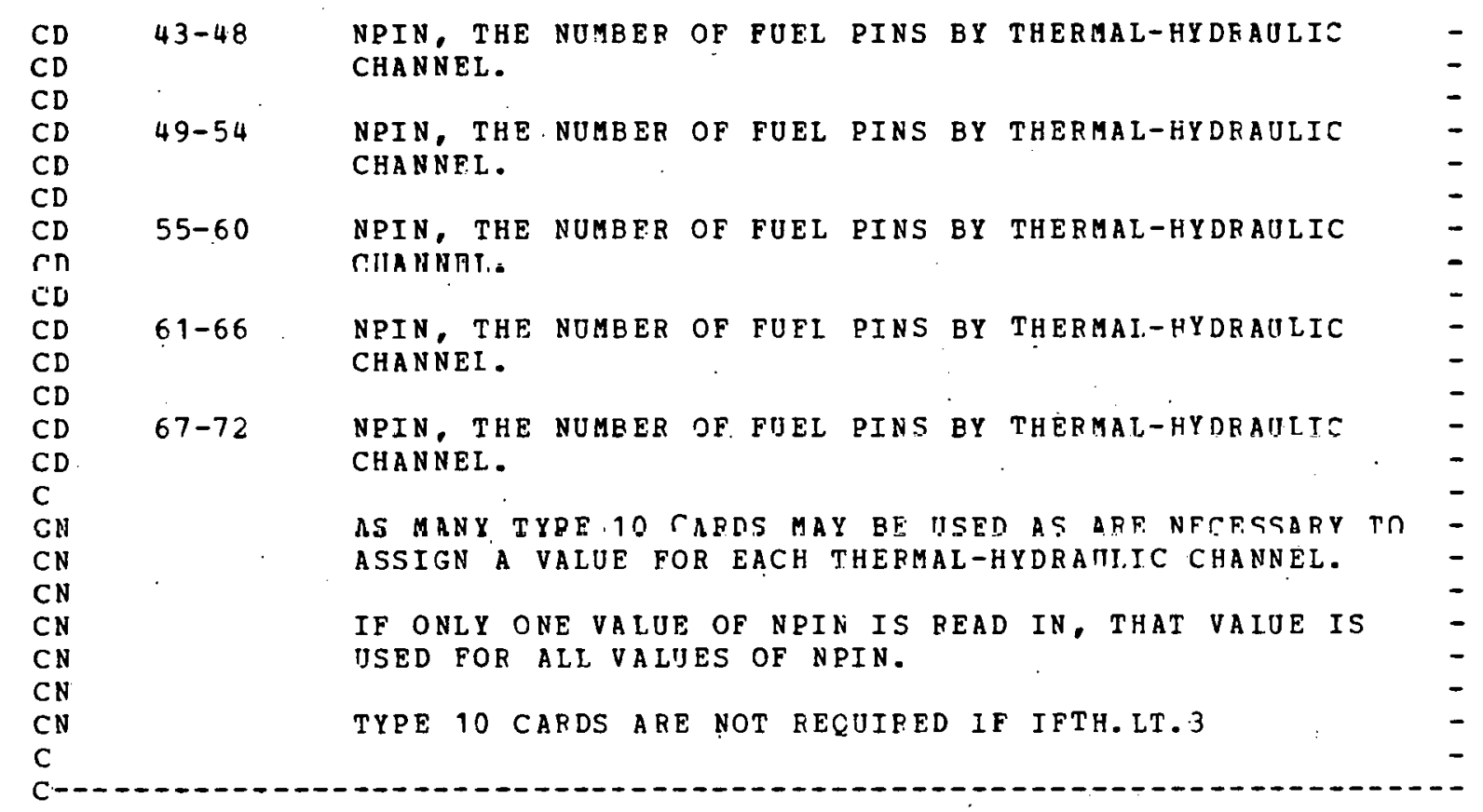

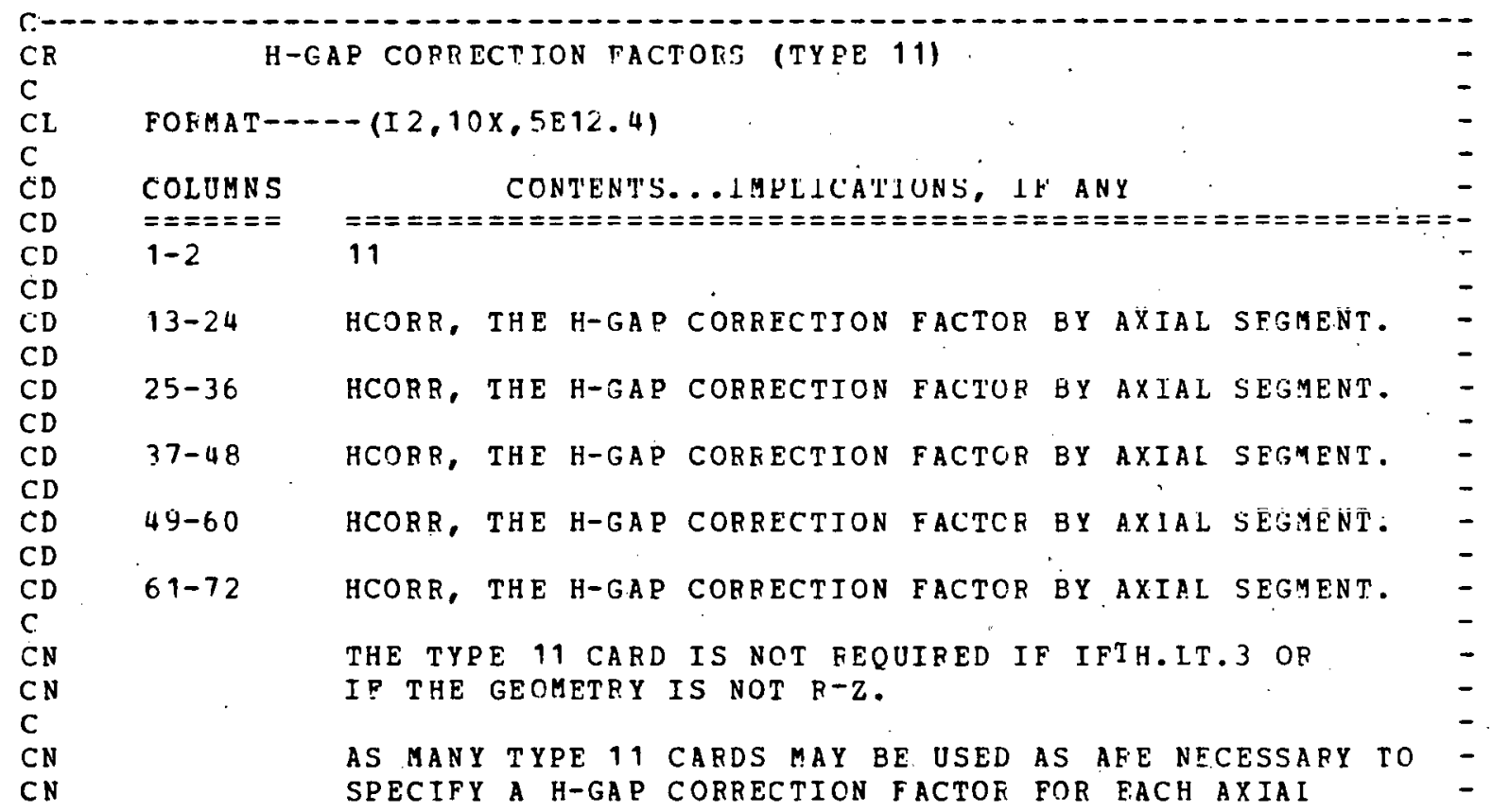




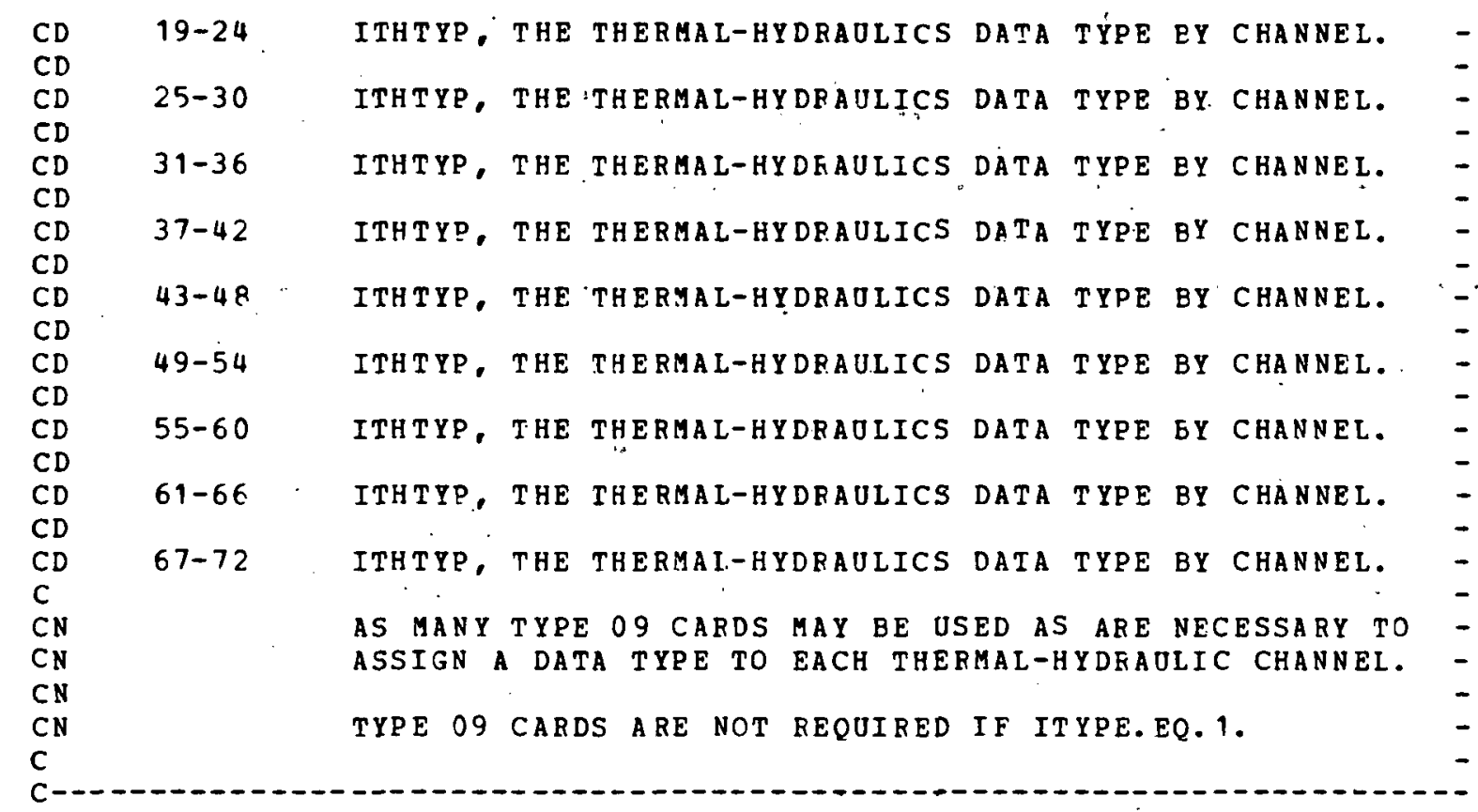

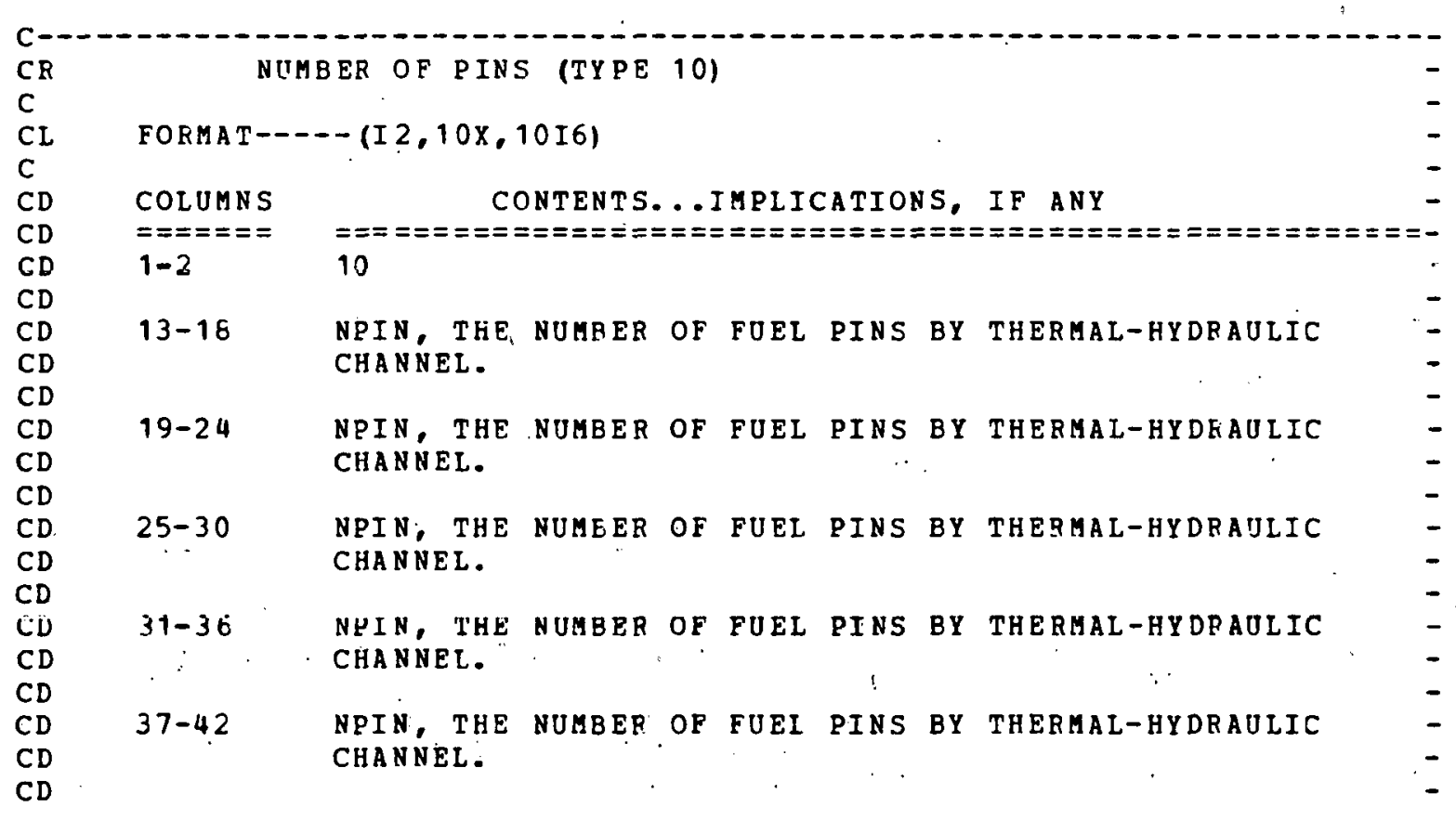




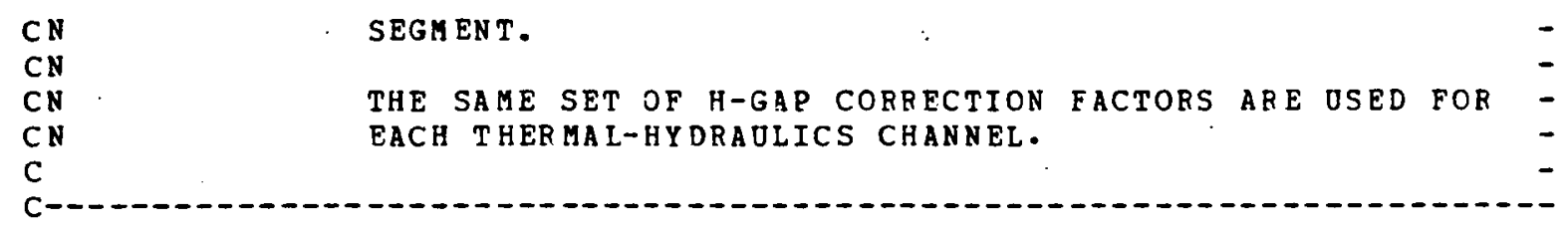

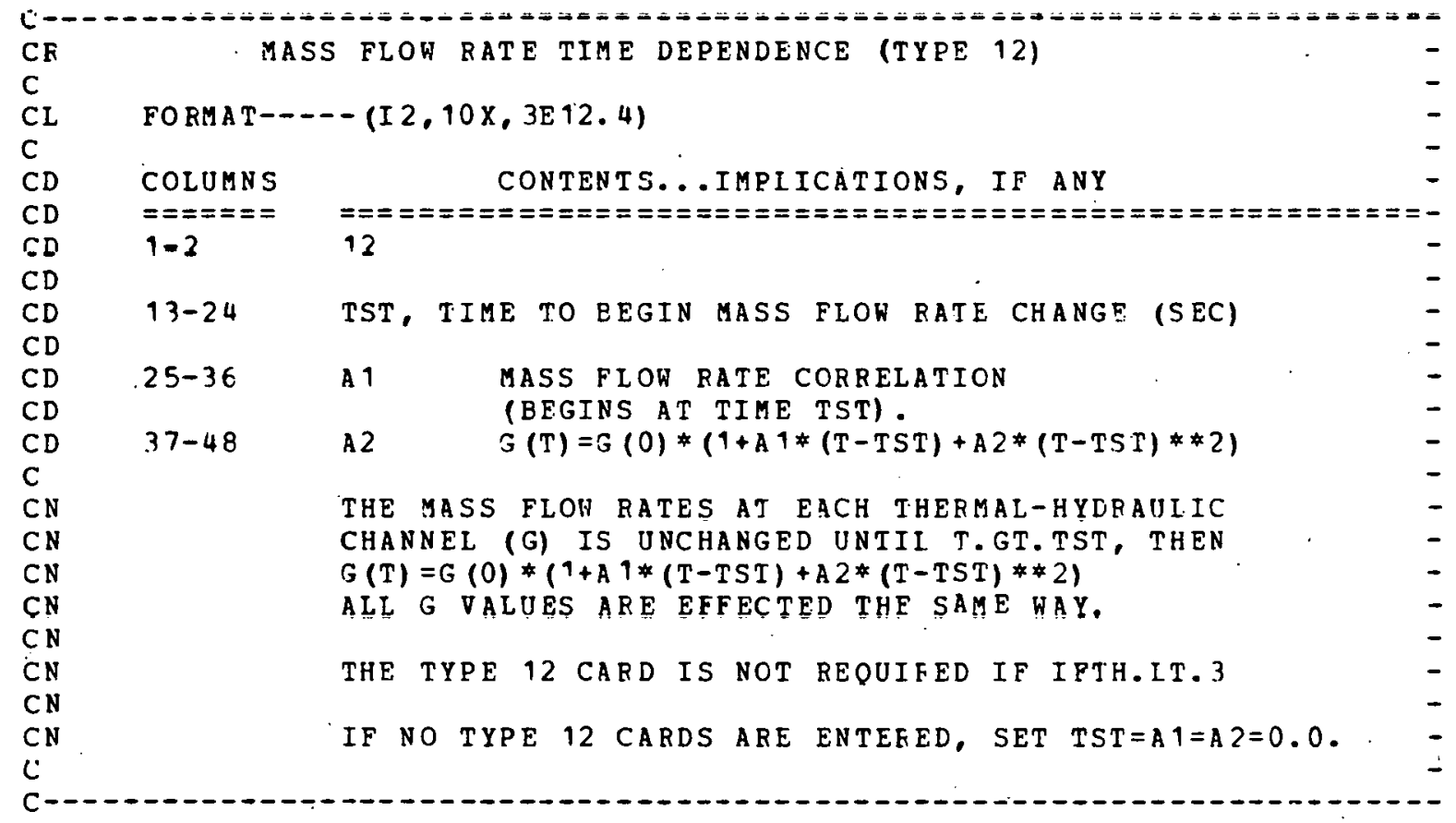

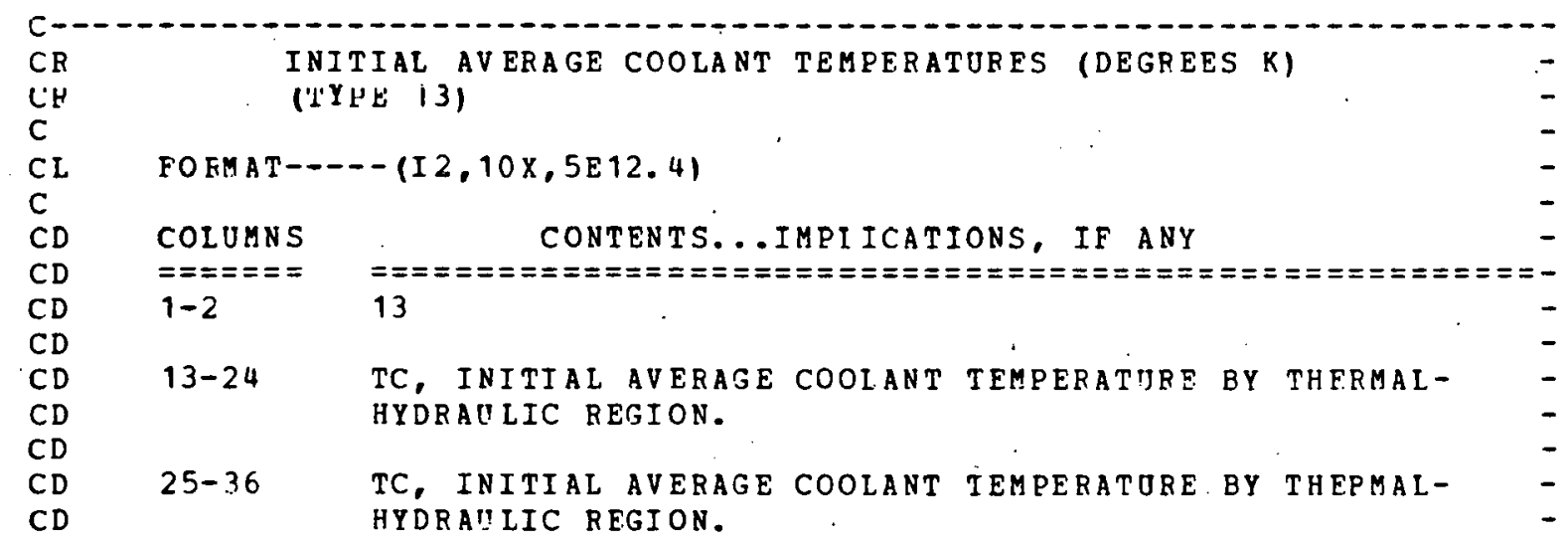




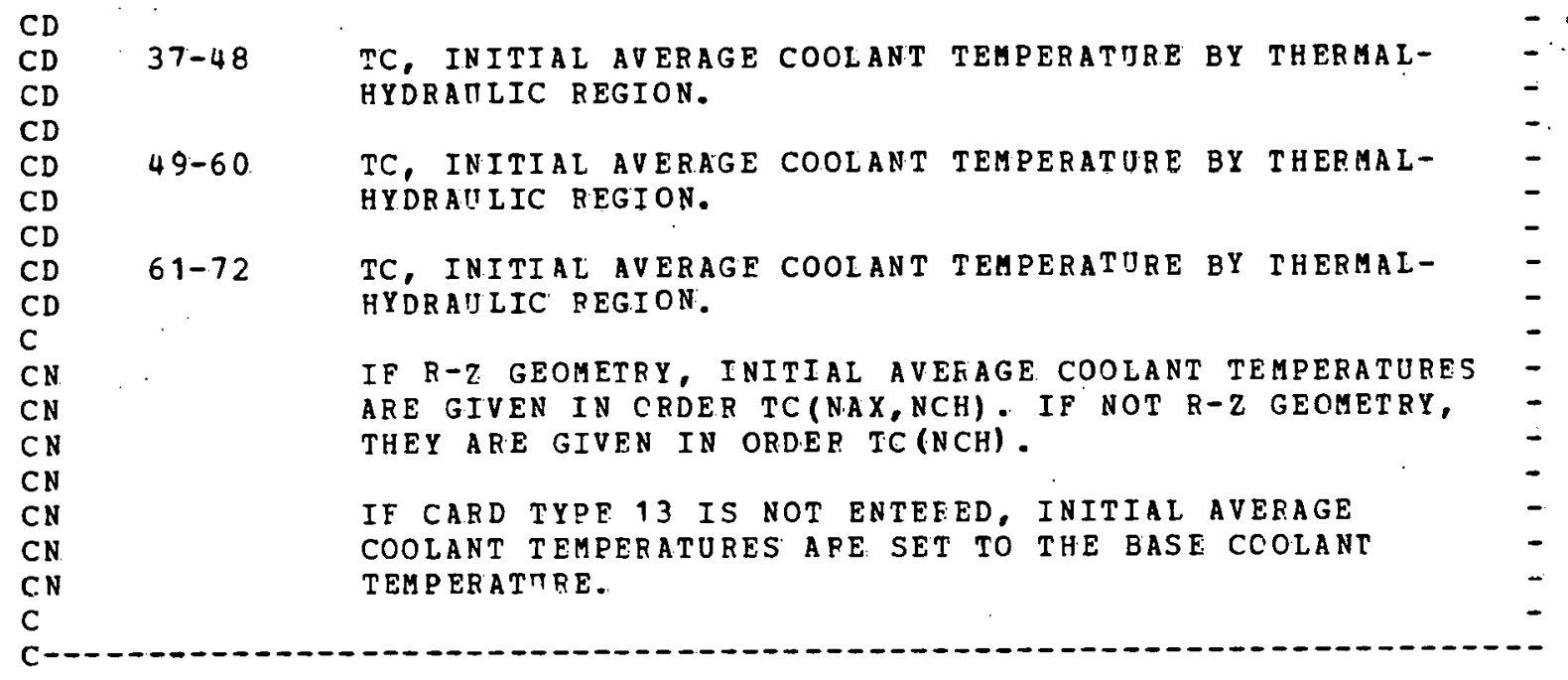

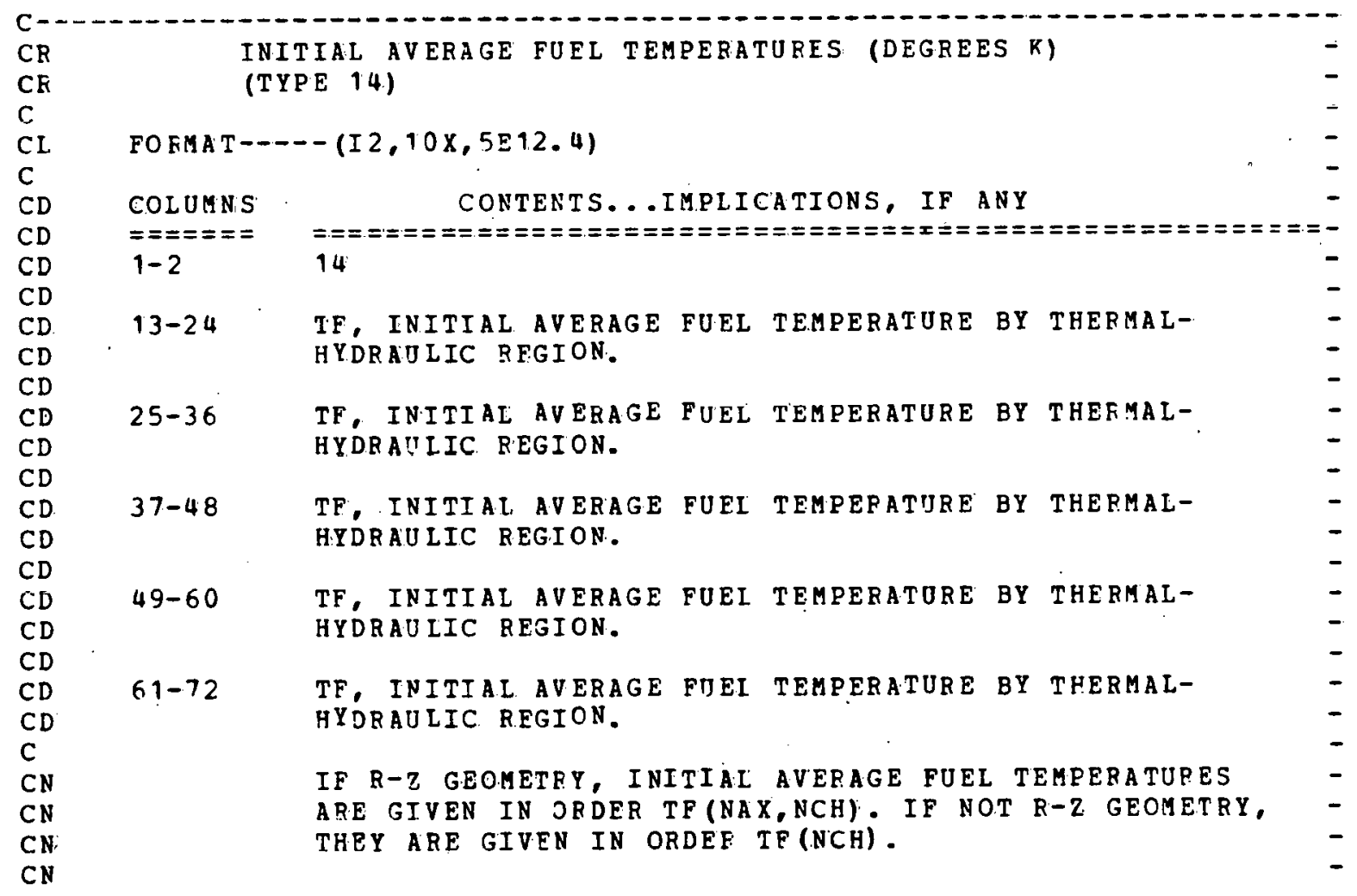


266

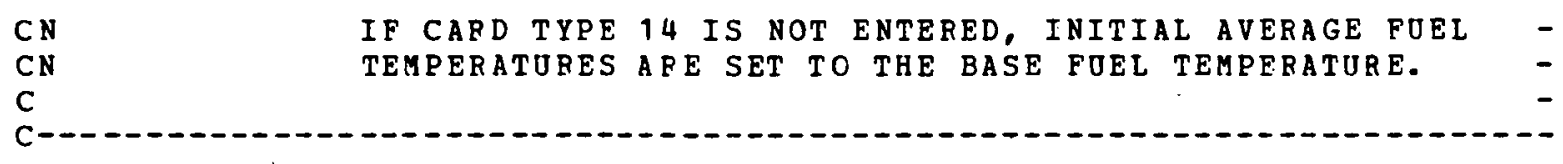


APPENDIX C

Listing of Binary Tnterface Files 


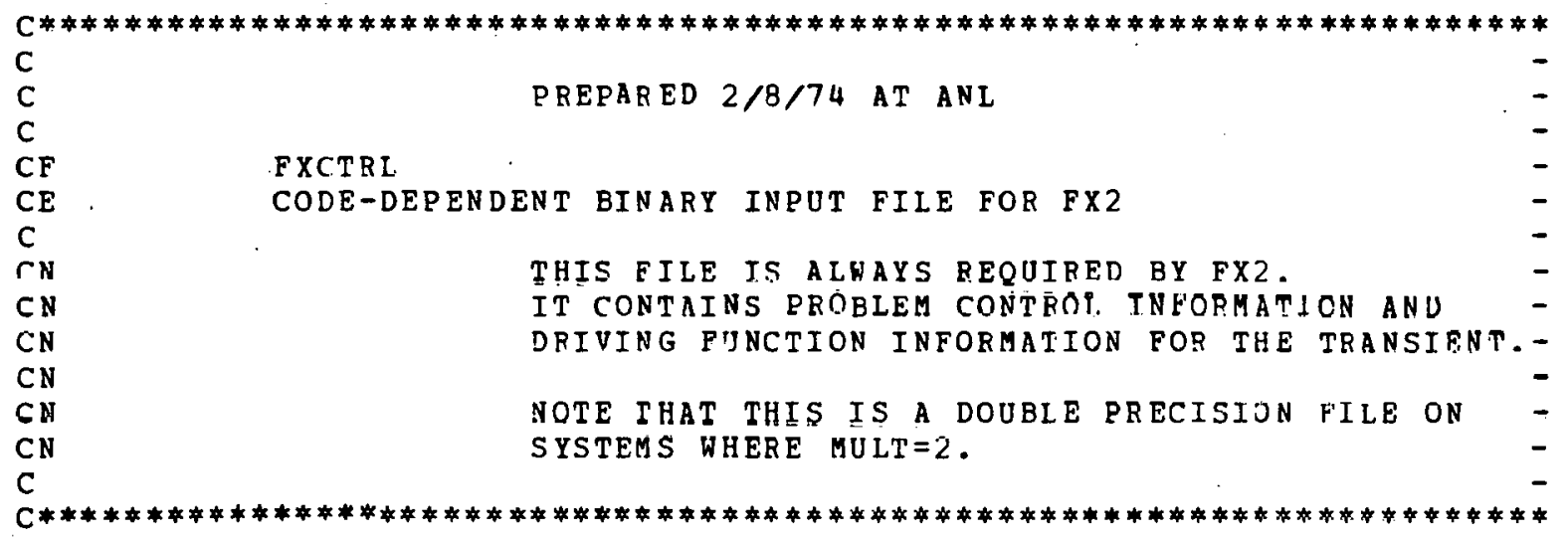

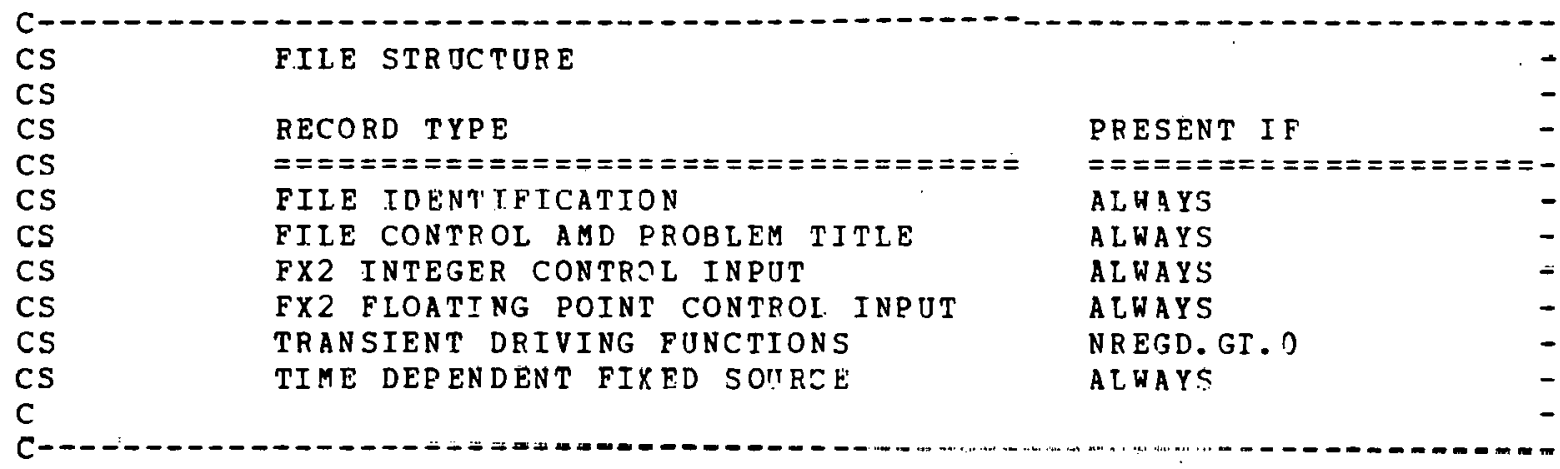

CD NREGD NUMEER OF DRIVING FUNCTIONS.

CR
$C$
$C C$


$C D$

$=2$, A6 WORD IS DOUBLE WORD.

$-$

C

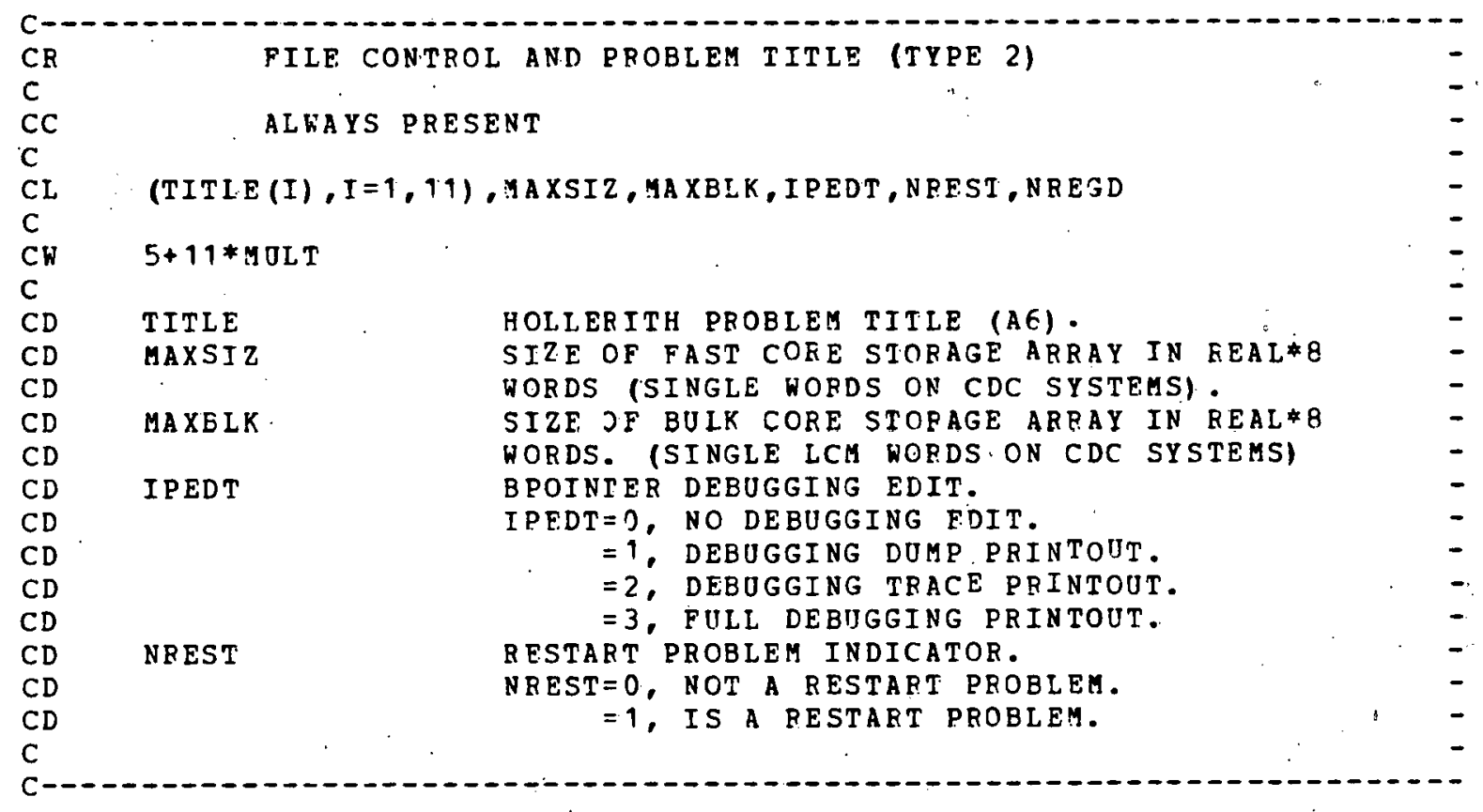

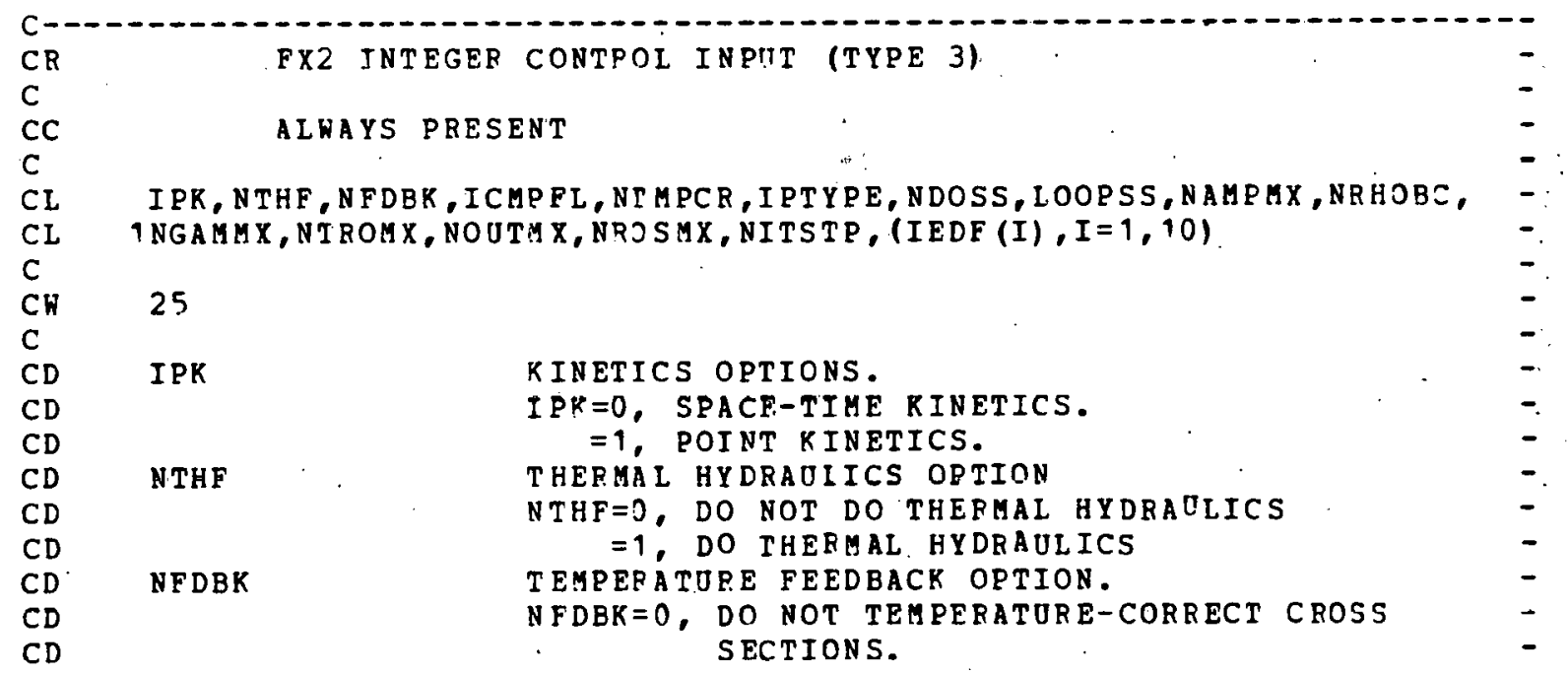


IEDF (3)

$\operatorname{IEDP}(4)$

IEDF (5)

IEDF (6)

$\operatorname{IEDF}(7)$

IEDF (8)

IEDF (9)

$\operatorname{IEDF}(10)$
SECTION HOMOGENIZATION. THERHAL-HYDRAULICS INPUT EDIT FLAG. IEDF $(3)=0$. DO NOT EDIT THERIAL-HYDRAULICS =1, PARTIAI. EDIT CF THERMAL-HYDRAULICS INPUT

$=2$, FULI EDIT OF THERMAL-HYDRAULICS. INPUT

UNUSED AT PRESENT

DISK EDIT FLAG.

$\operatorname{IEDF}(5)=0$, NO DISK EDIT

$=1$, BRIEF DISK EDIT

$=2$. DETAILED DISK EDIT POINTHISE POWERS, COOLANT AND FIEI. TEMPERATURES.

FUEL AND COOLANT TEMPERATURE EDIT FLAG IEDF(6) $=0$, DO NOI EDIT FUEL AND COOLANT TEMPERATUPES.

$=1$, EDIT FOEL AND COOLANT TEMPERAIURES AFTEE EACH SHAPE STEP.

REACTIVITY STEP SOMMARY ED.IT

$\operatorname{IEDF}(7)=0$, BRIEF EDIT.

$=1$, NORMAL EDIT. $=2$, DETAILED EDIT.

POWER DENSITY EDIT (EEACTIVITY STEP)

IEDF $(8)=0$, DO NOT EDIT POWER DENSITY AFTER REACTIVITY STEPS.

$=N$, EDIT POWER DENSITY AFTFP EVERY N REACTIVITY STEPS.

FLUX SHAPE CALCULATION EDIT

IEDF $(9)=0$, MINIMUA EDIT OF EACH FLUX SHAPE CALCULATION.

=1. EDIT ITERATION HISTORY AT EAzH FLUX SHAPE CALCULATION.

2. EDIT ITERATION HISTORY AND MUITIGROUP FIUX SHAPE AT EACH FLITX SHAPE CAICULATION.

POWER DENSITY EDIT (SHAPE STEP).

IEDF $(10)=0$, DO NOT EDIT POWER DENSITIES AFTER SHAPE STEPS

$=N$, EDIT POWER DENSITIES AFIER EVERY N SHAPE STEPS.

$\mathrm{C}$ 


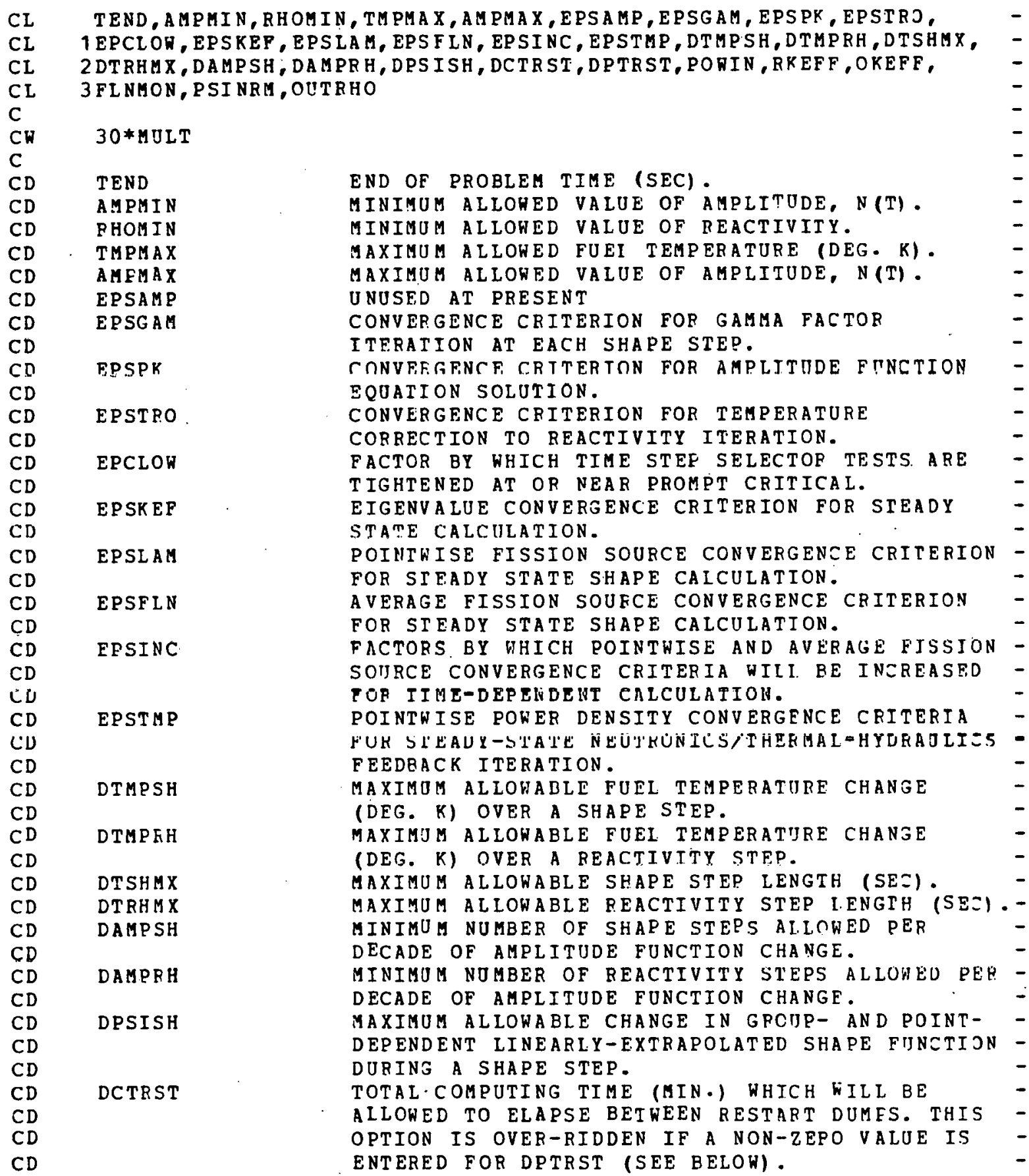




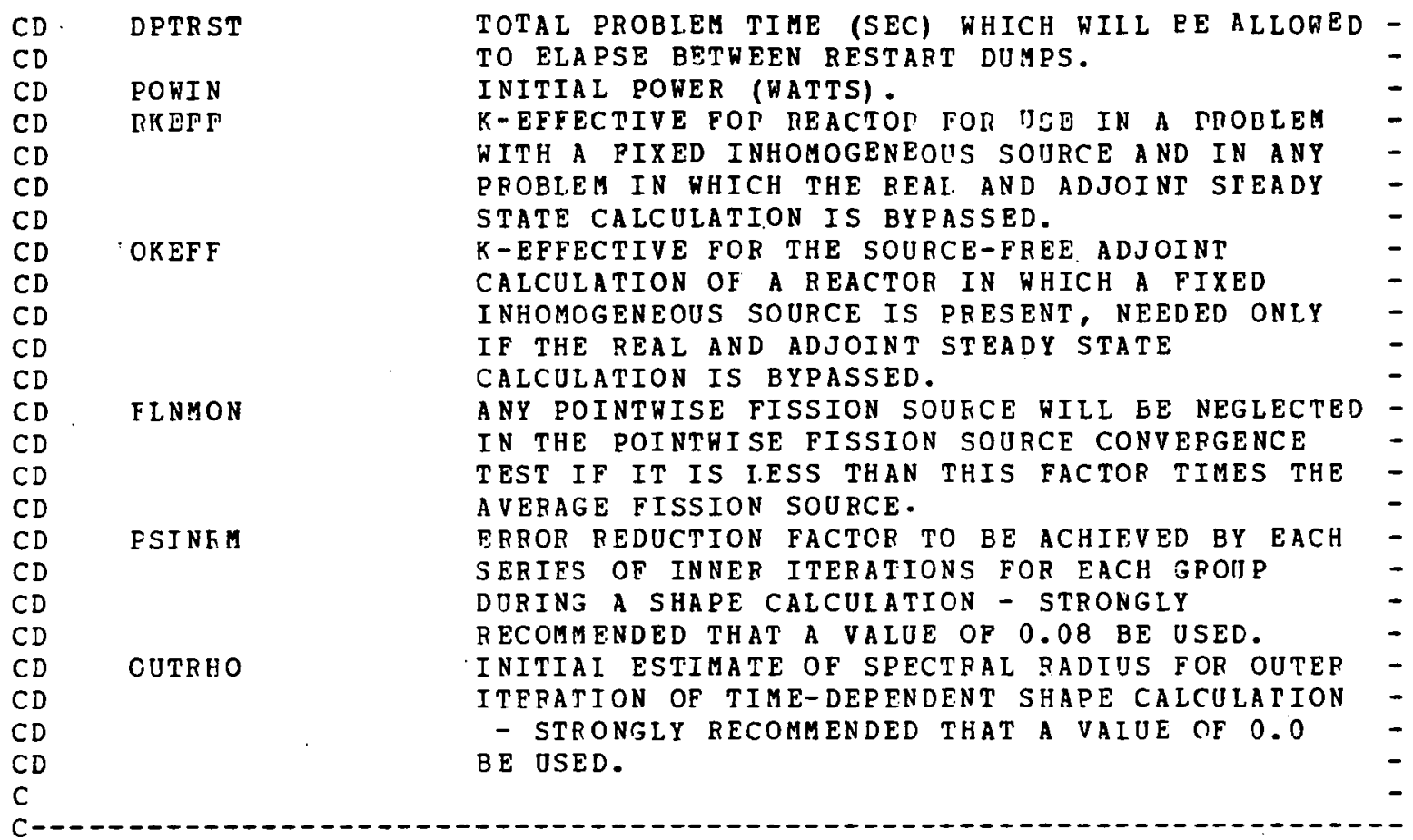

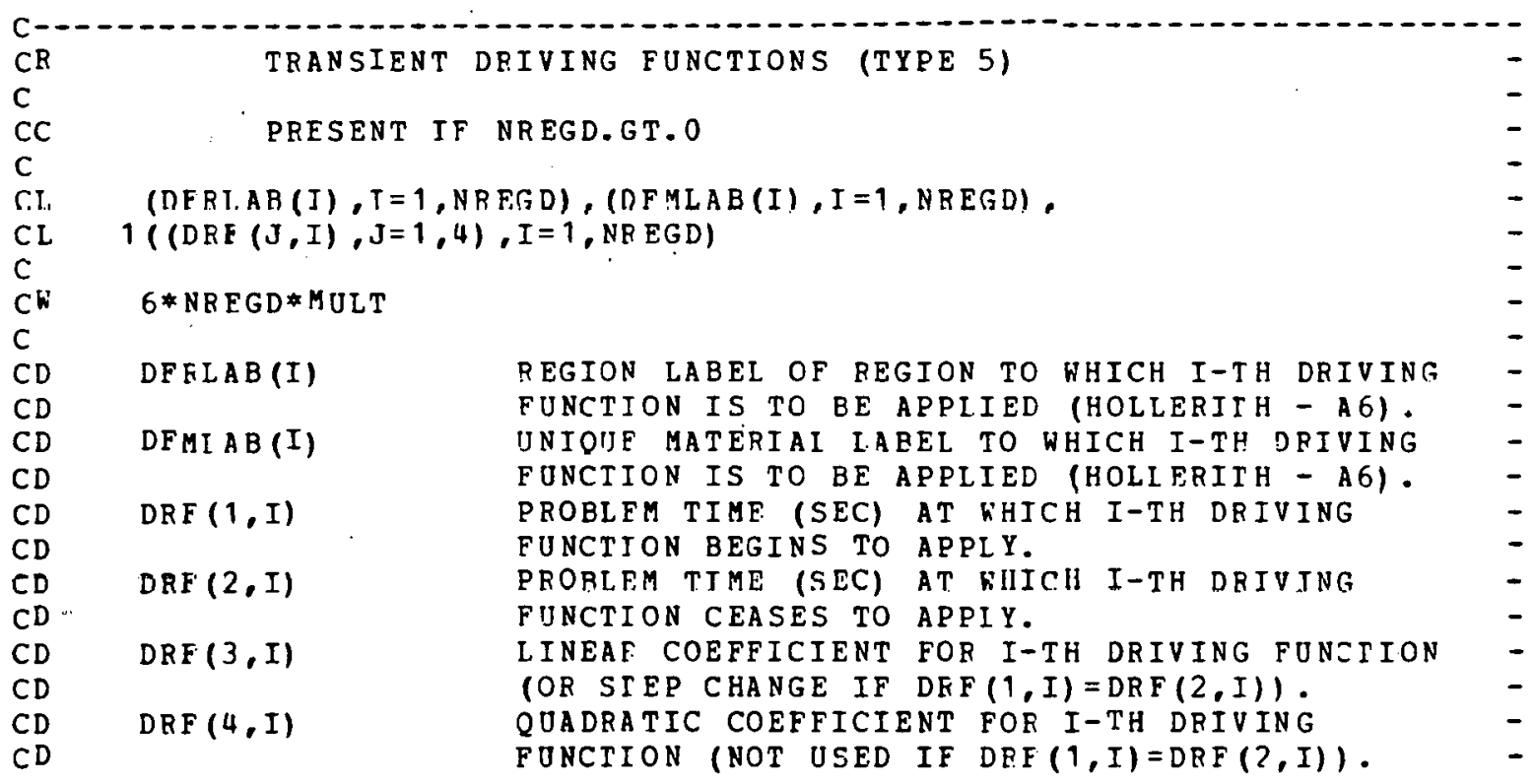




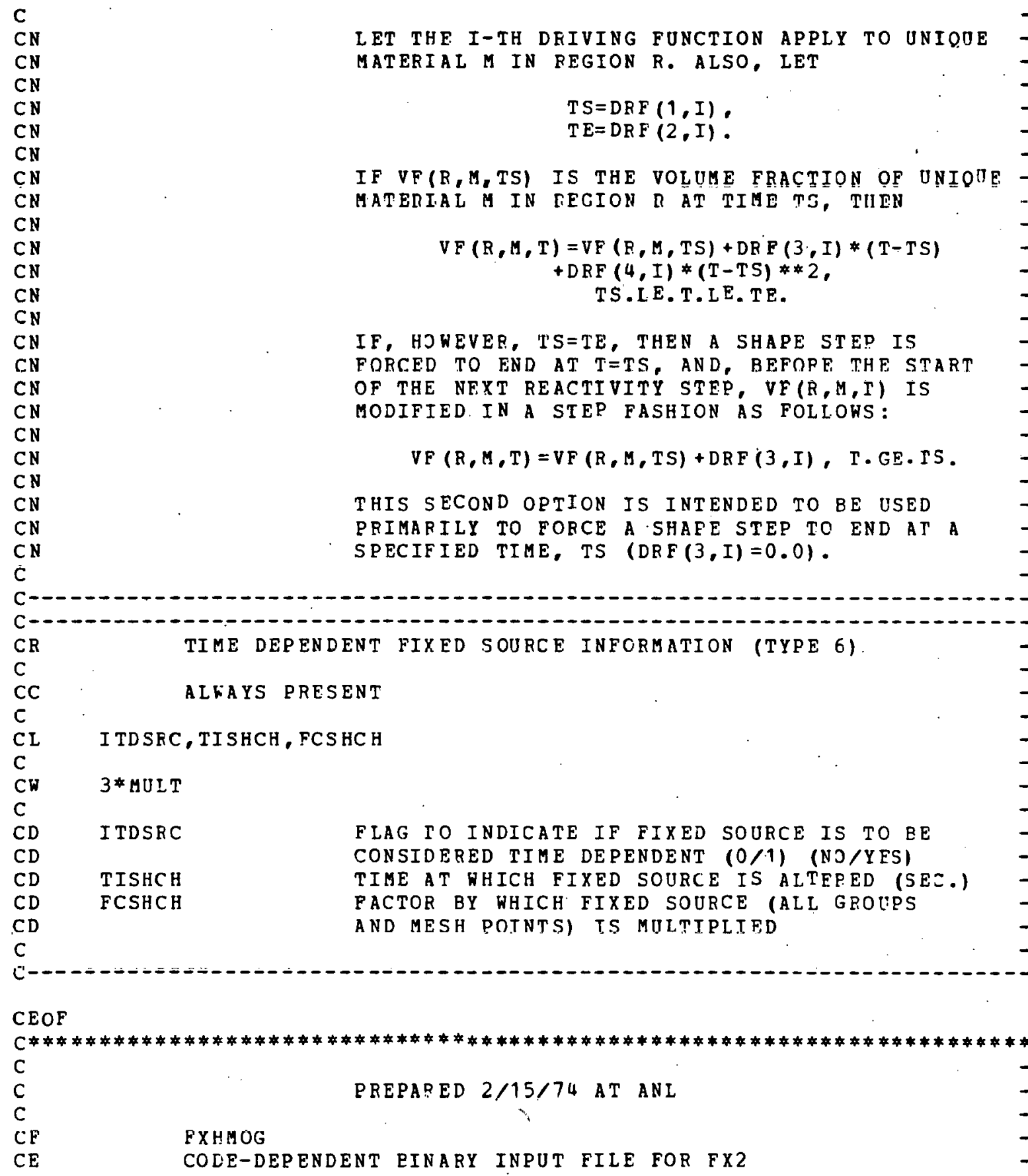


C

$\mathrm{CN}$
$\mathrm{CN}$
$\mathrm{CN}$
$\mathrm{CN}$
$\mathrm{CN}$
$\mathrm{CN}$
$\mathrm{CN}$
$\mathrm{CN}$
$\mathrm{CN}$
$\mathrm{C}$

$\mathrm{CN}$.

$\mathrm{CN}$

CN

$\mathrm{CN}$
RECORDS 1-3 AND 6 OF FXHMOG ARE ALHAYS

REQUIRED BY PX2. RECORDS 4 AND 5 ARE REQUIRED IF THE MACROSCOPIC CROSS SECTION FILE, XSZON, DOES NOT EXIST AS AN OLD FILE. FXHMOG CONTAINS ISOTOPE-TO-MATERIAL AMD MATERIAL-TO-REGION CROSS SECTION HOMOGENIZATION INFORMATION.

NOTE IHAT THIS IS A DOUBLE PRECISION FILE ON SYSTEMS WHERE MULT $=2$.
FIIE STRUCTURE

RECORD TYPE

= = = = = = = = = = = = = = = = = = = = = = = = =

FILE IDENTIFICATION

FILE CONTROL

MATERIAL, REGION, AND ZONE LABEIS

LIST OF ISOTOPE LABELS

ISOTOPE-TO-MATERIAL HOMOGENIZATION

IN FORHATION

MATERIAL-TO-REGION HOMOGENIZATION

INFORMATION
PRESENT IF

$==$ = = = = = = = = = = = = = = = =

ALWAYS

AL WAYS

ALWAYS

ALWAYS

AL WAYS

ALWAYS

$C D$

, CS

C

$\begin{array}{ll}C D & \text { MISLTH } \\ C D & \\ C D & \text { NISOU } \\ C D & \\ C D & \\ C D & \\ C D & \\ C D & \\ C D & \text { MMSLTH } \\ C D & \\ C D & \text { NREGM } \\ C D & \\ C D & \text { NHATU } \\ C D & \\ C D & \text { NTMATU } \\ C D & \\ C D & \text { NREG } \\ C D & \\ C D & \end{array}$

LENGTH OF ISOTOPE-TO-MATERIAL CROSS SECTION HOMOGENIZATION TABLE.

IF . GT. O, NUMBER OF DIFFERENT INIOUE ISOTOPES

A YUEAKING IN THE DIMIX ARRAY AND, HENCE, THE

LENGTH OF THE DITBL ARRAY.

IF . LT.0, RECORDS 4 AND 5 ARE DUMMY RECORDS AND

IABS(NISOU) IS THE LENGTH OF THE DUMMY DITBL

ARRAY.

LENGIH OF MATERIAL-TO-REGION CROSS SECTION HOMOGENIZATION TABLE.

NUMBER OF REGIONS FOR WHICH HOMOGENIZATION SPECIFICATIONS HAVE BEEN GIVEN, . GE. NREG. NULBER OF GENERIC MATERIALS USED IN FXHMOS, - IE.NMAT FROM XSCON.

NUMBER OF UNIQUE MATERIALS USED IN FXHMOG, . LE.NIMAT FROM XSCON.

NUMBER OF REGIONS USED TO DESCRIBE PROBLEM GEOMETRY (MUST BE CONSISTENT WITH NREG FROM GEODS I) . 

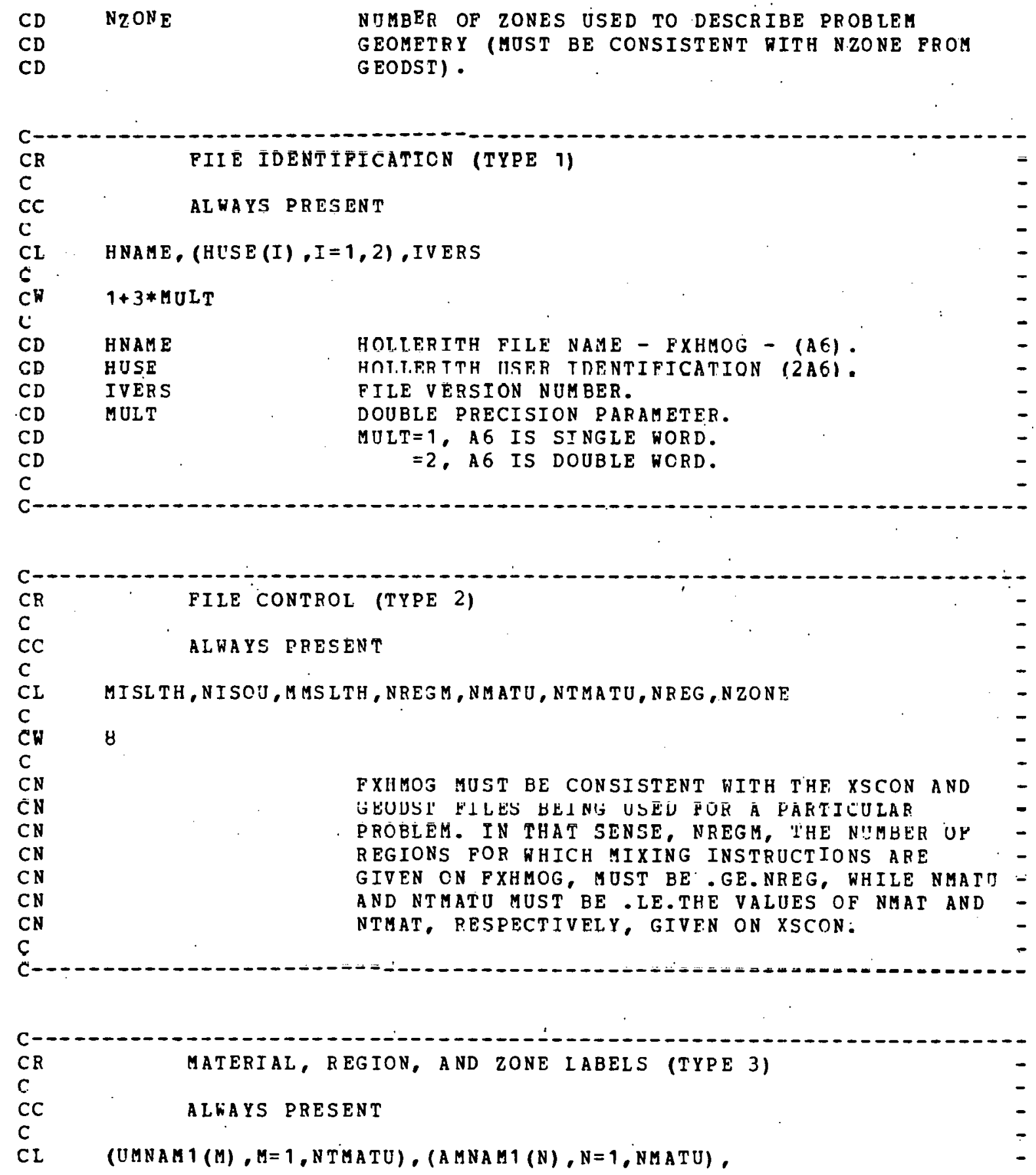


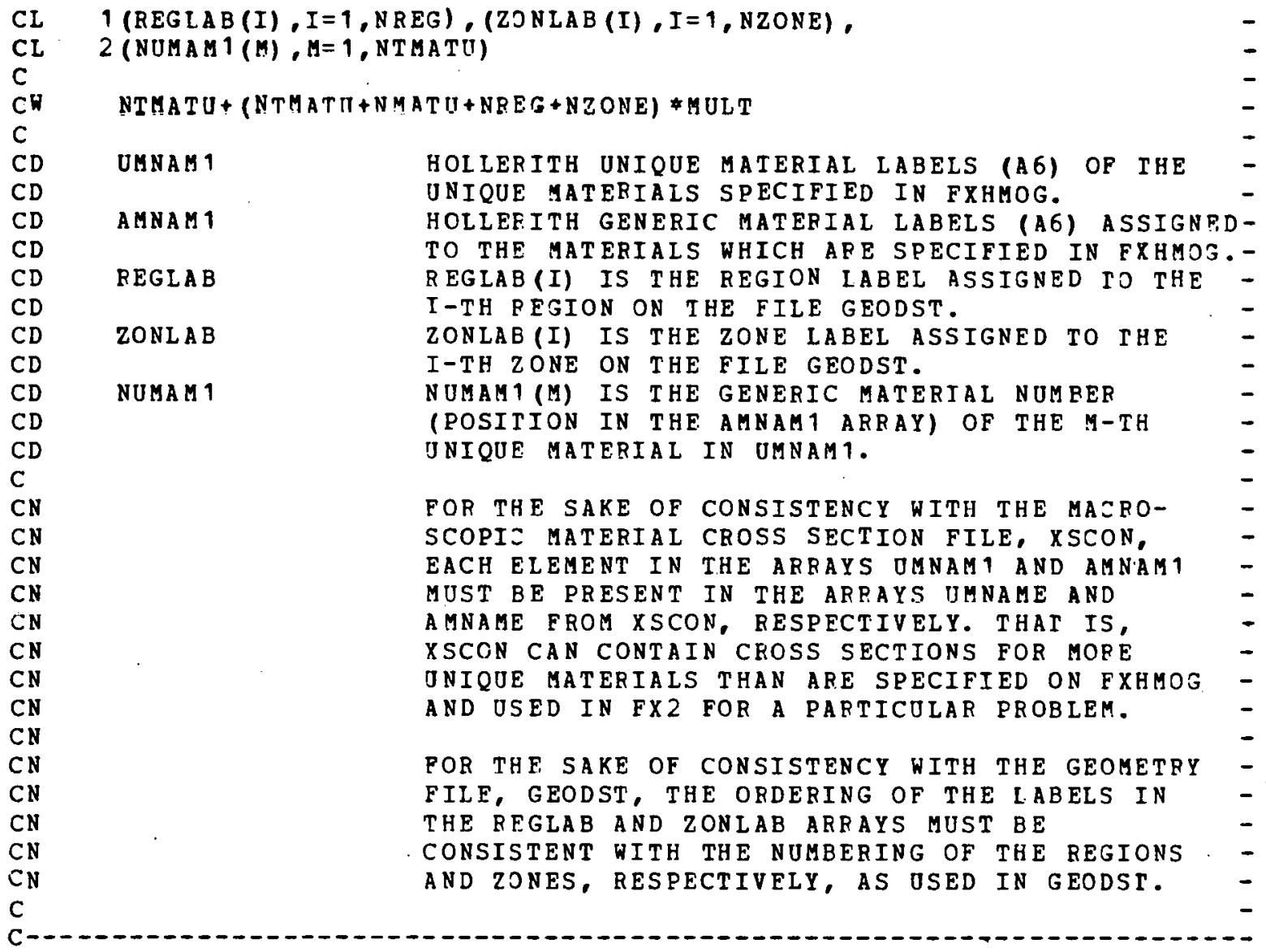

CR
$C$
$C C$


C

CN

$\mathrm{CN}$

$\mathrm{CN}$

CN

$\mathrm{CN}$

C

C-$$
\text { C- }
$$$$
\text { CR }
$$

C

r.

C

C L

CL

CL

C

$\mathrm{CW}$

$\mathrm{C}$

CD

CD

$C D$

$C D$

$C D$

CD

$C D$

$C D$

$C D$

CD

CD

$C D$

CD

$C D$

CD

CD

$C D$

CD

CD

$\mathrm{C}$

$\mathrm{CN}$

$\mathrm{CN}$

CN

$\mathrm{CN}$

$\mathrm{CN}$

$\mathrm{CN}$
RECORDS 4 (DITBI) AND 5 WIIL BE DUMMY RECORDS IF THIS FXHMOG WAS WRITTEN BY THE FX2 BCD INPUT PROCESSOR FOR A PROBLEM WHERE THE FILE XSCON HAD BEEN SPECIFIED AS AN OLD FILE. IN THIS CASE, THIS ISOTOPE-TO-MATERIAL HOMOGENIZATION INFORMATION WILL NOT BE NEEDED BY FX2.

ISOTOPE-TO-MATERIAL HOMOGENIZATION INFORMATION (TYPE 5 )

AL.WAYS PRESENT (DUMMY IF NISOD.IT.O)

$((D I M I X(K, I), K=1,4), I=1, M I S L T H),((\operatorname{ATMDEN}(K, I), K=1,2), I=1$, IISIIH), 1 (DMTBL $(M), M=1$, NTMATU), (MSPEC $(M), M=1, N$ TMATU),

2 (IFTMP (M), $M=1$, NTMATU)

2 *NTMATU+ (NTMATU+6*MISLTH) *MULT

DIMIX

ISOTOPE-TO-MATERIAL MIXING TABIE CONSISIING OF 4*MSPEC (M) ENTRTES FOF EACH UNIQUE MATERIAL, M. THESE ENTRIES ARE THE IABELS OF THE UNIQUE ISOTOFES WHICH COMPPISE UNIQUE

ATMDEN

DMTEI MATERIAL M AT FUUK IEUPETATURES. ATOMIC DENSITIES (ATOMS/BARN-CC) FOR THE MIXINJ TABLE. IT CONSISTS OF 2*MSPEC(M) ENTRIESFOR EACH UNIQUE MATERIAL M, CORPESPONDTNG TO THE 4*MSPEC(M) ENTRIES IN DIMIX. FOR A PARTICUIAR UNIQUE ISOTOPE IN MATERIAL $M$, IHE SAME ATOM DENSITY IS USED AT ALL FOUR TEMPERATURES.

UNIHL(M) IS THE UNIQUE MATERTAT, LADEL (AG) OF THE M-TH UNIQUE MATERIAL BEING SPECIFIED BY DIMIX AND ATMDEN.

MSPEC MSPEC(M) IS THE NUMBER OF UNIQUE ISOTOPES JSED TO CONSTRUCT UNIQUE MATERIAL M AT EACH TEMPERATURE.

TFTMP IFTMP $(M)=1$ IF 'THE M-IH UNIQUE MATERIAT IS TEMPERATURE-DEPENDENT; =0 IF IT IS NOT.

THE JNIQUE MATERIAL LABELED DMTEL (M) IS COMPOSED OF MSPEC(M) ONIQUE ISOTOEES AT EACH TEMPERATURE. THESE ISOTOPES AND THE CORRESPONDING ATOMIC DENSITIES ABE GIVEN IN ORDER IN THE ARRAYS DIMIX AND ATMDEN, RESPECTIVELY. THE FIRST ISOTOPE LABELS AND ATOMIC DENSITIES - 


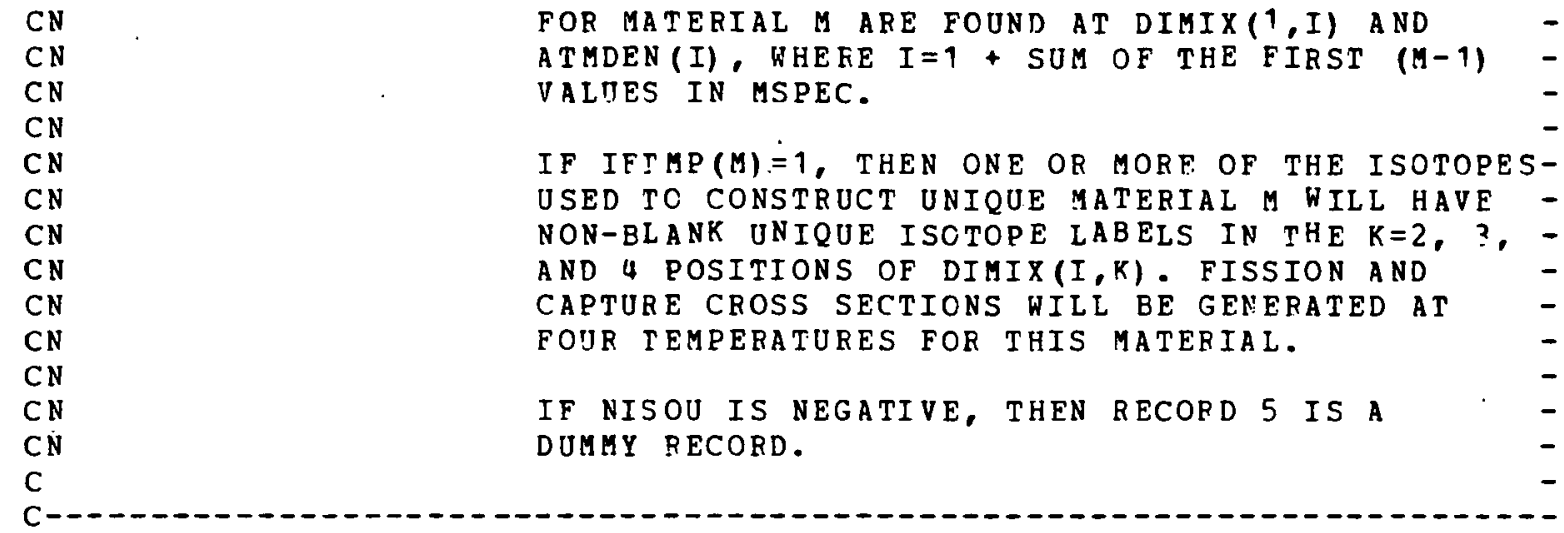

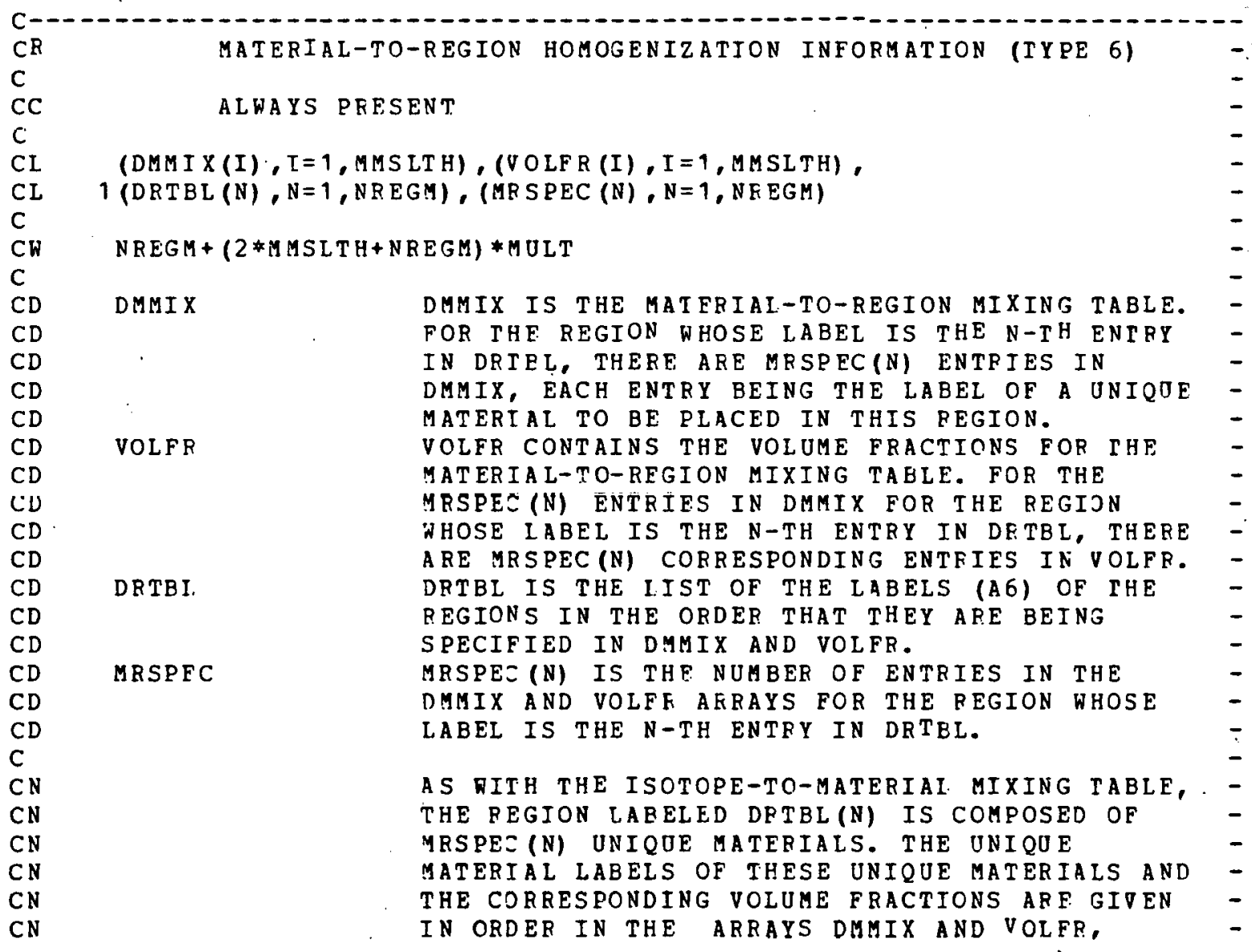




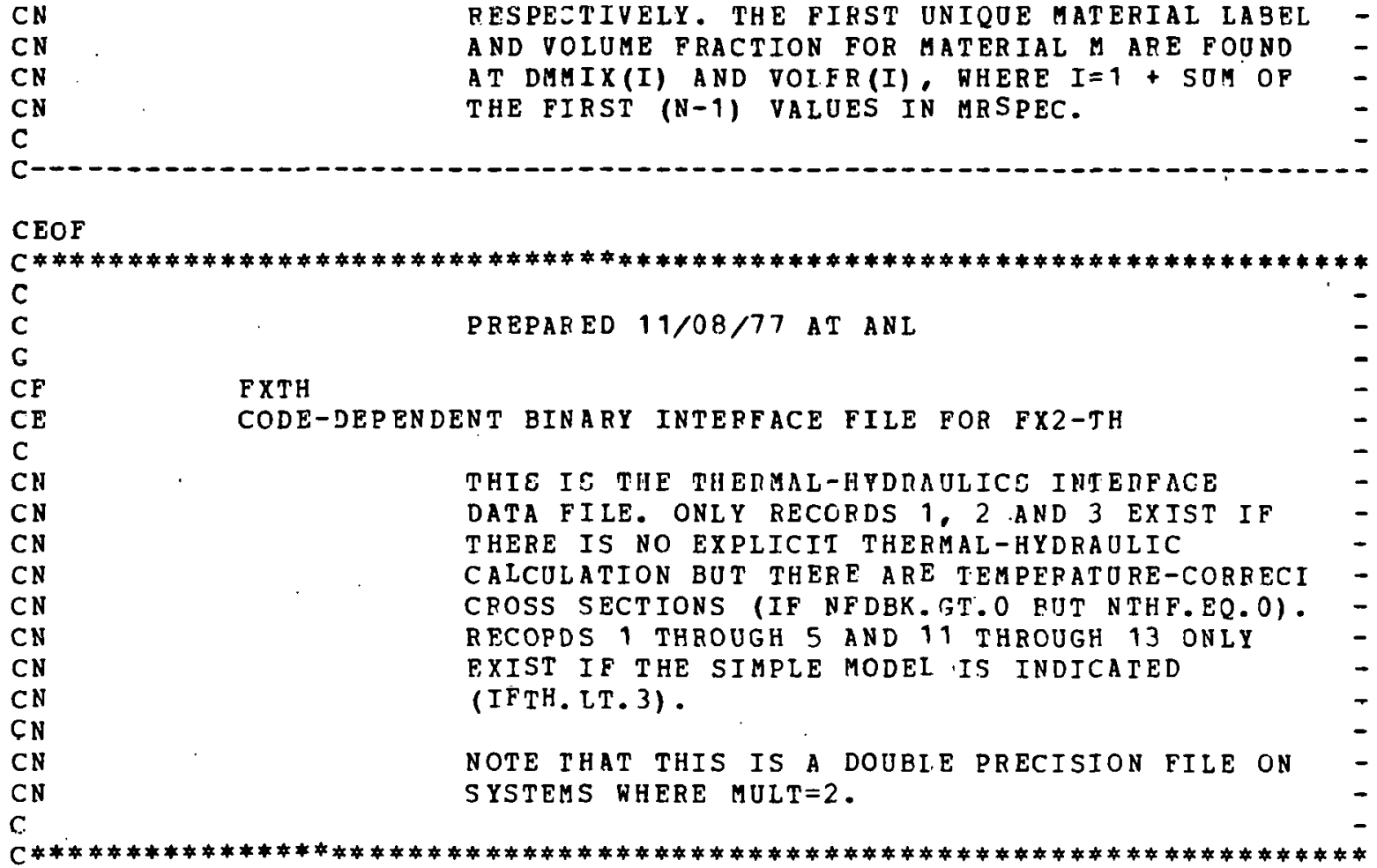

\begin{tabular}{|c|c|c|c|}
\hline $\mathrm{CS}$ & \multirow{2}{*}{\multicolumn{3}{|c|}{ FILE STRUCTURE }} \\
\hline CS & & & - \\
\hline CS & \multicolumn{2}{|l|}{ RECORD TYPE } & - \\
\hline CS & \multirow{2}{*}{ 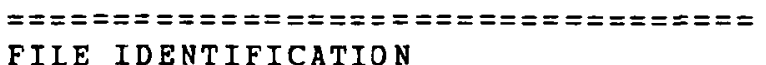 } & 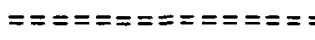 & \\
\hline CS & & ALWAYS PEESENT & - \\
\hline CS & FIIE CONTROL & ALWAYS PEESENT & - \\
\hline $\mathrm{CS}$ & \multirow{2}{*}{$\begin{array}{l}\text { THERMAL-HYDRAULICS MAP } \\
\text { THFRMAL-HYDRAULICS DATA TYPE }\end{array}$} & ALWAYS PRESENT & - \\
\hline CS & & NTHF. EQ. 1 & - \\
\hline $\operatorname{CS}$ & $\begin{array}{l}\text { THERMAL-HYDRAULICS } \text { DATA TYPE } \\
\text { MATERIAL CONSTANTS FILE }\end{array}$ & NTHF.EQ. 1 & - \\
\hline r.s & NIIMRER OF PTNS & NTHF - F. 1 . A ND. & - \\
\hline $\mathrm{CS}$ & \multirow{3}{*}{ INLET COOLANT TEMPERATURES } & IFTH.EQ. 3 & - \\
\hline CS & & NTHF.EQ. 1. AND. & - \\
\hline CS & & IFTH. EQ. 3 & - \\
\hline $\mathrm{CS}$ & \multirow[t]{2}{*}{ MASS FLOW RATES } & NTHF.EQ. 1. AND. & - \\
\hline CS & & IFTH. EQ. 3 & - \\
\hline $\mathrm{CS}$ & AYIAL MESH LENGTHS & $\mathrm{NTHF}, \mathrm{EQ} .1$ & - \\
\hline CS & \multirow[t]{2}{*}{ H-GAP CORRECT ION FACTORS } & NTHF.EQ. 1. AND. & - \\
\hline CS & & IFTH.EQ. 3 & - \\
\hline $\mathrm{CS}$ & NUMBER OF TYPE 13 AND TYPE 14 & NTHF.EQ. 1 & - \\
\hline
\end{tabular}




$\begin{array}{lll}\text { CS } & \text { INPIT CARDS } & \\ \text { CS } & \text { INITIAL AVERAGE COOLANT } & \text { NTHF.EQ.1 } \\ \text { CS } & \text { TEMPERATURES } & \\ \text { CS } & \text { INITIAL AVERAGE FJEL TEHEERATUKES } & \text { NTHE.Ë.1 } \\ \text { C } & & \end{array}$

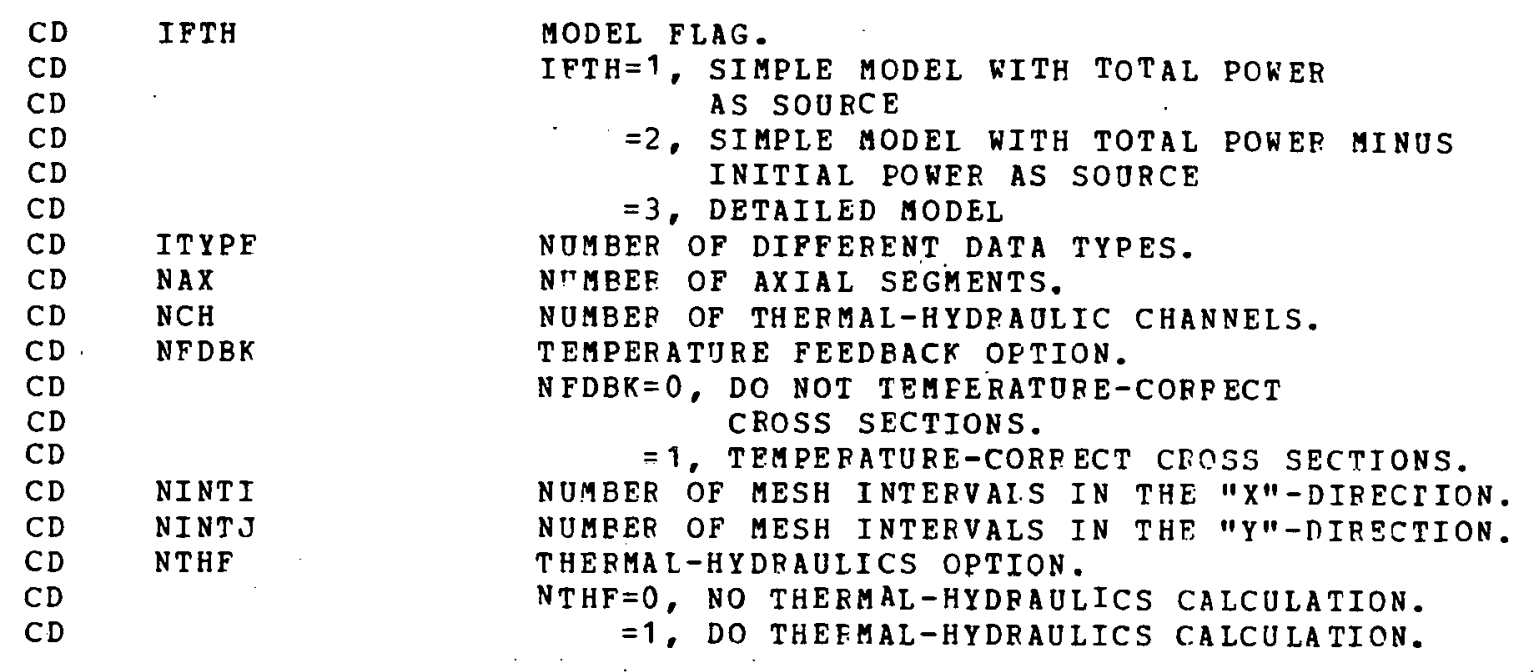
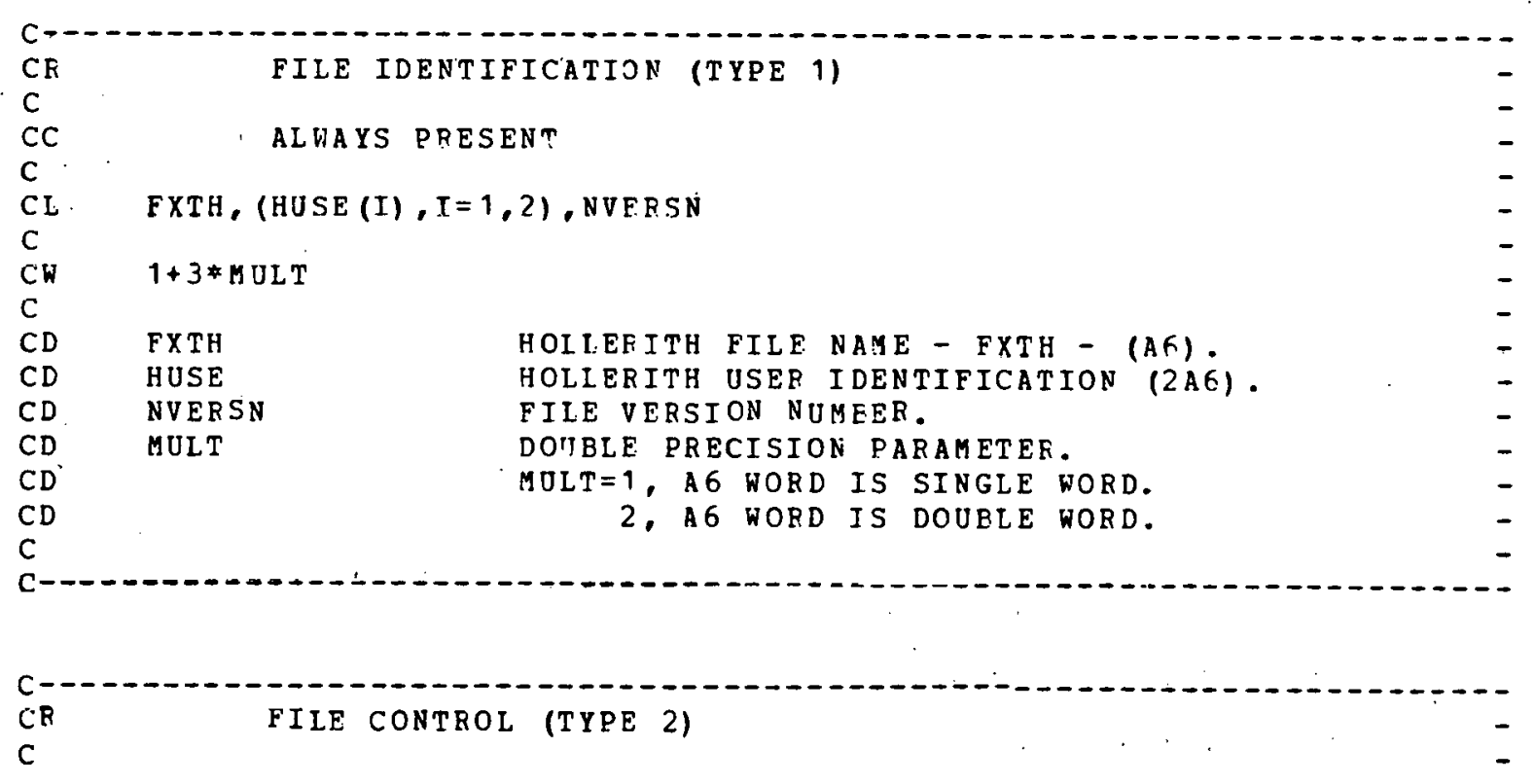

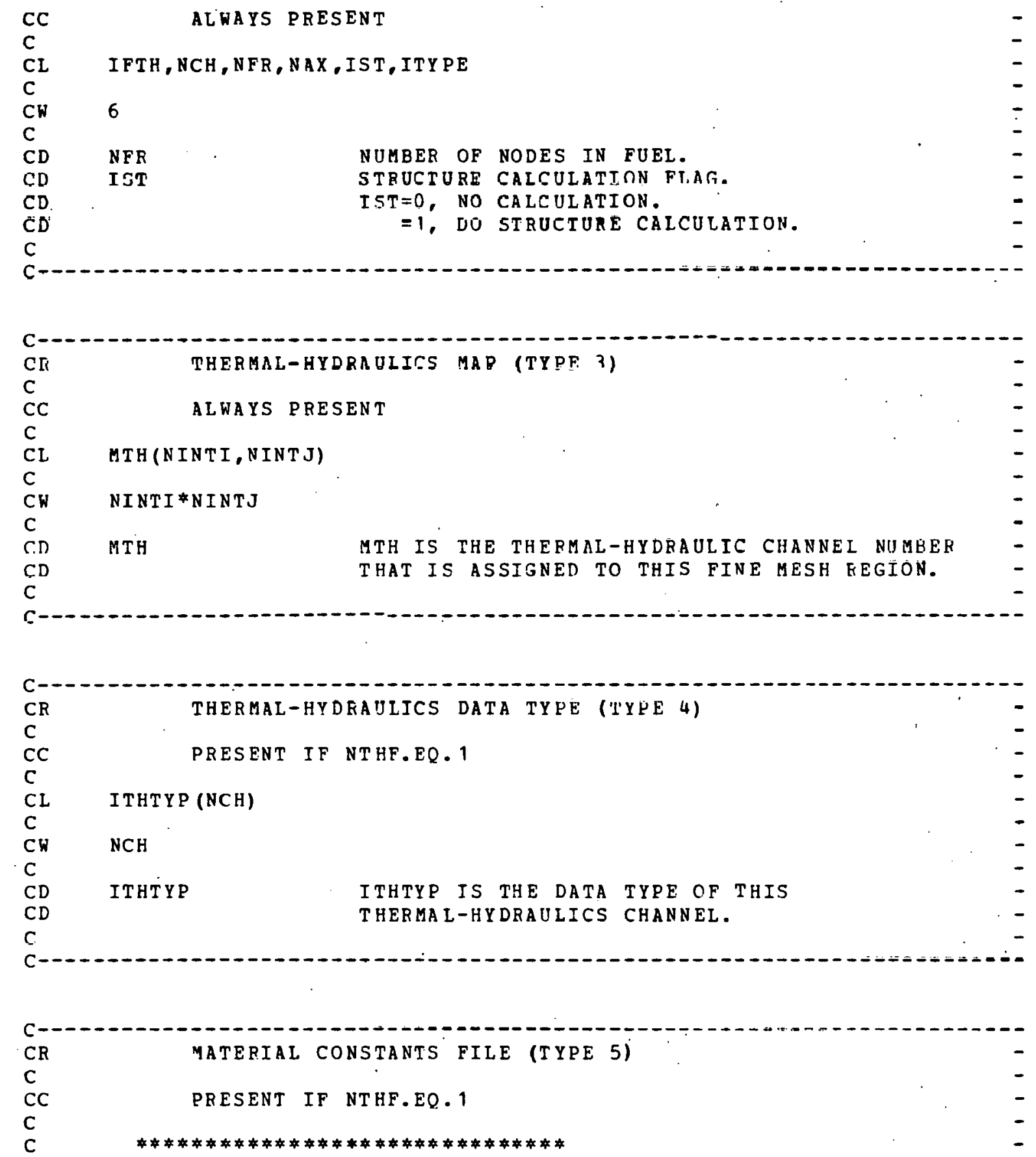


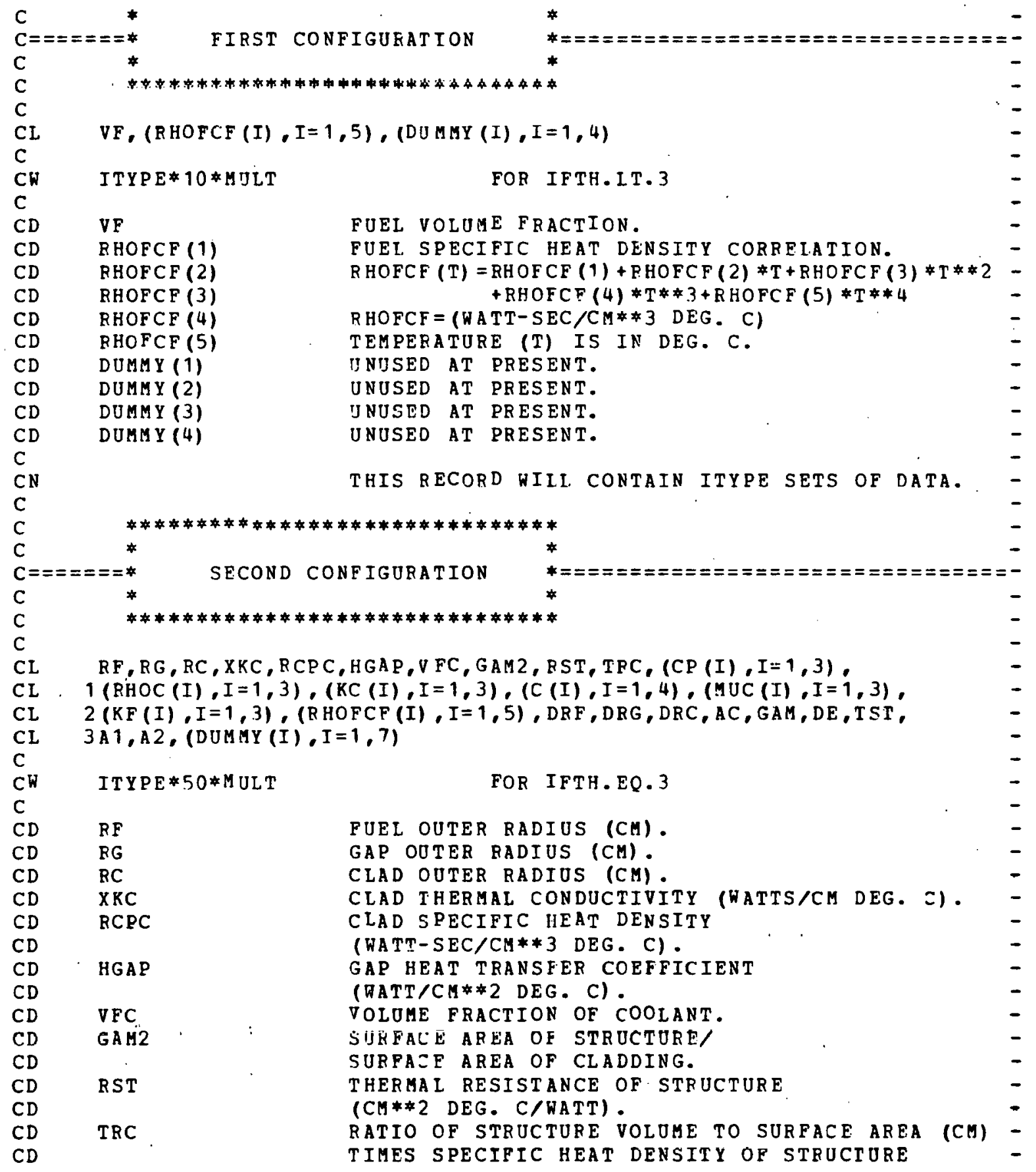




\begin{tabular}{|c|c|}
\hline \multicolumn{2}{|l|}{$C D$} \\
\hline$C D$ & $\mathrm{CP}(1)$ \\
\hline$C D$ & $C P(2)$ \\
\hline$C D$ & $\operatorname{cg}(3)$ \\
\hline $\begin{array}{l}C D \\
C D\end{array}$ & $\begin{array}{l}\text { RHOC (1) } \\
\text { RHOC (2) }\end{array}$ \\
\hline$C D$ & RHOC (3) \\
\hline$C D$ & KC (1) \\
\hline$C D$ & $\mathrm{KC}(2)$ \\
\hline$C D$ & $k C(3)$ \\
\hline$C D$ & $C(1)$ \\
\hline$C D$ & $C(2)$ \\
\hline$C D$ & $C(3)$ \\
\hline$C D$ & $C(4)$ \\
\hline \multicolumn{2}{|l|}{$C D$} \\
\hline$C D$ & $\operatorname{MUC}(1)$ \\
\hline$C D$ & $\operatorname{MUC}(2)$ \\
\hline$C D$ & $\operatorname{MUC}(3)$ \\
\hline$C D$ & $K F(1)$ \\
\hline$C D$ & $K F(2)$ \\
\hline$C D$ & KF (3) \\
\hline$C D$ & RHOFCF (1) \\
\hline$C D$ & EHOFCF (2) \\
\hline$C D$ & RHOFCF (3) \\
\hline$C D$ & RHOFCF (4) \\
\hline$C D$ & RHOFCF ( 5 \\
\hline$C D$ & DRF \\
\hline$C D$ & DRC \\
\hline$C D$ & DRC \\
\hline$C D$ & $A C$ \\
\hline$C D$ & GAM \\
\hline $\mathrm{CD}$ & $D E$ \\
\hline$C D$ & I ST \\
\hline$C D$ & A 1 \\
\hline$C D$ & A 2 \\
\hline \multicolumn{2}{|l|}{$C D$} \\
\hline$C D$ & DUMMY (1) \\
\hline$C D$ & DUMMY (2) \\
\hline$C D$ & DUMMY (3) \\
\hline$C D$ & DUMMY (4) \\
\hline$C D$ & DUMMY (5) \\
\hline$C D$ & DUMMY (6) \\
\hline$C D$ & DUMMY $(7)$ \\
\hline $\mathrm{C}$ & \\
\hline $\mathrm{CN}_{\mathrm{N}}$ & \\
\hline
\end{tabular}

(WATT-SEC/CM * 2 DEG. C). COOLANT SPECIFIC HEAT CORRELATION. $C P(T)=C P(1)+C P(2) * T+C P(3) * T * * 2$ $\mathrm{T}=\mathrm{DEG} . \mathrm{C}, \mathrm{CP}=(\mathrm{BATT}-\mathrm{SEC} / \mathrm{G} \mathrm{DEG} . \mathrm{C})$. COOLANT DENSITY CORRELATION. $\mathrm{RHOC}(\mathrm{T})=\mathrm{RHOC}(1)+\mathrm{RHOC}(2) * \mathrm{~T}+\mathrm{RHOC}(3) * \mathrm{~T} * 2 * 2:$ $T=D E G . C, \quad R H O C=(G / C M * 3)$. CONTANT THERMAL CONDUCTIVITY CORRELATION. $K C(T)=K C(1)+K C(2) * T+K C(3) * T * 2$ $T=D E G . C, K C=(W A T T S / C M D E G . C)$. HEAT TRANSFER COEFFICIENT CORRETATION. $\mathrm{NU}=\mathrm{C}(1)+\mathrm{C}(2) *(\mathrm{RE} * \mathrm{C}(3)) *(\mathrm{PR} * \mathrm{C}(4))$ NU, RE, AND PR ARE DIMENSIONLESS. NU IS NUSSELT NUMBER, RE IS REYNOIDS NUMBER, AND PP IS PRANDTI NUMBER. COOLANT VISCOSITY CORBELATION. $M U C(T)=\operatorname{MUC}(1)+\operatorname{MUC}(2) * T+M U C(3) * T * 2$ $\mathrm{T}=\mathrm{DEG}$. C, $\mathrm{MUC}=(\mathrm{G} / \mathrm{CM}-\mathrm{SEC})$. FUEL THERMAT, CONDUCTIVITY CORREATION. $K F(T)=K F(1)+K F(2) * T+K F(3) * T * * 2$ $\mathrm{T}=\mathrm{DEG}$. C, KF=(WATTS/CM DEG.C). FUEL SPECIFIC HEAT DENSITY CORRELATION. RHOFCF $(T)=R H O F C F(1)+R H O F C F(2) * T+D H O F C F(3) * T * 2$ $+\operatorname{RHOFCF}(4) * T * * 3+\mathrm{RHOFCF}(5) * \mathrm{~T} * 4 * 4$ $T=D E G . C, R H O \overline{F C F}=(W A T T-S E C / C M * 3$ DE'G. C). -

MESH SPACING IN FUEL (CM). MESH SPAOTNG TF RAP (CM). CLAD THICKNESS (CH). COOLANT FLON AREA $(C M * 2)$. SURFAEE TO VOLUME RATIO OF COOLANT CH $\$-1$. COOLANT HYDRAULIC DIAMETER (CH). TIME TO BEGIN MASS FLOW RATE CHANGE (SẼ). MASS FIOW RATE COPRELATION (BEGINS AT TIME TST).

$G(T)=G(D) *(1+A 1 *(T-T S T)+A 2 *(T-T S T) * 2)$

UNUSED AT PRESENT.

INUISED AT PRESENT.

UNOSED AT PRESENT.

TNUSED AT PRESENT.

IINUSED AT PRESENT.

UNUSED AT PRESENT.

UNUSED AT PRESENT.

THIS RECORD WILL CONTAIN ITYPE SETS OF DAIA. -

C

$\mathrm{c}-$ 

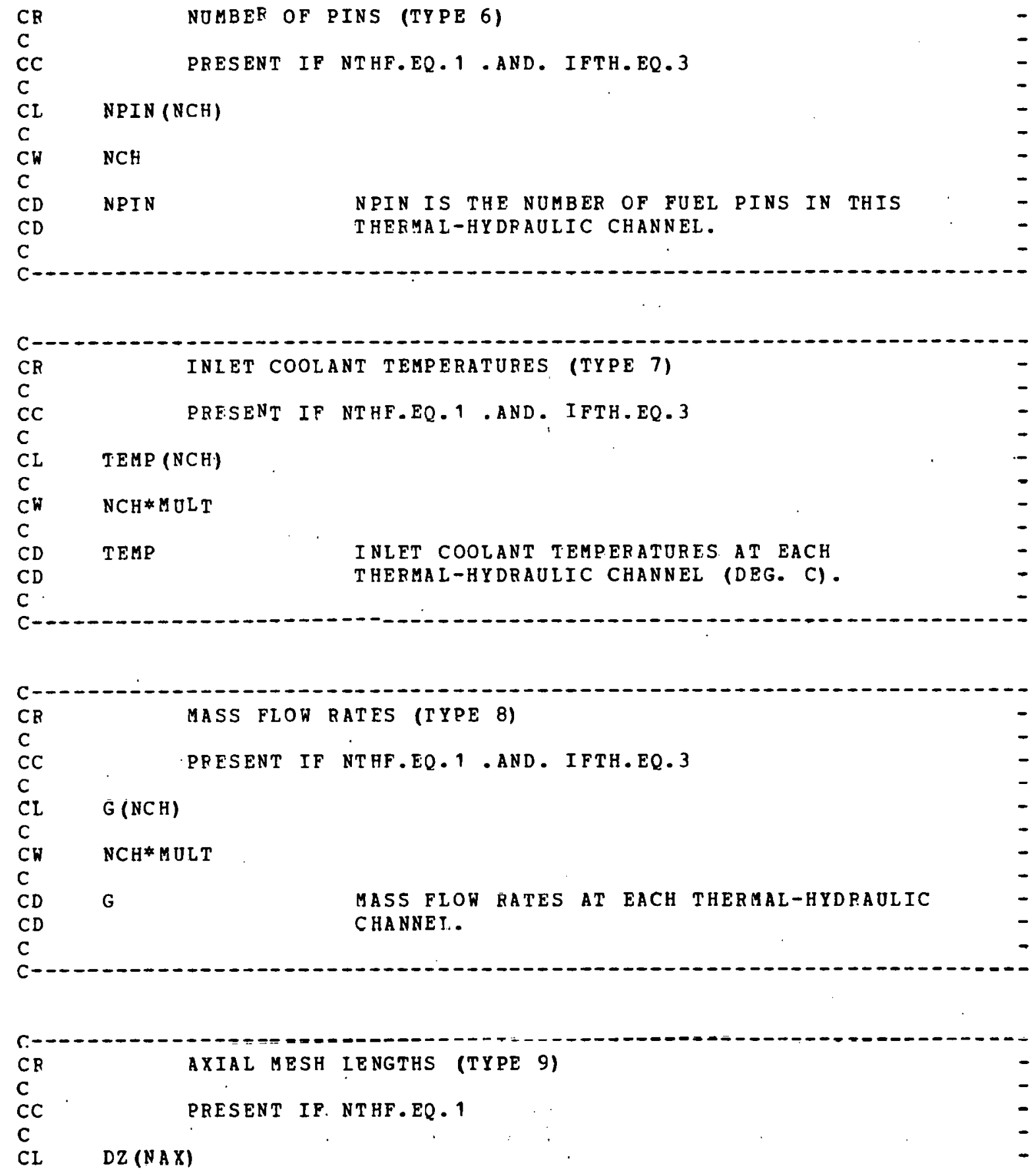

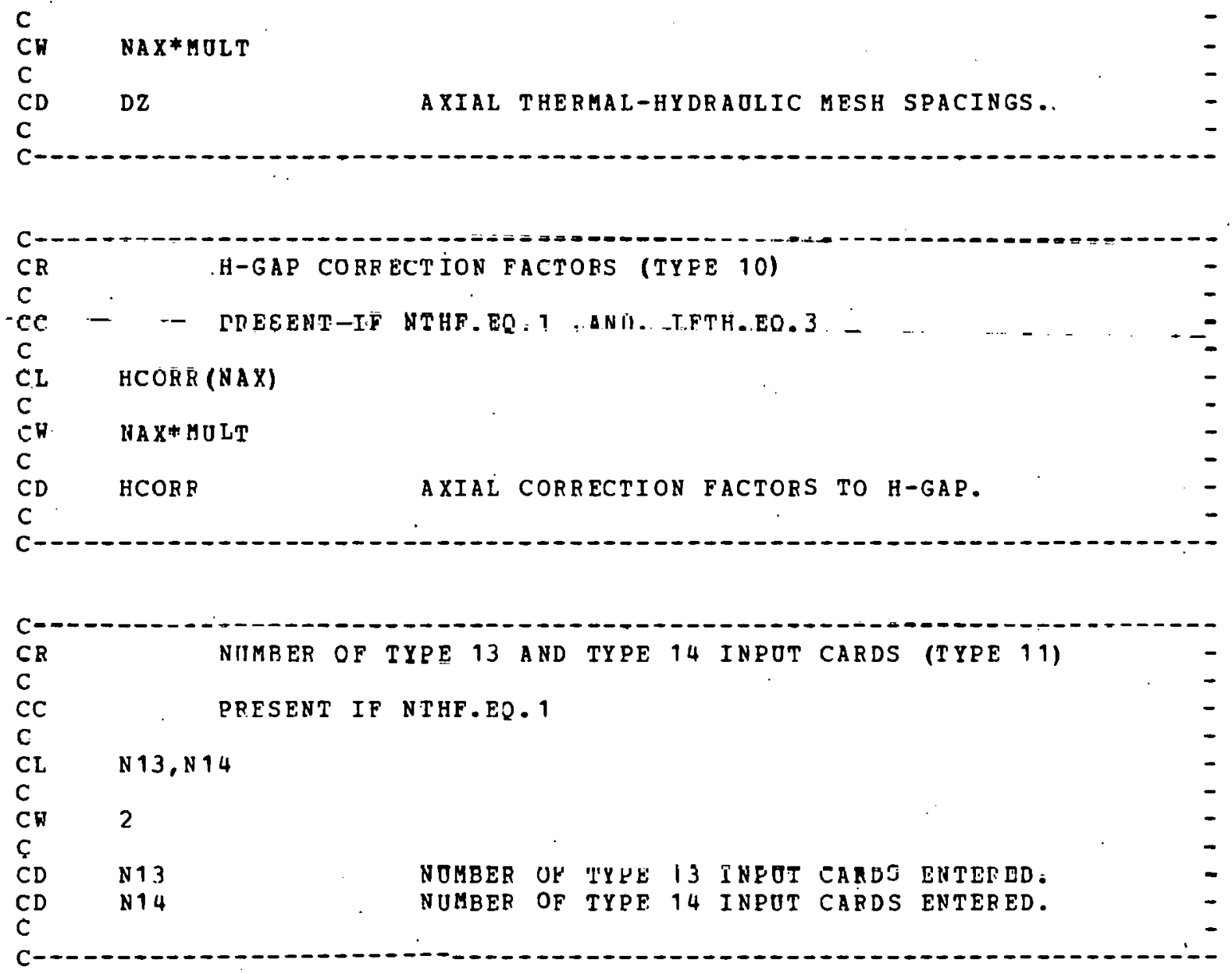

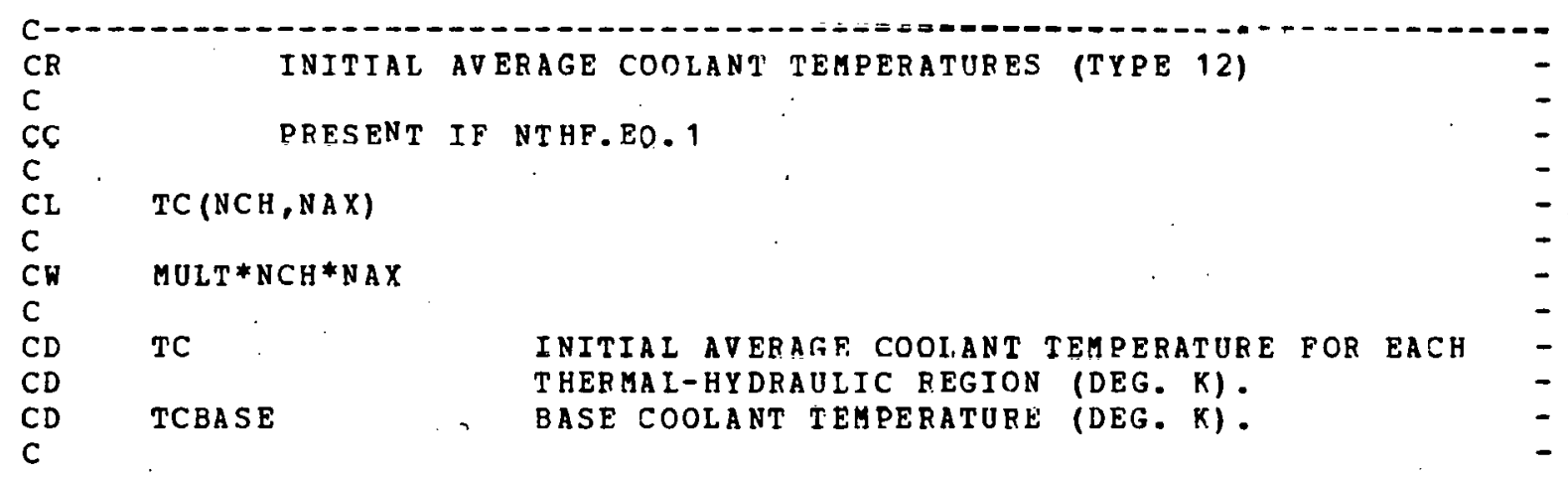



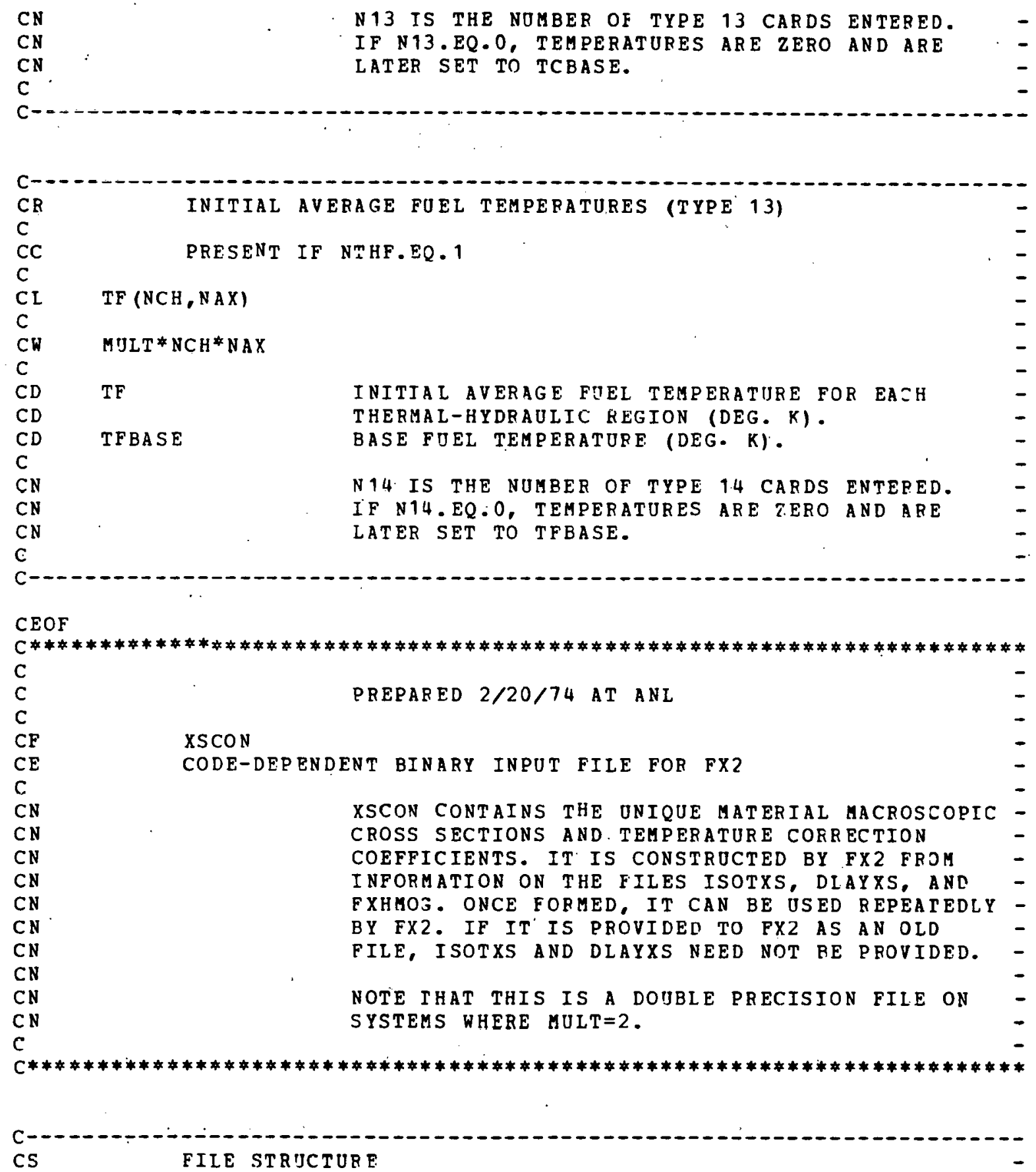


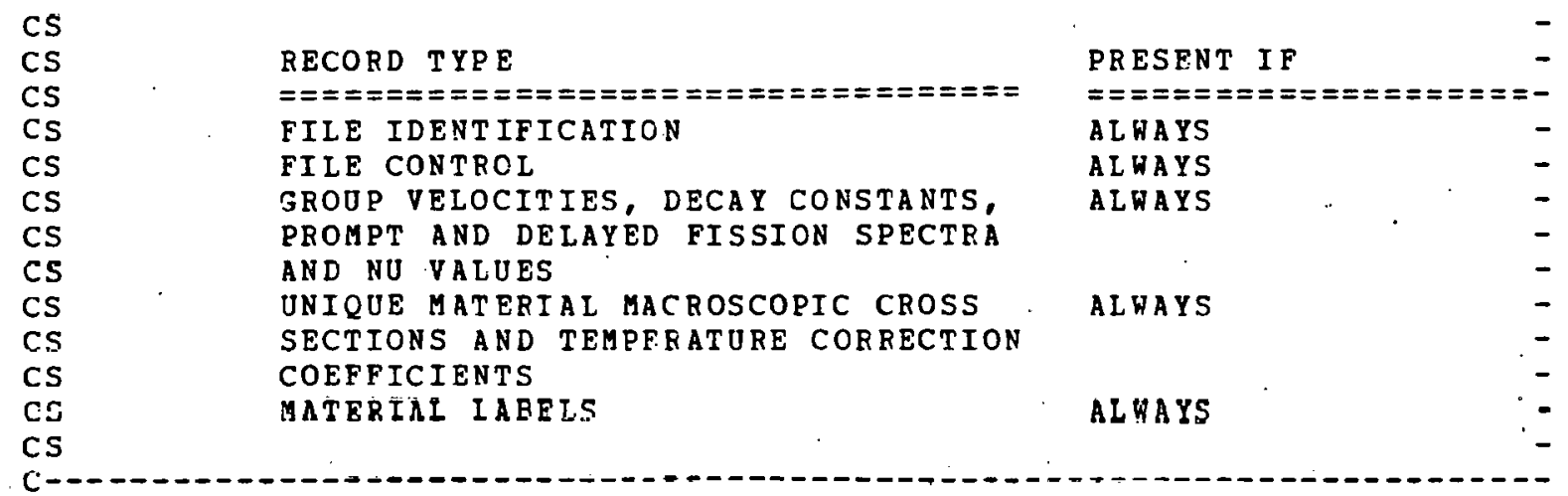

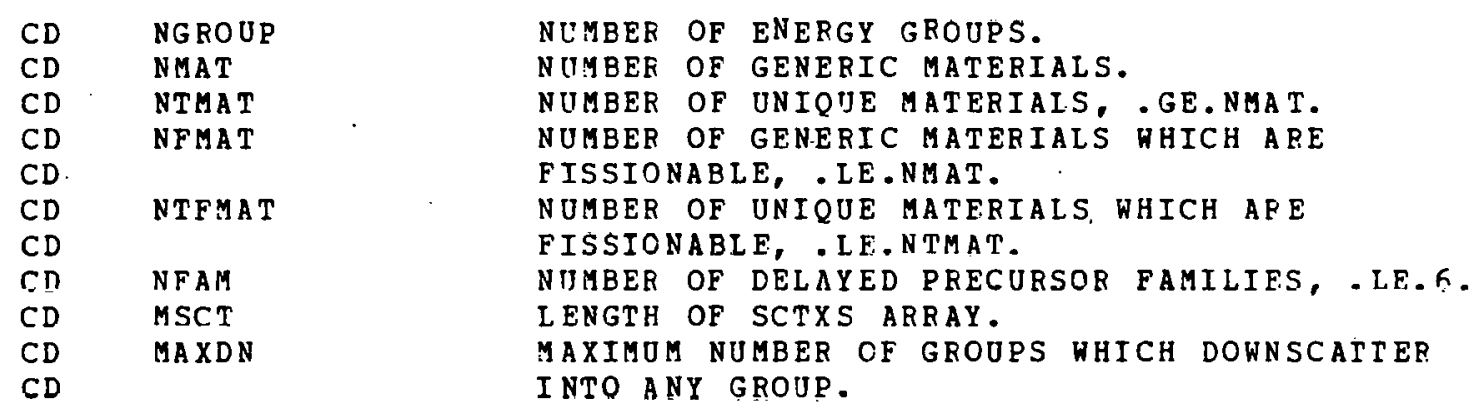

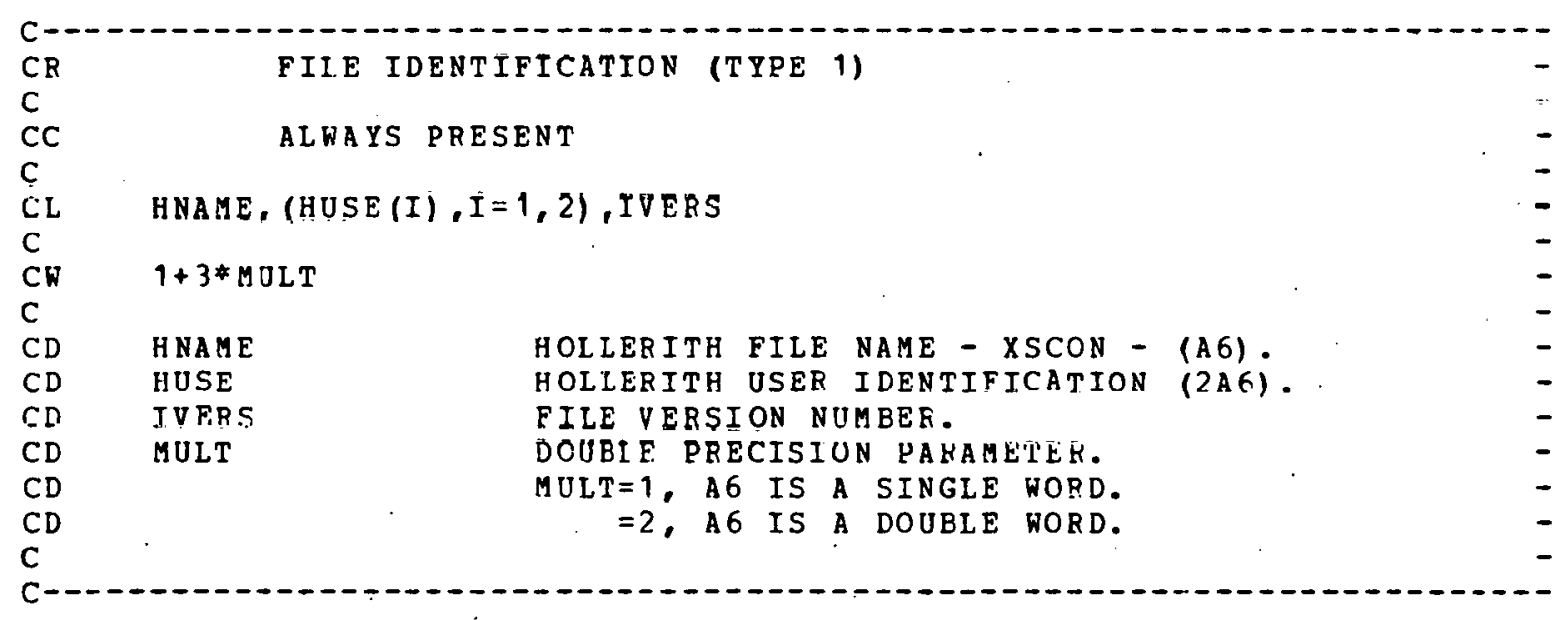

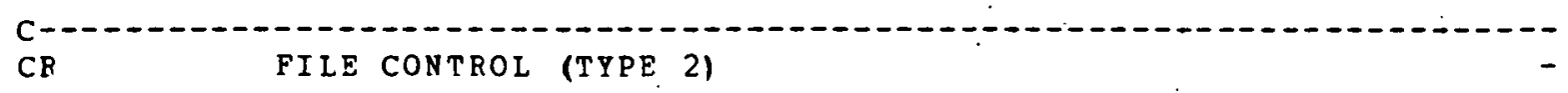




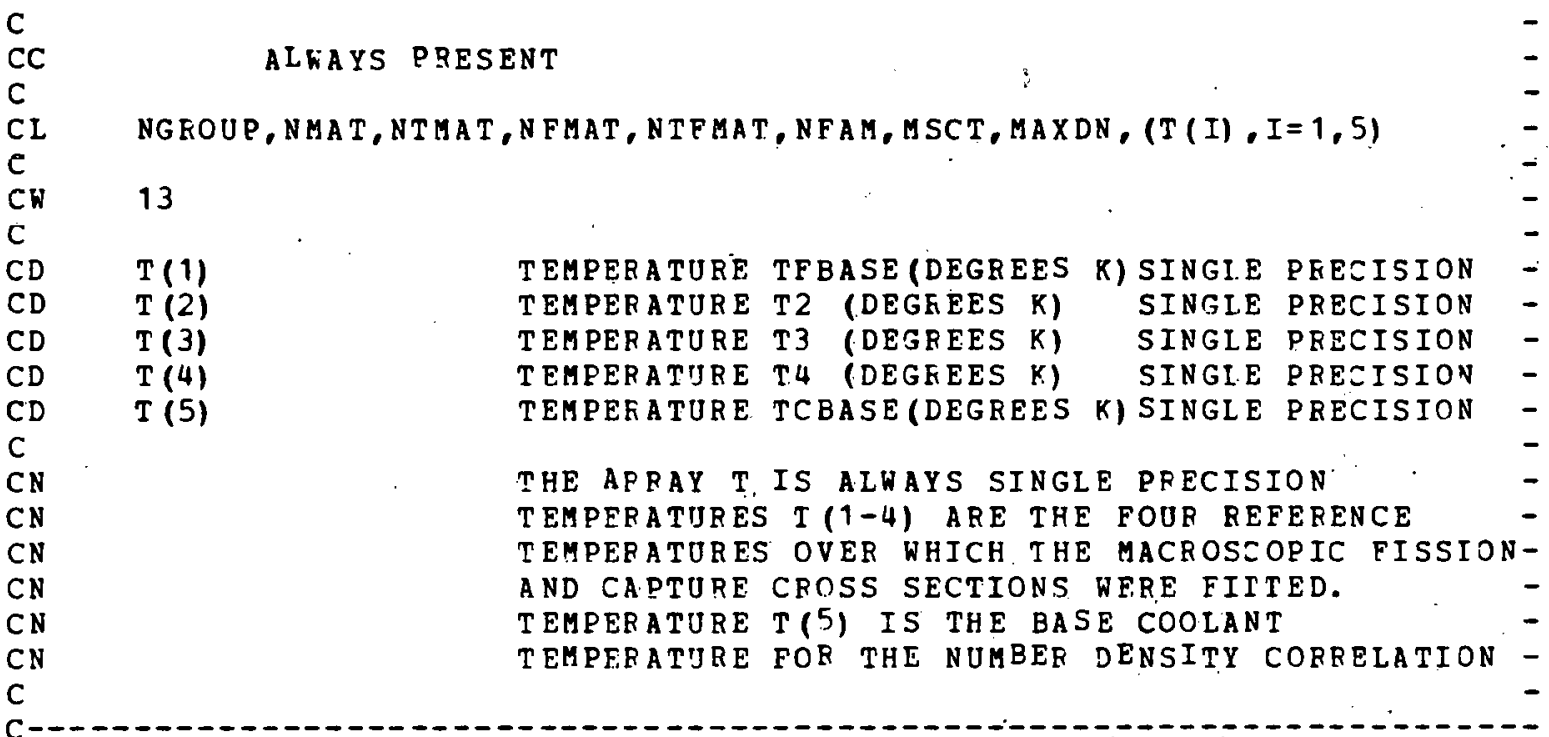

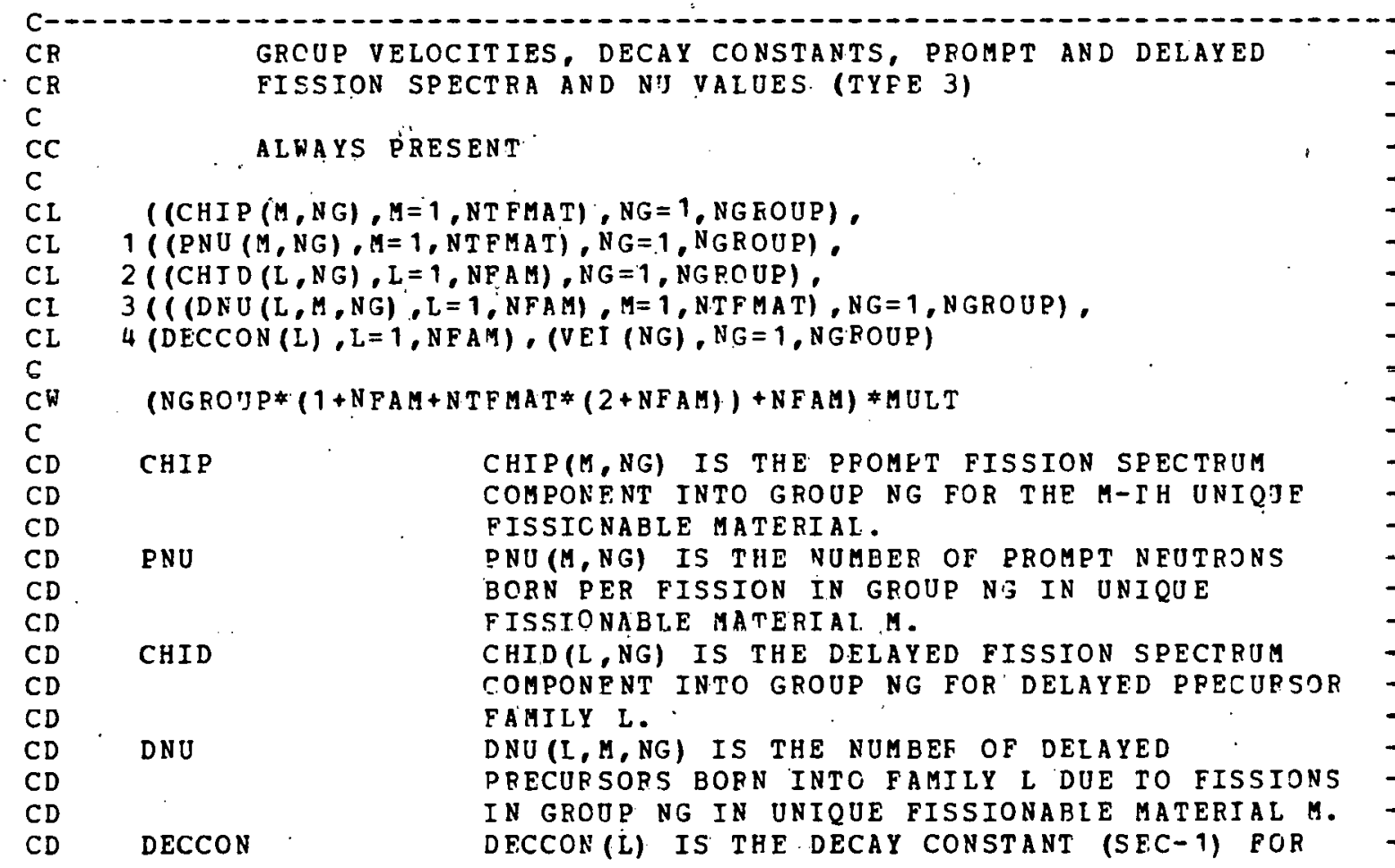




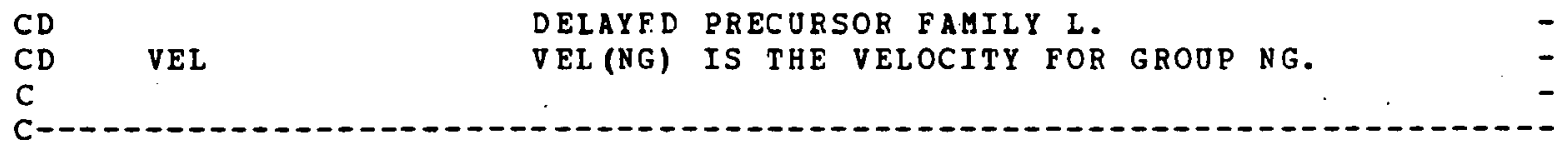

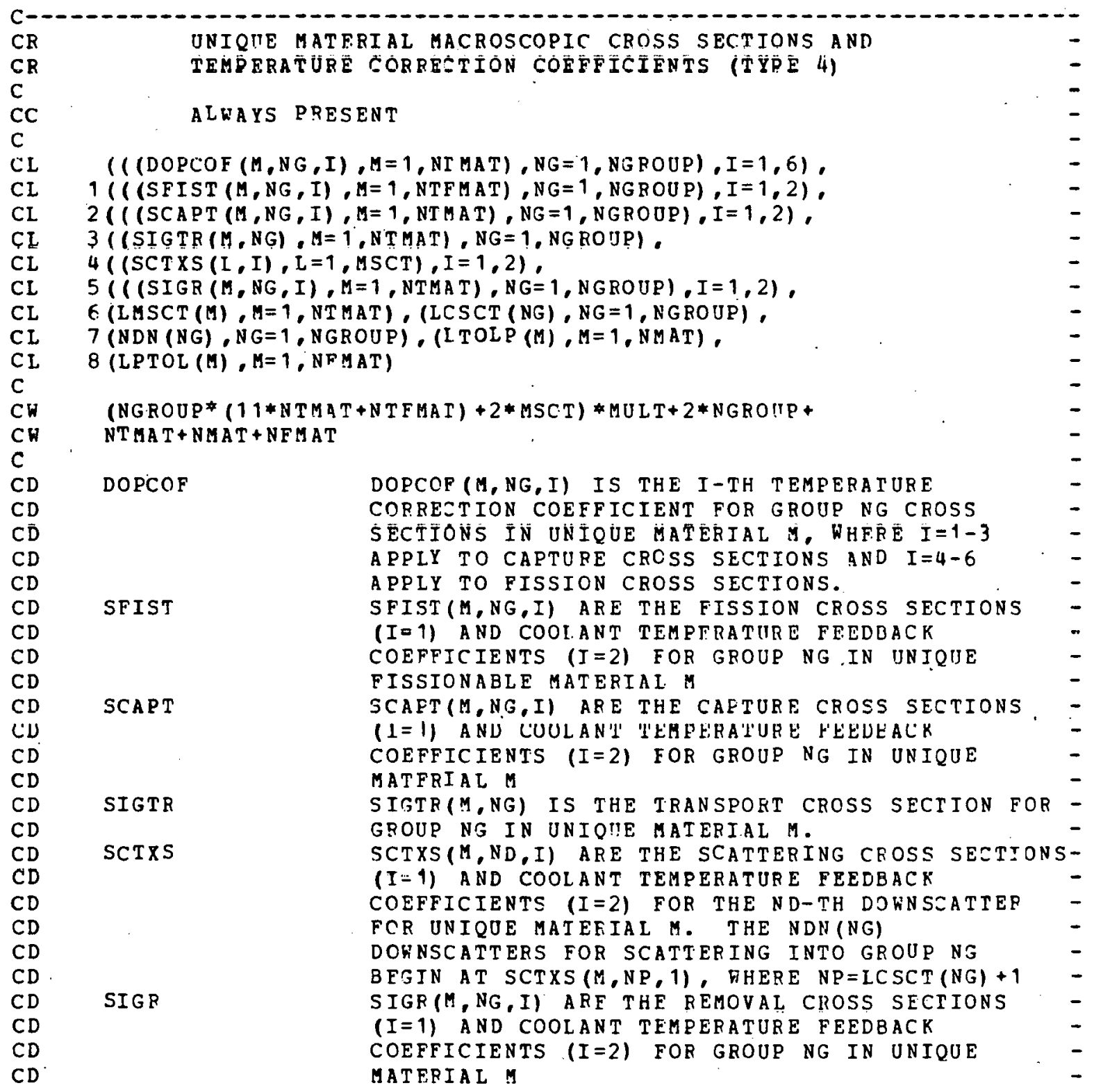


LMSCT(M) IS THE MAXIMUM NOMEER OF DONNSCATTER GROUPS. INTO ANY GROUP FOF UNIQTE MATERIAL M (= MAXDN).

LCSCT IS A POINTER INTO THE ARRAY SCTXS. THE NDN(NG) CROSS SECTIUNS POR SCATTERING INTO GROUP NG IN UNIQUE MATERIAL M ARE FOUND AT $\operatorname{SCTXS}(M, N D), N D=\operatorname{ICSCT}(N G)+1, \ldots$. $\mathrm{LCSCT}(\mathrm{NG})+\mathrm{NDN}(\mathrm{NG})$. NDN(NG) IS THE NUMBER OF GROJPS WHICH DONNS.CATTER INTO GROUP NG. LTOLP(M) IS THE GENERIC MATERTAL NUMBER OF THE M-TH MATERIAL TN THE AHNAME ARRAY; LTCLP(M)=M. LPTOL (M) IS THE GENERIC FISSIONABIE MATERIAL NUMBE OF THE M-TH MATERIAL IN THE AMNAME ARRAY; IPTOL:(M) =M, M. IE. NFMAT.

THE COEFFICIFNTS IN THE DOPCOF ARRAY ARE USED IN THE FOLLONING MANNER: LET THE FISSION AND CAPTURE CROSS SECTIONS FOR GROUP NG IN MATERIAL$M$ AT SOME FUEL TEMPERATUPE.T, BE REPRESENTED ASSIGFIS (NG, M,T) AND SIGCAP (NG,M,T) . WITHIN FX2, THEY WOULD BE COMPUTED AS FOLLOWS: -

$\operatorname{SICCAE~(NG,M,T)=SCAPT}(M, N G)$ + DOPCOF (M,NG, 1) (DSQRT (T) -DSORT (TBASE)) $+D O P C O F(M, N G, 2) *(D L C G(T / T B A S E))$

+ DOPCOF $(M, N G, 3)$ (TBASE-T) / (TBASE*T)

$\operatorname{SIGFIS(NG,M,T)=SFIST(M,NG)}$

+ DOPCOF $(M, N G, 4) *(D S Q R T(T)-D S Q R T(T B A S E))$

$+D O P C O F(M, N G, 5) *(D L O G(T / T B A S E))$

+ DOPCOF $(M, N G, 6) *(T B A S E-T) /($ TEASE*T) , M.LE.NTFMAT

HERE, T IS IN DEGREES KELVIN, AND TBASE IS THE LOWEST, OF THE FOUR TEMPERATURES AT WHICH MATEFIAL MACROSCOPIC CROSS SECTIONS WERE FORHED IN OKDER TO EXTRACT THE COEFFICIENTS IN THE DOPCOF ARBAY.

IN FX2-TH, THE NUMBEF DENSITY FOR EERTAIN ISOTOPES IS COPRELATED AS A LINEAR FUNCTION OF TIID COOLANT TEHPEBATIFF. THEREFORE, ALL MACROSCOPIC CROSS SECTIONS (SCATTERING,CAPTORE, TRANSPORT, AND FISSION) ARE CORRELATED TO THE COOIANT TEMPERATURE AS FOLLUWS: $\operatorname{SIG}(N G, M, T C)=\operatorname{SIGO}(N G, M)+\operatorname{SIG} 1(N G, M) *(T C-T C B A S E)$ A.HERE SIGO(NG,M) IS THE EASE CROSS SECTION, AND- 


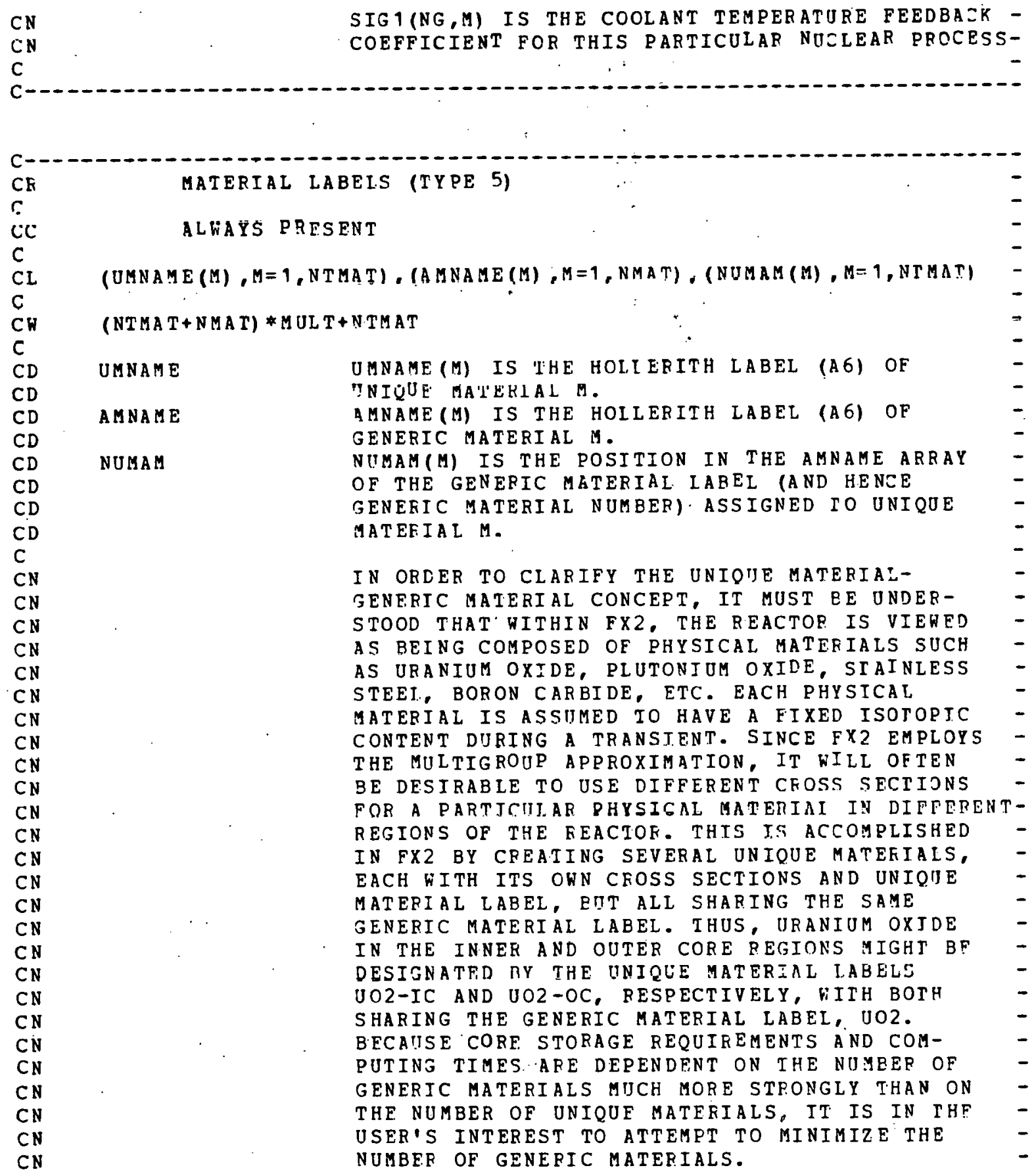



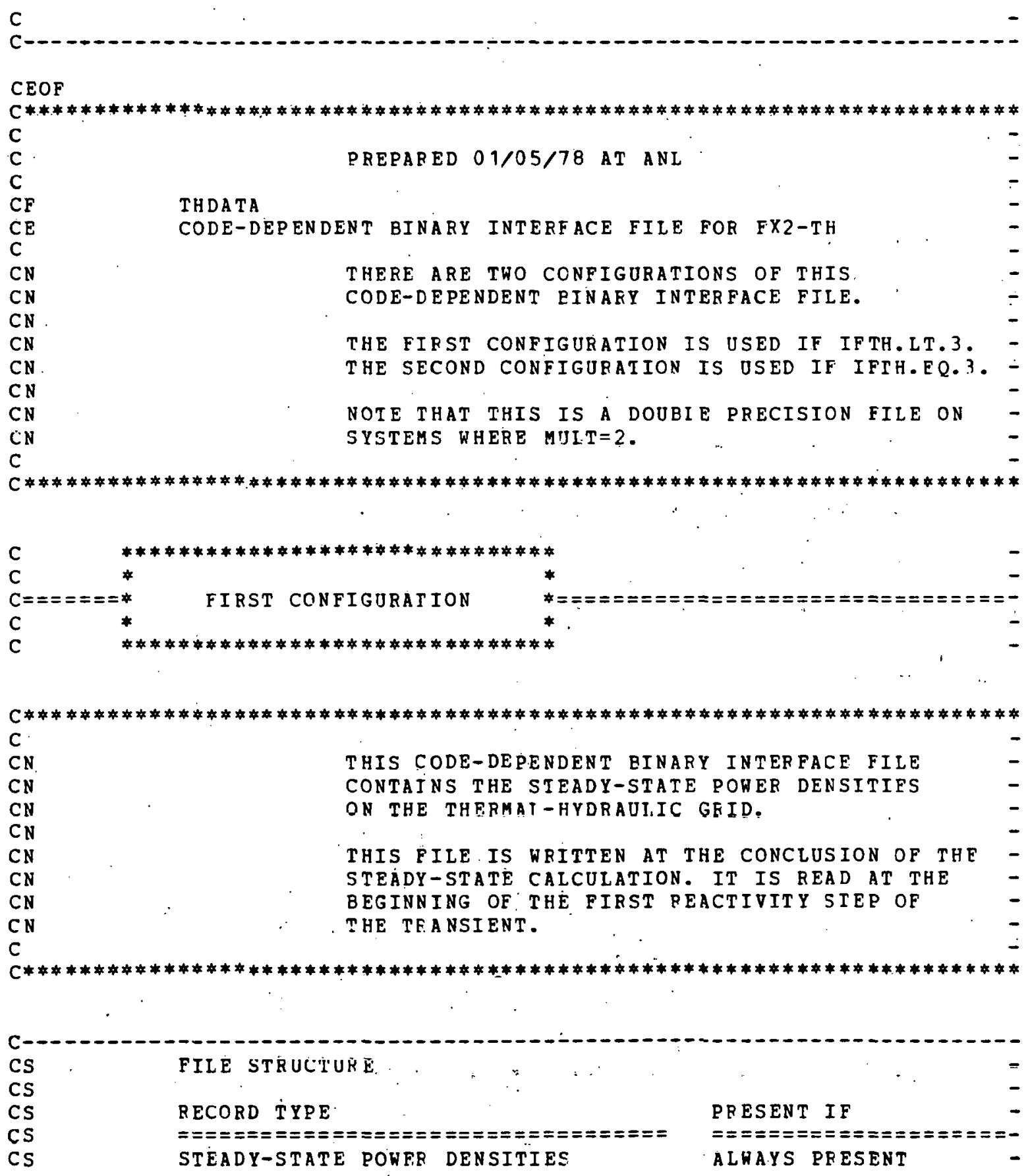
C

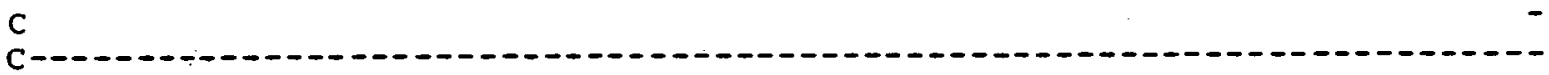

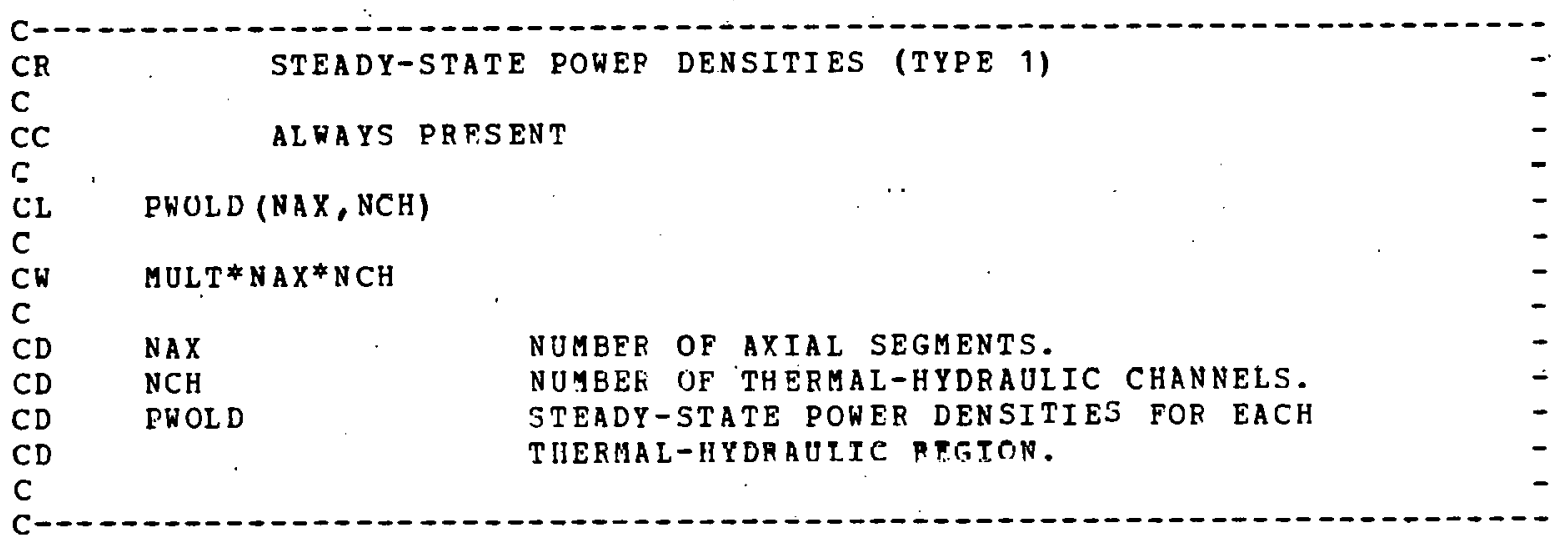
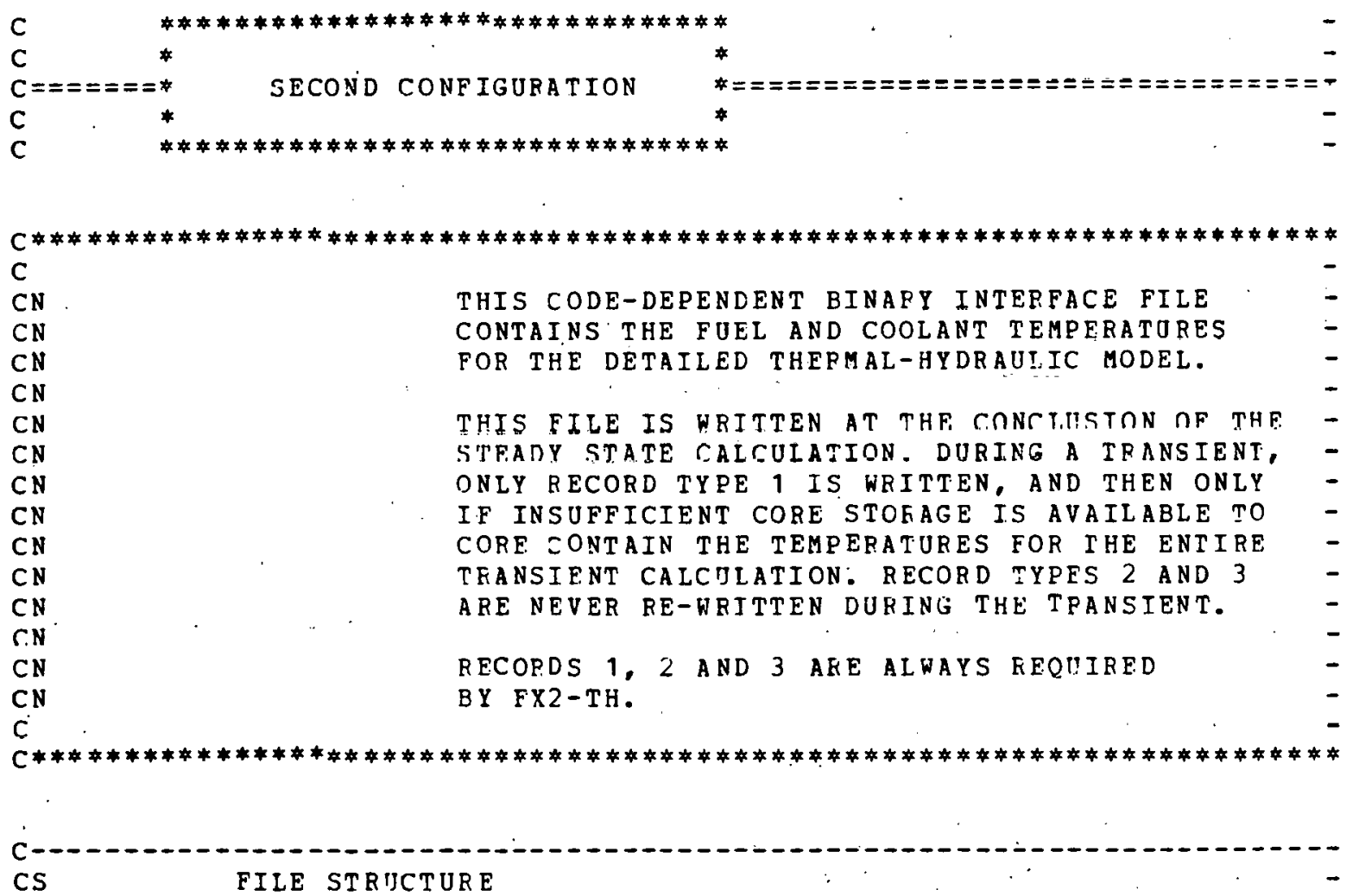


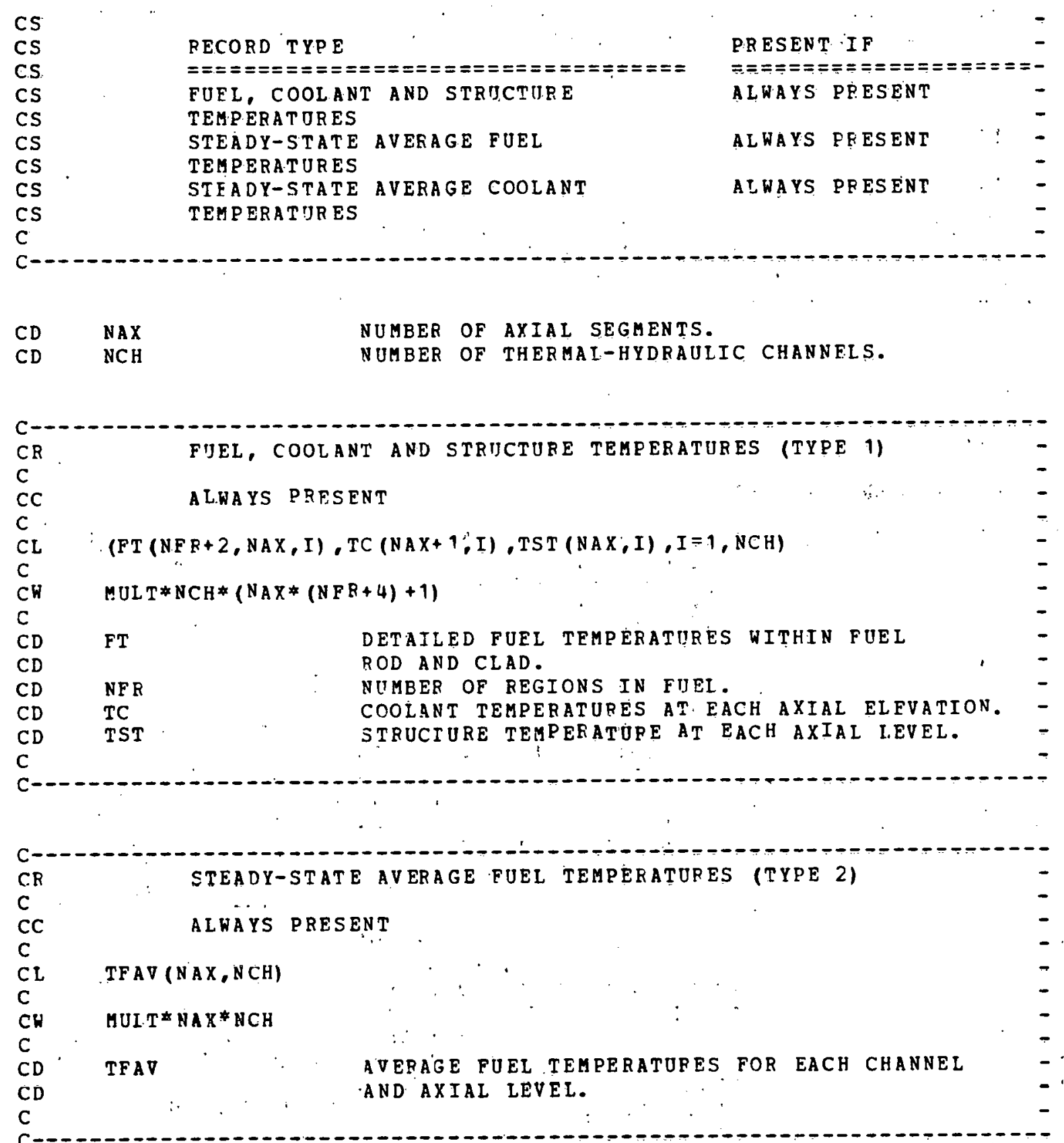

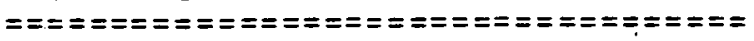
FUEL, COOLANT AND STRICTURE TEMPERAT ORES

STEADY-STATE AVERAGE FUEL

TEMPERATURES

STEADY-STATE AVERAGE COOLANT

=ニ=ニ= = =ニ= = = = = = = = = = = - 


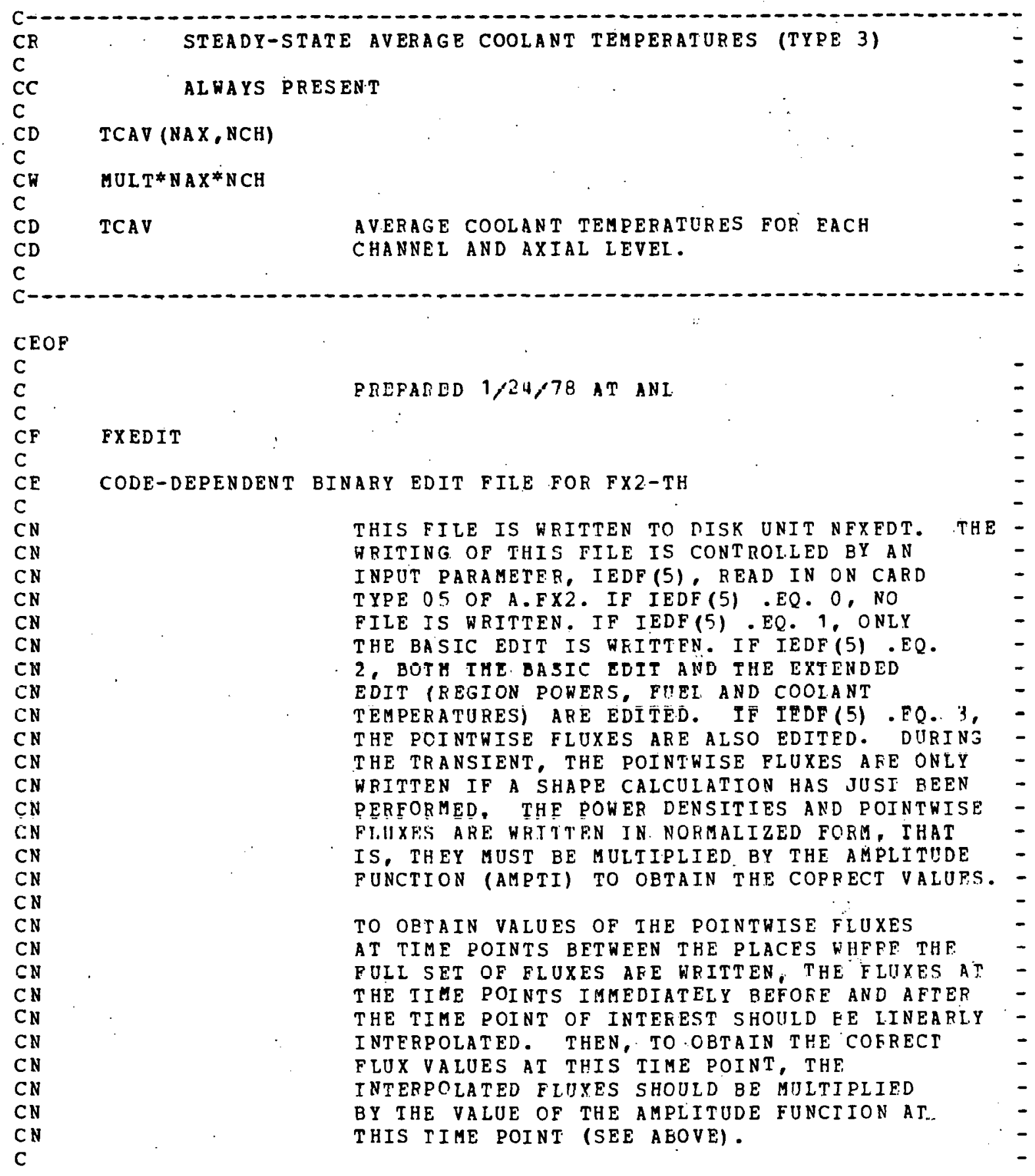




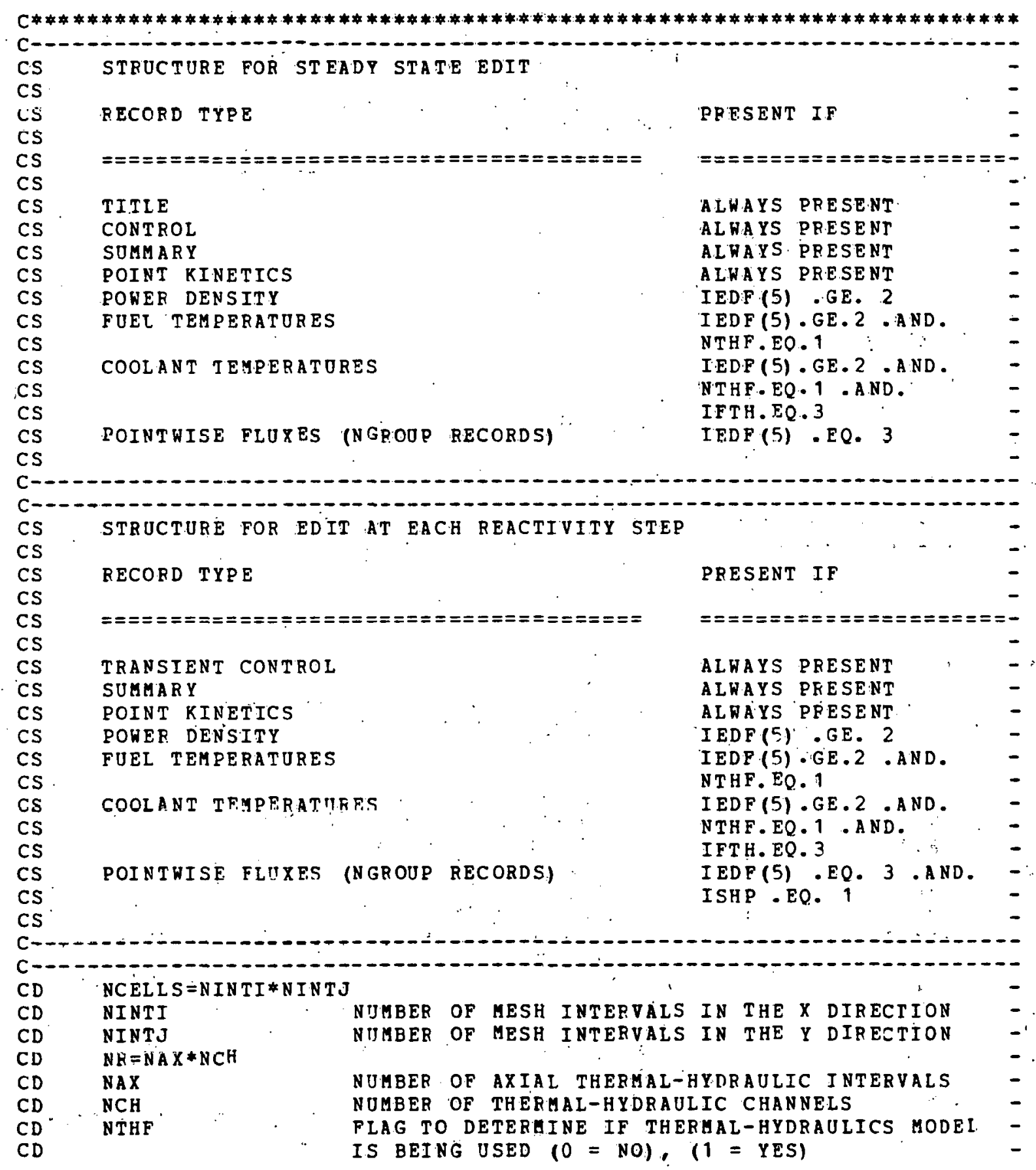




\begin{tabular}{|c|c|c|}
\hline $\begin{array}{l}C D \\
C D \\
C D\end{array}$ & IFTH & $\begin{array}{l}\text { FLAG TO DETERMINE WHICH THERMAL-HYDRAJLICS } \\
\text { MODEI IS BEING USED ( } 1 \text { OR } 2=\text { SIMPLE MODEL), } \\
\text { ( } 3 \text { = DETAILED MODEL). }\end{array}$ \\
\hline$C D$ & ISHP & FLAG TO DETERMINE IF SHAPE CALCULATION HAS \\
\hline$C D$ & & JUST BEEN MADE $(0=N O),(1=$ YES $)$. \\
\hline & & $-\infty$ \\
\hline $\begin{array}{l}\text { CR } \\
C\end{array}$ & TITLE & - \\
\hline $\mathrm{CC}$ & ALWAYS. PRESEI & NT \\
\hline C & & . \\
\hline CL & $(\operatorname{TITLE}(I), I=1,12)$ & I EDF (5) \\
\hline $\begin{array}{l}\mathrm{C} \\
\mathrm{CW}\end{array}$ & $12 * M U L T+1$ & 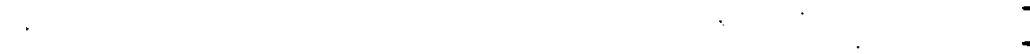 \\
\hline C & & 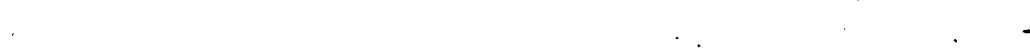 \\
\hline CD & TITI E & PROBLEM TITLE (IN 12 A 6 FORMAT) \\
\hline CD & IEDF (5) & CONTEOLS EDITING \\
\hline$C D$ & MUIT & DOURLE PRECISION PAEAMETER. \\
\hline CD & & MULT $=1$, A6 IS A SINGLE WORD. \\
\hline CD & & $=2$, A6 IS A DOUBLE WORD. \\
\hline C & & . $\quad$. \\
\hline & & ------ \\
\hline $\mathrm{C}-$ & ----------------1 & ------------------------ \\
\hline $\mathrm{CR}$ & CONTROL & - \\
\hline C & & \\
\hline $\mathrm{CC}$ & ALFAYS PRESE & NT \\
\hline C & & \\
\hline $\mathrm{CH}$ & 27 & 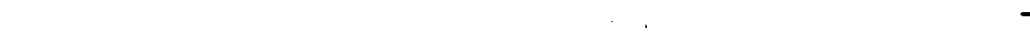 \\
\hline $\mathrm{C}$ & & \\
\hline$C D$ & NCH, NAX, NINTI, NIN? & TJ, NGEOUP, NMAT, NTMAT, NFMAT, NTFMAT, NFAM, MSCT, \\
\hline$C D$ & $1 \mathrm{MAXDN}, \mathrm{NREG}, \mathrm{NREGD}$, & NCMP, MNFPTS, NDSCT, NGEOM, NZONE, IMBL, J MBT, \\
\hline$C D$ & 2 IMER, JMBB, MAXSIZ, & NTHF, IFTH \\
\hline$C D$ & & \\
\hline$C D$ & NINTI & NO. OF X-DIRECTION MESH INTERVALS \\
\hline$C D$ & NINTJ & NO. OF Y-DIRFCTTON MESII JNTERVALS \\
\hline$C D$ & NGROUP & NO. OF NEJTRON ENERGY GROUPS \\
\hline$C D$ & NMAT & NO. OF GENERIC MATERIAIS \\
\hline$C D$ & NTMAT & NO. OF UNIQUE MATERIALS \\
\hline CD & NFMAT & NO. OF GENEPIC MATERIALS WHICH ARE FISSIONABI \\
\hline$C D$ & NTFMAT & NO. OF ÐNIQTE MATERIALS WHICH ARE FISSIONABLE \\
\hline CD & NFAM & NO. OF DELAYED PRECURSOR FAMILIES \\
\hline$C D$ & MSCT & WATERIAL SCATTERING CROSS SFCT \\
\hline CD & MAXDN & MAX. NO. OF DOWNSCATTER GROUPS FOR A GROISP \\
\hline$C D$ & N REG & NO. OF REGIONS \\
\hline$C D$ & N REGD & NO. OF REGIONS IN WHICH DRIVING FUNCTIONS \\
\hline$C D$ & $\cdot$ & ARE USED. \\
\hline$C D$ & NCMP & NO. OF COMPOSITIONS \\
\hline$C D$ & MNFPTS & NO. OF MESH INTERVALS WITH FISSIONABLE MATERIAL \\
\hline CD & NDSCT & TOTAL NUMBER OF. DOWNSCATTER GROUPS \\
\hline$C D$ & NGEOM & GEOMETRY TYPE \\
\hline
\end{tabular}




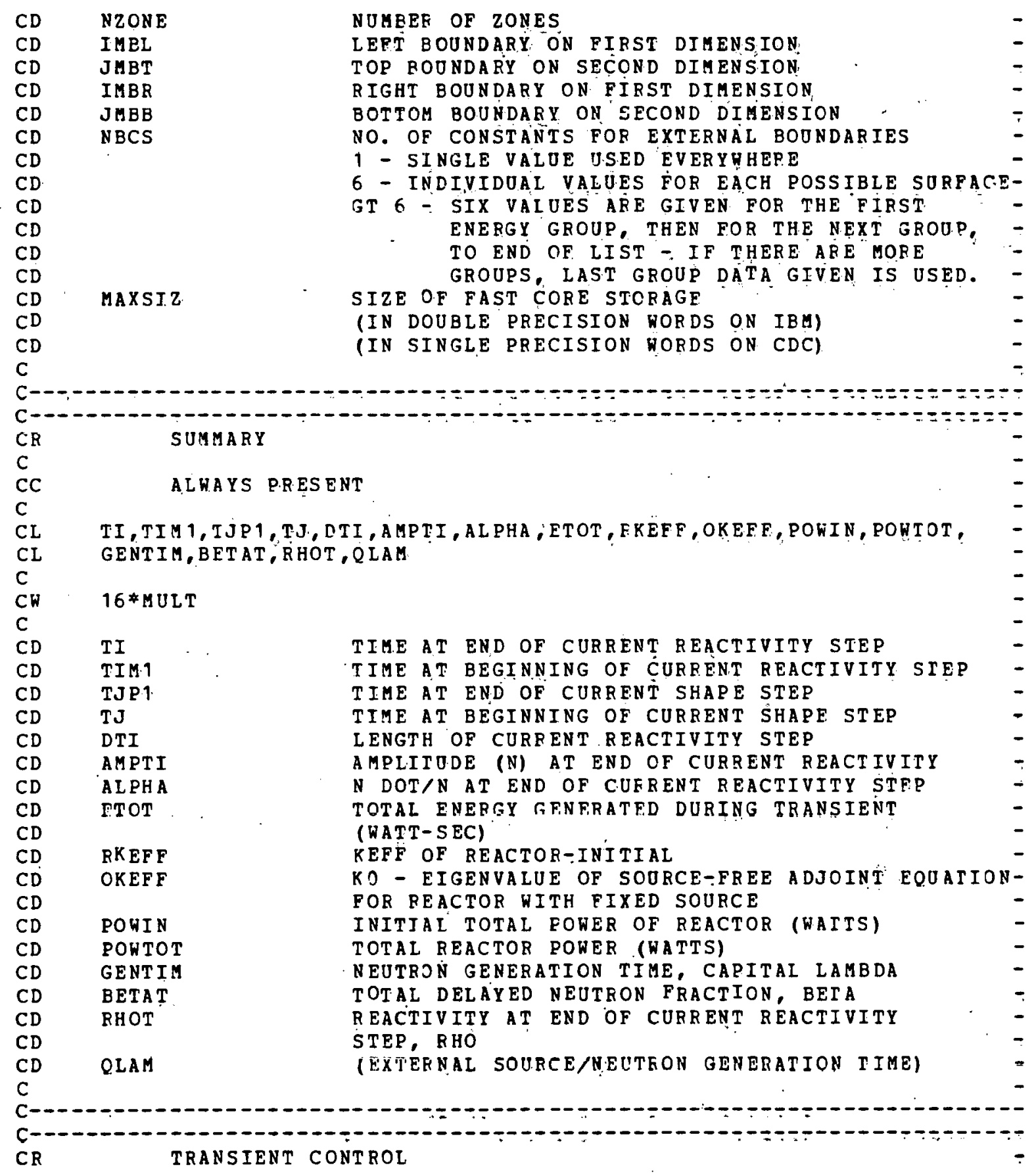




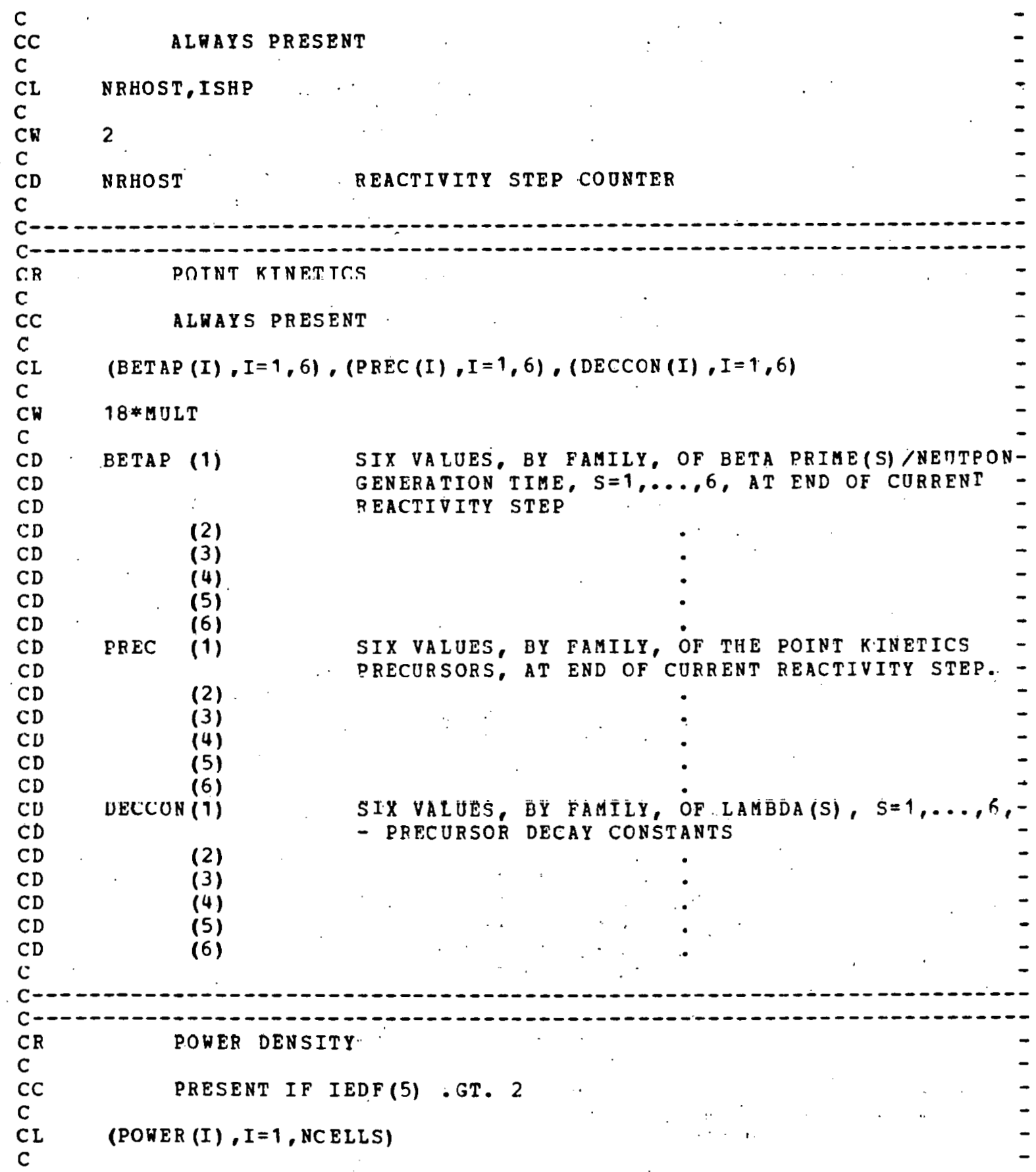




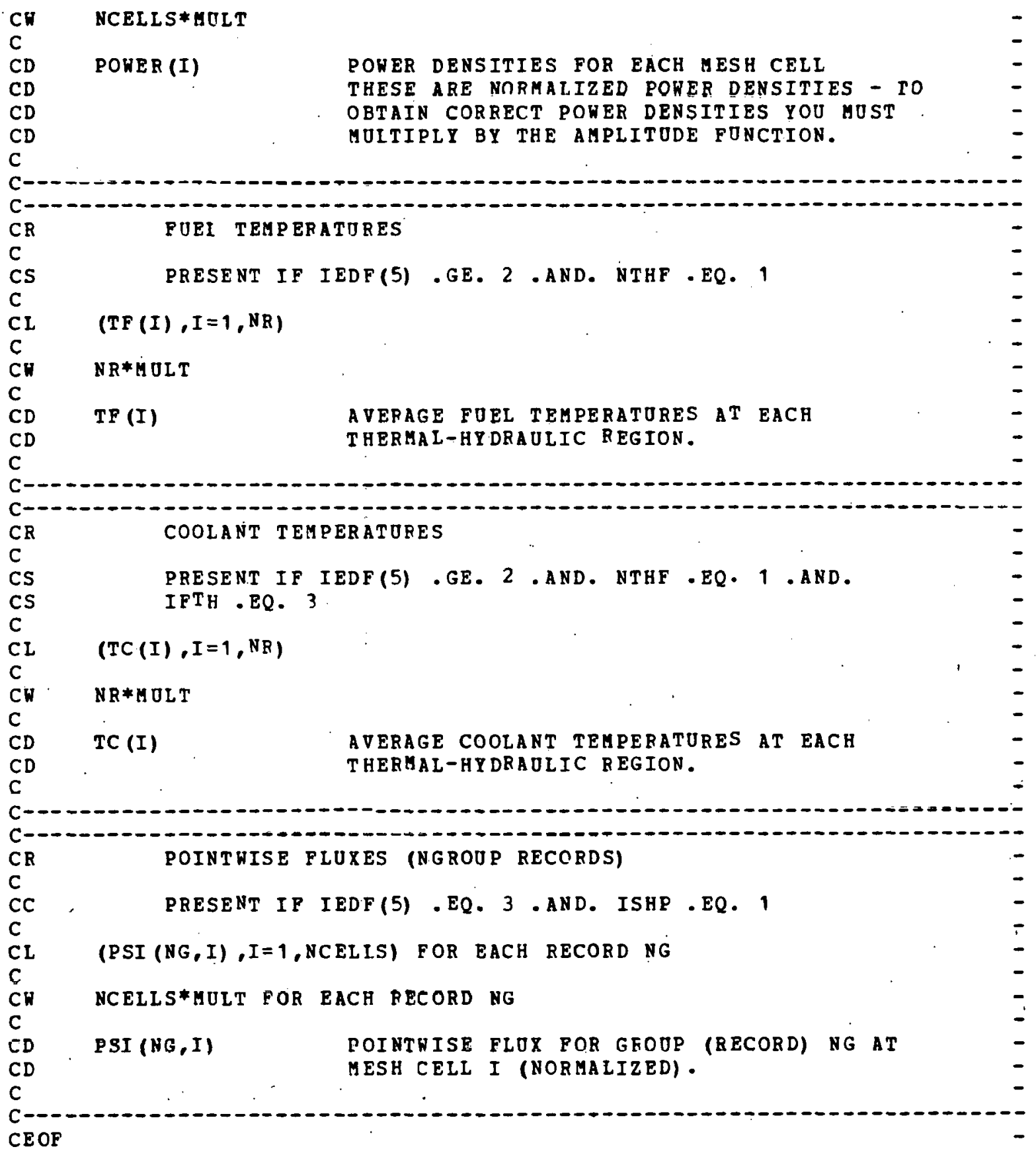


THIS PAGE

\section{WAS INTENTIONALLY LEFT BLANK}




\section{APPENDIX D}

Listing of Scratch Files 


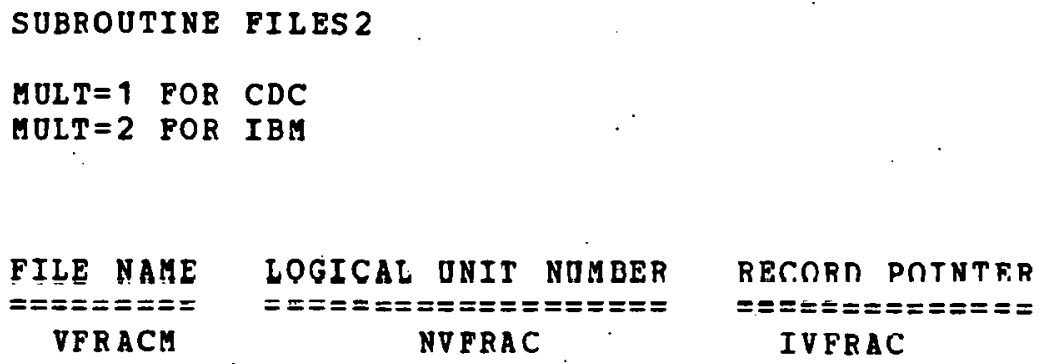

FILE NAME

$==ニ=ニ===$ POWDNG

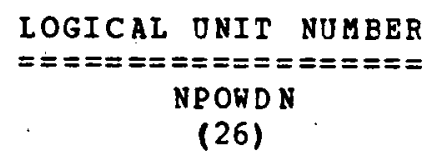




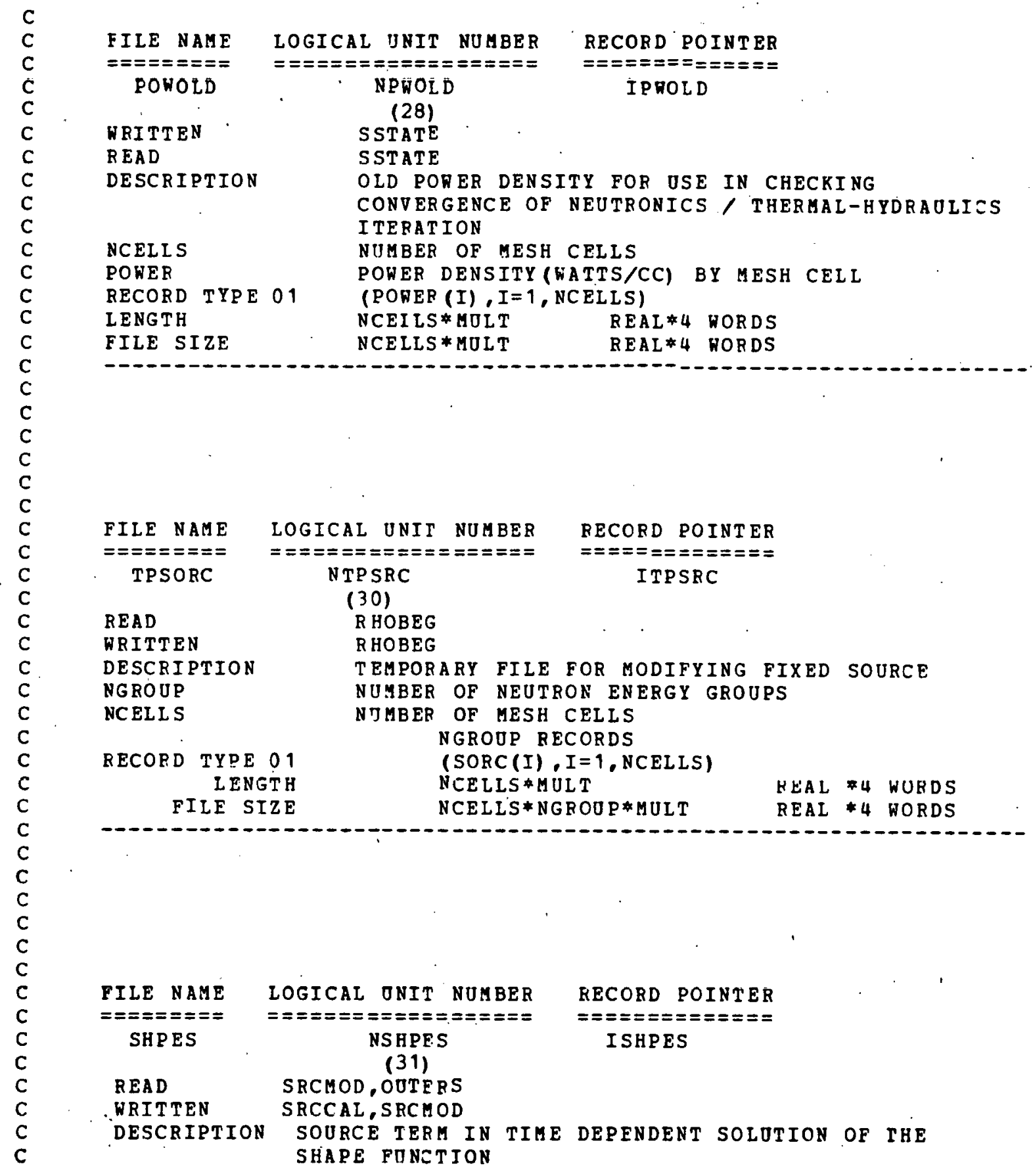




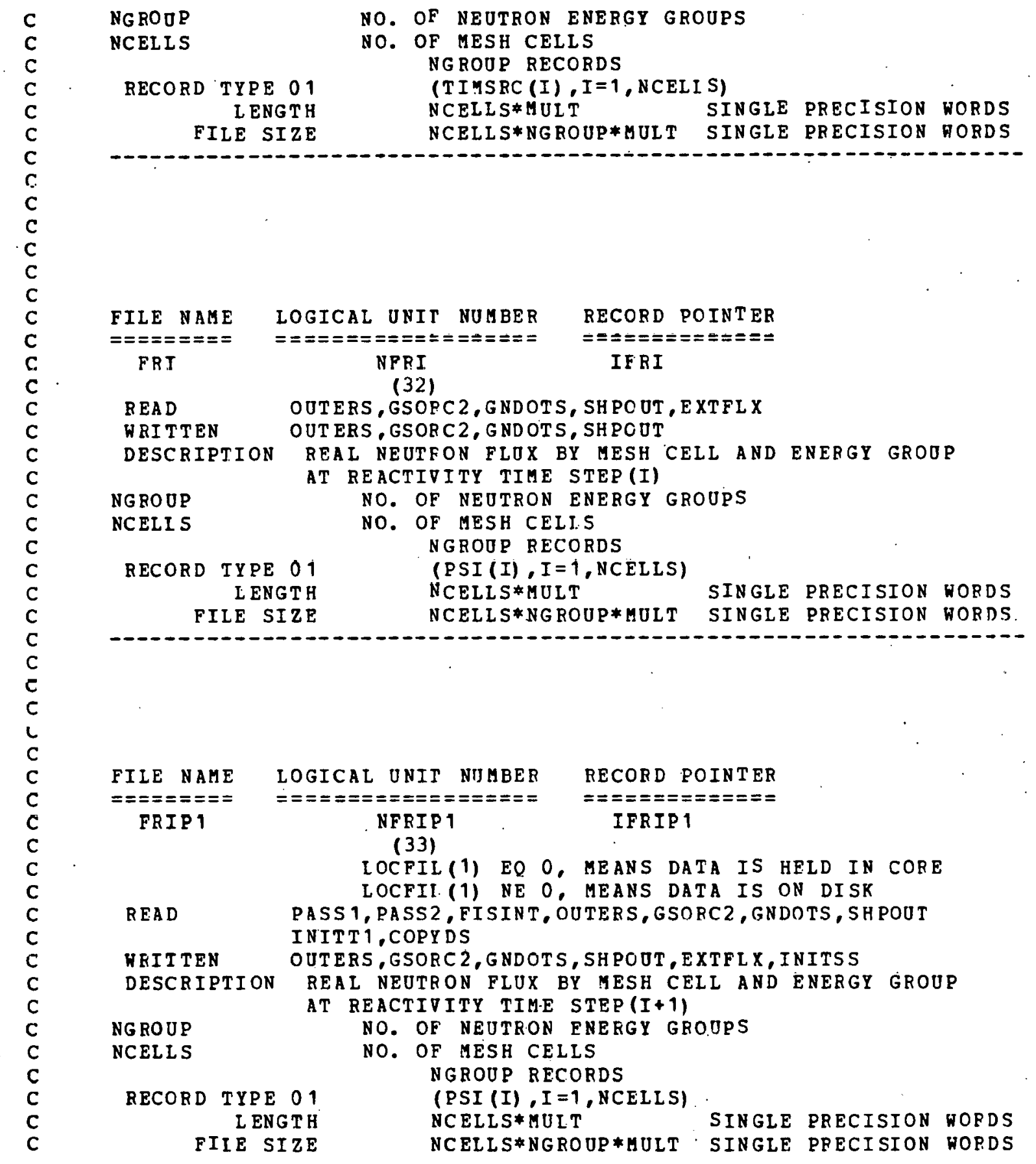




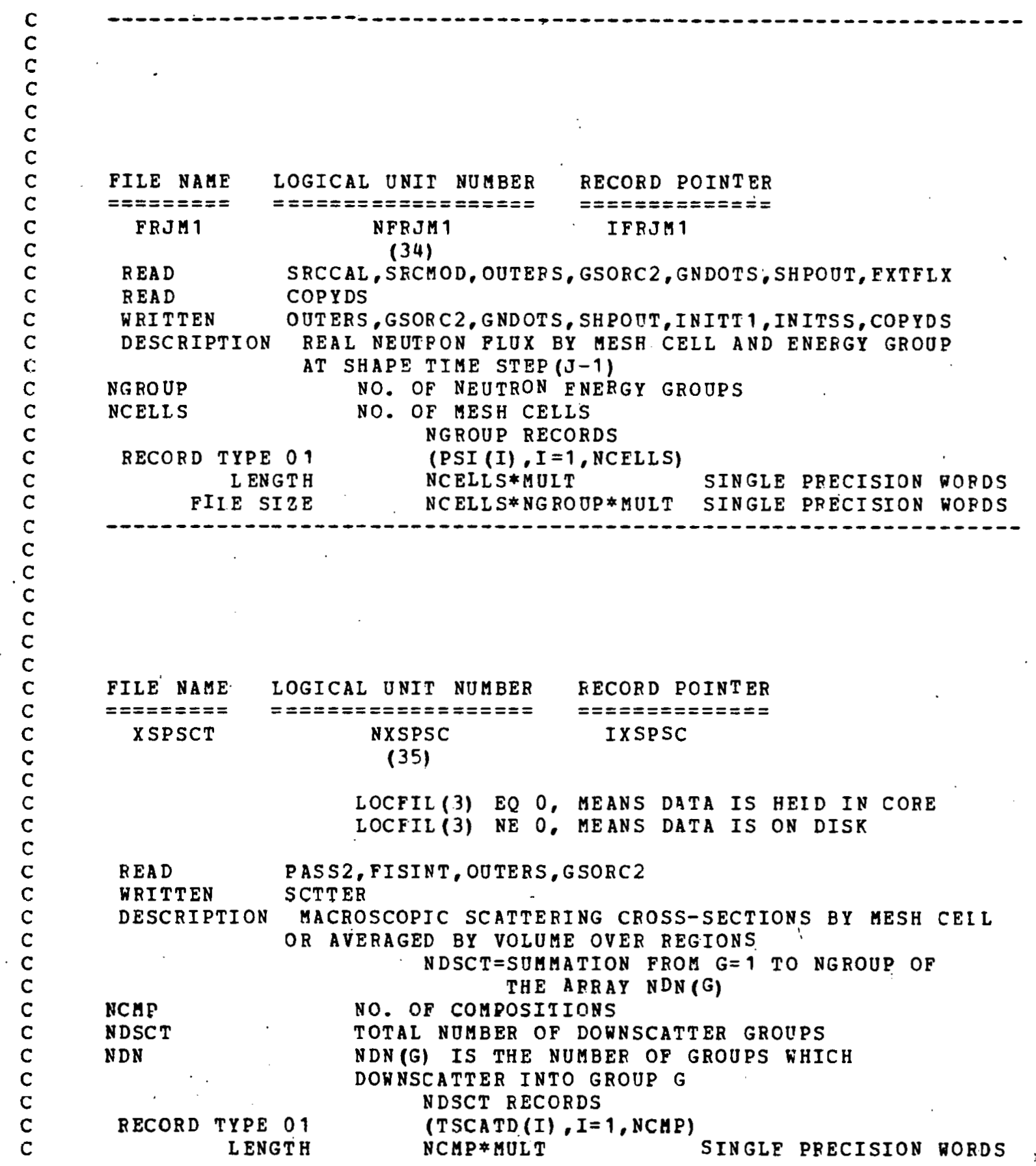




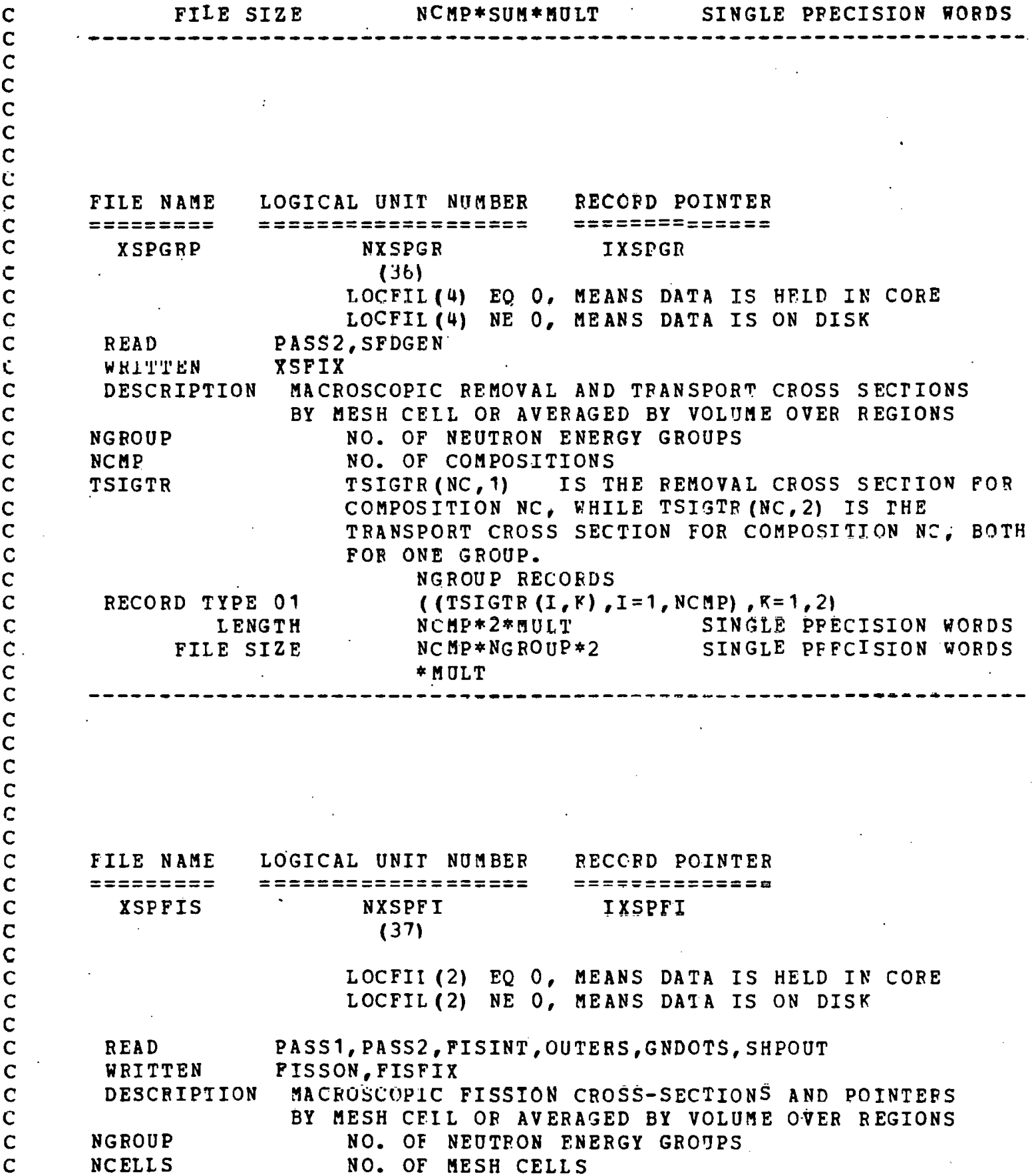




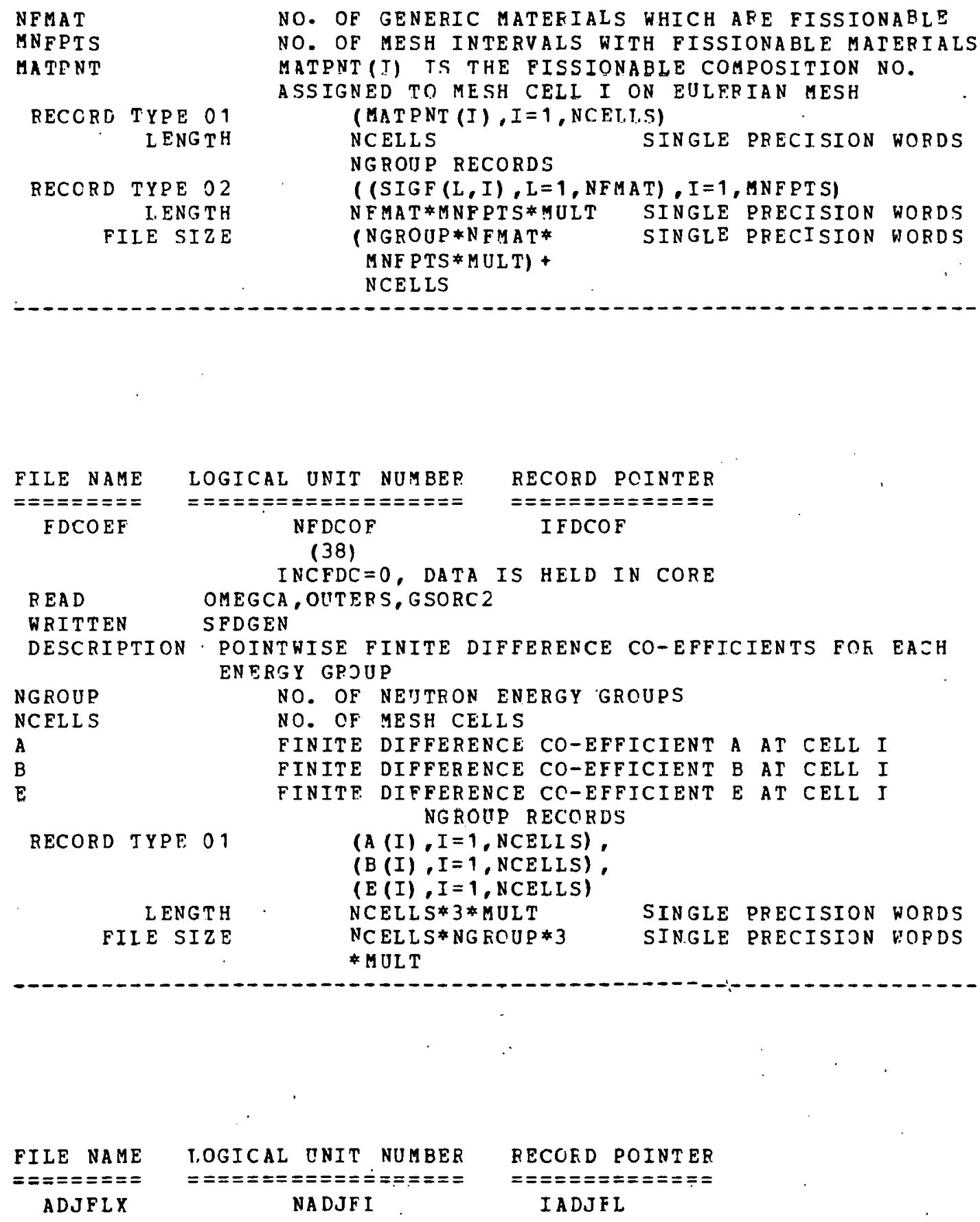




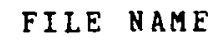




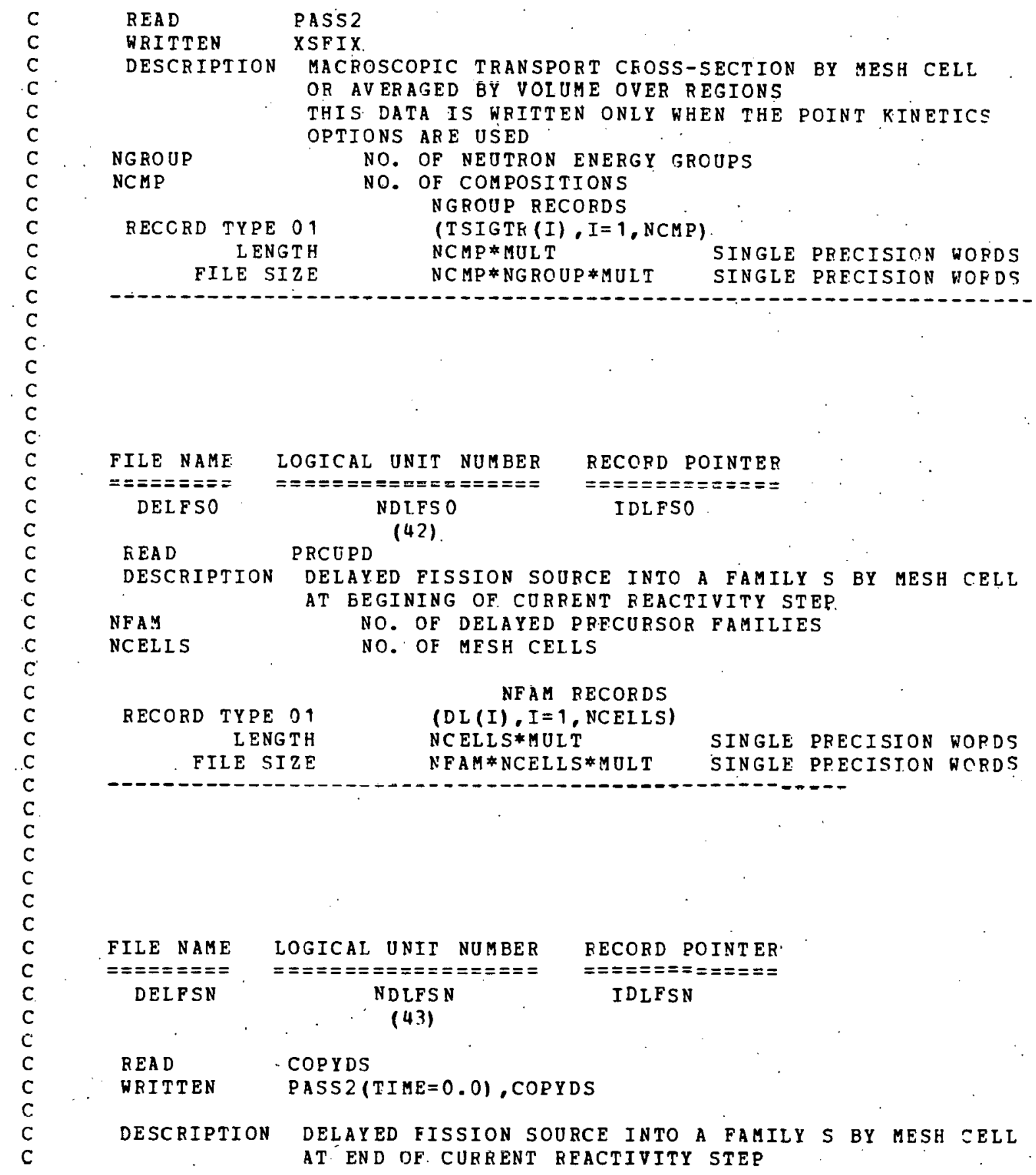




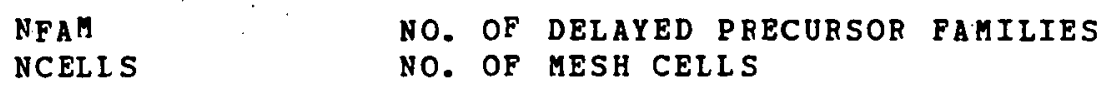

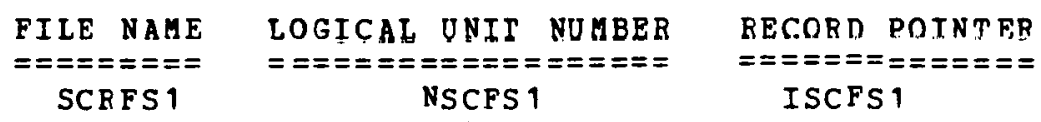




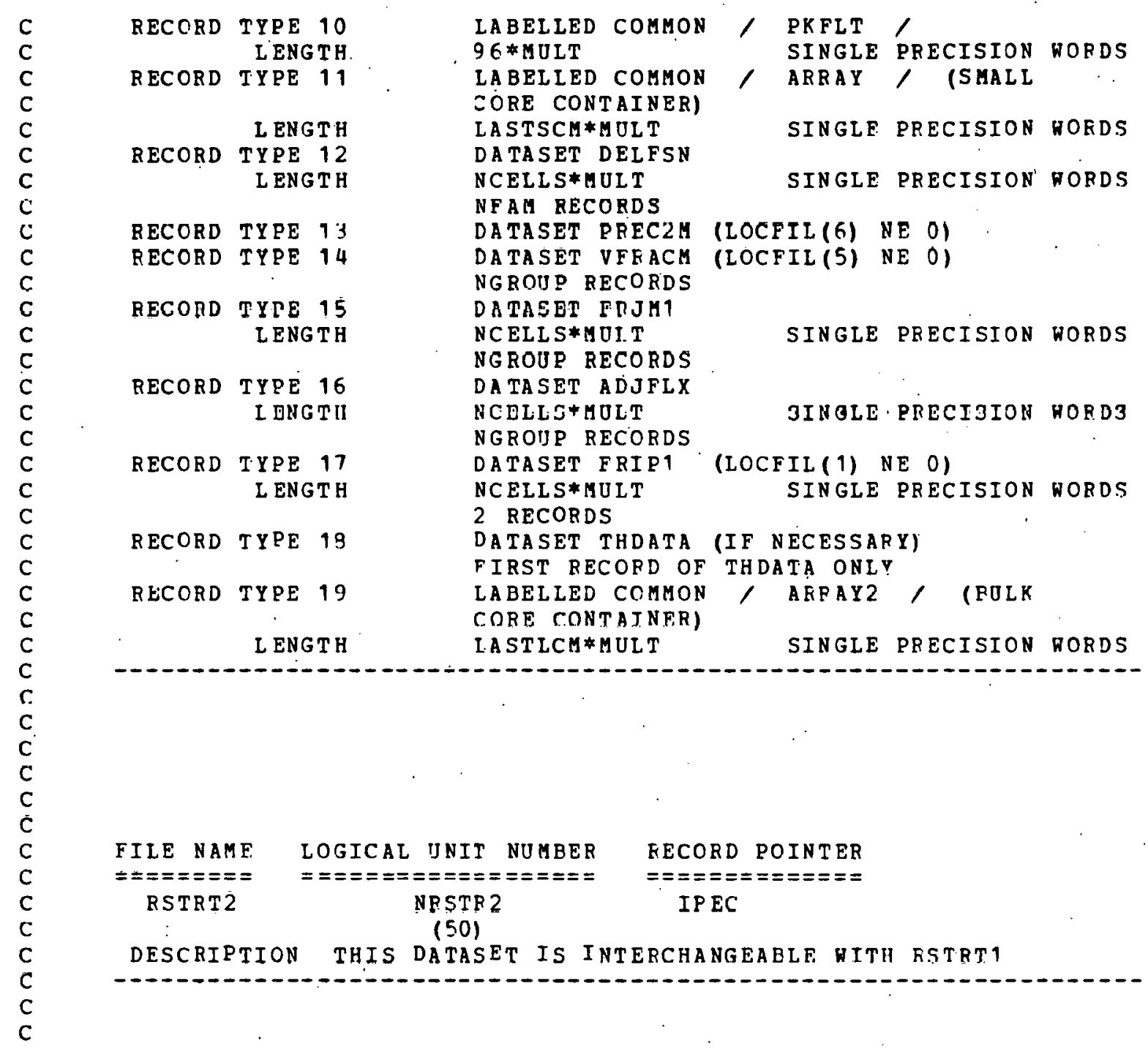


APPENDIX E

Listing of Common Blocks 


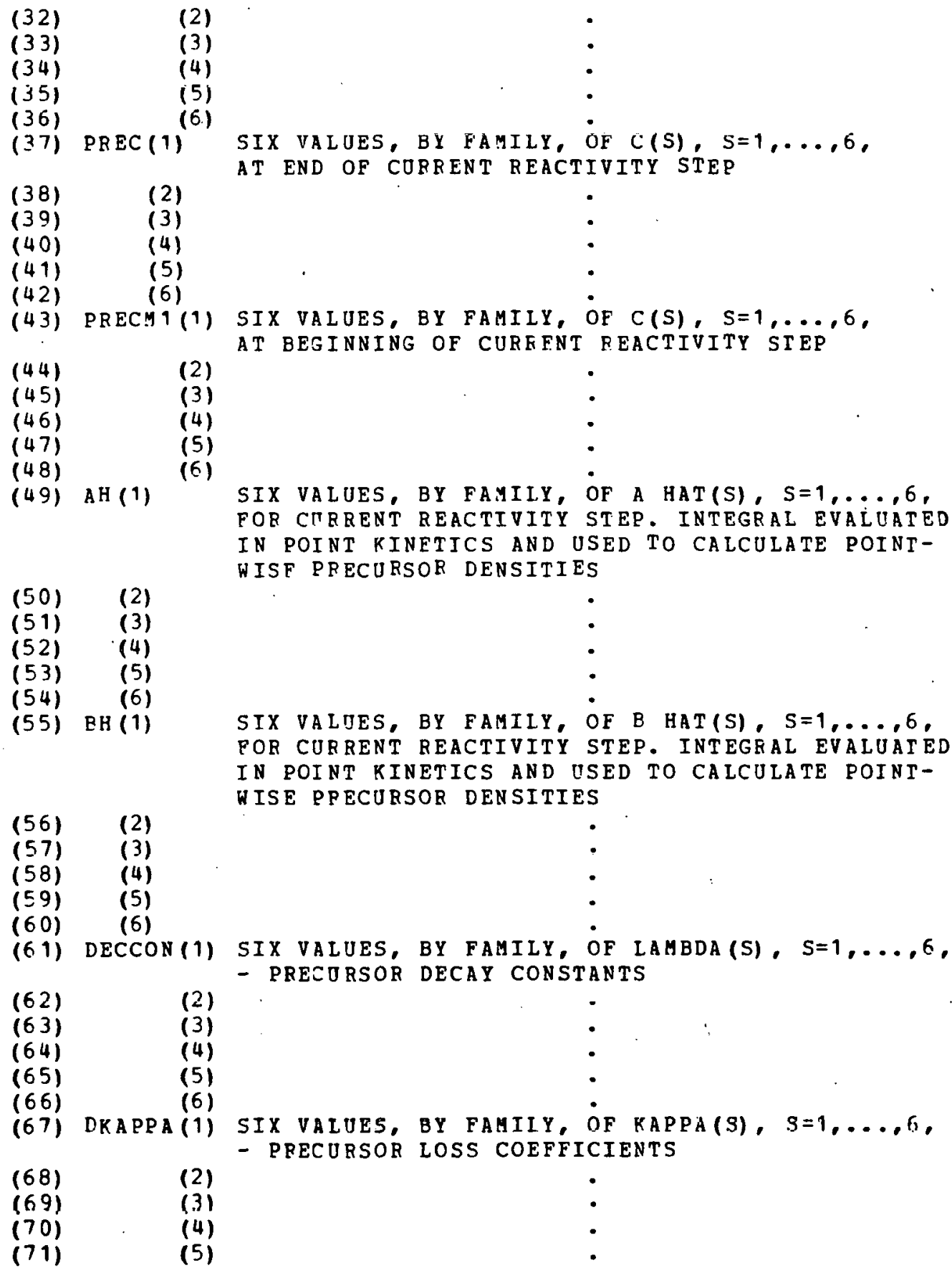




\begin{tabular}{|c|c|c|}
\hline C & (72) & \\
\hline C & (73) & EPSPK \\
\hline C & $(74)$ & EP ST \\
\hline C & & \\
\hline C & (75) & DELT \\
\hline $\begin{array}{l}\mathrm{C} \\
\mathrm{C}\end{array}$ & $(76)$ & ETOT \\
\hline$c$ & & 202 \\
\hline C & (77) & DTDT \\
\hline C & & \\
\hline $\begin{array}{l}\mathrm{C} \\
\mathrm{c}\end{array}$ & (78) & TFBA \\
\hline C & (79) & TCBAS \\
\hline C & & \\
\hline C & $(80)$ & FKEFF \\
\hline c & $(81)$ & OKEFE \\
\hline C & $(82)$ & GA.MMA \\
\hline C & $(83)$ & POWIN \\
\hline C & $(84)$ & POWTOI \\
\hline C & $(85)$ & EPSK \\
\hline C & & \\
\hline C & $(86)$ & EPSI \\
\hline C & $(87)$ & EP SF \\
\hline C & & \\
\hline C & $(88)$ & FLNM \\
\hline C & & \\
\hline $\begin{array}{l}\mathrm{C} \\
\mathrm{C}\end{array}$ & & \\
\hline C & $(89)$ & PSIN \\
\hline C & & \\
\hline C & & \\
\hline $\begin{array}{l}\mathrm{C} \\
\mathrm{C}\end{array}$ & $(90)$ & OUT \\
\hline C & $(91)$ & A MPMIN \\
\hline c & $(92)$ & PHOMI \\
\hline C & (93) & TMPMAX \\
\hline C & $(94)$ & A $1 \mathrm{PM}$ \\
\hline C & (95) & \\
\hline c & $(96)$ & ERSGA \\
\hline C & (97) & EPCL \\
\hline C & & \\
\hline C & $(98)$ & EPSII \\
\hline C & & \\
\hline $\begin{array}{l}\mathrm{C} \\
\mathrm{C}\end{array}$ & (99) & E \\
\hline C & & \\
\hline C & 001 & DTMPS \\
\hline
\end{tabular}

(6)

POINT KINETICS CONVERGENCE PARAMETERS REACTIVITY TEMPERATORE CORRECTION CONVERGENCE PARAMETER COEFFICIENT USED IN REACTIVITY TEMPERATURE CORKECT ION ITERATION TOTAL ENERGY GENERATED DURING TRANSIENT (WATT-SEC)

FIRST DERIVATIVE OF MAXIMUM TEMPERATURE CHANGE WITH RESPECT TO TIME

BASF FUEL TEMPEFATUEE (DEG.K) FOR TEMEERATIPE DEYENDENT CROSS SECIIONS

BASE COOLANT TEMPERATURE (DEG.K) FOR TEMPERATORE DEPENDENT CROSS SECTIONS

KEFF OF REACTOR-INITIAL

KU - EIGENVÁLUEE OF SOURCE-FREE ADJOINT EQUATIONS FOR REACTOR WITH FIXED SOURCE

GAMMA FACTOR FROM SHAPE CALCULATION

INITITAL TOTAI POWEE OF BEACTOR (WATTS) TOTAL REACTOR POWER (WATTS)

CONVERGENCE CRITERIA OF KEFF DURING INITIAL STEADY STATE SHAPE CALCUIATION CONVERGENCE CRITERIA ON RELATIVE FCINT ERROF OF FISSION SOURCES IN SHAPE CALCULATION CONVERGENCE CEITERIA ON RELATIVE SUM ERROR OF FISSION SOUPCES IN SHAPE CALC'JLATION RATIO OF MINIMUM FISSION SOJRCE VALUE AT A POINT WHICH WILL BE CONSIDERED IN RELATIVE POINT ERROR CALCULATION TO AVERAGE FISSION SOORCE DESIRED ERROR REDUCTION FACTOR FOR INNEP ITERATIONS ON A GROUP DURING AN OUIER ITERATION IN THE SHAPE CALCULATION SPECTRAL RADIUS OF OUTER ITERATION MATRIX IN THE SHAPE CALCULATION

MINIMUM ALLOWED VALUE OF AMPLITUDE

MINIMUA ALT.OHFE VATITE OE REACTIVITY

MAXIMUM ALLONED FIEL TEMPERATUPE

MAXIMUM ALLOWED AMPIITUDE FUNCTION VALUE (RESERVED FOR FUTURE USE)

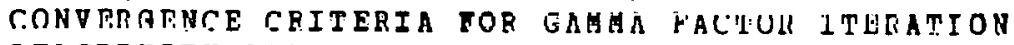
REACTIVITY STEP FACTOR BY WHICH TIME STEP SELECTION TESTS ARE TIGHTENED AT CR NEAR PROMPT CRITICAL

FACTOR BY WHICH EPSFLN AND EPSLAM ARE TO BE INCREASED FOR TIME-DEPENDENT CAICULATION CONVERGENCE CRITERIA FOR POINTWISE POWER DENSITY, STEADY STATE NEUTRONICS FEEDBAZK ITERAIION

MAXIMUM ALLOWED FUEL TEMPERATURE CHANGE 
(DEG. K) OVER A SHAPE STEP

MAXIMUM ALLOWED FUEL TEMPERATUPE CHANGE

(DEG. K) OVER A REACTIVITY STEP

MAXIMUM ALLOWED SHAPE STEP LENGTH (SEC)

MAXIMOM ALLOWED BEACTIVITY STEP LENGTH (SEC)

MAXIMUM ALLOHED AMPIITUDE FUNCTION CHANGE

OVER A SHAPE STFP

MAXIMOM ALLONED AMPLITUDE FUNCTION CHANGE

OVER A REACTIVITY STEP

TOTAL COMPUTING TIME (MIN.) WHICH WILL BE

ALTONED TO ELAPSE BEFORE ANOTHER RESTART DOMP

IS TAKEN

TOTAL PROBLEM TIME (SEC.) WHICH WILI. BE ALLON 8

TO ELAPSE BETWEEN THE TIMES THAT RESTART DOMPS

ARE TAKEN

MAXIMUM ALLOWABLE CHANGE IN GROOP- AND POINT-

DEPENDENT LINEARIY EXTRAPOLATED SHAPE FUNCTION

DURING A SHAPE STEP (RESERVED FOR FUTURE USE)

MAXIMUM OF THE ABSOIUTE VALUES OF DELTA PSI

OVER ALL MESH POINTS AND GROUPS/ (AVERAGE.

FIUXES *DELTA T), WHERE PSI IS THE NEUTRON

FLUX, AVERAGE FLUX IS THE SUM OF THE FLUXES/

(NO. OF MESH POINTS*NO. OF GROUPS), AND DELTA

$T$ IS THE PREVIOUS SHAPE STEP LENGTH

SUM OF MAXIMOM FUEL TEMPERATURE CHANGES OVER A SHAPE STEP

FACTOR USED TO REDUCE REACTIVITY STEP SIZE

GOING THROUGH PROMPT CRITICAL

FACTOR USED TO KEDOCE SHAPE STEP SIZE GOING

THROUGH PROMPT CRITICAL

HILI BE THE SMALLEST TIME AFTER THE END OF

THIS REACTIVITY STEP AT WHICH A DRIVING

FENCTION BEGINS OR ENDS

WILI BE THE SMALLEST TIME AFTER THE END OF

THIS SHAPE STEP AT WHICH a DRIVING FUNCTION

BEGINS OR ENDS

MAXIMUA FUEL TEMPERATURE OVER AIL THERMAL -

HYDRAUIIC REGIONS AT THIS REACTIVITY STEP

PREVIOUS REACTIVITY TIME STEP SIZE

PREVIOUS SHAPE TIME STEP SIZE

MAXIMUM FUEL TEMPERATURE CHANGE. OVER ALL MESH POINTS FOR THIS REACTIVITY STEP

EXTRAPOLATED AMPIITUDE

CPU TIME

PERIPHERAI PROCESSOR TIME

TIME IN SECONDS TO RON JOB

INITIAL REACTIVITY SUBTRACTED FROM RHOT(FLT(22))

WHEN IPK (INT (1)) = 3. THE PURPOSE IS TO START

THE TRANSIENT WITH REACTIVITY IDENTICALLY EQTAL 


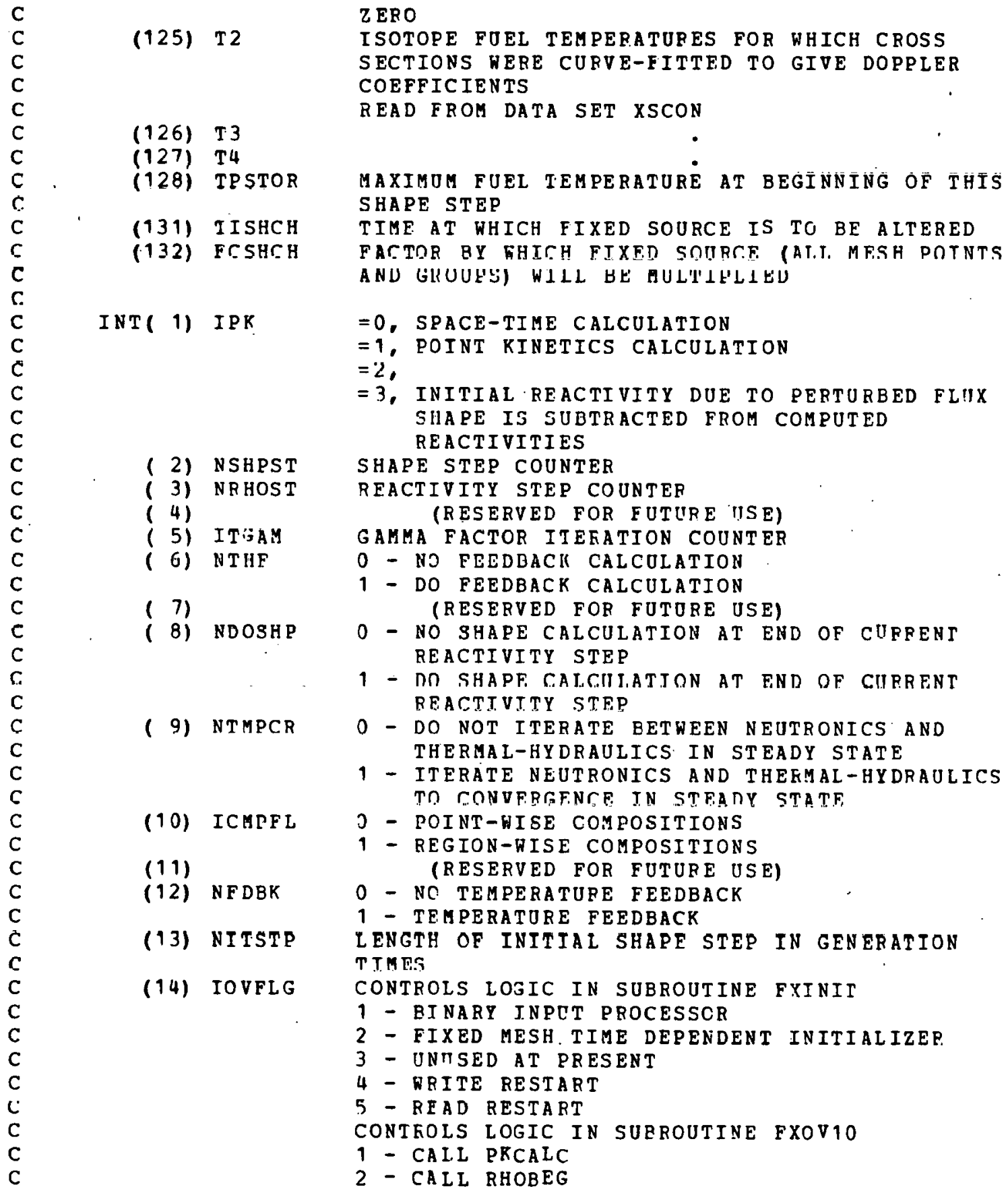


3 - CALL RHOEND

4 - CALL SHPEND

5 - CALL PKCALC

(15) NOSTEP

REACTIVITY GTEP COUNTER. IN CURRENT SHAPE SLEU

(16) ITRO

TEMPERATURE COPRECTION TO REACTIVITY

ITEFATION COUNTER

(17) IPKPT

POINTER IN POINT KINETICS. OPDATE INFORMATION BTFFER

(18) MAXORD

CURRENT ORDER OF SUBDIVISION OF REACTIVITY

(19) NERF

(20) NOUTMN.

TIME STEP INTO POINT KINETICS SIBBINTERVALS

ERROR FLAG

MINIMUM NUMBER OF OUTER ITERATIONS TO BE DONE

(21) NOUTMX

DURING A SHAPE CALCULATION

(22)

(23) ISHPOU

MAXIMUM NUMBER OF OUTER ITERATIONS TO BE DONE

DURING A SHAPE CALCULATION

(24) IPROB

(25) MKRTHY SOLUTION TYPE. FOF SHAPE CALCULATION

$$
\begin{aligned}
& \text { (RESERVED FOR FUTURE ISSE) } \\
& \text { OUTPIT EDIT SELECTOR FROOM SHAPE CALCULATION }
\end{aligned}
$$

CALCOIATION TYPE FOK SHAPE CALCULAIION

1 - INHOMOGENEOUS (FIXED SOURCE)

2 - EIGENVALUE (KEPE)

3 - TI EE-DEPENDENT

$0-$ REAL

1 - ADJOINT.

(26) IPTYPE

$=0$, HOMOGENEOUS PROEIEM

$=1$, FIXED SOURCE PRESENT

(27) NDOSS

O - DO STEADY STATE CALCULATION

1 - BYPASS STEADY STATE CaLCULATION

(28) LOOPSS

MAXIMUM NOBBER OF ITERATIONS BETWEEN NEUTRONICS

(29) MAXSCM

AND FEEDBACK CALCULATIONS AT STEADY STATE

(30) MAXLCM

NUMBER OF WORDS OF SCM AVAILABI.E

NTMBER OF WORDS OF LCH AVAILABLE.

(3 1) NGAMMX

MAXIMUM NUMBER OF GAMMA FACTOR ITERATIONS

ALLOWED AT A SHAPE STEP

(32) NTROMX

MAXIMUM NUMBER OF TEMPERATURE CORRECTION TO

REACTIVITY ITERATIONS TO BE DONE AT A

REACTIVITY STEP DURING UPDATE SWEEP

(33) NROSMX

MAXIMO NUMBER OF REACTIVITY STEPS ALLONED

IN A SHAPE STEP,

(34) IEDF (

INPOT BINARY FILE EDIT

0 - NO EDIT OF THE INPUT BINARY FILES

1 - EDIT INPUT BINARY FILES EXCEPT FOR MACROSCOPIC CROSS SECTIONS AND REGION MAP

2 - EDIT ALL INPUT BINARY FILES

(35) $\because$ ( 2) MICROSCOPIC CROSS SECTION EDIT:

O - PARTIAL EDIT OF MICROSCOPIC CROSS SECTION HOMOGENIZATION

1 - ALSO EDIT INPUT MICROSCOPIC CROSS SECTIONS DURING UICROSCOPIC CROSS SECTION HOHOGENIZATION 
(3) THERMAL HYDRAOLICS INPUT EDIT FLAG

0 - DO NOT EDIT THEEMAL-HYDEAULICS INPUT

1 - PARTIAL EDIT OF THERMAL-HYDRAIIL ICS INPUT

2 - FUIL EDIT OF THERMAL-HYDRAULICS INPJT

(37) (4) (RESERVED 'FOR FUTURE OSE)

(35) (5) DISK EDIT OPTIONS

O - NO DISK EDITING PERFORMED

1 -. BASIC EDIT WRITTEN TO DISK

2 - BASIC EDIT AND PONERS AND TEMPERATURES

3 - BASIC EDIT AND PONERS AND TEMPERATURES PLUS POINIWISE FLUXES

(37) (6) FUEL AND COOLANT TEMPERATURE EDIT FLAG

0 - DO NOT EDIT FIEI. AND COOLANT TEMPERATURES

1 - EDIT FUEL AND COOLANT TEMPERATURES AFTER EVERY SHAPE STEP

(40) ( I) KEALILITY STEP SUMMARY EDIT

0 - BRIEF EDIT

1 - NORMAL EDIT

2 - DETAILED EDIT

(41) (8) POWER DENSITY EDIT

(REACTIVITY STEP)

0 - DO NOT EDIT POWER. DENSITY

AFTER BEACTIVITY STEPS

$N$ - EDIT POWER DENSITY AFTER EVERY N REACTIVITY STEPS

(42) (9) FLUX SHAPE CALCULATION EDIT

0 - MTIMUM EDIT OF. EACH FLUX SHAPE CALZULATION

1 - EDIT ITERATION HISTORY AT EACH FLOX SHAPF. CAICULATION

2 - EDIT ITERATION HISTORY AND MULTIGROUP FLUX SHAPE AT EACH FLUX SHAPE CALCULATION

(43) (10) POWER DENSITY EDIT (SHAPE STEP)

0 - DO NOT EDIT POHEP DENSITY

AFTER SHAPE STEPS

N - EDIT POHER DENSITY AFTER EVERY N SHAPE STEPS

(44) LOCFIL (1) PSI STORAGE FLAG

(45) (2) TSIGF, MATPNT STORAGE FLAG

(46) (3) TSCATD STORAGE FIAG

(47)

(48) (5) VFAACM STORAGE FLAG

(49) (E) ZETAM STORAGE FLAG

(50)

(51) (8)

(52)

(8)

(53)

(10)

(54)

(57)

(58)

IRGOTO

THDATA FILE STORAGE FLAG

(RESERVED FOR

(RESERVED FOR FUTURE USE)

(RESERVED FOR EUTURE USE)

LOGIC FLOW FLAG (TIMDEP)

R FUTURE USE)

(59) NREST RESTAPT FLAG 
C

C

C

C

C

C

C

C

C

C

C

C

C

C

c

C

C

C

C

C

C
O - NO RESTART

1 - RESTART PROBLEM

(60) INITV

(61) NBS

(62) NTRIAG

(63) NTRIPT

(64) IPERB

(65) IFTH

(66) $\mathrm{NCH}$

(67) NFR

(6.8) NAX

( $\epsilon 9)$ IST

(70)

(71) I TYPE

(72) IEN

(73) ITDSRC

(74) ITDHAP

(75) ITDNOW

(76) LSTSCM

(77) LSTLCM

BODNDARY CONDITIONS

3 - DETAILED MODEL

FILE WAS WRITTEN
INITIALIZE THERMAL HYDRAULICS

TRIANGULAR GEOMETRY TYPE

LOWER TRIANGLE ORIENTATION

PERIODIC TRIANGULAR GEOMETRY

ALSO THETA - R PERIODICITY FLAG

THERHA I-HYDRAULICS CALCIILATION FLAG

1 - SIMPLE MODEL NITH ZEFO HEAT FLON APPROX.

2 - SIMPLE MODEL WITH CONSTANT HEAT FLOW APPROX.

NTHBER OF THERMAL-HYDRAUI IC CHANNELS

NUMBER OF REGIONS INSIDE THE FDEL PIN

NUMBER OF AXIAL THERMAL-HYDRAULIC SEGMENTS

STROCTURE CALCULATION FLAG

0 = NO STRUCTURE, 1 = STRUCTURE (RESERVED FOR FUTURE USE)

NUMBER OF DIFFERENT THERMAL-HYDRAOLIC DATA TYPES

IF IFTH=1, LEN $=10$. IF IFTH=2, LEN $=50$.

FEAG TO INDICATE IF FIXED SOURCE IS TIME DEPENDENT (0/4) (NO/YES)

FLAG TO INDICATE IF MODIFICATION TO FIXED SOURCE HAS BEEN MADE YET $(0 / 1)$ (NO/YES)

FLAG TO INDICATE IF FIXED SOURCE IS TO BE

MODIFIED THIS REACTIVITY STEP $(0 / 1)$ (NO/YES)

LENGTH OF SMALL CORE CONTAINER WHEN RESTART

LENGTH OF LARGE CORE CONTAINER WHEN RFSTART.

FIIE. NAS WEITTEN

NPTI , NPTJ , NINTI , NINTJ , NGROTP, NMAT ,

NTFMAT, NFAM , MSCT , MAXDN , NREG , NREGD :

NDSCT, NGEOM, NZONE , IMBL , JMBT , IMBR ,

MAXSIZ, LW4, LW8

NO. OF X-DIRECTION MESH LINES

NO. OF Y-DIRECTION MESH LINES

NO. OF X-DIRECTION MESH INTERVALS

NO. OF Y-DIRECTION MESH INTERVALS

NO. OF NETTRON ENERGY GROUPS

NO. OF GENERIC MATEKIALS

HO. OF ONIQUE MATERIALS

NO. OF GENEFIC MATERIALS WHICH ARE FISSIONABI.E

NO. OF ONIQUE MATERIALS WHICH ARE FISSIONABI,E

NO. OF DELAYED PRECURSCF FAMIIIES

MATERIAL SCATTERING CROSS SECTION ARRAY LENGTH

MAX. NO. OF DOWNSCATTER GROUPS FOR A GROUP

NO. OF REGIONS

NO. OF REGIONS IN WHICH DRIVING FUNCTIONS

ARE USED. 


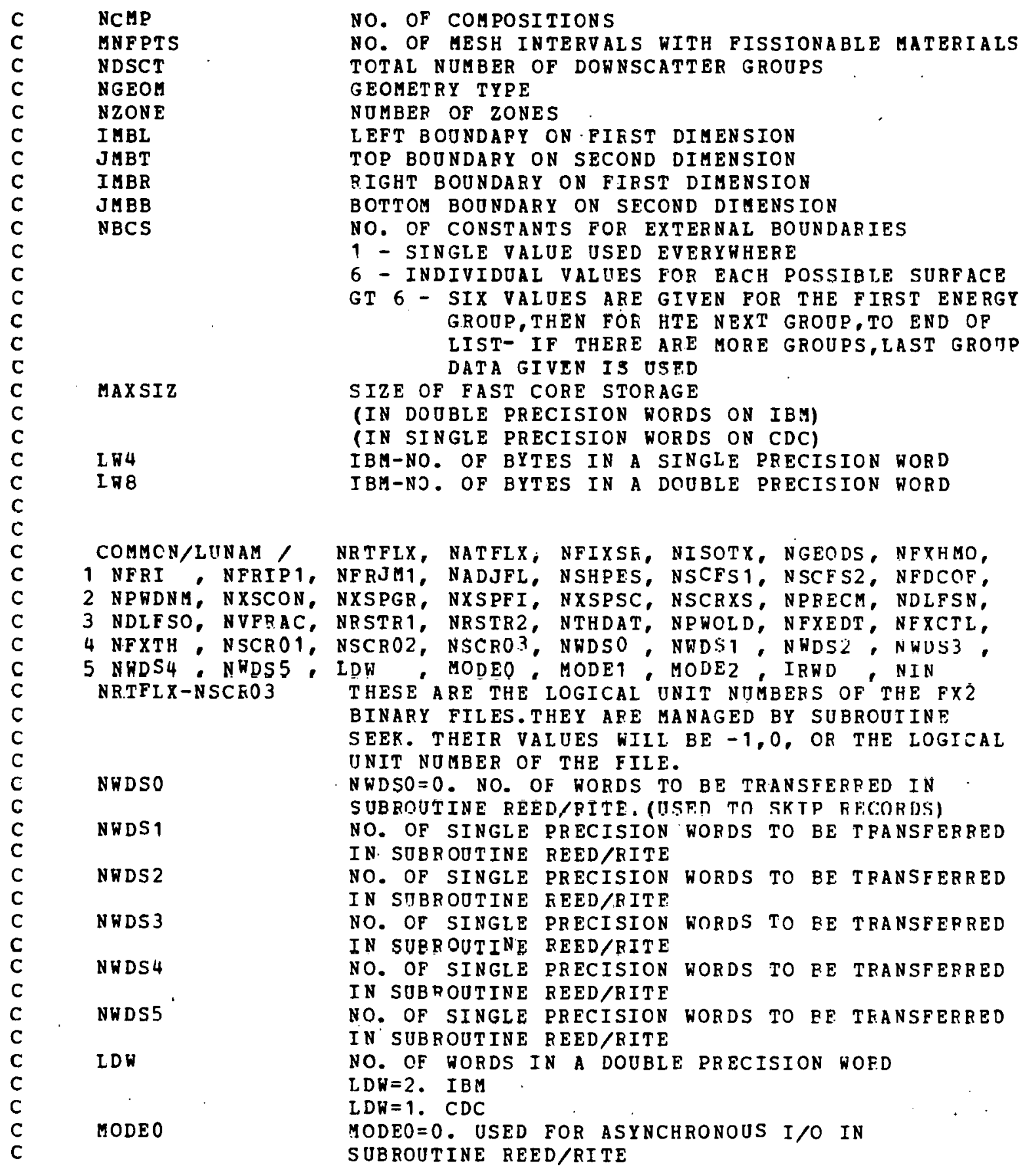




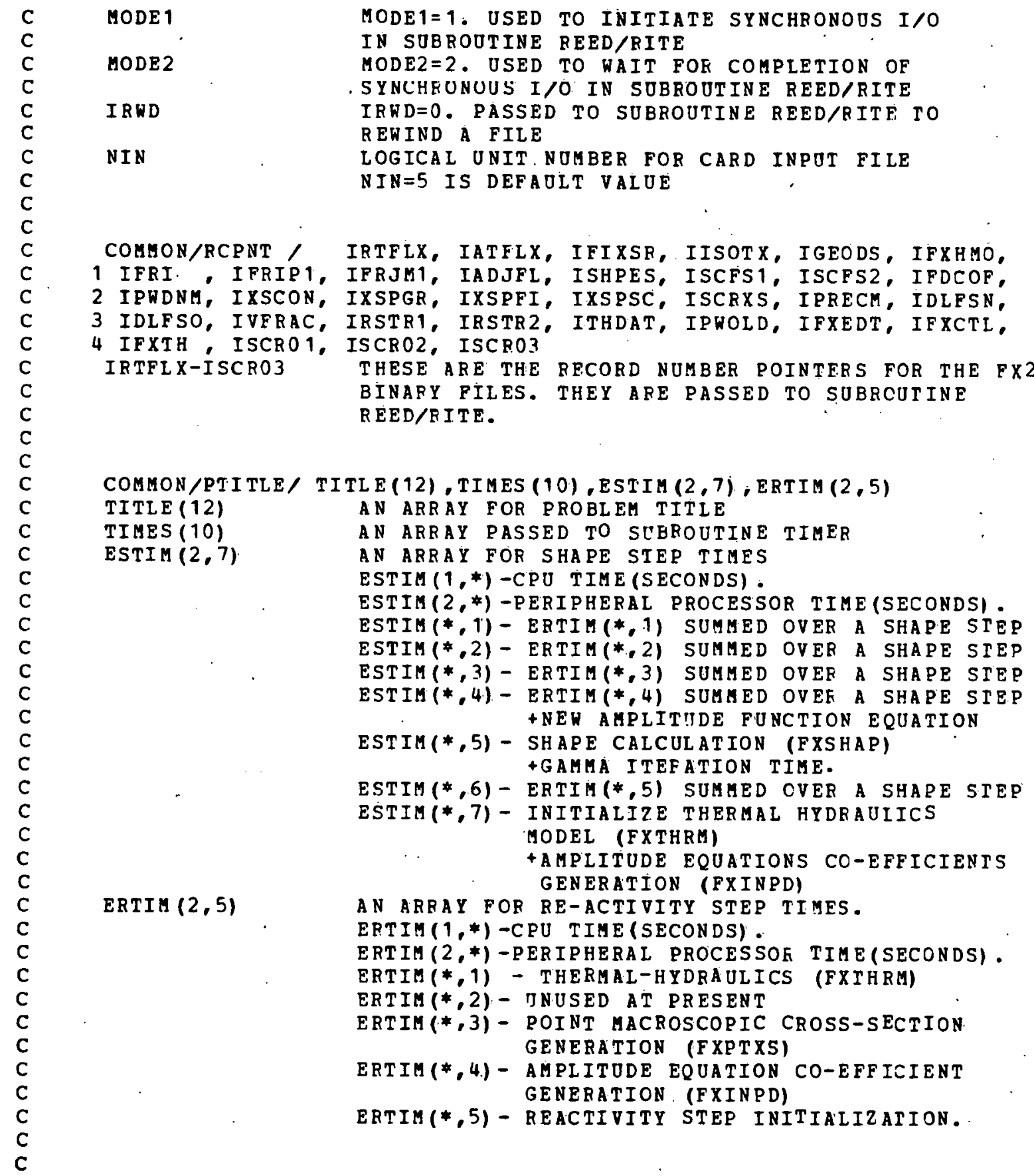




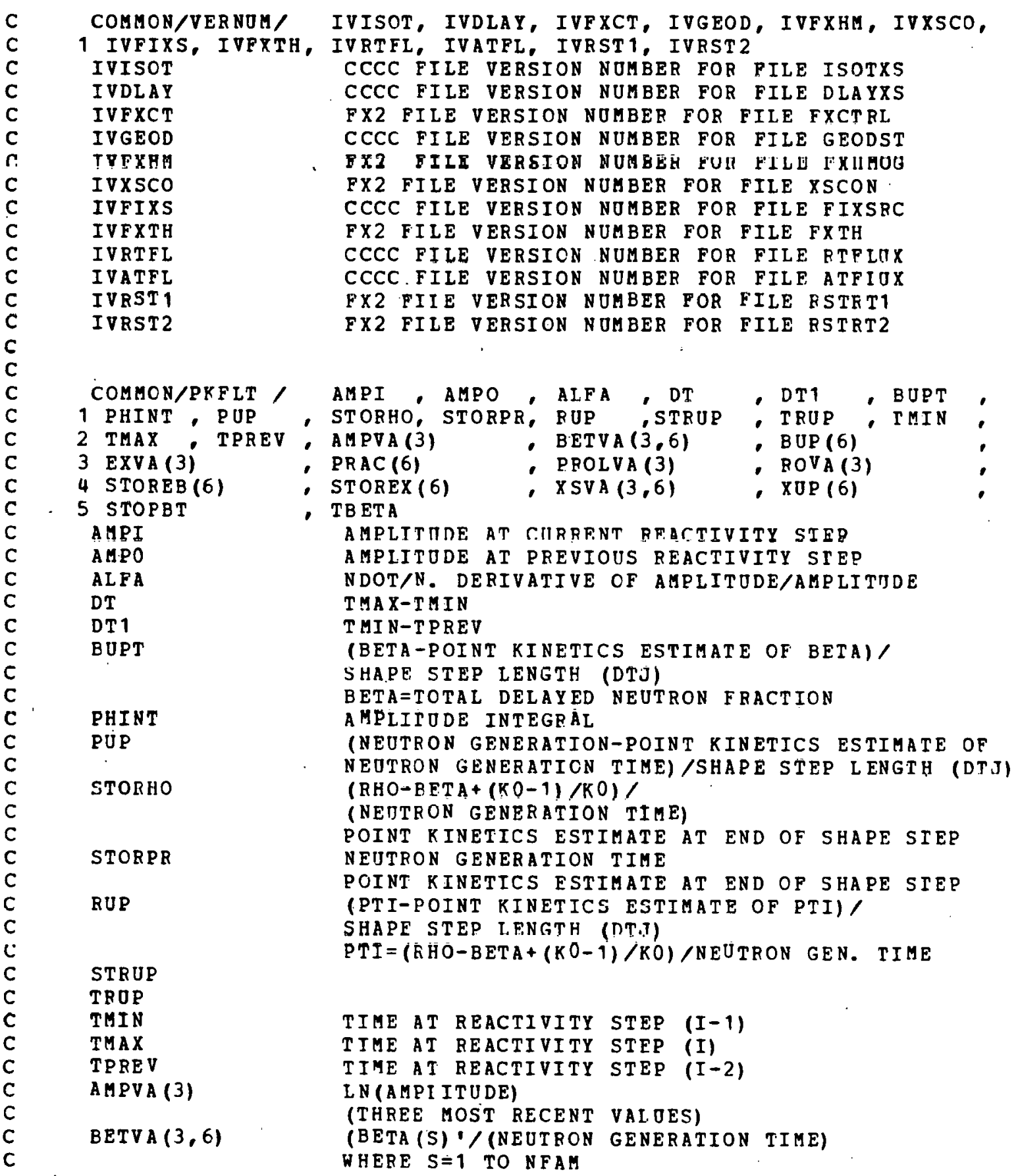




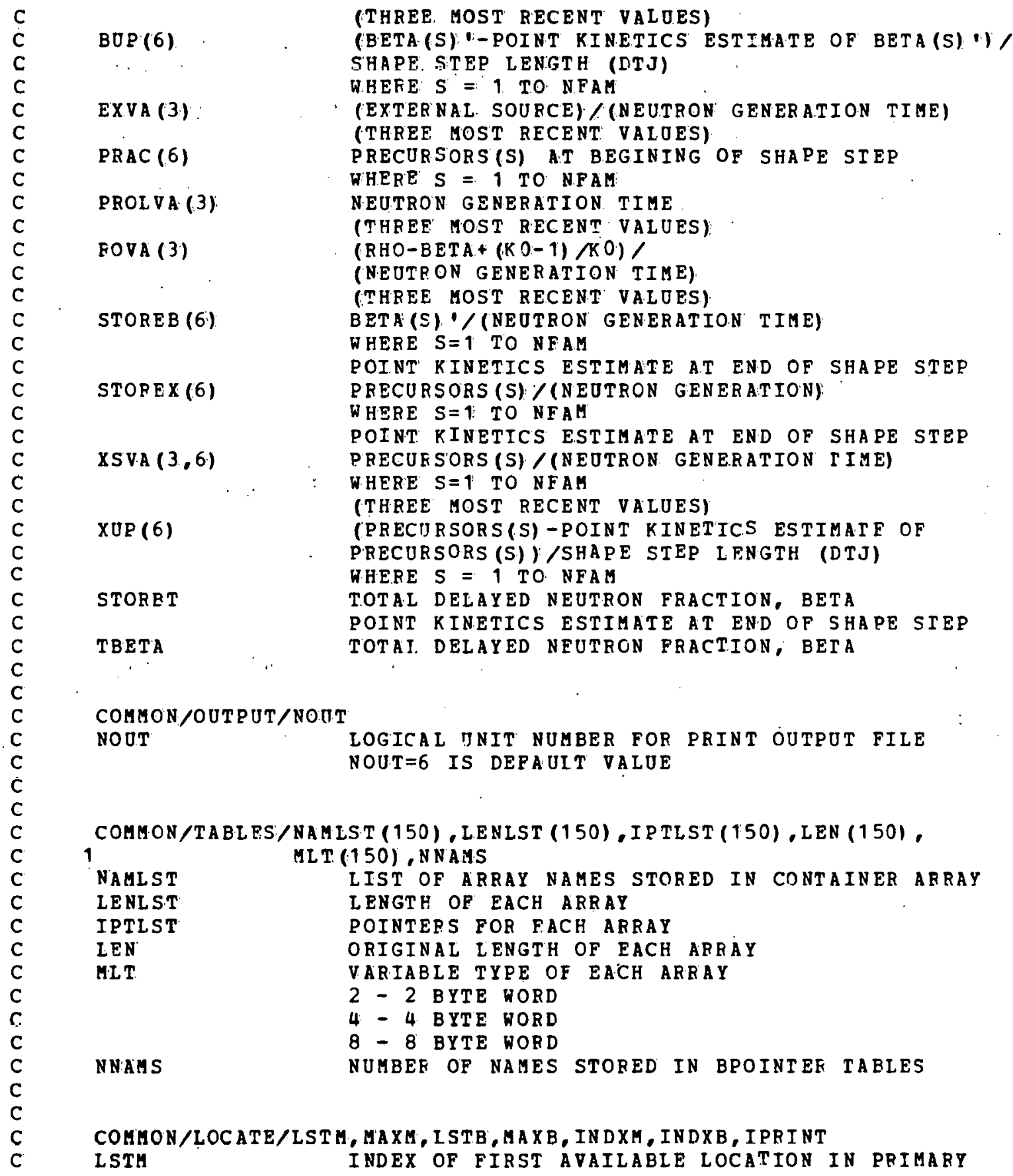


C
C
$C$
$C$
$C$
$C$
C
$C$
$C$
$C$
C
$C$
$C$
$C$
C
$C$
$C$
$C$
$C$
$C$
$C$
$C$
$C$
$C$
$C$
$C$
$C$
$C$
$C$
$C$
$C$
$C$
$C$
$C$

STORAGE ARRAY

MAXIMOM LENGTH OF THE PRIMARY STORAGE ARRAY

INDEX OF FIRST AVAILABLE LOCATION IN SECONDARY

STORAGE ARRAY

MAXIMUM LENGTH OF SECONDARY STORAGE ARRAY

$\operatorname{MAXB}$

ADDRESS (INDEX) ' OFFSET OF PRIMARY STORAGE

IN HOPDS

INDXB

ADDRESS (INDEX) ' OFFSET OF SECONDARY STORAGE

IN MORDS

IPRIN T

EDIT FLAG FOR POINTR ROUTINE

a - NO .PRTNTOIIT .

1 - DOMPS ONLY

2 - TRACE ONLY

DIIMPS AND TRACE

1 COMMON/ICMSIZ/
ILOCM
ILOCB
MAXBLK
MAXFM
MAXFB
MULT

ILOCM, IL OCB, MAXBLK, MAXFM, MAXFB, MULT, MAXNAM, MULT3, JLOCM, JLOCB

STARTING ADDRESS OF PRIMARY STORAGE ARRAY STARTING ADDRESS OF SECONDARY STORAGE APRAY LENGTH OF SECONDARY STORAGE ARBAY REQUESTED L. ENGTH OF PRIMARY STORAGE ARRAY BEFORE EXTENSION BY ADDRESS OFFSET LENGTH OF SECONDARY STORAGE ARRAY EEFCRE EXTENSION BY ADDRESS OFESET NO. OF WORDS IN A DOUBLE PRECISION WORD. MIIIT $=2$. IBM MULT $=1 . C D C$

MAXNAM MAXIMOM NO. OF NAMES WHICH CAN EE HELD IN POINTR TABLES (MAXNAM=150)

MULT3 MULT3= MULT* 3

JLOCM ADDRESS OF LABELLED COMMON BLOCK ARRAY JLOCB ADDRESS OF ECM CONTAINER ARRAY

COMMCN/BFLAGS/IFLAG (150)

IFLAG

MEMORY FLAG FOR FACH ARRAY

0 - PRIMARY STORAGE ARPAY

1 - SECONDARY STORAGE ARRAY 
APPENDIX F

Sample Problem 1 Output 


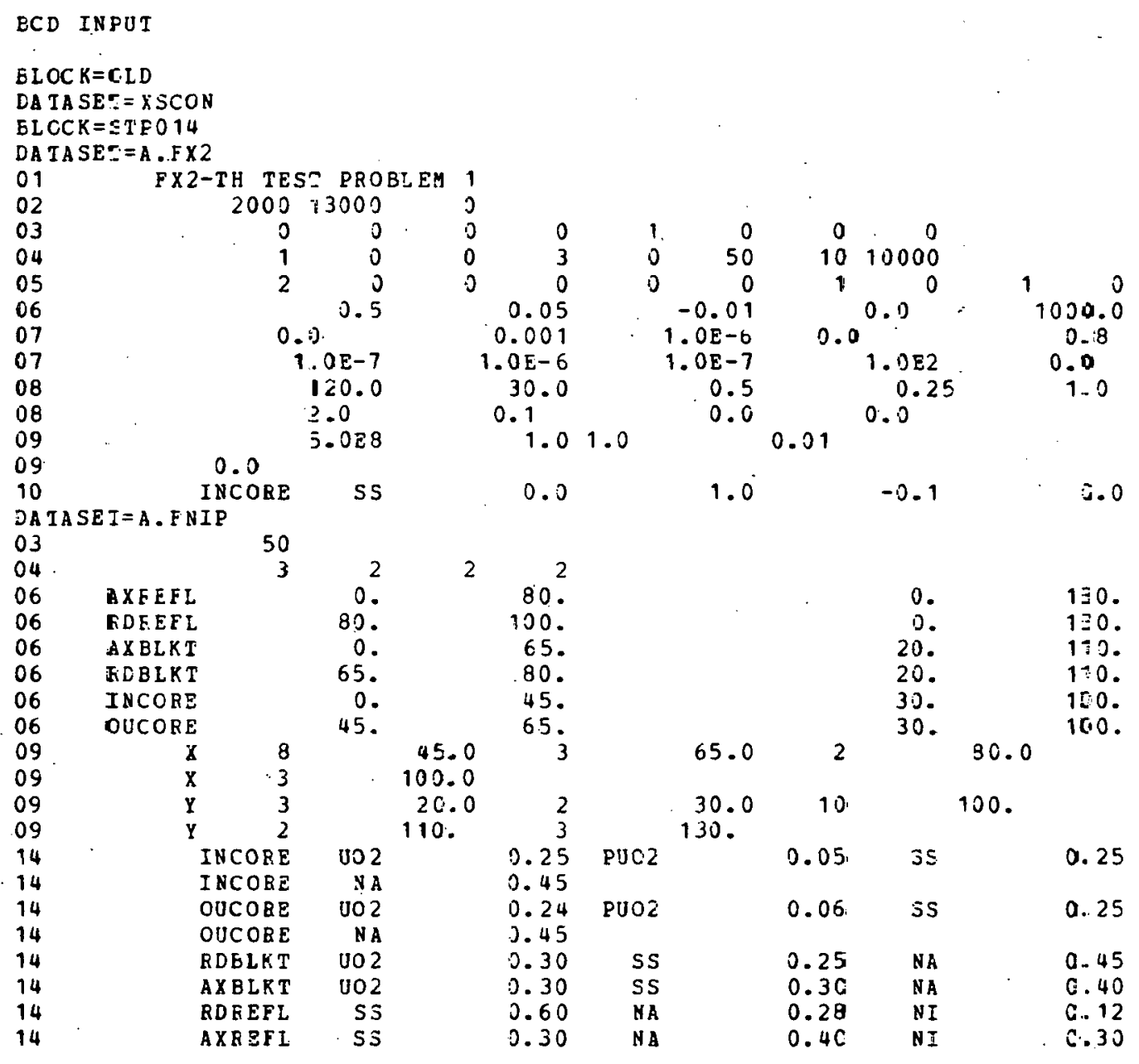




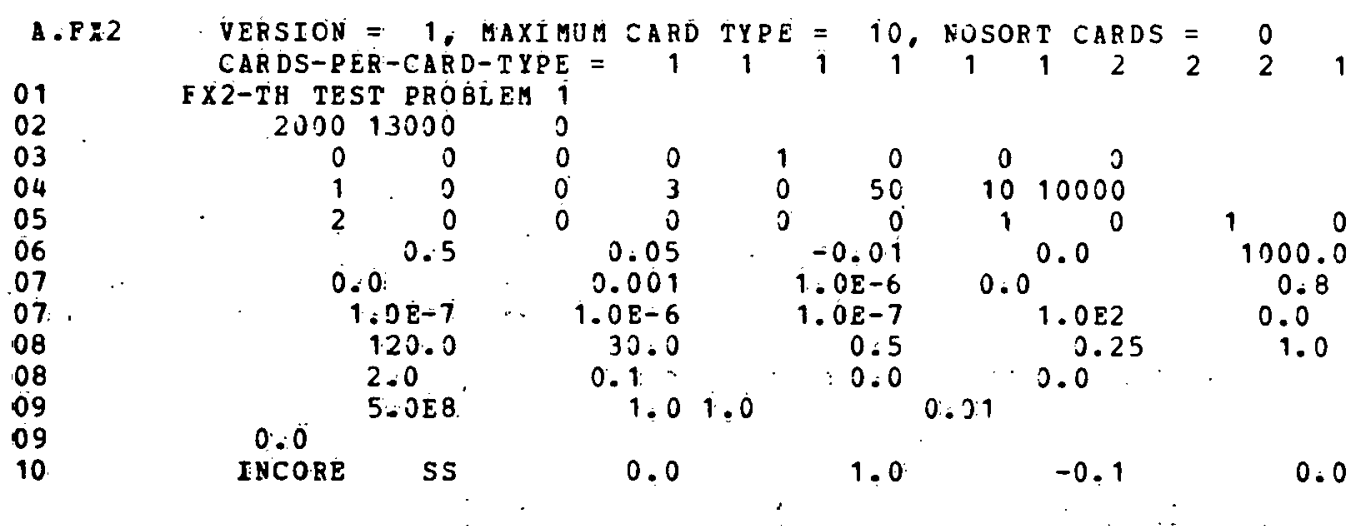

A.ENIP VERSION $=1$, AAXIMUE ZARD TYPE $=1.4$, NOSORT CARDS $=-0$

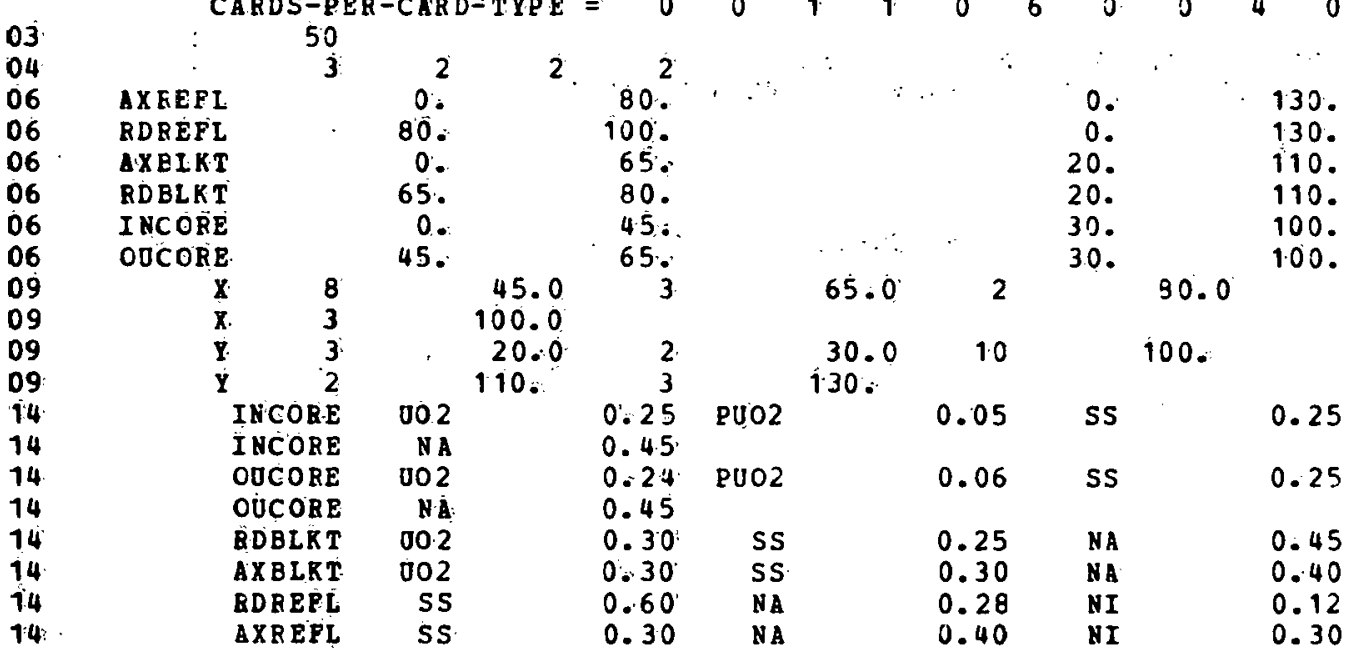


*** FX2 BCD INPUT PRCCESSOR

THE EOLLONING BINAPY DATASETS HIVE BEEN HRITTENNAME VERSION USER IDENIIPICATION

FXCT B L

GEODST

B 20219F X 2 PRO B2 $2219 F \times 2$ PEO B20219F $\times 2$ PEO

$B 20219 F \times 2$ PEO

RTFLUX

CENTKAI FROC ESSOR TIYE (SEC) PERIPHERAL PROCESSOB IIME (SEC) =
B $20219 F \times 2$ PRO

3CD INPOI PROCESSINZ COMPLETED

ATFLOX 
FX2-TH TEST PROBLEM -

INPUT FIIE FXCTRL, VERSTON 1, USER IUENTIFICATION = B20219FXZPRO HAS BEEN PROZESSED.

INPUT FILE GRODST, VERSICN - i, USER IDENIIFICATION = B20219FX2PRO HAS BEEN PROZESSED.

INPUT FILE FXHHOG, VERSION. 1, USER IDENTIFICATION = B20219FX2PRO HAS BEEN PROCESSED.

INPUT FILE $x S C O N$, VERSION 1, USER IDENTIFICATION = IEST.PFOB. 1 HAS BEEN PROZESSED.

INPUT PILE RTFLUX, VERSION. 1 ; USER IDENTIFICAIION = B20219FX2PRC HAS BEEN PROZESSED.

IMPUT FILE APFLUX, VERSICN 1, USER IDENTIFICATION = B2J219FX2PRO HAS BEEN PROCESSED. 
$\begin{array}{ll}\text { NREST } & 0 / 1 \text { NO/TES FESTAET PECBLEM } \\ \text { IPK } & 0 / 1 / 2 / 3 \text { SPACE-DEPENDENT } / \text { POI }\end{array}$

NTHF

NF DBK

ICMPFL

IPTYPE

SDOSS

SDOSS

ITDSRC

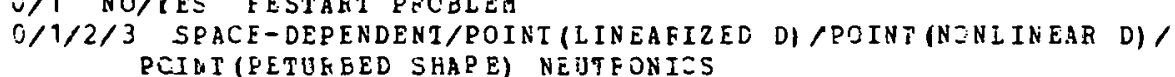

O/1 NO/YE MODIPY CPOSS SECTIONS FCR IEYEERATIRE DEOENDENCE

$0 / 1$ POINTAISE/REGIONHISE MATERIAL ZCQPOSITIONS

$0 / 1$ NO/YES INHOMOGENEOUS FIXED SOURCE PRESENT

$0 / 1$ YES/NO DO STEADY STATE CAECULATION

$O / 9$ NO/YES ITEAATE RETHEEN NEUTKONICS AND THERYAL HYOEAULICS IN STEAOY STATE

$0 / 1$ NJ/YES FIXED SOURCE IS TIME DEPENDENT

\section{STORAGE, ITERATION IIMITS}

$\begin{aligned} 2000 & \text { MAXSC: } \\ 13000 & \text { MAXLCA } \\ 1 & \text { IOOPSS } \\ 3 & \text { NGAYMX } \\ 50 & \text { NOJTMY } \\ 10 & \text { NROSYX } \\ 10000 & \text { NITST }\end{aligned}$

NC. OF WCEDS REQUESTED FOR SMALL ZOÑE ZONIAINER

NO. JF HOZES REOUEETED FOR LARGE CORE CONTAINER

MAY. NO. JF NEUTROR ICS/FEEDBACR IIERATIONS AI STEADY STATE

MAX. NO. JP GAMMA EACTOR ITERATIONS AT A SHAPE STEP

MAX. NO. JE OUTER ITEFATIONS DURING A FLUX SHAPE CAIZJLATION

MAX. NU. JF REACTI UIT STEPS IN A SHAPE STEP

\section{EDIT OPTIONS}

IEDF (1) 0/1/2 NOJE/FARTIAL/COMPLETE EDIT OF PROBLEY JESCRIPFION $0 / 1$ PARTEAL/COGPLETE EDIT OF CBOSS SETTION HOMOGENTZATION $0 / 1 / 2$ NOTE/FAFTIAL/COMPLETE EDIT OF THEKMAL-TYDRAULICS INPUT $0 / 1 / 2$ NO.JE/SIMPIE, DETAILED EDIT WFITTEN TO E JIT DISE

$0 /$ NONEYEVERY SHLPE SIEP EDIT FJEL AND COCLANT TEMPERATURES

$0 / 1 / 2$ BREEF/NORAAL/DETAILED BEACIIVITY STEP STYMRRY EDIT

O/N NONE,EVERY N GEACTIVITY STEPS POHER DENSLTY EDII

O/1/2 NCSE/PARTIAL/COAFLETE EDIT OF RAZH FLOI SHAPE ZALCOLAZION

$O / N$ NONE.EVERY N SHPE STEPS POHEF DENSITY EDIT

\section{PROBLEM LIMITS}


$0.100000 \mathrm{D}-02$ $0.1000000-05$ $0.800000 \mathrm{D}+00$ $0.1000000-06$ $0.10000 \mathrm{CD}-05$

$0.100000 D-06$

$0.1000000+03$

$0.1000000-02$

EPSigA FPS?K EPC $\rightarrow 0$ : EPSKE EPSEAM EP SZL ERSENC EPSTM

$0.120000 D+03$ $0.300000 D+02$ $0.500000 D+00$ $0.200000 D+00$ $0.500000 D+09$ C. $200000 D+01$ $0.100000 D+00$

DTMPSH DT MPRH DTSHMX DTRFII 8 DAMPSH DAMER B DPSISH

$\begin{array}{ll}0.500000 D+09 & \text { POHIN } \\ 1.000000000 & \text { RKEFF } \\ 1.000000000 & \text { OKEFF } \\ 0.100000 D-01 & \text { FLNSON } \\ 0.300000 D-01 & \text { PSINRY } \\ 0.0 & \text { OUTRHO } \\ 0.700000 D+03 & \text { TFBASE } \\ 0.500000 D+03 & \text { ICBASE } \\ 0.694650 D+01 & \text { DCTAST } \\ 0.1500000+01 & \text { DPTRST } \\ 0.0 & \text { TISHCH } \\ 0.100000 D+01 & \text { FCSHCH }\end{array}$

CONVEGGENCE CEITERIA

GAMMA FAZTOR CONVEKGENCE CRITERIA

AMPLITUDE FINCTION EOUATION SOLJTION ACCUẼEZY

FACTOR USED TO TIGHTEN TIME STEP SELECIORS NEAR PROMPT CRITICAL

EIGENVALJE CONVERUENCE CRITERIA, STEADY STATE F́LUX CALCULAIION

POINIVISE FISSION SOUKCE CONVEK GEXCE CRITEEIA FOR SHAPE CALCJLATION

AVERAGE ZISSION SCUFCE CGNVERGENCE CRITERIA FOR SHAPE CALCOLATION

FACIOR FDR LOOSENING E?SLAM AND EPSFLN FOR TIMZ-DEPENDENI CALCULAZION

POINIRISZ POWER DENSITY CONVERGENCE CRIIERIA, STEADY STATE ITEKATION

TIME STEP SELECTION CRITERI

MAX. ALLONED FUEL TEMPERATURE CHAKGE OVER SHAPE STEP (DEg.K)

MAX. ALLONED FOEL TEMPEGATURE CHAKGE OVER REACTIVITY STEP (DEG.K)

MAX. ALLCWED SHAPE STEP LENGTH (SEC)

MAX. ALLONED REACTIVITY STEP LENGIH (SEC)

NO. OF SBAPE STEPS/DECADE OF ALPLITUDE CHANSE

NO. OF REACTIVITY STEPS/DECADE OF AMPLITJDE CHANGE

MAX. ALLOHED POINTUISE FLUUX SHAPE CHANGE OVER SHAPE STEP

\section{OTHER FLOATING POINT PARAMETERS}

STEADY.STATE REACTOR POHER (WATTS)

R-EFPECT IVE (INITIAL GUESS IF NDOSS $=1$ )

EIGENVALLE OF SOURCE-FREE ADJOINT PROBLEL (USED IE IPIYPE=1)

IIN./AVE. CRITERIA FOR POINTWISE FISSION SOORCE TEST

ERROR REEUCTICN FACTOR FOR SHAPE CALCULATION INNER ITEKATIONS

INPIJ ESTIMATE OP SHAPE CALCULATION DOHINANEE RATIO

BASE FUEL TEMEERATUKE (DEG. K)

BASE COOLANT TEMPERATORE (DEG. K)

COSE COOLA TI TELERATURE (DEG. K)

PROBLEM TIME INTERVAL BETWEEN RESTART DUMPS (SEC)

IME THAT FIXED SOURCE NILI BE AITERED (SEE)

FACIOR BY WHICH PIXED SOURCE WILI BE MOLIIPLIED 
** PROELEM DESCEIPTION

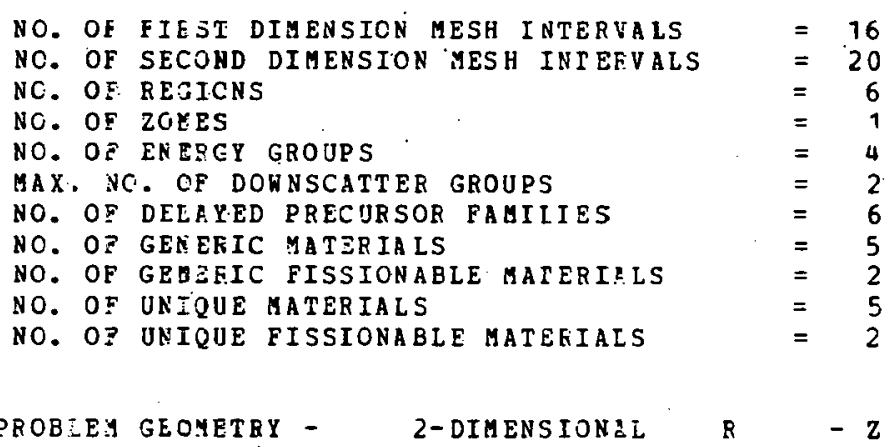

$\begin{array}{rlr}1 & 0.0 & 2 \\ 7 & 0.3375000+02 & 8 \\ 13 & 0.7250000+02 & 14\end{array}$

$\begin{array}{rr}7 & 0.3375000+02 \\ 13 & 0.725000 \mathrm{D}+02\end{array}$

\section{$0.5625000+01$ $0.3937500+02$} $0.8000000+02$

\section{R - DIRECTION MESH INTERVAL BOUNDAEIES}

$\begin{array}{rrrr}3 & 0.1125000+02 & 4 & 0.168750 D+02 \\ 9 & 0.4500000+02 & 10 & 0.516-66 ? \mathrm{D}+02 \\ 15 & 0.6666670+02 & 16 & 0.9333330+02\end{array}$

$0.2250000+02$
$0.5833330+02$ $0.8666670+02 \quad 16 \quad 0.9333330+02$

$17 \quad 0.1000000+03$

\section{- DIRECTION MESH INTERVAL BOJNDAEIES} $\begin{array}{rl}1 & 0.0 \\ 7 & 0.370000 D+02 \\ 13 & 0.790000 D+02 \\ 19 & 0.116667 D+03\end{array}$ $0.666667 D+01$
$0.440000 D+02$
$0.850000 D+02$
$0.123333 D+03$ 3
9
15
21

$0.133333 D+0.2$
$0.510000 D+02$ $0.510000 D+02$
$0.530000 D+02$

$4 \quad 0.2000000+0 z$ 0. $130000 \mathrm{D}+03$

BOONDAR? CONDITIONS (ORIGIN AI EONER LEFT)

0 - ZEEO FLUX 1 - ZERO CURPENT 2 - EXTRAPOLATED 3 - PEPIODIC OPPOSITE FALE 4 - PERIODIC NEXI ADJACENT FACE 5 - INVERTED PERIODIC ALONG FACE 
REGION NUBBER, LABEL, AND ASSZ JNMENT TO ZONE

\begin{tabular}{rrrr} 
FEGION ZONE & \multicolumn{2}{c}{ EEGION ZONE } \\
NO. LABEL NO. & NO. IABEL NO. \\
1 AXREFL & 1 & 2 RDREPL
\end{tabular}
FEGION $2 O N E$
NO. LABEL NO.
3 AXBLKT
E.ESTON $20 \mathrm{NE}$
ABEL NO.
4 RDELKT 1
REGLON ZONE
NO. LABEL NO.
5 INCORE 1

RESTON ZONE

N. LABEL NC.

6 JOCORE

BUCKLINGS EY ZONE ANO GROUP

(CM-2, LAST VALUE FOK ZONE USED FCR PEMAINING GROJPS)

BUCKLING FOR ALL ZONES, ALL GROJPS $0 . ?$ 
REACTOR FEGIONS BY MESH POINT 
REGION

NO. LABE:

1 AXREPI

2 RDREPI

$\therefore$

$3 \quad$ AXBLKT

RDB LKI

5. INCORE

6 OUCORE
ASSIGNGENI OF MATERIALS IO EETIONS DATERIAL

GENEEIこ

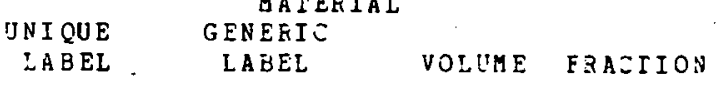

$0.300000 \mathrm{D}+00$

$0.4000000+00$

Si
NA

SS
NA

$0.600000 D+00$

SS

$0.2800000+00$

SS
NA

$\because 02$

บO2

$0.3000000+00$

$0.4000000+00$

$\mathrm{Ni}$
$\mathrm{Ni}$

UO2

NA

$0.3000000+00$

$0.450000 D+00$

PUO2

PUO2

SS .

$0.5000050-01$

$0.250000 D+00$

PUO 2

SS
PUO2

ss
$0.600000 \mathrm{D}-0$

$0.250000 D+00$

MATERIAL
UNIQUE
LABEL TEERI
IABEL

VOLUME FRACTION

NI

NI

$0.3000000+00$

N I

N I

$2.1200000+00$

SS

$5: 5$

$0.3000000+00$

ss

53

J. $250000 \mathrm{D}+00$

002 .. UJ

U32
NF.

$0.250000 D+00$

NA

$\mathrm{U} 02$

NA

Ua2
NF.

0.450000

0. $24000.00+30$.

$0.4500000+00$ 


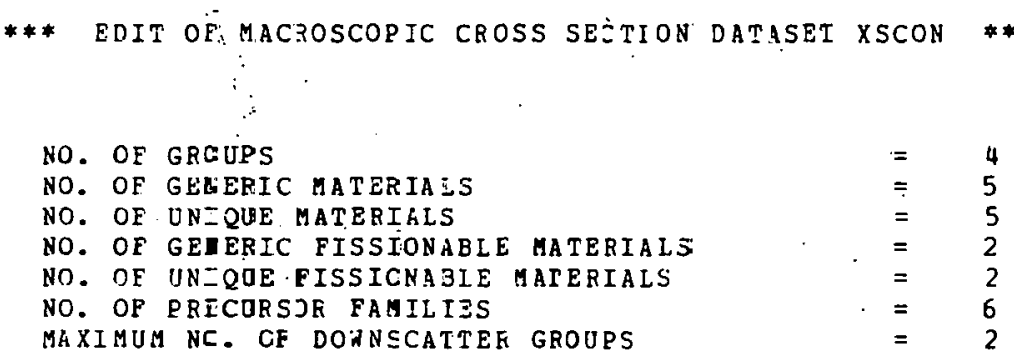

TEUPERATURES TO HHICH CROSS-SEZTIONS WERE FIJTED (DËG.K)
700.00
700.00
7000.00
700.00

GROUP BEUTKON VELOCITY

MAX DOHNSETTE.

$\begin{array}{ll}1 & 2.12455990+09 \\ 2 & 5.9584633 D+08 \\ 3 & 1.53272960+08 \\ 4 & 3.6674375 D+07\end{array}$

L, N, NOMBEz GF NEUTRGNS EMITTED INTO GEOUP NROM FAKILY L

$$
\begin{aligned}
& 5.881500 D-=1 \\
& 5.881500 D-=1 \\
& 5.881500 D-01 \\
& 5.881500 D-=1 \\
& 5.881500 D-=1 \\
& 5.881500 D-01
\end{aligned}
$$
$5.881500 D-01$

$1 \quad 1.3000000-02$ $3.5030000+00$

$\begin{array}{lll}1 & 2 & 4.081900 D-01 \\ 2 & 2 & 4.381500 D-01 \\ 3 & 2 & 4.381900 D-01 \\ 4 & 2 & 4.321900 D-01 \\ 5 & 2 & 4 . J 81500 D-01 \\ 6 & 2 & 4 . J 81900 D-01\end{array}$

* PEECURSOR DECAY CONSTANTS

FAMILY, DELAÏED MEUTPON FER UURSOR DECAY CONSTART FOR FAMILY 


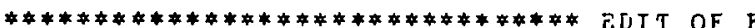

Duit of fissionable material pucz

IN $\mathrm{XSCON}$

UNIQUE MATERIAL LABEL - PUO2
GENEFIC MATERIAL LABEL - PJO2

$\begin{array}{cc} & \text { CAPTORE } \\ \text { GROUP } & \text { CROSS SECTION } \\ 1 & 4.219672 \mathrm{D}-02 \\ 2 & 1.053368 \mathrm{D}-02 \\ 3 & 2.410943 \mathrm{D}-02 \\ 4 & 1.188673 \mathrm{D}-01\end{array}$

TRANSFGRT

$1.9440120-01$ $4.0403350-01$ $5.0403350-01$ $5.5350350-01$ 8. $2618340-01$
FISSION CROSS SECTION

$4.8823480-02$ $3.5502010-02$ $4.6779342-J 2$ $1.8237160-0$
FISSION SPECTRUM (CHI)

5. $8815323-01$ $4.0819010-31$ j. $6383210-03$ $1.9472010-25$
R.SOMPT

NEUTRONS ( $2 N U$ )

\section{3. $2370500+00$} 2. $912616 D+03$ 2. $6559210+03$ 2. $8637930+00$

SCATTERING CFOSS SECTION (TOTAL)

IHTO GROUP FROH GROUP

$2 \quad 14.143188 \mathrm{D}-02$

$\begin{array}{lll}2 & 1 & 4.143188 D-02 \\ 3 & 1 & 1.445823 \mathrm{D}-04\end{array}$

20.0

$2.4010670-04$

$1.828452 D-23$

$1.393995 \mathrm{D}-03$

2. $168732 \mathrm{D}-03$

2. $168732 D-03$
$6.971079 D-04$

2. $240033 \mathrm{D}-04$
$26.033036 \mathrm{D}-03$

$31.300986 \mathrm{D}-03$

NO. CF DELAYED PRECURSORS BORN PER FISSION
L N , PRECURSORS BORN IN FAMILY L PER FISSION IN GROOR

1
2
3
4
5
6

$\begin{array}{ll}1 & 3 \\ 2 & 3 \\ 3 & 3 \\ 4 & 3 \\ 5 & 3 \\ 6 & 3\end{array}$

$2.394461 D-04$

1. $7682.04 \mathrm{D}-03$

1. $362965 \mathrm{D}-03$

$2.073075 \mathrm{D}-03$

$6.5204470-04$

2. $2072850-04$

* * this material has 2 epo doppler coefficients * *

* * COOLANT TEMPERATURE COEFFICIENTS * *

CAPTURE, REHOVAL, AND FISSION COEFFICIENTS ARE ALL ZERO

SCATTERING COEFFICIENTS ABE ALL ZREO

$2.3940710-04$

$1.764643 \mathrm{D}-03$

1. $3611310-03$

2. $067421 \mathrm{D}-03$

$6.4938110-04$

6. $4938110-04$
$2.2053500-04$ 
FX2-TH $07 / 77$ FX2-T: TEST EROBLEM 1

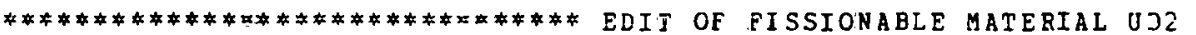

ENIQUE MATERIAI LABEL - VO2 GENERIC MATERIAL LABEL - 002

\begin{tabular}{|c|c|c|c|c|c|c|}
\hline ROOP & $\begin{array}{c}\text { CAPTURE } \\
\text { CROSS SECTIOY }\end{array}$ & $\begin{array}{c}\text { TRENSPGRT } \\
\text { CROSS SECTICN }\end{array}$ & $\begin{array}{c}\text { REMOVAL } \\
\text { CROSS SECTIJN }\end{array}$ & $\begin{array}{c}\text { FISSION } \\
\text { ZROSS SECTION }\end{array}$ & $\begin{array}{c}\text { FISSION } \\
\text { SPECTEUM (CHI) }\end{array}$ & $\begin{array}{c}\text { PROMPT } \\
\text { NEOT RONS (PNO) }\end{array}$ \\
\hline $\begin{array}{l}1 \\
2 \\
3 \\
4\end{array}$ & $\begin{array}{l}\text { 7. } 592895 \mathrm{D}-02 \\
1.075132 \mathrm{D}-02 \\
1.562200 \mathrm{D}-02 \\
2.916624 \mathrm{D}-02\end{array}$ & $\begin{array}{l}1.849744 D-01 \\
3.96502 \oplus D-01 \\
5.052330 D-01 \\
4.31003 * D-01\end{array}$ & $\begin{array}{l}\text { 8. } 944729 D-02 \\
\text { 1. } 107402 \mathrm{D}-02 \\
1.617540 \mathrm{D}-02 \\
3.112541 \mathrm{D}-02\end{array}$ & $\begin{array}{l}1.251835 D-02 \\
3.236011 D-04 \\
5.534008 D-04 \\
1.960172 D-03\end{array}$ & $\begin{array}{l}5.881532 D-01 \\
4.08 .901 D-01 \\
3.638: 321 D-03 \\
1.94 .201 D-05\end{array}$ & $\begin{array}{l}2.701577 D+00 \\
2.4447910+00 \\
2.414096 D+00 \\
2.413162 D+00\end{array}$ \\
\hline
\end{tabular}

SCATTERING CEOSS SECIICN (TOTAL)

INTO GROUP FEOM GROUE

$2 \quad 17.5 \equiv 4785 \mathrm{D}-02$

$3 \quad 15.1518490-04$

$\begin{array}{llll}1 & 5.151849 D-04 & 2 & 6.396771 D-0.3 \\ 2 & 4.057523 D-07 & 3 & 1.256830 D-03\end{array}$

NJ. OF DELAIED PRECLRSORS BCRK PER PISSION

$L$, $N$, FRECURSORS BORN IN FALILY L PEF FISSION IN GROUP $N$

$$
\begin{aligned}
& 5.374499 D-04 \\
& 5.604860 D-03 \\
& 6.60808: D-03 \\
& 1.581351 D-02 \\
& 9.137117 D-03 \\
& 3.04060: D-03
\end{aligned}
$$

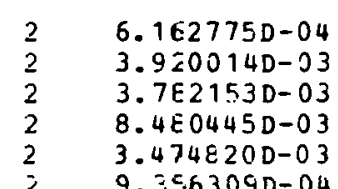

$\begin{array}{ll}2 & 3.4748200-03 \\ 2 & 9.356309 D-04\end{array}$

$\begin{array}{rr}1 & 3 \\ 2 & 3 \\ 3 & 3 \\ 4 & 3 \\ 5 & 3 \\ 6 & 3\end{array}$
6. $3525000-54$
$3.5145000-03$
$3.1020000-03$
6.715500D-1]3
6. $115000-13$

$2.112000 D-03$
$4.290000 D-1.14$

1
2
3
4
5
6

$6.3525000-04$

$3.5145000-03$

3. $102000 \mathrm{D}-03$

6. $715500 D-03$

2. $1120000-03$

4. $2900000-04$

* * this nategibl has zero doppieg coefficients ***

* * CDOLANT temperature coepfizjents ***

CAPTUEE, REUOVAL, AND FISSION COEFEICIENTS ARE ALI ZERO

SCATTERING COEFFICIENTS ARE ALl ZEBO 


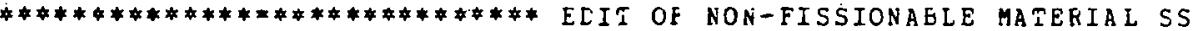

DNIOUE MATEFIAL LABEL GENEEIC MATEFIAL LABEL - SS

\begin{tabular}{|c|c|c|c|}
\hline & CAPTULE & TRANSPORT & REMOVAL \\
\hline FOO & CKOSS SECIION & OSS SECTION & SECT \\
\hline $\begin{array}{l}1 \\
2 \\
3\end{array}$ & $\begin{array}{l}5.654370 D-02 \\
3.606285 D-03 \\
3.642032 D-03 \\
S .163083[-03\end{array}$ & $\begin{array}{l}1.9480220-01 \\
2.832556 \mathrm{D}-01 \\
6.7157610-01 \\
7.8799990-01\end{array}$ & $\begin{array}{l}5.65437 C D-02 \\
3.6062850-03 \\
3.642032 D-03 \\
9.162383 D-03\end{array}$ \\
\hline
\end{tabular}

SCATTERING CFOSS SECIION (IOTAZ)

\section{INIO GROUP FROA GBOIJP}

$\begin{array}{lrr}2 & 1 & 5.503979 D-02 \\ 3 & 1 & 1.525620 \mathrm{D}-04\end{array}$

4

20.0

$23.1371530-03$

$3 \quad 1.214004 D-03$

* * this Matekial has zero dopplef COEEficients ***

* * Collant temperatufe coefficients * *

CAPTUEE, REMOVAL, AND FISSION COEFFICIENTS ABE ALL ZERO

SCATTERING CUEPFICIENTS ARE ALL ZEFO 


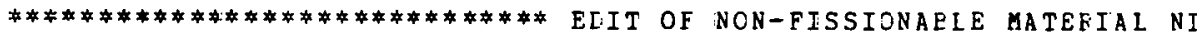

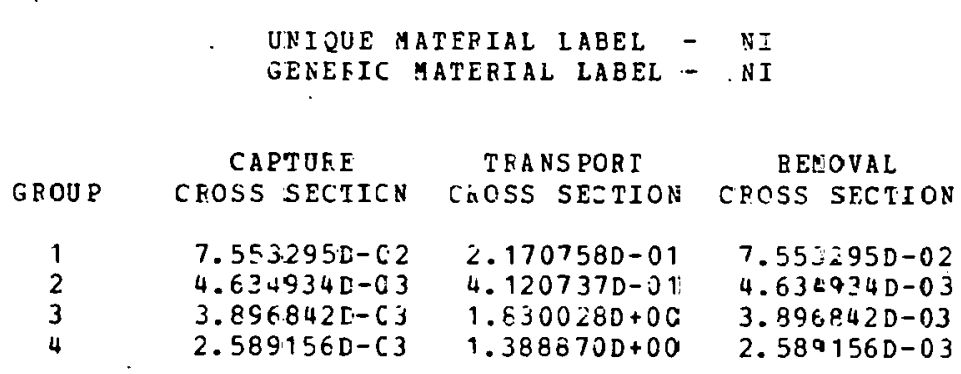

INTO GROUP EROK GROUE

$2 \quad 1 \quad 6.4 \equiv 9791 \mathrm{D}-02$

20.0

SCATTERING CROSS SECTION (TOTEL)

$2 \quad 3.786622 \mathrm{D}-0.3$

$3 \quad 2.4697090-03$

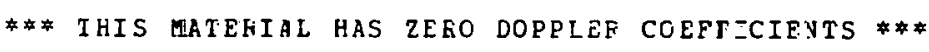

*** COOlaht temeeraiuré COEpficients *\#*

CAPTUGE, REYOVAL, AND FISSION CCFFFZCIENTS hRE ALL ZERO

SCATTERING COEFFICIENTS ARE ALL ZERO 


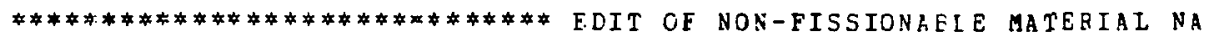

NTQUE MATEREAL LAEEL - NA

GENEEIC MATERIAL LABEL - NA

\begin{tabular}{|c|c|c|c|}
\hline \multirow[b]{2}{*}{ GROUP } & CAPTURE & TRANSPORT & RE YOVAL \\
\hline & CEOSS SECTION & CEOSS SECIION & CROSS SECTIOH \\
\hline 1 & $8.4117970-03$ & $4.0386370-02$ & $8.411797 \mathrm{D}-03$ \\
\hline $\begin{array}{l}2 \\
3\end{array}$ & $\begin{array}{l}1.9033960-03 \\
5.518917 D-04\end{array}$ & $\begin{array}{l}8.1243050-02 \\
1.5203160-01\end{array}$ & $\begin{array}{l}1.903396 D-03 \\
5.518917 D-0\end{array}$ \\
\hline 4 & $1.402493 D-04$ & $7.7511200-02$ & $1.4024930-$ \\
\hline
\end{tabular}

SCATTERING CROSS SEITION (TOTAL)

\section{INTO GROUP FEOM GROUP}

2.1 B.335418D-03

$3 \quad 1.739242 \mathrm{D}-07$

20.0
$2 \quad 1.8914390-.03$

$34: 6847+10-04$

* * Coolant tempefature COefficients ***

CAPTURE, REMOVAL, AND FISSSION CCEFFICIENTS ARE ALL ZERO

SCATIERING COEFFICIENTS AKE ALL Z ERO 


\section{* \#* :Xz STEADY STATE STORaje aliccatior}

CORE COHTINER

CORE SEQUEREMENTS STMMARY FCR
NUMBER OE VORDS IN ENTAINER

NUMBEP OF WORDS REQUITEU FOR PERMANENT IN-CERE hEFAYS

$=20 \mathrm{E}$

NUMEER CF WORDS REQUIRED FOF SCRATCH ARRAYS FCR IHIS EFOBLEM

$=51$

WUMEER OP WORDS REQUIRED FOK SCRATCH ARBAIS FCR IHIS EROJLEM

TOTAL NUMBER OF WORDI REQUIRED IN THIS CCNTIIBEF

$=\quad 3 E C$

$=4 \vdots 1$

COKE REQUIREYENTS SUMMAEY FOR LARGE CORE CONTAINER

NUMEER OF WORDS IN CENTAINER

$=13000$

NUABER OF WORDS REQUERED FOR PERAANENT IN-CEFE AERAYS

$=299$

NUMBER OF HORDS REQUERED FOE SCRATCH ARFAYS FJP THIS PẼOBLM

$=5372$

TOTAL NUMEER OF WORDS FEQUIRED IN THIS CONTAINLI.

$=5671$

NUMBEF OF ADDITIONAL FORDS REQUIRED TO STORE SCATTERING BAND OF FIUXES = 616

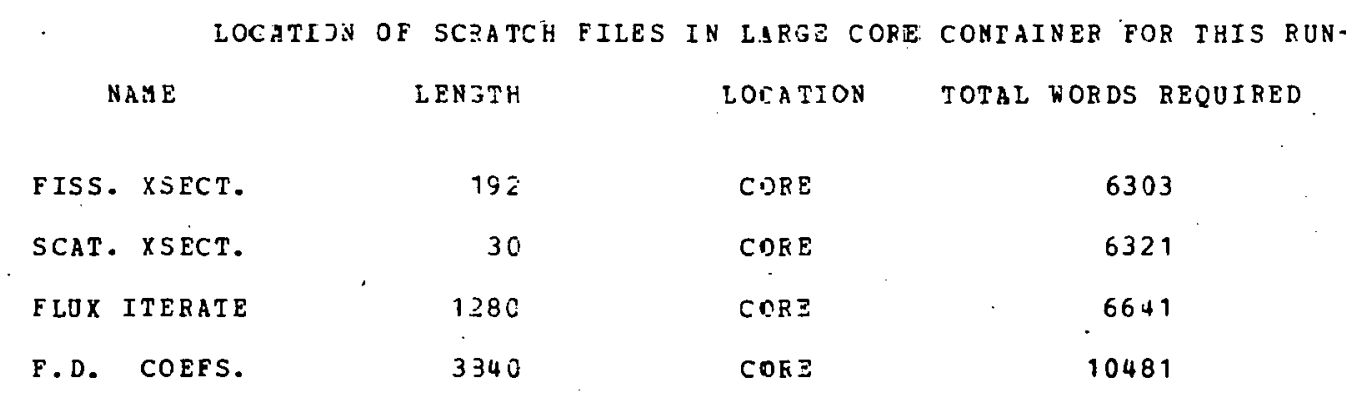

BINARY ZNPUT PROCESSING COMPLETミD

CENTRAL PROCESSOR TIME (SEC) 


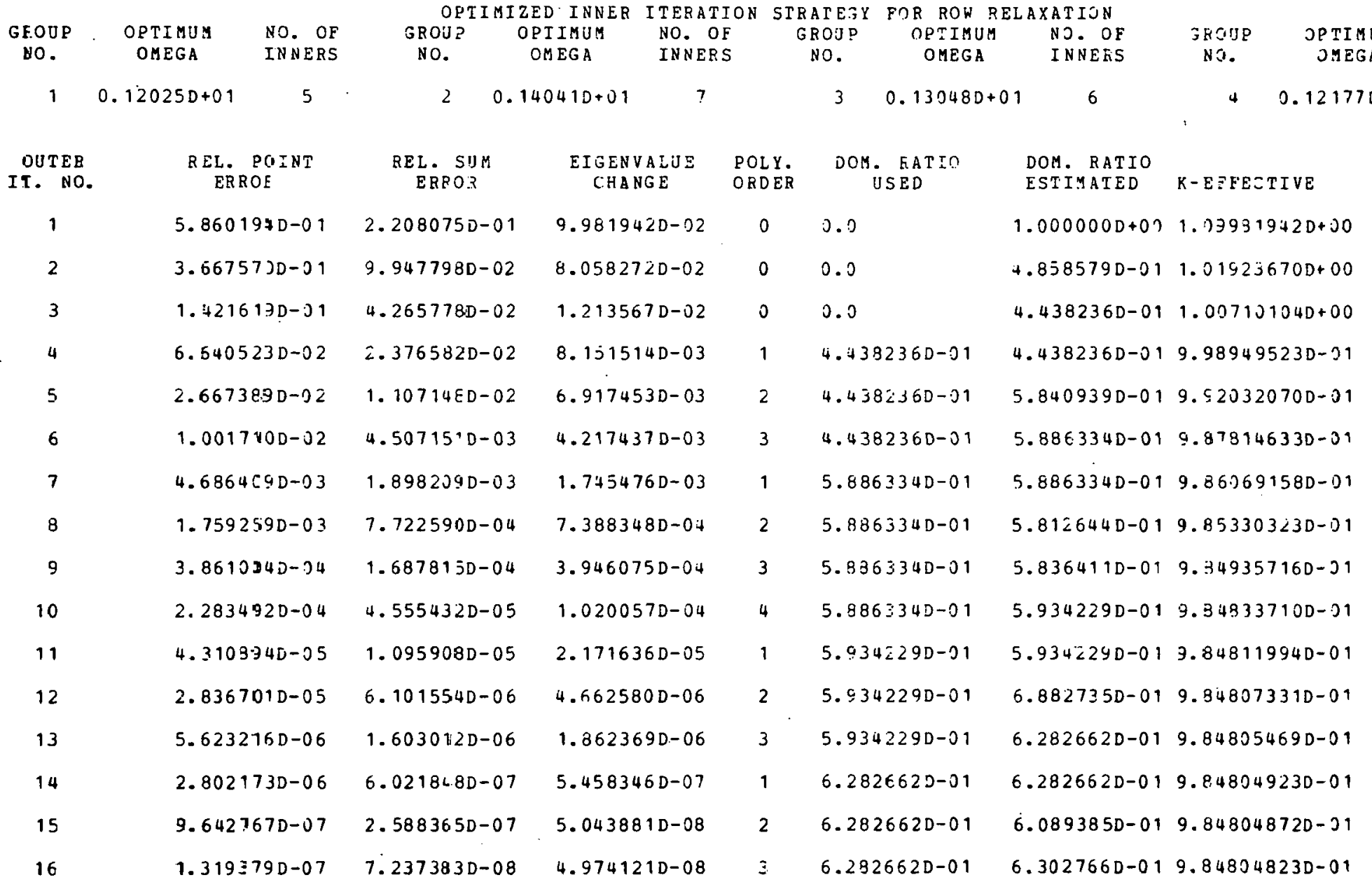

OUTER ITEFATIOES COMPLETED AT =TERATION 16, ITERATIONS HAVE CONDERGED

K-EFFECTIPE $=0.98480482264$ 


\begin{tabular}{|c|c|c|c|c|c|c|c|c|c|c|c|c|}
\hline \multirow{2}{*}{$\begin{array}{c}\text { GROUP } \\
\text { NO. } \\
1\end{array}$} & \multirow{2}{*}{$\begin{array}{c}\text { OPTIMUA } \\
\text { OMEGA } \\
0.12177 D+01\end{array}$} & $\begin{array}{l}\text { No. OF } \\
\text { INNERS }\end{array}$ & $\begin{array}{l}\text { OPT } \\
\text { GROUP } \\
\text { DO. }\end{array}$ & I.I I? & $\begin{array}{l}D \text { IN YER } \\
\text { TIMU: } \\
\text { MEGE }\end{array}$ & $\begin{array}{l}\text { IT } \Xi R A I \\
\text { NO. } \\
\text { INNEI }\end{array}$ & $\begin{array}{l}\text { ION SIE } \\
\mathrm{F} \\
\mathrm{S}\end{array}$ & $\begin{array}{l}\text { ATESY } \\
\text { ROUP } \\
\text { NO. }\end{array}$ & $\begin{array}{r}\text { FOP RIW } \\
\text { OPTIMJ } \\
\text { OMEG.S }\end{array}$ & $\begin{array}{l}\text { ELAXAT:ON } \\
\text { NO. OF } \\
\text { INSERS }\end{array}$ & $\begin{array}{l}\text { SPOUP } \\
\text { NO. }\end{array}$ & $\begin{array}{r}\text { OPTIM } \\
\text { JMES }\end{array}$ \\
\hline & & 5 & 2 & 0.1 & $048 D+01$ & 6 & & 3 & 0.140410 & .01 & 4 & 0.120 \\
\hline $\begin{array}{l}\text { OUTEE. } \\
\text { IT. NO. }\end{array}$ & \multicolumn{2}{|c|}{$\begin{array}{l}\text { REL. EOINT } \\
\text { ERRIR }\end{array}$} & \multicolumn{2}{|c|}{$\begin{array}{c}\text { REI. SUM } \\
\text { ERROR }\end{array}$} & \multicolumn{2}{|c|}{$\begin{array}{c}\text { EIGENVALCE } \\
\text { CHANGE }\end{array}$} & $\begin{array}{l}\text { POLY. } \\
\text { ORDER }\end{array}$ & \multicolumn{2}{|c|}{$\begin{array}{c}\text { DOM. RATIO } \\
\text { US } \equiv D\end{array}$} & $\begin{array}{l}\text { DDM. RATIO } \\
\text { ESIIMATED }\end{array}$ & \multicolumn{2}{|c|}{ K-EPFECTIVE } \\
\hline 1 & $5.224:$ & $9=8 D-01$ & $2.811394 \mathrm{D}$ & $-[1$ & $2.5 \uparrow 251$ & $17 D-02$ & 0 & 0.0 & & $1.0000000+00$ & 1.03993 & $30000+30$ \\
\hline 2 & 2.6960 & $210-01$ & $1.154570 \mathrm{D}$ & -01 & $3.79+81$ & $14 D-03$ & 0 & 0.0 & ' & 4. $315092 D-01$ & 1.01371 & $14810+00$ \\
\hline 3 & 9.5726 & $350-02$ & $4.585588 \mathrm{D}$ & -02 & $5.6) 778$ & $850-03$ & 0 & 0.0 & & 4. $17 ? 880 D-01$ & 1.00810 & $07020+00$ \\
\hline 4 & 4.7507 & $75.3 D-02$ & $2.294260 \mathrm{D}$ & $-0 ?$ & 8.12540 & $06 D-1) 3$ & 1 & 4.17 & $7783: 00-01$ & $4.1778300-01$ & 9.99978 & $8618 D-01$ \\
\hline 5 & 2.0070 & $0935-32$ & 1.05360010 & -02 & 7.30657 & $710-03$ & 2 & 4.17 & $778900-01$ & $5.73 \equiv 2+8 D-01$ & 9.92672 & $20470-01$ \\
\hline 6 & 8.1644 & $4300-03$ & 4.4375330 & -03 & 4.42685 & $52 \mathrm{C}-03$ & 3 & 4.17 & $77 \varepsilon 80[-)$. & $5.816931 \mathrm{D}-01$ & 9.88251 & $11950-31$ \\
\hline 7 & 3.9433 & $15 D-03$ & $1.951 \in 27 \mathrm{D}$ & $-0 \equiv$ & 1.93122 & $20[--i 3$ & 1 & 5.81 & $16 \subseteq 310-01$ & $5.8169310-01$ & 9.863 & $99740-01$ \\
\hline 8 & 1.5124 & $1250-03$ & $8.025597 \mathrm{D}$ & -04 & 8.81507 & $78 D-04$ & 2 & 5.81 & $169.31 D-21$ & $5.824214 \mathrm{D}-01$ & 9.85438 & $84660-01$ \\
\hline 9 & 3.4499 & $290-04$ & $1.855872 \mathrm{D}$ & -04 & 4.71836 & $650-0.4$ & 3 & 5.81 & $69310-01$ & $5.842387 D-01$ & 9.34956 & $55800-01$ \\
\hline 10 & 2.10878 & $323 D-04$ & 5.0986540 & -05 & 1.22711 & $15 D-04$ & 1 & 5.84 & $+23370-01$ & $5.8 \angle 2387 D-01$ & 9.8484 & $38080-01$ \\
\hline 11 & 4.5366 & $6473-05$ & 2.0941290 & -05 & 2.35338 & $81 D-05$ & 2 & 5.84 & $+2337.0-01$ & $5.8 \angle 8569 \mathrm{D}-01$ & 9.8432 & $02750-01$ \\
\hline $1<$ & 1.0777 & $760)-05$ & $5.3255=5 \mathrm{D}$ & -06 & 1.14657 & $76 D-05$ & 3 & 5.84 & $+23670-01$ & $5.930329 D-01$ & 9.84908 & $38080-01$ \\
\hline 13 & 9.5914 & $178 D-06$ & $1.9261 \equiv 50$ & -06 & 3.07736 & $65 \mathrm{D}-06$ & 1 & 5.93 & $303 \div 90-01$ & $5.930 \equiv 290-01$ & 9.84805 & $57300-01$ \\
\hline 14 & 1.9038 & $3320-06$ & $8.5053 E O D$ & -07 & 5.49505 & $560-07$ & 2 & 5.93 & $303 \approx 90-01$ & $E .07154$ ED-01 & 9.84805 & $51810-01$ \\
\hline 15 & 4.9115 & $510=07$ & $2.573311 \mathrm{D}$ & -07 & 2.82771 & $180-07$ & 3 & 5.93 & $3032.90-31$ & $6.193124 \mathrm{D}-01$ & 9.84804 & $4898 D-01$ \\
\hline 16 & 3.9700 & $87 D-07$ & $9.050388 \mathrm{D}$ & -08 & 6.37441 & $19 D-58$ & 1 & 6.19 & $331240-01$ & E. $193124 D-01$ & 9.84504 & $48340-01$ \\
\hline
\end{tabular}

OUTER ITEFATIONS COMPLETED AT ITERATION 16, ITERATIONS HAVE CONVERJED 


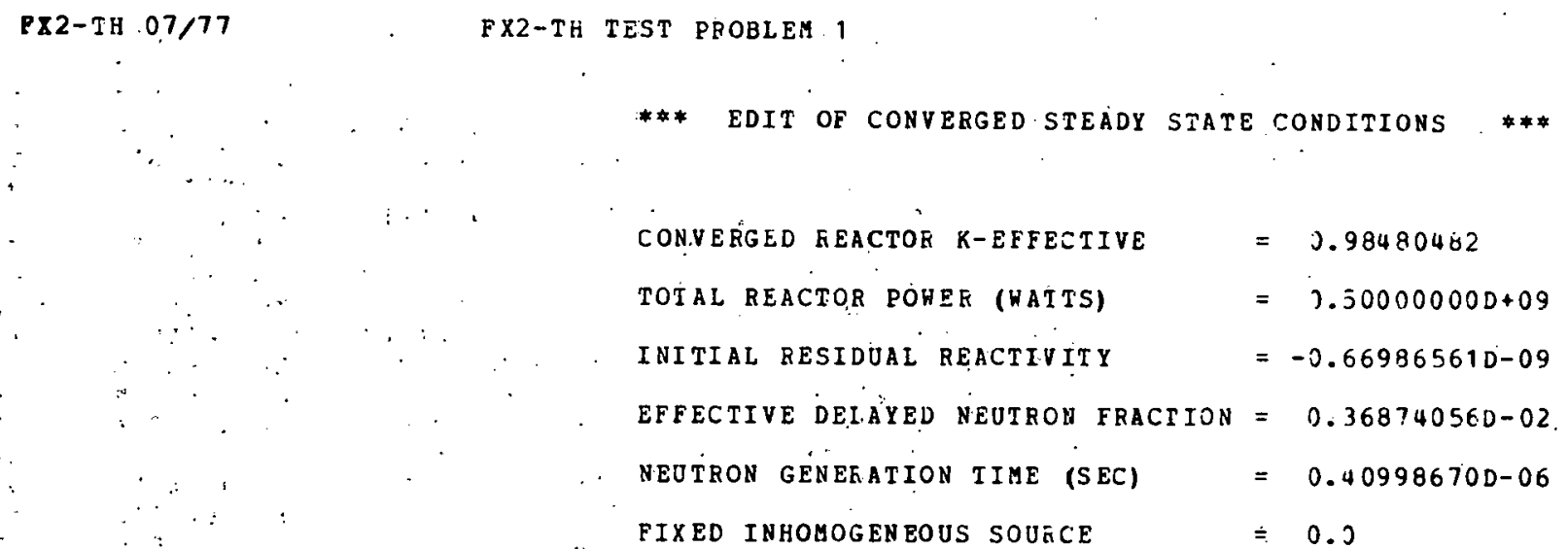

* * Edit of CONVERged.steady state conditions * *

$\begin{array}{ll}\text { CONVERGED GEACTOK K-EFFECTIVE } & =3.98480402 \\ \text { TOTAL REACTOR POHEK (WATTS) } & =3.500000000+09 \\ \text { INITIAL RESIDUAL REACTIVITY } & =-0.66986561 \text { D-09 } \\ \text { EFEECTIVE DEIAYED NEUTRON FRACIION } & =0.368740560-02 . \\ \text { NEOTRON GENEKATION TIME (SEC) } & =0.40998670 \mathrm{D}-06 \\ \text { FIXED INHOHOGENEOUS SOUACE } & =0.3\end{array}$

FAGILY

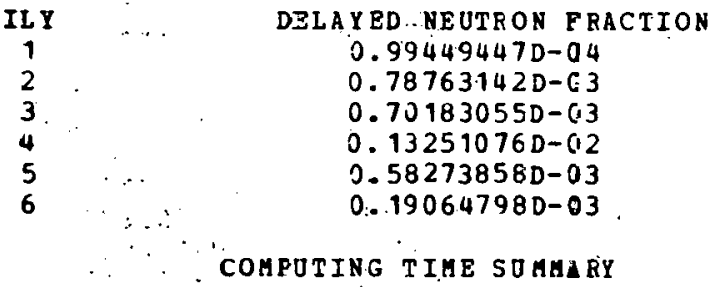

BCD CARD INROT PROCESSING

CROSS SECTION HOMOGENIZATION

BIBARY PILE ENPUT PROCESSING AND INITIALIZATION

GEHERATE CROSS SECTIONS

COAPUTE REAL AND ADJOINT NEUTRON RLUX

COAPUTE AHPLITUDE PUNCTION EOUATION COEFFICIENTS

COHPOTE THEBMAL/HYDRAULIC FEEDBACK

TOTAL FOR STEADY STATE CALCULATICA

TOTAL ELAPSED COAPUTING TIME (SEC) =

\author{
INTEGRATED PRECURSOR CONC. \\ $0.18659038 D+05$ \\ $0.611210930+05$ \\ $0.1258704 .0 D+05$ \\ $0.950610170+04$ \\ $0.107678770+04$ \\ $0.132860050+03$
}

CENTRAL PROEESSOR (SEC)

PERIPBERAL PROCESSOR (SEC)

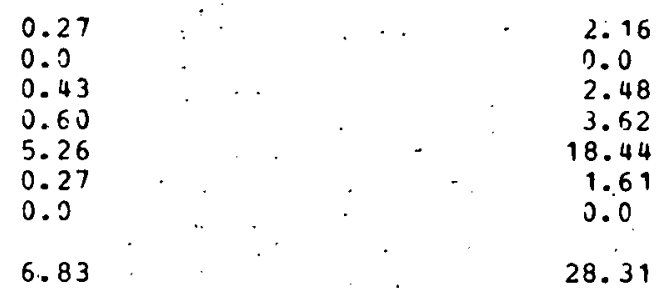

$35.29(\mathrm{CP}+\mathrm{PP})$ 
INITIFL POHER DEHSITY (HATTS, CMB) ON FIXED MESH

\begin{tabular}{|c|c|}
\hline AXIS & $\underset{1}{R-A X I S}$ \\
\hline 20 & 0.0 \\
\hline 19 & 0.0 \\
\hline 18 & 0.0 \\
\hline 17 & $3.5463 D+01$ \\
\hline 16 & $5.2339 D+01$ \\
\hline 15 & $5.2845 \mathrm{D}+02$ \\
\hline 14 & $6.49520+02$ \\
\hline 13 & $7.48330+02$ \\
\hline 12 & $8 . \div 7710+02$ \\
\hline 11 & $8.5341 D+02$ \\
\hline 10 & $8.53410+0<$ \\
\hline 9 & $8.17710+102$ \\
\hline 8 & $7.4833 D+02$ \\
\hline 7 & $6.49520+02$ \\
\hline 6 & $5.2845 \mathrm{D}+02$ \\
\hline 5 & $5.2339 D+01$ \\
\hline 4 & $3.54630+01$ \\
\hline 3 & 0.0 \\
\hline 2 & 0. \\
\hline 1 & 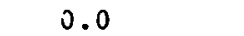 \\
\hline
\end{tabular}

Z-AXIS: R-AXIS

0.0
0.0
0.0
$1.9 C 19 D+01$
$2.8444 D+01$
$3.3029 D+02$
$4.0555 D+02$
$4.6679 D+02$
$5.0972 D+02$
$5.3179 D+02$
$5.3179 D+02$
$5.0972 D+02$
$4.6679 D+02$
$4.0555 D+02$
$3.3029 D+02$
$2.8494 D+01$
$1.9019 D+01$
0.0
0.0
0.0

R-AXIS

0.0
0.0

0. 3

3. $50940+01$

Э. $17800+01$

ᄃ. $2276 \mathrm{D}+02$

$E .4255 \mathrm{D}+02$

]. $40310+02$

3. $33960+02$

3. $4+29 D+02$

8. $44290+02$

8. $0896 \mathrm{D}+02$

7. $4031 \mathrm{D}+02$

$\epsilon .4255 D+02$

5. $2276 \mathrm{D}+02$

c. $17800+01$

3. $5084 \mathrm{D}+01$

O. 0

0.0

$R-A X I S$
11

0.0

0.0

0.0

1. $46870+01$

$2.1475 D+01$

2. $6112 \mathrm{D}+02$

3. $1858 \mathrm{D}+02$

3. $5560 \mathrm{D}+02$

3. $5560 D+02$

4. $15700+02$

$4.15700+02$

3. $667 \mathrm{D}+02$

$3.6560 \mathrm{D}+02$

3. $1858 \mathrm{D}+02$

2. $\leqslant 112 \mathrm{D}+02$

2. $1475 D+01$

1. $4687 D+01$

0.0

0.0
0.0
R-AXIS

3

0.0

0

$=.4332 D+01$

. $0675 \mathrm{D}+01$

E. $1147 D+02$

$5.2872 \mathrm{D}+02$

$7.2441 \mathrm{D}+02$

.. $91600+02$

. $2618 \mathrm{D}+02$

$7.9160 \mathrm{D}+02$

$7.24410+02$

$6.38720+02$

$5.11470+02$

5. $0675 D+01$

3. $4332 \mathrm{D}+0$

0.0

0.0

0.0

R-AXIS

12

.3

0.3

. $159 D+01$

i. $3505 \mathrm{D}+01$

i. $3721 \mathrm{D}+01$

ट. $1943 \mathrm{D}+01$

2. $-935 D+01$

3. $635 D+01$

$3.990+0$

$3.1999 D+01$

3. $C 635 D+0$

2.7935D+01

2. $3943 \mathrm{D}+01$

1. $3721 \mathrm{D}+0$

$1.3505 D+01$

0.0

0.0

0.0
0.0
E-AXIS

0.0

0.0

3. $3222 \mathrm{D}+01$

4. $9043 D+01$

4. $9475 \mathrm{D}+02$

$6.0824 D+02$

7. $3086 D+02$

7. $5590 \mathrm{D}+02$

7. $7936 \mathrm{D}+\mathrm{C} 2$

$7.3936 \mathrm{D}+\mathrm{C} 2$

$7.5590 \mathrm{D}+02$

b. $08240+02$

$4.9475 D+02$

4. $.043 D+D$

3. $\equiv 222 \mathrm{D}+\mathrm{D}$

0.0

0.2

0.

B-AXIS

13

0.0

0.0
0.0

$6.6555 D+00$

8. $3<95 D+00$

1. $0666 D+01$

1. $3798 D+01$

$1.5=39 D+0$

1. $6 E 52 D+01$

$1.7 \equiv 74 D+01$

$1.73740+01$

$1.6652 \mathrm{D}+0$

$1.5239 D+01$

1. $3198 \mathrm{D}+01$

1. $0656 D+01$

$8.3495 D+00$

$6.6555 \mathrm{D}+00$

0.0

0.0
R-A XIS

5

0.0

0.0

3. $17740+01$

4. $69200+J 1$

4. $72840+02$

$5.81430+02$

$6.70040+02$

7. $32250+02$

$7.6425 D+02$

$7.64250+02$

6. $70040+02$

$5.81430+02$

4. $72540+02$

4. $69200+01$

3. $1774 \mathrm{D}+01$

0.0

0.0

E- A X I S

14

0.0

0.0

0.0
0.0

0.0

0.0

0.0

0.0

0.0

0.0

0.0
0.0
0.0

0.0

0.0
0.0
0.0

0.0

0.0
0.0

0.0

0.0
0.0
$R-\underset{6}{\lambda}$ IS

R-AXIS

0.0

0.0

2. $7974 \mathrm{D}+01$

4. $14060+01$

$+.1488 D+02$

5. $1056 \mathrm{D}+02$

5. $8856 \mathrm{D}+02$.

$.71400+02$

5. $71400+02$

e. $4 ; 270+22$

5. $8856 D+02$

5. $10560+02$

4. $1488 D+02$

L. $1406 \mathrm{D}+01$

2. $7974 \mathrm{D}+01$

a. 0

0.0

0.0

0.0

$R-A X I S$

15

R-AXIS

0.0
0.0
0.0
0.0
0.0
0.0
0.0
0.0
0.0
0.0
0.0
0.0
0.0
0.0
0.0
0.0
0.0
0.0
0.0
0.0

0.0

3. 0

3.0

0.0

0.0

0.0

0.0

0.0

0.0

0.0

C. 0

G. 0

0. 0

0.0

ว.. 3

0. 3

0.3

0. 3

0.3

0.0 


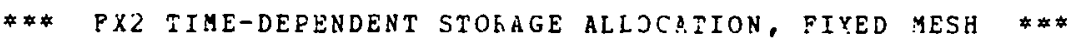

CORE REQUIREMENTS SUMMAKI FOR SMALL CORE CONTAINER

NUGBER CF WORDS IN CONMAINER

$=2000$

NOHBER OF WORDS REQJIEED FOR PERMANENT IN-CORE ARRAYS

$=334$

NUMEEE OF WORDS REQUIRED FOR SCRATCH ARKAYS FOH THIS PKOSLEM

$=1340$

TOTAL NUMBER OE WORES REDUIRED IN THIS CONTAINER

$=1674$

CORE REQUIREMENTS SJMMARY FOR LARGE COEE CORTAINER

NUMBER OF WORDS IN CONTAINEP

$=13000$

$=299$

$=5372$

NOUBER OE WORDS REQUIRED FOR SCRATCA ARRAYS FOR THIS PROELEM

$=5671$

NOHBER OF ADDITIONAL WORDS REQUIRED TO STORE SCATTERING BAND OF FLUXES $=320$

LOCATION OF SCRATCH FILES IN LARGE CORE CONTAINER FOR IHIS RUN-

$\begin{array}{lrcr}\text { NAYE } & \text { LENGTH } & \text { LOCATION } & \text { TOTAL WORDS R } \\ \text { XSPFIS } & 192 & \text { CORE } & 6023 \\ \text { XSPSCT } & 30 & \text { CORE } & 6041 \\ \text { XSPGEP } & 48 & \text { CORE } & 6077 \\ \text { FRIP1 } & 1280 & \text { CORE } & 6969 \\ \text { VPRACI } & 1600 & \text { CORE } & 8569 \\ \text { PREC2M } & 1920 & \text { CORE } & 9917 \\ \text { PDCOEF } & 3840 & \text { CORE } & 12129\end{array}$




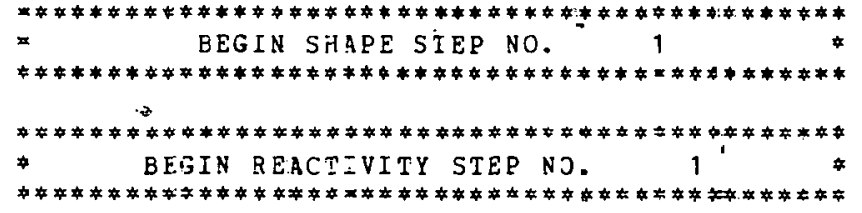

FIRST REACTIVIFY STE? = 10000 INIIIAL GENEPATION TIMES

REACTIVITI STEP BEGINS AT 0.3 SEC, ENDS AT 0.409087D-0Z SEC, LENGIH= J.409987D-J2 SEC

SHAPE STEP BEGINS AI J.0 SEC, ENDS AT 0.40Y\$87D-J2 5EC, LENGTH = 0.409937D-02 SEC

IHE FOLIOHENG DEIVING FUNCTIONS APPLY THIS REACEIVITY STEP

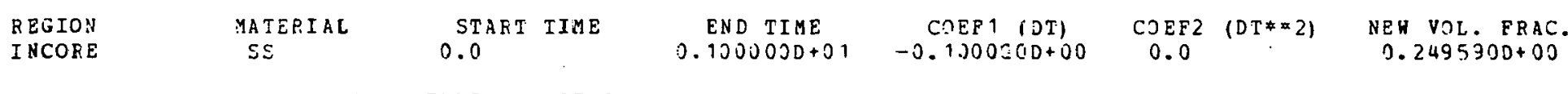

aMPLITUEE CHANGE OVEK.SHAFE STEP TERMINATES THIS STEE

\section{* * Summafy for Reactivity STEP NJMBZE 1 \\ STEP BEGAF AT 0.0 \\ SECONDS, ENDS AT 0.40993670[-02 SECONDS INTO TRANSIENT}

END-OF-STEP REACTIVITY

GENEEATION TIME (SECONDS)

MAXIMUY FUEL TEMP. CHANG̈̈ (DEG.K) $=0.0$

TOTAL REACTOF: POWER

COMPUTING TIME SUMMABY

TOTAL FOR TAIS STEP

TOTAL ELAPSED COMPITING TIME (SEC)
EFFECTZVE DELAZED NEUTZON FRACIION $=0.36874056 \mathrm{D}-02$ AMPIITEDE PUNCIION VELJE (N) $=0.10097890 \mathrm{D}+01$ TOTAL ENERGY FELEASE (HATT-SEC) $=0.205956710+07$

MAXIMU: FUEL TIJPERAIURE (DEG.K) $=0.3$

$$
=0.504894520+09
$$

CENTRAL PROEESSRR (SEC) PEKIPHERAL PROZESSOR (SEC)

$\begin{array}{lccc} & \text { PEKIPHERAL PROZESSOR } & \text { (SEC) } \\ 1.53 & 2.39 & & \\ 7.39 & & 30.82 & =\end{array}$




\begin{tabular}{|c|c|c|c|c|c|c|c|c|c|c|c|}
\hline & & & QP? & IMIZED INNER & ITETATIJN & STRAATEZY & $\Xi C R$ POH REL & XAT:ON & & & \\
\hline $\begin{array}{c}\text { GROUP } \\
\text { NO. }\end{array}$ & $\begin{array}{l}\text { CPTIMIS } \\
\text { OMEGA }\end{array}$ & $\begin{array}{l}\text { NO. OF } \\
\text { INNEPSS }\end{array}$ & $\begin{array}{l}\text { GROUP } \\
\text { No. }\end{array}$ & $\begin{array}{l}\text { OPI IMUM } \\
\text { OMEGA }\end{array}$ & $\begin{array}{l}\text { NO. OF } \\
\text { INTIERS }\end{array}$ & $\begin{array}{c}\text { GROUD } \\
\text { NO. }\end{array}$ & $\begin{array}{l}\text { ORT IMUM } \\
\text { OYESA }\end{array}$ & $\begin{array}{l}N 2 \text { OF } \\
\text { INNEFS }\end{array}$ & $\begin{array}{c}\text { GEOUP } \\
\text { ND. }\end{array}$ & $\begin{array}{l}\text { OP TIMUM } \\
\text { JMEGA }\end{array}$ & $\begin{array}{l}\text { MO. OF } \\
\text { INNERS }\end{array}$ \\
\hline 1 & $0.12025 \mathrm{D}+01$ & 5 & $\vdots$ & $0.140410+01$ & 7 & 3 & $0.132493+01$ & 6 & 4 & $0.121760+01$ & 5 \\
\hline
\end{tabular}

\begin{tabular}{|c|c|c|c|c|c|c|c|}
\hline $\begin{array}{l}\text { OUTEF } \\
\text { IT. NO. }\end{array}$ & $\begin{array}{c}\text { REL. DOINT } \\
\text { ER TOR. }\end{array}$ & $\begin{array}{l}\text { PEL: SUM } \\
\text { EREOK }\end{array}$ & $\begin{array}{c}\text { EIGENVALUE } \\
\text { CHANGE }\end{array}$ & $\begin{array}{l}\text { POI Y. } \\
\text { OREER }\end{array}$ & $\begin{array}{c}\text { SPEC. RAD. } \\
\text { USED }\end{array}$ & $\begin{array}{l}\text { SPEC. RAD. } \\
\text { ESTIMATED }\end{array}$ & GF.MMA FAこTOR. \\
\hline 1 & $2.413 \equiv 900-04$ & $8.5000+7 D-05$ & 0.0 & $\overline{0}$ & 0.0 & $1.0000000+00$ & $9.977098810-01$ \\
\hline$z$ & 5. $263 \approx 78 D-05$ & 8. $1040: 30-05$ & 0.0 & 0 & 0.0 & $9.534234 \mathrm{D}-01$ & $9.909976710-01$ \\
\hline 3 & 2.958 i $190-05$ & 8. $809553 D-06$ & 0.0 & 0 & 0.0 & $1.0871030-01$ & $1.000301850+30$ \\
\hline
\end{tabular}

CUTER ITERATYOAS COMPLETED AT ITEKATION 3, ITEEATIONS HAVE CONVEFGED

. GAMAA FACTOR FJR SHAPE CALCULATION = 1.00000184896

RHO STEP STEP LEVETH

No. (S EC)

$1 \quad 0.409986700-02$
* * AMELITUDE

ENDTIME NO. TEMP.

(SEC). CORE. ITER.

. $4099867.00-02$

0

\section{GEN. TIME $\operatorname{TISE} \vdots$}

$0.368773245-02$ $0.409952170-0.5$

\begin{tabular}{|c|c|c|}
\hline $\begin{array}{l}\text { UPDATED } \\
\text { REACTIVITY } \\
\text { REACTIVITY (\$) }\end{array}$ & $\begin{array}{l}\text { VALLE } \\
\text { ALPHA (DN /N) }\end{array}$ & AMPLITUDE \\
\hline $\begin{array}{l}0.36727527 D-04 \\
0.97593796 \mathrm{D}-02\end{array}$ & $0.239537410+0 i$ & $0.100952420+31$ \\
\hline
\end{tabular}


$\ldots$

\#* SUMYARY FOK SHAPE STEP NUIBER 1 ***

\author{
STEP BEGAN AT 0.0 SECONDS, ENDS AT $0.409386700-02$ SECONDS INTO TEANSIENT
}

STFZ EERJIRED 1 GaMAa PECTOR ITEGATIONS AND 1 REACTIVITY STEPS.

REACTOP POWER LEVEL $=0.50486$ 191C+09 FATTS

TOTAL ENEFGY RELEASE = $0.20597335 \mathrm{D}+07$ HATT-SIC

\section{COMPITING TIME SUEHARY}

THERMAI HYDRAULICS

GENEKATE CROSS SECTIONS

COMPUIE AMPLITUDE PUNETION EQUATJON COEFFICIENTS

SHAPE FUNCTION SOLUTION

SOLVE FOR AMPLITUDE FUNCIION, MISC.

TOTAL EOR THIS STEP

TOTAL ELAPSED COMPUITING IIME (SEC)
CENTRAL PPOEESSOR (SEE)

0.0
0.0
0.14
0.32
0.80
0.27
1.53
3.39

PERTPHERAL PROCESSOR (SEC)

$$
\begin{aligned}
& 0.0 \\
& 0.0 \\
& 0.25 \\
& 0.96 \\
& 3.37 \\
& 1.76 \\
& 6.34
\end{aligned}
$$

34.77 


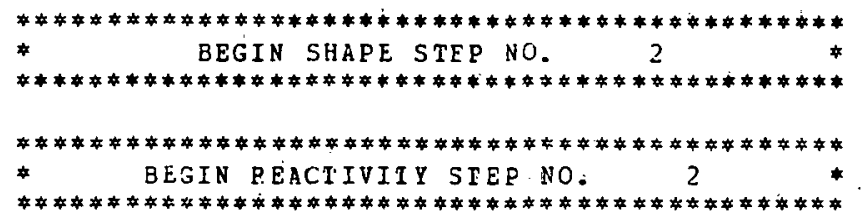

EEACIIVITY STEF SELECTION TEST STYMARY

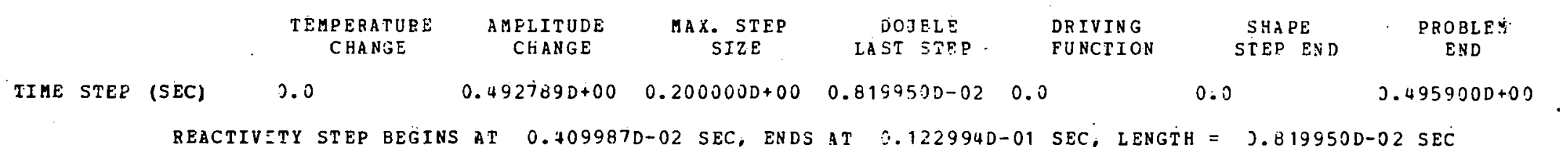

SHAPE STEP SELECTION TEST SIMMARY

$\begin{array}{ccclc}\text { FLUX SHAPE } & \text { MAX. STEP } & 10 \text { TIMES } & \text { DRIVING } & \text { PEOBLEM } \\ \text { CHANGE } & \text { SIZE } & \text { LAST STEP } & \text { FUNCTION } & \text { END }\end{array}$
TIME STEP (SËC)
$0.500000 D+30$
$0.4099750-010.0$
$0: 4559000+03$

SHAFE STEP BEGINS AT 0.409987D-02 SEC, ENDS ÁT 0.450973D-01 SEC; LENGTH=0.4099750-01 SEZ

THE FOLLOHING DRIVING FUNCTIONS APPLY THIS EEACTIVITY STEP

ALIPLTUDE CHÄNE OVER SHÁPE STEP TERHINATES THIS STEP

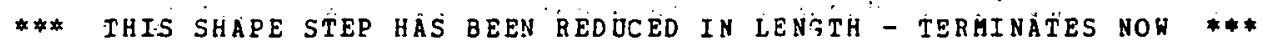

NEN LENGTH $=0.819950 D-02$ SEC; STEP END TIHE $=0: 1229940-01 \mathrm{SEC}$ 


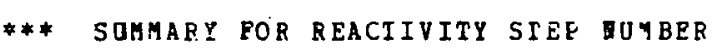

2

STEP BEGAM AT $0.4099867 C \mathrm{D}-02$ SECONDS,

\begin{abstract}
END-OF-STEP REACZIVITY
GENERATION TIME (SECONDS)

$=\quad$. $409883150-06$

AMPIITUDE FUNCTION DERIVATIVE (DN) $=0.254982660+01$

MAXIMUM FUEL IEMP. CHANGE (DEE.K) $=0.0$
\end{abstract}

$=0.110154530-03$

TOTAI EEACTOR POHER (WATTS)

COMPUT LNG TIME SUMARRY

TOTAL FOR CHIS STEP

TOTAL ELAPSED COMPUTING IIME CSEC
CENTRAL PROEESSOR (SEC)

$$
\begin{aligned}
& 0.37 \\
& 3.76
\end{aligned}
$$

3.76
EFFECTIVE DEIAYED NEUTRON FRACTION $=0.36983859 \mathrm{D}-02$ A MPLITUDE FINCTION VAIUE (N) $=0.103006150+01$

IOTAL ENBR:SY RELEASE (WATT-SEC) $=0.62417564 \mathrm{D}+07$

\section{$=0.51518323 D+29$}

$=0.3$ 
OJTER ITERATION SUGMARY REAL SOLUTIGN TIMEEP PPOBLEM PEOELEY TIME = 0.122394D-01 SECONDS

\begin{tabular}{|c|c|c|c|c|c|c|c|c|c|c|c|}
\hline & & & $O F I$ & IMIZEL INNER & ITEKAIION & SIFATEGZ & FO马 ROV RE! & XATIวN & & & \\
\hline $\begin{array}{c}\text { GRO:: P } \\
\text { NO. }\end{array}$ & $\begin{array}{c}\text { OPTIMUM } \\
\text { OMEGA }\end{array}$ & $\begin{array}{l}\text { NO. JF } \\
\text { INIERS }\end{array}$ & $\begin{array}{l}\text { GRO'J } \\
\text { NO.. }\end{array}$ & $\begin{array}{c}\text { OPIIMYM } \\
\text { CMEGA }\end{array}$ & $\begin{array}{l}\text { NO. OF } \\
\text { INNEKS }\end{array}$ & $\begin{array}{c}\text { SRCLP } \\
\text { NE: }\end{array}$ & $\begin{array}{c}\text { OPTIYYA } \\
\text { OMEGA }\end{array}$ & $\begin{array}{l}\text { NO. OF } \\
\text { INNERS }\end{array}$ & $\begin{array}{c}\text { GE.J } \\
\text { NI. }\end{array}$ & $\begin{array}{c}\text { OPTIMUS } \\
\text { JYESA }\end{array}$ & $\begin{array}{l}\text { NO. JF } \\
\text { INNEPS }\end{array}$ \\
\hline 1 & $0.12025 E+U 1$ & 5 & 2 & $.0 .140410+01$ & 7 & $\dot{2}$ & $0.13 j+9.0+01$ & $\dot{s}$ & 4 & $0.121760+01$ & .5 \\
\hline
\end{tabular}

\begin{tabular}{|c|c|c|c|c|c|c|c|}
\hline $\begin{array}{l}\text { OJTER } \\
\text { IT. NO. }\end{array}$ & $\begin{array}{l}\text { REL. POINT } \\
\text { ERRJ? }\end{array}$ & $\begin{array}{c}\text { REL. JUM } \\
\text { ERRJR }\end{array}$ & $\begin{array}{c}\text { EIGENYALUE } \\
\text { CHANGE }\end{array}$ & $\begin{array}{l}\text { FOI Y. } \\
\text { ORDEE }\end{array}$ & $\begin{array}{c}\text { SPEC. EAD. } \\
\text { USED }\end{array}$ & $\begin{array}{l}\text { SPEC. RAD. } \\
\text { ESTIMATED }\end{array}$ & GI:1A FAこTOR \\
\hline 1 & $3.8332+90-0.5$ & $1.5233630-05$ & 0.0 & ? & 0.2 & $1.0000000+00$ & $1.030003510+00$ \\
\hline 2 & $1.9597 .26 \mathrm{D}-05$ & $1.069660 \mathrm{D}-05$ & 0.0 & 0 & 0.0 & $7.0216480-01$ & $1.000000890+00$ \\
\hline is & $1.0529 .30 D-05$ & $5.3386710-06$ & 0.0 & 0 & 0.0 & $4.990969 D-J 1$ & $1.000000340+00$ \\
\hline
\end{tabular}

CUTER ITEBations CCMPLETEN AT ITERATION 3, ITERATIONS HAVE CONVEGGED

GAMA EACTOR FOR SHAPE CALCULATION = 1.00000033803

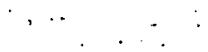

RHO STEP . STEP LENGTH

NO.

1

$$
\text { (SEC) }
$$

***APLIT:

NO. TEMP. CORE. ITER.

\begin{tabular}{|c|c|c|}
\hline$\cdot \dot{B}$ & $\begin{array}{l}\text { UPDATED } \\
\text { REACTY }\end{array}$ & VALUE \\
\hline N. $\operatorname{IIHE}(S \equiv C)$. & REACTIVITY (\$) - & 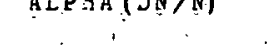 \\
\hline $\begin{array}{l}0.368838430-02 \\
0.409885120-0.5\end{array}$ & $\begin{array}{l}0.110158,8 D-03 \\
j .29866243 D-0.1\end{array}$ & $0.247 t 73900+01$ \\
\hline
\end{tabular}

A Y PLITUDE

$0.103006780+01$ 


\section{* F* SUMARY FOR SHAPE SIEP NJMEER}

2

STEP BEGAN AT 0.ட099867CD-02 SECONDS, ENDS AT $0.122993630-01$ SECONDS INIO TRANSIENT STEP REQUIREL 1 GAMMA FACTCB ITEKAIIONS AND 1 REACTZVIEY STEPS.

REACTOR PCAER LEVEL = $0.515187310+07$ WATTS

TOTAL ENESG? RELEASE $=\cdot 0.624177730+07$ WATT-SEC

COEPITIE: TIME SUMMARY

THERMAL SYYDRALICS

GENEKATE CROSS SECTIONS

COMPUTE AHPLITUDE PUNCION EQUATION COEFFECIENTS

SHAPE FUHCTION SOLUTIOH

SOLVE FOR A.IPLITUDE FTMCTION, HISC.

TOTAL FOR THIS STEP

TOTAL RLAPSEJ COMPUTING TIME (SEC) CENTRAL PROCESSOR (SEC)

$$
\begin{aligned}
& 0.0 \\
& 0.0 \\
& 0.13 \\
& 0.31 \\
& 0.62 \\
& 0.12 \\
& 1.13 \\
& 9.57
\end{aligned}
$$

PEX̃IPHERAL PROCESSOR (SEC) 


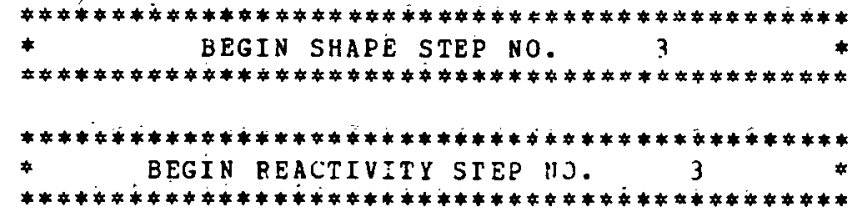

REACTIVITY STEP SELEZTIOUI TEST SUMAPY

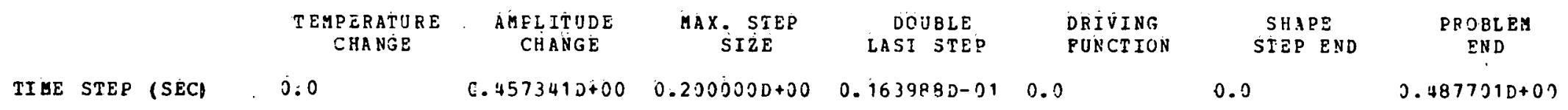

REACTIVITY STEP BEGINS AT 0.122994D-01 SEC, ENDS AT $3.236982 \mathrm{D}-01$ SEC, LENGTH = 0.163988D-J1 SEC

SHAFE STEP SELECTION TEST SIMMARY

\begin{tabular}{|c|c|c|c|c|c|}
\hline & $\begin{array}{c}\text { FLUX SHAPE } \\
\text { CHANGE }\end{array}$ & $\begin{array}{c}\text { MAX. STEP } \\
\text { SIZZ }\end{array}$ & $\begin{array}{r}10 \text { IIMES } \\
\text { LAST STEP }\end{array}$ & $\begin{array}{l}\text { DRIVING } \\
\text { FUNCTION }\end{array}$ & $\begin{array}{l}\text { PROELEM } \\
\text { END }\end{array}$ \\
\hline AE STEP (SEC) & $0.420176 \mathrm{D}+00$ & $0.5000000+20$ & $0.4099700+00$ & 0.9 & $0.4377010+00$ \\
\hline
\end{tabular}

S̈HAPE STEP BEGINS AT $0.1229940-01$ SEC, ENDS AT 0.422269D+DJ SEC, LENGTH = 0.4099.700+0' SEZ

THE FOLLONING DRIVING FUNCTIONS APPLY THIS REACTIVITI STEP

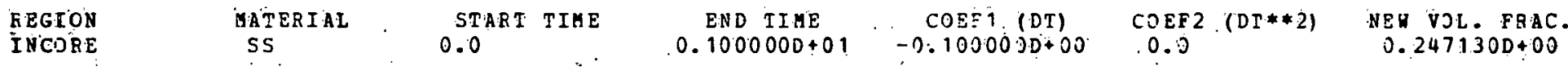

AMPLITUDE CHANGE OVER SHAPE STEP TERIINATES TRIS STEP

* * THIS SHAPE STEP HAS BEEN EEDUCEd IN LENGTH - TEgMINATES NOW **

NEN LENGTH $=0.163988 D-01 \mathrm{SEC}$, STEP END TIME $=0.286982 \mathrm{D}-01 \mathrm{SEC}$ 


\section{\# \# SUMMAFY FOR REACTIVITY STEP NUKBER $3 * 4 x$}

STEP BEGAN AT $0.1225936 .35-01$ SECOHDS, ENDS AT 0.28698154D-D1 SECONDS INTO TRASIENT

$\begin{array}{ll}\text { END-OF-STEP REACZTVITY } & =0.2559=236 \mathrm{D}-03 \\ \text { GENERATION TIME SECONDS) } & =0.40375107 \mathrm{D}-36\end{array}$ GENERATION TIME SECONDS)
A MPITUDE FUNCTISN DERIVAIIVE (DN) $=0.40375107 \mathrm{D}-36$
$=0.27339174 \mathrm{D}+31$

MAXIMUM FUEL TEYE. CHANGE (DEG.K) $=0.0$.

TOTAL REACTC
EEPEZTLVE DELAYED NEUTRON FRACTION = 0.36896876D-02 A.PLIT JDE FJNCTION VABUE (N) $=0.10739325 \mathrm{D}+01$ TOTAL ZNEBGY RELEASE INATT-SEC) $=0.14870432 \mathrm{D}+0 \mathrm{~B}$ MAXIMUS PUEL IEYPEFAZLRE (DEG.K) = 0.0

\section{$=0.53729164 D+C 9$}

CENTRAL PROCESSOR (SEC) PERIPHERAL PROEESSOE (SEC)

$$
0.39
$$

9.95

1. 28

$+1.27$

51.22
TOTAL POR THIS STEP
TOTAL ELADSED COMPUIING TIME (SEC) 


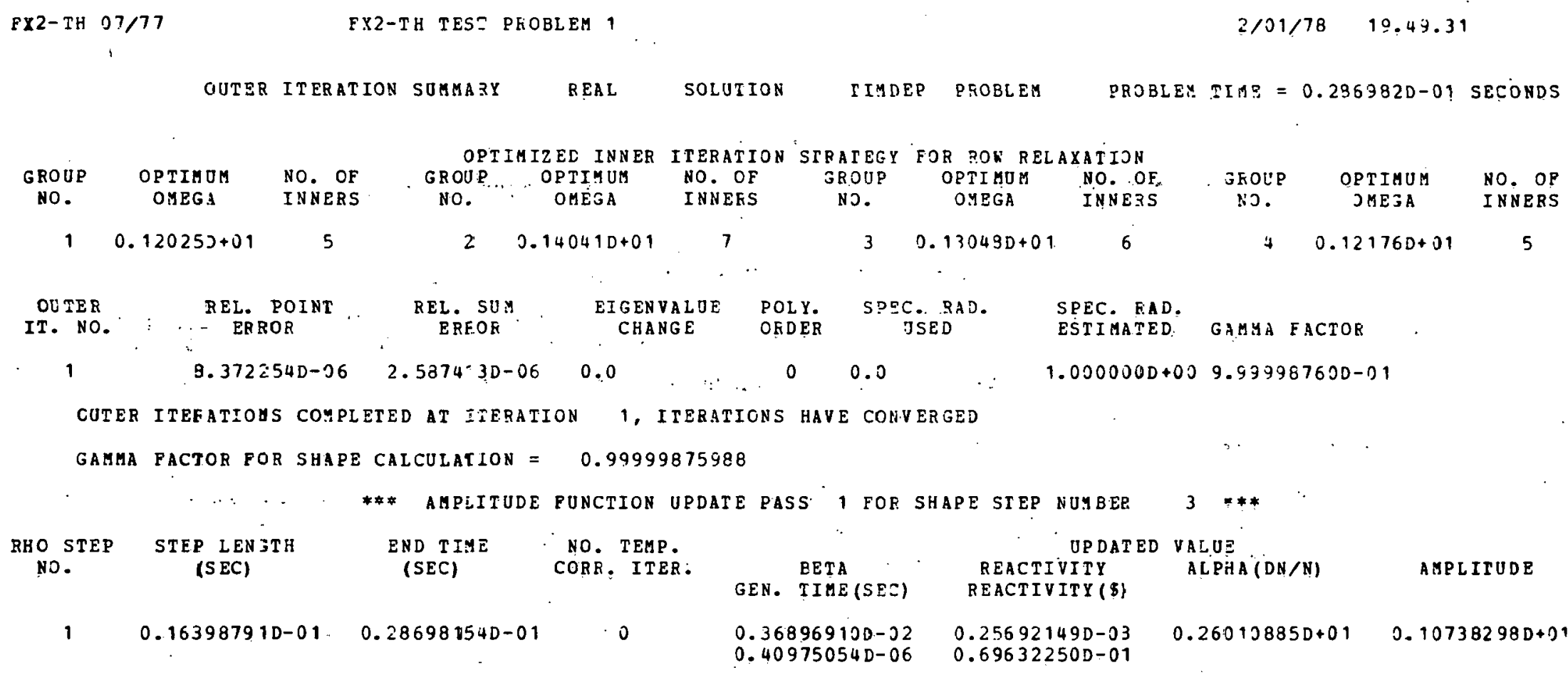

*** SUMHARY POR SHAPE STEP NUMBER, 3 \#**

STEP BEGAN AT 0.12299363D-01 SECONDS, ENDS AT 0.23699154D-01 SECONDS INTO TRANSIENT STEP REQUIRED 1 GaMHa FACTOR ITERATIONS AND 1 REACTIVITY STEPS.

REACTOR PORER LEVEI = $0.537290670+29$ HATTS

TOTAL ENERGY RELEASE $=0.148704300+08$ NATT-SEC

COMPJTING TIME SUMHARY * * * * * *NIRAL PROCESSOR" (SEC) * PERIPHERAL PROZESSOR (SEZ)

THERAAL, HYDRAJLI CS.

GERERATE CROSS SECTIONS

COHPOTE AMPLITUDE FUNCT
SHAPE FUNCTION SOLOTION

DQUATCN COEFFICIENTS

SOLVE FOR A HPLITUCE PUNCTION, MISC.

. 0.0

0.0
0.0

0.0
0.13

0.13
0.32

0.32
0.33

0.13

0.0

0.0

0.34
0.95

0.95

TOTAL FOR-THIS STEP

0.91 3.81

TOTAL ElaPSED COMPUTING TraE (SEC)

10,48

43.80

54.28 


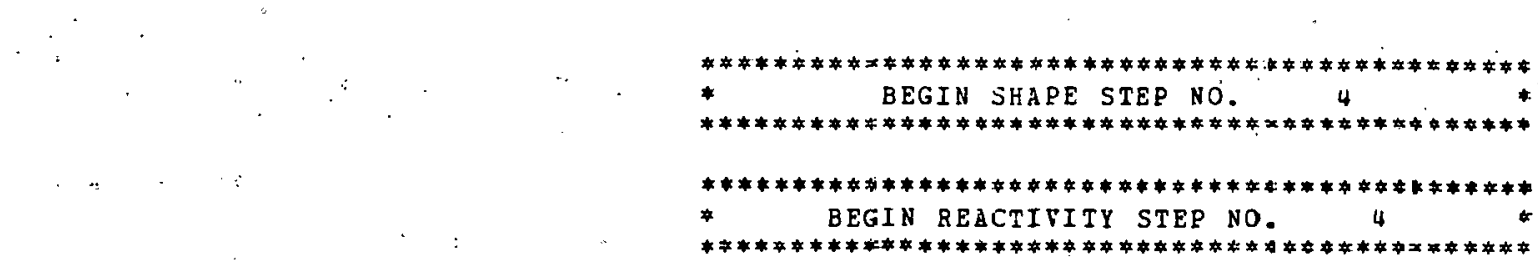

REACTIUITY STE? SELECIION TEST SUMMËRY

\begin{tabular}{|c|c|c|c|c|c|c|c|}
\hline & $\begin{array}{c}\text { IEUPERATUPE } \\
\text { CHANGE }\end{array}$ & $\begin{array}{l}\text { AMPLITUDE } \\
\text { CHANGE }\end{array}$ & $\begin{array}{c}\text { MAX: STEP } \\
\text { SIZE }\end{array}$ & $\begin{array}{l}\text { DOUBLE } \\
\text { LAST STEP }\end{array}$ & $\begin{array}{l}\text { DRIVIBG } \\
\text { FUNCT=ON }\end{array}$ & $\begin{array}{l}\text { SHAPE } \\
\text { SIEP END }\end{array}$ & $\begin{array}{c}\text { PROBLEM } \\
\text { END }\end{array}$ \\
\hline TIAE STEP (SEC) & $c .0$ & $0.4429790+00$. & $0.2000000+0.0$ & $0.32795 \Xi 0-01$ & 0.3 & 0.0 & $0.4713020+00$ \\
\hline
\end{tabular}

REACTIVITI STEP EEGINS AT 0.236982D-01 SEC, ENDS AT 0.614944D-01 SEC, IENGTH=0.327963D-01 SEC

SHAPE STEP SELECTION TEST SUMYARY

\begin{tabular}{|c|c|c|c|c|c|c|c|}
\hline & $\therefore$ & & $\begin{array}{l}\text { FLUX SEAPE } \\
\text { CHANGE }\end{array}$ & $\begin{array}{c}\text { MAX. STEP } \\
\text { SIZE }\end{array}$ & $\begin{array}{r}10 \text { TIIES } \\
\text { LAST STEP }\end{array}$ & $\begin{array}{l}\text { DRIVIUG } \\
\text { FINCTIDN }\end{array}$ & $\begin{array}{l}\text { PROBLEM } \\
\text { END }\end{array}$ \\
\hline I IME & STEP & (SEC) & $0.4218350+00$ & $0.5000000+00$ & $0.4099530+0 i$ & 0.3 & $0.471302 \mathrm{D}+00$ \\
\hline
\end{tabular}

“ SHAPE STEP BEGINS E.T $0.286982 \mathrm{D}-01$ SEC, ENDS AT $0.4505330+00$ SEC, LENGTH $=0.4218350+00$ SEZ

THE ROLLONING DRIVING FUNCTIONS APPLY THIS REACIIVITY STEP

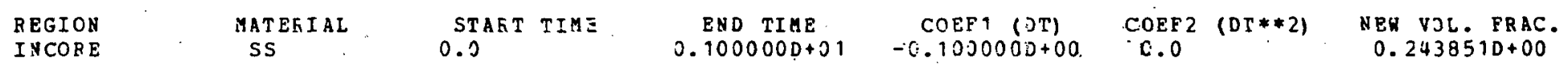

AMPLITLUD CHANGE OVER SHAPE STEP TERMINATES THIS STEP

*** THIS JHAPE STEP HAS BEEN FEDUCED IN IENGTH - TERAINATES TON ***

NEN LEMGTH $=0.327963$ D-01 SEC, SIEP END IIME $=0.6149440-1 \mathrm{SEC}$ 
STEP BEGAN AT 0.28698154D-01 SECONDS, ENDS AT 0.61494415D-01 SECONOS INIO TRANSIENT

\begin{abstract}
EXV-GF-STEP REACTIVITY
GENERATION TIME (STCONDS)

AYPLITUDE TINE (SECONDS)

MAXIAJUDE FIJNCTION DERIVATIVE (DN) $=0.33913540 \mathrm{D}+01$

MAXIMJU FUEL TEMP. CAANGE (DEG.K) $=0.0$
\end{abstract}

TOMAL REACTOK POWER (WATTS)

CENTRAL PEOZESSOR (SEC)

EFFFIIIVE TELAYED NEUTEON FRACTION

AMPLITJDE PINCTION VALUE (N)

TOTAL ENERGY RELEASE (HATT-SEZ)

$0.36923318 \mathrm{D}-02$ MAXIMUM F'IEL TEMPERATURE (DEG.K)

$0.333078670+08$

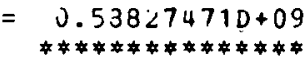

COMPUT ING TIME SIIMKA RY

TOTAL FOR THIS STEP

TOTAL ELAESED COMPUTING TIME (SEC)

$$
\begin{array}{r}
0.38 \\
10.86
\end{array}
$$

PERIPHEAAE PROCESSOR (SEC)

1.28

45.08

55.94 
OUTEE. ITFRATION SIMYARY PEAL SOIUTION TIMDZP PROBLEM PROBLEM TIME = 0.614944D-0.1 SECONDS

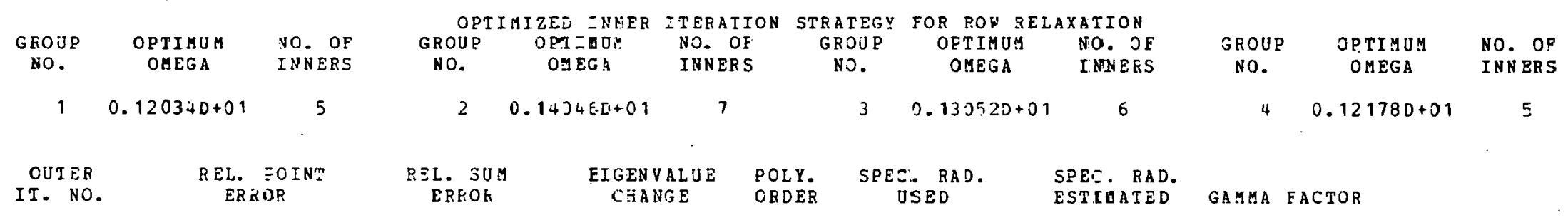

$2.2064660-05 \quad 5.8683490-C 6 \quad 0.0$

$0 \quad 0.0$

$1.0000000+009.999967640-01$

CUTEF ITERATIONS COMPLETED ET ITERATION 1, ITERATIONS HAVE ZONVZEGED

GAMMA PACTOR FOE SHAPE CALCEIATION $=0.99699676434$

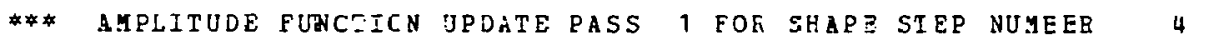

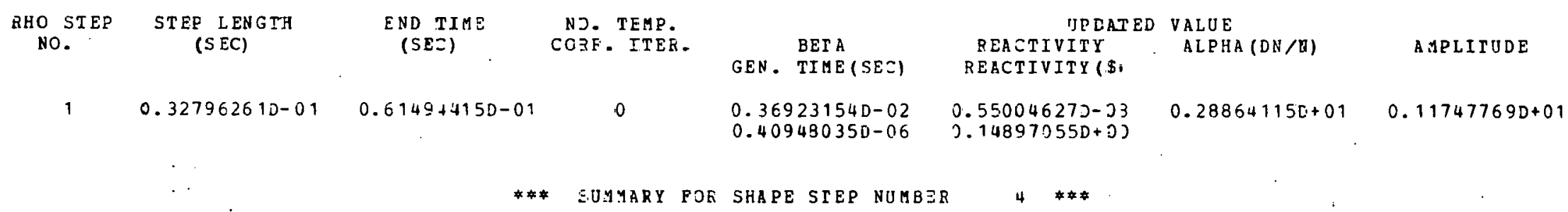

STEP BEGAN AT $0.2869815+2-01$ SECONES, ENDS AT $0.014944150-0^{\circ}$ SECONDS INTO TRANSIENT

STEP ERQUIRED 1 GaAEA FACTOR TTERATIONS AID 1 REACTIVITY STEPS.

REACTOR EOLER LEVEL = $0.58827150 D+09$ DATTS

TOTAL EMEEEY RELEASE $=0.3330784 E D+C 8$ WTT-SEC

COMPUTIN = TIME SUMMAKY

THERMAI. HYDRAULICS

GENERATE CROSS SECTIONS

COMPUTE AMPIITUDE FUNCTION EQUATICD COEFFICIENTS

SHAPE FUNCTION SOLOTIOA

SOLVE FOR AMPLITUDE FU UETION, MISC.

TOTAL FOR THIS STEP

\footnotetext{
tOTAL ELAPSE CCMPUTING IIME (SEC)
}

CENTRAL PROCESSCR (SEC)

PE?IPHERAL PROZESSOR (SEC)

s.

CENTAL. PROC

$\begin{array}{lcl}0.0 & 0.0 \\ 0.0 & 0.0 \\ 0.13 & 0.27 \\ 0.32 & 1.05 \\ 0.51 & 2.18 \\ 0.13 & 0.62 \\ 1.09 & 4.12 \\ 11.57 & + & \\ & & \\ & & \end{array}$




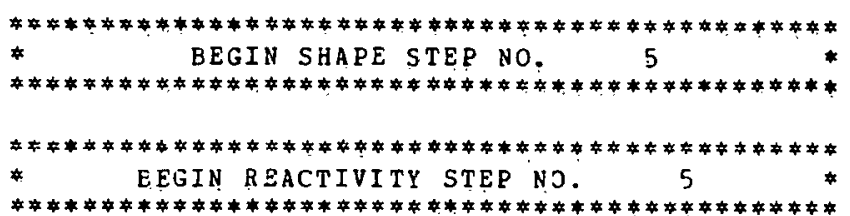

REACTIVITY STEP SELECTION IEST SIMMAPY

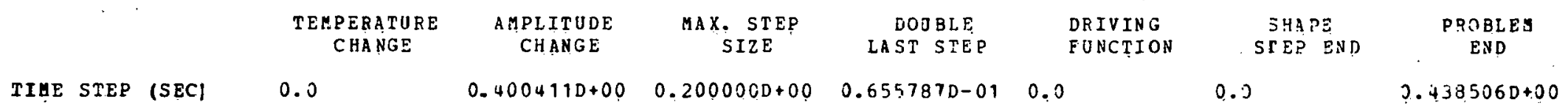

REACTIVITI STEP BEGINS AT 0.614944D-01 SEC, ENDS AT 0.127073D+00 SEC, LENGTH = 0.655787D-01 SEC

SHAPE STEP SEIECTION TEST SUMHARY

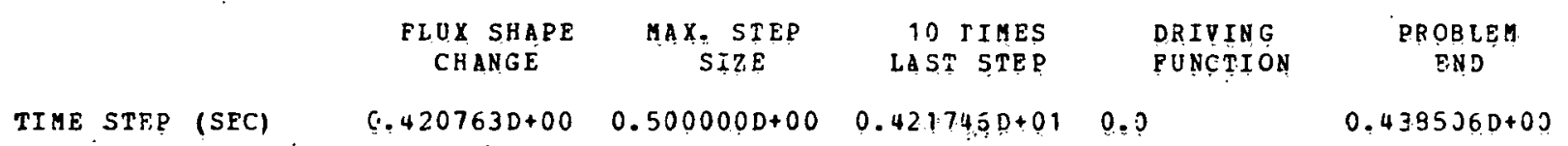

SHAPE STEP BEGINS AT $0.614944 \mathrm{D}-01$ SEC, ENDS AT $0.482257 \mathrm{D}+00$ SEC, LENGTH $=0.420763 \mathrm{D}+00 \mathrm{SEZ}$

THE FOLLONING DRIVING FUNCTIONS APPLY THIS REACTIVITY STEP

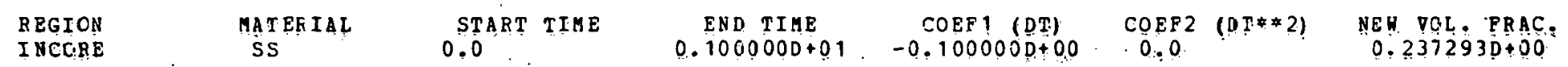

A APLITUDE CHANGE OVER SHAPE STEP TEYMMINATES THIS. STEE

*** THIS SHAPE STEP HAS BEEN REDUCED IN LENGTH - TERMINATES NOH ***

NEW LENGTH $=0.6557870-01 \mathrm{SEC}$, STEP END TISE $=0.1270730+00$ SEC 
*** SUMRARY FOR REACTIVITY SIEP NUGBER

STEP BEGAR AT C.61L.9441.5D-01 SECONDS,

END-OF-STEP REACTIVITY

GENERATION TIME (SECONDS)

AMPLITUDE FUNCTIDN DERIVAIIVE (DN) $=C .523227740 .01$

MAXIMUM. FOEL TERP. CHANGE (UBS.K) = 0.0

TOTAL REACTOE POWER (WATTS)
ENOS AT $0.127073150+00$ SECONDS INTO TRANSIENT

EFFECTIVE DEIAYED NEUTRON FRACTION = $0.369755280-02$ AMPLI?UDE FUNCIION VALUE (N) $=0.145283740+01$ YOTAL ENZRGY PELEASE (HATT-SEC) = $0.761820150+08$ MAXIMIY FIEL TEMPEKATURE (DEG.X)

$=0.3$

$$
=\quad 0.728677930+09
$$

CENTRAL PEOZESSOK (SEC)

0.41

11.99
PERIPHERAL PROCESSOR (SEC)

1.27
49.19

61.17
TOTAL FOR THIS STEP
TOTAL ELAPSED COMPUTIMI TIME (SEC!

$0 \cdot$




\begin{tabular}{|c|c|c|c|c|c|c|c|c|c|c|c|}
\hline & & & OP & I MIZED INNER & ITERATION & STRAIEGY & FOR POF REL & XATION & & & \\
\hline $\begin{array}{l}\text { GROUP } \\
\text { NO. }\end{array}$ & $\begin{array}{l}\text { OPTI HIJ } \\
\text { OHEGA }\end{array}$ & $\begin{array}{l}\text { NO. OF } \\
\text { INLERS }\end{array}$ & $\begin{array}{l}\text { GRCUP } \\
\text { NO. }\end{array}$ & $\begin{array}{l}\text { OFTIMUA } \\
\text { OMEGA }\end{array}$ & $\begin{array}{l}\text { NO. OE } \\
\text { INNEFS }\end{array}$ & $\begin{array}{c}\text { GROUP } \\
\text { NO. }\end{array}$ & $\begin{array}{l}\text { OPT I MUM } \\
\text { O:EGA }\end{array}$ & $\begin{array}{l}\text { NO. OF } \\
\text { INNERS }\end{array}$ & $\begin{array}{l}\text { ifOI! P } \\
\text { NO. }\end{array}$ & $\begin{array}{l}\text { OPI IMUM } \\
\text { OMEJA }\end{array}$ & $\begin{array}{l}\text { NO. OF } \\
\text { INNEPS }\end{array}$ \\
\hline 1 & $0.12034 D+01$ & 5 & 2 & $0.14046 D+01$ & 7 & 3 & $0.13 .5520+01$ & 6 & 4 & $0.12178 D+01$ & 5 \\
\hline
\end{tabular}

$\begin{array}{ccccccc}\text { OUTEK } & \text { REL. POINT } & \text { REL. SUM } & \text { EIGENVALUE } & \text { FOLY. } & \text { SPEC. RAD. } & \text { SPEC. EAD. } \\ \text { IT. NO. } & \text { ERROR } & \text { EREOR } & \text { CHANGE } & \text { OKDER } & \begin{array}{c}\text { OSED } \\ \text { ESTIMATED GAMM FAEIOF }\end{array} \\ 1 & 5.103032 D-05 & 9.2848310-06 & 0.0 & 0 & 0.0 & 1.0000000+009.99989674 D-01\end{array}$

OUTEE ITEFATIONS COMPLETED AT ETEKATION 1, ITERATIONS HAVE CONVEEGED

GAMMA FACTOR FOR STAPE CALCULATION $=0.99998967441$

** AMPETTUDE FUNCTION UPDATE PASS 1 FOR SHAPE STEP NOMBER 5 * *

\begin{tabular}{|c|c|c|c|c|c|c|c|}
\hline $\begin{array}{l}\text { RHO STËF } \\
\text { NO. }\end{array}$ & $\begin{array}{l}\text { STEP LEN } \\
\text { (SEC) }\end{array}$ & $\begin{array}{l}\text { END TIME } \\
\text { (SEC) }\end{array}$ & $\begin{array}{l}\text { NO. TEMP. } \\
\text { COEF. ITER: }\end{array}$ & $\begin{array}{ll} & \text { BETA } \\
\text { GEN. } & \text { TIME (SEC) }\end{array}$ & $\begin{array}{l}\text { UPDATED } \\
\text { REACTIVITY } \\
\text { REACTIVITY (\$) }\end{array}$ & $\begin{array}{l}\text { VALUE } \\
\text { ALPHA (DN/N) }\end{array}$ & AMPLIIJDE \\
\hline 1 & $0.65578739 D-01$ & $0.12707315 D+00$ & 0 & $\begin{array}{l}0.369760830-02 \\
0.408937010-06\end{array}$ & $\begin{array}{l}0.11346069 D-02 \\
0.30684886 D+00\end{array}$ & $0.36427226 D+01$ & $0.14523235 D+01$ \\
\hline
\end{tabular}

\section{* * SUMMARY POR SHAPE STEP NIMBER 5 ***}

STEP BEGAN ET $0.61494415 \mathrm{D}-01$ SECONDS, ENDS AT 0.12707315D+CJ SECCNDS INTO TRANSIENI

SIEP. RLQUIRED 1 GAUAA FACTOE ITERATIONS AND 1 REACTIVITY STEPS.

REACIOR POWEK LEVEL $=0.728677690+09$ WATTS

TCTAL ENEKGY RELEASE $=0.761822530+08$ WATT-SEC

\section{COMPUTING TIME SUMMARY}

\section{THERMAL HYDRAOLICS}

GEHERATE CROSS SECTIONS

COBPUTE AMPLITUDE FUNCTION EQDATION COEFFICIENTS

SHAPE PONCTION SOLJTION

SOLVE FOE AMPLITUDE FUNCTION, MISC.

TOTAI FOR THIS STEP

TOT:AL ELAPSED COMPUTING TIME (SEC)
CENTRAL PROZESSOR (SEC)

PERIPHERAL PROCESSCE (SEC)

$\begin{array}{ll}0.0 & 0.0 \\ 0.0 & 0.0 \\ 0.13 & 0.24 \\ 0.32 & 1.00 \\ 0.33 & 1.83 \\ 0.16 & 0.67 \\ 0.94 & 3.74\end{array}$

12.51

51.66

64.17 


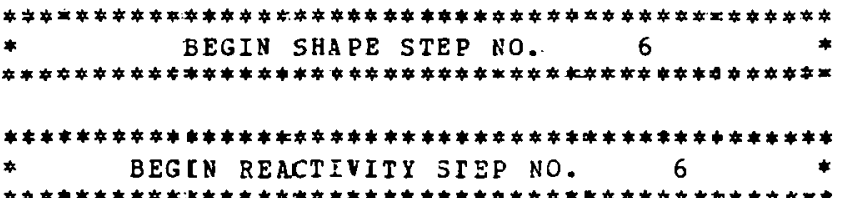

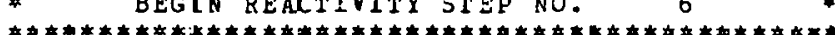

REACTIPITY STEP SELEETION TEST SUMMART

DEMPERATURE

$\begin{array}{lc}\text { AMFLITUDE } & \text { MAX. STEF } \\ \text { CHANGE } & S=2 E\end{array}$

$\begin{array}{cl}\text { DOIBBLE } & \text { DRIVING } \\ \text { LAST STEP } & \text { EUNCTION }\end{array}$

SHAPE
STEP END

PROBLEM

CERATURE

$3214200+00 \quad 0.2000 C 0 D+00 \quad 0.130851[+00 \quad 0.0$

0.0

$3.372927 D+00$

TIME STEF (SEC)

0.0

$0.127073 D+00 \mathrm{SEC}$, ENDS

$0.257924 \mathrm{D}+00$ SEC, LENGTH $=0.1308510+00 \mathrm{SEC}$

SHAPE STEP SEIECTION IESI JUMMARY

$\begin{array}{ccccc}\text { PIUX SHAPE } & \text { MAX. STEP } & \text { 10 IIHES } & \text { DRIVING } & \text { PROBIEM } \\ \text { CHANGE } & \text { SIZE } & \text { LAST STEP } & \text { FJNCTIOI } & \text {. END }\end{array}$

TIME STE? (SEC)

$0.417665 D+00 \quad 0.500003 D+00 \quad 0.419779 D-01 \quad 0.0$

$0.3729270+00$

SHAPE STEE BEGINS AT $0.127073 D+00$ SEC, ZNES AT $0.500000 D+O Q$ SEZ, LENGIH $=0.372927 D+00$ SEC

THE FOLIORING DRIVING FUUCIIONS APPLY THIS REACTIVITY STEP

BMPLTTIDE CHANGE OVER SBAPE STEP TEKMINATES THJS STEP 
STEP BEGAN AT $0.127073150+00$ SECONDS, ENDS AT $0.25792416 D+00$ SECONDS INTO TRANSIENT

$\begin{array}{ll}\text { END-UF-STEP REACPIVITY } & =0.22942095 \mathrm{D}-02 \\ \text { GENERATION TIME SECONDS) } & =0.407857190-06\end{array}$

A AEIITUDE FUNCTION DERIVAIIVE (DN) $=0.19354763 \mathrm{D}+02$

MAXIMUM FUEL TEM?. CHANGE (DEG.K! $=0.0$

TOTAL REACTOR POWER (WATTS)
COMPUTING TIME SOMMARY

TOTAL FOR THIS STEP

TOTAL ELAPSED COMPUTING TIME (SEC)
CENTRAL PROZFSSRR (SEC)

$$
0.44
$$

0.44

12.95
PERIPHERAL PROCESSOR (SEC)

EFFECTIVE DELAYED NEUTRON FEACT:ON $=0.37081274 D-02$ AMPLIJDE FUNCTION VALTE (N) $=0.278570830+01$ TOTAL ENERSY RELEASE (KATT-SEC) $=0.23636508 D+09$ MAXIMUM FUEL TEMPERATURE (DEG.K: = 0.3

$$
=0.14016901 \mathrm{D}+10
$$

1.32

65.93 
OUTEF: ITERATION SUMMARY REAL SOLUTION TIMDZP FROELEM PROBLEM TIME = 0.257924D+OO SECONDS

\begin{tabular}{|c|c|c|c|c|c|c|c|c|c|c|c|}
\hline & & & ORT & I IIZED I ANER & IT ERATION & STRAIEGY & FOR ROF PEL & XITION & & & \\
\hline $\begin{array}{l}\text { GROUP } \\
\text { NO. }\end{array}$ & $\begin{array}{l}\text { ORTIBUM } \\
\text { OHEGA }\end{array}$ & $\begin{array}{l}\text { NC. OF } \\
\text { INNE RS }\end{array}$ & $\begin{array}{l}\text { GROUP } \\
\text { NO. }\end{array}$ & $\begin{array}{l}\text { DPTIMOR } \\
\text { OMEGA }\end{array}$ & $\begin{array}{l}\text { NO. OF } \\
\text { IUN ERS }\end{array}$ & $\begin{array}{l}\text { GROUP } \\
\text { NO. }\end{array}$ & $\begin{array}{l}\text { OPTE BU Y } \\
\text { OYEGA }\end{array}$ & $\begin{array}{l}\text { NO, OF } \\
\text { INWERS }\end{array}$ & $\begin{array}{l}\text { GROUP } \\
\text { NO. }\end{array}$ & $\begin{array}{l}\text { OP TIMUM } \\
\text { OKEGA }\end{array}$ & $\begin{array}{l}\text { NO. O! } \\
\text { INNER }\end{array}$ \\
\hline 1 & $0.12034 \mathrm{D}+01$ & 5 & 2 & $0.14046[1+01$ & 7 & 3 & $0.1310520+01$ & 6 & 4 & $0.121790+01$ & 5 \\
\hline
\end{tabular}

\begin{tabular}{|c|c|c|c|c|c|c|c|}
\hline $\begin{array}{l}\text { OUTER } \\
\text { IT. NO. }\end{array}$ & 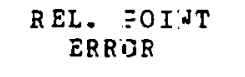 & $\begin{array}{l}\text { REL. SUM } \\
\text { E RROE }\end{array}$ & $\begin{array}{l}\text { EIGENVALUE } \\
\text { CHANGE }\end{array}$ & $\begin{array}{l}\text { POLY. } \\
\text { ORDER }\end{array}$ & $\begin{array}{c}\text { SFEC. PAD. } \\
\text { USED }\end{array}$ & $\begin{array}{l}\text { SPEC. RAD. } \\
\text { ESTIYATED }\end{array}$ & GAMMA PACTOA \\
\hline 1 & $1.986362 \mathrm{D}-34$ & $4.9402470-0.5$ & 0.0 & 0 & 0.0 & $1.0 .000000+00$ & $9.999509010-01$ \\
\hline 2 & $3.5549350-05$ & $3.540226 \mathrm{D}-05$ & 0.0 & 0 & 0.0 & 7. $166110 \mathrm{D}-01$ & $9.999999820-01$ \\
\hline 3 & $1.57141 .50-05$ & 4. $912283 D-05$ & 0.0 & 0 & 0.0 & $1 . \Xi 87584 \mathrm{D}-01$ & $1.00000178 D+00$ \\
\hline
\end{tabular}

OUTER ITERATIONS CCMPLETED AT ITERACION 3, ITERATIONS HAVE CONVERGED

GAMMA PACTC.R FOR SHAPE CALCULATION $=1.00000177690$

* * amplitude function tpdate pass 1 for shape step nugBer 6 ***

\begin{tabular}{|c|c|c|c|c|c|c|c|}
\hline $\begin{array}{l}\text { RHO STEP } \\
\text { NO. }\end{array}$ & $\begin{array}{l}\text { STEP LENGTH } \\
(\mathrm{SEC})\end{array}$ & $\begin{array}{l}\text { END TIME } \\
\text { (SEC) }\end{array}$ & $\begin{array}{l}\text { NO. TEMP. } \\
\text { CCRR. ITER. }\end{array}$ & $\begin{array}{l}\text { BEIA } \\
\text { GEN. TIME (SEC) }\end{array}$ & $\begin{array}{l}\text { OPDAIE } \\
\text { PEACTIVIEY } \\
\text { REACTIVITI(\$) }\end{array}$ & $\begin{array}{l}\text { VALUE } \\
\text { ALPHA }(D N / N)\end{array}$ & A MPL ITODE \\
\hline 1 & C. $130851000+00$ & $0.25792416 D+00$ & 0 & $\begin{array}{l}0.37083536 \mathrm{D}-02 \\
0.40784271 \mathrm{D}-06\end{array}$ & $\begin{array}{l}0.22948368 \mathrm{u}-02 \\
0.61882900 \mathrm{u}+00\end{array}$ & $0.69530252 D+01$ & $0.27864477 D+01$ \\
\hline
\end{tabular}


* * SUMMary For SHape STEP NUMBE?

$6 \$$ \#

STEP BEGAR AT $0.12707315 D+00$ SECONDS, ENDS AT $0.257924160+00$ SECONLS INTO TEANSIENI SIEP REQJIRED 1 GAMMA FACTOF ITERATIONS AND 1 REACTIVITY STEPS.

FEACTOP. POKER LEVEL $=0.140212530+13$ WATTS

TOTAL ENEPGY RELEASE $=0.20639370 \mathrm{D}+09$ NATT-SEC

COMPUI ING TIME SUMAARY

THERMAL HYDRAJIICS

GENERAT: CROSS SECTIONS

COMPUTE AMPLITUDE FUNCTION EQUATION COEFFICIENTS

SHAPE FINCTION SOLUMION

SOLVE FOE AMPLITUDE FUNCTION, MISC.

TOTAL FIR THIS STEP

TOTAL ELAPSED COMPUIING TI YE (SEC)
CENTEAL PEORESSRR (SEC)

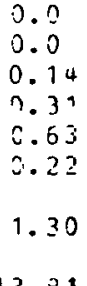

13.21
PEFIPHERA: PROCESSOR (SEC) 


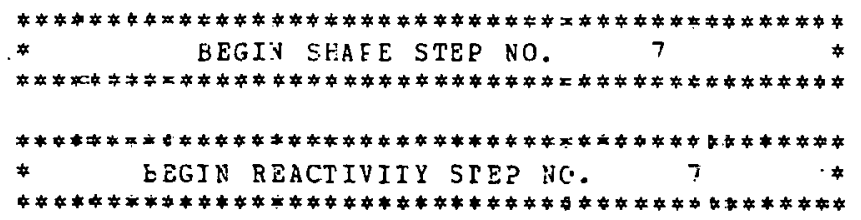

BEFCTIVITY STEP SELECTION TEST SUMBAPY

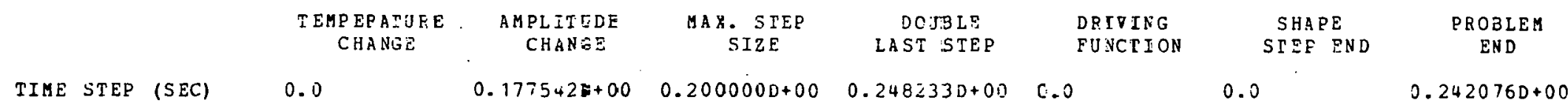

REACTIVIIY STEP BEGINS AT $=0.25 .7924$ D+00 SEC, ENDS AT 0.435466D+30 SEC, LENGTH = 0.177542D+J0 SE

SHAPE STEP SELECTION TESI SUMMARY

\begin{tabular}{|c|c|c|c|c|c|}
\hline & $\begin{array}{c}\text { FLUX SHLP } \\
\text { CHANGE }\end{array}$ & $\begin{array}{c}\text { MAX. STEP } \\
\text { JIZE }\end{array}$ & $\begin{array}{r}10 \mathrm{I}=\mathrm{MES} \\
\mathrm{L} \text { AS } \mathrm{STEP}\end{array}$ & $\begin{array}{l}\text { DRIVIN } \\
\text { FUNCII ON }\end{array}$ & $\begin{array}{l}\text { PROBLEM } \\
\text { END }\end{array}$ \\
\hline ME STEP (SEC) & $0.3931210+00$ & $0.500000 D+00$ & $0.39616 ! D+01$ & C. . $)$ & $0.2420760+00$ \\
\hline
\end{tabular}

SHAPE STEP BEGINS AT $0.2575240+00$ SEC, ENDS AT $0.5006000+1005 \mathrm{SC}, \mathrm{LENGTH}=0.2420760+03 \mathrm{SEZ}$

THE EOLLOWING ERIVING EUNCIIONS APPLY THIS REACIIVITI STEP

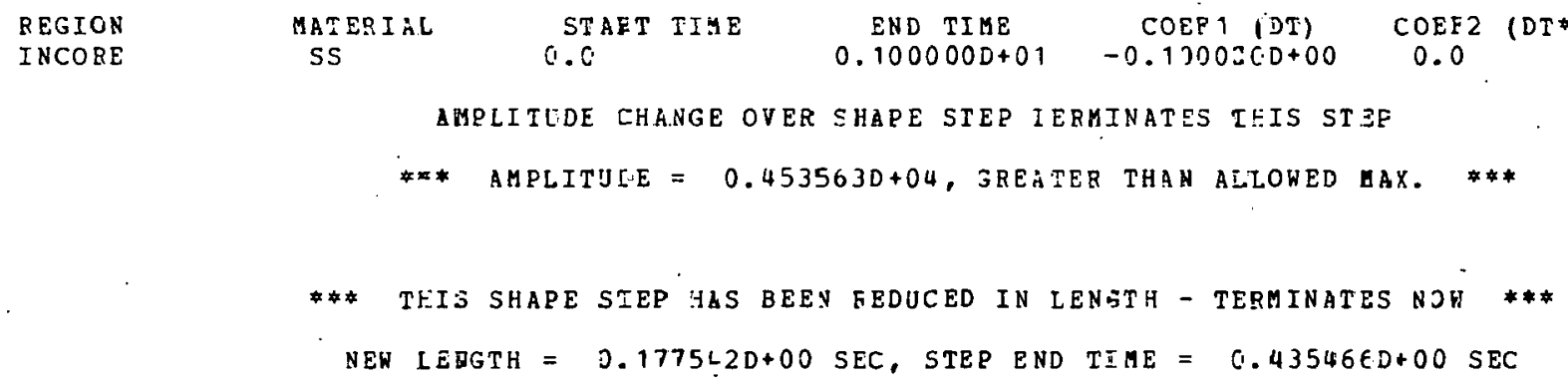

** Calculatiog vill be terminated before regdesazd time *** 
* * SumMafi for reactiviti SIEP number 7 \#

STEP BEGAN AT $0.257924160+00$ SECONDS, ENDS AT $0.435465960+00$ SECONDS INTO TRANSIENT

\begin{abstract}
END-OP-STEP REACTIVITT
GENERATION TIME ISECONDSI $\quad=0.40636727 \mathrm{D}-06$

GMPLITUDE FUNCTION DERIVATIVE (DN) $=0.15592132 D+0$ ?

DAXIMUM FUEL TEME. CHANGE (DEG.K) $=0.0$
\end{abstract}

TOTAL REACTOR POWER

COMPUTENG TIME SUMMARY

TOTAL FOR THIS STEP

TOTAI ELAPSED COHPUIING TIME (SEC)
EREECTIVE DELAYED NEOTRON FRACRTON =

EFFECTIVE DELAYED NETTRON PRACTION $=0.37228415 \mathrm{D}-02$

AMPLITUDE ENCTION VALUE (N) $=0.45356290 \mathrm{D}+0$

TOTAL ENERGY RELEASE (WATT-SEC) $=0.928319150+1$

MAXIMUM FUEL TEMPERATURE (DEG.K) $=0.0$

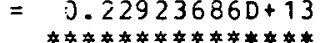

CENTRAL PROCESSOR (SEC) PERIPHERAL PROZESSOR (SEC)

0.81
14.62

73.45 


\begin{tabular}{|c|c|c|c|c|c|c|c|c|c|c|c|}
\hline & & & $O P$ & IUIZED INHER & ITERATIJN & STRATEJY & FOS ROW BEL & MATION & & & \\
\hline $\begin{array}{l}\text { GROUP } \\
\text { NO. }\end{array}$ & $\begin{array}{l}\text { OPTIQUM } \\
\text { OMEGA }\end{array}$ & $\begin{array}{l}\text { NO. OF } \\
\text { IN NERS }\end{array}$ & $\begin{array}{c}\text { GFOU P } \\
\text { NO. }\end{array}$ & $\begin{array}{c}\text { OPEIAUA } \\
\text { OAEGA }\end{array}$ & $\begin{array}{l}\text { NG. OF } \\
\text { INNERS }\end{array}$ & $\begin{array}{c}\text { GROUP } \\
\text { NO. }\end{array}$ & $\begin{array}{l}\text { OP I I ME } \\
\text { OMEG? }\end{array}$ & $\begin{array}{l}\text { OD. OF } \\
\text { INMERS }\end{array}$ & $\begin{array}{c}\text { GROUIP } \\
\text { NO. }\end{array}$ & $\begin{array}{l}\text { OPTIMUM } \\
\text { OMEGA }\end{array}$ & $\begin{array}{l}\text { NO. OF } \\
\text { INNERS }\end{array}$ \\
\hline 1 & $0.12103 D+01$ & 5 & 2 & $0.14685 D+01$ & 8 & 3 & $0.133900+01$ & 6 & 4 & $0.12182 D+01$ & 3 \\
\hline
\end{tabular}

\begin{tabular}{|c|c|c|c|c|c|c|c|}
\hline $\begin{array}{l}\text { OUTER } \\
\text { IT. NO. }\end{array}$ & $\begin{array}{l}\text { REL. PDINT } \\
\text { ERROS }\end{array}$ & $\begin{array}{l}\text { REL. SUM } \\
\text { ERROR }\end{array}$ & $\begin{array}{c}\text { EIGZNVALUE } \\
\text { CBANGE }\end{array}$ & $\begin{array}{l}\text { POLY. } \\
\text { ORDER }\end{array}$ & $\begin{array}{c}\text { SPEC. RAD. } \\
\text { OSED }\end{array}$ & $\begin{array}{l}\text { SEEC. RAD. } \\
\text { ESTIDATED }\end{array}$ & GAM A FACTOR \\
\hline 1 & $4.163393 D-04$ & 1. $238922 \mathrm{D}-04$ & 0.0 & 0 & 0.0 & $1.0000000+00$ & $9.9977503 .40-01$ \\
\hline 2 & 6. $162838 \mathrm{D}-05$ & 2. $10.5 \div 72 \mathrm{D}-04$ & 0.0 & 0 & 0.0 & $1.6993070+00$ & $9.99993781 \mathrm{D}-01$ \\
\hline 3 & $4.612425 D-05$ & $1.1153430-05$ & 0.0 & 0 & 0.0 & $5.29866 .5 \mathrm{D}-02$ & $1.000005460+00$ \\
\hline 4 & $1.77703 \leq D-35$ & $9.660 \Xi 31 D-06$ & 3.0 & 0 & 0.0 & $B: 651285 D-01$ & $1.000002320+00$ \\
\hline
\end{tabular}

OUTER ITERATIONS COMPLETED AT IIERATION 4 , ITERATIONS HAVE CONVERGED

GAMMA FACTOR FOR SHAPE CALCULATION = 1.00000232213

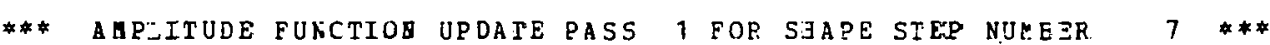

\begin{tabular}{|c|c|c|c|c|c|c|c|}
\hline $\begin{array}{l}\text { RHO STEP } \\
\text { NO. }\end{array}$ & $\begin{array}{c}\text { STEP LENGTF } \\
\text { (SEC) }\end{array}$ & $\begin{array}{l}\text { END IIIE } \\
\text { (SEC) }\end{array}$ & $\begin{array}{l}\text { NC. TEIIP. } \\
\text { CJER. ITER. }\end{array}$ & $\begin{array}{ll} & \text { EETA } \\
\text { GEN. } & \operatorname{TIME}(S E Z)\end{array}$ & $\begin{array}{l}\text { UPDATED } \\
\text { EEACTIVITY } \\
\text { BEACTEVIT (\$) }\end{array}$ & $\begin{array}{l}\text { VALUE } \\
\text { ALPHA (ON/N) }\end{array}$ & AMPLITUDE \\
\hline 1 & $0.177541800+00$ & $0.435465 \subseteq 60+00$ & 0 & $\begin{array}{l}0.372349620-02 \\
0.40627733 D-06\end{array}$ & $\begin{array}{r}0.385533438 D-.02 \\
0.10355439 D+.01\end{array}$ & $0.34684993 D+03$ & $0.47704425 D+04$ \\
\hline
\end{tabular}




\section{* * SuMMary for Shape step nitgar $\quad 7$ * * \\ STEP BEGAN AT $0.257924160+00$ SECONDS, ENDS AT $0.435465960+00$ SECONDS INTO TRANSIENT} STEP REGUIEED 1 GAMAA FACTOF ITERATIONS AND 1 REACTIVITY STEPS.

BEACTOR POWER LEVEI = $0.24116234 \mathrm{D}+13$ VATTS

TOTAL ENERGY RELEASE = $0.962096830+10$ FATI-SEC

\section{COMPUT ING TIME STMNAE}

\section{THERYAL HYDRAUIICS}

GENEEATZ CROSS SECTIONS

COBPUTE AMPLITLIDF FENCTION EQUATION COEFFICIENTS

SHAPE FINCTION SOLOTION

SOLVE FOE AMPLITUDE PUNCTION, AISC.

TOTAL FOR THIS STEP

TOTAL ELAPSED COMPUTING TIME (SEC)
CENTFAL PROZESSOR (SEC)

PEEIPHERAL PFOCESSOR (SEC)

$$
\begin{aligned}
& 0.0 \\
& 0.0 \\
& 0.13 \\
& 0.31 \\
& 0.95 \\
& 0.95 \\
& 2.37
\end{aligned}
$$

16.18

0.0
0.0
0.28
1.17
4.29
0.58
6.32

63.71 
EEAC. AMPL. GAMMA STEP ITER. IZER.

$\begin{array}{llll}* & 1 & 1 & 1 \\ * & 2 & 1 & 1 \\ * & 3 & 1 & 1 \\ * & 4 & 1 & 1 \\ * & 5 & 1 & 1 \\ \text { * } & 6 & 1 & 1 \\ \text { * } & 7 & 1 & 1\end{array}$

\section{TIME (SEC) REAL. STEP \\ ENGTH (SEC)}

4. $09987 D-03$

$1.229940-02$

2. $36982 \mathrm{D}-92$

6. $14944 \mathrm{D}-02$

1. $27673[-01$

2. $579245-01$

4. $354660-01$
4. $399870-013$

$8.199500-03$

1. $6.3988 \mathrm{D}-02$

3. $279630-02$

$6.557870-02$

1. 308510-0

1. $77542 \mathrm{D}-01$
REACTIVITY (DOLLARS)

$9.95938 \mathrm{D}-03$

$2.93662 \mathrm{D}-02$

$6.95322 \mathrm{D}-02$

1. $485710-01$

$3.065490-01$

$6.18 E 290-01$

$1.055540+00$
MAX. FIEL TEMP. (K) DELTA TEME. (K)

YAX. FUEL

0.0

0.0

0.0

0.0

0.0

0.0

0.0

0.0
TOTAL ENERGY (WATT-SEC)

$2.059730+06$

$5.24179 D+06$

$1.4870+0+07$

$3.33073 D+07$

$7.618230+07$

$2.0638+D+O B$

$9.62017 \mathrm{D}+09$
AMPLITJDE POHESTIRL

1. $30962 D+00 \quad 5.94362 D+08$

$1.05007 D+00 \quad 5.151930+09$

$1.373930+00 \quad 5.372910+08$

1. $17478 D+00 \quad 5.38272 D+08$

$1.452820+00$

$2.78645 \mathrm{D}+00$

$4.7704+D+03$ 
Distribution for ANL-78-97

Interna1:
J. A. Kyger
R. Avery
P. Kier
R. Lell
J. Ulrich
P. B. Abramson
C. H. Adams
C. Baker
W. P. Barthold
P. Bertoncini
C. Boley
L. L. Briggs
J. Brooks
J. Cahalan
Y. I. Chang
T. A. Daly
J. Deen
K. Derstine
D. Ehst
K. Evans
D. R. Ferguson
E. Fujita
P. Garner
M. Y. A. Gohar
L. J. Milton
Y. Orechwa
G. Palmiotti
E. M. Pennington

P. J. Persiani

P. Pizzica

B. Reynolds

R. R. Rudolph

D. Shaftman

J. Sienicki

R. A. Shober (30)

C. G. Stenberg

W. J. Sturm

S. F. Su

C. E. Ti11

B. J. Toppe1

R. Turski

D. Weber

W. Woodruff

R. J. Armani

E. F. Bennett

S. K. Bhattacharyya

M. M. Bretscher

R. G. Bucher

C. E. Cohn

R. J. Cornella

A. S. Cox

L. R. Dates

G. J. Dilorio

R. W. Doering

A. F. Engfer

R. E. Grajek

E. F. Groh

P. T. Guenther

C. Herzenberg

L. G. LeSage

F. E. LeVert

G. Lowe

F. H. Martens

R. D. McKnight

J. W. Meadows

P. A. Moldauer

J. Morman

N. O'Fallon

I. K. O1son

W. P. Poenitz

R. B. Pond

K. B. Porges

W. R. Robinson

G. K. Rusch

R. W. Schaefer

R. A. Scharping

A. B. Smith

D. L. Smith

D. M. Smith

D. C. Wade

J. F. Whalen

B. Yarlagadda

T. J. Yule

J. Matos

J. Snelgrove

A. Travelli

P. I. Amundson

C. L. Beck

S. G. Carpenter

P. Collins

G. A. Ducat

J. M. Gasidlo

R. W. Goin

G. Grasseschi

R. Kaiser

J. M. Larson

M. J. Lineberry

D. W. Maddison

P. B. McCarthy

H. F. McFarlane

D. N. Olsen

D. E. Puckett

F. W. Thalgott

Nationa1 Energy

Software Center (20)

. B. Krisciunas

ANL Contract File

ANL Libraries (5)

TIS Files (6) 


\section{External:}

DOE-TIC, for distribution per UC-79d (264)

Manager, Chicago Operations office Chief, Office of Patent Counsel, $\mathrm{CH}$ Director, Reactor Programs Div., $\mathrm{CH}$ Director, CH-INEL Director, DOE-RRT (2)

President, Argonne Universities Association Applied Physics Division Review Committee:

P. W. Dickson, Jr., Westinghouse Electric Corp.

R. L. Hellens, Combustion Engineering, Inc.

K. D. Lathrop, Los Alamos Scientific Lab.

W. B. Lowenstein, Electric Power Research Inst.

R. F. Redmond, Ohio State U.

R. Sher, Stanford U.

D. B. Wehmeyer, Detroit Fidisnn Co. 